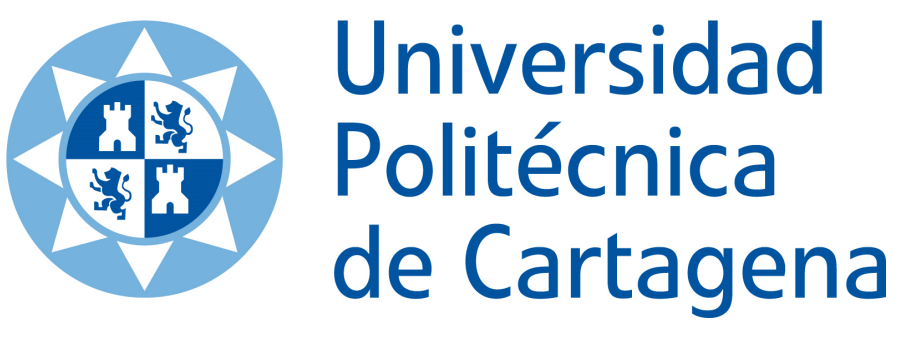

UNIVERSIDAD POLITÉCNICA DE CARTAGENA DEPARTAMENTO DE INGENIERÍA CIVIL

\title{
MODELOS DE COMPORTAMIENTO SECCIONAL DE HORMIGÓN ARMADO AJUSTADOS A ENSAYOS MEDIANTE ALGORITMOS METAHEURÍSTICOS
}

Andrés Tomás López López 



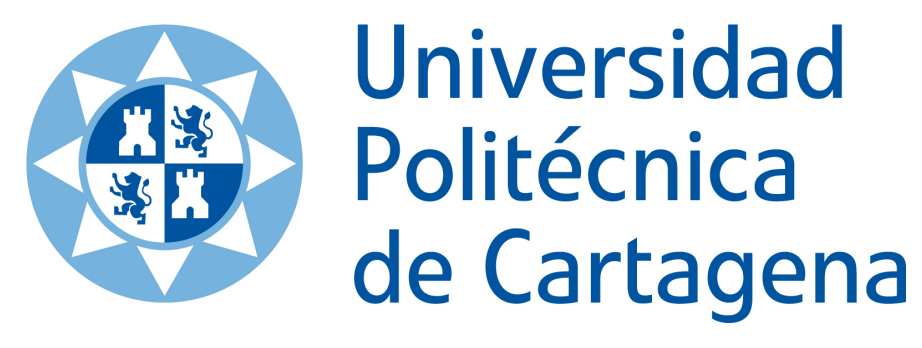

UNIVERSIDAD POLITÉCNICA DE CARTAGENA DEPARTAMENTO DE INGENIERÍA CIVIL

PROGRAMA DE DOCTORADO

MEDIO AMBIENTE Y MINERÍA SOSTENIBLE

\section{MODELOS DE COMPORTAMIENTO SECCIONAL DE HORMIGÓN ARMADO AJUSTADOS A ENSAYOS MEDIANTE ALGORITMOS METAHEURÍSTICOS}

TESIS DOCTORAL

Presentada por: D. Andrés Tomás López López

Dirigida por: Dr. Antonio Tomás Espín 



\section{Abstract}

Modelling concrete structures requires the implementation of numerical models capable of reproducing the nonlinearity of this problem adequately. In particular, the seismic behaviour of these structures requires the application of different types of models, such as models of sectional behaviour, hysteresis models and global models, being the interaction between them essential to simulate the problem in an integral way.

Seismic analysis of structures is a really time-consuming process, so that the models involved need to be accurate and efficient from the point of view of computing time. Therefore, it is important to obtain simple expressions that adequately reproduce the yield and ultimate behaviour of reinforced concrete sections, which is necessary to define the properties of the plastic hinges generated in the structure when acting the seismic load.

Obtaining these expressions involves proper calibration with experimental tests. In particular, if these expressions must be calibrated by an extensive database, specific numerical procedures are required, such as those based on optimization techniques, in order to achieve an optimum adjustment between the values offered by the expressions and the experimental results.

In this thesis, a database composed by more than 1000 experimental tests has been analysed and a set of tests is selected such that meet with seismic and construction requirements listed in the main construction codes; several empirical expressions relating to yielding and ultimate state of rectangular reinforced concrete sections have been calibrated; and the expressions obtained are implemented in the nonlinear analysis of several reinforced concrete frames, comparing the results obtained with different methods. 



\section{Resumen}

La modelización de estructuras de hormigón armado requiere el empleo de modelos numéricos capaces de reproducir convenientemente la no linealidad del problema. Concretamente, la obtención del comportamiento de estas estructuras frente a la acción sísmica exige la implementación de distintos tipos de modelos, como los modelos de comportamiento seccional, los modelos locales y los modelos globales, cuya interacción permite simular el problema de forma integral.

La modelización de estructuras en el análisis sísmico resulta especialmente exigente desde el punto de vista computacional, pues requiere el empleo de modelos que ofrezcan una adecuada precisión, pero con un coste computacional asumible. Resulta conveniente, por tanto, el desarrollo de expresiones sencillas que reproduzcan adecuadamente conceptos relativos a los estados de plastificación y último de secciones de hormigón armado, necesarios para caracterizar el comportamiento de las rótulas plásticas que se generan en la estructura al actuar la acción sísmica.

El desarrollo de este tipo de expresiones requiere una adecuada calibración con ensayos experimentales. En particular, si las bases de ensayos con las que se calibran son extensas, resulta necesario el empleo de métodos numéricos, como los basados en técnicas de optimización, para conseguir el mejor ajuste posible entre los resultados ofrecidos por las expresiones y los datos experimentales.

En esta tesis se ha analizado una base de datos compuesta por más de 1000 ensayos experimentales, obteniéndose a partir de ella una selección de ensayos que cumplan las prescripciones sísmico-constructivas impuestas por las principales normas y códigos de construcción; se han calibrado diversas expresiones empíricas que reproducen conceptos relativos a los estados de plastificación y último de secciones rectangulares de hormigón armado; y se han aplicado las expresiones obtenidas al análisis no lineal de pórticos planos de hormigón armado, contrastando los resultados obtenidos con los proporcionados por otros métodos. 

A mis padres y hermano 



\section{AGRADECIMIENTOS}

Estas líneas sirven para expresar mi profundo agradecimiento al Dr. Antonio Tomás Espín, al que, además de director de esta tesis, considero compañero y amigo. Su constante apoyo y estricta supervisión han sido fundamentales para el desarrollo de este trabajo. Siempre recordaré su capacidad de motivación y el rigor de sus planteamientos.

También quiero recordar al Dr. Gregorio Sánchez Olivares, profesor del Departamento de Ingeniería Civil, por su inestimable aportación en los aspectos numéricos de esta tesis y su continua disponibilidad para ayudarme.

Gracias a mis amigos: Javi, Carbonell, José y José Enrique, compañeros desinteresados que siempre me han apoyado y que han conseguido que no pierda la cabeza en este largo camino. Y también a Cristina, por su paciencia y cariño en los momentos más difíciles.

Finalmente, quiero agradecer a mis padres y hermano su apoyo incondicional. Ellos son los pilares de mi vida. A ellos les debo todo. 



\section{Índice}

LISTA DE TABLAS.

$x v i i$

LISTA DE FIGURAS

$x x i$

LISTA DE SÍMBOLOS

$x x x i$

\section{CAPÍTULO 1 INTRODUCCIÓN Y OBJETIVOS}

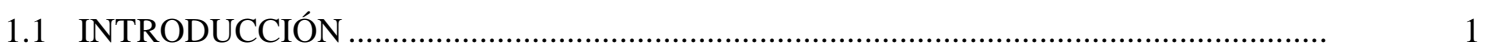

1.1.1 Modelización de estructuras de hormigón armado …….............................................. 1

1.1.2 Modelización de secciones de hormigón armado ..................................................... 2

1.2 OBJETIVOS DE LA TESIS ........................................................................................

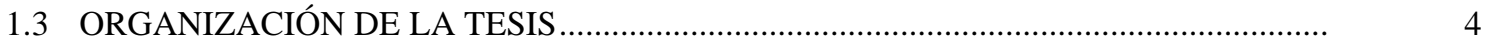

\section{CAPÍtulo 2 ESTADO DEL ARTE}

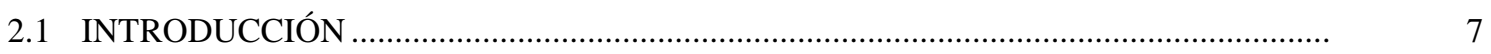

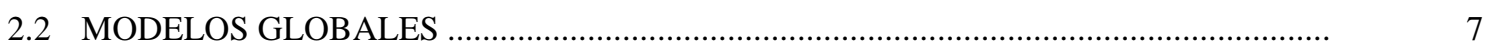

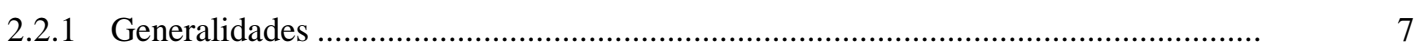

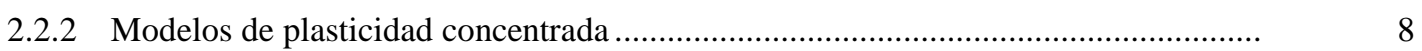

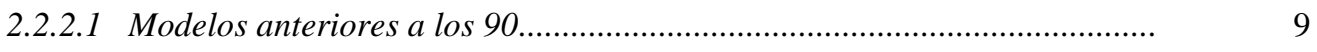

2.2.2.2 Últimas tendencias................................................................................ 10

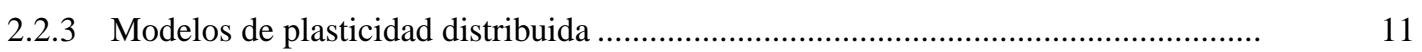


2.2.3.1 Modelos de plasticidad distribuida en términos de resultantes de tensiones y deformaciones

2.2.3.2 Modelos de fibras.

2.2.3.3 Últimas tendencias.

2.2.4 Modelos de disipación de energía concentrada.....

2.3.1 Generalidades

2.3.2 Últimas tendencias

2.4 MODELOS DE COMPORTAMIENTO SECCIONAL .................................................... 22

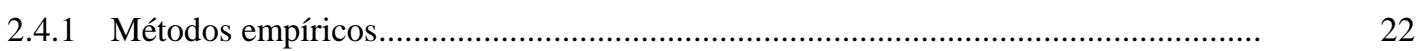

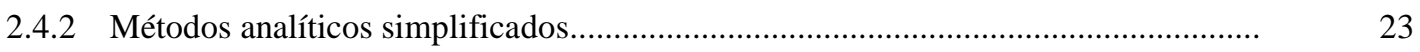

2.4.3 Métodos analíticos avanzados ........................................................................ 25

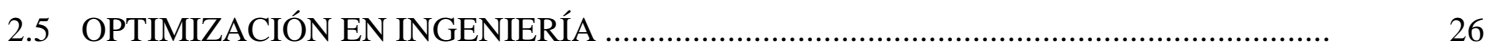

2.5.1 Algoritmos empleados en ingeniería estructural.............................................. 27

2.5.2 Algoritmos empleados en la calibración de modelos numéricos ............................. 29

2.6 PUBLICACIONES DEL DOCTORANDO RELACIONADAS CON ESTA TESIS.......... 33

\section{CAPÍTULO 3 BASES DE DATOS DE ENSAYOS EXPERIMENTALES}

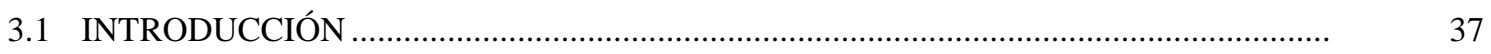

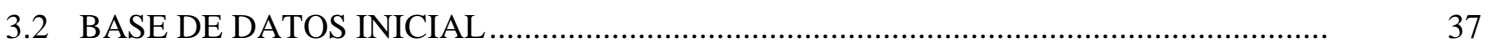

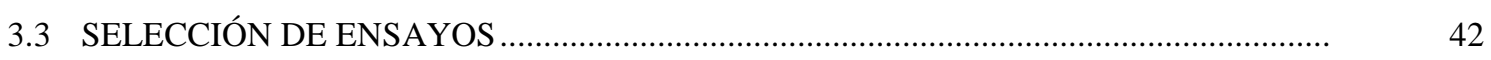

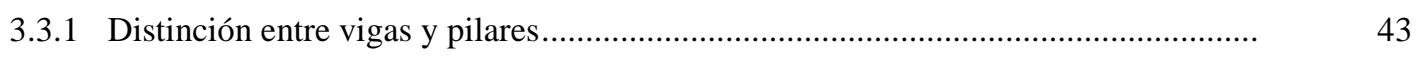

3.3.2 Dimensiones de la sección transversal...................................................................... 44

3.3.3 Resistencia a compresión simple del hormigón ..................................................... 46

3.3.4 Tipo de acero de las armaduras..................................................................... 46

3.3.5 Límite elástico del acero de la armadura longitudinal ......................................... 48

3.3.6 Límite elástico del acero de la armadura transversal .............................................. 48

3.3.7 Diámetro de la armadura longitudinal y transversal .............................................. 49

3.3.8 Cuantías de armadura longitudinal ............................................................... 51

3.3.9 Separación de armadura transversal................................................................. 54

\section{CAPÍTULO 4 AJUSTE DEL COMPORTAMIENTO DE PLASTIFICACIÓN Y ÚLTIMO DE SECCIONES RECTANGULARES DE HORMIGÓN ARMADO}


4.2.1 Expresiones de Panagiotakos y Fardis (2001) ………………………………........... 58

4.2.1.1 Curvatura de plastificación ........................................................................

4.2.1.2 Momento de plastificación...................................................................... 60

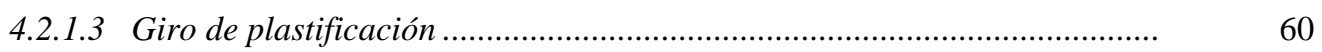

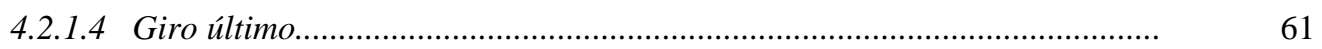

4.2.2 Expresiones de Biskinis y Fardis (2010a, 2010b) ................................................... 62

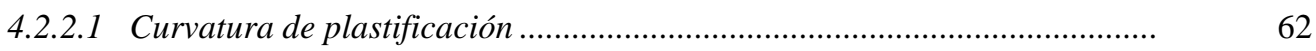

4.2.2.2 Momento de plastificación................................................................................

4.2.2.3 Giro de plastificación ................................................................................

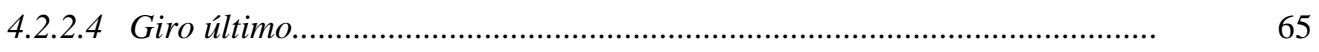

4.2.3 Expresiones en la normativa ..................................................................................... 66

4.3 EXPRESIONES DE AJUSTE CON LAS BASES DE ENSAYOS ................................... 67

4.3.1 Expresiones de ajuste con la selección de ensayos ..................................................... 68

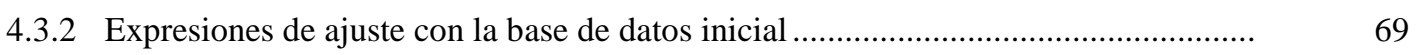

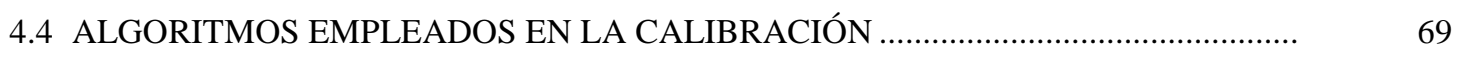

4.4.1 Ajuste con la selección de ensayos ........................................................................

4.4.2 Ajuste con la base de datos inicial .....................................................................

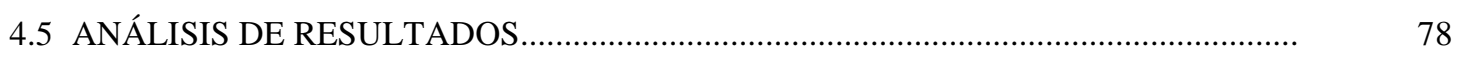

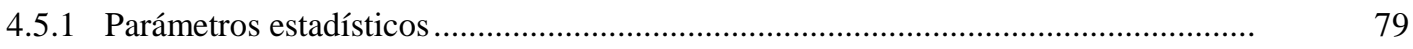

4.5.2 Estrategia de optimización para el ajuste de las expresiones con las

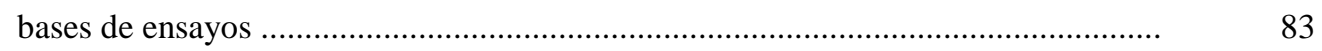

4.5.3 Expresiones ajustadas a la selección de ensayos .......................................................

4.5.3.1 Momento de plastificación......................................................................... 84

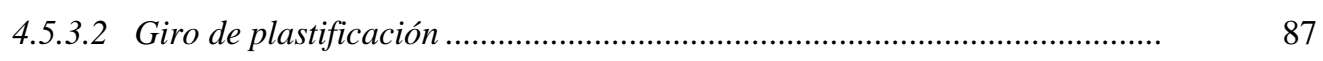

4.5.3.3 Giro último...............................................................................................

4.5.4 Expresiones ajustadas a la base de datos inicial .................................................... 95

4.5.4.1 Momento de plastificación........................................................................

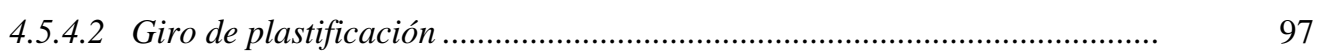

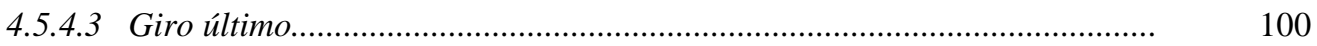

4.6 RESUMEN DE RESULTADOS ………………………………………………............ 102

CAPÍTULO 5 VALIDACIÓN DEL MÉTODO DE AJUSTE DEL COMPORTAMIENTO SECCIONAL. COMPARACIÓN DE RESULTADOS

5.1 INTRODUCCIÓN

5.2 ELEMENTO ESTRUCTURAL EMPLEADO EN LA VALIDACIÓN ……………….......... 108

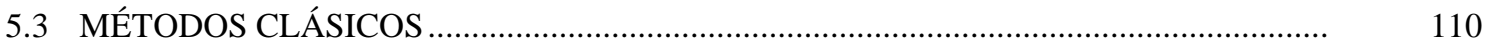


5.4 RESULTADOS

5.4.1 Diferencias en el momento de plastificación $M_{y}$.

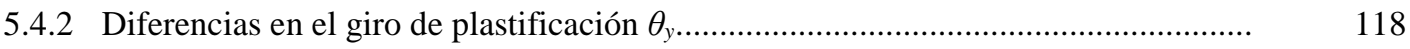

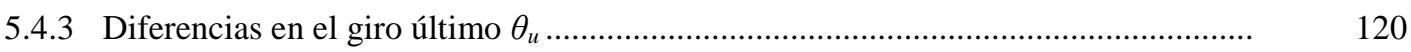

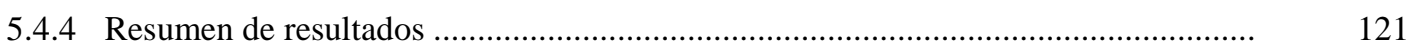

\section{CAPÍTULO 6 EJEMPLOS DE APLICACIÓN A ANÁLISIS SÍSMICO DE} ESTRUCTURAS

6.1 INTRODUCCIÓN

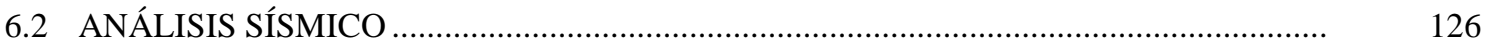

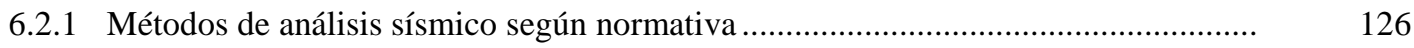

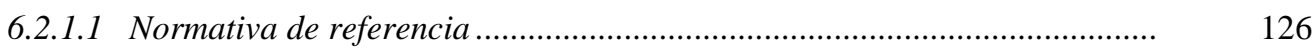

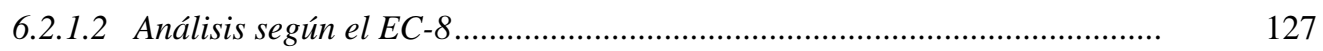

6.2.2 Análisis pushover: origen y evolución............................................................ 130

6.2.3 Indicaciones del EC-8 acerca del análisis pushover ........................................... 132

6.2.4 Análisis pushover de estructuras de hormigón armado mediante ETABS® ............ 138

6.2.4.1 Propiedades de los materiales .................................................................. 140

6.2.4.2 Definición de rótulas plásticas ................................................................ 140

6.2.4.3 Parámetros del análisis no lineal ........................................................ 145

6.3 EJEMPLOS DE APLICACIÓN .................................................................................. 147

6.3.1 Descripción de las estructuras analizadas ........................................................ 147

6.3.2 Relaciones momento-giro y curvas de plastificación ......................................... 154

6.3.3 Curvas de capacidad ................................................................................... 157

6.3.4 Resultados en el punto de plastificación global de la estructura.............................. 158

6.3.5 Resultados en el punto de colapso de la estructura ............................................ 164

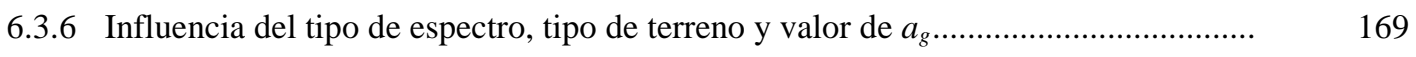

6.3.6.1 Pórtico regular de cinco plantas ............................................................ 171

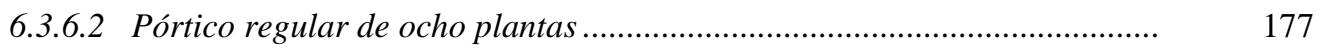

6.3.6.3 Pórtico espacial de cinco plantas .......................................................... 182

6.3.6.4 Pórtico espacial de ocho plantas ........................................................... 187

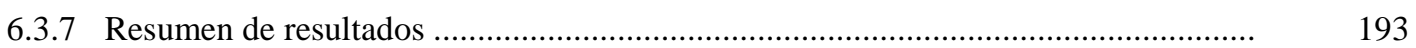




\section{CAPÍTULO 7 CONCLUSIONES}

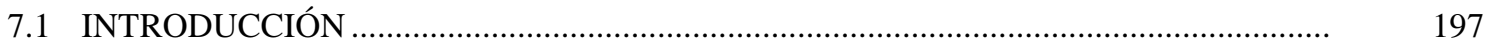

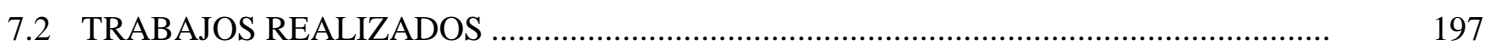

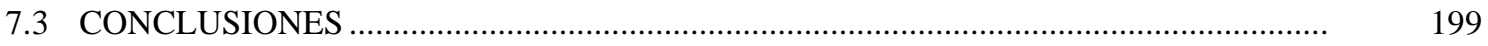

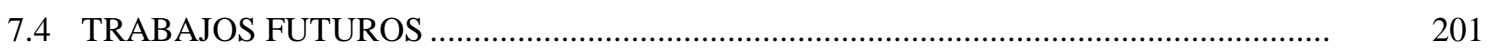

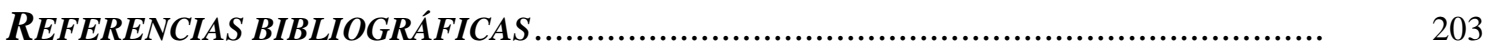

PUBLICACIONES DEL DOCTORANDO RELACIONADAS CON ESTA TESIS.................... 229 



\section{Lista de tablas}

Tabla 3.1 Rango de variación de las variables geométricas en la base de datos inicial (en $\mathrm{mm})$

Tabla 3.2 Rango de variación porcentual de las cuantías de armadura en la base de datos inicial

Tabla 3.3 Rango de variación de resistencias de materiales en la base de datos inicial (en MPa)

Tabla 3.4 Rango de variación de los diámetros de las barras de armado en la base de datos inicial (en $\mathrm{mm})$

Tabla 3.5 Restricciones geométricas en secciones rectangulares según EHE-08 (en mm).....

Tabla 3.6 Restricciones geométricas en secciones rectangulares según NCSE-02, $a_{c}=0,12 g$ (en $\mathrm{mm})$.

Tabla 3.7 Restricciones geométricas en secciones rectangulares según EC-2 y EC-8 (en $\mathrm{mm})$

Tabla 3.8 Restricciones geométricas en secciones rectangulares según ACI-318-08 (en $\mathrm{mm})$

Tabla 3.9 Rangos de utilización de la resistencia a compresión simple del hormigón $f_{c}$ según normativa (en MPa)

Tabla 3.10 Tipos de acero contemplados en la normativa

Tabla 3.11 Rangos de utilización del límite elástico del acero de la armadura longitudinal $\left(f_{y}, f_{y}{ }^{\prime}\right)$ según normativa (en MPa)

Tabla 3.12 Valores máximos para el límite elástico del acero de la armadura transversal $f_{y h}$ según normativa (en MPa)

Tabla 3.13 Rangos de utilización del diámetro de las barras de armadura según EHE-08 (en $\mathrm{mm})$

Tabla 3.14 Diámetro mínimo de las barras de armadura según NCSE-02 (en mm).....

Tabla 3.15 Rangos de utilización del diámetro de las barras de armadura según EC-2 (en $\mathrm{mm})$.

Tabla 3.16 Diámetro mínimo de las barras de armadura según EC-8 (en mm) 
Tabla 3.17 Rangos de utilización del diámetro de las barras de armadura según ACI-318-08 (en $\mathrm{mm})$

Tabla 3.18 Rango de variación de los diámetros de las barras de armadura considerados para la selección de ensayos (en mm).

Tabla 3.19 Armadura longitudinal mínima y máxima según EHE-08.

Tabla 3.20 Armadura longitudinal mínima y máxima según NCSE-02 .

Tabla 3.21 Armadura longitudinal mínima y máxima según EC-2.....

Tabla 3.22 Armadura longitudinal mínima y máxima según EC-8.

Tabla 3.23 Armadura longitudinal mínima y máxima según ACI-318-08

Tabla 3.24 Separación máxima en la armadura transversal según EHE-08 (en mm)

Tabla 3.25 Separación máxima en la armadura transversal según NCSE-02 (en mm).....

Tabla 3.26 Separación máxima en la armadura transversal según EC-2 (en mm)

Tabla 3.27 Separación máxima en la armadura transversal según EC-8 (en mm)

Tabla 3.28 Separación máxima en la armadura transversal según ACI-318-08 (en mm)......... 56

Tabla 4.1 Valores adoptados por los parámetros del algoritmo FA.....

Tabla 4.2 Medidas de síntesis para la variable $x=M_{y, \text { exp }} / M_{y, \text { pred }}$ según diferentes autores .....

Tabla 4.3 Parámetros para el análisis de la relación entre las variables $M_{y, \text { pred }}$ y $M_{y, \exp }$ según diferentes autores

Tabla 4.4 Medidas de síntesis para la variable $x=\theta_{y, \exp } / \theta_{y}$,pred según diferentes autores........

Tabla 4.5 Parámetros para el análisis de la relación entre las variables $\theta_{y, \exp }$ y $\theta_{y \text {, pred }}$ según diferentes autores.....

Tabla 4.6 Medidas de síntesis para la variable $x=\theta_{u \text {, exp }} / \theta_{u, p r e d}$ según diferentes autores........

Tabla 4.7 Parámetros para el análisis de la relación entre las variables $\theta_{u \text {,exp }}$ y $\theta_{u \text {,pred }}$ según diferentes autores .

Tabla 4.8 Medidas de síntesis para la variable $x=M_{y, \text { exp }} / M_{y, \text { pred }}$ según diferentes autores .....

Tabla 4.9 Parámetros para el análisis de la relación entre las variables $M_{y \text {,exp }}$ y $M_{y, \text { pred }}$ según diferentes autores ....

Tabla 4.10 Medidas de síntesis para la variable $x=\theta_{y}$, exp $/ \theta_{y}$,pred según diferentes autores........

Tabla 4.11 Parámetros para el análisis de la relación entre las variables $\theta_{y, \exp }$ y $\theta_{y, \text { pred }}$ según diferentes autores .....

Tabla 4.12 Medidas de síntesis para la variable $x=\theta_{u \text {, exp }} / \theta_{u \text {,pred }}$ según diferentes autores........

Tabla 4.13 Parámetros para el análisis de la relación entre las variables $\theta_{u \text {,exp }}$ y $\theta_{u \text {,pred }}$ según diferentes autores

Tabla 4.14 Mejoras obtenidas con las expresiones ajustadas a la selección de ensayos en los parámetros de dispersión respecto a otras expresiones (en \%).

Tabla 4.15 Mejoras obtenidas con las expresiones ajustadas a la selección de ensayos en los parámetros para el análisis de la relación $x_{\text {exp }}-x_{\text {pred }}$ respecto a otras expresiones (en $\%)$

Tabla 4.16 Mejoras obtenidas con las expresiones ajustadas a la base de datos inicial en los parámetros de dispersión respecto a otras expresiones (en \%). 
Tabla 4.17 Mejoras obtenidas con las expresiones ajustadas a la base de datos inicial en los parámetros para el análisis de la relación $x_{\text {exp }}-x_{\text {pred }}$ respecto a otras expresiones (en $\%)$

Tabla 5.1 Armadura de tracción y compresión y esfuerzo axil para los diferentes casos considerados en las secciones $300 \times 400$ y $350 \times 350 \mathrm{~mm}$

Tabla 5.2 Resultados obtenidos para el momento y giro de plastificación y giro último de la sección 300×400 mm según diferentes métodos.

Tabla 5.3 Resultados obtenidos para el momento y giro de plastificación y giro último de la sección $350 \times 350$ mm según diferentes métodos.

Tabla 6.1 Valores de parámetros para el espectro tipo 1

Tabla 6.2 Valores de parámetros para el espectro tipo 2 .

Tabla 6.3 Propiedades del hormigón.

Tabla 6.4 Propiedades del acero de las armaduras.

Tabla 6.5 Parámetros de las rótulas plásticas para vigas según la FEMA (2000)...

Tabla 6.6 Parámetros de las rótulas plásticas para pilares según la FEMA (2000).

Tabla 6.7 Porcentajes del giro último $\theta_{u}$ atribuidos en ETABS $®$ a los diferentes niveles de comportamiento

Tabla 6.8 Ancho tributario y cargas por metro lineal para los pórticos analizados.

Tabla 6.9 Características del pórtico regular de cinco plantas para las distribuciones de carga lateral uniforme y modal

Tabla 6.10 Características del pórtico regular de ocho plantas para las distribuciones de carga lateral uniforme y modal

Tabla 6.11 Características del pórtico espacial de cinco plantas para las distribuciones de carga lateral uniforme y modal

Tabla 6.12 Características del pórtico espacial de ocho plantas para las distribuciones de carga lateral uniforme y modal .....

Tabla 6.13 Resultados en el punto de plastificación global para el pórtico regular de cinco plantas

Tabla 6.14 Resultados en el punto de plastificación global para el pórtico regular de ocho plantas

Tabla 6.15 Resultados en el punto de plastificación global para el pórtico espacial de cinco plantas

Tabla 6.16 Resultados en el punto de plastificación global para el pórtico espacial de ocho plantas

Tabla 6.17 Resultados en el punto de colapso para el pórtico regular de cinco plantas......

Tabla 6.18 Resultados en el punto de colapso para el pórtico regular de ocho plantas........

Tabla 6.19 Resultados en el punto de colapso para el pórtico espacial de cinco plantas ...

Tabla 6.20 Resultados en el punto de colapso para el pórtico espacial de ocho plantas...........

Tabla 6.21 Valores de desplazamiento objetivo $d_{t}$ para el pórtico regular de cinco plantas ..... 
Tabla 6.22 Valores mínimos de aceleración $a_{g}$ para los que existen diferencias entre los diferentes tipos de rótulas plásticas

Tabla 6.23 Valores de desplazamiento objetivo $d_{t}$ para el pórtico regular de ocho plantas......

Tabla 6.24 Valores mínimos de aceleración $a_{g}$ para los que existen diferencias entre los diferentes tipos de rótulas plásticas

Tabla 6.25 Valores de desplazamiento objetivo $d_{t}$ para el pórtico espacial de cinco plantas....

Tabla 6.26 Valores mínimos de aceleración $a_{g}$ para los que existen diferencias entre los diferentes tipos de rótulas plásticas

Tabla 6.27 Valores de desplazamiento objetivo $d_{t}$ para el pórtico espacial de ocho plantas ....

Tabla 6.28 Valores mínimos de aceleración $a_{g}$ para los que existen diferencias entre los diferentes tipos de rótulas plásticas. 


\section{Lista de figuras}

Figura 1.1 Modelos de comportamiento no lineal. (a) Modelos globales.

(b) Modelos locales

Figura 2.1 Modelización de un elemento según diferentes modelos. (a) Modelo de dos componentes (b) Modelo ZAP (c) Modelo de fibras

Figura 2.2 Distribución temporal de modelos numéricos empleados para la simulación sísmica de estructuras de hormigón armado según diferentes enfoques

Figura 3.1 Configuración de ensayos para pilares. (a) Ménsula simple. (b) Ménsula doble....

Figura 3.2 Configuración de ensayos para vigas simplemente apoyadas. (a) Ensayo estándar.

(b) Ensayo con aplicación de carga en elemento rígido (stub)

Figura 3.3 Distribución de ensayos. (a) Según tipo de acero de las armaduras. (b) Según exista deslizamiento de la armadura longitudinal

Figura 3.4 Distribución de ensayos según el tipo de carga aplicada (monotónica o cíclica)....

Figura 3.5 Distribución de vigas y pilares considerados en la base de datos inicial

Figura 4.1 Relaciones esfuerzo-deformación en un elemento sometido a cargas monotónicas y cíclicas

Figura 4.2 Cromosoma de un individuo en un problema con tres variables de diseño

Figura 4.3 Individuos modificados mediante el operador de cruce multipunto con 5 puntos de división.

Figura 4.4 Disminución del parámetro de aleatoriedad $\alpha_{r}$ en función del parámetro $s_{\alpha}$

Figura 4.5 Ejemplo de diagrama box-plot obtenido con el programa Statgraphics®.

Figura 4.6 Diagramas box-plot para la variable $x=M_{y}$, exp $/ M_{y}$, pred. (a) Panagiotakos y Fardis (2001) y Biskinis y Fardis (2010a).

(b) Ecuación propuesta.

Figura 4.7 Coeficiente de variación $C V$ de la variable $x=M_{y}$,exp $/ M_{y}$,pred en función del porcentaje de variación $P_{i, 0}$ de los coeficientes iniciales $C_{i, 0}$. (a) Valor medio $\bar{x}$ entre 0,9 y 1,1 . (b) Valor medio $\bar{x}$ entre 0,95 y 1,05 . (c) Valor medio $\bar{x}$ entre 0,99 y 1,01

Figura 4.8 Comparación de valores experimentales y predichos para el momento de plastificación $M_{y}$. (a) Panagiotakos y Fardis (2001) y Biskinis y Fardis (2010a).

(b) Ecuación propuesta 
Figura 4.9 Diagramas box-plot para la variable $x=\theta_{y}$,exp $/ \theta_{y \text {,pred. }}$ (a) Panagiotakos y Fardis (2001). (b) EC-8. (c) Biskinis y Fardis (2010a). (c) Ecuación propuesta...

Figura 4.10 Coeficiente de variación $C V$ de la variable $x=\theta_{y}$,exp $/ \theta_{y, \text { pred }}$ en función del porcentaje de variación $P_{i, 0}$ de los coeficientes iniciales $C_{i, 0}$. (a) Valor medio $\bar{x}$ entre 0,9 y 1,1 . (b) Valor medio $\bar{x}$ entre 0,95 y 1,05 . (c) Valor medio $\bar{x}$ entre 0,99 y 1,01

Figura 4.11 Comparación de valores experimentales y predichos para el giro de plastificación $\theta_{y}$. (a) Panagiotakos y Fardis (2001). (b) EC-8. (c) Biskinis y Fardis (2010a).

(d) Ecuación propuesta

Figura 4.12 Diagramas box-plot para la variable $x=\theta_{u, \text { exp }} / \theta_{u \text {,pred. }}$ (a) Panagiotakos y Fardis (2001). (b) EC-8. (c) Biskinis y Fardis (2010b). (d) Ecuación propuesta

Figura 4.13 Coeficiente de variación $C V$ de la variable $x=\theta_{u}$,exp $/ \theta_{u \text {,pred }}$ en función del porcentaje de variación $P_{i, 0}$ de los coeficientes iniciales $C_{i, 0}$. (a) Valor medio $\bar{x}$ entre 0,9 y 1,1 . (b) Valor medio $\bar{x}$ entre 0,95 y 1,05 . (c) Valor medio $\bar{x}$ entre 0,99 y 1,01

Figura 4.14 Comparación de valores experimentales y predichos para el giro último $\theta_{u}$.

(a) Panagiotakos y Fardis (2001). (b) EC-8. (c) Biskinis y Fardis (2010b).

(d) Ecuación propuesta

Figura 4.15 Diagrama box-plot para la variable $x=M_{y}$, exp $/ M_{y}$, pred. (a) Panagiotakos y Fardis (2001) y Biskinis y Fardis (2010a). (b) Ecuación propuesta.

Figura 4.16 Coeficiente de variación $C V$ de la variable $x=M_{y}$,exp $/ M_{y \text {,pred }}$ en función del porcentaje de variación $P_{i, 0}$ de los coeficientes iniciales $C_{i, 0}$

Figura 4.17 Comparación de valores experimentales y predichos para el momento de plastificación $M_{y}$. (a) Panagiotakos y Fardis (2001) y Biskinis y Fardis (2010a).

(b) Ecuación propuesta

Figura 4.18 Diagramas box-plot para la variable $x=\theta_{y, \text { exp }} / \theta_{y, \text { pred. }}$ (a) Panagiotakos y Fardis (2001). (b) EC-8. (c) Biskinis y Fardis (2010a). (d) Ecuación propuesta

Figura 4.19 Coeficiente de variación $C V$ de la variable $x=\theta_{y}$,exp $/ \theta_{y}$,pred en función del porcentaje de variación $P_{i, 0}$ de los coeficientes iniciales $C_{i, 0}$

Figura 4.20 Comparación de valores experimentales y predichos para el giro de plastificación $\theta_{y}$. (a) Panagiotakos y Fardis (2001). (b) EC-8. (c) Biskinis y Fardis (2010a).

(d) Ecuación propuesta

Figura 4.21 Coeficiente de variación $C V$ de la variable $x=\theta_{u}$ exp $/ \theta_{u \text {,pred }}$ en función del porcentaje de variación $P_{i, 0}$ de los coeficientes iniciales $C_{i, 0}$

Figura 4.22 Diagrama box-plot para la variable $x=\theta_{u, \text { exp }} / \theta_{u \text {, pred. }}$ (a) Panagiotakos y Fardis (2001). (b) EC-8. (c) Biskinis y Fardis (2010b). (d) Ecuación propuesta

Figura 4.23 Comparación de valores experimentales y predichos para el giro último $\theta_{u}$.

(a) Panagiotakos y Fardis (2001). (b) EC-8. (c) Biskinis y Fardis (2010b).

(d) Ecuación propuesta

Figura 5.1 Elemento en voladizo y distribución de curvaturas a lo largo de su longitud 
Figura 5.2 Secciones consideradas para el elemento en voladizo (unidades en $\mathrm{mm}$ )

Figura 5.3 Discretización de la sección para integrar la ecuación constitutiva por tramos ......

Figura 5.4 Distribución real e idealizada de curvaturas en el elemento al plastificar únicamente la sección de empotramiento

Figura 5.5 Ratio entre el momento de plastificación obtenido con métodos clásicos $M_{y, m c}$ y con la expresión ajustada a la selección de ensayos $M_{y, p r e d}$ en función del esfuerzo axil y la cuantía de armadura de tracción. (a) Sección 300×400mm. (b) Sección $350 \times 350 \mathrm{~mm}$

Figura 5.6 Ratio entre el momento de plastificación obtenido con métodos clásicos $M_{y, m c} \mathrm{y}$ con la expresión ajustada a la selección de ensayos $M_{y, p r e d}$ en función del esfuerzo axil y la cuantía de armadura de compresión. (a) Sección 300×400mm.

(b) Sección $350 \times 350 \mathrm{~mm}$

Figura 5.7 Ratio entre el momento de plastificación obtenido con la expresión de Biskinis y Fardis (2010a) $M_{y, B F}$ y con la expresión ajustada a la selección de ensayos $M_{y, p r e d}$ en función del esfuerzo axil y la cuantía de armadura de tracción. (a) Sección 300×400 mm. (b) Sección 350×350 mm.

Figura 5.8 Ratio entre el momento de plastificación obtenido con la expresión de Biskinis y Fardis (2010a) $M_{y, B F}$ y con la expresión ajustada a la selección de ensayos $M_{y, p r e d}$ en función del esfuerzo axil y la cuantía de armadura de compresión. (a) Sección $300 \times 400$ mm. (b) Sección 350×350 mm

Figura 5.9 Ratio entre el giro de plastificación obtenido con métodos clásicos $\theta_{y, m c}$ y con la expresión ajustada a la selección de ensayos $\theta_{y, p r e d}$ en función del esfuerzo axil y la cuantía de armadura de tracción. (a) Sección 300×400mm. (b) Sección $350 \times 350 \mathrm{~mm}$

Figura 5.10 Ratio entre el giro de plastificación obtenido con métodos clásicos $\theta_{y, m c}$ y con la expresión ajustada a la selección de ensayos $\theta_{y \text {,pred }}$ en función del esfuerzo axil y la cuantía de armadura de compresión. (a) Sección 300×400mm. (b) Sección $350 \times 350 \mathrm{~mm}$

Figura 5.11 Ratio entre el giro de plastificación obtenido con la expresión de Biskinis y Fardis (2010a) $\theta_{y, B F}$ y con la expresión ajustada a la selección de ensayos $\theta_{y, p r e d}$ en función del esfuerzo axil y la cuantía de armadura de tracción. (a) Sección $300 \times 400$ mm. (b) Sección 350×350 mm

Figura 5.12 Ratio entre el giro de plastificación obtenido con la expresión de Biskinis y Fardis (2010a) $\theta_{y, B F}$ y con la expresión ajustada a la selección de ensayos $\theta_{y, p r e d}$ en función del esfuerzo axil y la cuantía de armadura de compresión. (a) Sección $300 \times 400$ mm. (b) Sección 350×350 mm.

Figura 5.13 Ratio entre el giro último obtenido con métodos clásicos $\theta_{u, m c}$ y con la expresión ajustada a la selección de ensayos $\theta_{u, p r e d}$ en función del esfuerzo axil y la cuantía de armadura de tracción. (a) Sección 300×400mm. (b) Sección 350×350mm ..... 
Figura 5.14 Ratio entre el giro último obtenido con métodos clásicos $\theta_{u, m c}$ y con la expresión ajustada a la selección de ensayos $\theta_{u, p r e d}$ en función del esfuerzo axil y la cuantía de armadura de compresión. (a) Sección 300×400 mm.

(b) Sección $350 \times 350 \mathrm{~mm}$

Figura 5.15 Ratio entre el giro último obtenido con la expresión de Biskinis y Fardis (2010b) $\theta_{u, B F}$ y con la expresión ajustada a la selección de ensayos $\theta_{u, p r e d}$ en función del esfuerzo axil y la cuantía de armadura de tracción. (a) Sección 300×400 mm.

(b) Sección 350×350 mm

Figura 5.16 Ratio entre el giro último obtenido con la expresión de Biskinis y Fardis (2010b) $\theta_{u, B F}$ y con la expresión ajustada a la selección de ensayos $\theta_{u \text {,pred }}$ en función del esfuerzo axil y la cuantía de armadura de compresión. (a) Sección 300×400mm. (b) Sección $350 \times 350 \mathrm{~mm}$

Figura 6.1 Curva de capacidad real y elasto-plástica para el sistema equivalente de un grado de libertad

Figura 6.2 Determinación del desplazamiento objetivo para el sistema equivalente de un grado de libertad. (a) Rango de periodo corto. (b) Rango de periodos medio y largo

Figura 6.3 Representación gráfica del espectro de respuesta elástica horizontal propuesto por el EC-8

Figura 6.4 Espectro de respuesta elástica horizontal propuesto por el EC-8 en función del tipo de terreno. (a) Espectro tipo 1. (b) Espectro tipo 2

Figura 6.5 Relación constitutiva tensión-deformación del hormigón a compresión

Figura 6.6 Relación constitutiva tensión-deformación del acero de las armaduras a tracción .

Figura 6.7 Definición de las posiciones de las rótulas plásticas

Figura 6.8 Relación momento-giro para rótulas plásticas de hormigón armado según la FEMA (2000)

Figura 6.9 Relación momento-giro adoptada por ETABS $®$ para las rótulas plásticas tipo lumped......

Figura 6.10 Relación momento-giro elasto-plástica para las rótulas plásticas definidas con las expresiones de comportamiento seccional

Figura 6.11 Alzados de los pórticos regulares. (a) Pórtico de cinco plantas. (b) Pórtico de ocho plantas

Figura 6.12 Secciones transversales de los elementos del pórtico regular de cinco plantas.

(a) Pilares. (b) Vigas

Figura 6.13 Secciones transversales de los elementos del pórtico regular de ocho plantas.

(a) Pilares. (b) Vigas

Figura 6.14 Estructuras espaciales. (a) Cinco plantas. (b) Ocho plantas

Figura 6.15 Dimensiones en metros de las plantas de las estructuras espaciales y alineación del pórtico A-A' considerado para el análisis pushover. 
Figura 6.16 Alzado de los pórticos espaciales. (a) Estructura de cinco plantas. (b) Estructura de ocho plantas

Figura 6.17 Secciones transversales de los elementos del pórtico espacial de cinco plantas.

(a) Pilares. (b) Vigas

Figura 6.18 Secciones transversales de los elementos del pórtico espacial de ocho plantas.

(a) Pilares. (b) Vigas

Figura 6.19 Relaciones momento-giro para las rótulas plásticas generadas en las vigas.

(a) Viga $500 \times 300 \mathrm{~mm}$. (b) Viga $300 \times 400 \mathrm{~mm}$

Figura 6.20 Relaciones momento-giro para las rótulas plásticas generadas en los pilares.

(a) Pilar P1. (b) Pilar P2. (c) Pilar P3. (d) Pilar P4.

Figura 6.21 Curvas de plastificación para los pilares de los pórticos. (a) Pilar P1. (b) Pilar P2. (c) Pilar P3. (d) Pilar P4

Figura 6.22 Curvas de capacidad para el pórtico regular de cinco plantas. (a) Carga lateral uniforme. (b) Carga lateral modal

Figura 6.23 Curvas de capacidad para el pórtico regular de ocho plantas. (a) Carga lateral uniforme. (b) Carga lateral modal

Figura 6.24 Curvas de capacidad para el pórtico espacial de cinco plantas. (a) Carga lateral uniforme. (b) Carga lateral modal

Figura 6.25 Curvas de capacidad para el pórtico espacial de ocho plantas. (a) Carga lateral uniforme. (b) Carga lateral modal

Figura 6.26 Colores asociados a los niveles de comportamiento IO, LS y CP y a los puntos B, C, D y E de la curva de respuesta de las rótulas plásticas

Figura 6.27 Distribución de rótulas plásticas para el pórtico regular de cinco plantas con cargas laterales uniformes en el punto de plastificación global. (a) ETABS®.

(b) Biskinis y Fardis (2010a, 2010b). (c) Expresiones calibradas.

Figura 6.28 Distribución de rótulas plásticas para el pórtico regular de ocho plantas con cargas laterales uniformes en el punto de plastificación global. (a) ETABS®.

(b) Biskinis y Fardis (2010a, 2010b). (c) Expresiones calibradas.

Figura 6.29 Distribución de rótulas plásticas para el pórtico espacial de cinco plantas con cargas laterales uniformes en el punto de plastificación global. (a) ETABSß. (b) Biskinis y Fardis (2010a, 2010b). (c) Expresiones calibradas.

Figura 6.30 Distribución de rótulas plásticas para el pórtico espacial de ocho plantas con cargas laterales uniformes en el punto de plastificación global. (a) ETABS®.

(b) Biskinis y Fardis (2010a, 2010b). (c) Expresiones calibradas.

Figura 6.31 Distribución de rótulas plásticas para el pórtico regular de cinco plantas con cargas laterales modales en el punto de plastificación global. (a) ETABS®.

(b) Biskinis y Fardis (2010a, 2010b). (c) Expresiones calibradas.

Figura 6.32 Distribución de rótulas plásticas para el pórtico regular de ocho plantas con cargas laterales modales en el punto de plastificación global. (a) ETABS®.

(b) Biskinis y Fardis (2010a, 2010b). (c) Expresiones calibradas. 
Figura 6.33 Distribución de rótulas plásticas para el pórtico espacial de cinco plantas con cargas laterales modales en el punto de plastificación global. (a) ETABS®.

(b) Biskinis y Fardis (2010a, 2010b). (c) Expresiones calibradas.

Figura 6.34 Distribución de rótulas plásticas para el pórtico espacial de ocho plantas con cargas laterales modales en el punto de plastificación global. (a) ETABS®.

(b) Biskinis y Fardis (2010a, 2010b). (c) Expresiones calibradas.

Figura 6.35 Distribución de rótulas plásticas para el pórtico regular de cinco plantas con cargas laterales uniformes en el punto de colapso. (a) ETABS®. (b) Biskinis y Fardis (2010a, 2010b). (c) Expresiones calibradas

Figura 6.36 Distribución de rótulas plásticas para el pórtico regular de ocho plantas con cargas laterales uniformes en el punto de colapso. (a) ETABS®. (b) Biskinis y Fardis (2010a, 2010b). (c) Expresiones calibradas

Figura 6.37 Distribución de rótulas plásticas para el pórtico espacial de cinco plantas con cargas laterales uniformes en el punto de colapso. (a) ETABS®. (b) Biskinis y Fardis (2010a, 2010b). (c) Expresiones calibradas

Figura 6.38 Distribución de rótulas plásticas para el pórtico espacial de ocho plantas con cargas laterales uniformes en el punto de colapso. (a) ETABS®. (b) Biskinis y Fardis (2010a, 2010b). (c) Expresiones calibradas

Figura 6.39 Distribución de rótulas plásticas para el pórtico regular de cinco plantas con cargas laterales modales en el punto de colapso. (a) ETABS®. (b) Biskinis y Fardis (2010a, 2010b). (c) Expresiones calibradas

Figura 6.40 Distribución de rótulas plásticas para el pórtico regular de ocho plantas con cargas laterales modales en el punto de colapso. (a) ETABS®. (b) Biskinis y Fardis (2010a, 2010b). (c) Expresiones calibradas

Figura 6.41 Distribución de rótulas plásticas para el pórtico espacial de cinco plantas con cargas laterales modales en el punto de colapso. (a) ETABS®. (b) Biskinis y Fardis (2010a, 2010b). (c) Expresiones calibradas

Figura 6.42 Distribución de rótulas plásticas para el pórtico espacial de ocho plantas con cargas laterales modales en el punto de colapso. (a) ETABS®. (b) Biskinis y Fardis (2010a, 2010b). (c) Expresiones calibradas

Figura 6.43 Desplazamientos laterales del pórtico regular de cinco plantas para el espectro tipo 2 y $a_{g}=0,5 g$. (a) Terreno tipo A. (b) Terreno tipo C. (c) Terreno tipo D.........

Figura 6.44 Desplazamientos laterales del pórtico regular de cinco plantas para el espectro tipo 1 y $a_{g}=1,0 g$. (a) Terreno tipo A. (b) Terreno tipo C. (c) Terreno tipo D.........

Figura 6.45 Drifts para el pórtico regular de cinco plantas considerando el espectro tipo 2 y $a_{g}=0,5 g$. (a) Terreno tipo A. (b) Terreno tipo C. (c) Terreno tipo D

Figura 6.46 Drifts para el pórtico regular de cinco plantas considerando el espectro tipo 1 y $a_{g}=1,0 g$. (a) Terreno tipo A. (b) Terreno tipo C. (c) Terreno tipo D 
Figura 6.47 Distribución de rótulas plásticas para el pórtico regular de cinco plantas considerando el espectro tipo $2, a_{g}=0,5 g$ y terreno tipo A. (a) ETABS $®$.

(b) Biskinis y Fardis (2010a, 2010b). (c) Expresiones calibradas.

Figura 6.48 Distribución de rótulas plásticas para el pórtico regular de cinco plantas considerando el espectro tipo 2, $a_{g}=0,5 g$ y terreno tipo C. (a) ETABS $®$.

(b) Biskinis y Fardis (2010a, 2010b). (c) Expresiones calibradas.

Figura 6.49 Distribución de rótulas plásticas para el pórtico regular de cinco plantas considerando el espectro tipo $2, a_{g}=0,5 g$ y terreno tipo D. (a) ETABS®.

(b) Biskinis y Fardis (2010a, 2010b). (c) Expresiones calibradas.

Figura 6.50 Distribución de rótulas plásticas para el pórtico regular de cinco plantas considerando el espectro tipo $1, a_{g}=1,0 g$ y terreno tipo A. (a) ETABS®.

(b) Biskinis y Fardis (2010a, 2010b). (c) Expresiones calibradas.

Figura 6.51 Distribución de rótulas plásticas para el pórtico regular de cinco plantas considerando el espectro tipo $1, a_{g}=1,0 g$ y terreno tipo C. (a) ETABS $®$.

(b) Biskinis y Fardis (2010a, 2010b). (c) Expresiones calibradas.

Figura 6.52 Distribución de rótulas plásticas para el pórtico regular de cinco plantas considerando el espectro tipo $1, a_{g}=1,0 g$ y terreno tipo D. (a) ETABS®.

(b) Biskinis y Fardis (2010a, 2010b). (c) Expresiones calibradas.

Figura 6.53 Desplazamientos laterales del pórtico regular de ocho plantas para el espectro tipo 2 y $a_{g}=0,5 g$. (a) Terreno tipo A. (b) Terreno tipo C. (c) Terreno tipo D

Figura 6.54 Desplazamientos laterales del pórtico regular de ocho plantas para el espectro tipo 1 y $a_{g}=1,0 g$. (a) Terreno tipo A. (b) Terreno tipo C. (c) Terreno tipo D

Figura 6.55 Drifts para el pórtico regular de ocho plantas considerando el espectro tipo 2 y $a_{g}=0,5 g$. (a) Terreno tipo A. (b) Terreno tipo C. (c) Terreno tipo D

Figura 6.56 Drifts para el pórtico regular de ocho plantas considerando el espectro tipo 1 y $a_{g}=1,0 g$. (a) Terreno tipo A. (b) Terreno tipo C. (c) Terreno tipo D

Figura 6.57 Distribución de rótulas plásticas para el pórtico regular de ocho plantas considerando el espectro tipo $2, a_{g}=0,5 g$ y terreno tipo A. (a) ETABS®.

(b) Biskinis y Fardis (2010a, 2010b). (c) Expresiones calibradas.

Figura 6.58 Distribución de rótulas plásticas para el pórtico regular de ocho plantas considerando el espectro tipo 2, $a_{g}=0,5 g$ y terreno tipo C. (a) ETABS®.

(b) Biskinis y Fardis (2010a, 2010b). (c) Expresiones calibradas.

Figura 6.59 Distribución de rótulas plásticas para el pórtico regular de ocho plantas considerando el espectro tipo 2, $a_{g}=0,5 g$ y terreno tipo D. (a) ETABS®.

(b) Biskinis y Fardis (2010a, 2010b). (c) Expresiones calibradas.

Figura 6.60 Distribución de rótulas plásticas para el pórtico regular de ocho plantas considerando el espectro tipo $1, a_{g}=1,0 g$ y terreno tipo A. (a) ETABS®.

(b) Biskinis y Fardis (2010a, 2010b). (c) Expresiones calibradas. 
Figura 6.61 Distribución de rótulas plásticas para el pórtico regular de ocho plantas considerando el espectro tipo $1, a_{g}=1,0 g$ y terreno tipo C. (a) ETABS $®$.

(b) Biskinis y Fardis (2010a, 2010b). (c) Expresiones calibradas.

Figura 6.62 Distribución de rótulas plásticas para el pórtico regular de ocho plantas considerando el espectro tipo $1, a_{g}=1,0 g$ y terreno tipo D. (a) ETABS®.

(b) Biskinis y Fardis (2010a, 2010b). (c) Expresiones calibradas.

Figura 6.63 Desplazamientos laterales del pórtico espacial de cinco plantas para el espectro tipo 2 y $a_{g}=0,5 g$. (a) Terreno tipo A. (b) Terreno tipo C. (c) Terreno tipo D........

Figura 6.64 Desplazamientos laterales del pórtico espacial de cinco plantas para el espectro tipo 1 y $a_{g}=1,0 g$. (a) Terreno tipo A. (b) Terreno tipo C. (c) Terreno tipo D........

Figura 6.65 Drifts para el pórtico espacial de cinco plantas considerando el espectro tipo 2 y $a_{g}=0,5 g$. (a) Terreno tipo A. (b) Terreno tipo C. (c) Terreno tipo D

Figura 6.66 Drifts para el pórtico espacial de cinco plantas considerando el espectro tipo 1 y $a_{g}=1,0 g$. (a) Terreno tipo A. (b) Terreno tipo C. (c) Terreno tipo D

Figura 6.67 Distribución de rótulas plásticas para el pórtico espacial de cinco plantas considerando el espectro tipo 2, $a_{g}=0,5 g$ y terreno tipo A. (a) ETABS®.

(b) Biskinis y Fardis (2010a, 2010b). (c) Expresiones calibradas.

Figura 6.68 Distribución de rótulas plásticas para el pórtico espacial de cinco plantas considerando el espectro tipo 2, $a_{g}=0,5 g$ y terreno tipo C. (a) ETABS®.

(b) Biskinis y Fardis (2010a, 2010b). (c) Expresiones calibradas.

Figura 6.69 Distribución de rótulas plásticas para el pórtico espacial de cinco plantas considerando el espectro tipo 2, $a_{g}=0,5 g$ y terreno tipo D. (a) ETABS®.

(b) Biskinis y Fardis (2010a, 2010b). (c) Expresiones calibradas.

Figura 6.70 Distribución de rótulas plásticas para el pórtico espacial de cinco plantas considerando el espectro tipo $1, a_{g}=1,0 g$ y terreno tipo A. (a) ETABS $®$.

(b) Biskinis y Fardis (2010a, 2010b). (c) Expresiones calibradas.

Figura 6.71 Distribución de rótulas plásticas para el pórtico espacial de cinco plantas considerando el espectro tipo $1, a_{g}=1,0 g$ y terreno tipo C. (a) ETABS $®$.

(b) Biskinis y Fardis (2010a, 2010b). (c) Expresiones calibradas.

Figura 6.72 Distribución de rótulas plásticas para el pórtico espacial de cinco plantas considerando el espectro tipo $1, a_{g}=1,0 g$ y terreno tipo D. (a) ETABS®.

(b) Biskinis y Fardis (2010a, 2010b). (c) Expresiones calibradas.

Figura 6.73 Desplazamientos laterales del pórtico espacial de ocho plantas para el espectro tipo 2 y $a_{g}=0,5 g$. (a) Terreno tipo A. (b) Terreno tipo C. (c) Terreno tipo D........

Figura 6.74 Desplazamientos laterales del pórtico espacial de ocho plantas para el espectro tipo 1 y $a_{g}=1,0 g$. (a) Terreno tipo A. (b) Terreno tipo C. (c) Terreno tipo D

Figura 6.75 Drifts para el pórtico espacial de ocho plantas con espectro tipo 2 y $a_{g}=0,5 g$.

(a) Terreno tipo A. (b) Terreno tipo C. (c) Terreno tipo D

Figura 6.76 Drifts para el pórtico espacial de ocho plantas con espectro tipo 1 y $a_{g}=1,0 g$.

(a) Terreno tipo A. (b) Terreno tipo C. (c) Terreno tipo D 
Figura 6.77 Distribución de rótulas plásticas para el pórtico espacial de ocho plantas considerando el espectro tipo $2, a_{g}=0,5 g$ y terreno tipo A. (a) ETABS $®$. (b) Biskinis y Fardis (2010a, 2010b). (c) Expresiones calibradas....

Figura 6.78 Distribución de rótulas plásticas para el pórtico espacial de ocho plantas considerando el espectro tipo 2, $a_{g}=0,5 g$ y terreno tipo C. (a) ETABS $®$. (b) Biskinis y Fardis (2010a, 2010b). (c) Expresiones calibradas.

Figura 6.79 Distribución de rótulas plásticas para el pórtico espacial de ocho plantas considerando el espectro tipo $2, a_{g}=0,5 g$ y terreno tipo D. (a) ETABS®. (b) Biskinis y Fardis (2010a, 2010b). (c) Expresiones calibradas.

Figura 6.80 Distribución de rótulas plásticas para el pórtico espacial de ocho plantas considerando el espectro tipo $1, a_{g}=1,0 g$ y terreno tipo A. (a) ETABS®. (b) Biskinis y Fardis (2010a, 2010b). (c) Expresiones calibradas.

Figura 6.81 Distribución de rótulas plásticas para el pórtico espacial de ocho plantas considerando el espectro tipo $1, a_{g}=1,0 g$ y terreno tipo C. (a) ETABS $₫$. (b) Biskinis y Fardis (2010a, 2010b). (c) Expresiones calibradas.

Figura 6.82 Distribución de rótulas plásticas para el pórtico espacial de ocho plantas considerando el espectro tipo $1, a_{g}=1,0 g$ y terreno tipo D. (a) ETABS $®$. 



\section{Lista de símbolos}

\section{MAYÚSCULAS ROMANAS}

A

$A_{c}$

$A_{c t}$

$A_{\text {máx }}$

$A_{\text {min }}$

$A_{s}$

$A_{s}{ }^{\prime}$

$A_{s, \text { máx }}$

$A_{s^{\prime}, \text { máx }}$

$A_{s, \text { min }}$

$A_{s^{\prime}, \min }$

$A_{s, \text { min mec }}$

$C_{i}$

$C_{i, 0}$

$C M$

CV

$E_{c}$

$E_{m}{ }^{*}$

$E_{s}$

$F^{*}$

$F_{b}$

$F_{i}$

Área bruta de la sección transversal del elemento.

Área de hormigón de la sección transversal del elemento.

Área de hormigón dentro de la zona de tracción, definida en el EC-2..

Área total máxima de armadura longitudinal en pilares.

Área total mínima de armadura longitudinal en pilares.

Área de armadura longitudinal de tracción dispuesta en la sección.

Área de armadura longitudinal de compresión dispuesta en la sección.

Área máxima de armadura longitudinal de tracción en vigas.

Área máxima de armadura longitudinal de compresión en vigas.

Área mínima de armadura longitudinal de tracción en vigas.

Área mínima de armadura longitudinal de compresión en vigas.

Área mínima de armadura longitudinal de tracción en vigas para evitar la rotura frágil según la instrucción EHE-08.

Coeficientes de ajuste de las expresiones de comportamiento seccional con las bases de ensayos.

Valores iniciales de los coeficientes de ajuste $C_{i}$ de las expresiones de comportamiento seccional con las bases de ensayos.

Variable que indica el tipo de carga impuesta en el ensayo: 0 para carga monotónica

o 1 para carga cíclica.

Coeficiente de variación de la variable $x$.

Módulo de deformación longitudinal secante del hormigón.

Energía de deformación del sistema real equivalente de un grado de libertad al generarse el mecanismo plástico.

Módulo de deformación longitudinal del acero de las armaduras.

Fuerza actuante en la base del sistema equivalente de un grado de libertad.

Esfuerzo cortante sísmico en la base de la estructura.

Fuerza lateral aplicada en la planta $i$-ésima de la estructura en el análisis pushover. 
$F_{i}$

$F_{y}{ }^{*}$

$G_{k, j}$

$I(\mathbf{x})$

L

$L_{p}$

$L_{s}$

M

$M_{s}$

$M_{y}$

$M_{y, B F}$

$M_{y, \exp }$

$M_{y, m c}$

$M_{y, p r e d}$

$M_{u}$

N

$N_{\text {máx }}$

$P_{D L R}$

$P F_{1}$

$P_{i, 0}$

$Q_{k, i}$

$Q_{1}$

$Q_{3}$

RI

$R^{2}$

S

$S_{a} / g$
Fuerza lateral normalizada aplicada en la planta $i$-ésima de la estructura en el análisis pushover.

Fuerza actuante en la base del sistema equivalente real de un grado de libertad al generarse el mecanismo plástico.

Valor característico de las acciones permanentes.

Intensidad de la luz emitida por una luciérnaga situada en la posición $\mathbf{x}$.

Longitud del elemento en voladizo.

Longitud de la rótula plástica generada en un elemento estructural.

Distancia entre las secciones de momento flector máximo y nulo en el elemento, determinada como el cociente entre el momento flector $M$ y el esfuerzo cortante $V$ actuantes en la sección de máximo momento flector.

Momento flector actuante en la sección.

Magnitud de ondas superficiales.

Momento flector de plastificación de la sección.

Momento de plastificación de la sección obtenido mediante la expresión de Biskinis y Fardis (2010a).

Valor experimental del momento de plastificación $M_{y}$ de la sección.

Momento de plastificación de la sección obtenido mediante métodos clásicos.

Valor del momento de plastificación $M_{y}$ obtenido mediante las expresiones de comportamiento seccional.

Momento flector último de la sección.

Esfuerzo axil actuante en la sección (positivo si es de compresión y negativo en caso contrario).

Número máximo de iteraciones en el proceso de optimización.

Probabilidad de superación de la acción sísmica correspondiente al requisito de limitación de daño del EC-8.

Factor de participación modal correspondiente al modo fundamental de vibración.

Porcentaje de variación de los valores iniciales de los coeficientes de ajuste $C_{i, 0}$.

Valor característico de las acciones variables.

Primer cuartil de la variable $x$.

Tercer cuartil de la variable $x$

Rango intercuartílico de la variable $x$.

Coeficiente de determinación para la relación entre los valores obtenidos mediante las expresiones de comportamiento seccional $x_{\text {pred }}$ y los valores experimentales $x_{\text {exp }}$ de una variable.

Coeficiente de suelo definido en el EC-8.

Aceleración del sistema equivalente de un grado de libertad, normalizada respecto a la aceración de la gravedad $g$. 
$S_{d}$

$S_{d}{ }^{\prime}$

$S_{e}\left(T^{*}\right)$

$S L$

ST

T

$T^{*}$

$T_{B}$

$T_{C}$

$T_{D}$

$T_{D L R}$

$T P$

$T_{1}$

V

$V_{R, c}$

$V_{R d, c}$

W
Valor absoluto de la suma de las diferencias entre los valores obtenidos mediante las expresiones de comportamiento seccional $x_{\text {pred }} \mathrm{y}$ los valores experimentales $x_{\exp }$ de una variable.

Desplazamiento del sistema equivalente de un grado de libertad.

Ordenada del espectro elástico de respuesta horizontal del EC-8 correspondiente al periodo $T^{*}$.

Variable que indica si, en el ensayo correspondiente, se produce el deslizamiento de la armadura longitudinal respecto al hormigón en la sección de anclaje $(S L=1)$ o no se produce $(S L=0)$.

Variable que depende del tipo de acero empleado en las armaduras: 1 para acero dúctil laminado en caliente (hot-rolled ductile steel); 2 para acero con tratamiento térmico (heat-treated steel); y 3 para acero conformado en frío (brittle cold-worked steel).

Periodo de vibración de un sistema lineal de un grado de libertad.

Periodo de vibración del sistema equivalente de un grado de libertad con comportamiento elástico ilimitado.

Límite inferior del periodo para el tramo de aceleración espectral constante del espectro de respuesta elástica horizontal propuesto por el EC-8.

Límite superior del periodo para el tramo de aceleración espectral constante del espectro de respuesta elástica horizontal propuesto por el EC-8.

Valor que define el comienzo del tramo de respuesta de desplazamiento constante del espectro de respuesta elástica horizontal propuesto por el EC-8.

Periodo de retorno de la acción sísmica correspondiente al requisito de limitación de daño del EC-8.

Variable que indica el tipo de elemento estructural: 0 para vigas y columnas o 1 para muros de cortante.

Periodo fundamental de vibración de la estructura.

Esfuerzo cortante de diseño.

Valor real de la resistencia a esfuerzo cortante de un elemento estructural sin armadura transversal.

Esfuerzo cortante que produce la fisuración diagonal de la sección, definido por el EC-2 como el valor de cálculo del esfuerzo cortante máximo que resiste un elemento estructural sin armadura transversal.

Peso total de la estructura, obtenido al considerar las masas que modelizan los efectos de inercia de la acción sísmica. 


\section{MINÚSCULAS ROMANAS}

$a^{\prime}$

$a_{c}$

$a_{c y}$

$a_{g}$

$a_{g R}$

$a_{s l}$

$a_{s t}$

$a_{v}$

$a_{w, n r}$

$a_{w, r}$

b

b'

$b_{c}$

$b_{i}$

$b_{t}$

$b_{w}$

$\operatorname{cov}\left(x_{\text {pred }}, x_{\text {exp }}\right)$

$d$

d'

$d^{*}$
Ordenada en el origen de la recta de regresión que relaciona los valores obtenidos mediante las expresiones de comportamiento seccional $x_{\text {pred }}$ y los valores experimentales $x_{\exp }$ de una variable.

Aceleración sísmica de cálculo, definida en la norma NCSE-02.

Parámetro que depende del tipo de carga empleada en el ensayo: 1 si la carga es monotónica y 0,6 si es cíclica.

Valor de cálculo de la aceleración del suelo en un terreno tipo A según el EC-8.

Aceleración máxima de referencia del suelo en un terreno tipo A según el EC-8.

Parámetro que indica si en el ensayo correspondiente se produce el deslizamiento entre la armadura longitudinal de tracción y el hormigón $\left(a_{s l}=1\right)$ o no $\left(a_{s l}=0\right)$.

Parámetro que depende del tipo de acero empleado en las armaduras: 1,5 para acero dúctil laminado en caliente (hot-rolled ductile steel); 1,25 para acero con tratamiento térmico (heat-treated steel); y 0,8 para acero conformado en frío (brittle coldworked steel).

Parámetro que considera la influencia del esfuerzo cortante en el giro de plastificación debido a la deformación por flexión: 0 si $V_{R, c}>M_{y} / L_{s}$ y 1 en caso contrario.

Parámetro que considera secciones con geometría diferente a la rectangular, siendo 1 para secciones en $\mathrm{T}, \mathrm{H}, \mathrm{U}$ o rectangular hueca y 0 para secciones rectangulares.

Parámetro que considera el tipo de elemento: 1 para muros de cortante y 0 para vigas y pilares.

Ancho de la sección transversal del elemento.

Coeficiente de regresión de la recta que relaciona los valores obtenidos mediante las expresiones de comportamiento seccional $x_{\text {pred }} \mathrm{y}$ los valores experimentales $x_{\text {exp }}$ de una variable.

Ancho del núcleo de hormigón de la sección tras el desprendimiento del recubrimiento o spalling.

Distancia entre dos barras consecutivas de armadura longitudinal atadas por horquillas o por esquinas de cercos.

Anchura media de la zona de tensión, definida en el EC-2.

Ancho del alma de la sección (para secciones tipo U o rectangular hueca este valor corresponde a la suma de los dos anchos).

Covarianza entre los valores obtenidos mediante las expresiones de comportamiento seccional $x_{\text {pred }} \mathrm{y}$ los valores experimentales $x_{\text {exp }}$ de una variable.

Canto útil de la sección transversal del elemento.

Recubrimiento mecánico de la armadura longitudinal.

Desplazamiento del sistema equivalente de un grado de libertad. 
$d^{*}{ }_{e t} \quad$ Desplazamiento objetivo del sistema equivalente de un grado de libertad con comportamiento elástico ilimitado.

$d_{m}^{*} \quad$ Desplazamiento del sistema real equivalente de un grado de libertad al generarse el mecanismo plástico.

$d^{*}$ Desplazamiento correspondiente al límite elástico del sistema elasto-plástico equivalente de un grado de libertad.

$d_{r}$

Desplome o drift entre plantas, obtenido como la diferencia entre los desplazamientos laterales superior e inferior de la planta.

$d_{t} \quad$ Desplazamiento objetivo de la estructura.

$d_{t}^{*}$

Desplazamiento objetivo del sistema elasto-plástico equivalente de un grado de libertad.

Resistencia a compresión simple del hormigón.

Valor medio de la resistencia efectiva a tracción del hormigón en el momento en que se espera que aparezcan las primeras fisuras, definido en el EC-2.

Valor medio de la resistencia del hormigón a tracción.

Valor de la función objetivo a optimizar para una luciérnaga situada en la posición $\mathbf{x}$.

Límite elástico del acero de la armadura longitudinal de tracción.

Límite elástico del acero de la armadura longitudinal de compresión

Límite elástico del acero de la armadura transversal.

Valor característico del límite elástico del acero de la armadura longitudinal de tracción.

Límite elástico del acero de la armadura vertical de alma.

Tensión de rotura a tracción del acero de las armaduras.

Aceleración de la gravedad.

Canto de la sección transversal del elemento.

Altura de una planta de la estructura.

Canto del núcleo de hormigón de la sección tras el desprendimiento del recubrimiento o spalling.

Coeficiente que considera el efecto de tensiones no uniformes autoequilibradas, definido en el EC-2.

Coeficiente que considera la distribución de las tensiones dentro de la sección inmediatamente antes de la fisuración y del cambio del brazo mecánico, definido en el EC-2.

Número de componentes de los vectores de posición $\mathbf{x}_{i}$ y $\mathbf{x}_{j}$ de las luciérnagas $i$ y $j$, coincidente con el número de variables del problema a optimizar.

Masa del sistema equivalente de un grado de libertad.

Masa de la planta $i$-ésima de la estructura en el análisis pushover.

Ratio entre el módulo de deformación longitudinal del acero de las armaduras $E_{s}$ y el módulo de deformación longitudinal secante del hormigón $E_{c}$.

Número de ensayos considerado para una variable. 
$n_{f} \quad$ Tamaño de la población de luciérnagas.

Coeficiente de comportamiento, definido en el EC-8.

Relación entre las aceleraciones experimentadas por los sistemas equivalentes de un grado de libertad con comportamiento elástico ilimitado y con resistencia limitada. Coeficiente de correlación lineal entre los valores obtenidos mediante las expresiones de comportamiento seccional $x_{\text {pred }} \mathrm{y}$ los valores experimentales $x_{\text {exp }}$ de una variable.

Generador de números aleatorios uniformemente distribuidos en el intervalo $[0,1]$.

Distancia entre las luciérnagas $i$ y $j$.

Desviación típica muestral de la variable $x$.

Separación de la armadura transversal.

Desplazamientos de las masas $m_{i}$ y $m_{j}$ para el modo fundamental de vibración de la estructura.

Velocidad a la que disminuye el parámetro de aleatoriedad $\alpha_{r}$. Espesor del ala comprimida en secciones tipo T, L, H o U.

Parámetro de aleatoriedad obtenido al modificar el término $\alpha_{r}(r d-1 / 2)$.

Parámetro que determina el instante del proceso de optimización donde el parámetro de aleatoriedad $\alpha_{r}$ comienza a reducirse.

Velocidad media de las ondas de corte.

Variable definida, para cada ensayo, mediante el cociente entre el valor experimental $x_{\exp }$ y el obtenido mediante la expresión de comportamiento seccional correspondiente $x_{\text {pred. }}$

Valor medio de la variable $x$

Valor experimental de una variable.

Valor medio de los valores experimentales $x_{\text {exp }}$ de una variable

Valor experimental de una variable correspondiente al ensayo $i$.

Componente $k$-ésima del vector de posición $\mathbf{x}_{i}$ de la luciérnaga $i$.

Valor de una variable obtenido mediante las expresiones de comportamiento seccional para el ensayo $i$.

Componente $k$-ésima del vector de posición $\mathbf{x}_{j}$ de la luciérnaga $j$.

Valor de una variable obtenido mediante las expresiones de comportamiento seccional.

Valor medio de los valores obtenidos mediante las expresiones de comportamiento seccional $x_{\text {pred }}$ para una variable

Distancia entre las fuerzas resultantes de tracción y compresión en la sección, obtenida como la diferencia entre el canto útil de la sección transversal $d$ y el recubrimiento mecánico $d$ ' para vigas y pilares, o 0,8 veces el canto de la sección transversal $h$ para muros de cortante.

Alturas de las masas $m_{i}$ y $m_{j}$ respecto al nivel de aplicación de la acción sísmica. 


\section{MINÚSCULAS ROMANAS (VECTORES Y MATRICES)}

$\mathbf{x}_{i}, \mathbf{x}_{j} \quad$ Vectores de posición de las luciérnagas $i$ y $j$.

\section{MINÚSCULAS GRIEGAS}

$\alpha$

$\alpha$

$\alpha_{1}$

$\beta$

$\beta_{\min }$

$\gamma$

ห

$\gamma_{c}$

$\gamma_{c}{ }^{\prime}$

$\gamma_{e l}$

$\gamma_{s}$

$\delta$

$\delta$

$\varepsilon_{c 0}$

$\varepsilon_{s u}$

$\varepsilon_{y}$$$
\eta
$$

$\theta_{A B}$

$\theta_{e l}$

$\theta_{p}$

$\theta_{y}$

$\theta_{y ; B F}$

$\theta_{\text {y,exp }}$

$\theta_{y, m c}$

$\theta_{y, \text { pred }}$

$\theta_{y 1}$

$\theta_{y 2}$
Factor de efectividad de confinamiento.

Parámetro que introduce aleatoriedad en el movimiento de las luciérnagas por el espacio de diseño.

Coeficiente de masa modal correspondiente al modo fundamental de vibración.

Coeficiente que representa la atracción entre luciérnagas.

Valor mínimo de la atracción entre luciérnagas $\beta$, para grandes distancias entre ellas.

Coeficiente de absorción de luz del medio donde se encuentran las luciérnagas.

Coeficiente de importancia de la estructura.

Coeficiente parcial de seguridad del hormigón.

Peso específico del hormigón.

Coeficiente cuyo valor depende de si el elemento estructural es de carácter sísmico primario $\left(\gamma_{e l}=1,5\right)$ o secundario $\left(\gamma_{e l}=1\right)$.

Peso específico del acero de las armaduras.

Desplazamiento del nudo de control de la estructura en el análisis pushover.

Ratio entre el recubrimiento mecánico $d$ ' y el canto útil de la sección $d$.

Deformación de rotura a compresión simple del hormigón.

Deformación de rotura a tracción del acero de las armaduras.

Deformación de plastificación a tracción del acero de las armaduras, obtenida como

el cociente entre el límite elástico del acero $f_{y}$ y el módulo de deformación longitudinal $E_{s}$.

Coeficiente de corrección del amortiguamiento de la estructura, con valor $\eta=1$ para un amortiguamiento viscoso del $5 \%$ del amortiguamiento crítico.

Giro de la sección.

Giro de la sección de empotramiento del elemento en voladizo.

Componente elástica del giro último de la sección.

Componente plástica del giro último de la sección.

Giro de plastificación de la sección.

Giro de plastificación de la sección obtenido mediante la expresión de Biskinis y Fardis (2010a).

Valor experimental del giro de plastificación $\theta_{y}$ de la sección.

Giro de plastificación de la sección obtenido mediante métodos clásicos.

Valor del giro de plastificación $\theta_{y}$ obtenido mediante las expresiones de comportamiento seccional.

Giro de plastificación debido a la deformación por flexión.

Giro de plastificación debido a la distorsión por esfuerzo cortante. 
$\theta_{y 3} \quad$ Giro de plastificación debido al deslizamiento de la armadura longitudinal de tracción respecto del hormigón.

$\theta_{u} \quad$ Giro último de la sección.

$\theta_{u, B F} \quad$ Giro último de la sección obtenido mediante la expresión de Biskinis y Fardis (2010b).

$\theta_{u, \exp } \quad$ Valor experimental del giro último $\theta_{u}$ de la sección.

$\theta_{u, m c} \quad$ Giro último de la sección obtenido mediante métodos clásicos.

$\theta_{u, \text { pred }} \quad$ Valor del giro último $\theta_{u}$ obtenido mediante las expresiones de comportamiento seccional.

$\mu_{\phi} \quad$ Coeficiente de ductilidad en curvaturas.

$v \quad$ Esfuerzo axil normalizado, obtenido mediante el cociente entre el esfuerzo axil $N$ actuante en la sección y $A f_{c}$, siendo $A$ el área bruta de la sección.

v' Coeficiente de Poisson del hormigón.

$\xi_{y} \quad$ Profundidad de la fibra neutra en el instante de plastificación de la sección.

$\rho \quad$ Cuantía de armadura longitudinal de tracción, definida como el cociente entre el área de armadura longitudinal de tracción $A_{s}$ y $b d$.

$\rho$

Cuantía de armadura longitudinal de compresión, definida como el cociente entre el área de armadura longitudinal de compresión $A_{s}{ }^{\prime}$ y $b d$.

Cuantía de armadura de tracción para que se produzca simultáneamente la plastificación de la armadura de tracción y la máxima deformación de compresión admisible para el hormigón.

Cuantía de armadura diagonal en elementos armados diagonalmente, obtenida como el cociente entre el área de armadura dispuesta a lo largo de una diagonal de la sección y $b d$.

Cuantía de armadura transversal, determinada como el cociente entre el área de armadura transversal $A_{s h}$ y $b s_{h}$.

Cuantía de armadura vertical de alma o web vertical reinforcement ratio, obtenida como el cociente entre el área de armadura longitudinal total situada entre la armadura longitudinal de tracción y compresión y $b d$. Desviación típica poblacional de la variable $x$.

$\sigma_{c p}$ Tensión normal en el hormigón por esfuerzo axil o pretensado, definida en el EC-2.

$\sigma_{s}$ Valor absoluto de la tensión máxima permitida en la armadura inmediatamente después de la formación de la fisura, definido en el EC-2. Desviación típica poblacional de los valores experimentales $x_{\text {exp }}$ de una variable. Desviación típica poblacional de los valores obtenidos mediante las expresiones de comportamiento seccional $x_{\text {pred }}$. Curvatura de la sección. 


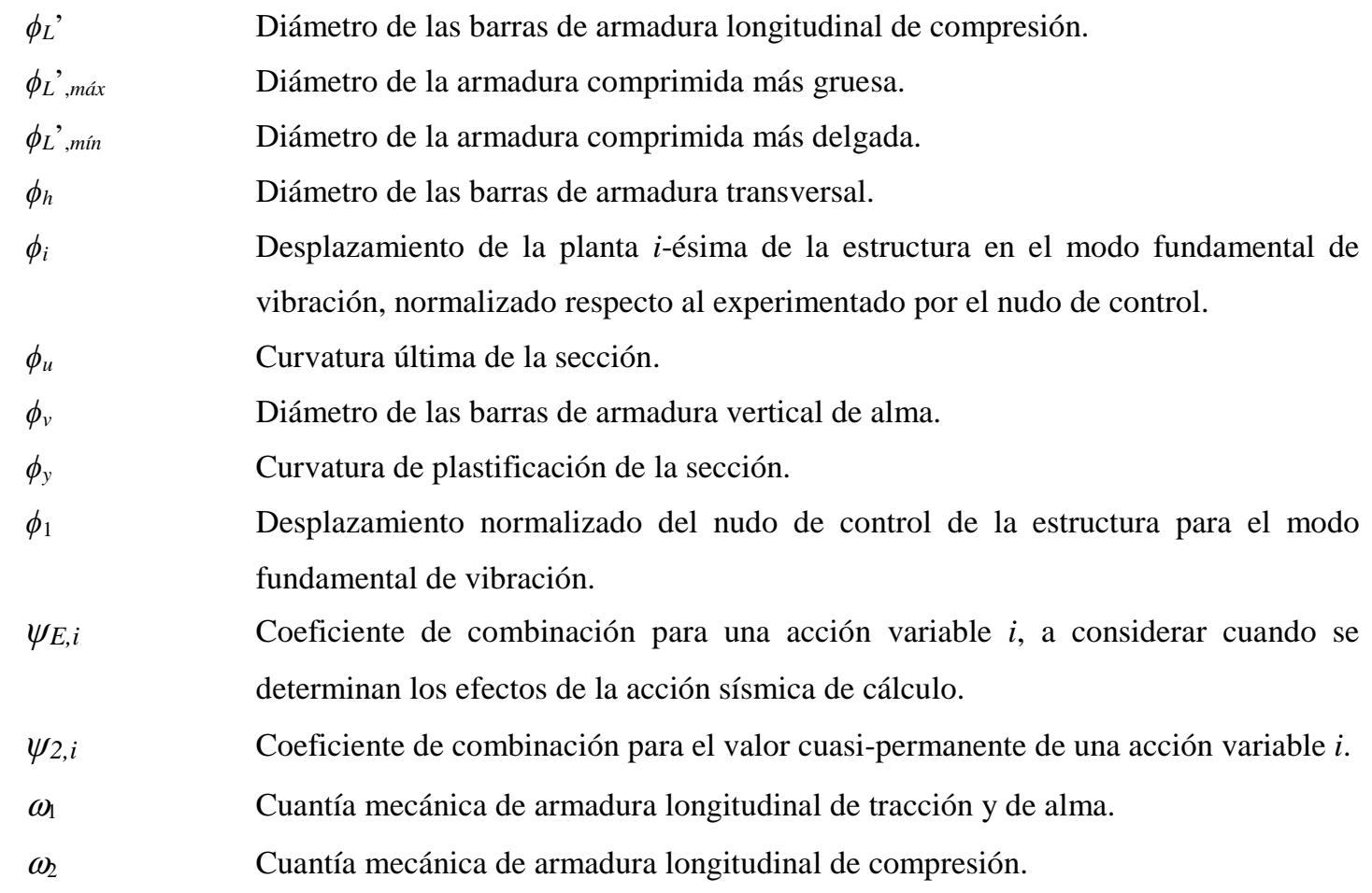

\section{MAYÚSCULAS GRIEGAS}
Factor de transformación que relaciona la estructura real y el sistema equivalente de un grado de libertad.
$\sum$
Suma.

\section{ACRÓNIMOS}
ACHE
Asociación Científico-técnica del Hormigón Estructural.
ACI American Concrete Institute.
ASCE
American Society of Civil Engineers.
ATC
Applied Technology Council.
$\mathrm{C}$
Sección tipo conforming según la FEMA (2000).
CEB
Comité Européen du Béton.
CP
Nivel de comportamiento collapse prevention, definido en la FEMA (2000).
EC Eurocódigo.
FEMA Federal Emergency Management Agency.
FIB
Fedération Internationale du Béton.
$\mathrm{NC}$
Sección tipo nonconforming según la FEMA (2000).
NCSE-02
Norma de Construcción Sismorresistente: Parte General y Edificación.
NEHRP National Earthquake Hazards Reduction Program.
IO
Nivel de comportamiento immediate occupancy, definido en la FEMA (2000).
LS
Nivel de comportamiento life safety, definido en la FEMA (2000).
$\mathrm{RC}$
Reinforced Concrete. 



\section{Introducción y objetivos}

\subsection{INTRODUCCIÓN}

\subsubsection{Modelización de estructuras de hormigón armado}

El análisis y diseño de estructuras requieren la aplicación de procedimientos de cálculo capaces de caracterizar convenientemente la respuesta de las mismas. Para abordar problemas complejos resulta necesario emplear modelos numéricos que simulen la realidad de la forma más precisa posible. La necesidad de considerar aspectos de comportamiento cada vez más complejos, unido al continuo avance en el campo computacional, han propiciado el desarrollo de modelos numéricos avanzados para abordar el problema con cierta garantía. Especial consideración merecen los modelos empleados en análisis no lineal, donde aspectos como la no linealidad geométrica y del material repercuten en la respuesta estructural.

El análisis sísmico constituye un caso particular de análisis no lineal que debe ser abordado con rigor. El comportamiento de las estructuras frente a la acción sísmica adquiere especial relevancia por la repercusión que genera en la sociedad la pérdida de vidas humanas y los elevados costes económicos derivados de un inadecuado diseño estructural.

El hormigón es uno de los materiales de construcción más utilizados en todo el mundo. El complejo comportamiento de este material en régimen no lineal exige el empleo de modelos numéricos cada vez más sofisticados para modelizar su comportamiento.

La modelización numérica de las estructuras de hormigón armado sometidas a la acción sísmica se puede abordar desde diferentes enfoques. Así, se encuentran modelos de comportamiento seccional, capaces de proporcionar información acerca de conceptos relativos a los estados de plastificación y último de la sección; modelos locales, encargados de simular el comportamiento de histéresis de las rótulas plásticas generadas en la estructura durante el transcurso de la acción sísmica; y modelos globales, 
confeccionados para simular el comportamiento general de la estructura. La interacción entre los diferentes tipos de modelos resulta fundamental para abordar el problema de forma integral. En la Figura 1.1a se muestra, a modo de ejemplo, el esquema de un elemento estructural en el que se generan rótulas plásticas, modelizado mediante dos modelos globales, mostrándose en la Figura 1.1.b el diagrama de histéresis momento flector-giro $(M-\theta)$ que representa el comportamiento de una de las rótulas mediante modelos locales.

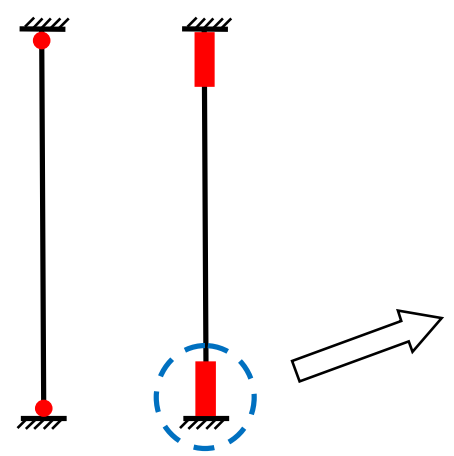

(a)

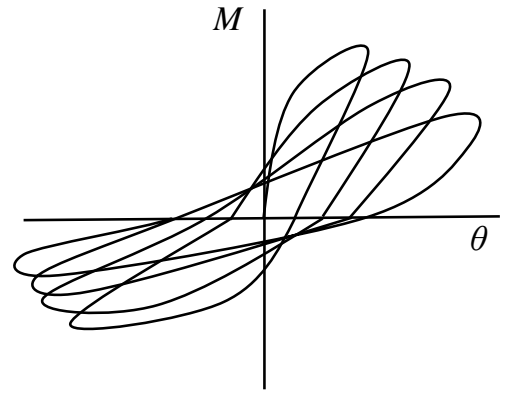

(b)

Figura 1.1 Modelos de comportamiento no lineal. (a) Modelos globales. (b) Modelos locales.

\subsubsection{Modelización de secciones de hormigón armado}

El desarrollo de modelos locales que reflejen el comportamiento de histéresis de las rótulas plásticas requiere la obtención de expresiones matemáticas que reproduzcan conceptos como el momento, la curvatura y el giro en los estados de plastificación y último de la sección. Dichos conceptos se obtienen aplicando modelos de comportamiento seccional, considerando aspectos como la geometría de la sección, las características de los materiales o la configuración de la armadura.

Los conceptos correspondientes a los estados de plastificación y último pueden obtenerse, fundamentalmente, siguiendo dos estrategias:

a) desarrollando subrutinas que imponen condiciones de equilibrio en la sección mediante procesos iterativos, $\mathrm{o}$

b) empleando expresiones calibradas con ensayos experimentales.

Las expresiones de comportamiento seccional, aunque resultan adecuadas para el diseño, tienen su principal aplicación en la evaluación de estructuras existentes, resultando especialmente útiles para el planteamiento de medidas de adecuación sísmica. Por otro lado, la relativa sencillez de estas expresiones y el hecho de estar exentas de rutinas de cálculo hacen que resulten muy eficientes desde el punto de vista computacional, por lo que su aplicación resulta de gran utilidad en la modelización sísmica. 
Para que las expresiones de comportamiento seccional ofrezcan unas adecuadas prestaciones, resulta fundamental su correcta calibración con resultados experimentales. La calibración de estas expresiones se ha abordado tradicionalmente mediante métodos sencillos, como el ajuste por mínimos cuadrados y el método de prueba y error. Estos métodos han ofrecido resultados satisfactorios cuando las expresiones no resultan excesivamente complejas, o cuando la extensión de la base de datos con la que se calibran es reducida. Sin embargo, si se pretende calibrar expresiones de marcado carácter no lineal, que presentan un elevado número de parámetros, con bases de datos extensas, resulta conveniente la utilización de otros métodos numéricos que permitan automatizar el proceso y obtengan un ajuste con un nivel de precisión adecuado. Para alcanzar este objetivo, las técnicas de optimización se erigen como métodos numéricos adecuados, pues con su utilización se obtienen valores de los parámetros que proporcionan el mejor ajuste posible entre los valores ofrecidos por las expresiones y los resultados experimentales.

A pesar de que las técnicas de optimización han experimentado importantes avances en los últimos años, no resulta común su aplicación a la calibración de modelos numéricos. De entre las técnicas de optimización aplicadas a la calibración, los algoritmos metaheurísticos suelen emplearse con mayor frecuencia, debido a su facilidad de convergencia hacia resultados satisfactorios.

Las bases de datos con las que se calibran las expresiones de comportamiento seccional deben ser representativas desde el punto de vista estadístico. Si además, dichas bases de datos contienen exclusivamente elementos estructurales que cumplan con las prescripciones constructivas y sísmicas impuestas por las principales normas y códigos de diseño, las expresiones calibradas de esta forma reproducirían adecuadamente el comportamiento de elementos habitualmente construidos en la práctica, pudiéndose aplicar en el proyecto de estructuras situadas en zonas sísmicas.

\subsection{OBJETIVOS DE LA TESIS}

La calibración de expresiones de comportamiento seccional con bases extensas de datos experimentales requiere el empleo de estrategias de optimización que proporcionen los valores de los parámetros que maximizan el ajuste entre los resultados experimentales y los obtenidos con dichas expresiones. Para medir la bondad del ajuste, resulta necesario evaluar una serie de conceptos estadísticos, los cuales permiten comparar los resultados obtenidos respecto a otros métodos.

Con objeto de obtener expresiones de comportamiento seccional con un ajuste óptimo a la base de ensayos disponible y que puedan ser aplicadas a edificios convencionales de hormigón armado situados en zonas sísmicas, pueden plantearse los siguientes objetivos para esta tesis: 
a) Realizar el estado del arte de las investigaciones más significativas en materia de modelización numérica del comportamiento sísmico de estructuras de hormigón armado, y del empleo de técnicas de optimización en la calibración de modelos numéricos.

b) Analizar una base de datos existente en la literatura compuesta por más de 1000 ensayos experimentales, seleccionando, a partir de ella, un conjunto de elementos que cumplan las prescripciones constructivas y de carácter sísmico impuestas por diversa normativa de diseño.

c) Estudiar las expresiones de comportamiento seccional desarrolladas por diversos autores, realizando un análisis de sensibilidad de los diferentes parámetros.

d) Plantear estrategias numéricas mediante técnicas de optimización para abordar la calibración de las expresiones con la base de datos experimentales disponible.

e) Validar las expresiones calibradas mediante el análisis comparativo de diferentes métodos de modelización del comportamiento de secciones de hormigón armado y de las rótulas plásticas generadas durante el análisis pushover de pórticos de hormigón armado.

\subsection{ORGANIZACIÓN DE LA TESIS}

Esta tesis se ha estructurado en siete capítulos, incluyendo este primer capítulo de introducción y objetivos.

En el capítulo 2 se realiza una revisión de las investigaciones desarrolladas en el campo de la modelización numérica del comportamiento sísmico de las estructuras de hormigón armado, analizando los distintos enfoques mediante los que se puede abordar este tema. Adicionalmente, se revisan las técnicas de optimización empleadas para la calibración de modelos numéricos. Posteriormente se citan las publicaciones relacionadas con la tesis presentada por el doctorando durante el desarrollo de la misma.

El capítulo 3 se ha dedicado a la descripción de la base de ensayos existente en la literatura, detallándose los parámetros y los aspectos sísmicos y constructivos de la normativa considerada para realizar la selección de ensayos.

En el capítulo 4 se ha realizado la descripción de las expresiones de comportamiento seccional que se utilizan para realizar la calibración con la base de ensayos, detallando las particularidades de los algoritmos empleados en la misma. Se muestran las expresiones resultantes y se analizan los resultados obtenidos mediante una serie de parámetros estadísticos.

En el capítulo 5 se analiza el comportamiento relativo a los estados de plastificación y último de dos secciones de hormigón armado bajo diferentes situaciones, comparando los resultados obtenidos al aplicar las expresiones calibradas en esta tesis con los de otros métodos. 
En el capítulo 6 se realizan diversos análisis pushover sobre cuatro pórticos planos, modelizando las rótulas plásticas que aparecen durante el análisis mediante diferentes métodos y comparando los resultados obtenidos.

Finalmente, en el capítulo 7 se establece una serie de conclusiones y se plantean ciertos aspectos que constituyen futuras líneas de investigación. 



\section{Estado del arte}

\subsection{INTRODUCCIÓN}

En este capítulo se realiza una revisión de los diferentes modelos numéricos disponibles en la literatura para la simulación numérica de estructuras de hormigón armado sometidas a acciones de naturaleza sísmica.

Es necesario destacar que, desde sus inicios, este problema comenzó a abordarse desde diferentes ópticas, en función del aspecto concreto que se deseara modelizar. Por tanto, existe una extensa variedad de modelos que pueden agruparse, según lo que se quiera modelizar, en tres grupos claramente diferenciados pero que, al mismo tiempo, interactúan entre sí:

a) modelos globales,

b) modelos locales, $y$

c) modelos de comportamiento seccional.

El objetivo de esta revisión no es una descripción exhaustiva de todos los modelos disponibles, sino establecer una panorámica de las investigaciones más importantes en los tres grandes campos de estudio en los que se puede dividir la modelización numérica del comportamiento sísmico de estructuras de hormigón armado.

Adicionalmente, se describen los algoritmos de optimización de mayor relevancia y su aplicación a la calibración de modelos numéricos con resultados experimentales.

\subsection{MODELOS GLOBALES}

\subsubsection{Generalidades}

Desde sus inicios, la modelización sísmica de estructuras de hormigón armado se ha concebido desde diferentes enfoques, desarrollándose modelos que estudian el problema desde un punto de vista general (modelos globales) y otros que simulan el comportamiento de ciertas zonas localizadas (modelos locales) o de la sección de un elemento estructural (modelos de comportamiento seccional). Con objeto de abordar el 
problema de una forma completa e integrada, resulta indispensable una correcta interacción entre estos tres tipos de modelos, siendo necesaria, en muchas ocasiones, la implementación de unos modelos dentro de otros.

El objetivo fundamental de los modelos globales consiste en reflejar el comportamiento general de la estructura durante el transcurso de las solicitaciones de carácter sísmico, simulando el comportamiento de los diferentes elementos de carácter estructural que la componen (vigas, pilares, muros de cortante, etc.) mediante un planteamiento general. Así, los modelos globales tienen como misión principal evaluar aspectos tales como los desplazamientos, esfuerzos y ductilidad en la estructura, concebida ésta como un sistema cuya respuesta viene definida por el comportamiento de cada una de sus partes.

El comportamiento sísmico de una estructura de hormigón armado puede ser, en ciertas ocasiones, un problema fuertemente condicionado por los efectos de naturaleza no lineal desde el punto de vista de la geometría, resultando imprescindible la consideración de ciertos efectos en los modelos globales, como los efectos P-Delta o los grandes desplazamientos.

Para la elaboración de modelos globales, resulta necesario establecer ciertas hipótesis de comportamiento de los diferentes elementos de carácter estructural que componen la estructura. Las hipótesis de comportamiento de mayor relevancia resultan ser la localización, longitud y posible propagación en el elemento de las rótulas plásticas o plastic hinges que se generen durante el transcurso de la acción sísmica. El establecimiento de estas hipótesis resulta fundamental a la hora de confeccionar el modelo, influyendo en dos aspectos muy importantes: el grado de aproximación a la realidad de los elementos estructurales y el coste computacional del modelo. En general, modelos muy sofisticados en los que se quiera simular de forma precisa el comportamiento de los elementos estructurales presentan un elevado coste computacional.

Desde su aparición en los años 60, muchos han sido los modelos de carácter global desarrollados para el estudio del comportamiento general de estructuras de hormigón armado sometidas a la acción sísmica. Atendiendo a los criterios de localización y características de las rótulas plásticas desarrolladas en un elemento, los modelos globales pueden dividirse en tres grupos claramente diferenciados:

a) modelos de plasticidad concentrada o lumped models,

b) modelos de plasticidad distribuida o distributed plasticity models, y

c) modelos de disipación de energía concentrada.

\subsubsection{Modelos de plasticidad concentrada}

Los modelos de plasticidad concentrada o lumped models adoptan, como hipótesis principales, los siguientes aspectos: 
a) la formación de rótulas plásticas se produce únicamente en los extremos del elemento, permaneciendo el resto en estado elástico, y

b) las rótulas plásticas desarrolladas presentan una longitud nula.

Las hipótesis adoptadas por los modelos de plasticidad concentrada no se corresponden con la realidad de estructuras de hormigón armado sometidas a acciones de naturaleza sísmica, ya que, en realidad, las zonas donde se desarrolla el comportamiento no lineal del material se propagan desde los extremos hacia el interior de los elementos mediante la formación de rótulas plásticas de una cierta longitud. Sin embargo, a pesar de su carácter aproximado, los modelos de plasticidad concentrada resultan muy eficientes desde el punto de vista computacional, proporcionando resultados suficientemente precisos en un tiempo aceptable. Este aspecto hace que sean unos de los modelos más usados en la actualidad para la modelización sísmica de estructuras de hormigón armado.

\subsubsection{Modelos anteriores a los 90}

En general, la modelización numérica de elementos de hormigón armado mediante los modelos de plasticidad concentrada depende de la formulación empleada. En función de dicha formulación, los elementos se pueden asemejar a sistemas de muelles actuando en serie o en paralelo. El primer modelo de plasticidad concentrada del que se tiene constancia es el denominado modelo de dos componentes, desarrollado por Clough, Benuska y Wilson (1965). Este modelo supone que los elementos estructurales están compuestos por dos componentes actuando en paralelo: una componente enteramente elástica y una componente elasto-plástica con rótulas plásticas situadas en los extremos del elemento. Algunos autores, como Giberson (1969), Powell (1972) y Mahin y Bertero (1975), destacaron como principal inconveniente de este modelo que sólo podía utilizarse con diagramas de histéresis de tipo bilineal. Posteriormente, Giberson (1967) propuso el modelo de una componente, estableciendo que un elemento estructural está constituido por un elemento elástico lineal y dos muelles rotacionales de carácter no lineal para simular las deformaciones plásticas en sus extremos, estando todas las componentes del sistema en serie. La simplicidad de este modelo y la posibilidad de utilizarlo con cualquier tipo de diagrama de histéresis hacen que, a día de hoy, siga siendo ampliamente utilizado.

A finales de los años 60 y mediados de los 70, los modelos globales fueron incorporando paulatinamente conceptos de mayor complejidad, mejorando la aproximación a la realidad del comportamiento sísmico de las estructuras de hormigón armado. En esta línea, Aoyama y Sugano (1968) fueron los primeros investigadores en considerar una relación trilineal momento flector-giro para modelizar la fisuración del hormigón previa a la plastificación de las armaduras, desarrollando el denominado modelo de cuatro componentes. Este modelo permitía concebir un elemento estructural como un conjunto compuesto por tres elementos elasto-plásticos para modelizar la 
fisuración del hormigón y la plastificación de las armaduras y un elemento enteramente elástico para modelizar la parte que permanece elástica y lineal. Uno de los modelos de plasticidad concentrada más influyentes corresponde a Otani (1974). Este modelo permitía reflejar aspectos de difícil consideración para la época, como el deslizamiento de las armaduras en los extremos del elemento estructural y el carácter indeformable del nudo viga-pilar. Si bien este modelo resulta complicado e ineficiente para su aplicación al diseño, sus novedosas aportaciones lo convirtieron en punto de partida para la modelización de ciertos aspectos en investigaciones posteriores.

No fue hasta mediados de los años 80 cuando los modelos globales comenzaron a considerar la influencia del esfuerzo cortante en el comportamiento sísmico de un elemento estructural. Así, Keshavarzian y Schnobrich (1984) propusieron una mejora del modelo de una componente que permitía considerar, de forma directa, la interacción esfuerzo axil-momento flector y el efecto del esfuerzo cortante en la formulación de la matriz de flexibilidad del elemento.

Los modelos de plasticidad concentrada desarrollados hasta mediados de los años 80 permitían exclusivamente la modelización de elementos para su análisis en estructuras situadas en el plano. Para solventar esta limitación, Lai, Will y Otani (1984) confeccionaron un modelo capaz de simular el comportamiento sísmico de un elemento considerando la interacción esfuerzo axil-flexión biaxial. Posteriormente, Powell y Chen (1986) propusieron un modelo que consideraba la interacción entre esfuerzo axil, momento torsor y flexión biaxial en la modelización de pilares en 3D.

\subsubsection{2 Últimas tendencias}

En los últimos años, el empleo de modelos de plasticidad concentrada resulta muy común para simular el comportamiento de estructuras de hormigón armado sometidas a la acción sísmica, debido a su relativa simplicidad y sus ventajas desde el punto de vista de la rapidez computacional. Resulta necesario incidir en el hecho de que los modelos de plasticidad concentrada que se emplean en la actualidad están fuertemente influenciados por los primeros modelos de plasticidad concentrada que surgieron, siendo, en muchas ocasiones, modificaciones y mejoras de los mismos.

Siguiendo este argumento, Hopper (2009) empleó un modelo de plasticidad concentrada basado en el modelo de una componente, incorporando en los extremos del elemento dos segmentos rígidos que simulan el carácter indeformable del nudo vigapilar. Mazza y Mazza (2010) desarrollaron un modelo de dos componentes, apto para el análisis de estructuras porticadas tridimensionales, ofreciendo la posibilidad de utilizar un diagrama momento-curvatura de tipo bilineal dependiente del esfuerzo axil. Los mismos autores (Mazza y Mazza, 2011) presentaron mejoras en el modelo anterior que permitían modelizar el comportamiento de una estructura frente a la componente vertical de la acción sísmica, y en un trabajo posterior (Mazza y Mazza, 2012) 
emplearon el mismo modelo para estudiar el efecto que la dirección de la acción sísmica induce en el comportamiento global de la estructura.

El empleo de modelos de plasticidad concentrada para la modelización del colapso de estructuras frente a acciones de tipo dinámico también ha sido objeto de estudio por parte de algunos investigadores. En concreto, Kaewkulchai y Williamson (2004) desarrollaron un modelo que permitía considerar en el proceso de colapso aspectos como la interacción axil-momento flector y el daño experimentado por el hormigón, mientras que Isobe y Tsuda (2003) estudiaron, de forma específica, el colapso de edificios de hormigón armado frente a acciones de tipo sísmico.

Algunos estudios se han centrado en comparar las prestaciones de diferentes modelos. Así, López-Almansa, Alfarah y Oller (2014) estudiaron la capacidad de un pórtico plano de hormigón armado, contrastando los resultados ofrecidos por un modelo de plasticidad concentrada con los obtenidos aplicando modelos más avanzados.

\subsubsection{Modelos de plasticidad distribuida}

A diferencia de los modelos de plasticidad concentrada, en los modelos de plasticidad distribuida se puede producir la plastificación de la sección en cualquier parte del elemento. La plastificación comienza en las secciones extremas y se propaga posteriormente hacia el interior del elemento, adquiriendo las rótulas plásticas una longitud finita y variable durante el transcurso de la acción sísmica.

Los modelos de plasticidad distribuida ofrecen una descripción más precisa del comportamiento sísmico de elementos de hormigón armado que la ofrecida por los modelos de plasticidad concentrada, debido a que permiten modelizar la degradación de rigidez progresiva que experimenta el elemento a medida que las rótulas plásticas se propagan a lo largo de su longitud. En este tipo de modelos, el comportamiento constitutivo de la sección transversal del elemento se puede obtener de dos formas diferentes:

a) en términos de resultantes de tensiones y deformaciones, y

b) explícitamente, mediante una discretización de la sección en fibras (modelos de fibras).

$\mathrm{Su}$ compleja formulación y mayor coste computacional hacen que, en ciertas ocasiones, la aplicación de los modelos de plasticidad distribuida resulte menos conveniente que la de los modelos de plasticidad concentrada.

\subsubsection{Modelos de plasticidad distribuida en términos de resultantes de tensiones y deformaciones}

Los primeros modelos de plasticidad distribuida en los que el comportamiento constitutivo de la sección transversal se obtiene en términos de resultantes de tensiones y deformaciones surgieron a mediados de los años 70. Así, Takayanagi y Schnobrich (1976) desarrollaron el modelo de múltiples subelementos, concebido para la 
modelización de sistemas de muros de cortante acoplados. Los mismos investigadores (Takayanagi y Schnobrich, 1979) modificaron posteriormente este modelo, implementando en sus propiedades de histéresis las características específicas de los sistemas de muros acoplados.

El primer modelo de plasticidad distribuida que considera la longitud de las rótulas plásticas desarrolladas en el elemento se debe a los trabajos de Soleimani (1979) y Soleimani, Popov y Bertero (1979). Estos autores desarrollaron el modelo ZAP, según el cual un elemento está constituido por los siguientes subelementos: zona elástica, rótulas plásticas en sus extremos, muelles rotacionales junto a las rótulas plásticas y elementos rígidos a continuación. Este modelo permitía considerar la deformación debida al deslizamiento de las armaduras y el carácter indeformable del nudo viga-pilar. En años posteriores tuvo lugar la aparición de algunas mejoras del modelo ZAP. De este modo, Keshavarzian y Schnobrich (1984) aplicaron el modelo ZAP a pilares con esfuerzos axiles variables, considerando la interacción momento flector-esfuerzo axil. Darvall y Mendis (1985) presentaron un método alternativo para el cálculo de las longitudes de las rótulas plásticas, empleando diferentes diagramas de histéresis. Filippou e Issa (1988) modelizaron elementos tipo viga empleando una superposición de subelementos diferente a la del modelo ZAP.

La importancia del tipo de formulación empleada en los modelos de plasticidad distribuida en los que el análisis se realiza en términos de resultantes quedó patente en los años 80 y 90, con el desarrollo de modelos con formulación basada en desplazamientos (Hughes, 1987; Zienkiewicz y Taylor, 2000) y en fuerzas (Spacone, Filippou y Taucer, 1996). Otras aportaciones (Neuenhofer y Filippou, 1997) realizan una reflexión acerca de las ventajas de la formulación basada en fuerzas respecto a la basada en desplazamientos.

\subsubsection{Modelos de fibras}

La década de los 70 coincidió con el desarrollo de los modelos de fibras, en los que se establece una doble discretización en un elemento estructural: se realiza una subdivisión en una serie de secciones transversales, las cuales se subdividen, a su vez, en pequeñas áreas de dimensión finita (fibras). En el caso de flexión uniaxial, la discretización de la sección se realiza mediante capas (modelos de capas o layer models), estableciéndose una doble subdivisión en áreas rectangulares o fibras para el caso de flexión biaxial (modelos de fibras o fiber models). Cada fibra representa una porción de hormigón o acero, dependiendo de su posición en la sección, obteniéndose el comportamiento constitutivo global de la sección mediante la integración de las respuestas de todas las fibras.

Los modelos de fibras utilizan diferentes procedimientos en su formulación. Los primeros modelos empleaban una formulación del elemento basada en rigidez, constituyendo el trabajo de Aktan, Pecknold y Sozen (1974) un ejemplo en el que se 
emplearon funciones de forma clásicas. Posteriormente comenzó el auge de los modelos de fibras con formulación en flexibilidad, en los que la flexibilidad del elemento se obtiene mediante la integración de las flexibilidades de las distintas secciones consideradas a lo largo de su longitud. Destacan en esta línea los trabajos de Kaba y Mahin (1984), quienes propusieron un modelo para su aplicación a pilares con flexión uniaxial que presentaba varias inconsistencias según Cardinetti (2011), y Zeris y Mahin (1988 y 1991), que aplicaron la formulación del modelo de Kaba y Mahin al caso de pilares con flexión biaxial. También resultan frecuentes los modelos de fibras con formulación mixta, los cuales desarrollan un procedimiento que combina las formulaciones en rigidez y flexibilidad. Un ejemplo es la aportación de Spacone, Ciampi y Filippou (1996), quienes obtienen las fuerzas nodales resistentes en un elemento estructural mediante un proceso iterativo.

Los modelos de fibras más sofisticados permiten la simulación de otros tipos de deformaciones de naturaleza no lineal, como es el caso de la deformación debida a esfuerzos cortantes. Dentro de este grupo se incluyen los modelos basados en modelos de bielas y tirantes, en los que se incorpora una estructura de tirantes para resistir el esfuerzo cortante (Guedes, Pegon y Pinto, 1994; Martinelli, 1998); modelos de microplanos, basados en una restricción cinemática según la cual la deformación externa de un elemento queda ligada a una serie de microplanos previamente seleccionados (Petrangeli, Pinto y Ciampi, 1999); modelos basados en modelos de fisuración difusa (Vecchio y Collins, 1988; Vecchio, 2000; Rose, 2001; Remino, 2004); y modelos basados en modelos de daño del hormigón (Mazars, 1984; La Borderie, 1991; Mazars et al., 2006).

\subsubsection{3 Últimas tendencias}

En la última década, los modelos de plasticidad distribuida en los que el análisis de la sección se realiza en términos de resultantes de tensiones y deformaciones han tenido numerosas aportaciones, si bien los modelos de fibras son los más avanzados y empleados actualmente. La temática tratada por ambos tipos de modelos es muy variada. Así, Kyakula y Wilkinson (2004) desarrollaron un modelo en el que las vigas y los pilares de la estructura están constituidos por subelementos conectados en serie para considerar la plasticidad en cualquier parte del elemento; Scott y Fenves (2006) plantearon un método que permitía considerar la longitud de la rótula plástica de un modo numéricamente estable; Roh, Reinhorn y Lee (2012) propusieron un modelo que permitía considerar varios tipos de distribución de curvaturas a lo largo de la longitud de la rótula plástica, ofreciendo un mayor grado de aproximación a la realidad del elemento; y Botez, Bredean e Ioani (2014) contrastaron los resultados obtenidos mediante modelos de plasticidad concentrada y distribuida al simular el colapso de dos edificios de hormigón armado. 
Resulta interesante la introducción de fenómenos de compleja modelización en los modelos de fibras empleados en los últimos años, así como su aplicación a muros de cortante. Así, Légeron, Paultre y Mazars (2005) desarrollaron un modelo de capas en el que se consideraba el daño experimentado por el hormigón, aplicándolo al diseño de estructuras mediante la filosofía Performance Based Earthquake Engineering (PBEE) (FEMA, 1997), y Men et al. (2006) utilizaron un modelo de fibras para la modelización sísmica de muros empleando elementos tipo shell.

La importancia de los modelos constitutivos escogidos para el hormigón y el acero de las armaduras en los modelos de fibras quedó patente en el trabajo de Zhou et al. (2011), quienes simularon el comportamiento sísmico de pilares empleando el modelo modificado de Kent y Park para el hormigón (Mander, Priestley y Park, 1988) y el modelo de Légeron para el acero de las armaduras (Légeron, Paultre y Mazars, 2005). En los últimos años, resulta común la combinación de modelos constitutivos que consideran el daño experimentado por el hormigón con los modelos de fibras. Algunos ejemplos son los trabajos de Grassl y Jirasek (2006), Voyiadjis, Taqieddin y Kattan (2008) y Li y Hatzigeorgiou (2012).

En los últimos años resulta cada vez más común el desarrollo de modelos de fibras empleados para el diseño sísmico-probabilístico basado en prestaciones o probabilistic performance-based seismic design (FIB, 2012b), como el modelo propuesto por Heo y Kunnath (2013) para evaluar el daño en los elementos estructurales de pórticos planos de hormigón armado.

La modelización de pilas de puente también se ha abordado recientemente mediante modelos de fibras. Así, Feng, Kowalsky y Nau (2014) desarrollaron un modelo para estudiar el comportamiento no lineal de pilas con sección circular, comparando los resultados con lo obtenido en ensayos estáticos y dinámicos.

\subsubsection{Modelos de disipación de energía concentrada}

Desde el trabajo pionero planteado por Kachanov (1958), la mecánica de la degradación se ha convertido en uno de los campos de investigación más activos en lo referente a la mecánica del sólido. La idea fundamental de esta teoría es la introducción de una nueva variable interna, el daño, que mide la densidad superficial de microfisuras y microporos del material y su influencia en sus propiedades. De acuerdo con Cipollina, LópezInojosa y Flórez-López (1995), esta idea básica resulta tan simple y general que ha sido usada para la modelización, hasta la fractura local, de la mayoría de los materiales de construcción. Sin embargo, la mecánica de la degradación, por sí sola, no resulta lo más adecuado para el análisis de muchas estructuras en el campo de la ingeniería civil, debido a que los modelos basados en esta teoría sólo pueden ser empleados para el análisis de estructuras relativamente simples.

Como consecuencia de los estudios desarrollados en la Universidad de los Andes (Venezuela) en el campo del análisis estructural (Cipollina, 1992; Cipollina, Febres- 
Cedillo y Flórez-López, 1992; Flórez-López, 1993a; Flórez-López, 1993b; LópezInojosa, 1995; Flórez-López, 1995; Cipollina y Flórez-López, 1995) surgen, con objeto de abordar el análisis sísmico de estructuras de hormigón armado, los modelos de disipación de energía concentrada. Estos modelos presentan una formulación que generaliza los modelos de plasticidad concentrada, incluyendo en los mismos los efectos del daño en el material y obteniendo, por consiguiente, unos resultados más cercanos a la realidad. A pesar de que ofrecen resultados bastante aceptables, su compleja formulación implica que estos modelos presenten una elevada exigencia desde el punto de vista computacional, aspecto que limita su campo de aplicación a estructuras que no contengan un elevado número de elementos.

Cipollina, López-Inojosa y Flórez-López (1995) desarrollaron un modelo de disipación de energía concentrada basado en el trabajo de Flórez-López (1993b), que permitía la modelización de pórticos planos. Posteriormente, el mismo Flórez-López (1996) presentó un modelo aplicable para acciones sísmicas en el que se proponen dos conjuntos de variables de daño del hormigón, estando cada conjunto definido para un sentido de carga.

Con objeto de considerar los efectos de fatiga en el material, surgen algunos modelos que generalizan el criterio clásico de Griffith (Griffith, 1921), empleado en la mecánica de la fractura, según el cual la propagación de fisuras sólo es posible si la energía liberada en el desarrollo de dichas fisuras es igual o superior a la resistencia a la fisuración del material. En este sentido, Thomson, Bendito y Flórez-López (1998) obtuvieron una nueva ley de daño que permitía considerar el efecto del número de ciclos en el deterioro del hormigón.

No fue hasta la aparición del modelo desarrollado por Marante y Flórez-López (2002) cuando se pudo aplicar los modelos de disipación de energía concentrada a estructuras 3D con elementos sometidos a flexión biaxial. Posteriormente, los mismos Marante y Flórez-López (2003) propusieron una mejora del modelo anterior, considerando la influencia de un esfuerzo axil variable y un momento torsor en el comportamiento del elemento.

Thomson y Flórez-López (2004) y Thomson et al. (2009) fueron los primeros investigadores en extender el campo de aplicación de los modelos de disipación de energía concentrada a los muros de cortante, considerando efectos de cierta complejidad, como la disminución de rigidez y resistencia debido a la fisuración diagonal producida por el esfuerzo cortante y las deformaciones permanentes debidas a la plastificación del acero de la armadura transversal.

En los últimos años, los modelos de disipación de energía concentrada están experimentando una tendencia hacia la consideración de fenómenos cada vez más complejos. Así, Adriano y Proença (2008) desarrollaron un modelo en el que se introdujeron tres nuevos conceptos: la consideración de deformaciones residuales debidas tanto al comportamiento del hormigón como del acero, el empleo de una 
función de daño alternativa y la introducción de una variable de giro debido al deslizamiento entre caras de microfisuras. Alva y Homce (2010) confeccionaron un modelo en el que se realiza una modificación de la función de resistencia de fisura respecto a los modelos anteriormente desarrollados, introduciendo un parámetro adicional que depende de la cuantía de armadura longitudinal, la forma de la sección transversal o el tipo de hormigón. Otros autores (Grassl et al., 2013) estudiaron el comportamiento de elementos estructurales sometidos a tracción y a estados de compresión multiaxial.

Ciertos estudios incluyeron modelos de disipación de energía concentrada aplicados a elementos en los que la geometría era un factor determinante. En esta línea, Perdomo et al. (2013) desarrollaron modelos para elementos con diferentes proporciones entre sus dimensiones (vigas, pilares, muros y elementos cortos), estableciendo conclusiones de su comportamiento sísmico en función de dichas proporciones. Amorim, Proença y Flórez-López (2013) propusieron un modelo aplicado a arcos de hormigón armado, permitiendo considerar incluso los efectos de la no linealidad geométrica.

Los modelos de disipación de energía concentrada se han aplicado también al estudio del colapso de estructuras frente a cargas sísmicas. En esta línea, Faleiro, Barbat y Oller (2005) presentaron un algoritmo para el análisis de estructuras de hormigón armado cuyos resultados predecían de forma aproximada el colapso de un pórtico de dos plantas y un vano. Faleiro, Oller y Barbat (2008) desarrollaron posteriormente un modelo de plasticidad concentrada en el que introdujeron un índice de daño local para evaluar el estado de daño de cada elemento y un índice de daño global para reflejar el estado de daño de toda la estructura, resultando estos índices de gran utilidad para evaluar la máxima carga sísmica que puede soportar una estructura. Posteriormente, Amorim, Proença y Flórez-López (2014) propusieron un modelo para simular el colapso sísmico de pórticos y arcos de hormigón armado.

La Figura 2.1 muestra el esquema de modelización de un elemento según algunos modelos globales. Si se desea disponer de más referencias acerca de los modelos globales, así como de una descripción más detallada de los mismos, véase López-López (2012).

\subsection{MODELOS LOCALES}

\subsubsection{Generalidades}

La modelización numérica de estructuras de hormigón armado frente a acciones de tipo sísmico requiere una adecuada simulación del comportamiento de ciertas zonas específicas de la estructura, como los nudos entre vigas y pilares o los empotramientos de pilares y muros en su base. Estas zonas son susceptibles de albergar la formación de rótulas plásticas durante el transcurso de la acción sísmica. La aparición de dichas 
rótulas influye considerablemente en el comportamiento global de la estructura, por lo que resulta imprescindible simular adecuadamente su comportamiento.

Para realizar una correcta modelización de las rótulas plásticas, resulta fundamental reflejar adecuadamente el comportamiento de histéresis de las mismas, simulando ciertos fenómenos característicos que tienen lugar en estructuras de hormigón armado cuando actúa la acción sísmica, esto es, la degradación de resistencia y rigidez del hormigón, el endurecimiento del acero de las armaduras, el efecto pinching o aumento repentino de la rigidez del tramo de recarga debido al cierre de fisuras en el hormigón, la caída de resistencia, o el efecto Bauschinger experimentado por el acero de las armaduras, según el cual se produce una disminución de su límite elástico en el sentido contrario de carga.
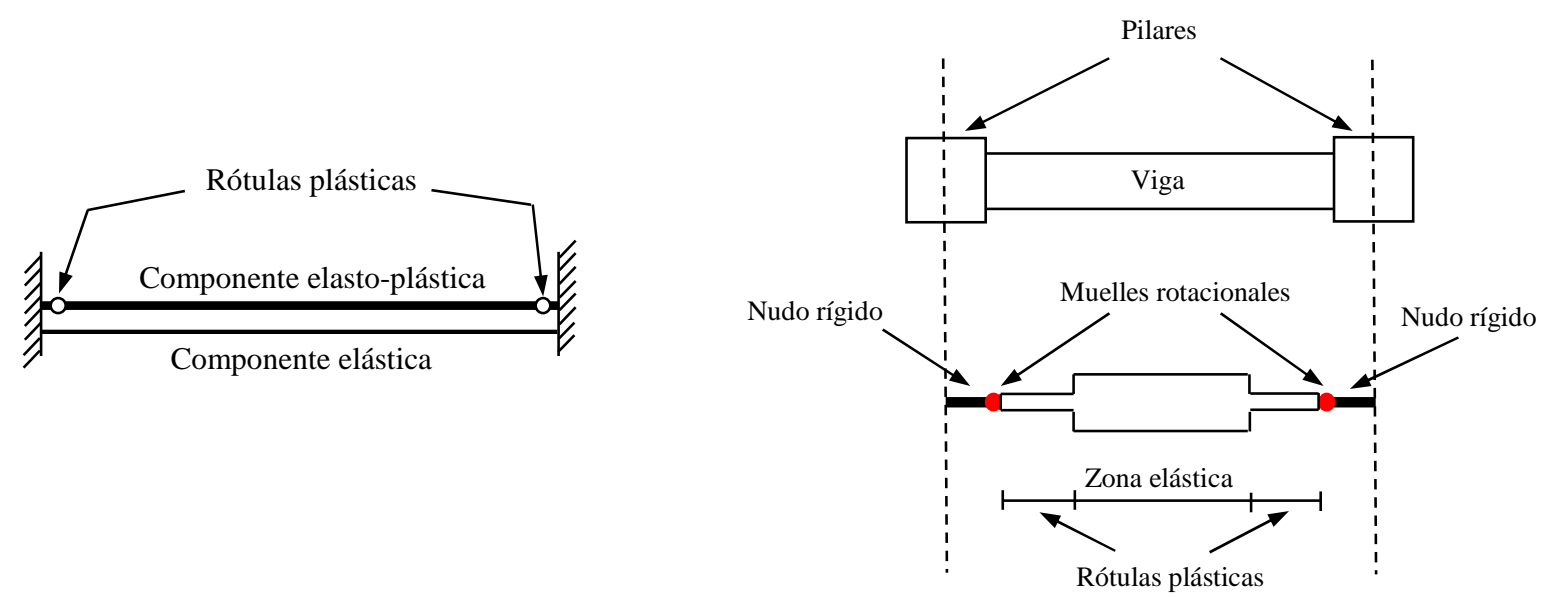

(a)

(b)

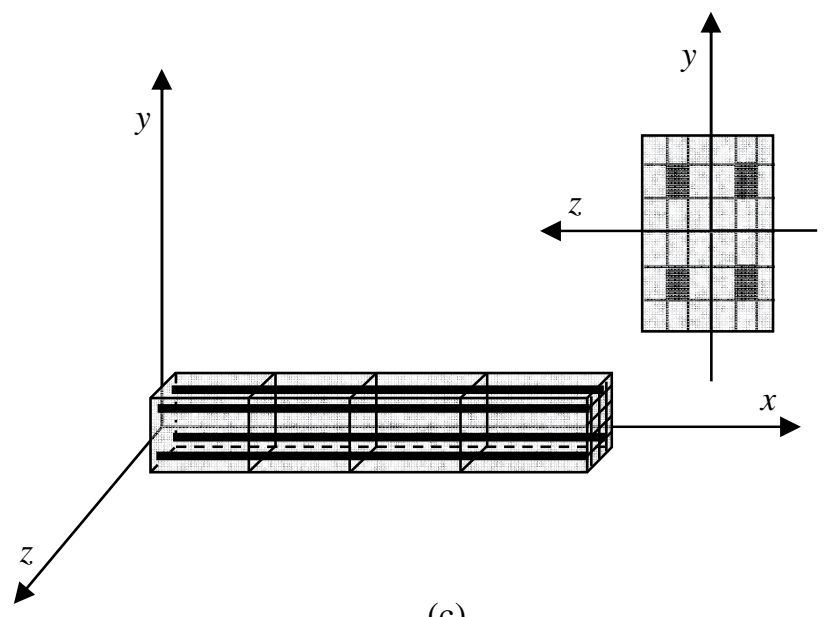

(c)

Figura 2.1 Modelización de un elemento según diferentes modelos. (a) Modelo de dos componentes (b) Modelo ZAP (c) Modelo de fibras. 
El comportamiento de histéresis de las rótulas plásticas se ha de simular mediante modelos locales, conocidos también como diagramas de histéresis, que puedan ser posteriormente implementados en modelos globales para, de este modo, obtener el comportamiento general de la estructura frente a la acción sísmica.

Tras la aparición de los primeros diagramas de histéresis, cuya excesiva simplicidad los hacía únicamente aplicables a elementos de acero, se han ido desarrollando modificaciones de los mismos para mejorar la precisión de los resultados y adaptarlos al comportamiento del hormigón armado. Los avances en los métodos numéricos y el aumento de la capacidad computacional provocan constantes mejoras en los diagramas de histéresis. Esta circunstancia implica que ciertos diagramas, que supusieron en su momento grandes avances, se consideren hoy simplistas.

Gran parte de las aplicaciones de los modelos numéricos empleados para la simulación sísmica de estructuras de hormigón armado se han desarrollado para estructuras situadas en el plano, resistiendo sus elementos momentos flectores que actúan según un único eje. En efecto, los primeros diagramas de histéresis surgieron para modelizar el comportamiento local de este tipo de elementos, con objeto de establecer una primera aproximación a la realidad del problema para estructuras sencillas. El primer diagrama de histéresis reconocido fue desarrollado por Ramberg y Osgood (1943), empleado para caracterizar el comportamiento de histéresis de piezas de acero en el campo de la ingeniería aeronáutica. De acuerdo con García (1998), este diagrama era de limitada aplicación a elementos estructurales de hormigón armado al no considerar los efectos de degradación de rigidez y resistencia del hormigón. Un diagrama de histéresis de especial trascendencia a lo largo de la historia es el diagrama bilineal, desarrollado por Goodman y Klumpp (1955), según el cual la relación esfuerzo-deformación está constituida por dos tramos lineales: un primer tramo elástico y un segundo tramo que simula el proceso de endurecimiento por deformación posterior a la plastificación del material. Posteriormente, Tanabashi (1956) desarrolló el diagrama elasto-plástico ordinario, que puede ser considerado como un caso particular del diagrama bilineal, en el que la pendiente del tramo de endurecimiento por deformación es nula. A pesar de que su empleo resulta más adecuado en elementos de acero, Veletsos, Newmark y Chelapati (1965) recurrieron a este diagrama para obtener de forma aproximada la respuesta de osciladores lineales simples de hormigón armado sujetos a diferentes tipos de excitaciones sísmicas.

Los años 60 y 70 coincidieron con la aparición de mejoras en los diagramas elasto-plástico ordinario y bilineal, las cuales dieron lugar a diagramas de histéresis aplicables a elementos estructurales de hormigón armado. Siguiendo esta estrategia, Clough (1966) desarrolló el diagrama elasto-plástico degradado y el diagrama bilineal degradado. Estos diagramas se diferenciaban de sus antecesores en que la pendiente del tramo de recarga es inferior a la del tramo elástico, aspecto que permite caracterizar la degradación del hormigón con los sucesivos ciclos de carga. Posteriormente, Imbeault y 
Nielsen (1973) desarrollaron el diagrama de degradación bilineal, modificando los valores de las pendientes de los tramos de descarga y recarga respecto al diagrama bilineal.

No fue hasta la década de los 70 cuando los diagramas de histéresis comenzaron a introducir novedades importantes respecto a los primeros modelos propuestos. Así, Takeda, Sozen y Nielsen (1970) desarrollaron el denominado diagrama de Takeda, proporcionando las siguientes mejoras:

a) existencia de tres tramos en la relación esfuerzo-deformación: tramo elástico, tramo fisurado y tramo de endurecimiento por deformación;

b) formulación obtenida calibrada con resultados experimentales; y

c) consideración de la deformación producida por el esfuerzo cortante.

La robustez de este diagrama hace que pueda ser considerado como uno de los diagramas de histéresis de referencia, habiendo sido objeto de modificaciones y mejoras por diversos investigadores a lo largo de los años. Así, Otani (1975) propuso el diagrama de Takeda simplificado, obtenido mediante la eliminación de las reglas del diagrama de Takeda relativas a la fisuración del hormigón. Otro trabajo que introdujo conceptos novedosos fue el desarrollado por Anderson y Townsend (1977), quienes desarrollaron el diagrama de conexión de degradación trilineal, en el que la pendiente del tramo inicial de carga era inferior a la del tramo elástico para considerar el efecto de fenómenos como la fisuración del hormigón y la deformación producida por el esfuerzo cortante.

La mitad de la década de los 70 dio lugar a la aparición de los primeros diagramas de histéresis empleados para el análisis 3D de estructuras sujetas a acciones sísmicas con componentes en dos direcciones ortogonales, basados en la teoría de la plasticidad clásica (Prager y Hodge, 1951), y que permitían considerar las interacciones inelásticas de los diferentes esfuerzos actuantes en la sección del elemento mediante la definición de superficies de interacción. Estos diagramas presentaban una formulación compleja y, por consiguiente, resultaban exigentes desde el punto de vista computacional, aspectos que limitaban su campo de aplicación a ejemplos sencillos. Dentro de este grupo, destacan las aportaciones de Pecknold (1974), que propuso el diagrama biaxial bilineal, y Takizawa y Aoyama (1976), quienes desarrollaron el diagrama biaxial de degradación trilineal.

Otros diagramas de histéresis cuya importancia es necesario destacar son los basados en el diagrama Bouc-Wen, desarrollado inicialmente por Bouc (1971) y generalizado posteriormente en el trabajo de Wen (1976). De acuerdo con Ismail, Ikhouane y Rodellar (2009), este modelo ha sido empleado en muchas ocasiones para modelizar el comportamiento histerético de diferentes dispositivos, especialmente en el campo de la ingeniería civil y mecánica (Badoni y Makris, 1996; Oldfield, Ouyang y Mottershead, 2005; Ikhouane, Mañosa y Rodellar, 2007). Algunas modificaciones posteriores del diagrama de Bouc-Wen las constituyen los trabajos de Baber y Wen 
(1981), quienes consideraron la degradación de resistencia y rigidez del hormigón en función de la energía disipada en los sucesivos ciclos de histéresis, y Baber y Noori (1985), que realizaron una aproximación a la modelización del efecto pinching, proponiendo el conocido como diagrama de Bouc-Wen-Baber-Noori.

El efecto pinching también fue tratado en el diagrama Sina, desarrollado por Saiidi y Sozen (1979). En el mismo trabajo, estos autores propusieron un diagrama de histéresis alternativo, el Q-hyst, que puede ser considerado como una modificación del diagrama bilineal. De acuerdo con Saiidi (1982), el diagrama Q-Hyst es un diagrama relativamente simple que se ajusta bien a resultados experimentales, lo que le convertía, en el momento de su aparición, en un diagrama adecuado para estructuras donde podían desarrollarse un elevado número de rótulas plásticas. Posteriormente, Filippou, Popov y Bertero (1983) propusieron un modelo de histéresis concebido exclusivamente para nudos entre vigas y pilares, simulando el efecto de la adherencia hormigón-acero y su deterioro a lo largo de los sucesivos ciclos de carga.

Aunque la influencia del efecto del esfuerzo cortante fue considerada anteriormente por otros autores de forma indirecta, algunos investigadores (Oesterle et al., 1979; Maruyama y Jirsa, 1979; Takayanagi, Derecho y Corley, 1979; Saatcioglu, Ozcebe y Lee, 1988; Saatcioglu y Ozcebe, 1988) se centraron en el tratamiento de este problema, concluyendo que las distorsiones inelásticas producidas por los esfuerzos cortantes podrían ser importantes en zonas de formación de rótulas plásticas, incluso cuando el comportamiento global del elemento está gobernado por esfuerzos de flexión. Partiendo de estos trabajos, Ozcebe y Saatcioglu (1989) desarrollaron un diagrama de histéresis concebido específicamente para modelizar la respuesta histerética de elementos frente a esfuerzo cortante. Adicionalmente, Alath y Kunnath (1995) desarrollaron un diagrama de histéresis para considerar la deformación debida al esfuerzo cortante de nudos viga-pilar, mientras que Biddah y Ghobarah (1999) consideraron la influencia conjunta del esfuerzo cortante y la pérdida de adherencia hormigón-acero. Otros estudios desarrollados para modelizar la influencia del esfuerzo cortante en el comportamiento histerético de los nudos viga-pilar corresponden a Hwang y Lee (1999), Youssef y Ghobarah (2001), Lowes y Altoontash (2003) y Altoontash (2004).

El efecto del amortiguamiento efectivo existente en un elemento estructural a lo largo de los diferentes ciclos de histéresis ha sido también objeto de estudio por parte de Fardis y Panagiotakos (1996), quienes cuantificaron este concepto en función de la ductilidad y los parámetros del modelo.

\subsection{2 Últimas tendencias}

Los modelos locales desarrollados en los últimos años parten, en muchos casos, de la base proporcionada por modelos anteriores, aportando novedades para su aplicación a numerosas líneas de investigación y considerando aspectos cada vez más complejos que 
mejoren la simulación del comportamiento histerético de un elemento estructural. Así, Galal y Ghobarah (2003) propusieron un modelo basado en la teoría de la plasticidad para considerar la influencia de la flexión biaxial y Shin y LaFave (2004) modelizaron los nudos viga-pilar considerando el giro debido a la plastificación de la armadura longitudinal.

Algunos investigadores desarrollaron diagramas de histéresis obtenidos mediante la superposición de modelos previamente propuestos para considerar diferentes efectos. En esta línea, Ibarra, Medina y Krawinkler (2005) desarrollaron el diagrama bilineal modificado, el diagrama peak oriented y el diagrama pinching. Posteriormente, Sezen y Chowdhury (2009) propusieron un diagrama de histéresis que se obtenía mediante la combinación de otros tres diagramas que consideraban las deformaciones debidas a la flexión, el esfuerzo cortante y el deslizamiento de armaduras en el nudo viga-pilar, apoyándose en los trabajos de Saatcioglu, Alsiwat y Ozcebe (1992), Sezen y Moehle (2003) y Setzler (2005).

La interacción flexión-esfuerzo cortante también ha sido considerada en trabajos recientes. Un ejemplo lo constituye el diagrama de Xu y Zhang (2011), concebido para modelizar el comportamiento no lineal de pilares de hormigón armado bajo carga axil constante.

En la última década surgen algunos modelos locales que consideran el daño experimentado por el hormigón. Destacan los trabajos de Wang y Shah (2006), quienes desarrollaron un modelo en el que se cuantificaba el daño experimentado por el hormigón en función de los ciclos de carga, y Zhang, Xu y Tang (2011), los cuales estudiaron el comportamiento de pilares considerando la interacción flexión-esfuerzo cortante y la acumulación de daño en el material a lo largo de los ciclos de carga.

Otras aportaciones presentan aplicaciones variadas. Favvata, Izzuddin y Karayannis (2008) modelizan los nudos exteriores viga-pilar, incorporando en sus características de histéresis la degradación de rigidez y el efecto pinching, y Novelli (2008) estudió la influencia de las proporciones de la sección transversal y el nivel de esfuerzo axil en el comportamiento histerético del elemento.

Algunos modelos locales fueron concebidos para simular el comportamiento de elementos con ausencia de detalles adecuados para zonas sísmicas. Chung et al. (2007) modelizaron pilares típicos de la ciudad de Hong Kong, sujetos a elevados esfuerzos axiles y con bajas cuantías volumétricas de armadura transversal. Sengupta y Li (2011, 2013) propusieron un modelo para simular el comportamiento de histéresis de nudos viga-pilar con bajas cuantías de armadura transversal.

Algunos modelos, incluso, permiten considerar el efecto que la duración de la acción sísmica ejerce en el comportamiento de histéresis. Un ejemplo de este planteamiento lo constituye el modelo de Ou et al. (2014), aplicado a pilas de puente.

Si se desea disponer de más referencias acerca de los modelos locales, así como de una descripción más detallada de los mismos, véase López-López (2012). 


\subsection{MODELOS DE COMPORTAMIENTO SECCIONAL}

La correcta determinación del comportamiento seccional de un elemento de hormigón armado resulta necesaria para el posterior desarrollo de un modelo de histéresis que simule de forma precisa el comportamiento sísmico de las posibles rótulas plásticas que se desarrollen en la estructura. Concretamente, se hace necesario el desarrollo de modelos capaces de determinar conceptos relacionados con la capacidad resistente y de deformación de una sección en los estados de plastificación y último, como la curvatura, el momento y el giro.

En la literatura técnica, es posible encontrar diversos métodos para la determinación de la capacidad de deformación en los estados de plastificación y último de una sección de hormigón armado, clasificados según tres grupos claramente diferenciados:

a) métodos empíricos,

b) métodos analíticos simplificados, $\mathrm{y}$

c) métodos analíticos avanzados.

El primer grupo lo constituyen modelos que, si bien presentan un cierto contenido teórico, se obtienen fundamentalmente mediante un ajuste con resultados experimentales. Los métodos analíticos simplificados engloban modelos donde los fundamentos teóricos tienen una mayor presencia, aunque también incluyen la obtención de ciertos aspectos mediante ensayos experimentales. Los métodos analíticos avanzados permiten modelizar de forma más precisa ciertos fenómenos de mayor complejidad, típicos del hormigón armado, como la fisuración, el efecto tensionstiffening, la adherencia acero-hormigón, el pandeo de la armadura longitudinal o el confinamiento debido a la armadura transversal, entre otros.

\subsubsection{Métodos empíricos}

Los ensayos experimentales constituyen, en opinión de algunos investigadores (Panagiotakos y Fardis, 2001), el mejor recurso para la validación, calibración y desarrollo de modelos que permitan obtener la capacidad de deformación de la sección transversal de un elemento estructural.

En esta línea, Park y Ang (1985) y Priestley (1998) ofrecen una primera aproximación de la curvatura y el giro de plastificación de una sección. Estos índices de deformación se obtienen en función de la deformación de plastificación de la armadura longitudinal $\varepsilon_{y}$ y de las dimensiones del elemento (longitud y canto de la sección), a través de simples cocientes, utilizando diferentes coeficientes de ajuste con resultados experimentales en función de que el elemento sea una viga, un pilar con sección rectangular o circular o un muro de cortante. Posteriormente, Panagiotakos y Fardis (2001) plantean expresiones para la obtención del giro de plastificación y el giro último de secciones rectangulares de hormigón armado en función de las características 
geométricas y mecánicas del elemento, calibrándolas con una base de datos de más de 1000 ensayos. En este trabajo, se proponen tres expresiones para el giro último, dependiendo de si el tipo de carga a la que se somete el elemento es monotónica, cíclica o ambos, y otra expresión adicional que considera explícitamente la influencia del número de ciclos de histéresis experimentados por dicho elemento.

El trabajo inicial propuesto por Panagiotakos y Fardis (2001) fue objeto de posteriores mejoras y ajustes, como es el caso de las expresiones propuestas por la Fedération Internationale du Béton (FIB, 2003a y 2003b) para la obtención del giro último en elementos sometidos a cargas monotónicas y cíclicas o las modificaciones realizadas por Fardis y Biskinis (2003), quienes reajustaron la expresión del giro último para cargas monotónicas o cíclicas y el giro de plastificación a una base de datos de más de 1300 ensayos, suponiendo que la situación de rotura se produce con un 20\% de caída en la rama post-pico de la curva constitutiva.

Las últimas modificaciones al trabajo inicial de Panagiotakos y Fardis (2001) se deben a Biskinis y Fardis (2010a, 2010b), quienes modificaron las expresiones anteriores para el giro último y el giro de plastificación, extendiendo su campo de aplicación a secciones no sólo rectangulares, sino también en $\mathrm{T}, \mathrm{H}, \mathrm{U}$ y secciones rectangulares huecas. La base de datos empleada para la calibración de las expresiones incluye hormigones con fibras poliméricas o elementos estructurales rehabilitados con encamisado de hormigón. Además, estos autores ofrecen expresiones para la obtención del giro de plastificación y último para el caso de flexión biaxial.

Otro modelo empírico a destacar, aunque de menor aplicación según Barrera (2012), es el desarrollado por Lam et al. (2003), en el que se propone una expresión para el giro último a partir de 15 ensayos experimentales de soportes de hormigón convencional resistiendo a flexo-compresión cíclica.

\subsubsection{Métodos analíticos simplificados}

Para determinar la capacidad de deformación de una sección en los estados de plastificación y último se requiere, en ciertas ocasiones, la elaboración de modelos con una mayor presencia de conceptos teóricos. Los métodos analíticos simplificados, basados en los modelos de soporte en voladizo, presentan esta característica, obteniendo ciertos índices de deformación mediante la consideración de la distribución de curvaturas a lo largo de la longitud de la rótula plástica $L_{p}$ desarrollada en el elemento.

Park y Paulay (1974) y Paulay y Priestley (1992) fueron algunos de los primeros investigadores en proponer expresiones para la curvatura, momento y giro de plastificación y la curvatura y momento último de una sección que, aunque aproximadas, resultaban de ciertos fundamentos teóricos. Según estos autores, la curvatura de plastificación se puede producir por la plastificación de la armadura de tracción o por alcanzarse la máxima deformación admisible del hormigón a compresión. 
Posteriormente, algunos investigadores propusieron expresiones para la obtención de la curvatura de plastificación y última, asumiendo un diagrama momento-curvatura de tipo bilineal. Algunos ejemplos son Priestley y Park (1987) y Lehman y Moehle (2000), quienes plantearon, apoyándose en los fundamentos teóricos desarrollados por los primeros trabajos, expresiones ajustadas a resultados procedentes de ensayos de pilas para puentes.

Uno de los métodos analíticos simplificados de mayor relevancia corresponde a Panagiotakos y Fardis (2001), quienes introdujeron nuevas expresiones para la determinación de la curvatura y momento de plastificación y la curvatura y giro últimos. Según estos autores, la curvatura última se obtiene como el menor valor de la curvatura que produce la rotura de la armadura longitudinal de tracción y la que produce el agotamiento del hormigón a compresión, considerando el efecto spalling o desprendimiento del recubrimiento de la sección. Como continuación a este trabajo, Fardis y Biskinis (2003) reajustaron las expresiones desarrolladas a una base de datos mayor, y Biskinis y Fardis (2010a, 2010b) ampliaron el campo de aplicación de las expresiones anteriores a secciones en $\mathrm{T}, \mathrm{H}, \mathrm{U}$ y rectangulares huecas, proponiendo incluso un método de cálculo del momento último considerando el efecto spalling.

Una adecuada estimación de la longitud de la rótula plástica desarrollada en un elemento resulta fundamental para la determinación del giro último de su sección. Dicha estimación resulta difícil, debido a la influencia de distintos efectos de compleja modelización, lo que obliga, en muchas ocasiones, a recurrir a expresiones calibradas con resultados experimentales. Una primera expresión para determinar la longitud de la rótula plástica, propuesta por Baker y Amarakone (1964), se obtuvo a través de 90 ensayos experimentales realizados en 13 países diferentes. Posteriormente, otros autores consideraron ciertos aspectos específicos, como el efecto producido por la rama de endurecimiento por deformación del acero en la resistencia última del elemento (Mattock, 1965; Corley, 1966; Mattock, 1967) o una relación entre el momento de plastificación y último de 0,85 (Sawyer, 1964).

En la década de los 80 y 90 surge el trabajo de diversos investigadores (Mander, Priestley y Park, 1984; Zahn, 1985; Priestley y Park, 1987; Paulay y Priestley, 1992; Priestley, Seible y Calvi, 1996; Lehman, 1998; Lehman y Moehle, 2000; Panagiotakos y Fardis, 2001), que conciben la obtención de la longitud de la rótula plástica considerando dos términos, uno debido a la flexión y otro debido al deslizamiento de armaduras en los extremos del elemento. Otras expresiones más sofisticadas corresponden a Ceroni et al. (2005), quienes obtuvieron la longitud de la rótula plástica mediante un modelo de fibras, o la expresión propuesta por Bae (2005), que considera la distribución de deformaciones en el contorno del núcleo confinado a lo largo del soporte para obtener la longitud de la rótula plástica. Otros autores (Panagiotakos y Fardis, 2001; Fardis y Biskinis, 2003 y Biskinis y Fardis, 2010a y 2010b) ofrecen expresiones 
particularizadas en función de si el elemento está sometido a cargas monotónicas o cíclicas.

\subsubsection{Métodos analíticos avanzados}

La obtención de la capacidad de deformación de una sección puede requerir el empleo de modelos analíticos más precisos, que permitan considerar ciertos fenómenos de mayor complejidad sin la necesidad de recurrir a ensayos experimentales. A este grupo pertenecen los modelos analíticos avanzados, que se centran, fundamentalmente, en la determinación de los giros de plastificación y último de una sección.

Los modelos analíticos avanzados ofrecen la posibilidad de modelizar de forma precisa efectos como la influencia de la fisuración debida al esfuerzo cortante y su modelización discreta o difusa, la adherencia hormigón-acero, el uso de diferentes modelos constitutivos para el hormigón y el acero, el efecto del pandeo de la armadura longitudinal o el confinamiento de la armadura transversal.

Las décadas de los 80 y 90 propiciaron la aparición de una serie de modelos aplicables a elementos sometidos a flexión sin carga axial. En este grupo, destacan los modelos de Stuttgart, Nápoles, Darmstadt-Leipzig, Delft, Zürich y Shakir-Rogowsky.

El modelo de Stuttgart fue propuesto por Langer (1987) y extendido posteriormente por Li (1995). Este modelo permite el cálculo del giro último para secciones de vigas de hormigón armado y pretensado a partir de la relación momentocurvatura, obtenida ésta en función de la geometría de la sección, su configuración de armadura y las relaciones constitutivas tensión-deformación para el hormigón y el acero. El modelo de Nápoles, desarrollado por Cosenza, Greco y Manfredi (1991, 1992) y Manfredi (1995) permite determinar el giro de plastificación de una sección, asumiendo como hipótesis principales la diferente deformación entre el hormigón y el acero, que el efecto tension-stiffening se restringe a una zona concreta con esfuerzos constantes y que la distancia entre fisuras es constante.

El modelo de Darmstad-Leipzig, desarrollado por Pommerening (1996), se aplica para obtener el giro último de una sección, calculando separadamente el debido a la flexión y al esfuerzo cortante, y considerando las alteraciones que la fisuración introduce en la relación constitutiva del acero para armar y pretensar. Bigaj y Walraven (1993) desarrollaron el modelo de Delft mediante un estudio analítico y experimental, permitiendo considerar el efecto escala de la sección y el efecto localization en la zona donde se desarrolla la rótula plástica para obtener el giro último de una sección. El modelo de Zürich se centra en el estudio del campo de tensiones discontinuas (Schlaich y Schäfer, 1984; Muttoni, Schwartz y Thürlimann, 1989; Sigrist, 1995), aplicándolo a la obtención del giro último en vigas. Finalmente, destaca la aportación de Shakir y Rogowsky (2000), que obtuvieron el giro último de una sección mediante la integración de las curvaturas a lo largo de la longitud de la rótula plástica. 
La cantidad de modelos analíticos avanzados aplicables a elementos sometidos a flexión y esfuerzo axil es escasa. Destaca el modelo de Bae (2005), que permite obtener el desplazamiento máximo de un soporte en voladizo, considerando la influencia del confinamiento del hormigón y el pandeo de la armadura longitudinal.

La Figura 2.2 muestra, a modo de resumen, un esquema general de los principales grupos de modelos desarrollados a lo largo del tiempo para la modelización sísmica de estructuras de hormigón armado.

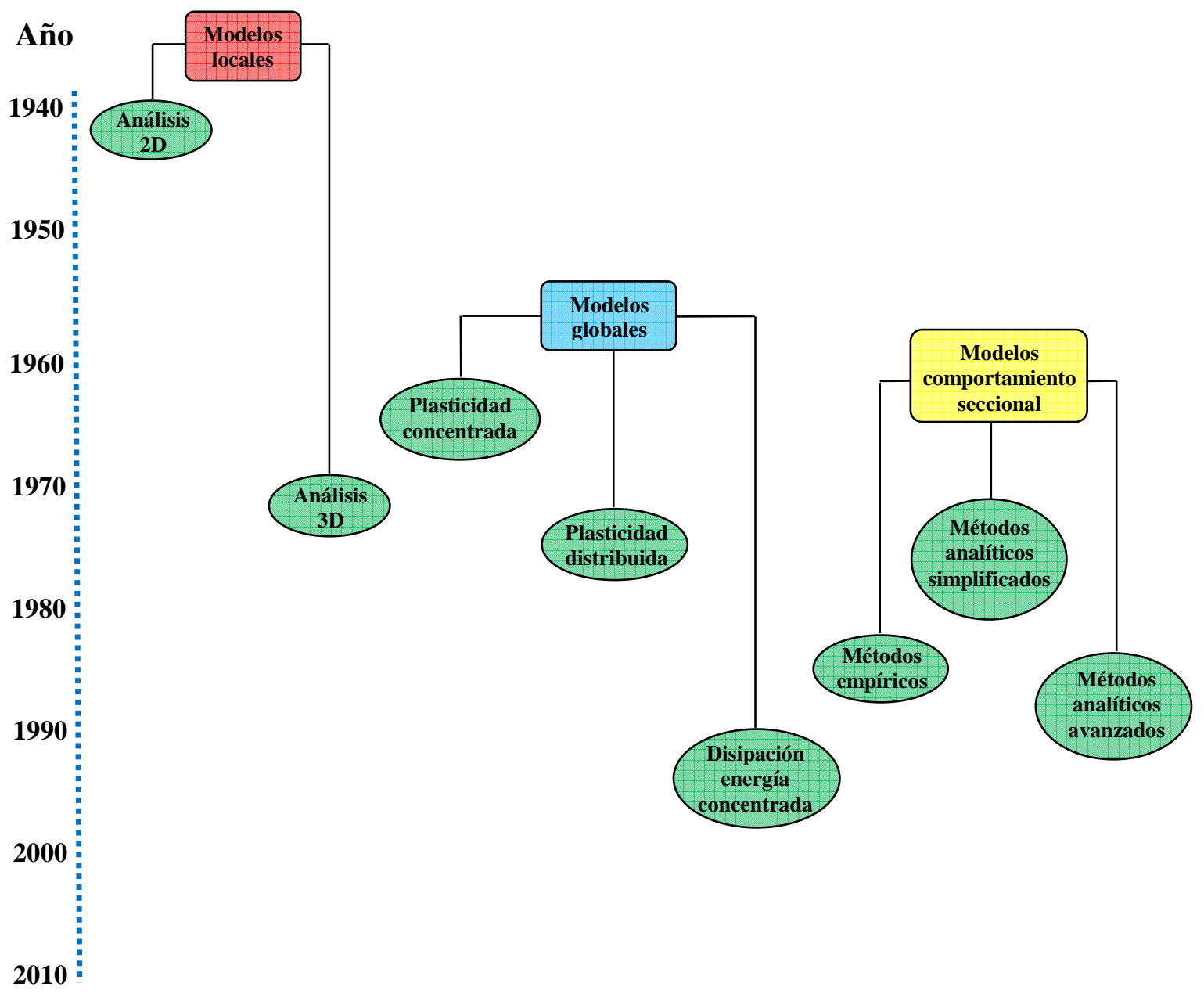

Figura 2.2 Distribución temporal de modelos numéricos empleados para la simulación sísmica de estructuras de hormigón armado según diferentes enfoques.

\subsection{OPTIMIZACIÓN EN INGENIERÍA}

Los problemas en ingeniería se suelen resolver mediante procedimientos que dependen de la experiencia previa del ingeniero. Es habitual aplicar estrategias de prueba y error que incrementan el tiempo de resolución y obtienen soluciones que, aun siendo buenas, no son las mejores. Un avance en este planteamiento se manifiesta mediante el desarrollo de estrategias numéricas por parte del ingeniero estructural que permitan 
obtener geometrías que mejoren el comportamiento resistente de algunas estructuras. Véase, por ejemplo, los trabajos de mejora del comportamiento de láminas a través de ligeros cambios en su forma implementados por Tomás y Martí (2010), o los métodos de búsqueda de forma en puentes arco espaciales desarrollados por Jorquera y Manterola (2012), entre otros. La aparición de las técnicas de optimización supone un avance en este planteamiento, permitiendo la implementación de procesos automatizados que reducen los tiempos de cálculo y proporcionan la solución óptima del problema, constituyendo una herramienta útil para el análisis y diseño en diversos ámbitos de la ingeniería.

\subsubsection{Algoritmos empleados en ingeniería estructural}

Desde la aparición de los primeros algoritmos de optimización se han desarrollado múltiples procedimientos, con distinto nivel de complejidad, para obtener la solución de diversos problemas estructurales. Mientras que los primeros procedimientos resolvían los problemas mediante la aplicación de métodos exactos que obtenían la solución óptima, el planteamiento de una extensa variedad de problemas complejos, que debían resolverse de forma eficiente, propició el desarrollo de procedimientos capaces de obtener buenas soluciones, aunque no fuesen las óptimas. Estos métodos, en los que la rapidez del proceso es tan importante como la calidad de la solución obtenida, se denominan heurísticos o aproximados. En palabras de Díaz (1996):

"Un método heurístico es un procedimiento para resolver un problema de optimización bien definido mediante una aproximación intuitiva, en la que la estructura del problema se utiliza de forma inteligente para obtener una buena solución”.

No se puede garantizar la existencia de métodos exactos para encontrar la solución óptima de problemas complejos. En caso de que existan, estos métodos invierten un tiempo computacional de un orden de magnitud muy superior al requerido por los métodos heurísticos, resultando inviable en muchas ocasiones su aplicación.

En los últimos años se ha observado un empleo creciente de los algoritmos metaheurísticos, al estar concebidos para resolver problemas que no pueden ser abordados por los métodos heurísticos tradicionales. Las principales características de estos algoritmos son la intensificación, que permite buscar alrededor de la mejor solución encontrada en una iteración, y la diversificación, proporcionada por la inclusión de técnicas estocásticas que introducen aleatoriedad en el proceso de búsqueda de la solución, permitiendo una exploración más amplia y precisa del espacio de diseño. Los métodos metaheurísticos proporcionan un marco general para crear nuevos algoritmos híbridos que combinan diferentes conceptos derivados de la inteligencia artificial, la evolución biológica y los mecanismos estadísticos (Osman y Kelly, 1996). 
La mayoría de algoritmos metaheurísticos imitan procesos naturales para resolver problemas complejos de optimización, como el comportamiento de grupos de animales o procesos de evolución de especies. Así, los algoritmos genéticos o Genetic Algorithms (GA) simulan el proceso de evolución biológica, considerando un conjunto de soluciones iniciales y generando sucesivas generaciones de soluciones basándose en el principio de supervivencia de las especies mejor adaptadas (Holland, 1975). Aunque las versiones estándar de estos algoritmos no son convenientes para resolver problemas complejos y exigentes desde el punto de vista computacional, trabajos como los de Saka (2007), Nimtawat y Nanakorn (2009) y Farhat, Nakamura y Takahashi (2009) demuestran que los algoritmos genéticos se han empleado frecuentemente en el campo de la ingeniería estructural. El algoritmo Particle Swarm Optimization (PSO) está basado en el comportamiento social de grupos de animales, como bancos de peces, colonias de insectos o bandadas de pájaros. Ejemplos de la aplicación de este algoritmo para la resolución de problemas de ingeniería estructural son los trabajos de Li, Huang y Liu (2009), Gomes (2011) y Dogn y Saka (2012). El algoritmo Ant Colony Optimization (ACO) simula el comportamiento de colonias de hormigas en su camino hacia las fuentes de alimento por la ruta más corta. Kaveh y Talatahari (2010a) y Aydogdu y Saka (2012) emplearon este algoritmo para el diseño de pórticos de acero, mientras que Martínez, González-Vidosa y Hospitaler (2011) utilizaron una variante de este algoritmo para la optimización económica de pilas de hormigón armado con sección rectangular hueca empleadas en viaductos de carretera.

Otros algoritmos más recientes inspirados en la naturaleza son el Cuckoo Search (CS), que simula el proceso de reproducción de pájaros cuco (Yang y Deb, 2009); el Hunting Search (HuS), inspirado en el comportamiento grupal de animales cazadores (Oftadeh, Mahjoob y Shariatpanahi, 2010); el Bat Algorithm (BA), basado en la capacidad de los murciélagos para conocer su entorno mediante la emisión de sonidos (Yang, 2010); y el Firefly Algorithm (FA), que simula el comportamiento de las luciérnagas durante su vuelo (Yang, 2009). En la actualidad se está comprobando que el algoritmo FA mejora las prestaciones de los anteriores, en particular de los GA y PSO.

Existen otros algoritmos metaheurísticos basados en ciertos procesos físicos, como el Simulated Annealing (SA), que simula el proceso de recocido del acero y la cerámica (Kirtrick, Gelatt y Vecchi, 1983); el Big Bang-Big Crunch (BB-BC), basado en la teoría del Big-Bang y Big-Crunch de evolución del universo (Erol y Eksin, 2006); y el Charged System Search (CSS), que usa la ley de Coulomb de la electrostática y las leyes de Newton para la mecánica (Kaveh y Talatahari, 2010b).

Otro algoritmo metaheurístico empleado en estudios recientes es el Harmony Search (HS), basado en el proceso seguido por un músico para encontrar las notas musicales que generan la mejor armonía posible (Geem, Kim y Loganathan, 2001). Este algoritmo fue empleado por Carbas y Saka (2012) en la optimización topológica de 
cúpulas y por Kaveh y Ahangaran (2012) en la optimización del coste de forjados mixtos.

Por último, Salajegheh y Gholizadeh (2012) destacan el empleo de técnicas de optimización basadas en redes neuronales o neural networks para simplificar la resolución de problemas complejos, las cuales imitan los mecanismos de interconexión de neuronas que caracterizan el funcionamiento del sistema nervioso de los animales. Algunos trabajos en los que se han empleado estos algoritmos corresponden a Topcu y Saridemir (2008) y Gholizadeh, Sheidaii y Farajzadeh (2012).

\subsubsection{Algoritmos empleados en la calibración de modelos numéricos}

Desde los inicios en la simulación del comportamiento de estructuras mediante modelos numéricos, la experiencia ha ido poniendo de manifiesto que, para que dichos modelos reflejen fidedignamente la realidad del problema, es indispensable realizar una adecuada calibración con resultados de carácter experimental. A modo de ejemplo puede citarse el trabajo de Ivorra, Pallarés y Adam (2009), en el cual se estudia el comportamiento sísmico de un campanario mediante un modelo calibrado con los resultados procedentes de ensayos dinámicos realizados sobre la estructura real, o el modelo de Orakcal y Wallace (2004), desarrollado para simular el comportamiento no lineal a flexión de muros esbeltos de hormigón armado.

Resulta necesario destacar que, a pesar de que las técnicas de optimización han experimentado un importante avance en los últimos años, se dispone de escasas referencias a trabajos en los que estas técnicas se emplean como una herramienta para la calibración de modelos numéricos que caracterizan el comportamiento de elementos de hormigón armado.

A lo largo del tiempo, la calibración de modelos numéricos ha sido abordada mediante diferentes enfoques. En aquellos casos donde se dispone de modelos con pocos parámetros y un reducido número de ensayos experimentales, la experiencia del investigador y la adopción de valores propuestos previamente en el tratamiento de problemas similares puede ser suficiente para conseguir un adecuado ajuste entre los resultados proporcionados por el modelo y los obtenidos experimentalmente. En otras ocasiones, la calibración de un cierto modelo está basada en un proceso de ajuste manual, requiriendo la experiencia del usuario en ese campo y un tedioso procedimiento de calibración de prueba y error. Un ejemplo lo constituye el modelo de Caner y Bazant (2000), desarrollado para caracterizar el comportamiento constitutivo del hormigón mediante sus propiedades microscópicas, obtenidas mediante la teoría de los microplanos o microplane concept.

De acuerdo con Kucerová (2007), el método de prueba y error se ha empleado comúnmente para la calibración de modelos numéricos, debido al desconocimiento de la variedad de técnicas disponibles para abordar este problema de forma eficaz. Sin embargo, y de acuerdo con Rodrigues et al. (2012), cuando se pretende calibrar un 
modelo que presenta un elevado número de parámetros y un fuerte carácter no lineal con una extensa base de datos experimentales, resulta necesario realizar un análisis inverso mediante el empleo de técnicas de optimización.

El análisis inverso constituye una estrategia de calibración, según la cual los valores de los parámetros del modelo se obtienen a partir de los resultados experimentales de que se dispone. Dentro del análisis inverso se pueden seguir dos procedimientos diferentes:

a) análisis inverso mediante estrategia directa, y

b) análisis inverso mediante estrategia inversa.

En el análisis inverso mediante estrategia directa, la calibración de los parámetros del modelo se realiza minimizando una función constituida por las diferencias entre los resultados del modelo y los resultados experimentales. Según Stavroulakis et al. (2003), aunque se pueden emplear diversas funciones a minimizar, los valores de los parámetros del modelo se obtienen normalmente empleando el criterio de mínimos cuadrados de las diferencias entre los valores obtenidos con el modelo y los experimentales. La principal ventaja del análisis inverso mediante estrategia directa consiste en la posibilidad de aplicarlo a la gran mayoría de casos, destacando además su alta capacidad para encontrar la solución del problema en caso de que ésta exista.

El análisis inverso mediante estrategia inversa requiere la existencia de una relación inversa, la cual obtiene directamente los valores de los parámetros del modelo a partir de los resultados experimentales. El principal inconveniente de esta estrategia es que dicha relación no siempre existe, debiendo realizarse en ciertas ocasiones aproximaciones a la misma, lo cual requiere de la intuición y experiencia del investigador. La ventaja más importante de este planteamiento es que, si la relación inversa existe o se ha realizado una buena aproximación, la obtención de los parámetros del modelo es muy rápida desde el punto de vista computacional.

Dentro de las técnicas de optimización empleadas para la calibración de modelos numéricos mediante análisis inverso con estrategia directa, destacan dos grandes grupos:

a) optimización matemática, y

b) optimización heurística.

La optimización mediante análisis inverso con estrategia directa se ha abordado tradicionalmente mediante el empleo de métodos de optimización matemática basados en el gradiente, habiéndose empleado estas técnicas para la calibración de modelos que caracterizan el comportamiento a tracción y flexión del hormigón. En esta línea, de Oliveira e Sousa y Gettu (2006) obtuvieron una relación tensión-abertura de fisura para caracterizar el comportamiento de piezas de hormigón sometidas a ensayos de tracción uniaxial, introduciendo funciones de peso en la estrategia de optimización al aparecer grandes aberturas de fisura en los elementos. Roelfstra y Wittmann (1986) calibraron modelos para simular el comportamiento de endurecimiento por deformación de piezas 
de hormigón sometidas a tracción uniaxial. Østergaard (2003) planteó un modelo capaz de simular el comportamiento de fisuración del hormigón a edades tempranas, desarrollando un algoritmo de optimización para realizar la calibración inversa con resultados experimentales procedentes de ensayos de tracción uniaxial y flexión con carga aplicada en tres puntos. Posteriormente, Amirineni (2009) empleó el algoritmo desarrollado por Østergaard (2003) para calibrar la curva tensión-abertura de fisura de hormigones con fibras, determinando en diferentes pasos el módulo elástico del hormigón, la resistencia a tracción y la pendiente de la rama inicial de dicha curva.

El comportamiento constitutivo del hormigón a compresión también ha sido estudiado a través de modelos calibrados mediante análisis inverso con estrategia directa. Un ejemplo lo constituye el trabajo de Van Dorsselaer et al. (2013), quienes realizaron un análisis de sensibilidad para identificar los parámetros del modelo y aplicaron un algoritmo de optimización para obtener los coeficientes que mejor ajuste proporcionaban entre los resultados del modelo y los de una serie de ensayos de losas de hormigón frente a punzonamiento.

Existen ciertos modelos calibrados mediante optimización inversa con estrategia directa para simular el comportamiento diferido del hormigón. Por un lado, Peng y Liu (2013) calibraron un modelo de elementos finitos para caracterizar la influencia de la difusión de la humedad en la retracción de secado del hormigón, identificando el coeficiente de difusión como parámetro más relevante y empleando para la calibración el módulo de optimización del software ANSYS®. Villmann et al. (2013) estudiaron el fenómeno de la retracción, considerando la deformación elástica y viscoelástica, la fisuración y la rama de reblandecimiento del comportamiento constitutivo del hormigón y calibrando los parámetros del modelo a partir de los resultados ofrecidos por elementos de hormigón sometidos a ensayos de secado.

Las técnicas de optimización también se han empleado para la calibración de modelos que simulan el comportamiento dinámico de estructuras. Así, Mosavi et al. (2014) realizaron un análisis inverso para calibrar los parámetros de un modelo de elementos finitos que simula los diferentes estados de vibración de un puente mixto de hormigón y acero. Los valores óptimos de los coeficientes se obtienen realizando un análisis de sensibilidad mediante la minimización de una función multivariable. Otro ejemplo de aplicación del método del gradiente lo constituye el trabajo de Torrenti, Pijaudier-Cabot y Reynouard (2010), quienes aplicaron el algoritmo de LevenbergMarquardt, presente en Marquardt (1963), para calibrar los parámetros de un modelo que proporciona las deformaciones verticales de piezas de hormigón, utilizando los resultados de ensayos correspondientes a tres elementos de dimensiones diferentes para considerar el efecto escala en las estructuras.

Aunque la optimización matemática ha sido empleada en ciertos casos con resultados satisfactorios, en la actualidad se presenta frecuentemente la dificultad de obtener las derivadas de ciertas funciones, incluso aunque éstas sean diferenciables, 
siendo necesario recurrir a estrategias numéricas basadas en diferencias finitas que aumentan la carga computacional y reducen la eficiencia del proceso. Como alternativa, las técnicas de optimización de tipo heurístico pueden ser aplicadas con éxito en estos casos. Aunque no garantizan la obtención de un óptimo global, el empleo de técnicas de búsqueda estocásticas resulta muy útil para explorar espacios de diseño más amplios que los examinados por las técnicas de optimización basadas en el gradiente (Salajegheh y Gholizadeh, 2012), encontrando soluciones que, si no constituyen el óptimo global, están muy cerca de él.

Las técnicas de optimización de tipo heurístico resultan más eficaces desde el punto de vista computacional, aspecto que hace que sean las más aplicadas hoy en día para acometer la calibración de modelos complejos. Concretamente, resulta muy común el empleo de algoritmos genéticos. Así, Sengupta y Li (2013) propusieron una modificación del modelo de histéresis de Bouc-Wen-Baber-Noori para modelizar el comportamiento de nudos viga-pilar con insuficiente armadura transversal. Estos autores recalibraron los parámetros del modelo inicial, considerando la interacción entre fenómenos como el efecto pinching, la degradación de resistencia o el endurecimiento por deformación de las armaduras. Otros autores, como Villmann, Villmann y Slowik (2004), emplearon algoritmos genéticos para obtener los valores de los parámetros que permiten modelizar la rama de pérdida de resistencia en la curva fuerza-desplazamiento de piezas de hormigón, introduciendo un operador de búsqueda local entre los operadores de generación de descendencia y de selección. Dicho operador agrupa las soluciones con menores valores de la función de adaptación en las diferentes iteraciones del algoritmo. Posteriormente, Bretschneider, Villmann y Slowik (2007) calibraron modelos que permiten obtener aspectos como la curva tensión-deformación de piezas de hormigón ensayadas a tracción directa; la curva fuerza-deformación vertical de piezas ensayadas a flexión simple, incluyendo el tramo de endurecimiento por deformación; y la curva tensión-abertura de fisura. Otra aplicación de los algoritmos genéticos corresponde al trabajo de Gharaibeh et al. (2012), quienes calibraron los coeficientes de modelos que reproducen el comportamiento de pavimentos de hormigón con capa asfáltica, hormigón con juntas y hormigón armado para diferentes niveles de tráfico.

Los algoritmos metaheurísticos se han empleado también para calibrar los parámetros de modelos correspondientes a presas de hormigón. En esta línea, Li et al. (2003) emplearon el algoritmo Ant Colony Optimization (ACO) para identificar y calibrar los parámetros de un modelo aplicable a presas de gravedad, poniendo de manifiesto la facilidad de convergencia de este algoritmo hacia el mínimo global respecto a los métodos basados en el gradiente. Posteriormente, Tian y Zhou (2004) realizaron un análisis inverso para obtener los parámetros mecánicos de un modelo de presa de gravedad aplicando el mismo tipo de algoritmo. Aunque el algoritmo ACO está concebido fundamentalmente para la resolución de problemas de tipo discreto, en este estudio se realizó una adaptación de dicho algoritmo, discretizando el espacio de 
búsqueda de los parámetros y obteniendo unos valores adecuados para los parámetros visibilidad e intensidad del camino. Otro ejemplo es el trabajo de Lin et al. (2014), quienes estudiaron la estabilidad global de grandes presas arco realizando una modificación del algoritmo ACO para obtener los parámetros que caracterizan el hormigón y el terreno de cimentación. Dichos parámetros se obtienen mediante análisis inverso a partir de las deformaciones medidas en presas mediante monitorización.

En la actualidad, la estrategia de optimización más empleada para la calibración de modelos mediante el análisis inverso con estrategia inversa la constituyen las redes neuronales artificiales. En esta línea, Kucerová y Mares (2010) y Kucerová y Leps (2014) emplearon diferentes procesos basados en redes neuronales artificiales para el ajuste de modelos constitutivos que permitían simular el comportamiento del hormigón mediante la teoría de microplanos o microplane concept. Otro ejemplo de aplicación de esta técnica de optimización corresponde al trabajo de Lehký, Kersner y Novák (2012), quienes plantearon un proceso de identificación de parámetros por separado para determinar la energía de fractura de elementos de hormigón a partir de resultados procedentes de ensayos de vigas a flexión.

En ciertas ocasiones, puede ser conveniente el empleo de estrategias que combinen la optimización matemática y la heurística. Siguiendo este planteamiento, Rodrigues et al. (2012) desarrollaron un modelo para la simulación del comportamiento fuerza-deformación de elementos de hormigón armado sometidos a cargas según dos direcciones ortogonales. La modelización del comportamiento global requiere una función de interacción del comportamiento de histéresis de cada eje por separado, la cual se calibró mediante una estrategia de optimización que combina un método basado en el gradiente y un algoritmo genético. Concretamente, el método empleado basado en el gradiente es el algoritmo de Levenberg-Marquardt.

Resulta conveniente destacar que no se han encontrado referencias a trabajos en los que los algoritmos metaheurísticos de tipo Swarm Intelligence se apliquen para la calibración de modelos numéricos de hormigón armado. Así, Agrawal, Chandwani y Nagar (2014) ponen de manifiesto que ciertos algoritmos empleados comúnmente en la actualidad, como el Particle Swarm Optimization (PSO), Ant Colony Optimization (ACO), Bat Algorithm (BA) y Firefly Algorithm (FA), se emplean generalmente en el campo de la ingeniería estructural para minimizar el peso de las estructuras de acero y el coste de las estructuras de hormigón, pero no para calibrar modelos numéricos.

\subsection{PUBLICACIONES DEL DOCTORANDO RELACIONADAS CON ESTA TESIS}

Los primeros trabajos relacionados con el contenido de esta tesis se materializaron en un artículo enviado en octubre de 2013 a la revista Computers and Concrete, el cual se encuentra actualmente en revisión, y una comunicación presentada al congreso Euro- 
Concrete 2014, celebrado en la ciudad austriaca de St. Anton am Alberg en marzo de 2014. En ellos se planteó una estrategia de optimización, basada en algoritmos genéticos, para calibrar expresiones de comportamiento seccional empleando una selección de ensayos de elementos de hormigón armado cumpliendo las prescripciones constructivas y sísmicas impuestas por las principales normas y códigos de diseño nacionales e internacionales. La selección de ensayos se obtiene a partir de una base de datos publicada en la literatura con más de 1000 ensayos. Las expresiones propuestas permiten reducir la dispersión de los resultados respecto a lo ofrecido previamente por otros autores, presentando además una aplicación directa a elementos estructurales habitualmente empleados en edificios de hormigón armado situados en zonas sísmicas. La comunicación presentada al congreso Euro-Concrete 2014 se publicó en el libro Computational Modelling of Concrete Structures, referenciado en el catálogo ISI Proceedings.

En el VI Congreso ACHE, celebrado en Madrid en junio de 2014, se presentó una comunicación, en línea con los trabajos anteriores, aunque esta vez centrándose en una descripción detallada de ciertos aspectos relativos a la selección de ensayos y las normas y códigos considerados para realizarla. Para ello, se justifican los parámetros considerados para efectuar la selección, se describen los tipos de elementos que la componen y se detallan los rangos de valores adoptados por los parámetros de dichos elementos. En lo que respecta a la normativa considerada, se presentan tres grupos, cada uno constituido por una norma/código principal y su correspondiente norma/código sísmico. Los grupos considerados son una pareja de normas para España, compuesta por la instrucción EHE 08 y la NCSE-02; otra pareja de códigos a nivel europeo, constituida por el EC-2 y el EC-8; y un último grupo, compuesto por el código americano ACI-318.

Posteriormente, en marzo de 2015, se publica un trabajo en la revista DYNA Ingeniería e Industria, enfocado a la normativa española, y se envía un artículo a la revista Engineering Structures, extendido a la normativa europea y americana, que se encuentra en revisión. En estos estudios, se amplían los trabajos previos estableciendo una comparación adicional de los resultados ofrecidos por las expresiones calibradas con los obtenidos al aplicar las expresiones propuestas por el EC-8 para la obtención del giro de plastificación y último de la sección, consiguiéndose una mejora en una serie de parámetros estadísticos.

Para finalizar, se ha remitido una comunicación a la $3^{\text {rd }}$ International Conference on Mechanical Models in Structural Engineering, a celebrar en junio de 2015 en la ciudad de Sevilla. En este estudio se realizan diversos análisis pushover de dos pórticos de hormigón armado, modelizando las rótulas plásticas generadas en la estructura mediante diferentes métodos, y comparando posteriormente los resultados obtenidos en los puntos de plastificación global y de colapso de la estructura. Del mismo modo, se ha enviado a la revista Computers and Structures una ampliación de este trabajo, incluyendo una evaluación de la influencia de los valores de la aceleración del suelo en 
las diferencias obtenidas al implementar rótulas plásticas modelizadas mediante diferentes métodos. Para ello, se aplica el método N2 incluido en el EC-8 para obtener el desplazamiento objetivo de la estructura, considerando tres valores diferentes de aceleración del suelo. 



\section{Bases de datos de ensayos experimentales}

\subsection{INTRODUCCIÓN}

El desarrollo de modelos y expresiones numéricas aptos para la descripción del comportamiento sísmico de estructuras de hormigón armado exige un adecuado ajuste de los mismos con datos de tipo experimental. Para ello, es necesario recurrir a una cantidad adecuada de ensayos experimentales relativos a elementos que puedan emplearse en este tipo de estructuras.

En este capítulo se realiza la descripción de una base de datos que contiene ensayos correspondientes a más de 1000 elementos estructurales de hormigón armado, para describir a continuación el proceso seguido en la obtención, a partir de dicha base de datos, de una selección de ensayos correspondientes a elementos que cumplen una serie de requisitos constructivos impuestos por las principales normas y códigos nacionales e internaciones. Ambas bases de datos, la completa y la seleccionada, se emplean para la calibración de las expresiones de comportamiento seccional que se describen en capítulos posteriores.

\subsection{BASE DE DATOS INICIAL}

La base de datos de ensayos experimentales que se utiliza en esta tesis se encuentra disponible en el trabajo desarrollado por Panagiotakos y Fardis (2001). Está compuesta por 1012 elementos de hormigón armado, ensayados frente a esfuerzos de flexión uniaxial, con o sin presencia de esfuerzo axil.

Esta base de datos se elaboró con objeto de recopilar información de elementos estructurales tipo vigas, pilares y muros de cortante. Del total de elementos, 270 se pueden considerar representativos de elementos tipo viga, puesto que presentan armadura longitudinal no simétrica y se ensayan en ausencia de esfuerzo axil. Además, estos elementos presentan sección rectangular, a excepción de dos, que tienen sección en T. 681 elementos pueden ser considerados como elementos tipo pilar, pues contienen 
armadura longitudinal con una configuración simétrica, sección cuadrada o rectangular y están ensayados con o sin esfuerzo axil. Dentro de este grupo, 23 presentan armadura diagonal, pudiendo estar combinada con barras convencionales de armadura longitudinal. Finalmente, 61 elementos corresponden a muros de cortante con sección rectangular o en T.

Los elementos de esta base de datos inicial presentan tres tipos de configuración estructural: ménsula simple, ménsula doble y viga simplemente apoyada, predominando las dos primeras configuraciones (Figura 3.1). En algunos ensayos se produce un cierto deslizamiento de la armadura longitudinal en la sección de máximo momento flector, generándose un giro en el extremo del elemento que provoca un drift, o diferencia de desplazamientos laterales entre sus extremos, y aumenta la curvatura media medida cerca de los mismos. Los elementos ensayados siguiendo la configuración de viga simplemente apoyada se encuentran solicitados con una fuerza transversal situada en el centro de vano. En estos elementos, debido a la simetría presente en su configuración, no se produce deslizamiento de la armadura longitudinal en la sección de máximo momento flector, excepto cuando la carga se aplica a través de un elemento rígido o stub (Figura 3.2) con una dimensión suficiente a lo largo del eje longitudinal de la viga como para que se pueda producir el deslizamiento de la armadura longitudinal a ambos lados de la sección de centro de vano.

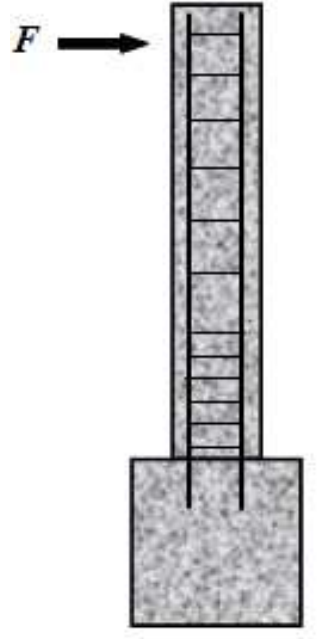

(a)

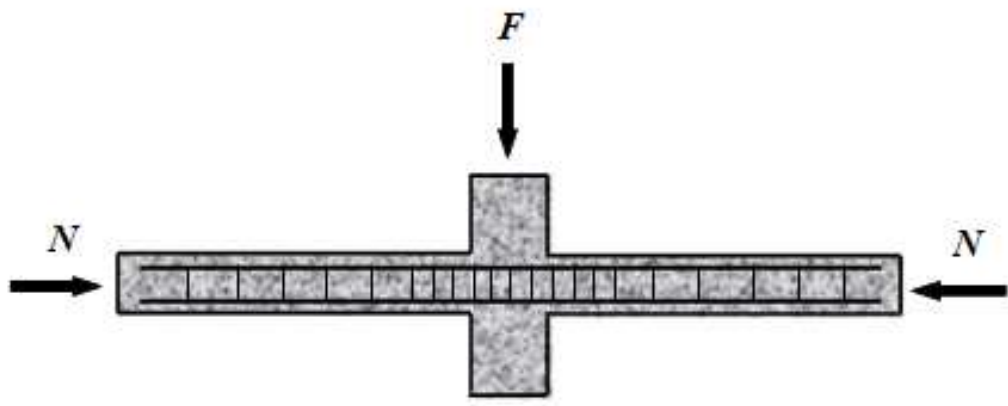

(b)

Figura 3.1 Configuración de ensayos para pilares. (a) Ménsula simple. (b) Ménsula doble.

Un aspecto importante a considerar en la base de datos inicial lo constituye la identificación del instante último o instante en el que se considera el fallo de un elemento. En el trabajo de Panagiotakos y Fardis (2001) se identifica el fallo del elemento cuando se detecta un cambio importante en la relación fuerza lateral aplicada-deformación. 


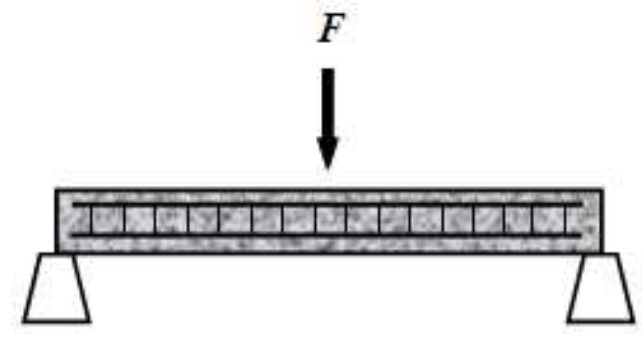

(a)

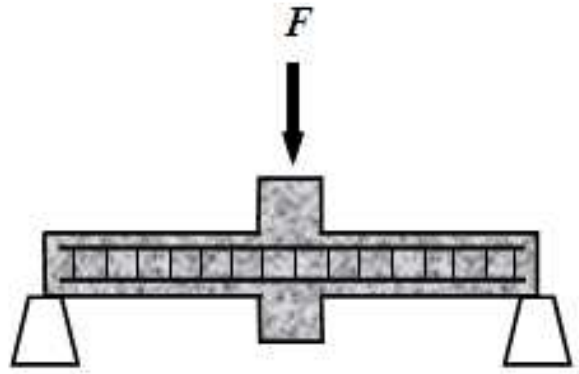

(b)

Figura 3.2 Configuración de ensayos para vigas simplemente apoyadas. (a) Ensayo estándar.

(b) Ensayo con aplicación de carga en elemento rígido (stub).

Para el caso de cargas monotónicas, se considera que el elemento alcanza el colapso cuando se produce una caída del $15 \%$ tras alcanzarse la máxima fuerza lateral aplicada en el ensayo. Si la carga aplicada es de tipo cíclico, se identifica el fallo del elemento cuando se produce una disminución importante en la pendiente de recarga y en el área de los ciclos de histéresis con respecto a los ciclos anteriores. Normalmente, el fallo del elemento se produce, al igual que para el caso de la carga monotónica, con una disminución del $15 \%$ en la máxima fuerza lateral aplicada en el ensayo.

Las variables consideradas en la base de datos inicial son:

$C M$ variable que indica el tipo de carga impuesta en el ensayo: 0 para carga monotónica o 1 para carga cíclica,

$S L$ variable que indica si, en el ensayo correspondiente, se produce el deslizamiento de la armadura longitudinal de tracción respecto al hormigón en la sección de anclaje $(S L=1)$ o no se produce $(S L=0)$,

ST variable que depende del tipo de acero empleado en las armaduras: 1 para acero dúctil laminado en caliente (hot-rolled ductile steel); 2 para acero con tratamiento térmico (heat-treated steel); y 3 para acero conformado en frío (brittle cold-worked steel),

$T P$ variable que indica el tipo de elemento estructural: 0 para vigas y pilares o 1 para muros de cortante,

$b \quad$ ancho de la sección transversal del elemento,

$d$ canto útil de la sección transversal del elemento,

$f_{c}$ resistencia a compresión simple del hormigón,

$f_{y}$ límite elástico del acero de la armadura longitudinal de tracción,

$f_{y}$, límite elástico del acero de la armadura longitudinal de compresión,

$f_{y h}$ límite elástico del acero de la armadura transversal,

$f_{y v} \quad$ límite elástico del acero de la armadura vertical de alma,

$h$ canto de la sección transversal del elemento, 
$L_{s} \quad$ distancia entre las secciones de momento flector máximo y nulo en el elemento, determinada como el cociente entre el momento flector $M$ y el esfuerzo cortante $V$ actuantes en la sección de máximo momento flector,

$s_{h} \quad$ separación de armadura transversal,

$\phi_{h} \quad$ diámetro de las barras de armadura transversal,

$\phi_{L} \quad$ diámetro de las barras de armadura longitudinal de tracción,

$\phi_{L}$ ' diámetro de las barras de armadura longitudinal de compresión,

$\phi_{v} \quad$ diámetro de las barras de armadura vertical de alma,

$v \quad$ esfuerzo axil normalizado, obtenido mediante el cociente entre el esfuerzo axil $N$ actuante en la sección y $A f_{c}$, siendo $A$ el área bruta de la sección,

$\rho \quad$ cuantía de armadura longitudinal de tracción, definida como el cociente entre el área de armadura longitudinal de tracción $A_{s} \mathrm{y} b d$,

$\rho$ ' cuantía de armadura longitudinal de compresión, definida como el cociente entre el área de armadura longitudinal de compresión $A_{s}$ ' y $b d$,

$\rho_{d} \quad$ cuantía de armadura diagonal en elementos armados diagonalmente, obtenida como el cociente entre el área de armadura dispuesta a lo largo de una diagonal de la sección y $b d$,

$\rho_{h} \quad$ cuantía de armadura de confinamiento, determinada como el cociente entre el área de armadura transversal $A_{s h} \mathrm{y} b s_{h}, \mathrm{y}$

$\rho_{v} \quad$ cuantía de armadura vertical de alma o web vertical reinforcement ratio, obtenida como el cociente entre el área de armadura longitudinal total situada entre la armadura longitudinal de tracción y compresión y $b d$.

Las Tablas 3.1 a 3.4 muestran los rangos de valores adoptados por las variables en la base de datos inicial. Algunos elementos presentan altos valores en el ancho $b$ y el canto $h$ de la sección al tratarse de muros de cortante (Tabla 3.1), además de una separación de armadura transversal excesivamente grande $\left(s_{h}=1000 \mathrm{~mm}\right)$.

Tabla 3.1 Rango de variación de las variables geométricas en la base de datos inicial (en mm).

\begin{tabular}{cc}
\hline Variable & Rango de variación \\
\hline$L_{s}$ & $150-6622$ \\
$b$ & $75-1000$ \\
$h$ & $102-2750$ \\
$d$ & $72-2625$ \\
$s_{h}$ & $18-1000$ \\
\hline
\end{tabular}

Además, algunos elementos presentan cuantías de armadura nulas (Tabla 3.2), por lo que no pueden ser considerados como elementos estructurales en la práctica constructiva. Puede observarse en la Tabla 3.3 cómo algunos elementos presentan hormigones de alta resistencia $\left(f_{\mathrm{c}} \geq 50 \mathrm{MPa}\right)$ y aceros con altos límites elásticos $\left(f_{y}, f_{y}\right.$, $\left.f_{y v}{ }^{\prime}=1204 \mathrm{MPa} ; f_{y h}=1424 \mathrm{MPa}\right)$. 
Tabla 3.2 Rango de variación porcentual de las cuantías de armadura en la base de datos inicial.

\begin{tabular}{cc}
\hline Variable & Rango de variación \\
\hline$\rho$ & $0,00-4,33$ \\
$\rho^{\prime}$ & $0,00-3,52$ \\
$\rho_{v}$ & $0,00-5,02$ \\
$\rho_{w}$ & $0,00-3,67$ \\
$\rho_{d}$ & $0,00-1,18$ \\
\hline
\end{tabular}

Tabla 3.3 Rango de variación de resistencias de materiales en la base de datos inicial (en MPa).

\begin{tabular}{cc}
\hline Variable & Rango de variación \\
\hline$f_{c}$ & $11-118$ \\
$f_{y}$ & $282-1204$ \\
$f_{y}{ }^{\prime}$ & $279-1204$ \\
$f_{y v}{ }^{\prime}$ & $316-1204$ \\
$f_{y h}$ & $220-1424$ \\
\hline
\end{tabular}

En lo relativo al diámetro de las barras de armadura (Tabla 3.4), destaca la presencia de elementos con diámetros rara vez empleados en estructuras de hormigón (inferiores a $6 \mathrm{~mm}$ y superiores a $32 \mathrm{~mm}$ ).

Tabla 3.4 Rango de variación de los diámetros de las barras de armado en la base de datos inicial (en mm).

\begin{tabular}{cc}
\hline Variable & Rango de variación \\
\hline$\phi_{L}$ & $5,7-35,7$ \\
$\phi_{L}{ }^{\prime}$ & $5,7-35,7$ \\
$\phi_{v}$ & $3,0-31,8$ \\
$\phi_{h}$ & $3,0-19,0$ \\
\hline
\end{tabular}

El valor del axil normalizado $v$ adopta valores comprendidos en el intervalo [-0,009; $0,900]$ para considerar, de este modo, los elementos sometidos a esfuerzos de tracción $(v$ $<0)$ y de compresión $(v>0)$.

El acero empleado en los 1012 ensayos de la base de datos inicial se puede agrupar en tres tipos: 824 ensayos emplean acero dúctil laminado en caliente con una tasa de endurecimiento por deformación $f_{u} / f_{y}=1,5 \mathrm{y}$ una deformación de rotura a tracción $\varepsilon_{s u}=$ $15 \% ; 129$ ensayos utilizan acero con tratamiento térmico, con $f_{u} / f_{y}=1,2$ y $\varepsilon_{s u}=8 \%$; y 59 elementos presentan acero conformado en frío, con $f_{u} / f_{y}=1,1$ y $\varepsilon_{s u}=4 \%$. La Figura 3.3 ofrece la distribución porcentual de los diferentes tipos de acero en los elementos de la base de datos inicial y la consideración o no del deslizamiento de la armadura longitudinal respecto al hormigón. Además, la carga cíclica es el tipo de carga predominante en los ensayos presentes en la base de datos inicial (Figura 3.4). 


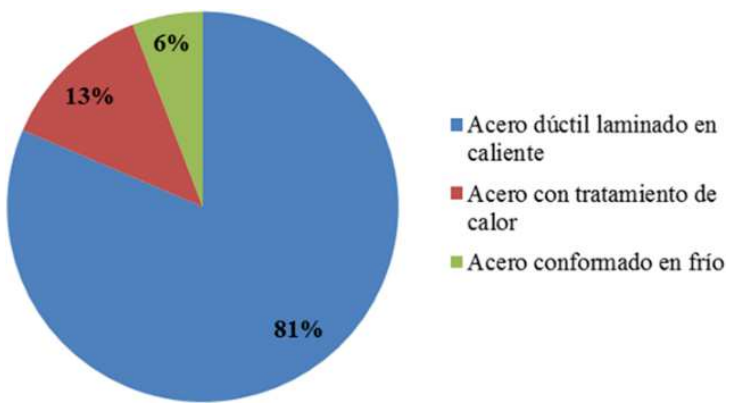

(a)

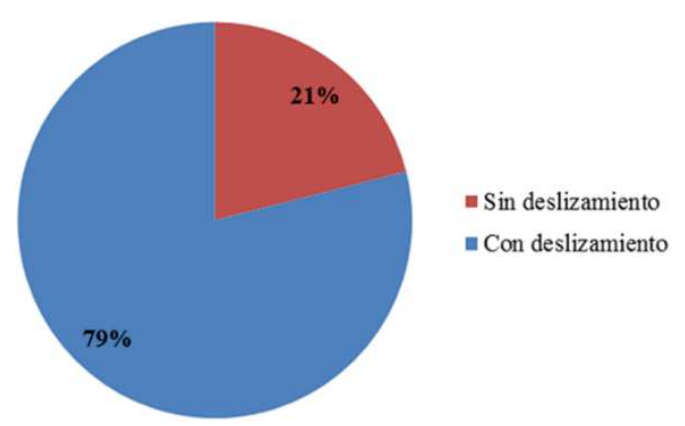

(b)

Figura 3.3 Distribución de ensayos. (a) Según tipo de acero de las armaduras. (b) Según exista deslizamiento de la armadura longitudinal.

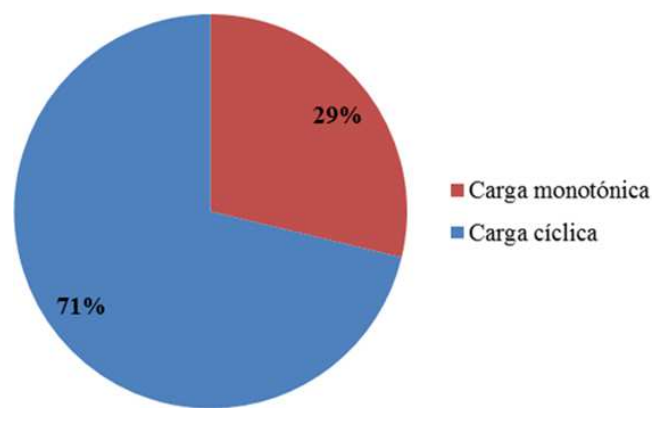

Figura 3.4 Distribución de ensayos según el tipo de carga aplicada (monotónica o cíclica).

\subsection{SELECCIÓN DE ENSAYOS}

El desarrollo de modelos y expresiones numéricas para simular el comportamiento sísmico de elementos de hormigón armado requiere una adecuada calibración de dichos modelos con datos experimentales. Para abordar este cometido es esencial elegir no sólo datos experimentales de suficiente calidad, sino también un número representativo de los mismos.

Con el propósito de cumplir estos requisitos, se ha confeccionado una selección de ensayos partiendo de la base de datos descrita en el apartado 3.2. Para elaborar esta selección, se han impuesto los condicionantes constructivos y de carácter sísmico establecidos por las principales normas y códigos de diseño nacionales e internacionales, con objeto de obtener un conjunto de ensayos que correspondan a elementos estructurales que puedan emplearse habitualmente en el diseño de construcciones convencionales de hormigón armado situadas en zonas sísmicas.

La normativa de diseño considerada se agrupa en tres parejas, una nacional, otra a nivel europeo y otra internacional (norteamericana), cada una de ellas compuesta por una norma/código principal y su correspondiente norma/código sísmico:

a) EHE-08 (Comisión Permanente del Hormigón, 2008) y NCSE-02 (Comisión Permanente de Normas Sismorresistentes, 2002), 
b) EC-2 (European Committee for Standardization, 2004a) y EC-8 (European Committee for Standardization, 2004b), y

c) ACI-318-08 (ACI Committe 318, 2008).

En la consideración de la instrucción EHE-08 se han incluido las indicaciones del Anexo 10, de carácter sísmico. Por otro lado, conviene destacar el hecho de que la normativa sísmica americana está compuesta por un amplio conjunto de normas, dependiendo de la zona de Estados Unidos que se considere, así como del nivel de riesgo sísmico que se quiera asumir. Por tanto, debido a este carácter heterogéneo, y a que el propio código ACI-318-08 posee un extenso capítulo dedicado a consideraciones de tipo sísmico, se ha considerado como "pareja" del código ACI-318-08 su parte sísmica (capítulo 21).

Por otra parte, el hecho de que la selección de ensayos atienda a criterios meramente constructivos hace que no se haya considerado el código modelo CM-2010 (FIB, 2012a), al ser éste de carácter más científico.

Debido al amplio número de variables presente en la base de datos inicial de Panagiotakos y Fardis (2001), se han escogido cinco grupos de variables para cumplir las restricciones impuestas por la normativa considerada: dimensiones de la sección transversal, características mecánicas de los materiales, diámetro de las barras de armado, cuantías de armadura y espaciamiento de la armadura transversal. El criterio seguido para el establecimiento de los rangos de variación de las diferentes variables es el siguiente:

a) en cada pareja de normas/códigos considerada se establece el rango de variación de cada variable, de forma que ese rango cumpla, simultáneamente, con lo establecido en las dos normas/códigos, y

b) a partir de los tres rangos de variación para cada variable, correspondientes con las tres parejas consideradas de normas/códigos, se obtiene un nuevo y definitivo rango de variación, de modo que siempre se satisfagan los requerimientos constructivos de, al menos, una de las tres parejas consideradas de normas/códigos.

Con este planteamiento se obtiene una selección de ensayos lo más extensa posible y adaptada a las exigencias de alguna de las tres parejas de normas/códigos consideradas.

\subsubsection{Distinción entre vigas y pilares}

Resulta necesario eliminar de la base de datos inicial los ensayos correspondientes a los muros de cortante y a las piezas traccionadas, con objeto de purgar la base de datos para considerar sólo los elementos flectados y los comprimidos, de tal modo que estos elementos puedan asimilarse a vigas y pilares. Por tanto, de los 1012 ensayos existentes en la base de datos inicial, se han eliminado los siguientes:

a) 61 ensayos correspondientes a muros de cortante, $y$

b) 4 ensayos correspondientes a elementos traccionados. 
De los 947 ensayos resultantes, es necesario a continuación distinguir cuáles corresponden a elementos tipo viga y cuáles a elementos tipo pilar, puesto que algunas variables, como la relación entre el ancho y el canto de la sección o las cuantías de armadura longitudinal, dependen de si el elemento ensayado se corresponde con un tipo u otro. Así, el criterio seguido para realizar esta distinción ha sido el contemplado por la instrucción EHE-08 (Anexo 10) y los códigos EC-8 y ACI-318-08 (capítulo 21). Según esta normativa, un elemento con un axil normalizado $v \leq 0,1$ puede considerarse viga, correspondiéndose con un pilar en caso contrario. Con este criterio se obtienen 465 vigas y 482 pilares.

Además de esta clasificación, y con objeto de adecuar la base de datos inicial a los elementos empleados habitualmente en edificación, se consideran los pilares con armadura simétrica y las vigas con armadura no simétrica. Los casos detectados que no cumplen con este requisito son:

a) 4 pilares con armadura no simétrica, lo que supone el 0,42\% del total de ensayos y el $0,83 \%$ del total de pilares, y

b) 199 vigas con armadura simétrica, lo que supone el $21 \%$ del total de ensayos y el $42,8 \%$ del total de vigas.

Debido al escaso porcentaje que representan estos 4 pilares sobre el total, se ha procedido a eliminarlos. Distinto ocurre con las vigas con armadura simétrica, que suponen un porcentaje importante sobre el total de ensayos. Se ha decidido conservarlas puesto que se trata de una configuración de armado que puede aparecer en la práctica constructiva. Por tanto, la distribución final de vigas y pilares considerada en la base de datos inicial es la mostrada en la Figura 3.5.

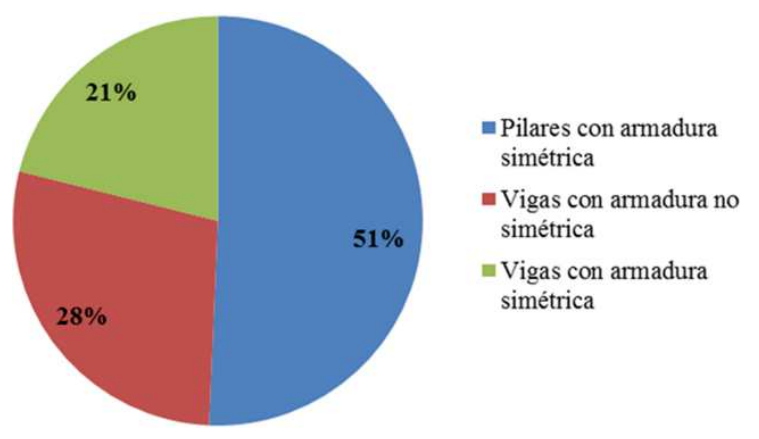

Figura 3.5 Distribución de vigas y pilares considerados en la base de datos inicial.

\subsubsection{Dimensiones de la sección transversal}

Los condicionantes que han de cumplir las dimensiones de la sección de un elemento estructural situado en zona símica están regulados, fundamentalmente, mediante las especificaciones de la normativa sísmica correspondiente. Estas limitaciones se imponen, generalmente, a secciones de geometría rectangular, dependiendo normalmente del nivel de ductilidad que se quiera alcanzar. 
En general, cuanto mayor es el nivel de ductilidad mayores son los valores mínimos impuestos para las dimensiones de la sección transversal. Con objeto de eliminar la menor cantidad posible de ensayos de la base de datos inicial, se ha impuesto el nivel de ductilidad menos restrictivo de la normativa, obteniéndose así valores mínimos menores, permitiendo que la variable correspondiente adopte el mayor rango de variación posible y que sea compatible con unos mínimos requisitos de carácter sísmico.

En la Tabla 3.5 se muestran los diferentes condicionantes impuestos por la instrucción EHE-08 en la geometría de secciones rectangulares. Las limitaciones $b \geq 0,3 h$ y dimensión mayor $\leq 2,5$ dimensión menor se corresponden con restricciones para considerar un elemento estructural como viga o pilar, respectivamente. El resto de restricciones mostradas se corresponden con el nivel de ductilidad alta, que es el menor nivel de ductilidad para el que la instrucción aporta requerimientos constructivos.

Tabla 3.5. Restricciones geométricas en secciones rectangulares según EHE-08 (en mm).

\begin{tabular}{cc}
\hline Vigas & Pilares \\
\hline$b \geq 0,3 h$ & Dimensión mayor $\leq 2,5$ dimensión menor \\
$b \geq 200$ & $\operatorname{mín}(b, h) \geq 250$ \\
\hline
\end{tabular}

En el caso de la norma NCSE-02 (Tabla 3.6), ésta impone, para el caso de vigas, unos valores mínimos del ancho de descuelgue en un forjado en función del nivel de ductilidad a alcanzar. De nuevo, se muestran las restricciones correspondientes con el nivel de ductilidad alta, que es el menor nivel de ductilidad para el que la norma aporta requerimientos constructivos. En lo que respecta a los pilares, se establecen unos valores mínimos en las dimensiones de la sección en función de la aceleración sísmica de cálculo $a_{c}$. Los valores mostrados en la Tabla 3.6 se corresponden con una aceleración $a_{c}=0,12 g$.

Tabla 3.6 Restricciones geométricas en secciones rectangulares según NCSE- $02, a_{c}=0,12 g$ (en $\mathrm{mm}$ ).

\begin{tabular}{cc}
\hline Vigas & Pilares \\
\hline$b \geq 200$ & $\operatorname{mín}(b, h) \geq 250$ \\
\hline
\end{tabular}

En la Tabla 3.7 se muestran las restricciones geométricas contempladas por los eurocódigos EC-2 y EC-8. El EC-2 no establece, para el caso de vigas, ninguna restricción en las dimensiones de la sección. Para el caso de pilares, este código resulta ser menos restrictivo que la instrucción EHE-08. En el caso del EC-8, de nuevo la menor ductilidad para la que el código aporta requerimientos constructivos es la ductilidad alta.

El código americano ACI-318-08 establece ciertas limitaciones en la geometría de la sección de elementos que constituyan pórticos especiales resistentes a flexión, los cuales deben garantizar unas prestaciones especiales de ductilidad (Tabla 3.8). 
Tabla 3.7 Restricciones geométricas en secciones rectangulares según EC-2 y EC-8 (en mm).

\begin{tabular}{ccc}
\hline Código & Vigas & Pilares \\
\hline EC-2 & Sin limitación & Dimensión mayor $\leq$ 4dimensión menor \\
EC-8 & $b \geq 200$ & $\operatorname{mín}(b, h) \geq 250$ \\
\hline
\end{tabular}

Tabla 3.8 Restricciones geométricas en secciones rectangulares según ACI-318-08 (en mm).

\begin{tabular}{cc}
\hline Vigas & Pilares \\
\hline \multirow{2}{*}{$b \geq \operatorname{mín}(0,3 h ; 250)$} & $\operatorname{mín}(b, h) \geq 300$ \\
& Dimensión menor $\geq 0,4$ dimensión mayor \\
\hline
\end{tabular}

\subsubsection{Resistencia a compresión simple del hormigón}

Los rangos de utilización para la resistencia a compresión simple del hormigón $f_{c}$ según la diversa normativa utilizada se muestran en la Tabla 3.9. Un aspecto a destacar es que el único código que no especifica un valor mínimo de resistencia a compresión simple del hormigón para su empleo en elementos de carácter estructural es el EC-2. Por tanto, se considera que la restricción $f_{c} \geq 12 \mathrm{MPa}$ impuesta en este código corresponde a elementos de carácter no estructural, como el hormigón de nivelación o limpieza de excavaciones. La limitación establecida por el EC-8 atiende a criterios sísmicos, debiendo aplicarse a elementos estructurales con ductilidad media. El código ACI-318-08 establece dos restricciones: una de carácter general $\left(f_{c} \geq 17 \mathrm{MPa}\right)$ y otra relativa a condicionantes de tipo sísmico $\left(f_{c} \geq 21 \mathrm{MPa}\right)$.

Tabla 3.9 Rangos de utilización de la resistencia a compresión simple del hormigón $f_{c}$ según normativa (en MPa).

\begin{tabular}{cc}
\hline Norma/Código & $f_{c}$ \\
\hline EHE-08 & $25-100$ \\
NCSE-02 & Sin limitaciones \\
EC-2 & $12-90$ \\
EC-8 & $\geq 16$ \\
ACI-318-08 & $\geq 17$ (con carácter general) \\
& $\geq 21$ (en zona sísmica) \\
\hline
\end{tabular}

El rango de valores impuesto finalmente en la selección de ensayos para la resistencia a compresión simple del hormigón es

$$
16 \leq f_{c} \leq 100 \mathrm{MPa}
$$

\subsubsection{Tipo de acero de las armaduras}

Las características de las armaduras de un elemento de hormigón armado quedan definidas, fundamentalmente, por el tipo de acero. Este aspecto condiciona los valores de ciertos conceptos relativos al estado de plastificación (límite elástico y deformación de 
plastificación) y al estado último (resistencia y deformación última) de las barras que constituyen la armadura.

En estructuras sismorresistentes de hormigón armado, se hace necesario el empleo de aceros con unas características adecuadas de ductilidad, de modo que los diferentes elementos presenten una suficiente capacidad de disipación de energía al transcurrir la acción sísmica.

La diversa normativa considerada establece generalmente una clasificación del acero en función de su nivel de ductilidad (Tabla 3.10), estableciendo que en estructuras proyectadas en zonas sísmicas deben emplearse aceros con un adecuado nivel de ductilidad, como los aceros SD en la instrucción EHE-08 y los de clase B y C en los códigos EC-2 y EC-8. La norma NCSE-02 no establece ninguna limitación especial, remitiéndose a los tipos de acero considerados en la normativa española. Los tipos de acero contemplados por el EC-8 se deben a la necesidad de alcanzar, como mínimo, un nivel de ductilidad media en el elemento, pudiendo incluso alcanzarse un nivel de ductilidad alta mediante el uso de aceros de tipo $\mathrm{C}$ o alta ductilidad.

Tabla 3.10 Tipos de acero contemplados en la normativa.

\begin{tabular}{cc}
\hline Norma/Código & Tipo de acero \\
\hline EHE-08 & Acero soldable (S) y acero soldable con características especiales de ductilidad (SD) \\
NCSE-02 & Acero soldable con características especiales de ductilidad (SD) \\
EC-2 & Acero de baja ductilidad (A), ductilidad media (B) y alta ductilidad (C) \\
EC-8 & Acero de ductilidad media (B) y alta ductilidad (C) \\
ACI-318-08 & Acero al carbón (S) \\
& Acero de baja aleación (W) \\
& Acero inoxidable \\
& Acero de rieles y ejes (R)
\end{tabular}

El código ACI-318-08 contempla aceros de diversa naturaleza. El acero al carbón (S) se emplea para la elaboración de barras corrugadas, siendo el tipo de acero más empleado actualmente en hormigón armado en Estados Unidos. El acero de baja aleación (W) se destina a la obtención de barras corrugadas empleadas en situaciones especiales donde se requieren propiedades controladas de tracción y soldabilidad. La aplicación del acero inoxidable se concreta en la elaboración de barras que requieran una alta resistencia a la corrosión o una permeabilidad magnética controlada. Finalmente, el empleo de acero de rieles y ejes se justifica para obtener barras que cumplan requisitos más estrictos en los ensayos de doblado.

Debido a que la base de datos inicial contiene aceros dúctiles laminados en caliente, con tratamiento térmico y conformados en frío, sin especificar condiciones de ductilidad, es necesario considerar para el tipo de acero, como excepción, el CM-2010, el cual contempla las anteriores categorías. Por tanto, el tipo de acero no constituye una variable 
que excluya elementos de la base de datos inicial para la elaboración de la selección de ensayos.

\subsubsection{Límite elástico del acero de la armadura longitudinal}

El valor del límite elástico del acero que constituye la armadura longitudinal es el aspecto clave para determinar su estado de plastificación, influyendo notablemente en el valor de conceptos como la curvatura, el momento, el giro de plastificación y el nivel de ductilidad de la sección transversal del elemento.

La normativa de carácter sísmico, esto es, la NCSE-02 y el EC-8, no impone condiciones al valor del límite elástico de la armadura longitudinal (Tabla 3.11), por lo que únicamente establece restricciones relativas a la ductilidad del acero.

Tabla 3.11 Rangos de utilización del límite elástico del acero de la armadura longitudinal $\left(f_{y}\right.$, $f_{y}^{\prime}$ ') según normativa (en $\mathrm{MPa}$ ).

\begin{tabular}{cc}
\hline Norma/Código & $f_{y}, f_{y}$, \\
\hline EHE-08 & $\geq 400$ \\
& $\geq 500$ \\
NCSE-02 & Sin limitaciones \\
EC-2 & $400-600$ \\
EC-8 & Sin limitaciones \\
ACI-318-08 & $280-550$ \\
\hline
\end{tabular}

El rango de valores escogido finalmente para el límite elástico del acero de la armadura longitudinal es

$$
280 \leq f_{y}, f_{y}^{\prime} \leq 600 \mathrm{MPa}
$$

\subsubsection{Límite elástico del acero de la armadura transversal}

La plastificación del acero de la armadura transversal marca un cambio importante en su comportamiento resistente, ejerciendo una fuerte influencia en el efecto de confinamiento del hormigón. Esto hace que el límite elástico de esta armadura intervenga directamente en la obtención de conceptos relacionados con el instante de rotura del elemento, como el giro y el momento último de la sección transversal.

La Tabla 3.12 muestra los rangos de utilización que la diversa normativa establece para el valor del límite elástico del acero de la armadura transversal. Algunas normas o códigos imponen valores máximos en el valor de esta variable. Para el caso de la instrucción EHE-08, la restricción impuesta se debe a una limitación de la deformación de la armadura transversal del $2 \%$, con lo que se consigue controlar su estado tensional en servicio y, consecuentemente, el nivel de fisuración experimentado por el elemento. El EC-2 establece el valor del límite elástico de la armadura transversal en función del valor del límite elástico de la armadura longitudinal. 
Tabla 3.12 Valores máximos para el límite elástico del acero de la armadura transversal $f_{y h}$ según normativa (en MPa).

\begin{tabular}{cc}
\hline Norma/Código & $f_{\text {yh }}$ \\
\hline EHE-08 & $\leq 400$ \\
NCSE-02 & Sin limitaciones \\
EC-2 & $\leq 0,8 f_{y}$ \\
EC-8 & Sin limitaciones \\
ACI-318-08 & $\leq 420$ \\
\hline
\end{tabular}

\subsubsection{Diámetro de la armadura longitudinal y transversal}

Las barras de acero corrugado que constituyen la armadura longitudinal y transversal de los elementos que componen la base de datos inicial presentan diámetros muy dispares, por lo que el diámetro ha resultado ser una variable especialmente selectiva en la confección de la selección de ensayos.

Por una parte, las barras de los elementos de la selección no deben presentar diámetros excesivamente grandes, pues esos diámetros dificultarían la ejecución de ciertos procesos de puesta en obra, como el enderezado, corte y doblado, además de incrementar excesivamente las longitudes de solape y de anclaje. Por otro lado, diámetros demasiado pequeños tampoco resulta conveniente, debido a la reducida capacidad mecánica y a los problemas que presentan en la soldadura.

La Tabla 3.13 muestra las limitaciones impuestas por la EHE-08 a los diámetros de las barras de armadura. La instrucción propone evitar, siempre que sea posible, el empleo de barras con un diámetro de $6 \mathrm{~mm}$ cuando se aplique cualquier proceso de soldadura, resistente o no resistente, en la elaboración y montaje de la armadura pasiva, por lo que el uso de este diámetro queda reservado a la elaboración de mallas electrosoldadas y armaduras básicas electrosoldadas en celosía.

Tabla 3.13 Rangos de utilización del diámetro de las barras de armadura según EHE-08 (en $\mathrm{mm})$.

\begin{tabular}{cccc}
\hline $\begin{array}{c}\text { Tipo de } \\
\text { elemento }\end{array}$ & $\begin{array}{c}\text { Armadura longitudinal } \\
\text { tracción }\left(\phi_{L}\right)\end{array}$ & $\begin{array}{c}\text { Armadura longitudinal } \\
\text { compresión }\left(\phi_{L}{ }^{\prime}\right)\end{array}$ & $\begin{array}{c}\text { Armadura } \\
\text { transversal }\left(\phi_{h}\right)\end{array}$ \\
\hline Vigas & $6-40$ & $6-40$ & $\begin{array}{c}\text { máx }\left(1 / 4 \phi_{L^{\prime} \text { máx }} ; 6\right)- \\
40\end{array}$ \\
Pilares & $6-40$ & $12-40$ & $\begin{array}{c}\text { máx }\left(1 / 4 \phi_{L^{\prime}}^{\prime} \text { máx }^{\prime} ; 6\right)- \\
40\end{array}$ \\
\hline
\end{tabular}

La norma NCSE-02 sólo impone limitaciones en el valor del diámetro de las barras de armadura transversal (Tabla 3.14). Esta condición se impone en los extremos de vigas y pilares para que exista un adecuado confinamiento del hormigón, aspecto que mejora el 
comportamiento de la rótulas plásticas que puedan generarse en estas zonas al actuar la acción sísmica.

Tabla 3.14 Diámetro mínimo de las barras de armadura según NCSE-02 (en mm).

\begin{tabular}{cccc}
\hline $\begin{array}{c}\text { Tipo de } \\
\text { elemento }\end{array}$ & $\begin{array}{c}\text { Armadura longitudinal } \\
\text { tracción }\left(\phi_{L}\right)\end{array}$ & $\begin{array}{c}\text { Armadura longitudinal } \\
\text { compresión }\left(\phi_{L}{ }^{\prime}\right)\end{array}$ & $\begin{array}{c}\text { Armadura } \\
\text { transversal }\left(\phi_{h}\right)\end{array}$ \\
\hline Vigas & Sin limitación & Sin limitación & $\geq 6$ \\
Pilares & Sin limitación & Sin limitación & $\geq 6$ \\
\hline
\end{tabular}

En las limitaciones impuestas por el EC-2 (Tabla 3.15) se considera un rango de valores del diámetro de la armadura longitudinal atendiendo a criterios relacionados con el control de la fisuración en el elemento estructural. De forma similar a la limitación impuesta por la norma NCSE-02, de la Tabla 3.16 se deduce que el EC-8 sólo impone restricciones en el valor del diámetro de las barras de armadura transversal, con objeto de garantizar unas adecuadas condiciones de ductilidad en las rótulas plásticas que puedan desarrollarse en los extremos de vigas y pilares. En lo que respecta al código ACI-31808, los diámetros adoptados son los considerados por la norma ASTM A615/A615M-13 (ASTM, 2008) (Tabla 3.17).

Tabla 3.15 Rangos de utilización del diámetro de las barras de armadura según EC-2 (en $\mathrm{mm})$.

\begin{tabular}{cccc}
\hline $\begin{array}{c}\text { Tipo de } \\
\text { elemento }\end{array}$ & $\begin{array}{c}\text { Armadura longitudinal } \\
\text { tracción }\left(\phi_{L}\right)\end{array}$ & $\begin{array}{c}\text { Armadura longitudinal } \\
\text { compresión }\left(\phi_{L}{ }^{\prime}\right)\end{array}$ & $\begin{array}{c}\text { Armadura transversal } \\
\left(\phi_{h}\right)\end{array}$ \\
\hline Vigas & $4-40$ & $4-40$ & $\begin{array}{c}\text { Sin limitación } \\
\text { máx }\left[1 / 4 \text { máx }\left(\phi_{L} ; \phi_{L}{ }^{\prime}\right) ;\right. \\
\text { Pilares }\end{array}$ \\
\hline
\end{tabular}

Tabla 3.16 Diámetro mínimo de las barras de armadura según EC-8 (en mm).

\begin{tabular}{cccc}
\hline $\begin{array}{c}\text { Tipo de } \\
\text { elemento }\end{array}$ & $\begin{array}{c}\text { Armadura longitudinal } \\
\text { tracción }\left(\phi_{L}\right)\end{array}$ & $\begin{array}{c}\text { Armadura longitudinal } \\
\text { compresión }\left(\phi_{L}{ }^{\prime}\right)\end{array}$ & $\begin{array}{c}\text { Armadura transversal } \\
\left(\phi_{h}\right)\end{array}$ \\
\hline Vigas & Sin limitación & Sin limitación & $\geq 6$ \\
Pilares & Sin limitación & Sin limitación & $\geq 6$ \\
\hline
\end{tabular}

Tabla 3.17 Rangos de utilización del diámetro de las barras de armadura según ACI-318-08 (en $\mathrm{mm}$ ).

\begin{tabular}{cccc}
\hline $\begin{array}{c}\text { Tipo de } \\
\text { elemento }\end{array}$ & $\begin{array}{c}\text { Armadura longitudinal } \\
\text { tracción }\left(\phi_{L}\right)\end{array}$ & $\begin{array}{c}\text { Armadura longitudinal } \\
\text { compresión }\left(\phi_{L}{ }^{\prime}\right)\end{array}$ & $\begin{array}{c}\text { Armadura transversal } \\
\left(\phi_{h}\right)\end{array}$ \\
\hline Vigas & $9,5-57,3$ & $9,5-57,3$ & $9,5-57,3$ \\
Pilares & $9,5-57,3$ & $9,5-57,3$ & $9,5-57,3$ \\
\hline
\end{tabular}


En las Tablas 3.13, 3.15 y 3.17 puede observarse los altos límites superiores contemplados por la instrucción EHE-08 y los códigos EC-2 y ACI-318-08 en los diámetros de las barras de armadura. Para conseguir una selección de ensayos correspondientes a elementos que se empleen normalmente en casos prácticos, se establecen los siguientes límites superiores para el diámetro de las barras:

$$
\begin{gathered}
\phi_{L}, \phi_{L}{ }^{\prime} \leq 32 \mathrm{~mm} \\
\phi_{h} \leq 14 \mathrm{~mm}
\end{gathered}
$$

Adicionalmente, el límite inferior considerado para el diámetro de la armadura longitudinal es

$$
\phi_{L}, \phi_{L}^{\prime} \geq 6 \mathrm{~mm}
$$

El rango final de valores adoptado para los diámetros de las barras de armadura en la realización de la selección de ensayos es el mostrado en la Tabla 3.18.

Tabla 3.18 Rango de variación de los diámetros de las barras de armadura considerados para la selección de ensayos (en mm).

\begin{tabular}{cccc}
\hline $\begin{array}{c}\text { Tipo de } \\
\text { elemento }\end{array}$ & $\begin{array}{c}\text { Armadura longitudinal } \\
\text { tracción }\left(\phi_{L}\right)\end{array}$ & $\begin{array}{c}\text { Armadura longitudinal } \\
\text { compresión }\left(\phi_{L}{ }^{\prime}\right)\end{array}$ & $\begin{array}{c}\text { Armadura transversal } \\
\left(\phi_{h}\right)\end{array}$ \\
\hline Vigas & $6-32$ & $6-32$ & $\operatorname{máx}\left(1 / 4 \phi_{\left.L^{\prime}{ }_{\text {máx }} ; 6\right)-14}\right.$ \\
Pilares & $6-32$ & $8-32$ & $\begin{array}{c}\text { máx }\left[\left(1 / 4 \phi_{L^{\prime} \text { máx }} ; 1 / 4\right.\right. \\
\left.\text { máx }\left(\phi_{L} ; \phi_{L^{\prime}}\right) ; 6\right]-14\end{array}$ \\
\hline
\end{tabular}

\subsubsection{Cuantías de armadura longitudinal}

Las cuantías mínimas de armadura longitudinal se imponen para limitar la fisuración debida a los fenómenos reológicos en el hormigón o para dotar al elemento de un adecuado nivel de ductilidad. Por otro lado, las cuantías máximas están relacionadas con la disminución de ductilidad que, durante el proceso de rotura del elemento, puede provocar un exceso de armadura.

Las variables relativas a la armadura longitudinal que se han considerado son:

$A_{s, m i n} \quad$ área mínima de armadura longitudinal de tracción en vigas,

$A_{s, m a ́ x} \quad$ área máxima de armadura longitudinal de tracción en vigas,

$A_{s^{\prime}, \text { min }}$ área mínima de armadura longitudinal de compresión en vigas,

$A_{s}{ }^{\prime}$,máx área máxima de armadura longitudinal de compresión en vigas,

$A_{\text {min }} \quad$ área total mínima de armadura longitudinal en pilares, y

$A_{\text {máx }} \quad$ área total máxima de armadura longitudinal en pilares.

Las cuantías de armadura longitudinal especificadas en la normativa se pueden agrupar atendiendo a criterios de tipo constructivo y/o de tipo sísmico.

Los criterios de tipo sísmico están relacionados con el nivel de ductilidad que se desee alcanzar en el elemento estructural. Así por ejemplo, ciertas normas y códigos contemplan que la cuantía máxima de armadura de tracción en vigas sea menor cuanto mayor sea el nivel de ductilidad a alcanzar en el elemento. En los casos en que, para una 
cierta variable de armadura longitudinal, la normativa establezca restricciones relativas a criterios constructivos y sísmicos, para la selección de ensayos se ha impuesto un rango de valores que permita el cumplimiento de ambos criterios. Cuando las limitaciones en las variables de armadura longitudinal atiendan sólo a criterios de tipo sísmico, se ha escogido el nivel de ductilidad que ofrezca el mayor rango de variación posible en estas variables.

En la Tabla 3.19 se muestran las expresiones para obtener los valores mínimos y máximos de la armadura longitudinal según la instrucción EHE-08. La limitación relativa al área máxima de armadura de tracción $A_{s, m a ́ x}$ se establece para dotar al elemento de un nivel alto de ductilidad. La expresión mostrada para la armadura mínima geométrica de tracción corresponde a elementos que contienen acero con un límite elástico de $500 \mathrm{MPa}$, ya que elementos con acero de límite elástico $400 \mathrm{MPa}$ requieren un valor de armadura mínima geométrica más exigente $(0,0033 b h)$, lo que excluiría a más elementos de la selección de ensayos.

Tabla 3.19 Armadura longitudinal mínima y máxima según EHE-08.

\begin{tabular}{cccccc}
\hline & Vigas & \multicolumn{3}{c}{ Pilares } \\
$A_{s, \text { mín }}$ & $A_{s, \text { máx }}$ & $A_{s^{\prime}, \text { mín }}$ & $A_{s^{\prime}, \text { máx }}$ & $A_{\text {mín }}$ & $A_{\text {máx }}$ \\
\hline máx $\left(0,0028 b h ; A_{s, \text { mín mec }} ; 2 \phi 14\right)$ & $\left(\rho^{\prime}+72 f_{c} / f_{y}{ }^{2}\right) b h$ & $0,33 A_{s}$ & Sin limitación & $0,01 b h$ & $0,06 b h$ \\
\hline
\end{tabular}

La norma NCSE-02, a diferencia del resto de normas y códigos, no contempla un valor máximo de área de armadura de tracción en vigas $A_{s, m a ́ x}$ (Tabla 3.20). Además, no se ha considerado el requerimiento impuesto por esta norma en vigas, por el cual se debe disponer una armadura mínima en ambas caras, correspondiente con una cierta fracción de la máxima armadura de momentos flectores negativos. Esto no se ha considerado al no presentar la base de datos inicial configuraciones de ensayos con uniones entre vigas y pilares, por lo que los valores de la armadura de flectores negativos en los extremos de la viga no están definidos.

Tabla 3.20 Armadura longitudinal mínima y máxima según NCSE-02.

\begin{tabular}{cccccc}
\hline & \multicolumn{2}{c}{ Vigas } & \multicolumn{3}{c}{ Pilares } \\
$A_{s, \text { min }}$ & $A_{s, \text { máx }}$ & $A_{s^{\prime}, \text { mín }}$ & $A_{s^{\prime}, \text { máx }}$ & $A_{\text {mín }}$ & $A_{\text {máx }}$ \\
\hline $\operatorname{máx}(2 \phi 14 ; 0,004 b h)$ & Sin limitación & $2 \phi 14$ & $0,025 b h$ & $0,01 b h$ & $0,06 b h$ \\
\hline
\end{tabular}

Las limitaciones impuestas a la armadura longitudinal por el EC-2 aparecen en la Tabla 3.21. Puede observarse cómo, a diferencia del resto de normas y códigos, el EC-2 no impone ningún valor mínimo al área de armadura de compresión en vigas $A_{s}$, ${ }^{\prime i n}$. 
Tabla 3.21 Armadura longitudinal mínima y máxima según EC-2.

\begin{tabular}{cccccc}
\hline & \multicolumn{2}{c}{ Vigas } & \multicolumn{2}{c}{ Pilares } \\
$A_{s, \text { min }}$ & $A_{s, \text { máx }}$ & $A_{s^{\prime}, \text { mín }}$ & $A_{s^{\prime}, \text { máx }}$ & $A_{\text {mín }}$ & $A_{\text {máx }}$ \\
\hline $\operatorname{máx}\left(0,26 f_{c t m} / f_{y k} b_{t} d ;\right.$ & $0,04 A_{c}$ & Sin limitación & $0,04 A_{c}$ & $\operatorname{máx}\left(0,1 N / f_{y} ; 0,002 A_{c}\right)$ & $0,04 A_{c}$ \\
$\left.0,0013 b_{t} d ; k_{c} k f_{c t, e f f} A_{c t} / \sigma_{s}\right)$ & & & & \\
\hline
\end{tabular}

El EC-8 establece dos limitaciones de valor mínimo de armadura de tracción para vigas $A_{s, \min }: A_{s} \geq f_{c t m} / f_{y} 0,5 b d$ para condiciones de ductilidad media y $A_{s} \geq 2 \phi 14$ para condiciones de ductilidad alta (Tabla 3.22). Como se pretende que el rango de valores sea lo más amplio posible, se ha escogido el valor menos restrictivo, esto es, el menor de los dos. La limitación correspondiente al valor máximo de la armadura de tracción en vigas $A_{s, m a ́ x}$ es la misma para los niveles de ductilidad medio y alto. El valor mínimo de armadura de compresión en vigas $A_{s^{\prime}, \text { min }}$ se ha establecido, al igual que para el caso del valor mínimo de armadura de tracción en vigas $A_{s, \min }$, como el mínimo de los impuestos por el EC-8, esto es, $A_{s} / 2$ para ductilidad media y $2 \phi 14$ para alta. En cuanto al valor máximo de armadura longitudinal de compresión $A_{s}$,máx, el EC-8, al igual que sucede con la instrucción EHE-08, no establece ninguna restricción.

De forma análoga a lo realizado con la norma NCSE-02, no se han considerado los valores de armadura impuestos a partir de una fracción de la armadura de flectores negativos presente en los extremos del elemento.

Por último, en lo relativo a los pilares, las condiciones impuestas por el EC-8 en los valores máximo y mínimo de armadura longitudinal total $A_{\text {mín }}$ y $A_{\text {máx }}$, respectivamente, coinciden para niveles de ductilidad alto y medio.

Tabla 3.22 Armadura longitudinal mínima y máxima según EC-8.

\begin{tabular}{cccccc}
\hline & \multicolumn{2}{c}{ Vigas } & & \multicolumn{2}{c}{ Pilares } \\
$A_{s, \text { min }}$ & $A_{s, \text { máx }}$ & $A_{s}^{\prime}$, mín & $A_{s^{\prime}, \text { máx }}$ & $A_{\text {mín }}$ & $A_{\text {máx }}$ \\
\hline $\operatorname{mín}\left(f_{c t m} / f_{y} 0,5 b d ;\right.$ & {$\left[\rho^{\prime}+0,0018 f_{c} /\left(\mu_{\phi} \varepsilon_{y} f_{y}\right)\right] b d$} & $\operatorname{mín}\left(0,5 A_{s} ;\right.$ & Sin & & \\
$2 \phi 14)$ & & $2 \phi 14)$ & limitación & $0,01 b d$ & $0,04 b d$ \\
\hline
\end{tabular}

En la Tabla 3.23 se muestran las expresiones para obtener los valores mínimos y máximos de la armadura longitudinal según el código ACI-318-08. Las dos limitaciones relativas al valor mínimo del área de armadura de tracción en vigas $A_{s, \min }$ atienden a criterios de tipo constructivo y símico, por lo que, siendo necesario cumplir ambas limitaciones, se ha escogido el valor máximo de las dos en la confección de la selección de ensayos. El valor mínimo de la armadura de compresión en vigas $A_{s}{ }^{\prime}$, mín se ha fijado como el mínimo de las dos limitaciones de carácter sísmico. Aunque ACI-318-08 establece un valor máximo de armadura longitudinal total en pilares $A_{\text {máx }}$ de $0,06 b h$ por criterios de carácter sísmico, también impone un valor $0,08 b h$ atendiendo a criterios 
constructivos, por lo que nuevamente se ha escogido el menor de los dos para satisfacer ambos criterios.

Tabla 3.23 Armadura longitudinal mínima y máxima según ACI-318-08.

\begin{tabular}{cccccc}
\hline & \multicolumn{2}{c}{ Vigas } & \multicolumn{3}{c}{ Pilares } \\
$A_{s, \text { min }}$ & $A_{s, \text { max }}$ & $A_{s}{ }^{\prime}{ }_{\text {,min }}$ & $A_{s}{ }^{\prime}$, max & $A_{\text {min }}$ & $A_{\text {max }}$ \\
\hline $\operatorname{máx}\left(0,25 \sqrt{ } f_{d} f_{y} b_{w} d ; 1,4 b_{w} d / f_{y}\right)$ & $0,025 b d$ & $\operatorname{mín}\left(0,25 \sqrt{ } f_{d} / f_{y} b_{w} d ; 1,4 b_{w} d / f_{y}\right)$ & $0,025 b d$ & $0,01 b h$ & $0,06 b h$ \\
\hline
\end{tabular}

\subsubsection{Separación de armadura transversal}

La separación de la armadura transversal es otra variable influyente en la determinación del estado último de un elemento estructural. Dicha separación debe ser la adecuada tanto para resistir los esfuerzos cortantes que debe soportar el elemento como para proporcionar al hormigón un adecuado confinamiento en las zonas donde puedan generarse rótulas plásticas al actuar la acción sísmica.

Para la selección de ensayos, la separación de armadura transversal $s_{h}$ se ha considerado imponiendo, en todos los casos, un valor máximo. No se ha considerado un valor mínimo dado que, salvo escasas excepciones, los elementos de la base de datos inicial presentan una separación suficiente en su armadura transversal como para ejecutar correctamente su hormigonado y puesta en obra.

En general, la separación de la armadura transversal impuesta por la normativa depende del ancho $b$, canto $h$ y canto útil $d$ de la sección transversal del elemento, los diámetros de las barras de armadura de tracción, compresión y transversal $\phi_{L}, \phi_{L}$ ' y $\phi_{h}$, respectivamente, y el ángulo $\alpha$ de los cercos que componen la armadura transversal con el eje del elemento.

En la Tabla 3.24 se muestran las expresiones para obtener los valores máximos de la separación de la armadura transversal según la instrucción EHE-08. Se establece un máximo $\left(s_{h} \leq 15 \phi_{L}{ }^{\prime},{ }_{\text {min }}\right)$ para poder considerar en el cálculo las armaduras pasivas en compresión. Otro máximo está relacionado con la dimensión mínima de la sección transversal y otras limitaciones ( $s_{h} \leq h / 2$ en vigas y $s_{h} \leq 150 \mathrm{~mm}$ en pilares) se imponen en las zonas del elemento donde no se desarrollan rótulas plásticas, con objeto de proporcionarle una mínima capacidad para formar parte de un sistema primario de resistencia sísmica.

La instrucción EHE-08 establece dos niveles de ductilidad en un elemento estructural: ductilidad alta y muy alta. Al confeccionar la selección de ensayos, las restricciones de carácter sísmico impuestas por esta instrucción en el valor máximo de la separación de armadura transversal se corresponden con un nivel de ductilidad distinto en función de que el elemento considerado sea viga o pilar. Así, y con objeto de escoger el mayor rango posible de valores en la máxima separación de armadura transversal, las 
restricciones escogidas se corresponden con un nivel de ductilidad muy alta en vigas, mientras que para el caso de pilares se considera un nivel de ductilidad alta.

Tabla 3.24 Separación máxima en la armadura transversal según EHE-08 (en mm).

\begin{tabular}{cc}
\hline Vigas & Pilares \\
\hline$\leq 15 \phi_{L^{\prime}}{ }^{\prime}$ mín & $\leq 15 \phi_{L^{\prime}, \text { mín }}$ \\
$\leq \min (b, h)$ & $\leq \operatorname{mín}(b, h)$ \\
$\leq 300$ & $\leq 150$ \\
$\leq h / 2$ & $\leq 15 \operatorname{mín}\left(\phi_{L}, \phi_{L}{ }^{\prime}\right)$ \\
$\leq \operatorname{mín}\left(d / 4 ; 8 \operatorname{mín}\left(\phi_{L}, \phi_{L^{\prime}}{ }^{\prime}\right) ; 24 \phi_{h} ; 200\right)$ & $\leq \operatorname{mín}\left[\left(b_{c} / 3 ; 150 ; 8 \operatorname{mín}\left(\phi_{L}, \phi_{L^{\prime}}{ }^{\prime}\right)\right]\right.$ \\
\hline
\end{tabular}

Al igual que sucede para el caso de la instrucción EHE-08, la norma NCSE-02 impone, como se muestra en la Tabla 3.25, un valor máximo de separación de armadura transversal en vigas $s_{h} \leq h / 2$ y en pilares $s_{h} \leq 15 \operatorname{mín}\left(\phi_{L}, \phi_{L}{ }^{\prime}\right)$ en zonas del elemento donde no se generen rótulas plásticas para dotarlo de un nivel mínimo de ductilidad. Al igual que la instrucción EHE-08, la norma NCSE-02 define, para el caso de vigas, un criterio de máxima separación de armadura transversal en función del nivel de ductilidad escogido. Sin embargo, a diferencia de la EHE-08, la NCSE-02 establece que, a mayor nivel de ductilidad escogido, menor es la separación máxima de la armadura transversal, por lo que las limitaciones mostradas en la Tabla 3.25 se corresponden con ductilidad alta como el menor nivel de ductilidad posible. Para el caso de pilares, sin embargo, la norma NCSE-02 establece los valores máximos de separación de armadura transversal en función del valor de aceleración de cálculo $a_{c}$, siendo estos valores mayores cuanto menor es el valor de dicha aceleración. Por tanto, las limitaciones escogidas se corresponden con un nivel de aceleración de cálculo $a_{c}=0,12 g$. Como excepción a lo anterior, la norma NCSE-02 establece un valor de separación máxima para vigas cuando la aceleración de cálculo es elevada $\left(a_{c} \geq 0,16 g\right)$.

Tabla 3.25 Separación máxima en la armadura transversal según NCSE-02 (en mm).

\begin{tabular}{cc}
\hline Vigas & Pilares \\
\hline$\leq h / 2$ & $\leq 15 \operatorname{mín}\left(\phi_{L}, \phi_{L}{ }^{\prime}\right)$ \\
$\leq \operatorname{mín}\left(h / 4 ; 8 \phi_{L}{ }^{\prime} ; 150\right)$ & $\leq \operatorname{mín}(b, h) / 3$ \\
$\leq 100\left(a_{c} \geq 0,16 g\right)$ & $\leq 100\left(\phi_{L}, \phi_{L}{ }^{\prime}=12\right.$ ó 14$)$ \\
& $\leq 150\left(\phi_{L}, \phi_{L}{ }^{\prime} \geq 16\right)$ \\
\hline
\end{tabular}

El EC-2 establece, como puede observarse en la Tabla 3.26, y de forma análoga a la instrucción EHE-08, una separación máxima de armadura transversal $s_{h} \leq 15 \phi_{L}$ ' para poder considerar en el cálculo la armadura longitudinal de compresión. Además, puede destacarse que el EC-2 impone una menor restricción a la separación de la armadura 
transversal atendiendo a criterios constructivos en pilares $\left(s_{h} \leq 240 \mathrm{~mm}\right)$ respecto a la instrucción EHE-08 $\left(s_{h} \leq 150 \mathrm{~mm}\right)$.

Tabla 3.26 Separación máxima en la armadura transversal según EC-2 (en mm).

\begin{tabular}{cc}
\hline Vigas & Pilares \\
\hline$\leq 0,75 d(1+\operatorname{cotg} \alpha)$ & $\leq 0,6 \operatorname{mín}\left[20 \operatorname{mín}\left(\phi_{L}, \phi_{L}{ }^{\prime}\right) ; \operatorname{mín}(b, h) ; 400\right]$ \\
$\leq 15 \phi_{L}{ }^{\prime}$ & \\
\hline
\end{tabular}

En lo relativo a las limitaciones impuestas por el EC-8 (Tabla 3.27), las separaciones que se han escogido para la selección de ensayos se corresponden con el menor nivel de ductilidad posible (ductilidad media), puesto que, al igual que la norma NCSE-02, este código considera que a mayor nivel de ductilidad, menor ha de ser el valor máximo de separación de armadura transversal.

Tabla 3.27 Separación máxima en la armadura transversal según EC-8 (en mm).

\begin{tabular}{cc}
\hline Vigas & Pilares \\
\hline$\leq \operatorname{mín}\left[h / 4 ; 24 \phi_{h} ; 225 ; 8 \operatorname{mín}\left(\phi_{L}, \phi_{L}{ }^{\prime}\right)\right]$ & $\leq \operatorname{mín}\left[b_{c} / 2 ; 175 ; 8 \operatorname{mín}\left(\phi_{L}, \phi_{L}{ }^{\prime}\right)\right]$ \\
\hline
\end{tabular}

Por último, dentro de los requisitos impuestos por el código ACI-318-08 (Tabla 3.28), la limitación $s_{h} \leq \operatorname{mín}(d / 2 ; 600)$ corresponde a criterios de carácter constructivo. El código ACI-318-08 también considera un espaciamiento máximo de la armadura transversal para que las barras longitudinales de compresión se puedan considerar en el cálculo $\left(s_{h} \leq \operatorname{mín}\left[16 \operatorname{mín}\left(\phi_{L}, \phi_{L}{ }^{\prime}\right) ; 48 \phi_{h} ; \operatorname{mín}(b, h)\right]\right)$. El valor $s_{h} \leq d / 2$ corresponde a la limitación impuesta en vigas en las zonas del elemento estructural donde no se desarrollan rótulas plásticas, mientras que para los pilares esta limitación se corresponde con $s_{h} \leq$ mín[mín $\left.(b, h) / 4 ; 6 \operatorname{mín}\left(\phi_{L}, \phi_{L}{ }^{\prime}\right) ; 150\right]$. El resto de limitaciones, más restrictivas, vienen impuestas para proporcionar un nivel de ductilidad adecuado en las zonas donde puedan desarrollarse rótulas plásticas.

Tabla 3.28 Separación máxima en la armadura transversal según ACI-318-08 (en mm).

\begin{tabular}{cc}
\hline Vigas & Pilares \\
\hline$\leq \min (d / 2 ; 600)$ & $\leq \operatorname{mín}\left[16 \operatorname{mín}\left(\phi_{L}, \phi_{L}{ }^{\prime}\right) ; 48 \phi_{h} ; \operatorname{mín}(b, h)\right]$ \\
$\leq \operatorname{mín}\left[16 \min \left(\phi_{L}, \phi_{L}{ }^{\prime}\right) ; 48 \phi_{h} ; \operatorname{mín}(b, h)\right]$ & $\leq \operatorname{mín}\left[8 \operatorname{mín}\left(\phi_{L}, \phi_{L}{ }^{\prime}\right) ; 24 \phi_{h} ; \operatorname{mín}(b, h) / 2 ; 300\right]$ \\
$\leq d / 2$ & \\
$\leq \operatorname{mín}\left(d / 4 ; 8 \min \left(\phi_{L}, \phi_{L}{ }^{\prime}\right) ; 24 \phi_{h} ; 300\right)$ & $\leq \operatorname{mín}\left[\operatorname{mín}(b, h) / 4 ; 6 \operatorname{mín}\left(\phi_{L}, \phi_{L}{ }^{\prime}\right) ; 150\right]$ \\
\hline
\end{tabular}




\section{Ajuste del comportamiento de plastificación y último de secciones rectangulares de hormigón armado}

\subsection{INTRODUCCIÓN}

En el análisis y diseño de estructuras, el comportamiento de un elemento estructural se encuentra fuertemente vinculado a las propiedades resistentes y la capacidad de deformación de su sección transversal. Si lo que se pretende es abordar la modelización numérica de estructuras de hormigón armado sometidas a acciones de tipo sísmico, resulta necesario conocer ciertos aspectos relacionados con la capacidad resistente y de deformación de la sección transversal de los diferentes elementos estructurales en los estados de plastificación y último, como son la curvatura, el momento y el giro de plastificación; y la curvatura, el momento y el giro últimos.

Tradicionalmente, el estudio de estos conceptos se ha abordado mediante el desarrollo de diagramas momento-curvatura (Kwak y Kim, 2010; Petschke et al., 2013) o momento-giro (Otani, Nagai y Aoyama, 1996; Haskett et al., 2009), los cuales reproducen el comportamiento de una sección frente a cargas monotónicas. La modificación de estos diagramas permite obtener la curva envolvente o cyclic envelope (Deierlein, Reinhorn y Willford, 2010) de los sucesivos ciclos de carga que definen el comportamiento de una sección frente a cargas cíclicas (Figura 4.1).

La dificultad para modelizar de forma precisa el estado de plastificación y último de una sección de hormigón armado mediante modelos y expresiones numéricas hace que, en ocasiones, sea necesario calibrar dichos modelos con ensayos experimentales. Esta estrategia permite obtener modelos que ofrecen una adecuada precisión en sus resultados, prescindiendo al mismo tiempo de rutinas de cálculo o análisis sofisticados. Este último aspecto implica que dichos modelos resulten relativamente sencillos y competitivos desde el punto de vista del tiempo computacional. 


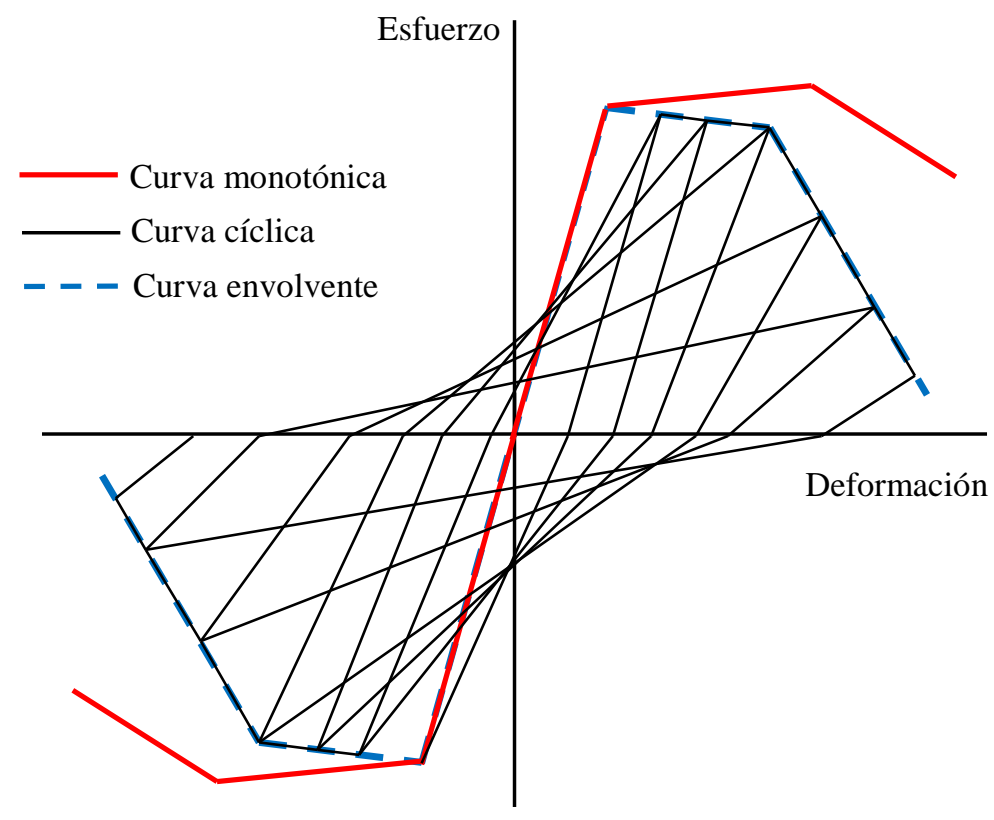

Figura 4.1 Relaciones esfuerzo-deformación en un elemento sometido a cargas monotónicas y cíclicas.

En esta tesis se proponen expresiones de comportamiento seccional aplicables a secciones rectangulares de hormigón armado, obtenidas a partir de las expresiones previamente desarrolladas en los trabajos de Panagiotakos y Fardis (2001) y Biskinis y Fardis (2010a, 2010b). Las expresiones propuestas por estos autores se describen en el apartado 4.2 .

\subsection{EXPRESIONES PREVIAS}

Panagiotakos y Fardis (2001) y Biskinis y Fardis (2010a, 2010b) desarrollaron expresiones para obtener conceptos relativos al estado de plastificación y último de la sección de un elemento estructural de hormigón armado. Concretamente, estas expresiones permiten obtener la curvatura, el momento y el giro de plastificación, así como el giro último. Aunque las expresiones están basadas en conceptos teóricos, algunas de ellas presentan ciertos términos empíricos al estar calibradas con una base de ensayos experimentales.

\subsubsection{Expresiones de Panagiotakos y Fardis (2001)}

Las expresiones desarrolladas en el trabajo de Panagiotakos y Fardis (2001) están ajustadas a la base de datos inicial descrita en el apartado 3.2 de esta tesis. Conviene destacar el hecho de que estas expresiones sólo son aplicables a secciones rectangulares de hormigón armado. 


\subsubsection{Curvatura de plastificación}

De acuerdo con Panagiotakos y Fardis (2001), el valor de la curvatura de plastificación $\phi_{y}$ se obtiene como el menor de los siguientes dos valores:

a) la curvatura producida por la plastificación del acero de la armadura de tracción

$$
\phi_{y}=\frac{f_{y}}{E_{s}\left(1-\xi_{y}\right) d}
$$

b) y la curvatura debida a una deformación de compresión excesiva en el hormigón

$$
\phi_{y}=\frac{1,8 f_{c}}{E_{c} \xi_{y} d}
$$

La profundidad de la fibra neutra en el instante de plastificación $\xi_{y}$ viene dada por

$$
\xi_{y}=\left(n^{2} A^{2}+2 n B\right)^{\frac{1}{2}}-n A
$$

Los parámetros $A$ y $B$ se obtienen mediante las ecuaciones (4.4) y (4.5) si la plastificación de la sección se produce por la plastificación del acero de la armadura de tracción, o empleando las ecuaciones (4.6) y (4.7) si la plastificación se produce por una deformación de compresión excesiva en el hormigón

$$
\begin{gathered}
A=\rho+\rho^{\prime}+\rho_{v}+\frac{N}{b d f_{y}} \\
B=\rho+\rho^{\prime} \delta^{\prime}+0,5 \rho_{v}\left(1+\delta^{\prime}\right)+\frac{N}{b d f_{y}} \\
A=\rho+\rho^{\prime}+\rho_{v}-\frac{N}{1,8 n b d f_{c}} \\
B=\rho+\rho^{\prime} \delta^{\prime}+0,5 \rho_{v}\left(1+\delta^{\prime}\right)
\end{gathered}
$$

donde

$E_{c} \quad$ módulo de deformación longitudinal secante del hormigón,

$E_{s}$ módulo de deformación longitudinal del acero de las armaduras,

$N \quad$ esfuerzo axil actuante en la sección (positivo si es de compresión y negativo en caso contrario),

$b$ ancho de la sección transversal del elemento,

$d$ canto útil de la sección transversal del elemento,

$f_{c}$ resistencia a compresión simple del hormigón,

$f_{y}$ límite elástico del acero de la armadura longitudinal de tracción,

$n$ ratio entre el módulo de deformación longitudinal del acero de las 
armaduras $E_{s}$ y el módulo de deformación longitudinal secante del hormigón $E_{c}$

$\delta$ ratio entre el recubrimiento mecánico $d^{\prime}$ y el canto útil de la sección $d$,

$\rho \quad$ cuantía de armadura longitudinal de tracción, definida como el cociente entre el área de armadura longitudinal de tracción $A_{s} \mathrm{y} b d$,

$\rho$ ' cuantía de armadura longitudinal de compresión, definida como el cociente entre el área de armadura longitudinal de compresión $A_{s}$ ' y $b d, \mathrm{y}$

$\rho_{v} \quad$ cuantía de armadura vertical de alma o web vertical reinforcement ratio, obtenida como el cociente entre el área de armadura longitudinal total situada entre la armadura longitudinal de tracción y compresión y $b d$.

\subsubsection{Momento de plastificación}

Panagiotakos y Fardis (2001) obtienen, mediante la condición de equilibrio, el momento de plastificación $M_{y}$ de una sección en función del valor de la curvatura de plastificación mediante la ecuación (4.8)

$$
\frac{M_{y}}{b d^{3}}=\phi_{y}\left\{E_{c} \frac{\xi_{y}^{2}}{2}\left(\frac{1+\delta^{\prime}}{2}-\frac{\xi_{y}}{3}\right)+\frac{E_{s}\left(1-\delta^{\prime}\right)}{2}\left[\left(1-\xi_{y}\right) \rho+\left(\xi_{y}-\delta^{\prime}\right) \rho^{\prime}+\frac{\rho_{v}}{6}\left(1-\delta^{\prime}\right)\right]\right\}
$$

\subsubsection{Giro de plastificación}

Al igual que sucede para el momento de plastificación, Panagiotakos y Fardis (2001) suponen que el giro de plastificación $\theta_{y}$ de una sección depende de la curvatura de plastificación. Estos autores obtienen el giro de plastificación mediante la suma de tres efectos:

a) el giro de plastificación debido a la deformación por flexión

$$
\theta_{y 1}=\phi_{y} \frac{L_{s}}{3}
$$

b) el giro de plastificación debido a la distorsión por esfuerzo cortante

$$
\theta_{y 2}=0,0025
$$

c) y el giro de plastificación debido al deslizamiento de la armadura longitudinal de tracción respecto del hormigón

$$
\theta_{y 3}=a_{s l} \frac{0,25 \varepsilon_{y} \phi_{L} f_{y}}{\left(d-d^{\prime}\right) \sqrt{f_{c}}}
$$

siendo

$L_{s} \quad$ distancia entre las secciones de momento flector máximo y nulo en el elemento, determinada como el cociente entre el momento flector $M$ y el esfuerzo cortante $V$ actuantes en la sección de máximo momento flector, 
$a_{s l}$ parámetro que indica si en el ensayo correspondiente se produce el deslizamiento entre la armadura longitudinal de tracción y el hormigón $\left(a_{s l}=\right.$ 1) o no $\left(a_{s l}=0\right)$, y

$\varepsilon_{y}$ deformación de plastificación a tracción del acero de las armaduras, obtenida como el cociente entre el límite elástico del acero $f_{y}$ y el módulo de deformación longitudinal $E_{s}$.

Por tanto, el giro de plastificación de la sección tiene por ecuación

$$
\theta_{y}=\phi_{y} \frac{L_{s}}{3}+0,0025+a_{s l} \frac{0,25 \varepsilon_{y} \phi_{L} f_{y}}{\left(d-d^{\prime}\right) \sqrt{f_{c}}}
$$

\subsubsection{Giro último}

La expresión propuesta por Panagiotakos y Fardis (2001) para el giro de una sección en el instante de rotura o giro último $\theta_{u}$ es

$$
\begin{aligned}
\theta_{u}(\%)= & a_{s t} a_{c y}\left(1+\frac{a_{s l}}{2,3}\right)\left(1-\frac{a_{w, r}}{3}\right)(0,2)^{v}\left[\frac{\max \left(0,01 ; \frac{\rho^{\prime} f_{y}^{\prime}}{f_{c}}\right)}{\max \left(0,01 ; \frac{\rho f_{y}}{f_{c}}\right)} f_{c}\right]^{0,275} \\
& \left(\frac{L_{s}}{h}\right)^{0,45}\left(\frac{100 \alpha \rho{ }_{h} f y h}{f_{c}}\right)_{1,3}{ }^{100} \rho_{d}
\end{aligned}
$$

donde

$a_{c y}$ parámetro que depende del tipo de carga empleada en el ensayo: 1 si la carga es monotónica y 0,6 si es cíclica,

$a_{s t} \quad$ parámetro que depende del tipo de acero: 1,5 para acero dúctil laminado en caliente (hot-rolled ductile steel); 1,25 para acero con tratamiento térmico (heat-treated steel); y 0,8 para acero conformado en frío (brittle coldworked steel),

$a_{w, r}$ parámetro que considera el tipo de elemento: 1 para muros de cortante y 0 para vigas y pilares,

$f_{y h} \quad$ límite elástico del acero de la armadura transversal,

$s_{h} \quad$ separación de la armadura transversal,

$\rho_{d}$ cuantía de armadura diagonal en elementos armados diagonalmente, obtenida como el cociente entre el área de armadura dispuesta a lo largo de una diagonal de la sección y $b d$, y

$\rho_{h} \quad$ cuantía de armadura transversal, determinada como el cociente entre el área de armadura transversal $A_{s h}$ y $b s_{h}$.

A su vez, el factor de efectividad de confinamiento $\alpha$, adoptado en el código 
modelo CM-2010 (FIB, 2012), se obtiene mediante

$$
\alpha=\left(1-\frac{s_{h}}{2 b_{c}}\right)\left(1-\frac{s_{h}}{2 h_{c}}\right)\left(1-\frac{\sum b_{i}^{2}}{6 b_{c} h_{c}}\right)
$$

siendo

$b_{c} \quad$ ancho del núcleo de hormigón de la sección tras el desprendimiento del recubrimiento o spalling,

$b_{i} \quad$ distancia entre dos barras consecutivas de armadura longitudinal atadas por horquillas o por esquinas de cercos,

$h_{c} \quad$ canto del núcleo de hormigón de la sección tras el desprendimiento del recubrimiento o spalling.

\subsubsection{Expresiones de Biskinis y Fardis (2010a, 2010b)}

Biskinis y Fardis (2010a, 2010b) realizaron ciertas modificaciones en las expresiones propuestas inicialmente por Panagiotakos y Fardis (2001). Las novedades introducidas corresponden a los siguientes aspectos:

a) la base de ensayos con la que se calibraron las expresiones contiene un mayor número de ensayos que la expuesta en Panagiotakos y Fardis (2001), incluyendo además elementos rehabilitados mediante bandas de polímeros reforzados con fibras FRP (fibre reinforced polymers) o recrecidos de hormigón, y

b) las expresiones propuestas son aplicables no sólo a secciones rectangulares, sino también a otras secciones con geometrías más complejas, tipo T, H, U y sección rectangular hueca, pudiendo aplicarse incluso a secciones del elemento situadas en zonas de solape de la armadura longitudinal.

El empleo de recrecidos de hormigón y bandas de fibras FRP constituye, de acuerdo con Tomás, Parra y Huesca (2015), una estrategia de refuerzo de estructuras de hormigón armado comúnmente empleada en intervenciones inmediatas post-sismo.

\subsubsection{Curvatura de plastificación}

De forma análoga a Panagiotakos y Fardis (2001), Biskinis y Fardis (2010a) obtienen el valor de la curvatura de plastificación de una sección como el menor de dos valores:

a) curvatura debida a la plastificación del acero de la armadura de tracción

$$
\phi_{y}=\frac{f_{y}}{E_{s}\left(1-\xi_{y}\right) d}
$$

b) curvatura debida a una deformación de compresión excesiva en el hormigón

$$
\phi_{y}=\frac{1,8 f_{c}}{E_{c} \xi_{y} d}
$$

La profundidad de la fibra neutra en el instante de plastificación de la sección $\xi_{y}$ 
viene definida por

$$
\xi_{y}=\left(n^{2} A^{2}+2 n B\right)^{\frac{1}{2}}-n A
$$

Las ecuaciones (4.18) y (4.19) determinan los parámetros $A$ y $B$ si la plastificación de la sección se produce por el acero de la armadura de tracción, mientras que las ecuaciones (4.20) y (4.21) se emplean si la plastificación se debe a una deformación de compresión excesiva en el hormigón.

$$
\begin{gathered}
A=\frac{b}{b_{w}}\left(\rho+\rho^{\prime}+\rho_{v}+\frac{N}{b d f_{y}}\right)+\frac{1}{n} \frac{t}{d}\left(\frac{b}{b_{w}}-1\right) \\
B=\frac{b}{b_{w}}\left(\rho+\rho^{\prime} \delta^{\prime}+\rho_{v} \frac{\left(1+\delta^{\prime}\right)}{2}+\frac{N}{b d f_{y}}\right)+\frac{1}{2 n}\left(\frac{t}{d}\right)^{2}\left(\frac{b}{b_{w}}-1\right) \\
A=\frac{b}{b_{w}}\left(\rho+\rho^{\prime}+\rho_{v}-\frac{N}{1,8 n b d f_{c}}\right)+\frac{1}{n} \frac{t}{d}\left(\frac{b}{b_{w}}-1\right) \\
B=\frac{b}{b_{w}}\left(\rho+\rho^{\prime} \delta^{\prime}+\rho_{v} \frac{\left(1+\delta^{\prime}\right)}{2}\right)+\frac{1}{2 n}\left(\frac{t}{d}\right)^{2}\left(\frac{b}{b_{w}}-1\right)
\end{gathered}
$$

siendo

$b_{w} \quad$ ancho del alma de la sección (para secciones tipo U o rectangular hueca este valor corresponde a la suma de los dos anchos), y

$t \quad$ espesor del ala comprimida en secciones tipo T, L, H o U.

Conviene mencionar que, para el caso de secciones rectangulares, las expresiones propuestas para el cálculo de la curvatura de plastificación por Biskinis y Fardis (2010a) coinciden con las desarrolladas por Panagiotakos y Fardis (2001).

\subsubsection{Momento de plastificación}

Biskinis y Fardis (2010a) obtienen el momento de plastificación de la sección a partir de la curvatura de plastificación, imponiendo el equilibrio de fuerzas normales a la sección mediante la ecuación (4.22). Las diferencias respecto a la ecuación (4.8) desarrollada por Panagiotakos y Fardis (2001) permiten considerar secciones con geometría distinta a la rectangular.

$$
\frac{M_{y}}{b d^{3}}=\phi_{y}\left\{\begin{array}{l}
E_{c}\left[\frac{\xi_{y}^{2}}{2}\left(\frac{1+\delta^{\prime}}{2}-\frac{\xi_{y}}{3}\right) \frac{b_{w}}{b}+\left(1-\frac{b_{w}}{b}\right)\left(\xi_{y}-\frac{t}{2 d}\right)\left(1-\frac{t}{2 d}\right) \frac{t}{2 d}\right] \\
+\frac{E_{s}\left(1-\delta^{\prime}\right)}{2}\left[\left(1-\xi_{y}\right) \rho+\left(\xi_{y}-\delta^{\prime}\right) \rho^{\prime}+\frac{\rho_{v}}{6}\left(1-\delta^{\prime}\right)\right]
\end{array}\right\}
$$

Del mismo modo que sucede para la curvatura de plastificación, aplicando la 
ecuación (4.22) a secciones rectangulares se obtiene la ecuación (4.8) expuesta en el trabajo de Panagiotakos y Fardis (2001).

\subsubsection{Giro de plastificación}

Siguiendo el mismo razonamiento que Panagiotakos y Fardis (2001), Biskinis y Fardis (2010a) plantean que el giro de plastificación de una sección se obtiene mediante la suma del giro debido a la deformación por flexión, el giro correspondiente a la distorsión por esfuerzo cortante y el giro inducido por el deslizamiento de la armadura longitudinal de tracción respecto del hormigón, dados por las ecuaciones (4.23), (4.24) y (4.25), respectivamente.

$$
\begin{aligned}
& \theta_{y 1}=\phi_{y} \frac{L_{s}+a_{v} z}{3} \\
& \theta_{y 2}=0,0014\left(1+1,5 \frac{h}{L_{s}}\right) \\
& \theta_{y 3}=a_{s l} \frac{\phi_{y} \phi_{L} f_{y}}{8 \sqrt{f_{c}}}
\end{aligned}
$$

Por tanto, el giro de plastificación se obtiene como

$$
\theta_{y}=\phi_{y} \frac{L_{s}+a_{v} z}{3}+0,0014\left(1+1,5 \frac{h}{L_{s}}\right)+a_{s l} \frac{\phi_{y} \phi_{L} f_{y}}{8 \sqrt{f_{c}}}
$$

donde

$V_{R d, c}$ esfuerzo cortante que produce la fisuración diagonal de la sección, definido por el EC-2 (European Committee for Standardization, 2004a) como el valor de cálculo del esfuerzo cortante máximo que resiste un elemento estructural sin armadura transversal,

$a_{v} \quad$ parámetro que considera la influencia del esfuerzo cortante en el giro de plastificación debido a la deformación por flexión: 0 si $V_{R d, c}>M_{y} / L_{s}$ y 1 en caso contrario, y

$z$ distancia entre las fuerzas resultantes de tracción y compresión en la sección, obtenida como la diferencia entre el canto útil de la sección transversal $d$ y el recubrimiento mecánico $d$ ' para vigas y pilares, o 0,8 veces el canto de la sección transversal $h$ para muros de cortante.

El valor de cálculo de la resistencia a esfuerzo cortante de un elemento sin armadura transversal $V_{R d, c}$ se obtiene, de acuerdo con el EC-2, mediante

$$
V_{R d, c}=\left[C_{R d, c} k\left(100 \rho f_{c}\right)^{\frac{1}{3}}+k_{1} \sigma_{c p}\right] b d
$$

con un valor mínimo de 


$$
V_{R d, c}=\left(v_{\min }+k_{1} \sigma_{c p}\right) b d
$$

donde

$$
\begin{gathered}
C_{R d, c}=\frac{0,18}{\gamma_{c}} \\
k=1+\sqrt{\frac{200}{d}} \leq 2,0 \\
k_{1}=0,15 \\
\sigma_{c p}=\frac{N}{A}<0,2 f_{c} \\
v_{\text {min }}=0,035 k^{\frac{3}{2}} f_{c}^{\frac{1}{2}}
\end{gathered}
$$

siendo $\gamma_{c}$ el coeficiente parcial de seguridad del hormigón y $\sigma_{c p}$ la tensión de compresión en el eje central debida a la carga axil. Con objeto de obtener el valor real de la resistencia a esfuerzo cortante $V_{R, c}$ del elemento sin armadura transversal, se ha adoptado $\gamma_{c}=1$.

Resulta relevante destacar que, de acuerdo con Biskinis y Fardis (2010a), los coeficientes de la ecuación (4.24), correspondiente al giro de plastificación debido a la influencia del esfuerzo cortante, son estrictamente empíricos, ya que se obtienen mediante la calibración con ensayos experimentales.

\subsubsection{Giro último}

La expresión propuesta por Biskinis y Fardis (2010b) para el giro último de una sección viene dada por la ecuación (4.34).

$$
\begin{aligned}
\theta_{u}= & a_{s t}\left(1-0,43 a_{c y}\right)\left(1+\frac{a_{s l}}{2}\right)\left(1-0,42 a_{w, r}\right)\left(1-\frac{2}{7} a_{w, n r}\right) 0,3^{v} \\
& {\left[\frac{\max \left(0,01 ; \omega_{2}\right)}{\max \left(0,01 ; \omega_{1}\right)} f_{c}\right]^{0,225}\left[\min \left(9, \frac{L_{s}}{h}\right)\right]^{0,35} 25^{\left(\frac{\alpha \rho_{h} f_{y h}}{f_{c}}\right)} 1,25^{100 \rho_{d}} }
\end{aligned}
$$

donde

$$
\begin{gathered}
\omega_{1}=\frac{\rho f_{y}+\rho_{v} f_{y v}}{f_{c}} \\
\omega_{2}=\frac{\rho^{\prime} f_{y}^{\prime}}{f_{c}}
\end{gathered}
$$

$a_{w, n r}$ parámetro que considera secciones con geometría diferente a la rectangular, siendo 1 para secciones en $\mathrm{T}, \mathrm{H}, \mathrm{U}$ o rectangular hueca y 0 para secciones rectangulares, 
$f_{y v} \quad$ límite elástico del acero de la armadura vertical de alma,

$\omega_{1}$ cuantía mecánica de armadura longitudinal de tracción y de alma, y

$\omega_{2}$ cuantía mecánica de armadura longitudinal de compresión

\subsubsection{Expresiones en la normativa}

La disponibilidad de expresiones que proporcionen conceptos relativos al comportamiento seccional de elementos de hormigón armado para los estados de plastificación y último en la normativa de diseño es escasa. No obstante, el código modelo CM-2010 (FIB, 2012a) y el EC-8 (European Committee for Standardization, 2005) proponen expresiones para determinar el giro de la sección de un elemento estructural en dichos estados.

El código modelo CM-2010 establece la verificación del estado límite último para la acción sísmica en elementos unidimensionales, determinando las máximas deformaciones inelásticas admisibles a flexión en las rótulas plásticas que se generan en los extremos de dichos elementos. Para ello, plantea expresiones basadas en los trabajos de Biskinis y Fardis (2010a, 2010b) para obtener el giro de plastificación $\theta_{y}$ y último $\theta_{u}$ de una sección.

Por otro lado, el código EC-8 tiene por objeto establecer una serie de prescripciones para la evaluación y adecuación sísmica de edificios existentes. Define los siguientes estados límite en función del nivel de daño de la estructura tras el transcurso de la acción sísmica:

a) estado límite de proximidad al colapso $N C$ : la estructura está seriamente dañada, con rigidez y resistencia lateral residuales bajas, pero los elementos verticales son capaces de resistir cargas verticales. La mayor parte de los elementos no estructurales han colapsado, produciéndose importantes desplazamientos relativos permanentes. La estructura está cerca del colapso, no siendo capaz de resistir otro terremoto.

b) estado límite de daños significativos $S D$ : la estructura se encuentra significativamente dañada, con poca rigidez y resistencia lateral residuales, siendo los elementos verticales capaces de soportar acciones verticales. Los elementos no estructurales han sufrido daños, aunque los tabiques y rellenos no han fallado fuera de su plano medio. Se producen desplazamientos relativos permanentes moderados. La estructura puede resistir terremotos moderados, aunque su reparación puede no resultar rentable.

c) estado límite de limitación de daños $D L$ : la estructura está ligeramente dañada, con elementos estructurales que no han experimentado una plastificación significativa y que mantienen sus propiedades de resistencia y rigidez. Los elementos no estructurales pueden presentar una fisuración generalizada, pero su reparación resulta económicamente viable. Los desplazamientos relativos permanentes son despreciables y la estructura no necesita medidas de 
reparación.

La información específica para evaluar estructuras de hormigón armado se encuentra en su Anexo A. Para analizar la capacidad de deformación de un elemento, el EC-8 propone expresiones que determinan el giro de la sección. Concretamente, la expresión propuesta para el giro de plastificación $\theta_{y}$ de una sección, correspondiente al estado de limitación de daños $D L$, es

$$
\theta_{y}=\phi_{y} \frac{L_{s}+a_{v} z}{3}+0,0014\left(1+1,5 \frac{h}{L_{s}}\right)+\frac{\varepsilon_{y}}{d-d^{\prime}} \frac{\phi_{L} f_{y}}{6 \sqrt{f_{c}}}
$$

El giro último $\theta_{u}$ de la sección, obtenido para el estado límite de proximidad al colapso $N C$, viene dado por

$$
\theta_{u}=\frac{1}{\gamma_{e l}} 0,016(0,3)^{v}\left[\frac{\max \left(0,01 ; \omega_{2}\right)}{\max \left(0,01 ; \omega_{1}\right)} f_{c}\right]^{0,225}\left[\min \left(9, \frac{L_{s}}{h}\right)\right]^{0,35} 25^{\left(\frac{\alpha \rho_{h} f_{y h}}{f_{c}}\right)} 1,25^{100 \rho_{d}}
$$

donde

$\gamma_{e l} \quad$ coeficiente cuyo valor depende de si el elemento estructural es de carácter sísmico primario $\left(\gamma_{e l}=1,5\right)$ o secundario $\left(\gamma_{e l}=1\right)$.

Conviene destacar que las ecuaciones (4.37) y (4.38) resultan similares a las propuestas por Biskinis y Fardis (2010a, 2010b).

\subsection{EXPRESIONES DE AJUSTE CON LAS BASES DE ENSAYOS}

Tras describir las expresiones propuestas por otros autores y las expuestas en el EC-8 para reproducir el comportamiento seccional en el estado de plastificación y último de la sección de un elemento de hormigón armado, en este apartado se plantean nuevas expresiones para la determinación de ciertos conceptos. El objetivo principal es modificar algunas expresiones existentes, introduciendo en ellas una serie de coeficientes que mejoren el ajuste a la selección de ensayos y a la base de datos inicial, descritas en el capítulo 3 de esta tesis.

Las expresiones sobre las que se aplican los coeficientes de ajuste son el momento de plastificación, el giro de plastificación y el giro último de la sección, planteadas por Biskinis y Fardis (2010a, 2010b). Estas expresiones son más recientes y sofisticadas que las desarrolladas por Panagiotakos y Fardis (2001), por lo que modelizan con mayor precisión el comportamiento seccional de un elemento de hormigón armado.

Debido a las buenas prestaciones que estas expresiones ofrecen (BenaventCliment, Escolano-Margarit y Morillas, 2014), y con objeto de respetar su formulación en la medida de lo posible, los coeficientes de ajuste propuestos se aplican únicamente a los términos más influyentes en la expresión correspondiente. Dichos términos se identifican tras realizar diversas pruebas de tanteo de valores. Conviene destacar, además, que no se añaden nuevos términos a las expresiones originales, tan sólo se 
mejoran los resultados mediante el empleo de coeficientes de ajuste.

Resulta relevante el hecho de que el número de valores medidos para la curvatura de plastificación en las bases de ensayos descritas en el capítulo 3 de esta tesis es muy inferior a los disponibles para el momento y el giro de plastificación. Este aspecto, unido a la fuerte dependencia del momento y el giro de plastificación respecto a la curvatura de plastificación, hace que no sea posible proporcionar expresiones para estos tres conceptos con un adecuado ajuste a sus correspondientes ensayos, por lo que se ha decidido conservar la expresión propuesta por Biskinis y Fardis (2010a) para la curvatura de plastificación.

\subsubsection{Expresiones de ajuste con la selección de ensayos}

La selección de ensayos constituye una base de datos de elementos que cumplen las prescripciones sísmico-constructivas impuestas por las principales normas y códigos de diseño. Por tanto, el objetivo principal de la calibración de las ecuaciones (4.22), (4.26) y (4.34) con dicha selección es la obtención de unas expresiones que reproduzcan un comportamiento más ajustado a elementos empleados en estructuras convencionales de hormigón armado situadas en zonas sísmicas. Adicionalmente, la homogeneidad de la selección de ensayos contribuye a que las expresiones calibradas ofrezcan una menor dispersión en los resultados respecto a las expresiones iniciales desarrolladas por Biskinis y Fardis (2010a, 2010b).

Tras realizar diversas pruebas introduciendo coeficientes en diferentes términos de la ecuación (4.22), la propuesta para ajustar el momento de plastificación a la selección de ensayos tiene por expresión

$$
\frac{M_{y}}{b d^{3}}=\phi_{y}\left\{E_{c} \frac{\xi_{y}^{2}}{2}\left(C_{1} \frac{1+\delta^{\prime}}{2}-C_{2} \frac{\xi_{y}}{3}\right)+\frac{E_{s}\left(1-\delta^{\prime}\right)}{2}\left[C_{3}\left(1-\xi_{y}\right) \rho+C_{4}\left(\xi_{y}-\delta^{\prime}\right) \rho^{\prime}+C_{5} \frac{\rho_{v}}{6}\left(1-\delta^{\prime}\right)\right]\right\}
$$

La expresión planteada para el giro de plastificación, introduciendo cinco coeficientes $C_{6}$ a $C_{10}$ en la ecuación (4.26), viene definida por

$$
\theta_{y}=\phi_{y} \frac{C_{6} L_{s}+C_{7} a_{v} z}{3}+C_{8}\left(1+C_{9} \frac{h}{L_{s}}\right)+C_{10} a_{s l} \frac{\phi_{y} \phi_{L} f_{y}}{8 \sqrt{f_{c}}}
$$

En la ecuación (4.40) se observa que los coeficientes propuestos afectan a los tres sumandos de la misma, esto es, el giro por flexión, el giro por efecto del esfuerzo cortante y el giro por deslizamiento de armaduras, pues en este caso los tres sumandos tienen influencia en el resultado.

Los coeficientes propuestos para calibrar la expresión del giro último desarrollada por Biskinis y Fardis (2010b) con la selección de ensayos se muestran en la ecuación (4.41). 


$$
\begin{aligned}
\theta_{u}= & a_{s t}\left(1-C_{11} a_{c y}\right)\left(1+\frac{a_{s l}}{2}\right)\left(1-0,42 a_{w, r}\right)\left(1-\frac{2}{7} a_{w, n r}\right)\left(C_{12}\right)^{v}\left[\frac{\max \left(0,01 ; \omega_{2}\right)}{\max \left(0,01 ; \omega_{1}\right)} f_{c}\right]^{C_{13}} \\
& {\left[\min \left(9, \frac{L_{s}}{h}\right)\right]^{C_{14}} 25^{\left(\frac{\alpha \rho_{h} f_{y h}}{f_{c}}\right)} C_{15}{ }^{100 \rho_{d}} }
\end{aligned}
$$

\subsubsection{Expresiones de ajuste con la base de datos inicial}

Tras plantear las expresiones para el ajuste con la selección de ensayos, en este apartado se propone una serie de coeficientes para calibrar el momento de plastificación, el giro de plastificación y el giro último con la base de datos inicial. Resulta conveniente destacar que la mayor homogeneidad de la selección de ensayos respecto a la base de datos inicial ha de introducir, previsiblemente, una menor dispersión en las expresiones ajustadas a dicha selección respecto a las calibradas con la base de datos inicial. Por tanto, la calibración de las ecuaciones (4.22), (4.26) y (4.34) con la base de datos inicial se aborda para corroborar esta previsión, por la cual las mejoras obtenidas han de resultar más significativas si se ajustan las ecuaciones a la selección de ensayos que si se ajustan a la base de datos inicial. La propuesta de expresiones correspondientes al momento y al giro de plastificación, introduciendo los coeficientes $C_{1}$ a $C_{10}$, es la misma que la realizada para la selección de ensayos mediante las ecuaciones (4.39) y (4.40), respectivamente. La expresión propuesta para abordar el ajuste del giro último con la base de datos inicial viene dada por la ecuación (4.42).

$$
\begin{aligned}
\theta_{u}= & C_{11} a_{s t}\left(1-C_{12} a_{c y}\right)\left(1+C_{13} a_{s l}\right)\left(1-C_{14} a_{w, r}\right)\left(1-\frac{2}{7} a_{w, n r}\right)\left(C_{15}\right)^{v}\left[\frac{\max \left(0,01 ; \omega_{2}\right)}{\max \left(0,01 ; \omega_{1}\right)} f_{c}\right]^{C_{16}} \\
& {\left[\min \left(9, \frac{L_{s}}{h}\right)\right]^{C_{17}} C_{18}\left(\frac{\alpha \rho_{h} f_{y h}}{f_{c}}\right) C_{19}{ }^{100 \rho_{d}} }
\end{aligned}
$$

En la ecuación (4.42) se considera un mayor número de coeficientes de ajuste respecto a los empleados para calibrar la expresión del giro último con la selección de ensayos dada por la ecuación (4.41). Esto se debe a que, para mejorar los resultados del giro último aplicado a la base de ensayos inicial respecto a los obtenidos con las expresiones propuestas por Panagiotakos y Fardis (2001) y Biskinis y Fardis (2010b), es necesario modificar un mayor número de términos en la expresión inicial desarrollada por Biskinis y Fardis (2010b). Además, se introduce el coeficiente $C_{14}$ que multiplica a la variable $a_{w r}$, que considera si el elemento es un muro de cortante, puesto que en la base de datos inicial hay 61 ensayos correspondientes a este tipo de elemento.

\subsection{ALGORITMOS EMPLEADOS EN LA CALIBRACIÓN}

Si lo que se pretende es obtener el mejor ajuste posible entre las bases de ensayos y las ecuaciones (4.39) a (4.42), resulta necesario recurrir al empleo de técnicas numéricas para determinar los valores de los coeficientes de ajuste propuestos, entre las cuales, las 
técnicas de optimización son unas herramientas muy útiles para este propósito.

En este apartado se realiza una descripción de los algoritmos empleados en cada proceso de calibración. A este respecto, conviene destacar el hecho de que el número de ensayos presentes en las bases de ensayos difiere según se considere la base de datos inicial o la selección de ensayos, por lo que este aspecto repercute de forma directa en el tiempo computacional invertido en el proceso de calibración y, consecuentemente, en la elección del tipo de algoritmo a emplear.

\subsubsection{Ajuste con la selección de ensayos}

Con objeto de abordar el ajuste de las ecuaciones (4.39) a (4.41) con la selección de ensayos, se emplea un proceso de optimización basado en algoritmos genéticos, pues, como se ha comentado en el Capítulo 2, este tipo de algoritmos han venido siendo ampliamente utilizados para la calibración de modelos numéricos con resultados satisfactorios. Concretamente, la herramienta empleada para la calibración es la toolbox implementada en el software MATLAB® (MATLAB, 2014).

De acuerdo con Salajegheh y Gholizadeh (2012), los algoritmos genéticos han sido extensamente empleados para resolver problemas complejos en el campo de la ingeniería estructural. Respecto al ajuste con la selección de ensayos, el número de ensayos presente en la selección, en torno a 200, hace que el proceso de ajuste sea abordable desde el punto de vista del tiempo computacional empleando este tipo de algoritmos.

Los algoritmos genéticos (Holland, 1975; Goldberg, 1989) constituyen una estrategia de optimización basada en una búsqueda estocástica del espacio de diseño, imitando el proceso natural de evolución biológica al establecer un símil entre ciertos aspectos numéricos y conceptos típicos de la genética. En este sentido, estos algoritmos operan sobre una población inicial de individuos o posibles soluciones del problema, originando sucesivas iteraciones o generaciones donde los mejores individuos se escogen aplicando el principio de supervivencia, según el cual sobreviven aquellos que mejor se adaptan al medio, es decir, cuya solución se aproxima más a la solución óptima del problema. Esta estrategia origina mejores aproximaciones a la solución óptima del problema a medida que se obtienen las sucesivas generaciones.

Durante el proceso de optimización, los diferentes individuos que constituyen una población se codifican mediante cadenas o cromosomas de valores binarios 0 y 1 , denominados genes. El conjunto de genes que constituyen un individuo se denomina genotipo y la decodificación del genotipo da lugar al fenotipo, que representa los valores reales de las variables del problema para dicho individuo. Así por ejemplo, en un problema definido mediante tres variables de diseño $x_{1}, x_{2}$ y $x_{3}$, un individuo se puede codificar mediante el cromosoma de la Figura 4.2. 


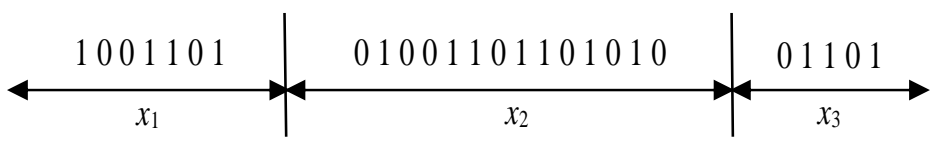

Figura 4.2 Cromosoma de un individuo en un problema con tres variables de diseño.

Tras la obtención de una solución real del problema mediante la decodificación del cromosoma que representa a un individuo, es posible evaluar la bondad de dicha solución empleando la función de adaptación o fitness function. Esta función considera conjuntamente la función objetivo y las restricciones del problema. Siguiendo la analogía con la genética, el valor de la función de adaptación representa la habilidad del individuo para sobrevivir en su hábitat. Además, esta función interviene en la selección de los individuos que generan descendencia, de modo que a los que presentan un mejor valor se les atribuye una mayor probabilidad de ser seleccionados.

Una vez que los individuos son seleccionados, generan descendientes mediante el cruce o intercambio de algunos fragmentos de su genotipo siguiendo ciertas pautas probabilísticas. Posteriormente, en los descendientes generados se produce una mutación o cambio en algunos de sus genes siguiendo, de nuevo, unos criterios específicos de probabilidad. Tras el cruce y la mutación de los descendientes, éstos se reinsertan en la población inicial, los genotipos de los individuos de la nueva población son decodificados y se evalúa nuevamente la función objetivo para cada uno de ellos.

Es de esperar que el comportamiento medio de los individuos de la nueva generación mejore el de la generación anterior, puesto que los mejores individuos sobreviven y los peores son excluidos. El proceso anteriormente descrito se repite hasta que el algoritmo obtenga la solución óptima del problema o se supere un cierto número de iteraciones previamente establecido.

Un esquema de algoritmo genético simple es el establecido por Goldberg (1989): $\mathrm{t}=0$

Generación de población $\mathrm{P}(\mathrm{t})$;

Evaluación de población $\mathrm{P}(\mathrm{t})$;

while $\mathrm{t}<$ nmaxgeneraciones

$\mathrm{t}=\mathrm{t}+1$;

Selección de individuos de $\mathrm{P}(\mathrm{t}-\mathrm{l})$

Reproducción de parejas de individuos en $\mathrm{P}(\mathrm{t})$;

Evaluación de $\mathrm{P}(\mathrm{t})$;

end

El proceso de optimización implementado en la toolbox de MATLAB® comienza con la generación aleatoria de una población inicial de individuos. La toolbox permite la división de la población inicial en una serie de subpoblaciones, aplicando el proceso de optimización a cada población de forma independiente. Además, ofrece la posibilidad de utilizar subrutinas que permiten la migración o intercambio de individuos entre ellas. 
La tasa y los patrones de migración de individuos entre subpoblaciones simulan la diversidad genética que se da en la naturaleza. De acuerdo con Mühlenbein, Schomisch, y Born (1991) y Starkweather, Whitley y Mathias (1990), esta estrategia mejora los resultados respecto a los obtenidos al resolver el problema empleando una única población. A este respecto, en el ajuste de las ecuaciones (4.39) a (4.41) con la selección de ensayos se ha considerado una población total dividida en 10 subpoblaciones, cada una con un tamaño constante de 5000 individuos. El número máximo de generaciones o iteraciones del proceso es 500, mientras que la tasa de migración entre subpoblaciones es del $50 \%$ de los individuos, produciéndose cada 10 generaciones.

Posteriormente a la constitución de la población inicial, resulta necesario emplear ciertos operadores para el desarrollo de las sucesivas generaciones, como son el operador selección, el operador cruce y el operador mutación.

El proceso de selección consiste en determinar el número de veces que se escoge un cierto individuo para crear descendientes en la siguiente generación. De entre los métodos disponibles en la toolbox para realizar este proceso (Chipperfield, Fleming y Fonseca, 1994), se escoge el muestreo estocástico universal o stochastic universal sampling (SUS). Este procedimiento genera una serie de $N$ puntos igualmente espaciados por el espacio de diseño, tantos como individuos se quieran escoger para crear descendencia. Posteriormente, los individuos de la población se redistribuyen aleatoriamente por el espacio de diseño, escogiéndose los $N$ individuos con valores de la función objetivo más próximos a los $N$ puntos inicialmente dispuestos en el espacio de diseño.

El operador de cruce permite intercambiar fragmentos de cromosomas entre individuos de una misma subpoblación. El tipo de cruce empleado en la toolbox es el cruce multipunto o multi-point crossover, el cual divide un cromosoma en varios fragmentos para realizar el intercambio (Figura 4.3).

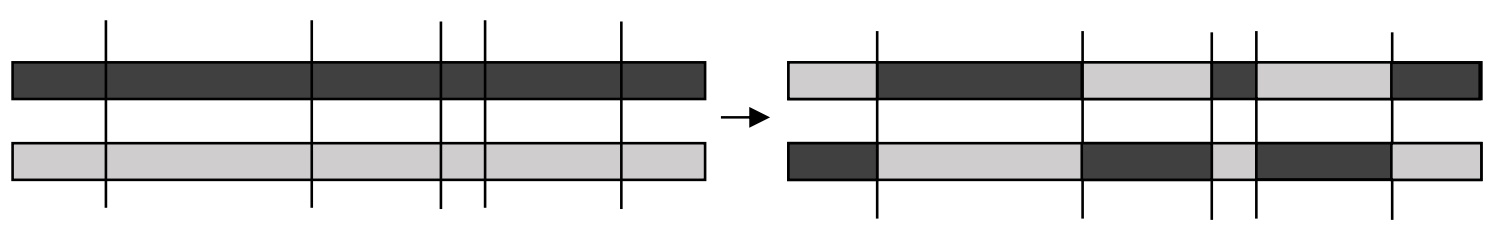

Figura 4.3 Individuos modificados mediante el operador de cruce multipunto con 5 puntos de división.

De acuerdo con Chipperfield, Fleming y Fonseca (1994), el operador de cruce multipunto permite realizar una amplia búsqueda del espacio de diseño, dificultando la convergencia hacia individuos que presenten buenos valores de la función objetivo en las primeras etapas del proceso de optimización. Como consecuencia, se consigue un proceso de búsqueda más robusto, disminuyendo la posibilidad de obtener una solución que constituya un óptimo local.

La mutación producida en los individuos obtenidos tras realizar el proceso de 
cruce se aplica de forma aleatoria, con una probabilidad baja, estos es, con valores comprendidos normalmente en el rango 0,001-0,01. El operador mutación evita que la probabilidad de búsqueda en cualquier región del espacio de diseño sea cero. Adicionalmente, permite recuperar genes que hayan podido eliminarse durante los procesos previos de selección y cruce y que contribuyan a mejorar las soluciones generadas. En la toolbox empleada se ha utilizado un operador de mutación que aplica una tasa de mutación linealmente decreciente desde 1 en el inicio del proceso, hasta 0 en la convergencia hacia la solución. La disminución de la tasa de mutación a medida que avanza el proceso es fundamental para garantizar la convergencia.

Con objeto de reinsertar los individuos en la población, se ha dispuesto una estrategia elitista, según la cual ciertos individuos están presentes en generaciones sucesivas. En concreto, se ha considerado un elitismo del $50 \%$ de los mejores individuos, reinsertándolos con los mejores de la generación anterior. Se considera que la solución óptima del problema es la obtenida tras realizar 10 pruebas con diferentes poblaciones iniciales generadas aleatoriamente.

\subsubsection{Ajuste con la base de datos inicial}

El ajuste de las ecuaciones (4.39), (4.40) y (4.42) con los 1012 ensayos existentes en la base de datos inicial exige emplear un algoritmo adecuado desde el punto de vista de la rapidez computacional y la facilidad de convergencia hacia el óptimo global. Las técnicas de optimización basadas en algoritmos metaheurísticos han evolucionado en los últimos años, existiendo una extensa variedad de algoritmos para la resolución de problemas.

De entre las técnicas más novedosas, se ha escogido el algoritmo de la luciérnaga o Firefly Algorithm (FA) para abordar el ajuste de las ecuaciones con la base de datos inicial. De acuerdo con Yang y He (2013), la eficiencia de este algoritmo se debe principalmente a los siguientes aspectos:

a) subdivisión automática del espacio de búsqueda de la solución en subgrupos, y

b) posibilidad de controlar la aleatoriedad del proceso de búsqueda de la solución a medida que se van produciendo las iteraciones.

Las ventajas del algoritmo FA frente a otros tipos de algoritmos han quedado patentes en diversos estudios. Así, Chatterjee y Mahanti (2012) obtuvieron mejoras en los resultados respecto a lo obtenido aplicando el algoritmo Artificial Bee Colony (ABC), mientras que Gandomi, Yang y Alavi (2011) resolvieron distintos problemas, obteniendo mejores resultados con menor tiempo computacional respecto a los algoritmos genéticos y los algoritmos Particle Swarm Optimization (PSO), Simulated Annealing (SA) y Harmony Search (HS). Otro aspecto a destacar es que no se han localizado trabajos en los que el algoritmo FA se emplee en la calibración de modelos numéricos de hormigón armado con ensayos experimentales, por lo que la utilización de este algoritmo en la tesis supone una ampliación del campo de aplicación de las técnicas 
de optimización.

El algoritmo FA simula el comportamiento de las luciérnagas durante su vuelo, imitando sus mecanismos de atracción en función de las señales luminosas que emiten. Las luciérnagas se relacionan y comunican entre sí siguiendo diversos patrones de bioluminiscencia. Algunos de los atributos que caracterizan estos patrones son la tasa de luminiscencia o rate of flashing y su duración.

El algoritmo FA empleado en esta tesis simplifica el comportamiento real de las luciérnagas al considerar las siguientes hipótesis:

a) todas las luciérnagas se atraen del mismo modo, sin considerar su sexo,

b) la atracción entre luciérnagas es proporcional a su brillo o intensidad de la luz $I$, disminuyendo ambos aspectos a medida que aumenta la distancia entre luciérnagas. Las luciérnagas menos brillantes se moverán hacia las que más brillen. Si no existen luciérnagas que brillen más que una dada, ésta se moverá aleatoriamente por el espacio de diseño, y

c) el brillo de una luciérnaga está determinado por su valor de la función objetivo.

En el algoritmo FA empleado, resulta relevante la formulación dispuesta para considerar la variación de la intensidad de la luz emitida por una luciérnaga con la distancia y la atracción entre luciérnagas. La intensidad $I$ de la luz emitida por una luciérnaga situada en una posición $\mathbf{x}$ es proporcional al valor de la función objetivo $f$ a optimizar, esto es

$$
I(\boldsymbol{x}) \alpha f(\boldsymbol{x})
$$

donde $\alpha$ representa la proporcionalidad entre ambos conceptos.

Además, la intensidad $I$ de la luz que emite una luciérnaga, percibida a una cierta distancia $r$, es inversamente proporcional al cuadrado de dicha distancia, es decir

$$
I \propto \frac{1}{r^{2}}
$$

Yang (2009) establece que la forma más simple de considerar la intensidad de la luz en función de la distancia $I(r)$ es

$$
I(r)=\frac{I_{0}}{r^{2}}
$$

donde $I_{0}$ es la intensidad de luz emitida por una luciérnaga.

El algoritmo FA considera, además, que el aire presente en el medio absorbe luz, disminuyendo la intensidad de la luz a medida que aumenta la distancia entre luciérnagas. Para considerar la influencia del medio, con un coeficiente de absorción de luz $\gamma$, la intensidad de la luz se define como

$$
I(r)=I_{0} e^{-\gamma r}
$$


Si se considera la influencia de la distancia y la absorción de luz por parte del medio, se tiene la expresión de la intensidad de la luz I implementada en el algoritmo FA empleado en esta tesis

$$
I(r)=I_{0} e^{-\not r^{2}}
$$

Respecto a la atracción entre luciérnagas $\beta$, ésta se define mediante

$$
\beta(r)=\beta_{0} e^{-\not r^{2}}
$$

siendo $\beta_{0}$ el valor de la atracción cuando la luciérnaga que emite la luz y la que la percibe se encuentran en la misma posición, esto es, $r=0$. En la mayoría de aplicaciones se adopta el valor $\beta_{0}=1$ (Yang, 2009). Conviene destacar el hecho de que la atracción que produce una cierta luciérnaga es relativa, debiendo considerarse desde la perspectiva del resto de luciérnagas, por lo que esta variable depende de la distancia $r_{i j}$ entre las luciérnagas $i$ y $j$. Dadas dos luciérnagas $i$ y $j$ situadas en las posiciones $\mathbf{x}_{i} \mathbf{y}$ $\mathbf{x}_{j}$, la distancia $r_{i j}$ entre ellas viene dada por

$$
r_{i j}=\left\|\mathbf{x}_{i}-\mathbf{x}_{j}\right\|=\sqrt{\sum_{k=1}^{m}\left(x_{i, k}-x_{j, k}\right)^{2}}
$$

siendo

$m \quad$ número de componentes de los vectores de posición $\mathbf{x}_{i}$ y $\mathbf{x}_{j}$, coincidente con el número de variables del problema,

$x_{i, k} \quad$ componente $k$-ésima de la posición $\mathbf{x}_{i}$ que ocupa la luciérnaga $i, \mathrm{y}$

$x_{j, k} \quad$ componente $k$-ésima de la posición $\mathbf{x}_{j}$ que ocupa la luciérnaga $j$.

La posición de una luciérnaga $i$ atraída por otra $j$ con más brillo es

$$
\mathbf{x}_{i}=\mathbf{x}_{i}+\beta_{0} e^{-\not r_{i j}^{2}}\left(\mathbf{x}_{j}-\mathbf{x}_{i}\right)+\alpha_{r}\left(r d-\frac{1}{2}\right)
$$

donde

rd generador de números aleatorios uniformemente distribuidos en el intervalo $[0,1], \mathrm{y}$

$\alpha_{r} \quad$ parámetro que introduce aleatoriedad en el movimiento de las luciérnagas por el espacio de diseño.

El parámetro $\gamma$ caracteriza la variación de la atracción entre luciérnagas a lo largo del proceso de optimización. De acuerdo con Yang (2009), este parámetro desempeña un papel crucial en el comportamiento general del algoritmo y, especialmente, en su velocidad de convergencia. En la mayoría de aplicaciones adopta valores comprendidos en el rango 0,01-100.

A continuación se muestra un esquema básico del algoritmo FA (Yang, 2009): 
Función objetivo $\mathrm{f}(\mathrm{x}), \mathrm{x}=\left(\mathrm{x}_{1}, \ldots \ldots \ldots, \mathrm{x}_{\mathrm{d}}\right)^{\mathrm{T}}$

Generación de población inicial de luciérnagas $\mathrm{x}_{\mathrm{i}}\left(\mathrm{i}=1,2, \ldots \ldots, \mathrm{n}_{\mathrm{f}}\right)$

Intensidad de luz $\mathrm{I}_{\mathrm{i}}$ en $\mathrm{x}_{\mathrm{i}}$ determinada por $\mathrm{f}\left(\mathrm{x}_{\mathrm{i}}\right)$

Definición del coeficiente de absorción de luz $\gamma$

while $\mathrm{t}<$ maxgeneraciones

for $\mathrm{i}=1$ : $\mathrm{n}_{\mathrm{f}}$ todas las $\mathrm{n}_{\mathrm{f}}$ luciérnagas

for $\mathrm{j}=1: \mathrm{i}$ todas las $\mathrm{n}_{\mathrm{f}}$ luciérnagas

if $\mathrm{I}_{\mathrm{j}}>\mathrm{I}_{\mathrm{i}}$

La luciérnaga i se mueve hacia la $\mathrm{j}$

end if

La atracción disminuye con la distancia r mediante una función del tipo e $\mathrm{e}^{-\gamma \mathrm{r}}$

Evaluación de nuevas soluciones y actualización de la intensidad de luz end for $\mathrm{j}$

end for $\mathrm{i}$

Evaluación de las luciérnagas y localización de la mejor

end while

Resultados y visualización

El algoritmo FA empleado presenta, además de los parámetros anteriormente descritos, los siguientes:

$N_{\text {máx }}$ número máximo de iteraciones en el proceso de optimización,

$n_{f}$ tamaño de la población de luciérnagas,

$s_{\alpha} \quad$ velocidad a la que disminuye el parámetro de aleatoriedad $\alpha_{r}$,

$t_{r} \quad$ parámetro que determina el instante del proceso de optimización donde el parámetro de aleatoriedad $\alpha_{r}$ comienza a reducirse, y

$\beta_{\text {min }}$ valor mínimo de la atracción entre luciérnagas $\beta$, para grandes distancias entre ellas.

La atracción entre luciérnagas considerada en el algoritmo de esta tesis difiere de la expresada en la ecuación (4.48), siendo

$$
\beta(r)=e^{-\gamma r^{2}}+\beta_{\min }\left(1-e^{-\gamma r^{2}}\right)
$$

Esta expresión se implementa para impedir que las luciérnagas lejanas a la que más brilla ( $r$ muy grande) sean excluidas en el proceso de búsqueda de la solución del problema por presentar una atracción nula, esto es, $\beta=0$, obligando a que se sientan atraídas con un valor mínimo $\beta_{\min }$ y se puedan desplazar mediante un cierto avance recogido en su vector de posición x. Otra modificación, dada por la ecuación (4.52), establece la posición $\mathbf{x}_{i}$ de una luciérnaga en cada iteración del proceso

$$
\mathbf{x}_{i}=\mathbf{x}_{i}+\beta(r)\left(\mathbf{x}_{j}-\mathbf{x}_{i}\right)+t m
$$


siendo $\mathrm{tm}$ un parámetro de aleatoriedad obtenido al modificar el término $\alpha_{r}(r d-$ 1/2) de la ecuación (4.50), considerando el tamaño de la población y la distancia entre luciérnagas. A este respecto, cuanto mayor es el tamaño de la población de luciérnagas, menor es la aleatoriedad introducida en el proceso, pues hay un mayor número de individuos que participan en la búsqueda de la solución. Respecto a la distancia entre luciérnagas, cuanto más alejada se encuentra una luciérnaga de la que más brilla, mayor aleatoriedad presentará en la búsqueda de la solución.

Si se observan las expresiones que determinan la atracción entre luciérnagas $\beta$ y la posición $\mathbf{x}_{i}$ de una luciérnaga $i$, dadas por las ecuaciones (4.51) y (4.52), respectivamente, se deduce que para medios totalmente transparentes, esto es, $\gamma=0$, el valor de la atracción $\beta$ es la unidad, por lo que la luciérnaga $i$ se desplaza hasta la posición de la luciérnaga $j$ más luminosa.

La Tabla 4.1 muestra los valores adoptados por los diferentes parámetros del algoritmo FA. Tras diversas pruebas realizadas, se concluye que una población de $n_{f}=$ 100 luciérnagas es suficientemente extensa para resolver el problema. Debido a la facilidad del algoritmo para converger, un número máximo de iteraciones $N_{\text {máx }}=1,5 n_{f}$ resulta suficiente para el ajuste de las expresiones, pudiendo adoptarse en algunos casos valores menores, como $0,5 n_{f}$ o $0,6 n_{f}$.

Tabla 4.1 Valores adoptados por los parámetros del algoritmo FA.

\begin{tabular}{cc}
\hline Parámetro & Valor \\
\hline$N_{\max }$ & $1,5 n_{f}$ \\
$t_{r}$ & 0,5 \\
$n_{f}$ & 100 \\
$s_{\alpha}$ & 1 \\
$\alpha_{r}$ & $0,001-0,01 n_{f}$ \\
$\beta_{\min }$ & 0,1 \\
$\beta_{0}$ & 1 \\
$\gamma$ & 0,5 \\
\hline
\end{tabular}

Con objeto de realizar una exploración del espacio de diseño lo más amplia posible, el parámetro de aleatoriedad $\alpha_{r}$ considerado ha sido el máximo de los valores recomendables, esto es, $\alpha_{r}=0,01 n_{f}$, mientras que el valor escogido para el parámetro $t_{r}$ $=0,5$ implica que la aleatoriedad en la búsqueda comienza a disminuir a mitad del proceso. El coeficiente de absorción considerado $\gamma=0,5$ supone un medio con una transparencia intermedia entre totalmente transparente $(\gamma=0)$ y completamente opaco $(\gamma$ $=1$ ). El valor adoptado por la atracción mínima $\beta_{\text {min }}=0,1$ ha demostrado ser apropiado para involucrar a todas las luciérnagas en la búsqueda de la solución.

La Figura 4.4 muestra la influencia del parámetro $s_{\alpha}$ en la disminución de la aleatoriedad a medida que avanza el proceso de optimización. Los dos valores que 
puede adoptar este parámetro, 0 y 1, implican la utilización de una función cuadrática diferente para reducir el valor del parámetro de aleatoriedad $\alpha_{r}$. De la Figura 4.4 se desprende que suponer $s_{\alpha}=0$ implica una mayor aleatoriedad en las últimas iteraciones del proceso, obteniéndose resultados con menor precisión que los obtenidos al adoptar $s_{\alpha}=1$. La adopción de $s_{\alpha}=0$ permite obtener una aproximación a la solución óptima, resultando útil para localizar máximos y mínimos locales en el espacio de búsqueda.

La complejidad de las expresiones propuestas para el ajuste a la base de datos inicial y del espacio de diseño, el cual presenta mínimos de carácter local, hace necesario el empleo de un algoritmo de optimización con unas características adecuadas. La naturaleza estocástica del algoritmo empleado exige considerar una importante componente de aleatoriedad, reduciéndola al final del proceso para mejorar la precisión de la solución obtenida. Aunque el algoritmo empleado no garantice la obtención de un óptimo global, tras la realización de diversas pruebas se ha constatado que la probabilidad de que se alcance dicho óptimo es del $90 \%$, circunstancia que denota una adecuada robustez en el algoritmo.

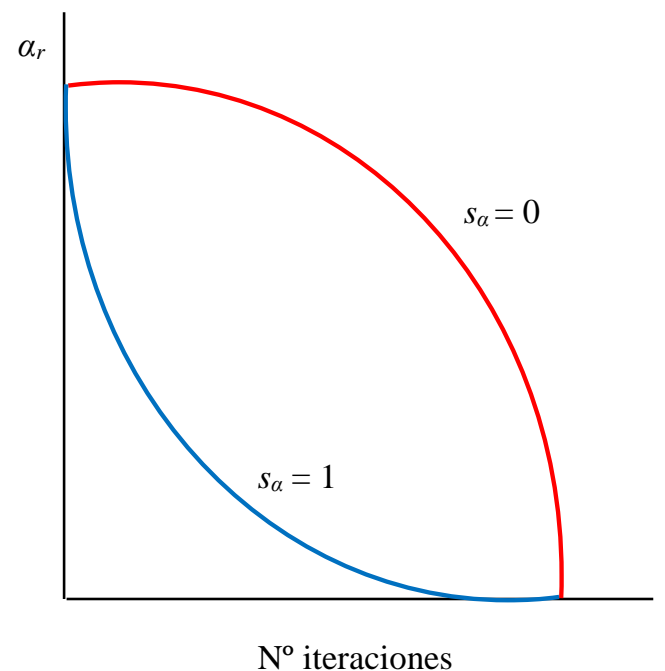

Figura 4.4 Disminución del parámetro de aleatoriedad $\alpha_{r}$ en función del parámetro $s_{\alpha}$.

\subsection{ANÁLISIS DE RESULTADOS}

En este apartado se presentan las expresiones para el momento de plastificación, el giro de plastificación y el giro último de secciones rectangulares de hormigón armado ajustadas con la base de datos inicial y la selección de ensayos. Previamente se define una serie de parámetros estadísticos necesarios para analizar los resultados obtenidos con estas expresiones, comparándolos posteriormente con los obtenidos aplicando las expresiones de Panagiotakos y Fardis (2001) y Biskinis y Fardis (2010a, 2010b). Resulta de interés establecer también una comparación con las expresiones propuestas en la normativa de diseño, para lo cual se emplean las ecuaciones del EC-8 descritas en 
el apartado 4.2.3. Se describe, además, el planteamiento adoptado en el proceso de optimización para realizar el ajuste.

\subsubsection{Parámetros estadísticos}

Para cada una de las expresiones a ajustar se considera una variable $x$ definida, para cada ensayo, mediante el cociente entre el valor presente en las bases de ensayos o valor experimental $x_{\text {exp }}$ y el valor obtenido con la expresión correspondiente o valor predicho $x_{\text {pred: }}$

$$
x=\frac{x_{\text {exp }}}{x_{\text {pred }}}
$$

La particularización de la variable $x$ para el momento de plastificación, el giro de plastificación y el giro último viene dada por las ecuaciones (4.54), (4.55) y (4.56), respectivamente.

$$
\begin{gathered}
x=\frac{M_{y, \text { exp }}}{M_{y, \text { pred }}} \\
x=\frac{\theta_{y, \text { exp }}}{\theta_{y, \text { pred }}} \\
x=\frac{\theta_{u, \text { exp }}}{\theta_{u, \text { pred }}}
\end{gathered}
$$

Los parámetros estadísticos considerados se clasifican según los siguientes grupos:

a) medidas de síntesis: se emplean para mostrar las características esenciales de una serie de valores adoptados por la variable. Dentro de este grupo se consideran las medidas de tendencia central, cuyos valores representan a todos los datos de que se dispone, y las medidas de dispersión, las cuales acompañan a las medidas de tendencia central y ofrecen información acerca de la dispersión de los valores de la variable respecto a estas últimas, y

b) parámetros para el análisis de la relación entre valores experimentales $x_{\exp } \mathrm{y}$ predichos $x_{\text {pred }}$.

Las medidas de tendencia central consideradas en este estudio son la media y la mediana, las cuales se emplean comúnmente en las publicaciones científicas. La expresión de la media $\bar{x}$ viene dada por

$$
\bar{x}=\frac{\sum_{i=1}^{n_{e}} \frac{x_{i, \mathrm{exp}}}{x_{i, \text { pred }}}}{n_{e}}
$$

donde 
$n_{e} \quad$ número de ensayos considerado,

$x_{i, \exp }$ valor experimental presente en el ensayo $i, \mathrm{y}$

$x_{i, p r e d}$ valor predicho para el ensayo $i$.

La mediana de un conjunto de valores se define como el valor que ocupa la posición central tras ordenarlos de menor a mayor.

Al presentar un valor correspondiente a una medida de tendencia central, resulta conveniente acompañarlo con alguna medida de dispersión que muestre su representatividad. De entre las medidas de dispersión existentes, en este trabajo se considera la desviación típica $\sigma$, el rango intercuartílico $R I$ y el coeficiente de variación $C V$. La desviación típica se emplea frecuentemente para acompañar a la media, ya que ambos parámetros presentan las mismas unidades. Su expresión, para una población, se corresponde con

$$
\sigma=\sqrt{\frac{\sum_{i=1}^{n_{e}}\left(\frac{x_{i, \text { exp }}}{x_{i, p r e d}}-\bar{x}\right)^{2}}{n_{e}}}
$$

Si se considera la selección de ensayos descrita en el capítulo 3 de esta tesis como una muestra de la población total, es decir, de la base de datos inicial, resulta necesario definir la desviación típica muestral $s$, aplicando la corrección de Bessel al dividir por $n_{e^{-1}}$ según la ecuación (4.59).

$$
s=\sqrt{\frac{\sum_{i=1}^{n_{e}}\left(\frac{x_{i, \text { exp }}}{x_{i, \text { pred }}}-\bar{x}\right)^{2}}{n_{e}-1}}
$$

Se define el rango intercuartílico $R I$ como la diferencia entre el tercer cuartil $Q_{3}$ y el primer cuartil $Q_{1}$. El primer y tercer cuartil son valores que dejan por debajo el $25 \mathrm{y}$ $75 \%$ de los valores de la variable, respectivamente. Por tanto, el rango intercuartílico representa un intervalo en el que se encuentra el $50 \%$ de los valores de la variable, resultando adecuado para mostrar la distribución de dichos valores en torno a la mediana.

El coeficiente de variación $C V$ viene dado por

$$
C V=\frac{\sigma}{\bar{x}}
$$

Con objeto de evaluar el grado de relación lineal entre los valores experimentales $x_{\text {exp }}$ de las bases de ensayos y los obtenidos con las expresiones ajustadas o valores predichos $x_{\text {pred }}$, se realiza un análisis de regresión. Previamente a la realización de dicho análisis, resulta necesario determinar si existe alguna relación de dependencia entre ambas variables. Para ello, se realiza el siguiente contraste de hipótesis: 
a) hipótesis nula $H_{0}$ : las variables analizadas son independientes, y

b) hipótesis alternativa $H_{1}$ : existe dependencia entre ambas variables.

La aceptación o rechazo de la hipótesis nula $H_{0}$ depende del p-valor o $p$-value. Este parámetro representa el riesgo de rechazar la hipótesis nula siendo ésta verdadera. Valores bajos del p-valor sugieren que es seguro rechazar la hipótesis nula y suponer, por tanto, que las variables analizadas presentan cierta dependencia. Concretamente, si el p-valor resulta inferior a un nivel de significación de 0,05 , se rechaza la hipótesis nula. Tras analizar la relación entre los valores experimentales y predichos para el momento y el giro de plastificación y el giro último con el programa Statgraphics ${ }^{\circledR}$ (Statgraphics, 2013), el p-valor obtenido para todos los casos es 0, por lo que se puede suponer una cierta dependencia entre ambos valores.

Para cuantificar en un índice numérico el grado de relación lineal existente entre las dos variables consideradas, se utiliza la covarianza $\operatorname{cov}\left(x_{\text {pred }}, x_{\text {exp }}\right)$ y el coeficiente de correlación lineal $r$. La covarianza viene dada por la expresión

$$
\operatorname{cov}\left(x_{\text {pred }}, x_{\text {exp }}\right)=\frac{\sum_{i=1}^{n_{e}}\left(x_{i, p r e d}-\overline{x_{\text {pred }}}\right)\left(x_{i, e x p}-\overline{x_{\text {exp }}}\right)}{n_{e}}
$$

donde

$\overline{x_{\exp }}$ valor medio de los valores experimentales, $\mathrm{y}$

$\overline{x_{\text {pred }}}$ valor medio de los valores predichos.

La covarianza presenta el inconveniente de depender de las dimensiones en que se expresan las variables. Con objeto de obtener un índice numérico adimensional, se define el coeficiente de correlación lineal $r$ como

$$
\begin{gathered}
r=\frac{\operatorname{cov}\left(x_{\text {pred }}, x_{\text {exp }}\right)}{\sigma_{x, \text { pred }} \sigma_{x, \exp }} \\
\sigma_{x, \text { pred }}=\sqrt{\frac{\sum_{i=1}^{n_{e}}\left(x_{i, p r e d}-\overline{x_{\text {pred }}}\right)^{2}}{n_{e}}} \\
\sigma_{x, \exp }=\sqrt{\frac{\sum_{i=1}^{n_{e}\left(x_{i, \text { exp }}-\overline{x_{\text {exp }}}\right)^{2}}}{n_{e}}}
\end{gathered}
$$

siendo

$\sigma_{x, \exp }$ desviación típica poblacional de los valores experimentales, y

$\sigma_{x, \text { pred }}$ desviación típica poblacional de los valores predichos.

Al igual que se ha comentado para la desviación típica, se puede obtener la covarianza muestral, aplicada a la selección de ensayos, dividiendo por $n_{e^{-1}}$ en la 
ecuación (4.61).

La recta de regresión que relaciona los valores experimentales y predichos tiene por ecuación

$$
x_{\text {exp }}=a^{\prime}+b^{\prime} x_{\text {pred }}
$$

siendo

$a^{\prime} \quad$ ordenada en el origen de la recta de regresión, y

b' coeficiente de regresión o pendiente de la recta de regresión.

Los coeficientes $a^{\prime}$ y $b^{\prime}$ se obtienen mediante las siguientes expresiones:

$$
\begin{gathered}
b^{\prime}=\frac{\operatorname{cov}\left(x_{\text {pred }}, x_{\text {exp }}\right)}{\sigma_{x, p r e d}} \\
a^{\prime}=\overline{x_{\text {exp }}}-b^{\prime} \overline{x_{\text {pred }}}
\end{gathered}
$$

Un ajuste perfecto entre los valores experimentales y predichos daría lugar a una recta de regresión $x_{\text {exp }}=x_{\text {pred }}$. Por tanto, cuanto más próximo sea el valor de $a$ ' a 0 y el de $b^{\prime}$ a 1 , más se aproximan los valores obtenidos con las expresiones ajustadas a los valores experimentales. Con objeto de evaluar la bondad del ajuste entre la recta de regresión y los puntos obtenidos al representar en unos ejes los valores experimentales y los predichos, se define el coeficiente de determinación $R^{2}$. Este coeficiente representa el porcentaje de puntos situados sobre la recta de regresión, adoptando valores entre 0 y 1. Para el caso de regresión lineal simple, el coeficiente de determinación puede obtenerse como

$$
R^{2}=r^{2}
$$

Otro parámetro que se emplea para medir el ajuste entre valores experimentales y predichos es el valor absoluto de la suma de sus diferencias $S_{d}$, cuya expresión se corresponde con

$$
S_{d}=\sum_{i=1}^{n_{e}}\left|x_{i, e x p}-x_{i, p r e d}\right|
$$

por lo que un valor reducido de este parámetro es indicativo de un adecuado ajuste entre ambos valores.

Finalmente se considera el diagrama box-plot o caja-bigotes para la variable $x$ definida en la ecuación (4.53). Un ejemplo de este diagrama puede verse en la Figura 4.5 . 


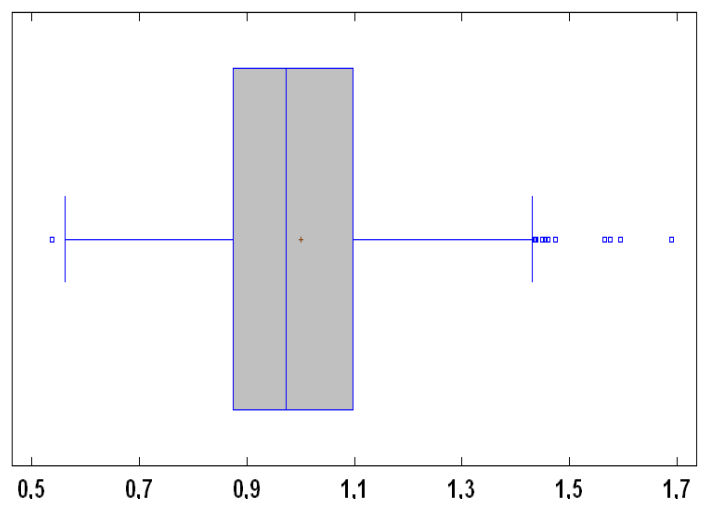

Figura 4.5 Ejemplo de diagrama box-plot obtenido con el programa Statgraphics ${ }^{\circledR}$.

El diagrama box-plot ofrece una representación gráfica sencilla de un conjunto de datos, aportando información acerca de su distribución respecto de la media, representada por el punto marrón, y la mediana, que coincide con la línea vertical situada dentro de la caja, tal y como muestra la Figura 4.5. Este diagrama permite además visualizar aspectos como el rango intercuartílico $R I$, representado por el ancho de la caja; unos valores mínimo y máximo, obtenidos a partir de los cuartiles $Q_{1}$ y $Q_{3}$, y representados por los extremos de los bigotes o líneas horizontales del diagrama; y la presencia de puntos atípicos o outliers, situados fuera de los bigotes.

\subsubsection{Estrategia de optimización para el ajuste de las expresiones con las bases de ensayos}

El motivo de proponer las expresiones del apartado 4.3 para el ajuste con las bases de ensayos es conseguir que los valores predichos $x_{\text {pred }}$ se aproximen tanto como sea posible a los presentes en las bases de ensayos $x_{\text {exp }}$, pues de ese modo se puede considerar que las expresiones reproducen de forma precisa el comportamiento de plastificación y último de la sección de un elemento estructural de hormigón armado.

Existen ciertos aspectos que dificultan el ajuste, como el elevado número de variables que presentan las expresiones, su marcado carácter no lineal y las extensas bases de ensayos empleadas. Estas circunstancias obligan a plantear un proceso de optimización para obtener el mejor ajuste posible.

Para maximizar la bondad del ajuste, es necesario cumplir las siguientes condiciones:

a) minimizar la dispersión de la variable $x$ (ecuación 4.53) respecto a su valor medio $\bar{x}, \mathrm{y}$

b) aproximar el valor medio $\bar{x}$ a 1 .

La estrategia adoptada en el proceso de optimización para obtener los valores de los coeficientes de ajuste $C_{i}$ de las ecuaciones (4.39) a (4.42) consiste en

a) emplear el coeficiente de variación $C V$ como función objetivo a minimizar, con objeto de reducir la dispersión, y 
b) establecer como restricción que el valor medio de la variable $\bar{x}$ adopte valores próximos a 1. Las posibles restricciones consideradas son

$$
\begin{gathered}
0,9 \leq \bar{x} \leq 1,1 \\
0,95 \leq \bar{x} \leq 1,05 \\
0,99 \leq \bar{x} \leq 1,01
\end{gathered}
$$

El coeficiente de variación $C V$ resultante tras el proceso de optimización depende de los valores que adoptan los coeficientes de ajuste $C_{i}$. Con objeto de respetar la estructura original de las expresiones de Biskinis y Fardis (2010a, 2010b), en las que se introducen dichos coeficientes de ajuste, y no modificarla mediante valores que disten excesivamente de los propuestos por estos autores, se impone en el proceso de optimización que los valores iniciales $C_{i, 0}$ de los coeficientes de ajuste $C_{i}$ sean los de las expresiones originales, estableciendo además la siguiente restricción adicional:

$$
\left(1-P_{i, 0}\right) C_{i, 0} \leq C_{i} \leq\left(1+P_{i, 0}\right) C_{i, 0}
$$

siendo $P_{i, 0}$ un porcentaje de variación del valor inicial $C_{i, 0}$.

La reducción del coeficiente de variación $C V$ se consigue al aumentar el porcentaje de variación $P_{i, 0}$, pues de esta forma se incrementa el rango de valores que pueden adoptar los coeficientes de ajuste $C_{i}$. El proceso finaliza cuando el coeficiente de variación alcanza un valor constante que se considera mínimo.

\subsubsection{Expresiones ajustadas a la selección de ensayos}

\subsubsection{Momento de plastificación}

La expresión propuesta para el momento de plastificación $M_{y}$ tras el ajuste con la selección de ensayos se corresponde con

$$
\frac{M_{y}}{b d^{3}}=\phi_{y}\left\{E_{c} \frac{\xi_{y}^{2}}{2}\left(1,40 \frac{1+\delta^{\prime}}{2}-1,90 \frac{\xi_{y}}{3}\right)+\frac{E_{s}\left(1-\delta^{\prime}\right)}{2}\left[1,50\left(1-\xi_{y}\right) \rho+0,10\left(\xi_{y}-\delta^{\prime}\right) \rho^{\prime}+0,10 \frac{\rho_{v}}{6}\left(1-\delta^{\prime}\right)\right]\right\}
$$

En relación con la expresión original propuesta por Biskinis y Fardis (2010a), en la ecuación (4.74) aumenta la importancia de ciertos aspectos geométricos, como el cociente $\delta$ ' entre el recubrimiento mecánico $d^{\prime}$ y el canto útil de la pieza $d$, y la profundidad de la fibra neutra en la plastificación de la sección $\xi_{y}$. En lo que respecta al armado de la sección, la cuantía de armadura de tracción $\rho$ aumenta de forma significativa su influencia en los resultados en detrimento de la armadura de compresión $\rho$.

Los diagramas box-plot obtenidos para la variable $x=M_{y, \text { exp }} / M_{y, \text { pred }}$ mediante las expresiones propuestas por diferentes autores son los mostrados en la Figura 4.6. Dichos diagramas presentan un eje horizontal cuya longitud es proporcional a la diferencia 
entre los valores extremos, aspecto que permite apreciar las distintas distribuciones de valores en torno a la media y a la mediana. Se observa que al aplicar la ecuación (4.74) se obtienen resultados con unos valores de media y mediana más próximos a $1 \mathrm{y}$ un diagrama con un ancho de caja inferior. Se obtienen además unos valores mínimo y máximo más próximos, aspecto que se manifiesta en la menor extensión de los bigotes.

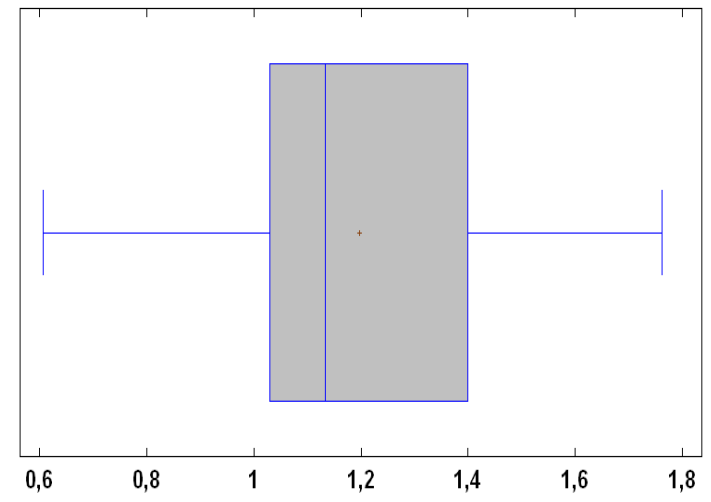

(a)

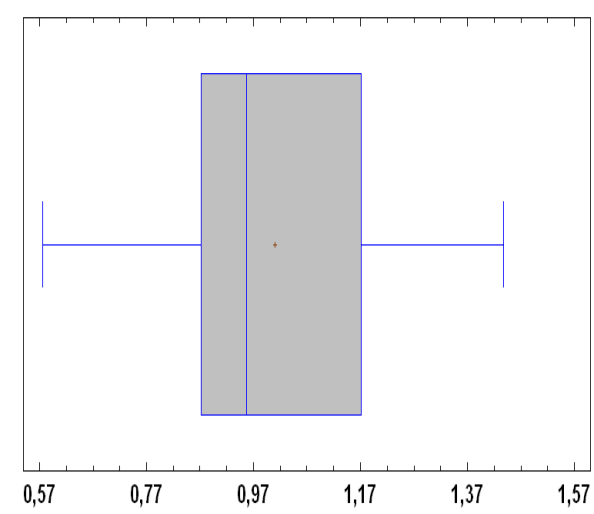

(b)

Figura 4.6 Diagramas box-plot para la variable $x=M_{y, \text { exp }} / M_{y, \text { pred }}$. (a) Panagiotakos y Fardis (2001) y Biskinis y Fardis (2010a). (b) Ecuación propuesta.

La Figura 4.7 muestra la relación entre el coeficiente de variación $C V$ obtenido para la variable $x=M_{y, \text { exp }} / M_{y, \text { pred }}$ y el porcentaje de variación $P_{i, 0}$ de los valores iniciales $C_{i, 0}$ adoptados en la expresión (4.22) de Biskinis y Fardis (2010a). Se observa cómo el coeficiente de variación disminuye a medida que aumenta el porcentaje de variación $P_{i, 0}$ puesto que se amplía el rango de valores que pueden adoptar los coeficientes de ajuste $C_{i}$. Conviene destacar que las restricciones impuestas en el valor medio $\bar{x}$ de la variable $M_{y, \text { exp }} / M_{y, p r e d}$, dadas por las ecuaciones (4.70) a (4.72), no influyen en el valor mínimo obtenido para el coeficiente de variación $C V$. Para los tres casos, se obtiene un coeficiente de variación $C V=18,56 \%$ para un porcentaje de variación $P_{i, 0}$ del $150 \%$ de los coeficientes iniciales $C_{i, 0}$.

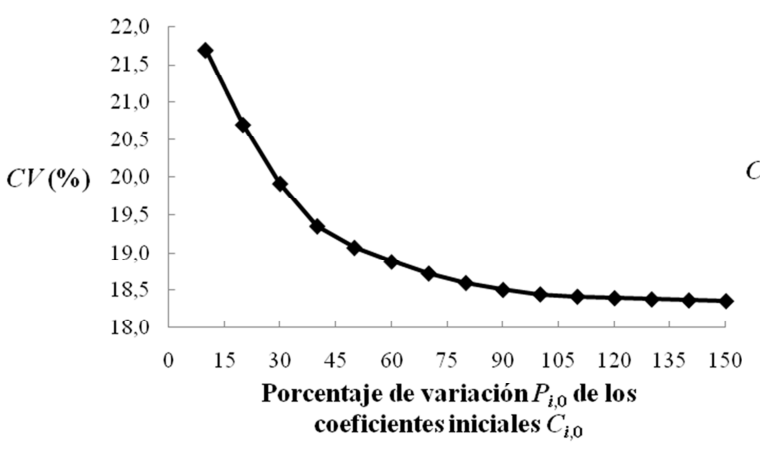

(a)

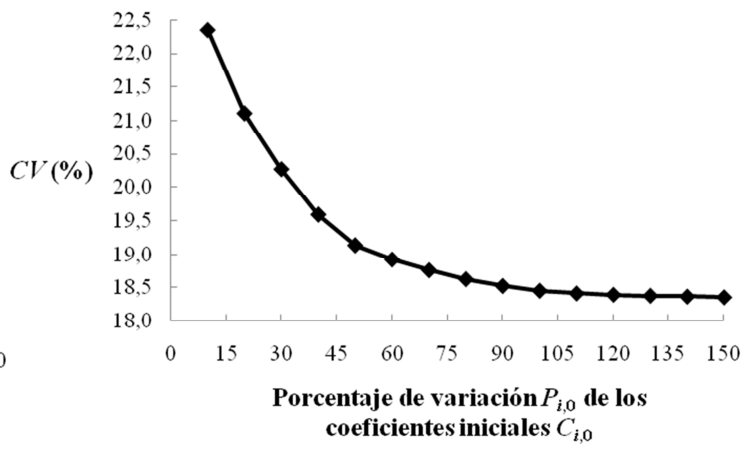

(b) 


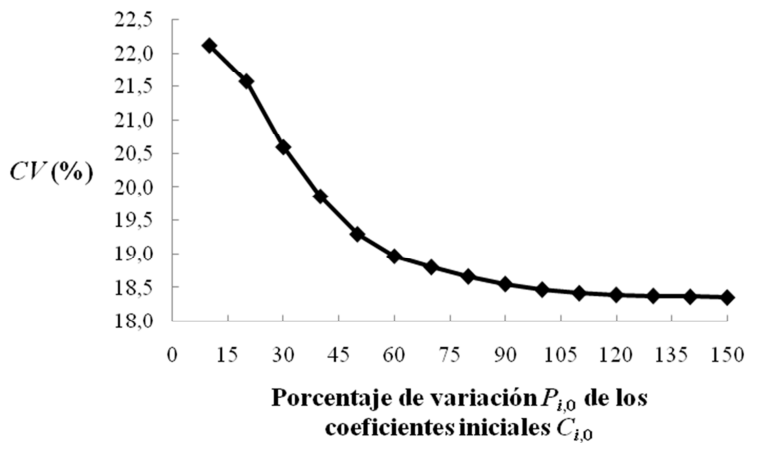

(c)

Figura 4.7 Coeficiente de variación $C V$ de la variable $x=M_{y}$,exp $/ M_{y}$,pred en función del porcentaje de variación $P_{i, 0}$ de los coeficientes iniciales $C_{i, 0}$. (a) Valor medio $\bar{x}$ entre 0,9 y 1,1 . (b) Valor medio $\bar{x}$ entre 0,95 y 1,05 . (c) Valor medio $\bar{x}$ entre 0,99 y 1,01 .

Los parámetros estadísticos obtenidos tras aplicar las expresiones propuestas por diferentes autores se muestran en las Tablas 4.2 y 4.3. Se concluye que los resultados obtenidos con la ecuación (4.74) suponen una mejora de aproximadamente el $16 \%$ en los valores de la media $\bar{x}$ y el coeficiente de variación $C V$ respecto a los obtenidos con las expresiones desarrolladas por otros autores. Se consigue un valor de la mediana más próximo a 1, obteniéndose además una disminución en el valor de la desviación típica muestral $s$ del $28 \%$ y un rango intercuartílico RI un $19 \%$ inferior, lo que implica una mayor concentración de los resultados respecto a la media y la mediana. A la vista de la Tabla 4.3, se consigue un valor de la covarianza $\operatorname{cov}\left(x_{\text {pred }}, x_{\text {exp }}\right)$ mayor que el obtenido aplicando las otras expresiones, circunstancia que denota una mayor dependencia lineal entre los valores experimentales y los predichos. Además, los coeficientes $a^{\prime}$ y $b^{\prime}$ de la recta de regresión obtenida se aproximan más a los valores 0 y 1 , respectivamente, por lo que dicha recta se acerca más a la igualdad $x_{\text {exp }}=x_{\text {pred }}$. Los coeficientes de correlación lineal $r$ y de determinación $R^{2}$, aunque mejoran, adoptan valores similares, mientras que se consigue una disminución del $19 \%$ en el valor de $S_{d}$.

Tabla 4.2 Medidas de síntesis para la variable $x=M_{y, \text { exp }} / M_{y, \text { pred }}$ según diferentes autores.

\begin{tabular}{lcccccc}
\hline & $\mathrm{N}^{\mathrm{o}}$ de ensayos & $\bar{x}$ & $s$ & Mediana & $R I$ & $C V(\%)$ \\
\hline $\begin{array}{l}\text { Panagiotakos y Fardis (2001) } \\
\text { Biskinis y Fardis (2010a) }\end{array}$ & 192 & 1,20 & 0,26 & 1,13 & 0,37 & 21,96 \\
Ecuación propuesta & & 1,01 & 0,19 & 0,96 & 0,30 & 18,56 \\
\hline
\end{tabular}

La relación entre los valores experimentales y predichos para el momento de plastificación puede verse gráficamente en la Figura 4.8. La gráfica correspondiente a los resultados obtenidos con la ecuación (4.74) muestra una mejor aproximación a la recta $M_{y, \text { exp }}=M_{y, \text { pred }}$ que la obtenida por otros autores, aspecto que evidencia un mejor ajuste entre valores experimentales y predichos. 
Tabla 4.3 Parámetros para el análisis de la relación entre las variables $M_{y, \text { pred }}$ y $M_{y, \text { exp }}$ según diferentes autores.

\begin{tabular}{lcccccc}
\hline & $\operatorname{cov}\left(x_{\text {pred }}, x_{\text {exp }}\right)$ & $a^{\prime}$ & $b^{\prime}$ & $r$ & $R^{2}$ & $S_{d}$ \\
\hline $\begin{array}{l}\text { Panagiotakos y Fardis (2001) } \\
\text { Biskinis y Fardis (2010a) }\end{array}$ & 37471 & 6,14 & 1,08 & 0,98 & 0,95 & 6356,35 \\
Ecuación propuesta & 41539 & $-1,44$ & 0,99 & 0,99 & 0,97 & 5138,05 \\
\hline
\end{tabular}

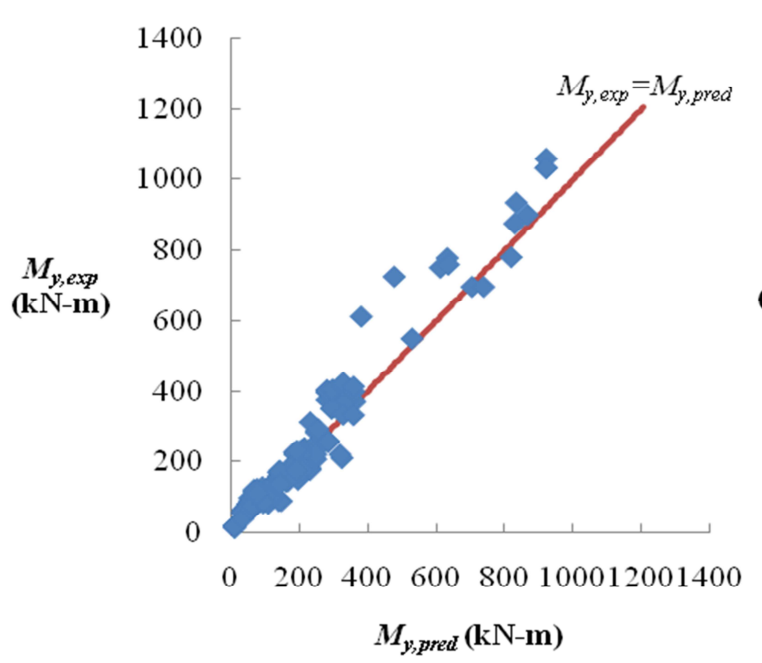

(a)

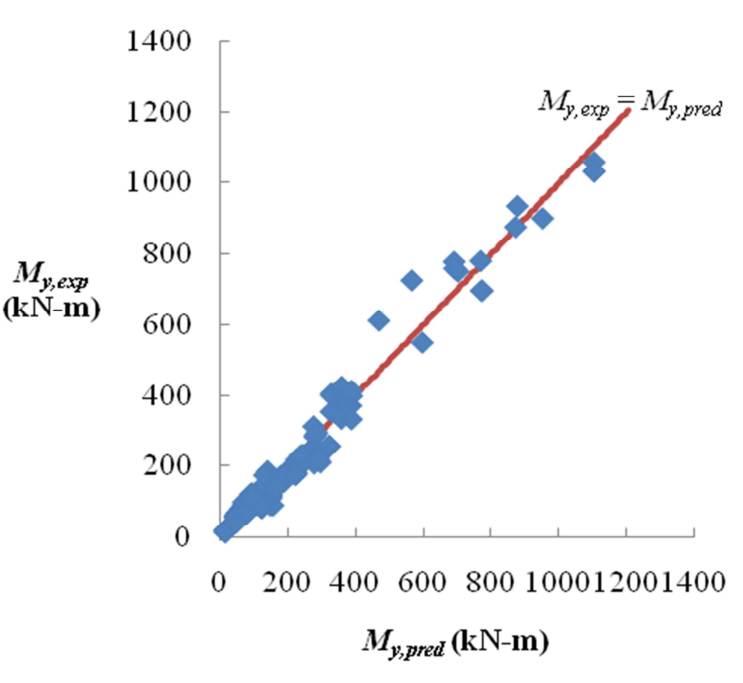

(b)

Figura 4.8 Comparación de valores experimentales y predichos para el momento de plastificación $M_{y}$. (a) Panagiotakos y Fardis (2001) y Biskinis y Fardis (2010a). (b) Ecuación propuesta.

\subsubsection{Giro de plastificación}

El ajuste de la ecuación (4.40) con la selección de ensayos proporciona la siguiente expresión para el giro de plastificación:

$$
\theta_{y}=\phi_{y} \frac{1,594 L_{s}+2,552 a_{v} z}{3}-0,0031\left(1-1,741 \frac{h}{L_{s}}\right)+1,270 a_{s l} \frac{\phi_{y} \phi_{L} f_{y}}{8 \sqrt{f_{c}}}
$$

A la vista de la ecuación (4.75), destaca la mayor influencia de la deformación debida a la flexión y al deslizamiento de la armadura longitudinal de tracción respecto del hormigón, correspondientes al primer y tercer término, respectivamente, en comparación con la ecuación (4.26) propuesta por Biskinis y Fardis (2010a). Significativo resulta también el cambio de signo en los coeficientes presentes en el giro debido a la deformación por esfuerzo cortante, representado por el segundo término, lo cual es posible al tratarse de un término puramente empírico.

En la Figura 4.9 se advierte la presencia de puntos atípicos o outliers en los diagramas box-plot obtenidos al aplicar la ecuación (4.12) de Panagiotakos y Fardis 
(2001), la ecuación incluida en el EC-8 y la ecuación (4.75). Sin embargo, los valores de los puntos atípicos son menores para el caso de esta última, lo cual resulta indicativo de un mejor ajuste entre resultados experimentales y predichos. El hecho de que al aplicar la expresión propuesta por Biskinis y Fardis (2010a) no se obtengan puntos atípicos se debe a que el ancho de caja del diagrama box-plot resulta superior al obtenido con la ecuación (4.75), de forma que ciertos valores no son considerados outliers al alcanzar los bigotes valores superiores. No obstante, los valores extremos obtenidos con ambas expresiones resultan similares.

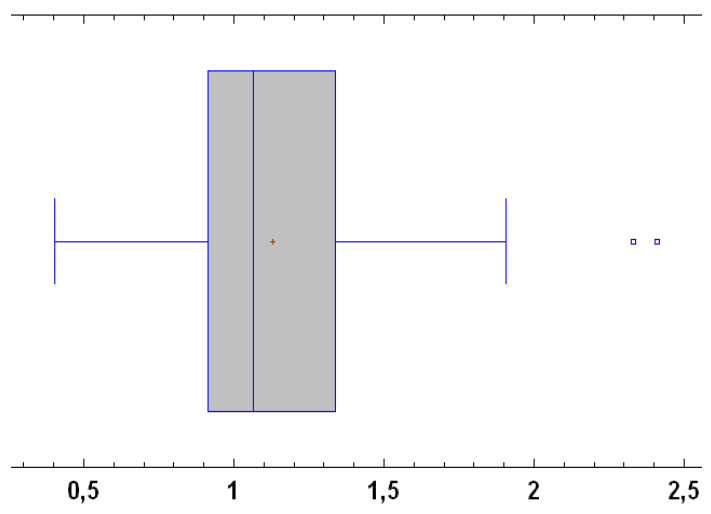

(a)

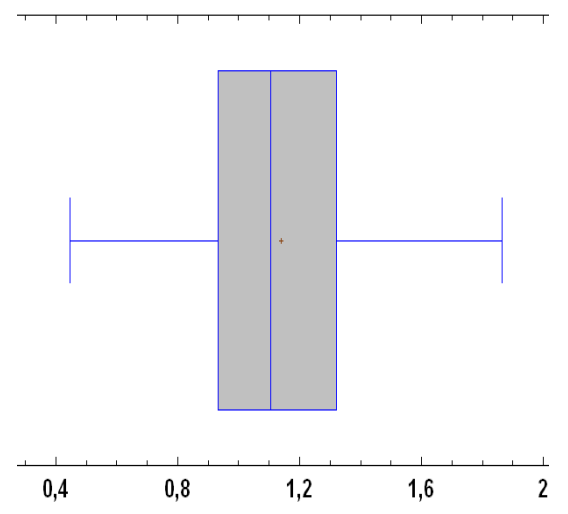

(c)

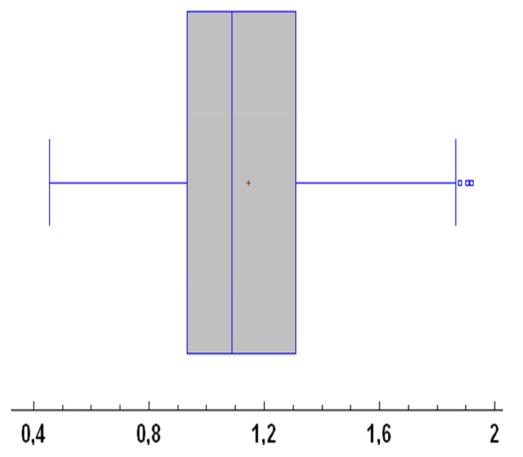

(b)

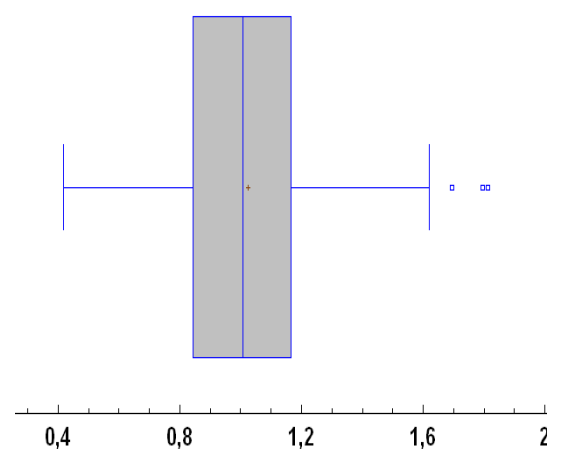

(d)

Figura 4.9 Diagramas box-plot para la variable $x=\theta_{y, \text { exp }} / \theta_{y \text {,pred. }}$ (a) Panagiotakos y Fardis (2001). (b) EC-8. (c) Biskinis y Fardis (2010a). (c) Ecuación propuesta.

En la Figura 4.10 se presenta la evolución del coeficiente de variación $C V$ de la variable $x=\theta_{y, \text { exp }} / \theta_{y \text {, pred }}$ al aumentar el porcentaje de variación $P_{i, 0}$ de los coeficientes iniciales $C_{i, 0}$ presentes en la expresión de Biskinis y Fardis (2010a). Se observa que la evolución es similar para los casos en que el valor medio $\bar{x}$ de la variable $\theta_{y, \text { exp }} / \theta_{y, \text { pred }}$ está comprendido en los intervalos [0,9-1,1] y [0,95-1,05]. En ambas gráficas se aprecia un claro descenso en el valor del coeficiente de variación $C V$ para porcentajes de variación $P_{i, 0}$ en torno al $220 \%$. La gráfica obtenida cuando el valor medio se encuentra 
entre los valores 0,99 y 1,01 muestra una serie de cambios bruscos de pendiente, alcanzándose valores mínimos del coeficiente de variación $C V$ próximos al $24,5 \%$. Conviene destacar el hecho de que para alcanzar un valor mínimo en el coeficiente de variación $C V$ de la variable $x=\theta_{y, \text { exp }} / \theta_{y, \text { pred }}$ es necesario considerar que los coeficientes de ajuste $C_{i}$ adoptan valores en el intervalo $\left[(1-3,5) C_{i, 0},(1+3,5) C_{i, 0}\right]$, más amplio que el intervalo obtenido para el momento de plastificación a partir de $P_{i, 0}=150 \%$, deduciéndose que el ajuste del giro de plastificación a la selección de ensayos exige una búsqueda más amplia en el espacio de diseño para obtener los coeficientes de ajuste $C_{i}$.

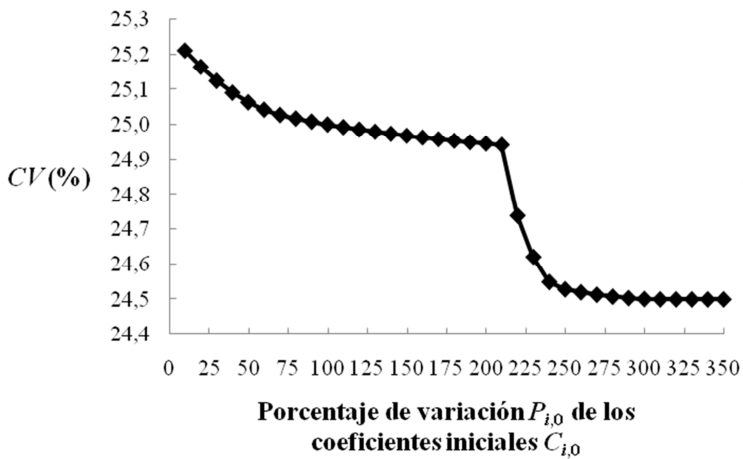

(a)

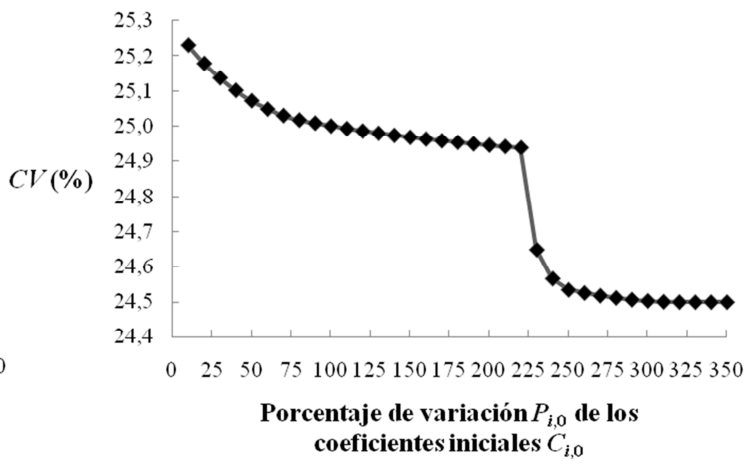

(b)

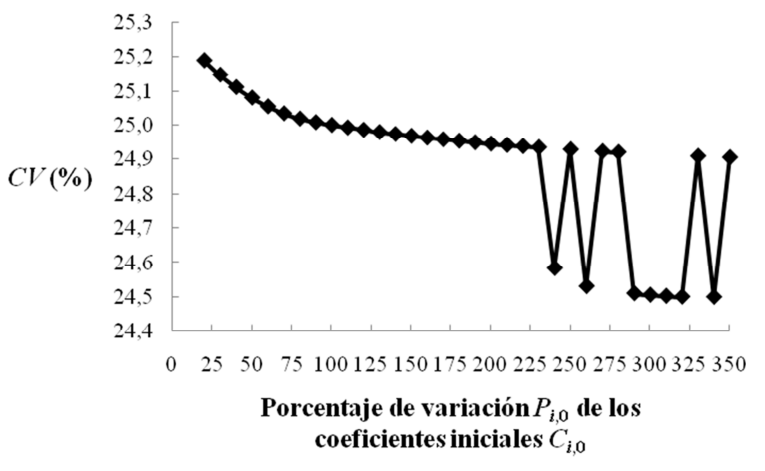

(c)

Figura 4.10 Coeficiente de variación $C V$ de la variable $x=\theta_{y}$,exp $/ \theta_{y \text {,pred }}$ en función del porcentaje de variación $P_{i, 0}$ de los coeficientes iniciales $C_{i, 0}$. (a) Valor medio $\bar{x}$ entre 0,9 y 1,1 . (b) Valor medio $\bar{x}$ entre 0,95 y 1,05 . (c) Valor medio $\bar{x}$ entre 0,99 y $1,01$.

En la Tabla 4.4 se muestran los resultados relativos a las medidas de síntesis para la variable $x=\theta_{y, \text { exp }} / \theta_{y, \text { pred. }}$. Al aplicar la ecuación (4.75), la desviación típica muestral $s$ disminuye entre el 14 y el $24 \%$ respecto a lo obtenido con otras expresiones, mientras que el coeficiente de variación $C V$ se reduce entre el 3 y el $16 \%$. Además, se obtienen valores de la media y la mediana más próximos a 1 , obteniéndose disminuciones en el rango intercuartílico $R I$ entre el 13 y el $25 \%$. Los coeficientes de ajuste $C_{i}$ presentes en la ecuación (4.75) corresponden a la restricción $0,95 \leq \bar{x} \leq 1,05$, puesto que se encuentran más próximos a los valores iniciales $C_{i}$ que los obtenidos aplicando la 
restricción $0,99 \leq \bar{x} \leq 1,01$.

Tabla 4.4 Medidas de síntesis para la variable $x=\theta_{y, \text { exp }} / \theta_{y \text {,pred }}$ según diferentes autores.

\begin{tabular}{lcccccc}
\hline & $\mathrm{N}^{\circ}$ de ensayos & $\bar{x}$ & $s$ & Mediana & RI & \multicolumn{1}{c}{$C V(\%)$} \\
\hline Panagiotakos y Fardis (2001) & & 1,13 & 0,33 & 1,07 & 0,44 & 29,07 \\
EC-8 & & 1,14 & 0,31 & 1,09 & 0,38 & 27,25 \\
Biskinis y Fardis (2010a) & 177 & 1,14 & 0,29 & 1,10 & 0,42 & 25,26 \\
Ecuación propuesta & & 1,04 & 0,25 & 1,03 & 0,33 & 24,50 \\
\hline
\end{tabular}

Los parámetros expuestos en la Tabla 4.5 implican que la ecuación (4.75) obtiene un valor de la covarianza $\operatorname{cov}\left(x_{\text {pred }}, x_{\text {exp }}\right)$ en torno a un $33 \%$ superior al obtenido con otras expresiones, además de unos coeficientes $a^{\prime}$ y $b^{\prime}$ más cercanos a los que darían lugar a una recta de regresión $x_{\text {exp }}=x_{\text {pred }}$. El coeficiente de correlación lineal $r$ supone una mejora del $16 \%$ respecto al obtenido con la expresión de Panagiotakos y Fardis (2001) y del $25 \%$ respecto a la expresión del EC-8, adoptando un valor similar al obtenido aplicando la expresión de Biskinis y Fardis (2010a). Se consigue una disminución de $S_{d}$ entre el 9 y el $16 \%$.

Tabla 4.5 Parámetros para el análisis de la relación entre las variables $\theta_{y}$ exp y $\theta_{y}$,pred según diferentes autores.

\begin{tabular}{lcccccc}
\hline & $\operatorname{cov}\left(x_{\text {pred, }} x_{\text {exp }}\right)$ & $a^{\prime}$ & $b^{\prime}$ & $r$ & $R^{2}$ & $S_{d}$ \\
\hline Panagiotakos y Fardis (2001) & 0,039 & 0,17 & 0,94 & 0,56 & 0,31 & 40,98 \\
EC-8 & 0,036 & 0,23 & 0,89 & 0,52 & 0,27 & 42,34 \\
Biskinis y Fardis (2010a) & 0,038 & $-0,13$ & 1,29 & 0,64 & 0,41 & 39,27 \\
Ecuación propuesta & 0,052 & 0,08 & 0,97 & 0,65 & 0,42 & 35,77 \\
\hline
\end{tabular}

La representación gráfica valores predichos-valores experimentales para el giro de plastificación se muestra en la Figura 4.11, donde la ecuación propuesta ofrece resultados más cercanos a la recta $\theta_{y, \text { exp }}=\theta_{y, \text { pred }}$.

\subsubsection{Giro último}

La expresión del giro último $\theta_{u}$ calibrada con la selección de ensayos es

$$
\begin{aligned}
\theta_{u}= & a_{s t}\left(1-0,167 a_{c y}\right)\left(1+\frac{a_{s l}}{2}\right)\left(1-0,42 a_{w, r}\right)\left(1-\frac{2}{7} a_{w, n r}\right)(0,237)^{r}\left[\frac{\max \left(0,01 ; \omega_{2}\right)}{\max \left(0,01 ; \omega_{1}\right)} f_{c}\right]^{0,093} \\
& {\left[\min \left(9, \frac{L_{s}}{h}\right)\right]^{0,537} 25^{\left(\frac{\alpha \rho_{h} f_{j h}}{f_{c}}\right.} 1,004^{100 \rho_{d}} }
\end{aligned}
$$

Los coeficientes de ajuste $C_{11}$ a $C_{15}$ obtenidos implican un cambio en la importancia relativa de algunos términos respecto de la ecuación (4.34) inicialmente 
propuesta por Biskinis y Fardis (2010b). Así, ciertos aspectos resultan menos influyentes en los resultados, como el tipo de carga considerada, monotónica o cíclica, mediante el parámetro $a_{c y}$; el esfuerzo axil de compresión, representado por el parámetro $v$; y las cuantías mecánicas de armadura longitudinal de tracción $\mathrm{y}$ compresión $\omega_{1}$ y $\omega_{2}$. Sin embargo, el término que considera la esbeltez de cortante $L_{s} / h$ adquiere mayor importancia.

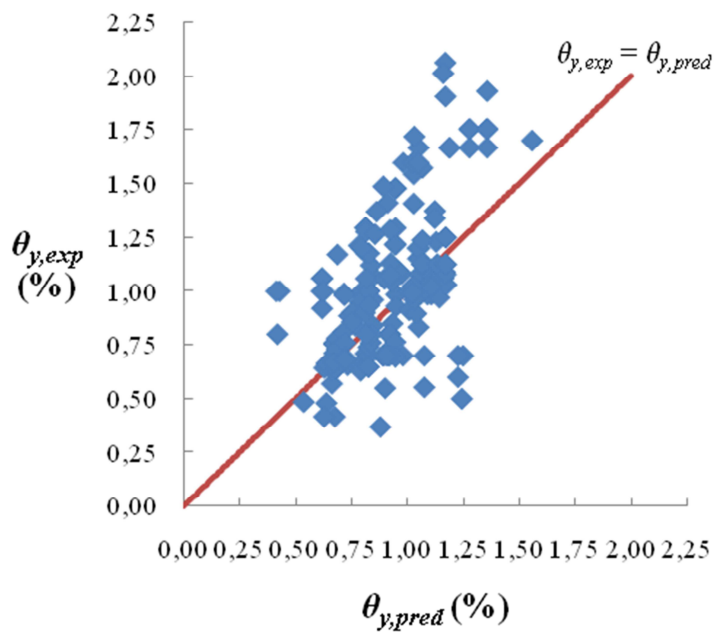

(a)

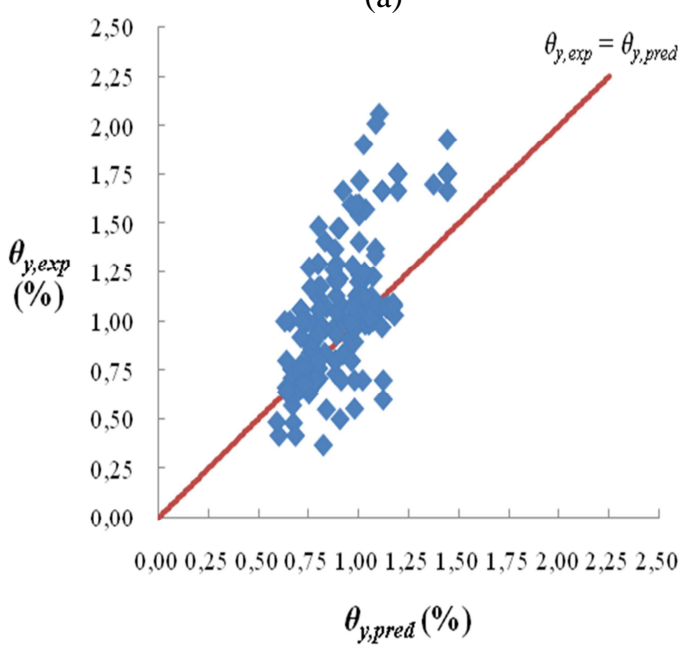

(c)

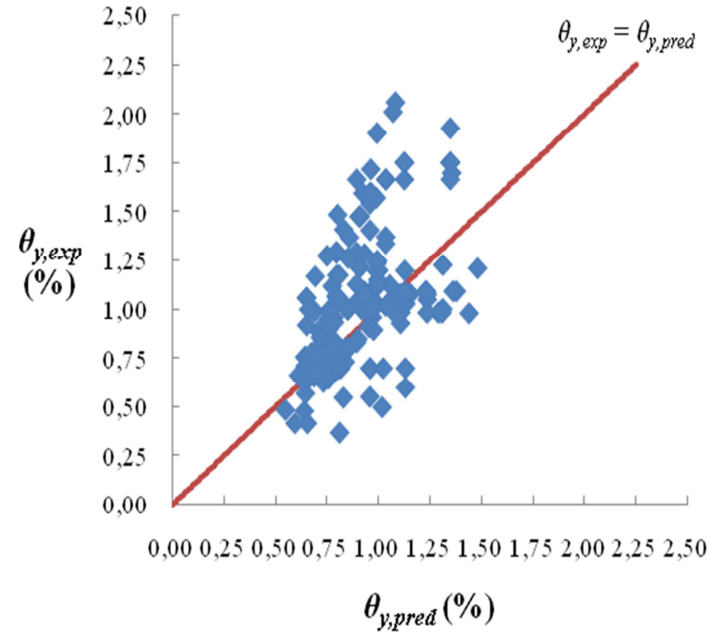

(b)

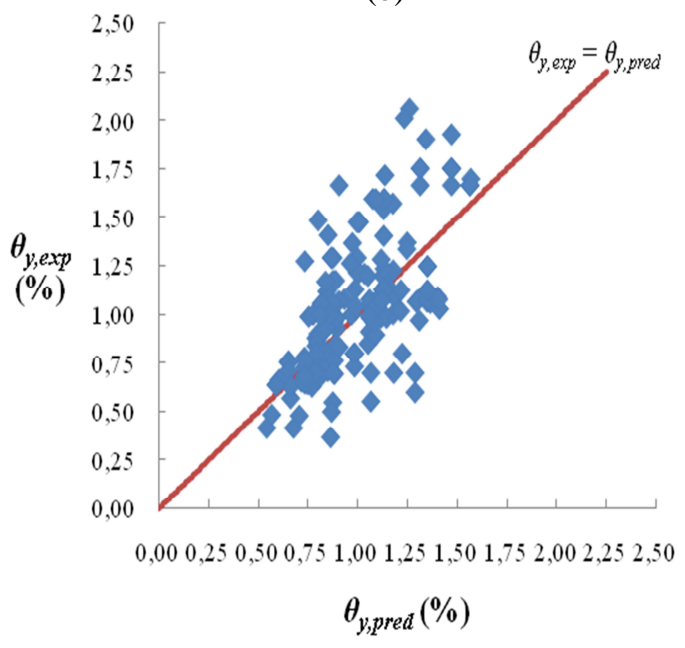

(d)

Figura 4.11 Comparación de valores experimentales y predichos para el giro de plastificación $\theta_{y}$. (a) Panagiotakos y Fardis (2001). (b) EC-8. (c) Biskinis y Fardis (2010a). (d) Ecuación propuesta.

Los diagramas box-plot de la Figura 4.12 obtenidos al aplicar las diferentes expresiones consideradas no presentan puntos atípicos para la variable $x=\theta_{u \text {, exp }} / \theta_{u \text {,pred }}$. Se aprecia un ancho de caja menor en los resultados obtenidos con la ecuación (4.76), lo cual es representativo de una mayor concentración de valores respecto a la mediana. Además, los valores extremos resultan inferiores a los obtenidos con el resto de 
expresiones.

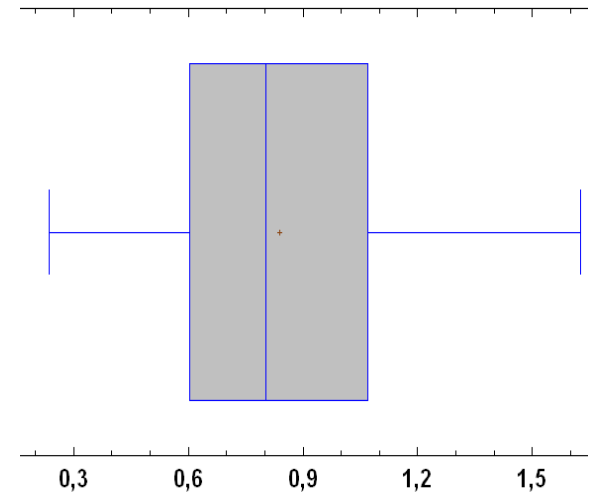

(a)

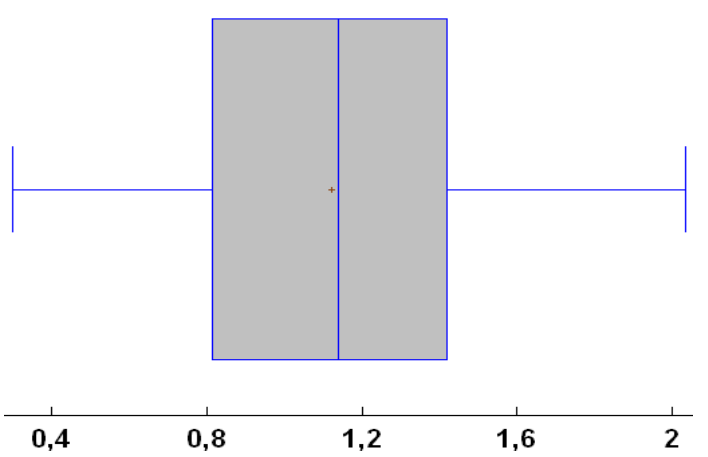

(c)

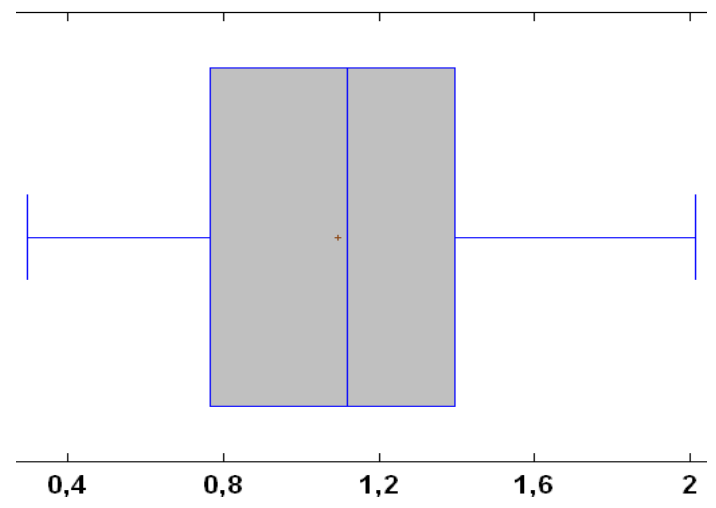

(b)

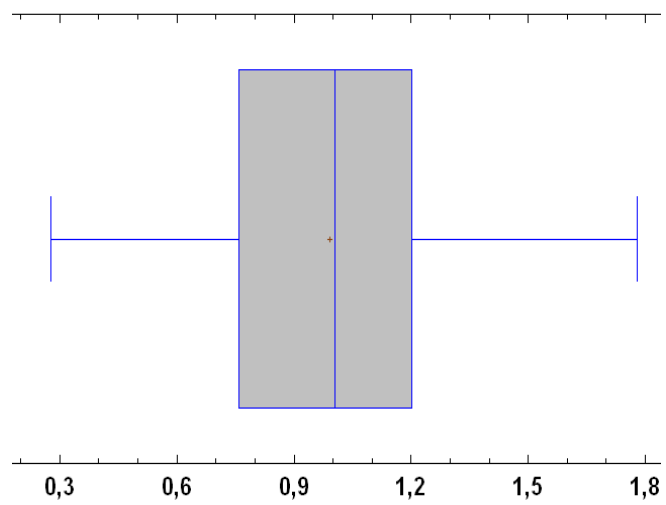

(d)

Figura 4.12 Diagramas box-plot para la variable $x=\theta_{u \text {,exp }}\left(\theta_{u \text {,pred }}\right.$. (a) Panagiotakos y Fardis (2001). (b) EC-8. (c) Biskinis y Fardis (2010b). (d) Ecuación propuesta.

En la Figura 4.13 se muestra la relación entre el coeficiente de variación $C V$ de la variable $x=\theta_{u \text {,exp }} / \theta_{u \text {,pred }}$ y el porcentaje de variación $P_{i, 0}$ de los coeficientes iniciales $C_{i, 0}$ presentes en la ecuación (4.34) de Biskinis y Fardis (2010b). Las tres restricciones impuestas en el valor medio $\bar{x}$ de la variable $\theta_{u, \text { exp }} / \theta_{u, p r e d}$ implican una evolución similar del coeficiente de variación $C V$, obteniéndose un valor mínimo $C V=31,5 \%$ para un porcentaje de variación $P_{i, 0}=100 \%$. Los coeficientes de ajuste $C_{i}$ presentes en la ecuación (4.76) corresponden a la restricción $0,99 \leq \bar{x} \leq 1,01$.

La Tabla 4.6 muestra los valores de las medidas de síntesis para la variable $x=$ $\theta_{u, \text { exp }} / \theta_{u, \text { pred. }}$. La ecuación (4.76) consigue una mejora en la media $\bar{x}$ entre el 9 y el $18 \%$ respecto al resto de expresiones, obteniéndose un valor próximo a 1. Se consigue también una disminución en $C V$ entre el 6 y el $14 \%$. Además, el valor obtenido para la mediana es 1 y el rango intercuartílico $R I$ disminuye un $28 \%$ respecto a Biskinis y Fardis (2010b) y la expresión del EC-8. Los resultados obtenidos tras analizar la relación entre $\theta_{u, \exp }$ y $\theta_{u \text {,pred }}$ (Tabla 4.7) implican un aumento de la covarianza del $88 \%$ 
respecto a la obtenida con la expresión del EC-8 y un incremento del coeficiente de correlación lineal $r$ entre el 18 y el $63 \%$ respecto a las otras expresiones empleadas. La recta de regresión conseguida mejora las obtenidas con otras expresiones y se consigue una reducción de $S_{d}$ entre el 17 y el $36 \%$.

La relación entre los valores experimentales y predichos para el giro último $\theta_{u}$ se muestra gráficamente en la Figura 4.14. Como sucede para el momento y el giro de plastificación, la ecuación (4.76) obtiene unos valores más cercanos a los valores experimentales que las ecuaciones desarrolladas por otros autores.

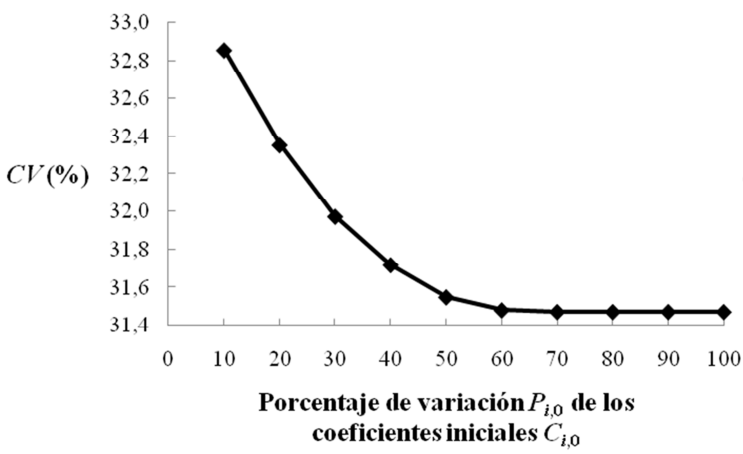

(a)

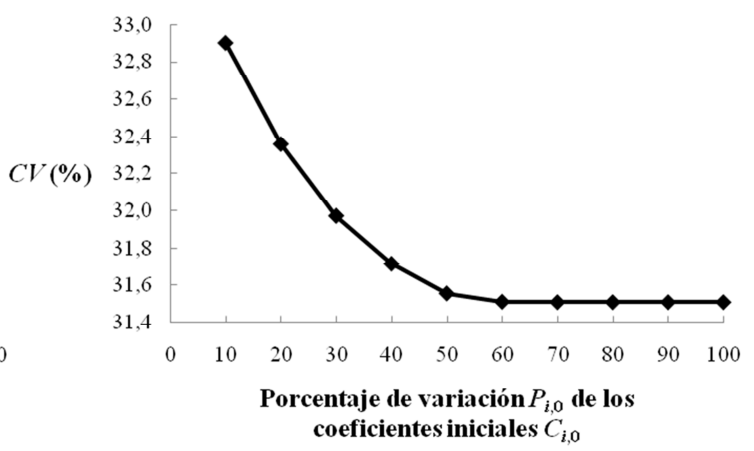

(b)

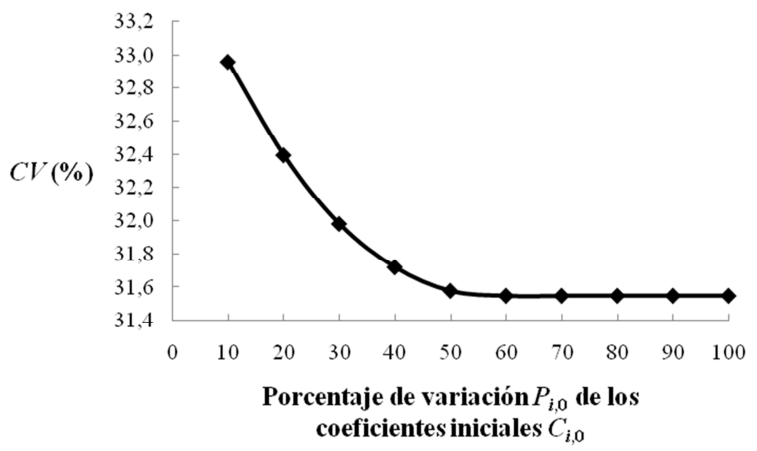

(c)

Figura 4.13 Coeficiente de variación $C V$ de la variable $x=\theta_{u \text {,exp }} / \theta_{u \text {,pred }}$ en función del porcentaje de variación $P_{i, 0}$ de los coeficientes iniciales $C_{i, 0}$. (a) Valor medio $\bar{x}$ entre 0,9 y 1,1 . (b) Valor medio $\bar{x}$ entre 0,95 y 1,05 . (c) Valor medio $\bar{x}$ entre 0,99 y $1,01$.

Tabla 4.6 Medidas de síntesis para la variable $x=\theta_{u, \text { exp }} / \theta_{u, p r e d}$ según diferentes autores.

\begin{tabular}{lcccccc}
\hline & $N^{\text {o de ensayos }}$ & $\bar{x}$ & $s$ & Mediana & $R I$ & $C V(\%)$ \\
\hline Panagiotakos y Fardis (2001) & & 0,84 & 0,31 & 0,80 & 0,47 & 36,57 \\
EC-8 & 155 & 1,09 & 0,38 & 1,12 & 0,62 & 35,12 \\
Biskinis y Fardis (2010b) & & 1,12 & 0,38 & 1,14 & 0,61 & 33,48 \\
Ecuación propuesta & & 0,99 & 0,31 & 1,00 & 0,44 & 31,55 \\
\hline
\end{tabular}


Tabla 4.7 Parámetros para el análisis de la relación entre las variables $\theta_{u, e x p}$ y $\theta_{u \text {,pred }}$ según diferentes autores.

\begin{tabular}{lcccccc}
\hline & $\operatorname{cov}\left(x_{\text {pred }}, x_{\text {exp }}\right)$ & $a^{\prime}$ & $b^{\prime}$ & $r$ & $R^{2}$ & $S_{d}$ \\
\hline Panagiotakos y Fardis (2001) & 1,791 & 2,57 & 0,40 & 0,41 & 0,17 & 294,57 \\
EC-8 & 0,887 & $-0,82$ & 1,28 & 0,52 & 0,27 & 235,09 \\
Biskinis y Fardis (2010b) & 1,087 & $-0,72$ & 1,29 & 0,57 & 0,33 & 228,84 \\
Ecuación propuesta & 1,665 & $-0,66$ & 1,12 & 0,67 & 0,45 & 189,21 \\
\hline
\end{tabular}

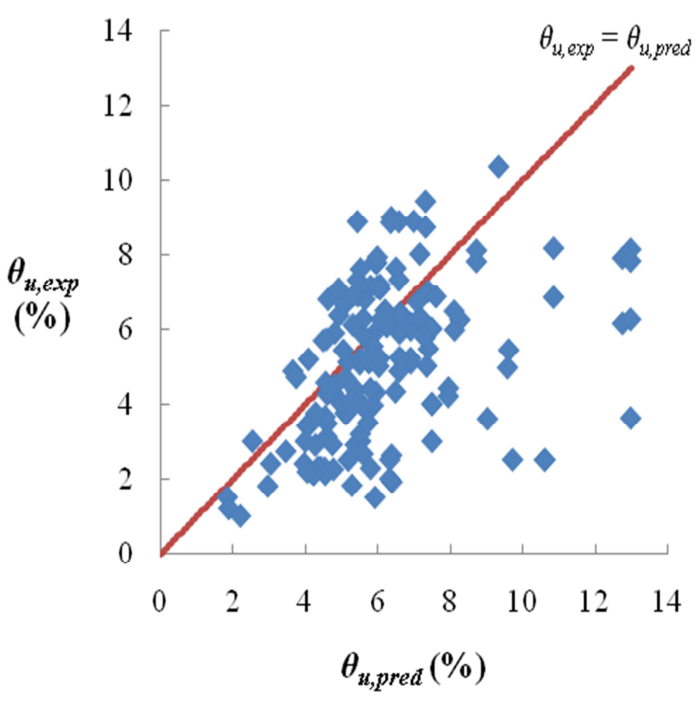

(a)

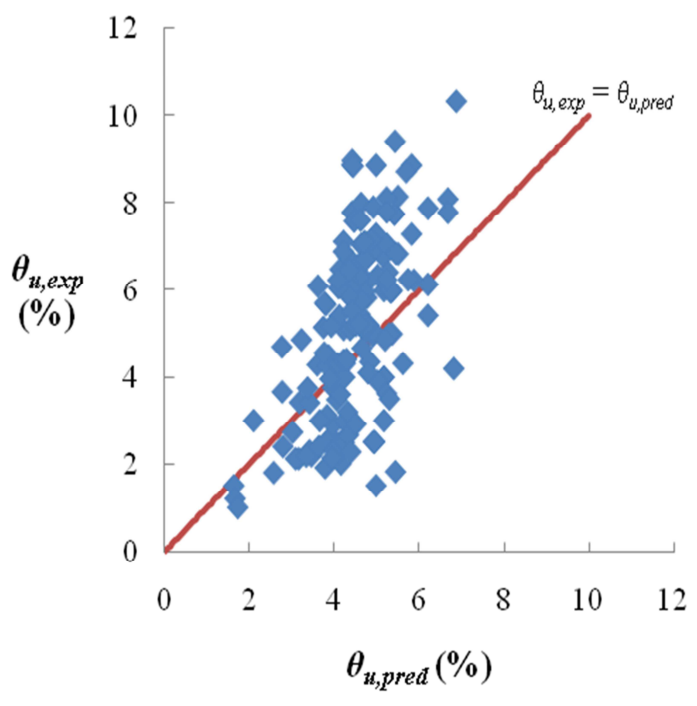

(c)

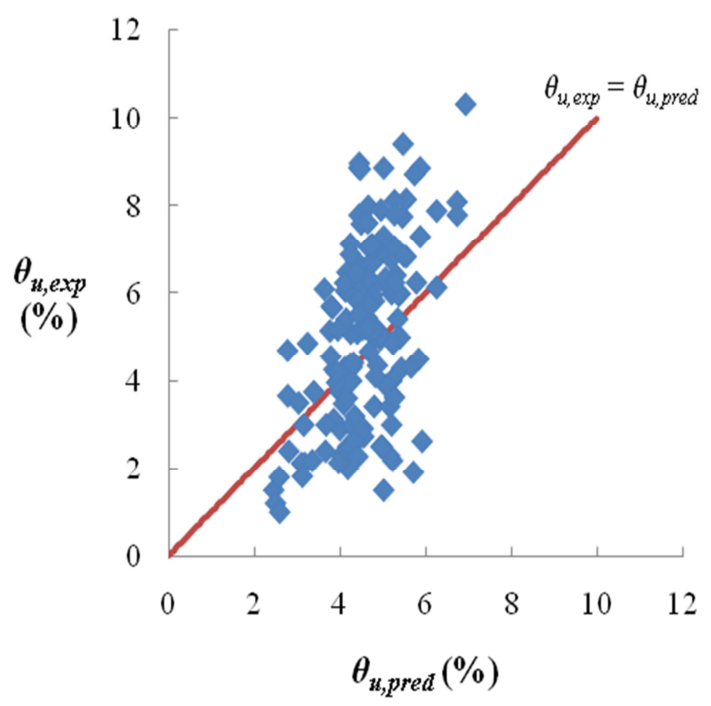

(b)

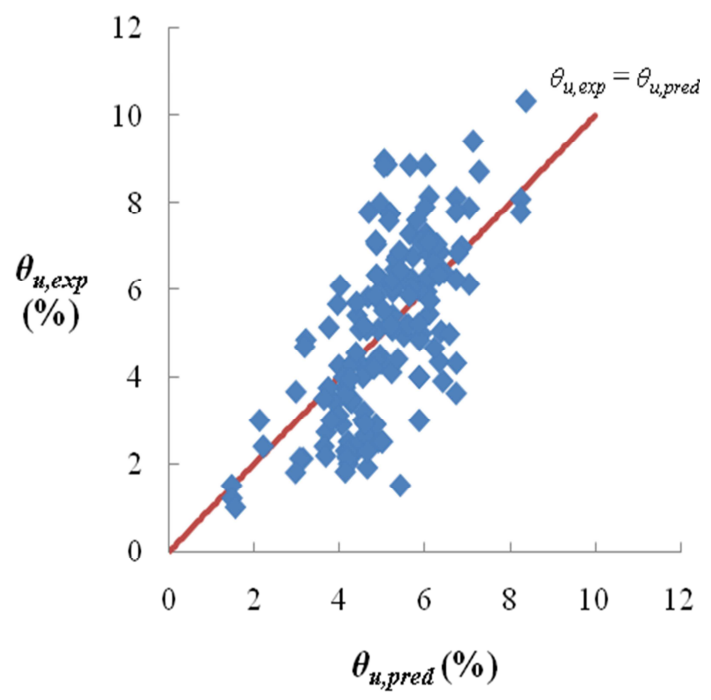

(d)

Figura 4.14 Comparación de valores experimentales y predichos para el giro último $\theta_{u}$. (a) Panagiotakos y Fardis (2001). (b) EC-8. (c) Biskinis y Fardis (2010b). (d) Ecuación propuesta. 


\subsubsection{Expresiones ajustadas a la base de datos inicial}

\subsubsection{Momento de plastificación}

El momento de plastificación $M_{y}$ ajustado a la base de datos inicial viene dado por

$$
\frac{M_{y}}{b d^{3}}=\phi_{y}\left\{E_{c} \frac{\xi_{y}^{2}}{2}\left(1,426 \frac{1+\delta^{\prime}}{2}-1,895 \frac{\xi_{y}}{3}\right)+\frac{E_{s}\left(1-\delta^{\prime}\right)}{2}\left[1,11 \not\left(1-\xi_{y}\right) \rho+0,54 \phi\left(\xi_{y}-\delta^{\prime}\right) \rho^{\prime}+0,100 \frac{\rho_{v}}{6}\left(1-\delta^{\prime}\right)\right]\right\}
$$

En la ecuación (4.77) se muestran unos coeficientes de ajuste $C_{i}$ para el cociente $\delta$ ' entre el recubrimiento mecánico $d$ ' y el canto útil de la pieza $d$ y la profundidad de la fibra neutra en la plastificación de la sección $\xi_{y}$ similares a los obtenidos en la ecuación (4.74) ajustada a la selección de ensayos, aumentando la influencia de estos términos respecto a la expresión (4.22) propuesta por Biskinis y Fardis (2010a). La influencia de la cuantía de armadura de tracción $\rho$ disminuye un $25 \%$ respecto a la expresión ajustada a la selección de ensayos, mientras que el efecto de la armadura de compresión $\rho$ ' es del orden de cinco veces superior.

La Figura 4.15 muestra que, a partir de los resultados obtenidos aplicando la ecuación (4.77), se obtiene un diagrama box-plot con un ancho de caja inferior y unos valores de la media y la mediana más cercanos a 1 . La mayor presencia de outliers al aplicar dicha ecuación no es indicativo de una mayor dispersión, pues dichos valores atípicos se generan porque los extremos de los bigotes alcanzan valores inferiores al presentar el diagrama un ancho de caja menor.

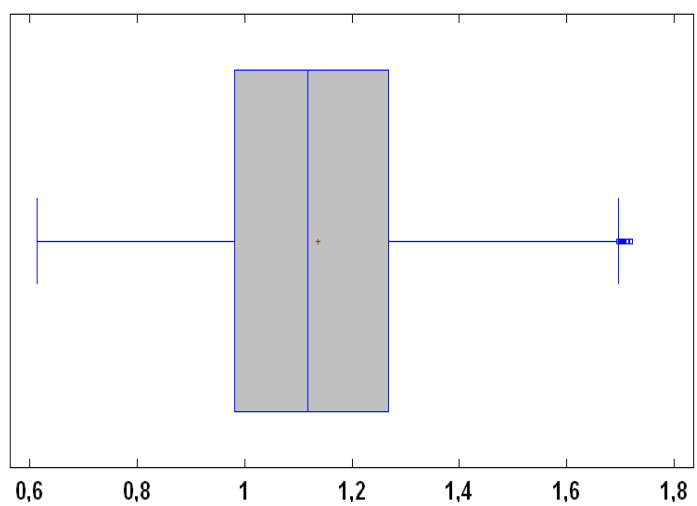

(a)

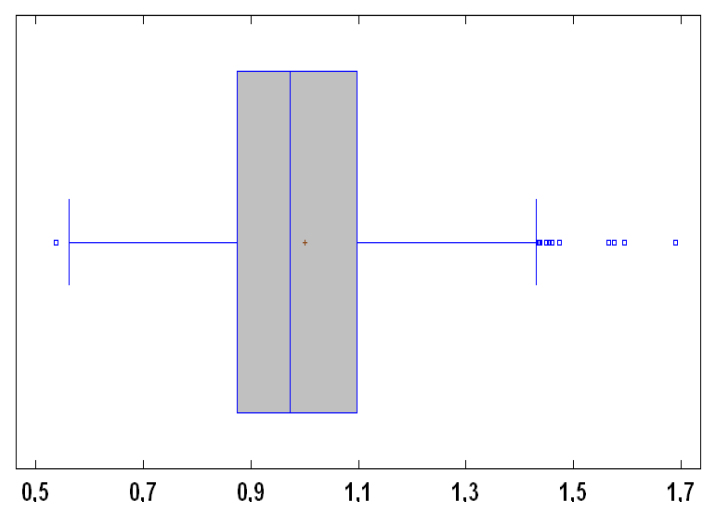

(b)

Figura 4.15 Diagrama box-plot para la variable $x=M_{y \text {,exp }} / M_{y, \text { pred. }}$ (a) Panagiotakos y Fardis (2001) y Biskinis y Fardis (2010a). (b) Ecuación propuesta.

La relación entre el coeficiente de variación $C V$ de la variable $x=M_{y, \text { exp }} / M_{y, p r e d} \mathrm{y}$ el porcentaje de variación $P_{i, 0}$ de los coeficientes iniciales $C_{i, 0}$, mostrada en la Figura 4.16, coincide para las tres restricciones impuestas en el valor medio $\bar{x}$ de dicha variable, alcanzándose un valor mínimo del coeficiente de variación $C V$ para un porcentaje de variación $P_{i, 0}=90 \%$. 
Las medidas de síntesis expuestas en la Tabla 4.8 implican que la ecuación (4.77) consigue una disminución del 7 y el $14 \%$ en el coeficiente de variación $C V$ y en la desviación típica poblacional $\sigma$, respectivamente. Se obtiene también una reducción del $24 \%$ en el rango intercuartílico $R I$.

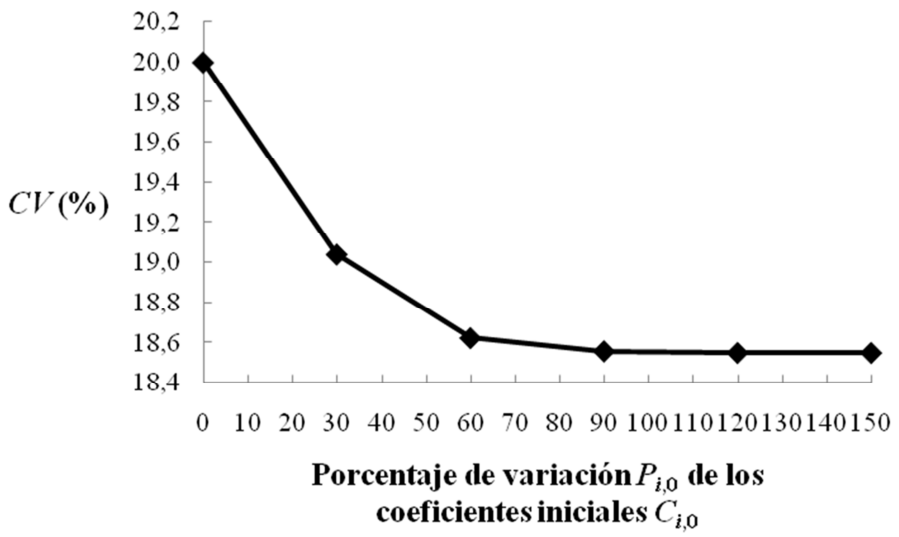

Figura 4.16 Coeficiente de variación $C V$ de la variable $x=M_{y}$,exp $/ M_{y, \text { pred }}$ en función del porcentaje de variación $P_{i, 0}$ de los coeficientes iniciales $C_{i, 0}$.

Tabla 4.8 Medidas de síntesis para la variable $x=M_{y, \text { exp }} / M_{y, p r e d}$ según diferentes autores.

\begin{tabular}{lcccccc}
\hline & $\mathrm{N}^{\mathrm{o}}$ de ensayos & $\bar{x}$ & $\sigma$ & Mediana & $R I$ & $C V(\%)$ \\
\hline $\begin{array}{l}\text { Panagiotakos y Fardis (2001) } \\
\text { Biskinis y Fardis (2010a) }\end{array}$ & 985 & 1,06 & 0,21 & 1,02 & 0,29 & 20 \\
Ecuación propuesta & & 1,00 & 0,18 & 0,97 & 0,22 & 18,56 \\
\hline
\end{tabular}

En los resultados mostrados en la Tabla 4.9, se observa que la covarianza $\operatorname{cov}\left(x_{\text {pred }}, x_{\text {exp }}\right)$ aumenta un $14 \%$ respecto a la obtenida mediante las expresiones de otros autores. Respecto a la recta de regresión, aunque el valor del coeficiente $a$ ' se aleja de cero, el valor del coeficiente de regresión $b$ ' se encuentra más próximo a 1. El valor absoluto de la suma de diferencias entre valores experimentales y predichos $S_{d}$ disminuye un $10 \%$.

Tabla 4.9 Parámetros para el análisis de la relación entre las variables $M_{y \text {,exp }}$ y $M_{y}$,pred según diferentes autores.

\begin{tabular}{lcccccc}
\hline & $\operatorname{cov}\left(x_{\text {pred }}, x_{\text {exp }}\right)$ & $a^{\prime}$ & $b^{\prime}$ & $r$ & $R^{2}$ & $S_{d}$ \\
\hline $\begin{array}{l}\text { Panagiotakos y Fardis (2001) } \\
\text { Biskinis y Fardis (2010a) }\end{array}$ & 370598 & $-1,15$ & 1,08 & 0,99 & 0,98 & 38375 \\
Ecuación propuesta & 423850 & 2,48 & 0,95 & 0,99 & 0,98 & 34520 \\
\hline
\end{tabular}

La Figura 4.17 muestra la relación entre los valores experimentales y predichos para el momento de plastificación $M_{y}$, donde puede apreciarse una mayor concentración 
de puntos en torno a la recta de $45^{\circ}$ para los resultados obtenidos con la ecuación (4.77).

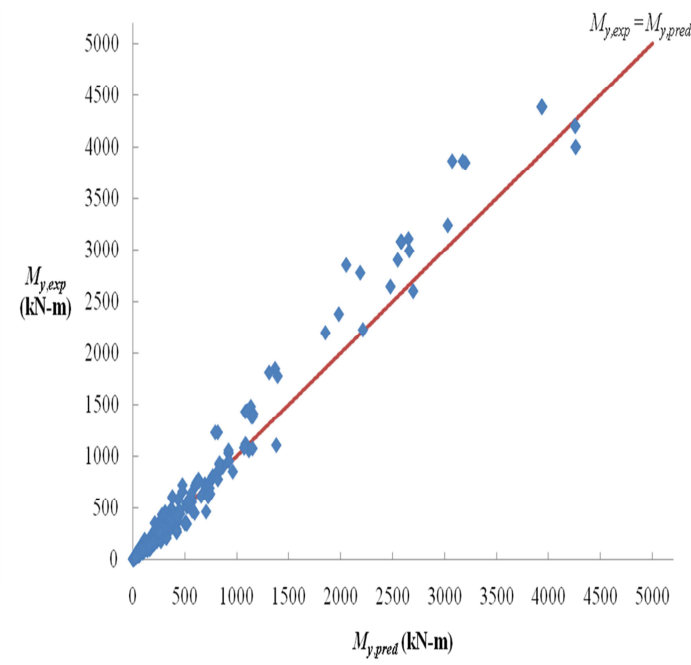

(a)

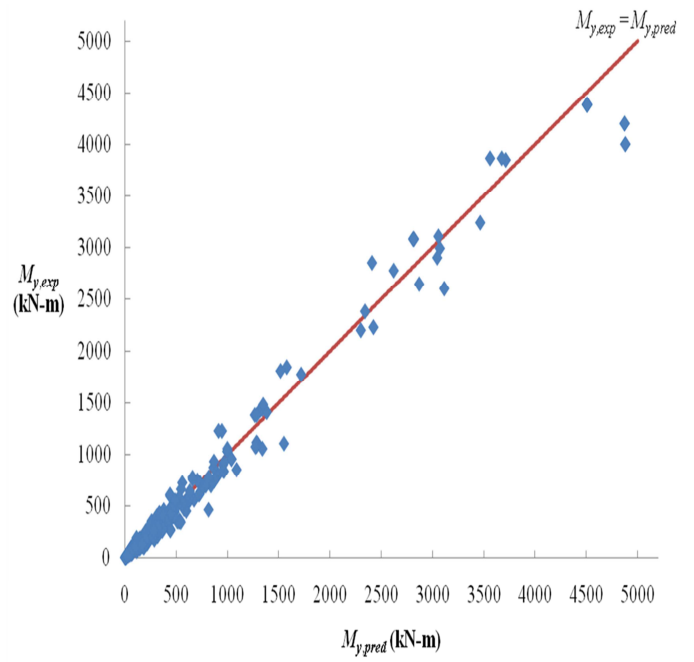

(b)

Figura 4.17 Comparación de valores experimentales y predichos para el momento de plastificación $M_{y}$. (a) Panagiotakos y Fardis (2001) y Biskinis y Fardis (2010a). (b) Ecuación propuesta.

\subsubsection{Giro de plastificación}

En la expresión del giro de plastificación $\theta_{y}$ ajustada a la base de datos inicial, dada por la ecuación (4.78), el giro de deformación por flexión disminuye su influencia respecto a la ecuación (4.75) ajustada a la selección de ensayos, mientras que el efecto del deslizamiento de la armadura de tracción aumenta un $27 \%$. Los signos de los coeficientes de ajuste $C_{i}$ aplicados en el giro por esfuerzo cortante coinciden con los propuestos en la ecuación (4.26) de Biskinis y Fardis (2010a).

$$
\theta_{y}=\phi_{y} \frac{0,831 L_{s}+0,411 a_{v} z}{3}+0,0024\left(1+0,843 \frac{h}{L_{s}}\right)+1,617 a_{s l} \frac{\phi_{y} \phi_{L} f_{y}}{8 \sqrt{f_{c}}}
$$

La Figura 4.18 muestra la distribución de los valores de la variable $x=\theta_{y}$,exp $/ \theta_{y}$, pred en torno a sus medidas de tendencia central. Aunque las expresiones de los diferentes autores obtienen valores similares para la media y la mediana, los valores extremos resultan inferiores si se aplica la expresión propuesta, lo cual denota un mejor ajuste entre resultados experimentales y predichos. La relación entre el coeficiente de variación $C V$ y el porcentaje de variación $P_{i, 0}$ de los coeficientes iniciales $C_{i, 0}$, mostrada en la Figura 4.19, no depende de las restricciones impuestas en el valor medio $\bar{x}$, obteniéndose un valor mínimo de $C V=35 \%$ para $P_{i, 0}=90 \%$. 


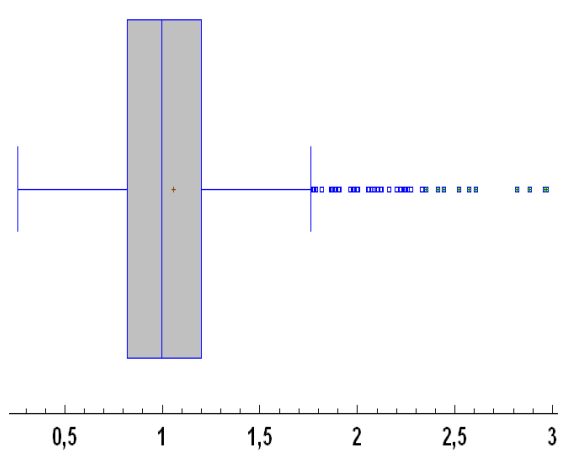

(a)

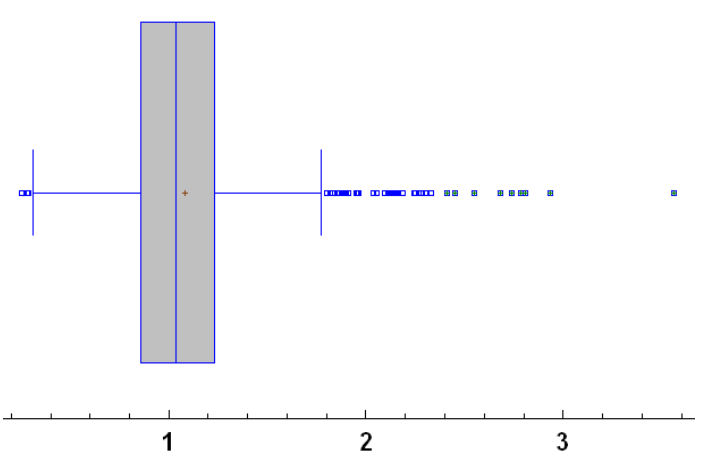

(c)

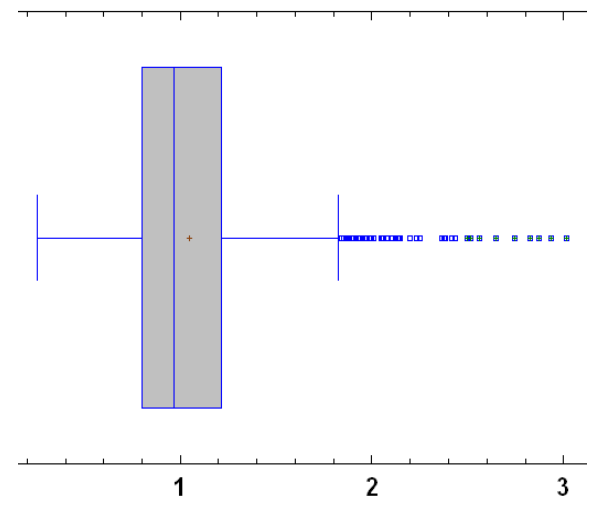

(b)

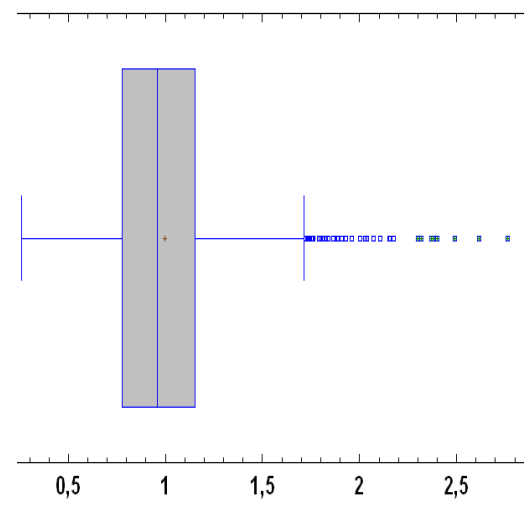

(d)

Figura 4.18 Diagramas box-plot para la variable $x=\theta_{y \text {,exp }} / \theta_{y \text {, pred. }}$ (a) Panagiotakos y Fardis (2001). (b) EC-8. (c) Biskinis y Fardis (2010a). (d) Ecuación propuesta.

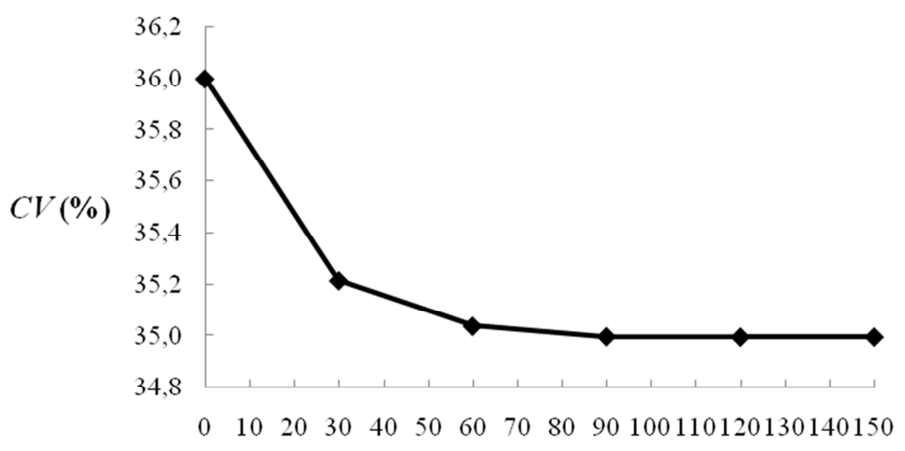

\footnotetext{
Porcentaje de variación $P_{i, 0}$ de los coeficientes iniciales $C_{i, 0}$
}

Figura 4.19 Coeficiente de variación $C V$ de la variable $x=\theta_{y, \text { exp }} / \theta_{y}$,pred en función del porcentaje de variación $P_{i, 0}$ de los coeficientes iniciales $C_{i, 0}$.

A la vista de la Tabla 4.10, los resultados obtenidos con la ecuación (4.78) suponen una disminución de la dispersión respecto a lo obtenido con otras expresiones, ya que se reduce la desviación típica poblacional $\sigma$ entre el 8 y el $10 \%$. Se obtiene además un valor medio $\bar{x}$ de la variable $\theta_{y}$,exp $/ \theta_{y}$, pred próximo a 1 . A partir de los 
resultados mostrados en la Tabla 4.11 acerca de la dependencia entre las variables $\theta_{y}$,pred y $\theta_{y}$, exp se deduce que no se obtienen diferencias significativas al aplicar las distintas expresiones consideradas, si bien se aumenta la covarianza un $15 \%$ respecto a lo obtenido con la expresión del EC-8.

Tabla 4.10 Medidas de síntesis para la variable $x=\theta_{y, \text { exp }} / \theta_{y, \text { pred }}$ según diferentes autores.

\begin{tabular}{lcccccc}
\hline & $\mathrm{N}^{\circ}$ de ensayos & $\bar{x}$ & $\sigma$ & Mediana & RI & \multicolumn{1}{c}{$C V(\%)$} \\
\hline Panagiotakos y Fardis (2001) & & 1,06 & 0,38 & 0,99 & 0,38 & 36,00 \\
EC-8 & & 1,05 & 0,39 & 0,97 & 0,41 & 37,51 \\
Biskinis y Fardis (2010a) & 963 & 1,07 & 0,39 & 1,02 & 0,37 & 35,99 \\
Ecuación propuesta & & 0,99 & 0,35 & 0,96 & 0,37 & 34,93 \\
\hline
\end{tabular}

Tabla 4.11 Parámetros para el análisis de la relación entre las variables $\theta_{y}$ exp y $\theta_{y, \text { pred }}$ según diferentes autores.

\begin{tabular}{lcccccc}
\hline & $\operatorname{cov}\left(x_{\text {exp }}, x_{\text {pred }}\right)$ & $a^{\prime}$ & $b^{\prime}$ & $r$ & $R^{2}$ & $S_{d}$ \\
\hline Panagiotakos y Fardis (2001) & 0,174 & 0,29 & 0,74 & 0,69 & 0,48 & 257,51 \\
EC-8 & 0,144 & 0,19 & 0,85 & 0,67 & 0,45 & 267,30 \\
Biskinis y Fardis (2010a) & 0,151 & 0,19 & 0,87 & 0,69 & 0,49 & 250,42 \\
Ecuación propuesta & 0,166 & 0,20 & 0,80 & 0,70 & 0,49 & 258,04 \\
\hline
\end{tabular}

La relación entre los valores experimentales y predichos para el giro de plastificación $\theta_{y}$ se muestra gráficamente en la Figura 4.20. Los resultados obtenidos con la ecuación (4.78) están más concentrados en torno a la recta $\theta_{y}$,exp $=\theta_{y \text {,pred }}$.

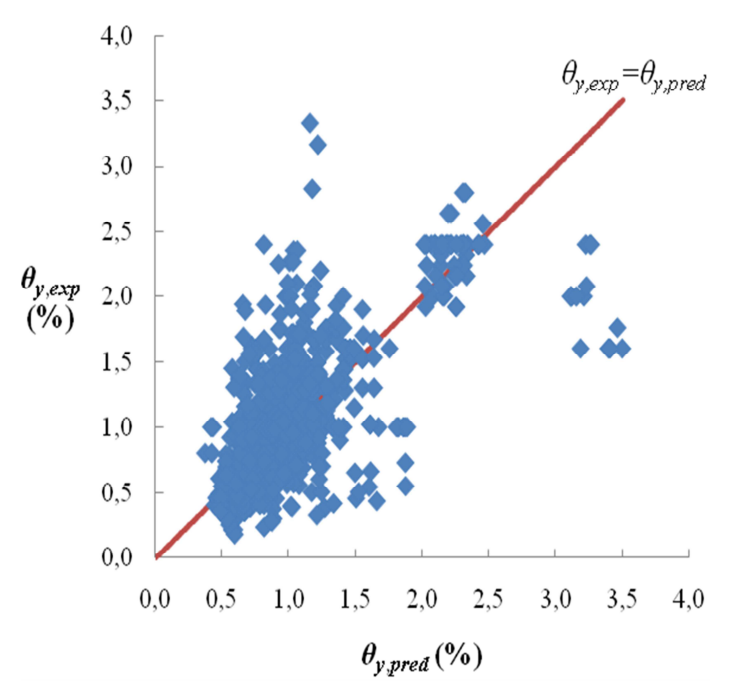

(a)

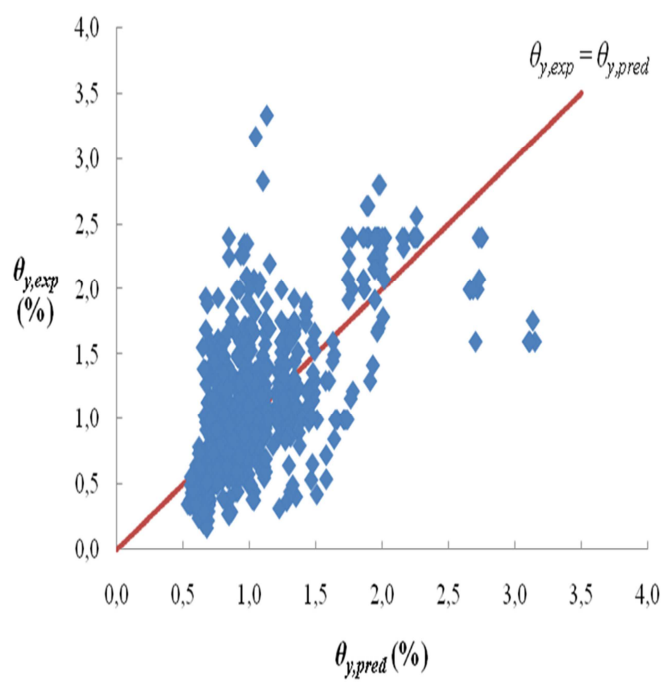

(b) 


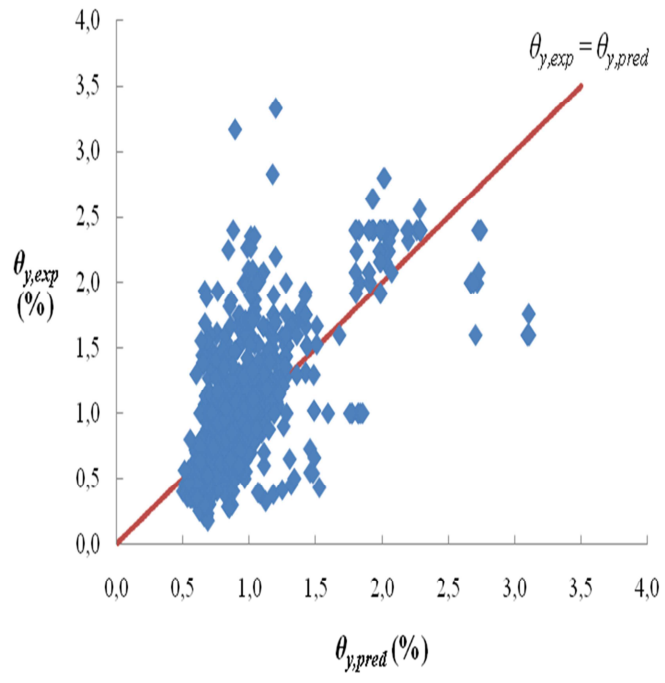

(c)

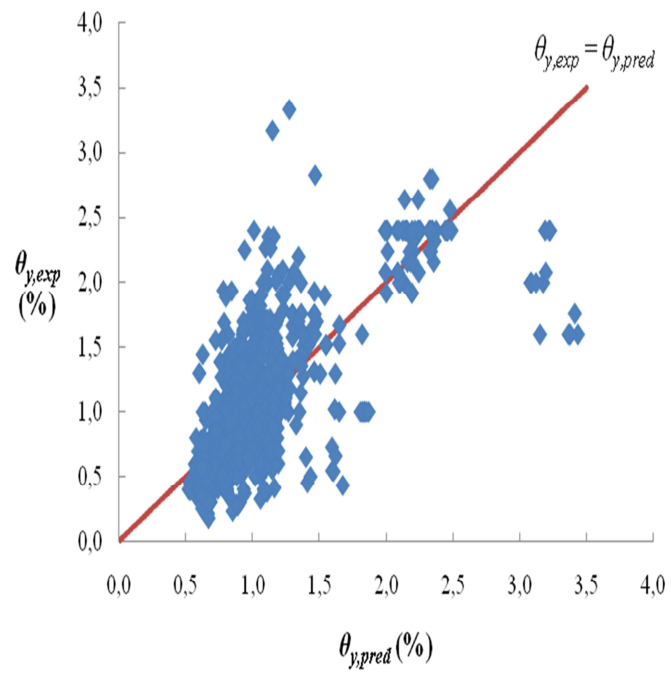

(d)

Figura 4.20 Comparación de valores experimentales y predichos para el giro de plastificación $\theta_{y .}$. (a) Panagiotakos y Fardis (2001). (b) EC-8. (c) Biskinis y Fardis (2010a). (d) Ecuación propuesta.

\subsubsection{Giro último}

La expresión propuesta para el giro último $\theta_{u}$, tras el ajuste con la base de datos inicial, se corresponde con

$$
\begin{aligned}
\theta_{u}(\%)= & 0,975 a_{s t}\left(1-0,546 a_{c y}\right)\left(1+0,716 a_{s l}\right)\left(1-0,398 a_{w, r}\right)\left(1-\frac{2}{7} a_{w, n r}\right)(0,311)^{v}\left[\frac{\max \left(0,01 ; \omega_{2}\right)}{\max \left(0,01 ; \omega_{1}\right)} f_{c}\right]^{0,299} \\
& {\left[\min \left(9, \frac{L_{s}}{h}\right)\right]^{0,279} 19,420^{\left(\frac{\alpha \rho_{h} f_{y h}}{f_{c}}\right)} 0,929^{100 \rho_{d}} }
\end{aligned}
$$

Los coeficientes de ajuste $C_{i}$ presentes en la ecuación (4.79) implican que el tipo de carga considerada mediante el parámetro $a_{c y}$, el esfuerzo axil normalizado $v$ y las cuantías mecánicas de armadura longitudinal de tracción y compresión $\omega_{1}$ y $\omega_{2}$ aumentan su influencia respecto a la ecuación (4.76) propuesta para la selección de ensayos. Sin embargo, el término que considera la esbeltez de cortante $L_{s} / h$ disminuye su influencia en los resultados en torno a un $50 \%$.

La relación entre el coeficiente de variación $C V$ de la variable $x=\theta_{u \text {, exp }} / \theta_{u \text {,pred }}$ y el porcentaje de variación $P_{i, 0}$ de los coeficientes iniciales $C_{i, 0}$ se muestra en la Figura 4.21. Al igual que sucede para el momento y el giro de plastificación ajustados con la base de datos inicial, la restricción impuesta en el valor medio $\bar{x}$ no influye en la evolución del coeficiente de variación $C V$ al aumentar el porcentaje de variación $P_{i, 0}$. Respecto a los diagramas box-plot, mostrados en la Figura 4.22, los valores extremos de la variable $x=\theta_{u}$, exp $/ \theta_{u \text {,pred }}$ obtenidos mediante la ecuación (4.79) son inferiores a los que resultan de aplicar las expresiones de otros autores, observándose los mayores 
valores extremos para lo obtenido aplicando la expresión del EC-8.

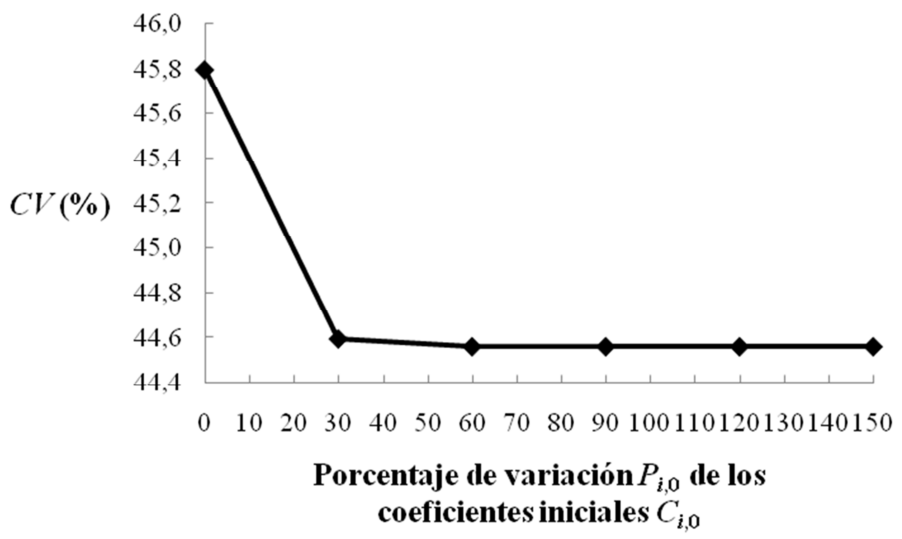

Figura 4.21 Coeficiente de variación $C V$ de la variable $x=\theta_{u \text {,exp }} / \theta_{u \text {,pred }}$ en función del porcentaje de variación $P_{i, 0}$ de los coeficientes iniciales $C_{i, 0}$.

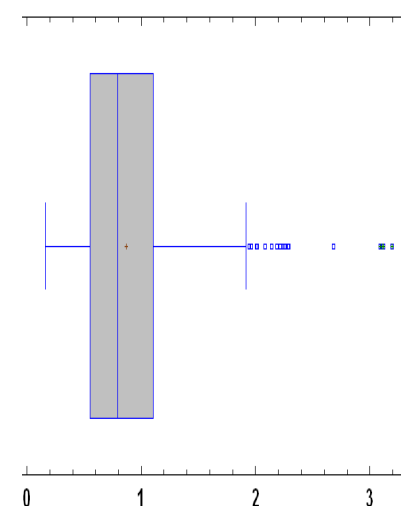

(a)

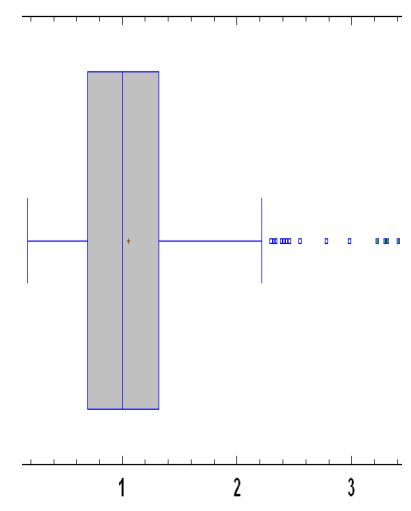

(c)

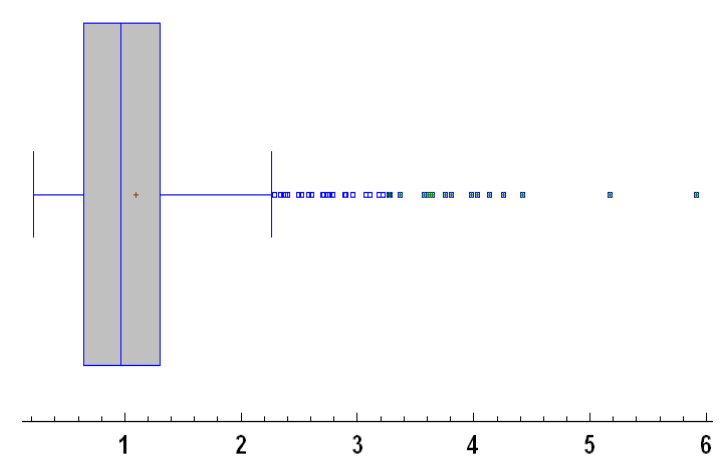

(b)

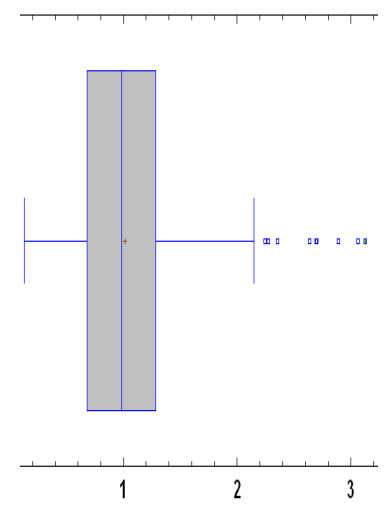

(d)

Figura 4.22 Diagrama box-plot para la variable $x=\theta_{u \text {,exp }} / \theta_{u, p r e d .}$ (a) Panagiotakos y Fardis (2001). (b) EC-8. (c) Biskinis y Fardis (2010b). (d) Ecuación propuesta.

Las medidas de síntesis para la variable $x=\theta_{u \text {, exp }} / \theta_{u \text {,pred }}$ se muestran en la Tabla 4.12. Con la ecuación (4.79) se consigue una reducción entre el 8 y el $34 \%$ en la 
desviación típica poblacional $\sigma$ respecto al resto de expresiones, disminuyendo el coeficiente de variación $C V$ entre el 3 y el $27 \%$. Además, se obtiene un valor medio $\bar{x}$ $=1$. Respecto a los resultados obtenidos al analizar la relación entre $\theta_{u \text {,exp }}$ y $\theta_{u \text {,pred }}$, expuestos en la Tabla 4.13, se obtiene un aumento de la covarianza $\operatorname{cov}\left(x_{\text {exp }}, x_{\text {pred }}\right)$ entre el 16 y el $33 \%$ respecto a Panagiotakos y Fardis (2001) y Biskinis y Fardis (2010b), triplicándose el valor obtenido aplicando la ecuación del EC-8. Se incrementa el coeficiente de regresión $r$ entre el 35 y el $56 \%$, obteniéndose además una recta de regresión con unos coeficientes $a^{\prime}$ y $b^{\prime}$ más próximos a 0 y 1 , respectivamente. El valor de $S_{d}$ disminuye un $23 \%$ respecto a Panagiotakos y Fardis (2001) y un $10 \%$ respecto a la expresión del EC-8.

Tabla 4.12 Medidas de síntesis para la variable $x=\theta_{u \text {,exp }} / \theta_{u \text {,pred }}$ según diferentes autores.

\begin{tabular}{lcccccc}
\hline & $\mathrm{N}^{\circ}$ de ensayos & $\bar{x}$ & $\sigma$ & Mediana & RI & CV (\%) \\
\hline Panagiotakos y Fardis (2001) & & 1,06 & 0,49 & 1,00 & 0,55 & 47,00 \\
EC-8 & & 1,09 & 0,67 & 0,97 & 0,65 & 60,66 \\
Biskinis y Fardis (2010b) & 875 & 1,05 & 0,48 & 1,00 & 0,63 & 45,80 \\
Ecuación propuesta & & 1,00 & 0,44 & 0,97 & 0,59 & 44,58 \\
\hline
\end{tabular}

Tabla 4.13 Parámetros para el análisis de la relación entre las variables $\theta_{u, \exp }$ y $\theta_{u, p r e d}$ según diferentes autores.

\begin{tabular}{lcccccc}
\hline & $\operatorname{cov}\left(x_{\text {exp }}, x_{\text {pred }}\right)$ & $a^{\prime}$ & $b^{\prime}$ & $r$ & $R^{2}$ & $S_{d}$ \\
\hline Panagiotakos y Fardis (2001) & 5,457 & 0,88 & 0,70 & 0,52 & 0,27 & 1895,67 \\
EC-8 & 2,111 & $-1,17$ & 1,37 & 0,45 & 0,20 & 1616,08 \\
Biskinis y Fardis (2010b) & 4,762 & $-1,83$ & 1,46 & 0,70 & 0,49 & 1451,57 \\
Ecuación propuesta & 6,333 & $-0,49$ & 1,10 & 0,70 & 0,49 & 1454,94 \\
\hline
\end{tabular}

La Figura 4.23 muestra gráficamente la relación entre los valores experimentales y predichos para el giro último $\theta_{u}$. Aunque las expresiones de los diferentes autores proporcionan resultados que presentan dispersión en torno a la recta $\theta_{u, \text { exp }}=\theta_{u \text {,pred }}$, la ecuación (4.79) mejora el ajuste respecto a dicha recta.

\subsection{RESUMEN DE RESULTADOS}

La principal aplicación de las expresiones de comportamiento seccional disponibles en la normativa de diseño y en la literatura técnica es la rehabilitación y el refuerzo sísmico de edificios. Sin embargo, la eficiencia de estas expresiones desde el punto de vista computacional, al estar exentas de rutinas de cálculo, y la precisión que ofrecen al estar calibradas con ensayos experimentales, hacen que resulten útiles para determinar conceptos asociados a los estados de plastificación y último de un elemento en el análisis sísmico de estructuras. 


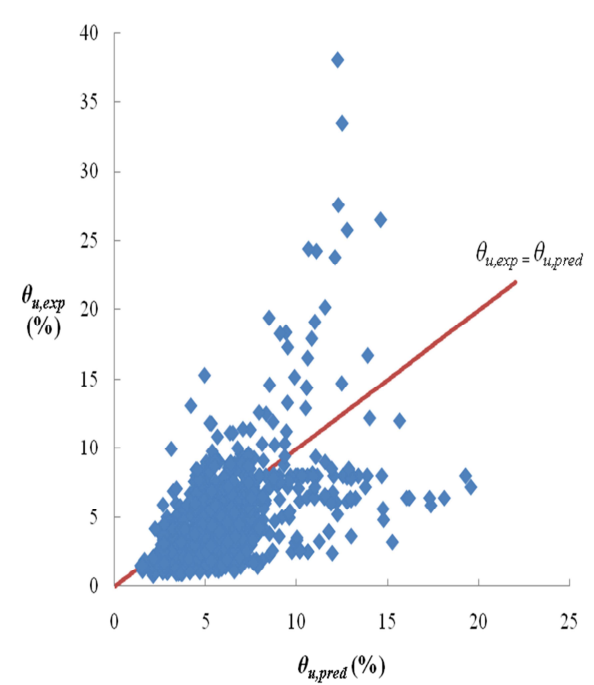

(a)

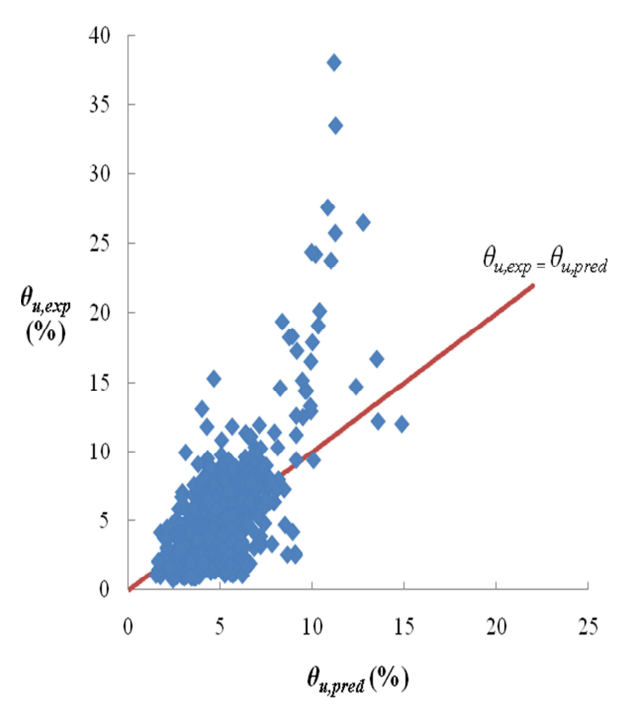

(c)

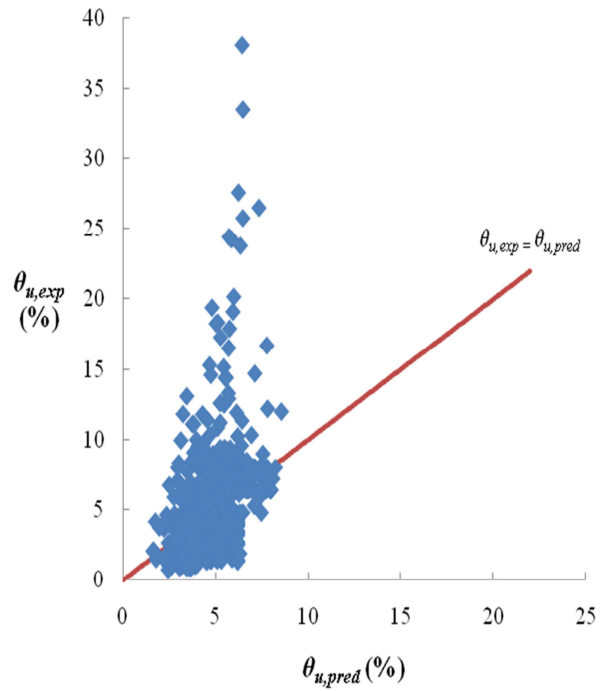

(b)

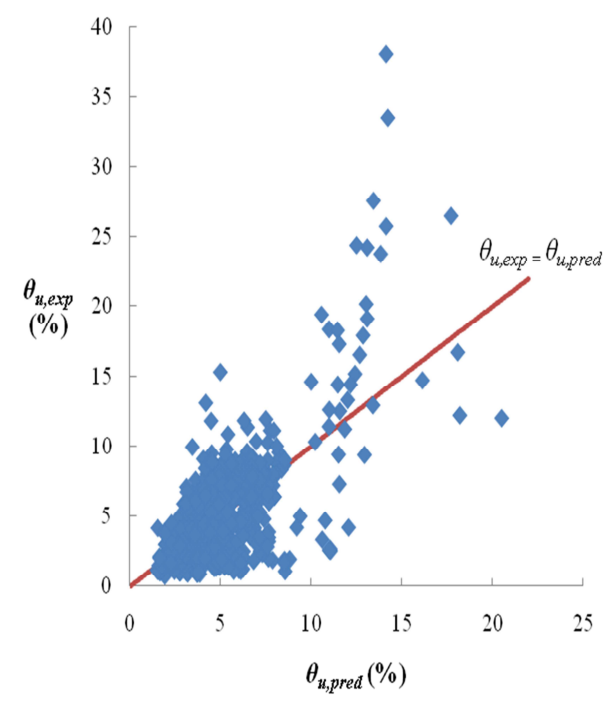

(d)

Figura 4.23 Comparación de valores experimentales y predichos para el giro último $\theta_{u}$. (a) Panagiotakos y Fardis (2001). (b) EC-8. (c) Biskinis y Fardis (2010b). (d) Ecuación propuesta.

En este capítulo se han propuesto expresiones para obtener el momento de plastificación $M_{y}$, el giro de plastificación $\theta_{y}$ y el giro último $\theta_{u}$ de secciones rectangulares de hormigón armado. Dichas expresiones se obtienen tras calibrar las ecuaciones propuestas en los trabajos de Biskinis y Fardis (2010a, 2010b) con las bases de ensayos descritas en el capítulo 3 de esta tesis, empleando una serie de coeficientes de ajuste $C_{i}$. Se han analizado los resultados obtenidos con las expresiones ajustadas, comparándolos posteriormente con los obtenidos aplicando las expresiones desarrolladas por Panagiotakos y Fardis (2001) y Biskinis y Fardis (2010a, 2010b) y las propuestas en el EC-8. Para analizar y comparar los resultados se han obtenido algunas 
medidas de síntesis para la variable $x=x_{\text {exp }} / x_{\text {pred }}$ y ciertos parámetros que cuantifican el grado de relación lineal entre los valores experimentales $x_{\exp } \mathrm{y}$ los obtenidos con las expresiones $x_{\text {pred }}$.

Dada la extensa cantidad de resultados obtenidos, resulta conveniente presentar una síntesis de los mismos. Así, las Tablas 4.14 a 4.17 muestran el rango de mejoras conseguidas con las expresiones ajustadas respecto al resto de expresiones consideradas para el momento de plastificación $M_{y}$, el giro de plastificación $\theta_{y}$ y el giro último $\theta_{u}$. Al analizar la variable $x=x_{\text {exp }} / x_{\text {pred }}$ se consiguen valores más próximos a 1 para las medidas de tendencia central, esto es, la media $\bar{x}$ y la mediana. Además, se reduce la dispersión en los resultados, ya que disminuyen la desviación típica $s$, el rango intercuartílico $R I$ y el coeficiente de variación $C V$. La disminución de la dispersión se puede ver gráficamente en los diagramas box-plot, apreciándose unos valores inferiores para los extremos de los bigotes y una disminución del ancho de caja al reducirse el rango intercuartílico $R I$.

El incremento obtenido en ciertos parámetros como la covarianza $\operatorname{cov}\left(x_{\text {exp }}, x_{\text {pred }}\right)$ y el coeficiente de correlación lineal $r$ indica un mayor grado de relación lineal entre los valores experimentales y predichos, mientras que la reducción del valor absoluto de la suma de diferencias entre valores experimentales y predichos $S_{d}$ es indicativo de un mejor ajuste entre ambos valores. Finalmente, la representación gráfica de los valores experimentales y predichos muestra que las expresiones ajustadas obtienen resultados más concentrados en torno a la recta $x_{\text {exp }}=x_{\text {pred }}$.

Tabla 4.14 Mejoras obtenidas con las expresiones ajustadas a la selección de ensayos en los parámetros de dispersión respecto a otras expresiones (en \%).

\begin{tabular}{lccc}
\hline & $s$ & $R I$ & $C V$ \\
\hline Panagiotakos y Fardis (2001) & $24-28$ & $6-25$ & $14-16$ \\
EC-8 & $18-19$ & $13-29$ & 10 \\
Biskinis y Fardis (2010a, 2010b ) & $14-28$ & $19-28$ & $3-16$ \\
\hline
\end{tabular}

Tabla 4.15 Mejoras obtenidas con las expresiones ajustadas a la selección de ensayos en los parámetros para el análisis de la relación $x_{\text {exp }}-x_{\text {pred }}$ respecto a otras expresiones (en \%).

\begin{tabular}{lcccc}
\hline & $\operatorname{cov}\left(x_{\text {exp }}, x_{\text {pred }}\right)$ & $r$ & $R^{2}$ & $S_{d}$ \\
\hline Panagiotakos y Fardis (2001) & $11-33$ & $1-62$ & $2-160$ & $13-36$ \\
EC-8 & $44-88$ & $25-29$ & $56-67$ & $16-20$ \\
Biskinis y Fardis (2010a, 2010b ) & $11-53$ & $1-16$ & $2-36$ & $9-19$ \\
\hline
\end{tabular}


Tabla 4.16 Mejoras obtenidas con las expresiones ajustadas a la base de datos inicial en los parámetros de dispersión respecto a otras expresiones (en \%).

\begin{tabular}{lccc}
\hline & $\sigma$ & $R I$ & $C V$ \\
\hline Panagiotakos y Fardis (2001) & $7-10$ & $3-24$ & $3-14$ \\
EC-8 & $10-34$ & $9-11$ & $7-27$ \\
Biskinis y Fardis (2010a, 2010b ) & $7-10$ & $5-24$ & $3-14$ \\
\hline
\end{tabular}

Tabla 4.17 Mejoras obtenidas con las expresiones ajustadas a la base de datos inicial en los parámetros para el análisis de la relación $x_{\text {exp }}-x_{\text {pred }}$ respecto a otras expresiones (en \%).

\begin{tabular}{lcccc}
\hline & $\operatorname{cov}\left(x_{\text {exp }}, x_{\text {pred }}\right)$ & $r$ & $R^{2}$ & $S_{d}$ \\
\hline Panagiotakos y Fardis (2001) & $14-16$ & $1-35$ & $2-83$ & $10-23$ \\
EC-8 & $15-300$ & $4-56$ & $9-145$ & $3-10$ \\
Biskinis y Fardis (2010a, 2010b ) & $10-33$ & 0 & 0 & 0 \\
\hline
\end{tabular}

La aproximación de la media $\bar{x}$ y la mediana a 1 , además de la reducción de la dispersión y el aumento del grado de relación lineal entre los valores experimentales $x_{\text {exp }}$ y los valores $x_{\text {pred }}$ obtenidos con las expresiones calibradas con las bases de ensayos implica que dichas expresiones ofrezcan un mejor ajuste entre ambos valores, proporcionando una precisión superior a la ofrecida por otras expresiones disponibles.

Los valores obtenidos para los parámetros estadísticos considerados confirman que las mejoras conseguidas resultan más significativas cuando el ajuste se realiza con la selección de ensayos, confirmando lo adecuado de confeccionar dicha selección. Las expresiones calibradas con la selección de ensayos resultan de especial utilidad para el ingeniero estructural, debido a su aplicación directa a edificios convencionales de hormigón armado situados en zonas sísmicas. 



\section{Validación del método de ajuste del comportamiento seccional. Comparación de resultados}

\subsection{INTRODUCCIÓN}

Tras efectuar el ajuste de ciertas expresiones de comportamiento seccional a la selección de ensayos descrita en el capítulo 3, en este capítulo se establece una comparación entre los resultados ofrecidos por dichas ecuaciones y los obtenidos con otros métodos o expresiones. Concretamente, los métodos considerados para realizar la comparación de resultados son los métodos clásicos y las expresiones propuestas por otros autores (Biskinis y Fardis, 2010a; Biskinis y Fardis, 2010b).

La consideración de los métodos anteriores para su comparación con las expresiones ajustadas a la selección de ensayos atiende a distintas consideraciones. Por un lado, se compara con los métodos clásicos, cuyos fundamentos teóricos se han empleado en numerosos estudios (Cosenza et al., 2009; Verderame et al., 2010; entre otros), para contrastar resultados relativos al comportamiento seccional de un elemento de hormigón armado. Por otro lado, resulta conveniente realizar el contraste con las expresiones propuestas por Biskinis y Fardis (2010a, 2010b), puesto que a partir de ellas se han obtenido las expresiones ajustadas a la selección de ensayos.

Con objeto de cotejar los resultados obtenidos empleando los diferentes métodos considerados, se analizan dos secciones rectangulares de hormigón armado, obteniéndose ciertos conceptos relativos al comportamiento de plastificación y último (momento y giro).

\subsection{ELEMENTO ESTRUCTURAL EMPLEADO EN LA VALIDACIÓN}

La Figura 5.1 muestra un elemento en voladizo de longitud $L=2 \mathrm{~m}$ sobre el que se aplica una carga transversal de tipo puntual en su extremo libre A, alcanzándose el 
momento flector último $M_{u}$ y la curvatura última $\phi_{u}$ en la sección de empotramiento B. En la citada figura se puede apreciar, además, la distribución real e idealizada de curvaturas a lo largo de su longitud. La curvatura de tipo plástico se extiende sobre la rótula plástica desarrollada en la sección de empotramiento, de longitud $L_{p}$, estando dicha longitud constituida por todas las secciones en las que el momento flector actuante es igual o superior al momento flector de plastificación de la sección $M_{y}$.

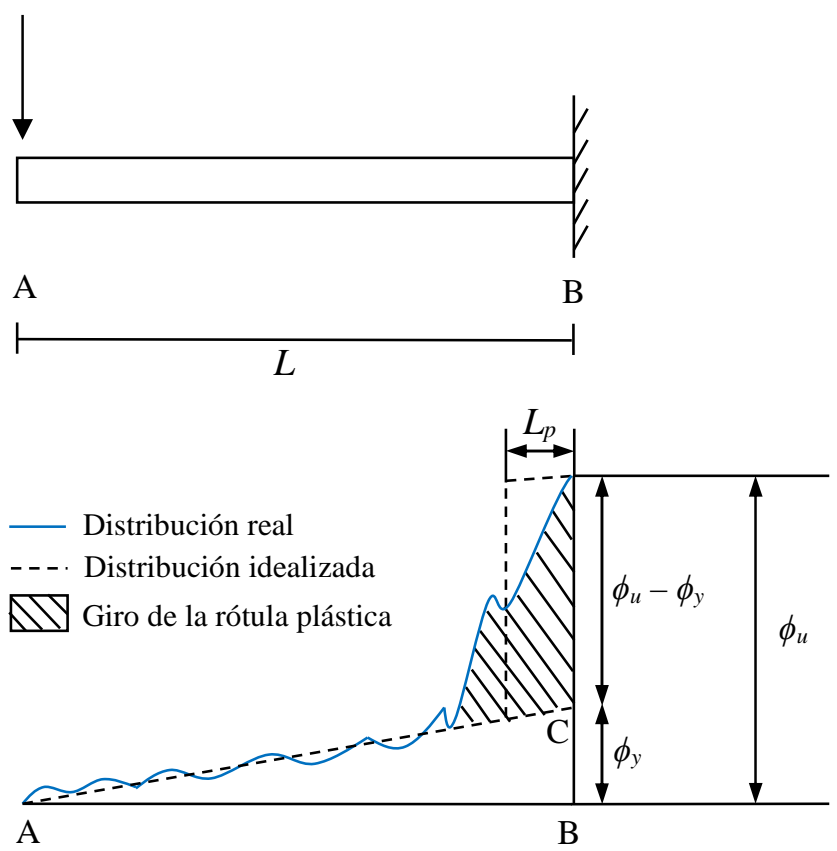

Figura 5.1 Elemento en voladizo y distribución de curvaturas a lo largo de su longitud.

Para contrastar los resultados obtenidos al emplear los métodos expuestos en el apartado 5.1, se consideran las dos secciones rectangulares mostradas en la Figura 5.2. para el elemento en voladizo descrito.

Con objeto de estudiar la influencia de la geometría de la sección en los resultados obtenidos aplicando los diferentes métodos considerados, las secciones de la Figura 5.2 presentan ciertas diferencias en sus proporciones. Así, la sección $300 \times 400 \mathrm{~mm}$ es de tipo viga, esto es, con sección rectangular, mientras que la sección $350 \times 350$ mm es de tipo pilar, con sección cuadrada. Aunque ambas secciones presentan áreas similares, la distinta geometría supone una diferencia en sus inercias del $28 \%$. Respecto al armado, la sección $300 \times 400 \mathrm{~mm}$ presenta tres barras para la armadura longitudinal de tracción $A_{s}$ y compresión $A_{s}$ ', mientras que la sección $350 \times 350 \mathrm{~mm}$ presenta cuatro. La armadura transversal es la misma para ambas secciones. La resistencia a compresión simple $f_{c}$ del hormigón es $25 \mathrm{MPa}$ y el acero de las armaduras es de tipo B $500 \mathrm{SD}$, con límite elástico de la armadura de tracción $f_{y}=500 \mathrm{MPa}$. 

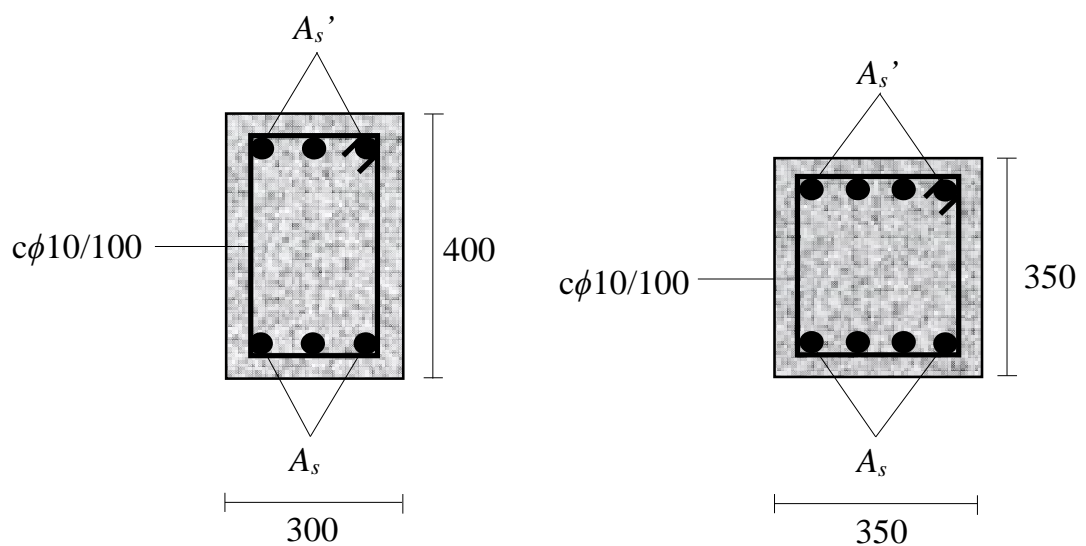

Figura 5.2 Secciones consideradas para el elemento en voladizo (unidades en mm).

Para cada una de estas secciones se analizan las diferencias obtenidas aplicando los métodos considerados, estudiando la influencia de los siguientes aspectos:

a) área de armadura longitudinal de tracción $A_{s}$,

b) área de armadura longitudinal de compresión $A_{s}$, y

c) esfuerzo axil $N$ actuante en la sección.

Para estudiar la influencia de la armadura de tracción en los resultados obtenidos se consideran tres casos: cuantía reducida, media y elevada de armadura de tracción. En todos estos casos, la armadura de compresión es constante. Si lo que se pretende es evaluar la influencia de la armadura de compresión, se consideran las mismas cuantías de armadura de compresión, esto es, reducida, media y elevada, siendo en este caso constante la armadura de tracción. La influencia del esfuerzo axil $N$ se estudia considerando cuatro casos: axil nulo, axil reducido, axil medio y axil elevado.

La Tabla 5.1 muestra, para las dos secciones de la Figura 5.2, los diferentes casos que se han analizado tras la combinación de todos los aspectos anteriormente expuestos. Los casos analizados presentan las siguientes características:

a) casos 1 a 12: armadura de compresión constante, aumentando la armadura de tracción cada cuatro casos

b) casos 13 a 24: armadura de tracción constante, aumentando la armadura de compresión cada cuatro casos.

Además, los diferentes niveles considerados de esfuerzo axil $N$ se repiten periódicamente cada cuatro casos.

Conviene destacar que para los 24 casos considerados en la Tabla 5.1 se cumplen las restricciones impuestas al confeccionar la selección de ensayos del capítulo 3. Este aspecto resulta imprescindible para la aplicación de las expresiones calibradas con dicha selección de ensayos. 
Tabla 5.1 Armadura de tracción y compresión y esfuerzo axil para los diferentes casos considerados en las secciones $300 \times 400$ y $350 \times 350 \mathrm{~mm}$.

\begin{tabular}{|c|c|c|c|c|c|c|}
\hline \multirow{2}{*}{ CASO } & \multicolumn{3}{|c|}{ Sección 300×400mm } & \multicolumn{3}{|c|}{ Sección $350 \times 350 \mathrm{~mm}$} \\
\hline & $A_{s}$ & $A_{s}{ }^{\prime}$ & $N(\mathrm{kN})$ & $A_{s}$ & $A_{s}{ }^{\prime}$ & $N(\mathrm{kN})$ \\
\hline 1 & $3 \phi 12$ & $3 \phi 12$ & 0 & $4 \phi 12$ & $4 \phi 12$ & 0 \\
\hline 2 & $3 \phi 12$ & $3 \phi 12$ & 300 & $4 \phi 12$ & $4 \phi 12$ & 200 \\
\hline 3 & $3 \phi 12$ & $3 \phi 12$ & 600 & $4 \phi 12$ & $4 \phi 12$ & 300 \\
\hline 4 & $3 \phi 12$ & $3 \phi 12$ & 900 & $4 \phi 12$ & $4 \phi 12$ & 400 \\
\hline 5 & $3 \phi 16$ & $3 \phi 12$ & 0 & $4 \phi 20$ & $4 \phi 12$ & 0 \\
\hline 6 & $3 \phi 16$ & $3 \phi 12$ & 300 & $4 \phi 20$ & $4 \phi 12$ & 200 \\
\hline 7 & $3 \phi 16$ & $3 \phi 12$ & 600 & $4 \phi 20$ & $4 \phi 12$ & 300 \\
\hline 8 & $3 \phi 16$ & $3 \phi 12$ & 900 & $4 \phi 20$ & $4 \phi 12$ & 400 \\
\hline 9 & $3 \phi 20$ & $3 \phi 12$ & 0 & $4 \phi 25$ & $4 \phi 12$ & 0 \\
\hline 10 & $3 \phi 20$ & $3 \phi 12$ & 300 & $4 \phi 25$ & $4 \phi 12$ & 200 \\
\hline 11 & $3 \phi 20$ & $3 \phi 12$ & 600 & $4 \phi 25$ & $4 \phi 12$ & 300 \\
\hline 12 & $3 \phi 20$ & $3 \phi 12$ & 900 & $4 \phi 25$ & $4 \phi 12$ & 400 \\
\hline 13 & $3 \phi 12$ & $3 \phi 12$ & 0 & $4 \phi 12$ & $4 \phi 12$ & 0 \\
\hline 14 & $3 \phi 12$ & $3 \phi 12$ & 300 & $4 \phi 12$ & $4 \phi 12$ & 200 \\
\hline 15 & $3 \phi 12$ & $3 \phi 12$ & 600 & $4 \phi 12$ & $4 \phi 12$ & 300 \\
\hline 16 & $3 \phi 12$ & $3 \phi 12$ & 900 & $4 \phi 12$ & $4 \phi 12$ & 400 \\
\hline 17 & $3 \phi 12$ & $3 \phi 16$ & 0 & $4 \phi 12$ & $4 \phi 20$ & 0 \\
\hline 18 & $3 \phi 12$ & $3 \phi 16$ & 300 & $4 \phi 12$ & $4 \phi 20$ & 200 \\
\hline 19 & $3 \phi 12$ & $3 \phi 16$ & 600 & $4 \phi 12$ & $4 \phi 20$ & 300 \\
\hline 20 & $3 \phi 12$ & $3 \phi 16$ & 900 & $4 \phi 12$ & $4 \phi 20$ & 400 \\
\hline 21 & $3 \phi 12$ & $3 \phi 20$ & 0 & $4 \phi 12$ & $4 \phi 25$ & 0 \\
\hline 22 & $3 \phi 12$ & $3 \phi 20$ & 300 & $4 \phi 12$ & $4 \phi 25$ & 200 \\
\hline 23 & $3 \phi 12$ & $3 \phi 20$ & 600 & $4 \phi 12$ & $4 \phi 25$ & 300 \\
\hline 24 & $3 \phi 12$ & $3 \phi 20$ & 900 & $4 \phi 12$ & $4 \phi 25$ & 400 \\
\hline
\end{tabular}

\subsection{MÉTODOS CLÁSICOS}

En este apartado se describen los fundamentos de los métodos clásicos empleados para la comparación con las expresiones ajustadas a la selección de ensayos descritas en el capítulo 4. Estos métodos consideran los siguientes aspectos:

a) los giros de plastificación y último de la sección de empotramiento del elemento mostrado en la Figura 5.1 se obtienen atendiendo a ciertos fundamentos teóricos correspondientes a elementos de hormigón armado, expuestos en Park y Paulay (1974), y 
b) los conceptos dependientes únicamente de las características de la sección del elemento, como la curvatura y el momento de plastificación y la curvatura última, se obtienen aplicando el Prontuario Informático del Hormigón (Corres et al., 2014), el cual constituye una herramienta comúnmente empleada y aceptada.

El giro de la sección de empotramiento del elemento en voladizo de la Figura 5.1 se puede obtener mediante la integración de la curvatura $\varphi$ a lo largo de su longitud, según

$$
\theta_{A B}=\int_{A}^{B} \varphi d x
$$

Sin embargo, la aplicación de la ecuación (5.1) requiere conocer la distribución real de curvaturas a lo largo del elemento, aspecto que no resulta sencillo debido a la presencia de fisuras por toda su longitud. Si lo que se pretende es obtener el giro de la sección de empotramiento en el instante último $\theta_{u}$, una aproximación al problema, de acuerdo con Park y Paulay (1974), es

$$
\theta_{u}=\theta_{e l}+\theta_{p}
$$

siendo

$\theta_{e l} \quad$ componente elástica del giro último de la sección, y

$\theta_{p} \quad$ componente plástica del giro último de la sección.

De la observación de la Figura 5.1 se deduce que el área del triángulo $\mathrm{ABC}$ se corresponde con el giro elástico de la sección $\theta_{e l}$. El área sombreada representa el giro plástico $\theta_{p}$. Si ésta se sustituye por un rectángulo de base $L_{p}$ y altura $\phi_{u}-\phi_{y}$, el giro último de la sección viene dado por

$$
\theta_{u}=\frac{1}{2} L \phi_{y}+\left(\phi_{u}-\phi_{y}\right) L_{p}
$$

Para el cálculo de la longitud de la rótula plástica $L_{p}$, existe una extensa variedad de expresiones disponibles en la literatura, casi todas ellas de carácter empírico. En esta tesis se emplean las siguientes:

a) Sawyer (1964)

$$
L_{p}=0,25 d+0,075 L_{s}
$$

b) Corley (1966)

$$
L_{p}=0,5 d+0,2 \sqrt{d} \frac{L_{s}}{d}
$$

c) Mattock (1967)

$$
L_{p}=0,5 d+0,05 L_{s}
$$


donde

$d \quad$ canto útil de la sección, $\mathrm{y}$

$L_{s} \quad$ distancia entre las secciones de momento flector máximo y nulo.

La aplicación de las ecuaciones (5.4) a (5.6) para el cálculo de la longitud de la rótula plástica $L_{p}$ implica la obtención de tres valores similares para el giro último $\theta_{u}$. Por tanto, se considera como giro último el promedio de dichos valores.

El Prontuario Informático del Hormigón efectua una integración de las ecuaciones constitutivas de los materiales a lo largo de la sección para determinar los valores del momento de plastificación $M_{y}$ y la curvatura de plastificación $\phi_{y}$ y última $\phi_{u}$. Concretamente, considera el diagrama parábola-rectángulo para definir el comportamiento constitutivo del hormigón y el diagrama bilineal para representar el efecto de las armaduras. Ambos diagramas pueden ser consultados en la instrucción EHE-08.

La integración de las tensiones normales se realiza mediante el algoritmo de Marín (Marín, 1988). Este algoritmo permite la integración de funciones polinómicas de dos variables $F(x, y)$ a lo largo de un recinto de integración $\mathrm{A}$

$$
\iint_{A} x^{p} y^{q} F(x, y) d A
$$

El recinto de integración, esto es, la sección del elemento, ha de estar encerrado por un contorno poligonal cerrado simplemente conexo, determinado por un conjunto de vértices de coordenadas $\left(x_{i}, y_{i}\right)$, con $i=1,2, \ldots, P$, siendo $P$ el último vértice. Los vértices han de estar numerados en sentido antihorario, debiendo coincidir las coordenadas del primer y último vértice.

La función polinómica $F(x, y)$, es decir, la ecuación constitutiva del material, se puede obtener mediante el sumatorio

$$
F(x, y)=\sum_{m=0}^{M} \sum_{n=0}^{N} a_{m, n} x^{m} y^{n}
$$

donde $a_{m, n}$ es un coeficiente genérico y $M$ y $N$ los máximos exponentes considerados en la función que se desea integrar. La integral doble de la ecuación (5.7) se puede determinar mediante

$$
\iint_{A} x^{p} y^{q} F(x, y) d A=\sum_{m=0}^{M} \sum_{n=0}^{N} a_{m, n} M_{p+m, q+n}
$$

siendo $M_{p+m, q+n}$ el momento de área de orden $p+m$ y $q+n$. Para la integración de las ecuaciones constitutivas, el prontuario supone $p=0$ y $q=0$, por lo que la ecuación (5.9) pasa a ser

$$
\iint_{A} F(x, y) d A=\sum_{m=0}^{M} \sum_{n=0}^{N} a_{m, n} M_{m, n}
$$

siendo ahora $M_{m, n}$ el momento de área de orden $m$ y $n$, definido por 


$$
M_{m, n}=\frac{m ! n !}{(m+n+2) !} \sum_{i=1}^{P} w_{i} \sum_{j=0}^{m} \sum_{k=0}^{n}\left(\begin{array}{c}
j+k \\
j
\end{array}\right)\left(\begin{array}{c}
m+n-j-k \\
n-k
\end{array}\right) x_{i}^{m-j} x_{i+1}^{j} y_{i}^{n-k} y_{i+1}^{k}
$$

donde

$\mathrm{w}_{\mathrm{i}}=\mathrm{x}_{\mathrm{i}} \mathrm{y}_{\mathrm{i}+1}-\mathrm{x}_{\mathrm{i}+1} \mathrm{y}_{\mathrm{i}}$

Tal y como se deduce de la ecuación (5.7), para poder aplicar el algoritmo de Marín es necesario que las ecuaciones constitutivas puedan definirse mediante términos polinómicos. Para salvar este inconveniente, el prontuario define dichas ecuaciones a través de puntos unidos mediante tramos rectos, esto es, polinomios de primer grado. Esta aproximación requiere que las ecuaciones constitutivas sean integradas por tramos, dividiéndose la sección en fragmentos paralelos a la fibra neutra, tal y como muestra la Figura 5.3.

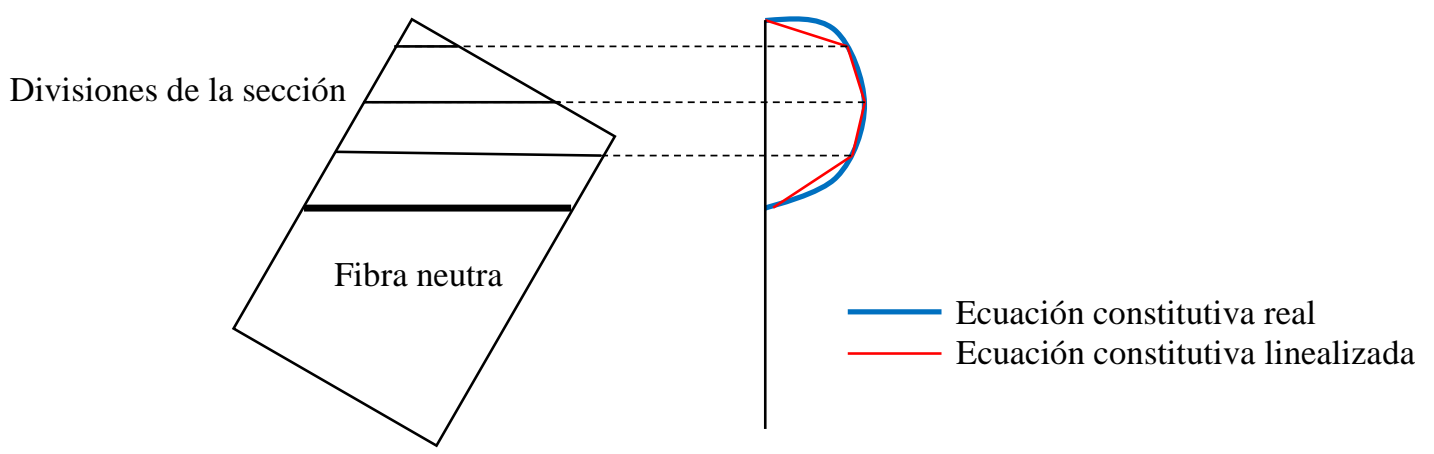

Figura 5.3 Discretización de la sección para integrar la ecuación constitutiva por tramos.

La integración de la ecuación constitutiva en cada una de las regiones de la sección permite obtener la resultante de tensiones en dicha región. La repetición de este proceso para todos los tramos de la ecuación constitutiva linealizada permite la integración total de la ecuación constitutiva real.

Si lo que se pretende obtener es el giro de plastificación de la sección de empotramiento $\theta_{y}$, se considera que dicha sección es la única plastificada en todo el elemento, obteniéndose la distribución de curvaturas real e idealizada que se muestra en la Figura 5.4. En este caso, se puede emplear la aproximación propuesta por Park y Paulay (1974):

$$
\theta_{y}=\frac{1}{2} L \phi_{y}
$$

\subsection{RESULTADOS}

Las Tablas 5.2 y 5.3 muestran los resultados obtenidos tras el análisis de las secciones expuestas en la Figura 5.2 con los diferentes métodos considerados. A este respecto, conviene destacar el hecho de que la curvatura de plastificación $\phi_{y}$ de la sección influye considerablemente en las diferencias obtenidas al aplicar los distintos métodos. Los tres 
métodos empleados consideran que la plastificación de la sección se produce por la plastificación de la armadura de tracción o por alcanzarse unas deformaciones de compresión excesivas en el hormigón.

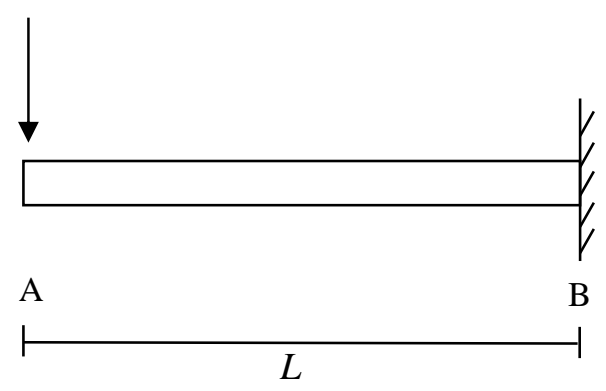

- Distribución real

- - -. Distribución idealizada

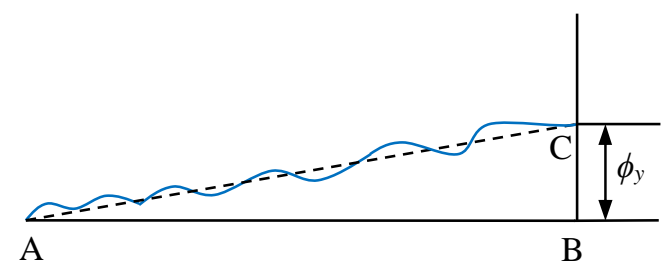

Figura 5.4 Distribución real e idealizada de curvaturas en el elemento al plastificar únicamente la sección de empotramiento.

Las mayores diferencias obtenidas para la curvatura de plastificación se dan entre la expresión de Biskinis y Fardis (2010a) y los métodos clásicos. En todos los casos analizados, la curvatura de plastificación obtenida con la expresión de Biskinis y Fardis (2010a) resulta inferior a la proporcionada por el Prontuario Informático del Hormigón. Concretamente, si la plastificación de la sección se produce por el hormigón, el prontuario supone que ésta tiene lugar al alcanzarse un valor de deformación de rotura a compresión simple $\varepsilon_{c 0}=2 \%$, mientras que la expresión de Biskinis y Fardis (2010a) considera una deformación máxima admisible por el hormigón del 1,6\%o antes de la plastificación.

Las diferencias obtenidas para la curvatura de plastificación entre el prontuario y la expresión de Biskinis y Fardis (2010a) son mayores al aumentar el esfuerzo axil y la cuantía de armadura de tracción. Así, con elevados valores del axil y de la cuantía de armadura de tracción, el prontuario considera que la plastificación se produce por la armadura, mientras que la expresión de Biskinis y Fardis (2010a) supone que la plastificación se produce por el hormigón, obteniéndose diferencias importantes. Por otro lado, las diferencias disminuyen a medida que aumenta la armadura de compresión y el esfuerzo axil, puesto que en estos casos ambos métodos consideran que la plastificación de la sección se produce por la armadura de tracción. 
Tabla 5.2 Resultados obtenidos para el momento y giro de plastificación y giro último de la sección 300×400 mm según diferentes métodos.

\begin{tabular}{cccccccccc}
\hline \multirow{2}{*}{ CASO } & \multicolumn{3}{c}{ Métodos clásicos } & \multicolumn{3}{c}{ Biskinis y Fardis (2010a, 2010b ) } & \multicolumn{3}{c}{ Expresiones ajustadas } \\
& $M_{y}(\mathrm{kNm})$ & $\theta_{y}(\%)$ & $\theta_{u}(\%)$ & $M_{y}(\mathrm{kNm})$ & $\theta_{y}(\%)$ & $\theta_{u}(\%)$ & $M_{y}(\mathrm{kNm})$ & $\theta_{y}(\%)$ & $\theta_{u}(\%)$ \\
\hline 1 & 55,0 & 0,998 & 1,525 & 55,3 & 0,902 & 6,252 & 76,4 & 0,902 & 8,072 \\
2 & 100,0 & 1,103 & 1,685 & 102,7 & 1,007 & 5,543 & 132,3 & 1,062 & 6,990 \\
3 & 138,6 & 1,277 & 1,746 & 146,8 & 1,090 & 4,914 & 182,7 & 1,190 & 6,052 \\
4 & 169,0 & 1,424 & 1,631 & 183,5 & 1,107 & 4,357 & 221,9 & 1,216 & 5,241 \\
5 & 94,5 & 0,970 & 1,536 & 96,5 & 1,000 & 5,507 & 131,5 & 1,280 & 7,671 \\
6 & 137,9 & 1,150 & 1,758 & 142,2 & 1,098 & 4,883 & 184,7 & 1,188 & 6,643 \\
7 & 173,9 & 1,313 & 1,657 & 185,1 & 1,181 & 4,329 & 233,2 & 1,315 & 5,752 \\
8 & 202,0 & 1,549 & 1,663 & 204,9 & 1,088 & 3,838 & 248,4 & 1,173 & 4,981 \\
9 & 145,3 & 1,098 & 1,677 & 148,2 & 1,209 & 4,994 & 200,5 & 1,449 & 7,378 \\
10 & 185,5 & 1,275 & 1,743 & 192,2 & 1,202 & 4,427 & 250,8 & 1,616 & 6,389 \\
11 & 217,2 & 1,422 & 1,630 & 225,4 & 1,233 & 3,925 & 285,6 & 1,671 & 5,532 \\
12 & 240,9 & 1,657 & 1,678 & 225,7 & 1,071 & 3,480 & 273,9 & 1,135 & 4,791 \\
13 & 55,0 & 0,998 & 1,525 & 55,3 & 0,902 & 6,252 & 76,4 & 0,902 & 8,072 \\
14 & 100,0 & 1,103 & 1,685 & 102,7 & 1,007 & 5,543 & 132,3 & 1,062 & 6,990 \\
15 & 138,6 & 1,277 & 1,746 & 146,8 & 1,090 & 4,914 & 182,7 & 1,190 & 6,052 \\
16 & 169,0 & 1,424 & 1,631 & 183,5 & 1,107 & 4,357 & 221,9 & 1,216 & 5,241 \\
17 & 54,8 & 0,934 & 1,477 & 55,2 & 0,899 & 7,131 & 75,1 & 0,897 & 8,533 \\
18 & 99,6 & 1,089 & 1,664 & 102,7 & 0,999 & 6,322 & 128,1 & 1,050 & 7,389 \\
19 & 139,3 & 1,287 & 1,851 & 147,1 & 1,079 & 5,605 & 176,5 & 1,173 & 6,398 \\
20 & 172,1 & 1,395 & 1,681 & 189,0 & 1,149 & 4,969 & 221,2 & 1,280 & 5,540 \\
21 & 54,6 & 0,936 & 1,481 & 55,1 & 0,896 & 7,900 & 73,7 & 0,892 & 8,913 \\
22 & 99,0 & 1,076 & 1,644 & 102,6 & 0,989 & 7,004 & 123,2 & 1,036 & 7,718 \\
23 & 139,1 & 1,232 & 1,823 & 147,3 & 1,066 & 6,209 & 169,4 & 1,154 & 6,683 \\
24 & 174,2 & 1,366 & 1,755 & 190,0 & 1,135 & 5,505 & 212,6 & 1,259 & 5,787 \\
\hline
\end{tabular}

\subsubsection{Diferencias en el momento de plastificación $M_{y}$}

Las Figuras 5.5 a 5.8 muestran las diferencias obtenidas para el momento de plastificación $M_{y}$ de la sección al aplicar diferentes métodos. De ellas se deduce que los valores obtenidos con la expresión ajustada a la selección de ensayos son, en todos los casos, superiores a los obtenidos con los otros dos métodos, debido a la influencia del coeficiente de ajuste $C_{3}=1,5$ que afecta a la cuantía de armadura de tracción $\rho$ en la expresión ajustada con la selección de ensayos (ecuación 4.74).

De la observación de la Figura 5.5 se concluye que al aumentar el esfuerzo axil y la cuantía de armadura de tracción se reducen las diferencias entre los resultados obtenidos con la expresión ajustada y los métodos clásicos. Aunque el momento de plastificación obtenido con la expresión ajustada es superior al proporcionado por los métodos clásicos, cuanto mayor es el esfuerzo axil y la cuantía de armadura de tracción, mayor es la curvatura de plastificación obtenida con el prontuario respecto a la obtenida con la expresión de Biskinis y Fardis (2010a), reduciéndose las diferencias obtenidas en el momento de plastificación. 
Tabla 5.3 Resultados obtenidos para el momento y giro de plastificación y giro último de la sección 350×350 mm según diferentes métodos.

\begin{tabular}{cccccccccc}
\hline \multirow{2}{*}{ CASO } & \multicolumn{3}{c}{ Métodos clásicos } & \multicolumn{3}{c}{ Biskinis y Fardis (2010a, 2010b ) } & \multicolumn{3}{c}{ Expresiones ajustadas } \\
& $M_{y}(\mathrm{kNm})$ & $\theta_{y}(\%)$ & $\theta_{u}(\%)$ & $M_{y}(\mathrm{kNm})$ & $\theta_{y}(\%)$ & $\theta_{u}(\%)$ & $M_{y}(\mathrm{kNm})$ & $\theta_{y}(\%)$ & $\theta_{u}(\%)$ \\
\hline 1 & 61,8 & 1,115 & 1,709 & 62,5 & 1,044 & 6,533 & 86,0 & 1,114 & 8,648 \\
2 & 88,1 & 1,271 & 1,884 & 90,1 & 1,122 & 6,039 & 118,0 & 1,234 & 7,872 \\
3 & 100,3 & 1,312 & 1,946 & 103,4 & 1,157 & 5,806 & 133,2 & 1,287 & 7,510 \\
4 & 111,8 & 1,355 & 2,008 & 116,4 & 1,189 & 5,582 & 148,0 & 1,336 & 7,165 \\
5 & 162,3 & 1,347 & 1,998 & 166,9 & 1,417 & 5,212 & 223,7 & 1,759 & 7,895 \\
6 & 185 & 1,490 & 1,949 & 192,1 & 1,382 & 4,818 & 252,0 & 1,877 & 7,186 \\
7 & 194,7 & 1,530 & 1,896 & 204,5 & 1,414 & 4,632 & 265,7 & 1,933 & 6,856 \\
8 & 204,6 & 1,613 & 1,891 & 216,4 & 1,444 & 4,453 & 278,7 & 1,985 & 6,541 \\
9 & 241,6 & 1,569 & 1,887 & 255,1 & 1,631 & 4,725 & 339,1 & 2,071 & 7,591 \\
10 & 260,9 & 1,752 & 1,913 & 254,6 & 1,562 & 4,367 & 332,6 & 1,963 & 6,910 \\
11 & 269 & 1,840 & 1,937 & 251,1 & 1,509 & 4,199 & 324,9 & 1,880 & 6,593 \\
12 & 277,2 & 2,001 & 2,024 & 247,8 & 1,358 & 4,037 & 317,5 & 1,800 & 6,290 \\
13 & 61,8 & 1,115 & 1,709 & 62,5 & 1,044 & 6,533 & 86,0 & 1,114 & 8,648 \\
14 & 88,1 & 1,271 & 1,884 & 90,1 & 1,122 & 6,039 & 118,0 & 1,234 & 7,872 \\
15 & 100,3 & 1,312 & 1,946 & 103,4 & 1,157 & 5,806 & 133,2 & 1,287 & 7,510 \\
16 & 11,8 & 1,355 & 2,008 & 116,4 & 1,189 & 5,582 & 148,0 & 1,336 & 7,165 \\
17 & 61,5 & 1,122 & 1,720 & 62,2 & 1,035 & 8,249 & 82,8 & 1,100 & 9,542 \\
18 & 86,6 & 1,170 & 1,793 & 89,6 & 1,103 & 7,626 & 110,3 & 1,204 & 8,686 \\
19 & 99 & 1,279 & 1,896 & 102,9 & 1,133 & 7,332 & 123,7 & 1,251 & 8,287 \\
20 & 110,6 & 1,306 & 1,936 & 116,0 & 1,162 & 7,049 & 136,8 & 1,295 & 7,907 \\
21 & 61,4 & 1,126 & 1,726 & 62,0 & 1,029 & 9,140 & 80,9 & 1,091 & 9,968 \\
22 & 85,7 & 1,162 & 1,781 & 89,1 & 1,090 & 8,449 & 105,2 & 1,184 & 9,073 \\
23 & 97 & 1,181 & 1,810 & 102,5 & 1,117 & 8,123 & 117,2 & 1,226 & 8,656 \\
24 & 109,2 & 1,286 & 1,906 & 115,6 & 1,144 & 7,810 & 129,1 & 1,267 & 8,259 \\
\hline
\end{tabular}

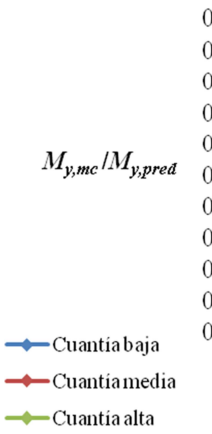

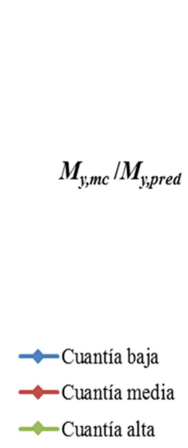

(a)

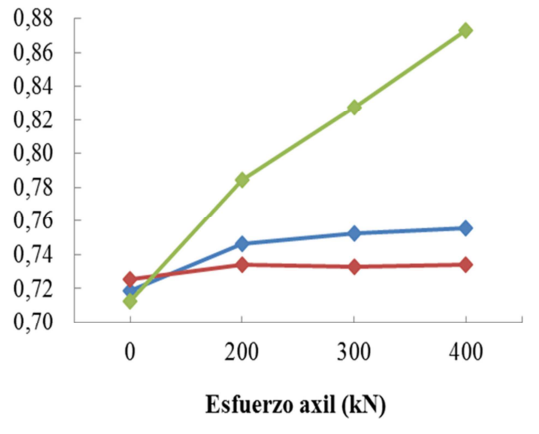

(b)

Figura 5.5 Ratio entre el momento de plastificación obtenido con métodos clásicos $M_{y, m c}$ y con la expresión ajustada a la selección de ensayos $M_{y, p r e d}$ en función del esfuerzo axil y la cuantía de armadura de tracción. (a) Sección 300×400 mm. (b) Sección 350×350 mm.

Al estudiar la influencia de la armadura de compresión, la Figura 5.6 muestra que a mayor esfuerzo axil y cuantía de armadura de compresión, menores son las diferencias 
obtenidas para el momento de plastificación. Además, cuantías elevadas de armadura de compresión inducen diferencias mayores en el momento de plastificación que cuantías elevadas de armadura de tracción.

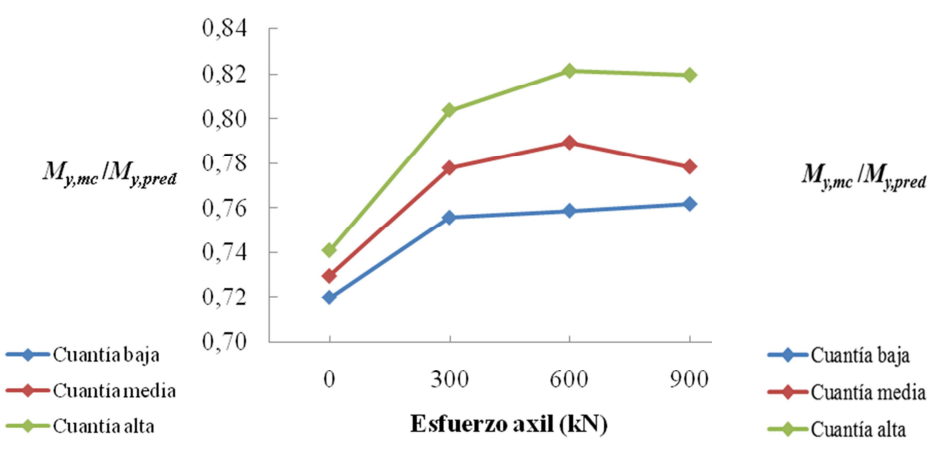

(a)

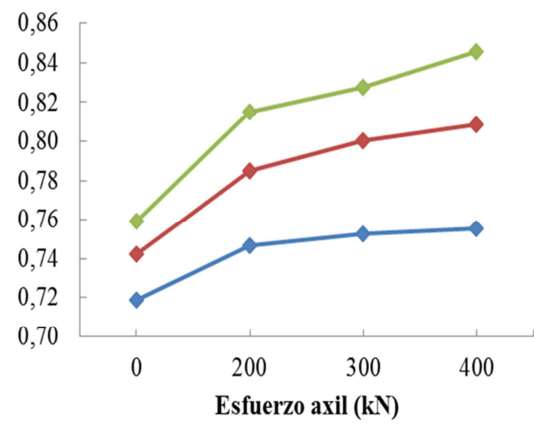

(b)

Figura 5.6 Ratio entre el momento de plastificación obtenido con métodos clásicos $M_{y, m c}$ y con la expresión ajustada a la selección de ensayos $M_{y, p r e d}$ en función del esfuerzo axil y la cuantía de armadura de compresión. (a) Sección 300×400mm. (b) Sección 350×350mm.

La Figura 5.7 muestra la comparación del momento de plastificación obtenido con la expresión ajustada y con la ecuación propuesta por Biskinis y Fardis (2010a). Se deduce que el aumento del esfuerzo axil reduce las diferencias entre ambos métodos. La cuantía de armadura de tracción no influye significativamente en las diferencias obtenidas, mientras que, de acuerdo con la Figura 5.8, aumentos de la cuantía de armadura de compresión disminuyen las diferencias.

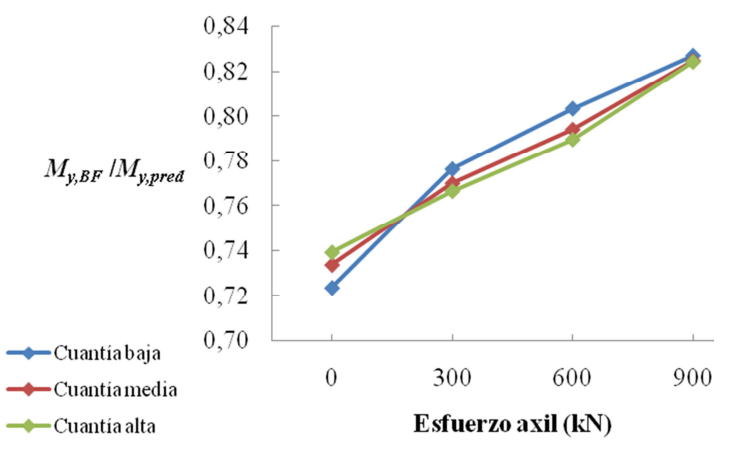

(a)

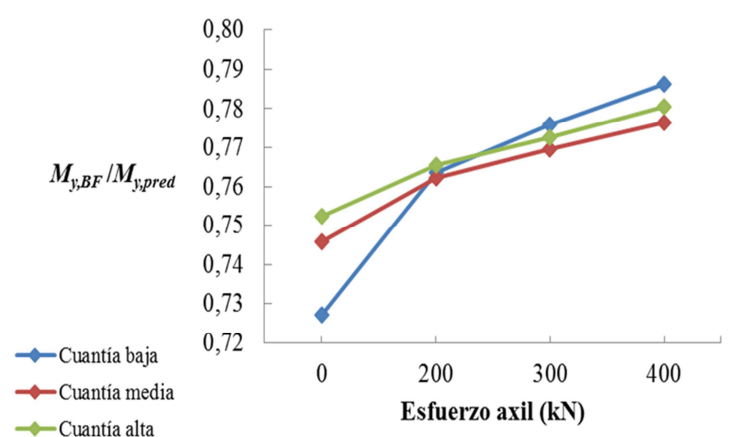

(b)

Figura 5.7 Ratio entre el momento de plastificación obtenido con la expresión de Biskinis y Fardis (2010a) $M_{y, B F}$ y con la expresión ajustada a la selección de ensayos $M_{y, p r e d}$ en función del esfuerzo axil y la cuantía de armadura de tracción. (a) Sección 300×400 mm. (b) Sección $350 \times 350 \mathrm{~mm}$. 


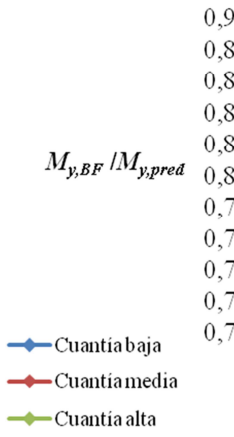

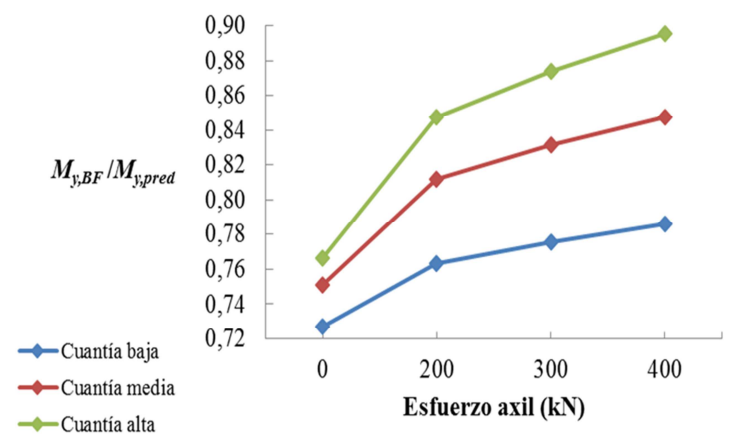

(b)

Figura 5.8 Ratio entre el momento de plastificación obtenido con la expresión de Biskinis y Fardis (2010a) $M_{y, B F}$ y con la expresión ajustada a la selección de ensayos $M_{y, p r e d}$ en función del esfuerzo axil y la cuantía de armadura de compresión. (a) Sección 300×400mm. (b) Sección $350 \times 350 \mathrm{~mm}$.

\subsubsection{Diferencias en el giro de plastificación $\theta_{y}$}

Las diferencias obtenidas al aplicar la expresión del giro de plastificación $\theta_{y}$ ajustada a la selección de ensayos (ecuación 4.75) y los métodos clásicos se muestran en las Figuras 5.9 y 5.10. De la Figura 5.9 se deduce que, para cuantía de armadura de tracción media-elevada y axil reducido-medio, el giro de plastificación obtenido con métodos clásicos es inferior al obtenido con la expresión ajustada, con diferencias comprendidas entre el $2 \%$ para el caso 11 de la sección $350 \times 350 \mathrm{~mm}$ y el $32 \%$ para el caso 9 de la sección $300 \times 400 \mathrm{~mm}$. Sin embargo, para cuantías reducidas de armadura de tracción, el giro obtenido con métodos clásicos resulta superior al obtenido con la expresión ajustada. Además, valores elevados de esfuerzo axil implican diferencias importantes entre ambos métodos, obteniéndose valores hasta el $46 \%$ superiores con métodos clásicos para la sección $300 \times 400 \mathrm{~mm}$ en el caso 12. Esto se debe a que la expresión ajustada supone la plastificación del hormigón, obteniéndose una curvatura de plastificación muy inferior a la obtenida con el prontuario. Respecto a la influencia de la armadura de compresión, los valores obtenidos con métodos clásicos son, en general, superiores a los obtenidos con la expresión ajustada, alcanzándose una diferencia máxima del $17 \%$ para el caso 16 de la sección 300×400 mm.

De acuerdo con las Figuras 5.11 y 5.12, la expresión de Biskinis y Fardis (2010a) proporciona, salvo algunos casos, valores inferiores a los obtenidos con la expresión ajustada. Se observa además que las diferencias aumentan al disminuir la cuantía de armadura de compresión y al incrementarse el esfuerzo axil. Por otro lado, la influencia de la armadura de tracción no sigue un patrón claro, obteniéndose diferencias máximas del $36 \%$ para el caso 11 de la sección $300 \times 400$ mm. De la observación de la Figura 5.12 se concluye que la cuantía de armadura de compresión no induce diferencias significativas en los resultados proporcionados por ambos métodos. 


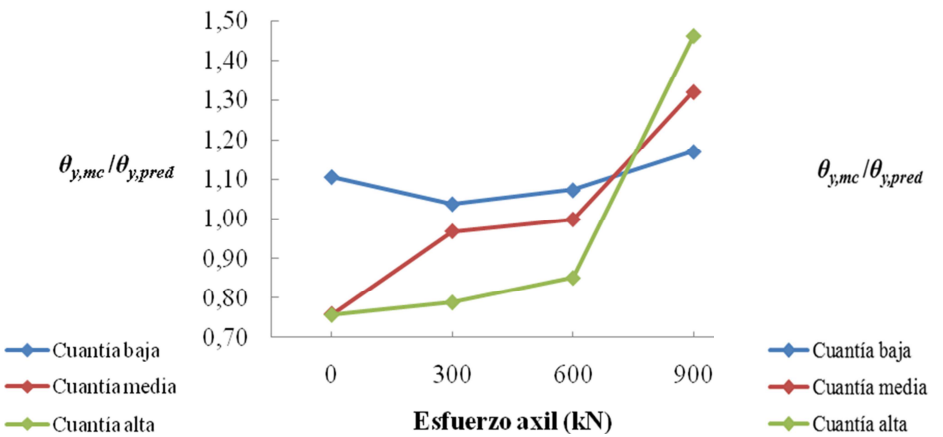

(a)

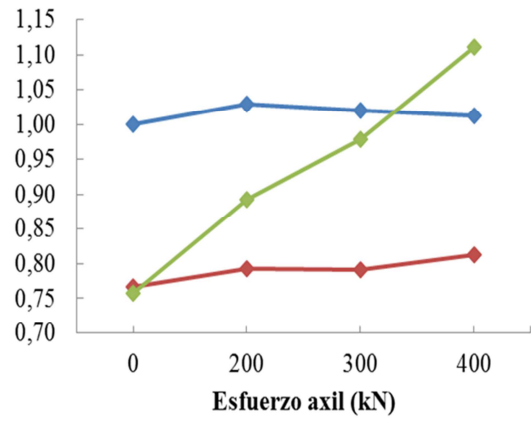

(b)

Figura 5.9 Ratio entre el giro de plastificación obtenido con métodos clásicos $\theta_{y, m c}$ y con la expresión ajustada a la selección de ensayos $\theta_{y, p r e d}$ en función del esfuerzo axil y la cuantía de armadura de tracción. (a) Sección 300×400mm. (b) Sección 350×350 mm.

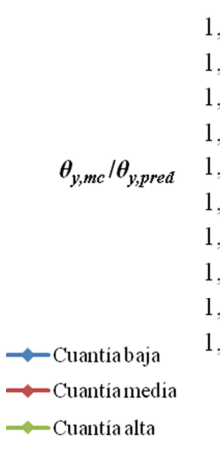

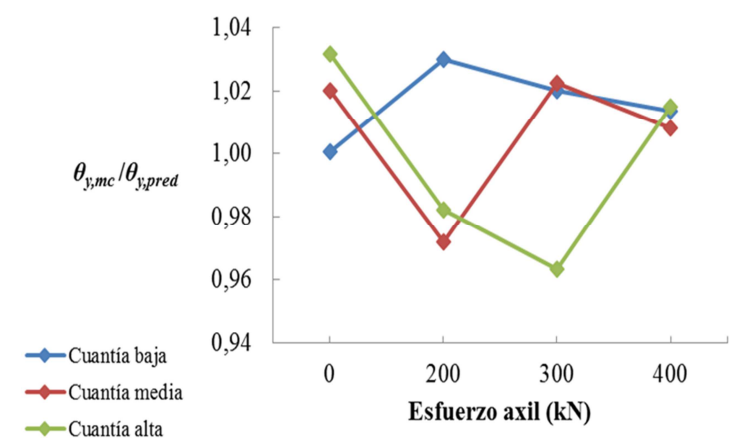

(a)

(b)

Figura 5.10 Ratio entre el giro de plastificación obtenido con métodos clásicos $\theta_{y, m c}$ y con la expresión ajustada a la selección de ensayos $\theta_{y, \text { pred }}$ en función del esfuerzo axil y la cuantía de armadura de compresión. (a) Sección 300×400mm. (b) Sección 350×350mm.

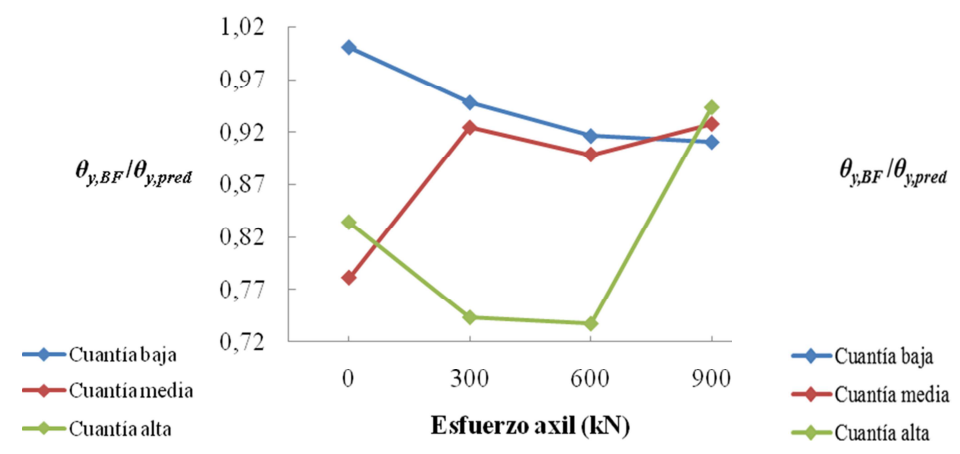

(a)

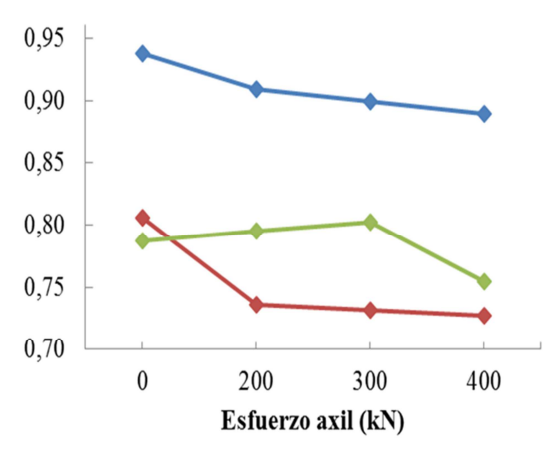

(b)

Figura 5.11 Ratio entre el giro de plastificación obtenido con la expresión de Biskinis y Fardis (2010a) $\theta_{y, B F}$ y con la expresión ajustada a la selección de ensayos $\theta_{y, p r e d}$ en función del esfuerzo axil y la cuantía de armadura de tracción. (a) Sección 300×400 mm. (b) Sección 350×350 mm. 


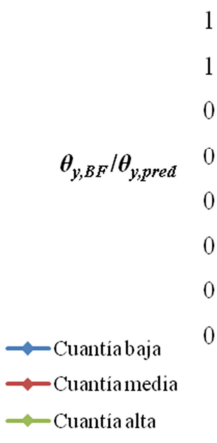

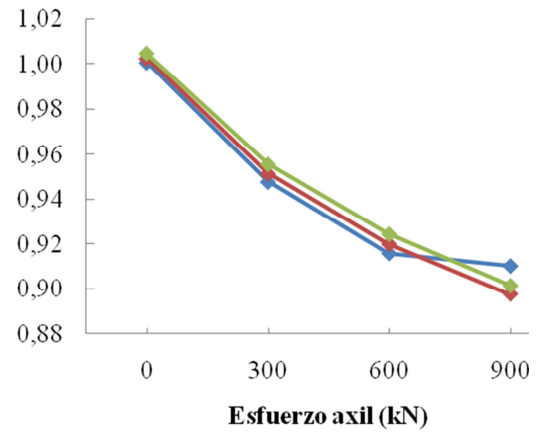

(a)

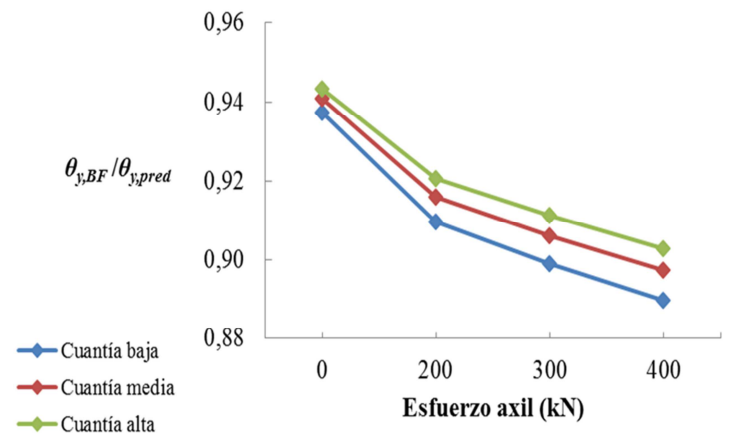

(b)

Figura 5.12 Ratio entre el giro de plastificación obtenido con la expresión de Biskinis y Fardis (2010a) $\theta_{y, B F}$ y con la expresión ajustada a la selección de ensayos $\theta_{y, p r e d}$ en función del esfuerzo axil y la cuantía de armadura de compresión. (a) Sección 300×400 mm. (b) Sección 350×350 $\mathrm{mm}$.

\subsubsection{Diferencias en el giro último $\theta_{u}$}

Observando las Figuras 5.13 a 5.16, se concluye que el giro último obtenido con la expresión ajustada resulta superior al obtenido con los otros dos métodos. Concretamente, los métodos clásicos ofrecen resultados muy inferiores, ya que no considera, entre otros aspectos, el confinamiento del hormigón proporcionado por la armadura transversal. La Figura 5.13 muestra que las diferencias disminuyen a medida que aumenta la armadura de tracción y el esfuerzo axil. Sin embargo, en la Figura 5.14 se aprecia que al aumentar la cuantía de armadura de compresión se producen unas mayores diferencias.

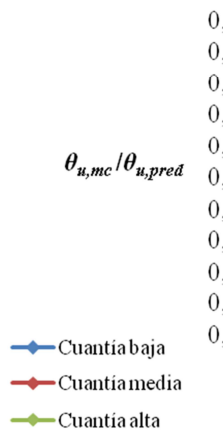

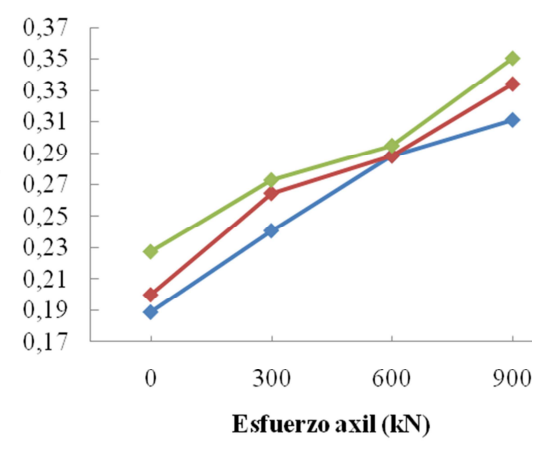

(a)

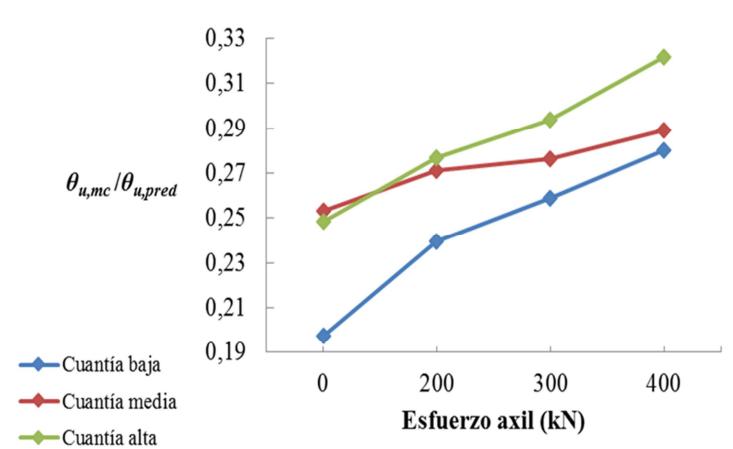

(b)

Figura 5.13 Ratio entre el giro último obtenido con métodos clásicos $\theta_{u, m c}$ y con la expresión ajustada a la selección de ensayos $\theta_{u, p r e d}$ en función del esfuerzo axil y la cuantía de armadura de tracción. (a) Sección 300×400 mm. (b) Sección 350×350 mm. 


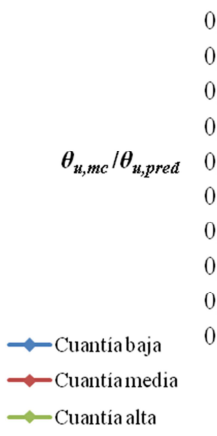

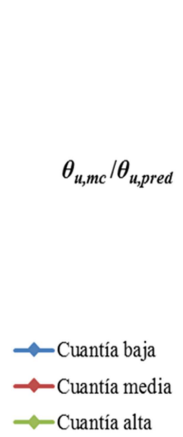

(a)

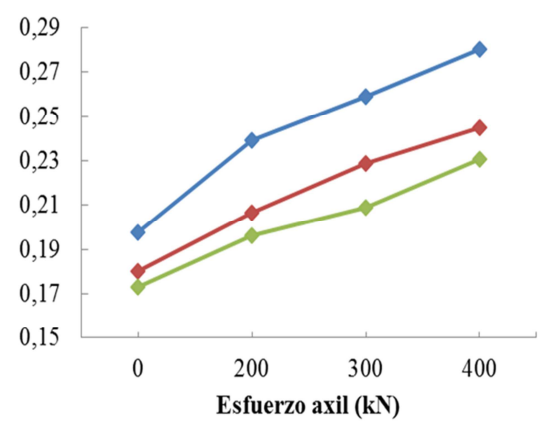

(b)

Figura 5.14 Ratio entre el giro último obtenido con métodos clásicos $\theta_{u, m c}$ y con la expresión ajustada a la selección de ensayos $\theta_{u, p r e d}$ en función del esfuerzo axil y la cuantía de armadura de compresión. (a) Sección 300×400 mm. (b) Sección 350×350 mm.

Tal y como muestra la Figura 5.15, las diferencias respecto a lo obtenido con la expresión propuesta por Biskinis y Fardis (2010b) aumentan a medida que se incrementa la armadura de tracción y disminuye el esfuerzo axil, mientras que aumentos de la armadura de compresión y de esfuerzo axil reducen las diferencias (Figura 5.16). La diferencia máxima es del $60 \%$, correspondiente al caso 9 de la sección $350 \times 350$ $\mathrm{mm}$.

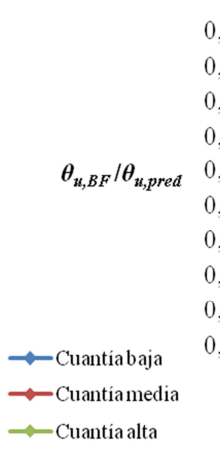

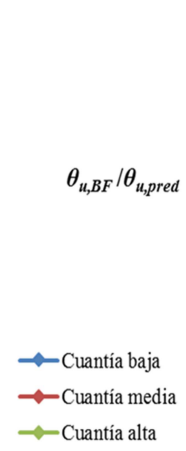

(a)

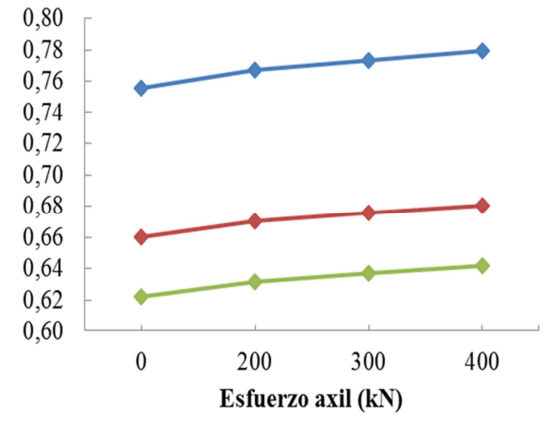

(b)

Figura 5.15 Ratio entre el giro último obtenido con la expresión de Biskinis y Fardis (2010b) $\theta_{u, B F}$ y con la expresión ajustada a la selección de ensayos $\theta_{u, p r e d}$ en función del esfuerzo axil y la cuantía de armadura de tracción. (a) Sección 300×400mm. (b) Sección 350×350mm.

\subsubsection{Resumen de resultados}

Tras analizar las diferencias en los resultados obtenidos aplicando diferentes métodos, se establecen, a modo de resumen, una serie de conclusiones. El momento de plastificación $M_{y}$ obtenido con la expresión ajustada a la selección de ensayos resulta superior al obtenido con métodos clásicos y la expresión de Biskinis y Fardis (2010a) en todos los casos analizados. Las diferencias están fuertemente influenciadas por el valor de la curvatura de plastificación $\phi_{y}$ y por el efecto del coeficiente de ajuste $C_{3}=1,5$ que 
afecta a la cuantía de armadura de tracción $\rho$ en la expresión ajustada a la selección de ensayos (ecuación 4.74). Las diferencias respecto a los métodos clásicos oscilan entre el $13 \%$ para el caso 12 de la sección $300 \times 400 \mathrm{~mm}$ y el $40 \%$ para el caso 9 de la sección $350 \times 350 \mathrm{~mm}$. Respecto a la expresión de Biskinis y Fardis (2010a), las diferencias están comprendidas entre el $11 \%$ para el caso 24 de la sección $350 \times 350 \mathrm{~mm}$ y el $38 \%$, correspondiente al caso 1 de la sección 300×400 mm.
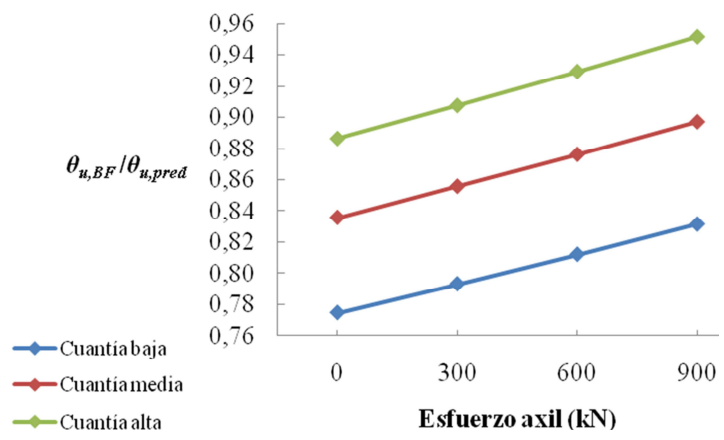

(a)
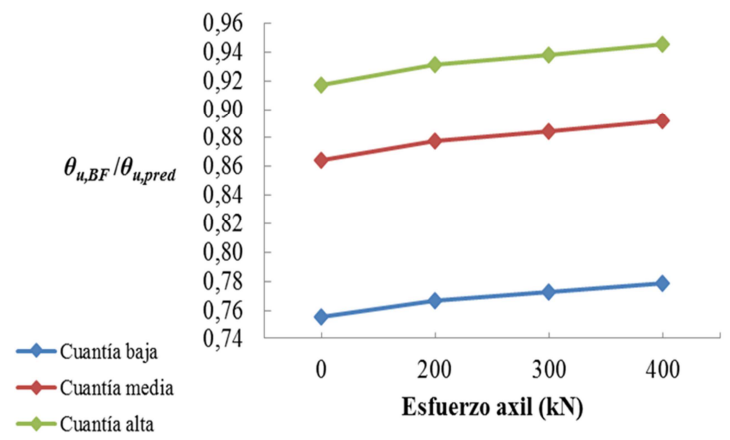

(b)

Figura 5.16 Ratio entre el giro último obtenido con la expresión de Biskinis y Fardis (2010b) $\theta_{u, B F}$ y con la expresión ajustada a la selección de ensayos $\theta_{u, p r e d}$ en función del esfuerzo axil y la cuantía de armadura de compresión. (a) Sección 300×400 mm. (b) Sección 350×350 mm.

En cuanto al giro de plastificación $\theta_{y}$, las diferencias obtenidas dependen del valor de la curvatura de plastificación $\phi_{y}$. Según el caso, el giro de plastificación obtenido con la expresión ajustada puede ser superior o inferior al obtenido con métodos clásicos, presentando diferencias entre el $1 \%$ para el caso 1 de la sección $350 \times 350 \mathrm{~mm}$ y el 46 $\%$, correspondiente al caso 12 de la sección $300 \times 400$ mm. Los valores obtenidos con la expresión de Biskinis y Fardis (2010a) resultan, según el caso, inferiores o iguales a los obtenidos con la expresión ajustada, obteniéndose una diferencia máxima del $36 \%$ para el caso 11 de la sección $300 \times 400 \mathrm{~mm}$.

Respecto al giro último $\theta_{u}$, la expresión ajustada obtiene valores entre 2,86 y 6 veces superiores a los obtenidos con métodos clásicos, correspondientes a los casos 12 y 21 de la sección $300 \times 400$ mm, respectivamente. Los valores obtenidos con la expresión ajustada son superiores a los ofrecidos por la expresión de Biskinis y Fardis (2010b), con diferencias comprendidas entre el $5 \%$ para el caso 24 de la sección $300 \times 400 \mathrm{~mm}$ y el $60 \%$ para el caso 9 de la sección $350 \times 350 \mathrm{~mm}$.

A partir de los resultados anteriormente expuestos, se puede concluir que las expresiones calibradas con la selección de ensayos proporcionan una capacidad resistente y de deformación de la sección superiores a las ofrecidas por otros métodos, ya que, salvo algunos casos analizados para el giro de plastificación $\theta_{y}$, las expresiones calibradas obtienen valores superiores del momento de plastificación $M_{y}$, giro de plastificación $\theta_{y}$ y giro último $\theta_{u}$. 
La mayor homogeneidad de la selección de ensayos respecto a la base de datos con la que se calibraron las expresiones de Biskinis y Fardis (2010a, 2010b) propicia que las expresiones calibradas con dicha selección ofrezcan mayor precisión en los resultados y mejor aproximación al comportamiento de elementos estructurales que se emplean habitualmente en la práctica constructiva.

Por otro lado, los resultados obtenidos ponen de manifiesto que las hipótesis adoptadas en los métodos clásicos suelen quedar muy del lado de la seguridad, infraestimando estos métodos la capacidad resistente y de deformación de un elemento estructural y resultando en ocasiones excesivamente conservadores. 



\section{Ejemplos de aplicación a análisis sísmico de estructuras}

\subsection{INTRODUCCIÓN}

Para modelizar convenientemente el comportamiento no lineal de las estructuras de hormigón armado resulta fundamental una adecuada caracterización de las rótulas plásticas o plastic hinges que pueden generarse en ciertas zonas de la estructura. A su vez, el comportamiento seccional de un elemento estructural en los estados de plastificación y último sirve de base para la determinación de las propiedades de las rótulas plásticas, por lo que la correcta utilización de conceptos como la curvatura, el momento y el giro experimentados por la sección en dichos estados resulta esencial para predecir de forma fidedigna la evolución de las rótulas plásticas a lo largo del análisis.

El objetivo principal de este capítulo es estudiar la influencia del comportamiento seccional de un elemento en las características de las rótulas plásticas que en él se desarrollan, evaluándose además ciertos aspectos que caracterizan el comportamiento global de la estructura en función de las propiedades de dichas rótulas. Para ello se ha efectuado una serie de análisis pushover de pórticos de hormigón armado, implementándose un tipo de rótula plástica para cada análisis realizado y comparándose posteriormente los resultados obtenidos. Los análisis se han realizado con el software ETABS $®$ (ETABS, 2013). Las propiedades de la rótulas plásticas se han obtenido empleando diferentes expresiones de comportamiento seccional, habiéndose considerado

a) rótulas plásticas que adopta por defecto el software ETABS $®$,

b) rótulas plásticas obtenidas a partir de las expresiones de Biskinis y Fardis (2010a, 2010b), y

c) rótulas plásticas modelizadas con las expresiones ajustadas a la selección de ensayos, expuestas en el capítulo 4 de esta tesis.

Los análisis pushover de los pórticos se han realizado siguiendo las prescripciones impuestas por el EC-8. 


\subsection{ANÁLISIS SÍSMICO}

\subsubsection{Métodos de análisis sísmico según normativa}

\subsubsection{Normativa de referencia}

El análisis sísmico de estructuras constituye un caso particular de los análisis no lineal y dinámico, considerados éstos en el sentido amplio de su definición. A pesar de que la acción sísmica induce en la estructura efectos de no linealidad geométrica y mecánica, la normativa de diseño considera métodos tanto de tipo lineal como no lineal para abordar este tipo de análisis.

Las prescripciones relativas a los tipos de análisis sísmico dependen de la norma o código considerado. Así, la instrucción EHE-08, aunque contempla en su anexo sísmico la aplicación de métodos lineales -como el análisis modal espectral y el método estático equivalente- y métodos no lineales -como el análisis dinámico no lineal y el análisis estático no lineal o pushover-, no establece criterios específicos para realizar dichos análisis, remitiéndose a la consulta de la normativa sísmica aplicable y/o literatura técnica. Por otro lado, la norma NCSE-02 contempla la aplicación del análisis modal espectral como método de referencia para abordar el cálculo sísmico. Otras alternativas contempladas en esta norma son el análisis dinámico, empleando un modelo lineal o no lineal de la estructura, y un método simplificado aplicable a estructuras comunes que cumplen ciertos condicionantes específicos.

El código modelo CM-2010 propone la aplicación del análisis lineal para determinar las deformaciones inelásticas a flexión de los elementos de la estructura debidas a la acción sísmica. Adicionalmente, plantea la realización del análisis dinámico lineal y el análisis modal espectral para determinar los valores máximos de dichas deformaciones. No obstante, este código considera el análisis dinámico no lineal como el método de referencia para abordar el análisis símico.

En lo que respecta a la normativa europea, las prescripciones relativas al análisis sísmico se incluyen en el EC-8. Dichas prescripciones se describen de forma detallada en el apartado 6.2.1.2 de esta tesis, por ser el EC-8 el código escogido para realizar los análisis pushover.

La normativa americana establece las indicaciones para realizar análisis sísmicos de forma más precisa. Si bien el código ACI-318-08 no establece prescripciones acerca de los métodos a emplear para abordar este tipo de análisis, otras normas específicas para la rehabilitación y refuerzo sísmico de edificios, como la FEMA (2000) y la ATC40 (Applied Technology Council, 1996), proponen la aplicación de una extensa variedad de procedimientos. Así, la FEMA (2000) contempla la aplicación de análisis de tipo lineal cuando las estructuras presentan una distribución regular de sus elementos. De entre los métodos lineales, se considera el análisis en el que se aplican fuerzas laterales, el análisis modal espectral y el análisis dinámico. Tanto en el análisis 
en el que se aplican las fuerzas laterales como en al análisis modal espectral, se aplica una distribución de fuerzas laterales que produce un desplazamiento igual al máximo esperado por el terremoto de diseño. En cuanto a los métodos no lineales, la FEMA (2000) contempla la aplicación del análisis estático o pushover y el análisis dinámico. Esta norma establece además unos criterios claros de aceptación, en función de que el análisis realizado sea de tipo lineal o no lineal. La norma ATC-40 se centra en el desarrollo del análisis estático no lineal o pushover, estableciendo de forma detallada diferentes procedimientos para la obtención de la curva de capacidad de la estructura y de la curva de demanda impuesta por la acción sísmica, así como del punto de desempeño o performance point, obtenido como la intersección de ambas curvas. Como métodos de análisis sísmico adicionales, plantea la aplicación del análisis de tipo lineal estático, denominado método de la fuerza lateral, y de otros como el análisis modal espectral, el análisis dinámico y los procedimientos que emplean el ratio demandacapacidad. Como métodos de análisis no lineal alternativos al pushover, esta norma propone la aplicación del método secante y del análisis dinámico.

\subsubsection{Análisis según el EC-8}

Al igual que el resto de normas y códigos comentados, el EC-8 considera métodos de tipo lineal y no lineal para abordar el análisis sísmico de estructuras. La elección del tipo de análisis a realizar depende de la precisión a alcanzar en los resultados.

El empleo de los métodos de tipo lineal que contempla el EC-8 para el análisis sísmico depende de la influencia de los diferentes modos de vibración en la respuesta de la estructura. Los métodos considerados son:

a) método de análisis de la fuerza lateral o lateral force method, aplicado a estructuras cuya respuesta no está afectada de forma significativa por los modos de vibración superiores al modo fundamental, y

b) análisis modal mediante espectros de respuesta o modal response spectrum analysis, empleado en estructuras cuya respuesta está influenciada por los modos de vibración superiores al modo fundamental.

Un caso particular de análisis modal mediante espectros de respuesta es el que considera un espectro de respuesta reducido respecto al elástico, denominado espectro de cálculo, introduciendo el coeficiente de comportamiento $q$. Este análisis, aun siendo de tipo lineal, permite reducir la acción sísmica considerada debido a la capacidad de la estructura para resistir en el rango no lineal.

A pesar de la disponibilidad de métodos de tipo lineal para abordar el análisis y diseño sísmico de estructuras, ciertos estudios (Bertero et al., 1991 y Krawinkler, 1995) pusieron de manifiesto la necesidad de establecer cambios en la metodología dispuesta en las principales normas y códigos sísmicos actuales, basada en la hipótesis de que las estructuras se comportan de forma elástica y lineal durante el transcurso de la acción sísmica. Entre otros aspectos, quedó patente la importancia de estimar el daño que la 
acción sísmica induce en las estructuras, aspecto que no puede ser considerado mediante análisis de tipo lineal (Mestrovic, Cizmar y Pende, 2008).

Con objeto de solventar estos inconvenientes, surge como alternativa el análisis sísmico de carácter no lineal. Este tipo de análisis no se ha realizado de forma frecuente, entre otros motivos, por limitaciones en la capacidad de cálculo. Sin embargo, los constantes avances experimentados desde el punto de vista computacional están propiciando que este tipo de análisis se aborde cada vez con mayor frecuencia, incluyéndose en algunas normas y códigos sísmicos ciertas prescripciones para su implementación. Así, el EC-8 contempla dos tipos de análisis sísmico no lineal:

a) análisis estático no lineal o pushover analysis, y

b) análisis dinámico no lineal o non-linear time history analysis.

El análisis dinámico no lineal presenta como principales inconvenientes su complejidad y la escasa regulación existente en la normativa de edificación acerca de su implementación, aspectos que limitan su aplicación en la práctica del ingeniero estructural.

Como alternativa al análisis dinámico no lineal y al análisis elástico y lineal que emplea el coeficiente de comportamiento $q$, el EC-8 propone la realización del análisis pushover. Este tipo de análisis constituye un método relativamente simple de evaluar el comportamiento no lineal de las estructuras frente a acciones de tipo sísmico, considerando la no linealidad geométrica y mecánica. Para considerar la no linealidad geométrica, resulta necesario introducir en el modelo los efectos P-delta existentes al alcanzar la estructura ciertos niveles de deformación. La no linealidad mecánica se considera introduciendo ciertas propiedades de los materiales, relativas a su comportamiento de plastificación y último, su posible carácter de endurecimiento por deformación y la degradación de su resistencia y rigidez. En lo relativo a la modelización de las cargas actuantes, se aplican cargas constantes de tipo gravitatorio y cargas laterales sobre las masas del modelo que se incrementan de forma monotónica, simulándose de esta forma las fuerzas de inercia inducidas por la acción sísmica. El método es capaz de describir la evolución de los mecanismos plásticos generados en la estructura y permite estimar los niveles de daño producidos por las fuerzas laterales.

El análisis pushover adopta como hipótesis más relevante que la respuesta de una estructura con múltiples grados de libertad se puede asemejar a la de un sistema de un grado de libertad o single degree of freedom system. Esta simplificación supone que la respuesta estructural está controlada por un único modo de vibración y que la forma de dicho modo permanece constante a lo largo del análisis, lo cual no se ajusta a la realidad de las estructuras sometidas a acciones sísmicas (Krawinkler, 1996). Sin embargo, Fajfar (2000b) puso de manifiesto que esta hipótesis proporciona una adecuada predicción de la respuesta máxima de estructuras de múltiples grados de libertad cuya respuesta está gobernada por el modo fundamental de vibración, ajustándose los 
resultados obtenidos a los proporcionados por ensayos experimentales y análisis dinámicos de tipo no lineal.

La principal utilidad del análisis pushover es que permite comparar la resistencia y capacidad de deformación de la estructura en el rango no lineal con la demanda impuesta por la acción sísmica considerada. De acuerdo con Krawinkler y Seneviratna (1998) y Nogueiro, Bento y Da Silva (2006), el análisis pushover proporciona la siguiente información adicional:

a) esfuerzos impuestos por la acción sísmica en elementos potencialmente frágiles, como esfuerzos axiles en pilares, momentos flectores en conexiones entre vigas y pilares y esfuerzos cortantes en vigas de hormigón armado y muros de mampostería sin armar,

b) estimación de la demanda de deformación en elementos que deben deformarse de forma inelástica para disipar parte de la energía inducida por la acción sísmica, pudiendo concretarse el diseño estructural mediante el control de las deformaciones,

c) posibilidad de evaluar la influencia del deterioro de la resistencia de ciertos elementos en el comportamiento global de la estructura,

d) obtención de la secuencia de plastificación y fallo de los elementos estructurales y de los niveles de comportamiento estructural o performance levels, pudiendo definirse diferentes niveles de diseño en función de las exigencias a cumplir,

e) identificación de las regiones críticas que presentan una alta demanda de deformación impuesta por la acción sísmica y que deben ser objeto de detalles estructurales específicos,

f) localización de discontinuidades de resistencia en planta o alzado que pueden inducir cambios en las características dinámicas de la estructura en el rango inelástico,

g) estimación de los desplazamientos entre plantas o drifts, aspecto que resulta de interés para controlar los niveles de daño inducidos en la estructura y evaluar los efectos P-delta, y

h) determinación de los desplazamientos laterales de las plantas de la estructura, aspecto que proporciona información acerca del riesgo de impacto con edificios colindantes.

A pesar de su utilidad, el análisis pushover convencional presenta como principales inconvenientes su incapacidad para considerar convenientemente la influencia de los diferentes modos de vibración que afectan a la respuesta de la estructura o la dificultad para modelizar la progresiva degradación de rigidez que experimenta el hormigón. De acuerdo con Krawinkler y Seneviratna (1998), presenta otros inconvenientes adicionales: 
a) es un método estático, por lo que no es capaz de predecir el comportamiento dinámico de la estructura con una adecuada precisión,

b) el patrón de cargas laterales introducido en la estructura repercute en los resultados obtenidos. Cada patrón tiende a favorecer un modo concreto de deformación, por lo que, de acuerdo con Krawinkler (1996), es necesario considerar más de una distribución de cargas laterales, y

c) la incorporación de efectos de torsión debido a la no uniformidad de la masa, la resistencia y la rigidez de la estructura influye en los resultados obtenidos.

\subsubsection{Análisis pushover: origen y evolución}

De acuerdo con Taghavipour, Majid y Liang (2013), el origen del análisis pushover se puede atribuir a los años 70, con los trabajos, entre otros, de Takeda, Sozen y Nielsen (1970), Freeman, Nicoletti y Tyrell (1975) y Saiidi y Sozen (1979, 1981). En estos estudios se desarrollaron curvas fuerza-desplazamiento para sistemas de hormigón armado de un grado de libertad, obteniéndose una primera aproximación a las curvas de capacidad proporcionadas por los análisis pushover realizados actualmente.

Aunque estos primeros trabajos comenzaron a sentar las bases de lo que el análisis pushover es hoy en día, López (2004) afirma que sólo a partir de finales de la década de los 80 comenzó a ganar importancia este tipo de análisis, empleándose como una aproximación adecuada para la evaluación del comportamiento sísmico de las estructuras. Así, el primer tipo de análisis pushover que se planteó es el conocido en la literatura como pushover clásico o convencional. En este análisis se introducen en la estructura ciertas distribuciones de carga lateral, adoptando como hipótesis principales que la estructura se deforma únicamente según el modo fundamental de vibración y que el patrón de cargas laterales permanece constante a lo largo del análisis. Siguiendo este planteamiento, Fajfar y Fischinger (1987, 1988) introdujeron una serie de mejoras, proponiendo el método N2. Este método ha ido evolucionando a lo largo de los años con las aportaciones, entre otros, de Fajfar y Gaspersic (1996) y Fajfar (2000a, 2000b), siendo el método propuesto por el EC-8 para la realización del análisis pushover. Otras aportaciones, como las planteadas por Lawson, Vance y Krawinkler (1994) y Krawinkler y Seneviratna (1998) se centraron en discutir acerca del posible campo de aplicación del pushover convencional y sus ventajas e inconvenientes respecto a lo ofrecido por los análisis elástico lineal y no lineal dinámico.

Aunque las primeras contribuciones relativas al análisis pushover fueron concebidas para su aplicación a estructuras planas o en 3D simétricas, Moghadam y Tso (1996) y Kilar y Fajfar (1997) plantearon por primera vez un método pushover para estructuras 3D asimétricas con objeto de considerar los efectos de torsión que aparecen en este tipo de estructuras. Otros trabajos posteriores, como los de Moghadam y Tso (1998, 2000), mejoraron este planteamiento al establecer diferentes procedimientos para obtener el desplazamiento objetivo o target displacement de la estructura. 
Con objeto de considerar la influencia de los modos de vibración superiores al modo fundamental en la respuesta de la estructura, surgen algunos métodos que se diferencian en la forma de determinar el vector de cargas laterales a aplicar en la estructura y en la determinación de la demanda impuesta por la acción sísmica. En esta línea, investigadores como Sasaki, Freeman y Paret (1998) y Chopra y Goel (2001, 2002) plantearon análisis pushover de tipo modal, aplicando una distribución de cargas laterales proporcional a la deformación de cada modo de vibración por separado y combinando las respuestas máximas obtenidas con cada modo de vibración mediante técnicas de combinación modal. Otras aportaciones en esta línea corresponden a Poursha, Khoshnoudian y Moghadam (2009), quienes desarrollaron el conocido como pushover consecutivo modal, en el que se desarrollan análisis pushover de tipo modal de forma consecutiva partiendo de estados de tensión y deformación correspondientes a etapas inmediatamente anteriores del análisis; Jan, Liu y Kao (2004), que plantearon el upper bound pushover analysis, obteniendo el patrón de cargas laterales al considerar únicamente la influencia del primer y segundo modo de vibración de la estructura; y Kim y Kurama (2008), quienes propusieron el mass proportional pushover, en el que la influencia de los modos de vibración superiores se considera mediante un único patrón constante de cargas laterales proporcional a las masas sísmicas de cada planta de la estructura.

Como alternativa a los métodos anteriormente descritos, otros autores (Bracci, Kunnath y Reinhorn, 1997; Gupta y Kunnath, 2000; Aydinoglu, 2003; López, 2004) comenzaron a desarrollar el pushover adaptativo o adaptive pushover. A diferencia de otros métodos, en este planteamiento la distribución de fuerzas laterales no es constante, sino que se va actualizando a lo largo del análisis en concordancia con las deformadas de los diferentes modos de vibración de la estructura y los factores de participación obtenidos tras realizar un análisis de autovalores en cada paso del proceso, pudiendo considerarse además la degradación de rigidez progresiva que experimenta la estructura. Concretamente, Antoniou y Pinho (2004a) estudiaron la modalidad de pushover adaptativo basado en fuerzas y Antoniou y Pinho (2004b) desarrollaron un enfoque basado en desplazamientos.

Aunque actualmente se tiende hacia el desarrollo de análisis pushover cada vez más sofisticados, el análisis pushover convencional se emplea con frecuencia, siendo el método considerado por las principales normas y códigos de diseño. Un ejemplo reciente de esta circunstancia lo constituye el trabajo de Taghavipour, Majid y Liang (2013), quienes aplicaron este tipo de análisis a estructuras de hormigón armado para estudiar la influencia del patrón de cargas laterales empleado. 


\subsubsection{Indicaciones del EC-8 acerca del análisis pushover}

Para considerar la influencia de la distribución vertical de cargas laterales en los resultados obtenidos en el análisis pushover, el EC-8 impone la aplicación de, al menos, los siguientes patrones de carga:

a) un patrón uniforme, constituido por fuerzas laterales proporcionales a las masas con independencia de su altura, lo que supone considerar una aceleración uniforme en la estructura. Taghavipour, Majid y Liang (2013) determinaron esta distribución de fuerzas como

$$
F_{i}=\frac{m_{i}}{\sum m_{i}}
$$

siendo

$F_{i} \quad$ fuerza lateral aplicada en la planta $i$-ésima,

$m_{i} \quad$ masa de la planta $i$-ésima, y

$\Sigma m_{i}$ suma de las masas de las plantas de la estructura.

b) un patrón modal, definido mediante la distribución de fuerzas laterales obtenidas al realizar un análisis elástico y lineal de la estructura.

La distribución modal de fuerzas laterales a aplicar en la estructura depende del método de análisis lineal que pueda aplicarse. Así, la aplicación del método de análisis de la fuerza lateral exige, de acuerdo con el EC-8, el cumplimiento de las siguientes condiciones:

a) el periodo fundamental $T_{1}$ de vibración de la estructura en las dos direcciones principales resulta inferior a los siguientes valores

$$
T_{1} \leq\left\{\begin{array}{c}
4 T_{c} \\
2,0 s
\end{array}\right\}
$$

donde $T_{c}$ es el límite superior del periodo para el tramo de aceleración espectral constante del espectro de respuesta elástica horizontal propuesto por el EC-8.

b) la estructura cumple los criterios de regularidad en alzado expuestos en el apartado 4.2.3.3. del EC-8.

El método de análisis de la fuerza lateral considera dos tipos de distribución vertical de fuerzas laterales:

a) una distribución obtenida al considerar los desplazamientos horizontales de cada planta de la estructura correspondientes al modo fundamental de vibración:

$$
F_{i}=F_{b} \frac{s_{i} m_{i}}{\Sigma s_{j} m_{j}}
$$


b) un patrón de cargas laterales obtenido al aproximar la deformada de la estructura en el modo fundamental de vibración a una distribución de desplazamientos horizontales que se incrementan linealmente con la altura:

$$
F_{i}=F_{b} \frac{z_{i} m_{i}}{\Sigma z_{j} m_{j}}
$$

siendo

$F_{b}$ esfuerzo cortante sísmico en la base,

$s_{i}, s_{j}$ desplazamientos de las masas $m_{i}$ y $m_{j}$ para el modo fundamental de vibración, y

$z_{i}, z_{j}$ alturas de las masas $m_{i}$ y $m_{j}$ respecto al nivel de aplicación de la acción sísmica.

La combinación de acciones a considerar para determinar la masa asociada a cada planta de la estructura viene dada por

$$
\begin{gathered}
\Sigma G_{k, j}+\Sigma \psi_{E, i} Q_{k, i} \\
\psi_{E, i}=\phi \psi_{2, i}
\end{gathered}
$$

donde

$G_{k, j} \quad$ valor característico de las acciones permanentes,

$Q_{k, i} \quad$ valor característico de las acciones variables,

$\phi \quad$ coeficiente cuyo valor viene dado por la Tabla 4.2 del EC-8,

$\psi_{E, i}$ coeficiente de combinación para una acción variable al determinar los efectos de la acción sísmica de cálculo, y

$\psi_{2, i}$ coeficiente de combinación para el valor cuasi-permanente de una acción variable.

El EC-8 establece ciertas indicaciones para obtener la curva de capacidad de la estructura al realizar el análisis pushover. Dicha curva representa la relación entre el esfuerzo cortante en la base $F_{b}$, obtenido como la suma de todos los esfuerzos cortantes actuantes en las secciones de empotramiento de los pilares, y el desplazamiento del nudo de control de la estructura $\delta$, supuesto en el centro de gravedad de la última planta.

Un aspecto importante al realizar el análisis pushover lo constituye la determinación del desplazamiento objetivo o target displacement impuesto en el nudo de control de la estructura, el cual representa la demanda sísmica debida al espectro de respuesta elástica horizontal. Para determinar el desplazamiento objetivo, el EC-8 propone la implementación del método N2 (Fajfar y Gaspersic, 1996). Este método presenta como principal característica la adopción conjunta de dos estrategias diferentes: el análisis pushover y la aproximación basada en el espectro de respuesta elástica horizontal. El método N2 parte de la curva de capacidad obtenida tras realizar el 
análisis pushover, evaluando posteriormente el desplazamiento objetivo mediante el empleo del espectro de respuesta elástica horizontal.

La utilización del espectro de respuesta elástica exige determinar la curva de capacidad del sistema de un grado de libertad equivalente a la estructura. Para ello, resulta necesario obtener la masa $m^{*}$ de dicho sistema y el factor de transformación $\Gamma$ que lo relaciona con la estructura real, aplicando las ecuaciones (6.7) a (6.9):

$$
\begin{gathered}
\bar{F}_{i}=m_{i} \phi_{i} \\
m^{*}=\Sigma \bar{F}_{i} \\
\Gamma=\frac{m^{*}}{\Sigma m_{i} \phi_{i}^{2}}
\end{gathered}
$$

donde

$\bar{F}_{i} \quad$ fuerza lateral normalizada actuante en la planta $i$-ésima, y

$\phi_{i} \quad$ desplazamiento de la planta $i$-ésima en el modo fundamental de vibración, normalizado respecto al experimentado por el nudo de control.

La curva de capacidad del sistema equivalente de un grado de libertad se obtiene al determinar la fuerza $F^{*}$ actuante en la base de dicho sistema y su desplazamiento $d^{*}$.

$$
\begin{aligned}
F^{*} & =\frac{F_{b}}{\Gamma} \\
d^{*} & =\frac{\delta}{\Gamma}
\end{aligned}
$$

Tras obtener la curva de capacidad para el sistema equivalente de un grado de libertad, resulta necesario idealizarla suponiendo un comportamiento elasto-plástico tal y como muestra la Figura 6.1.

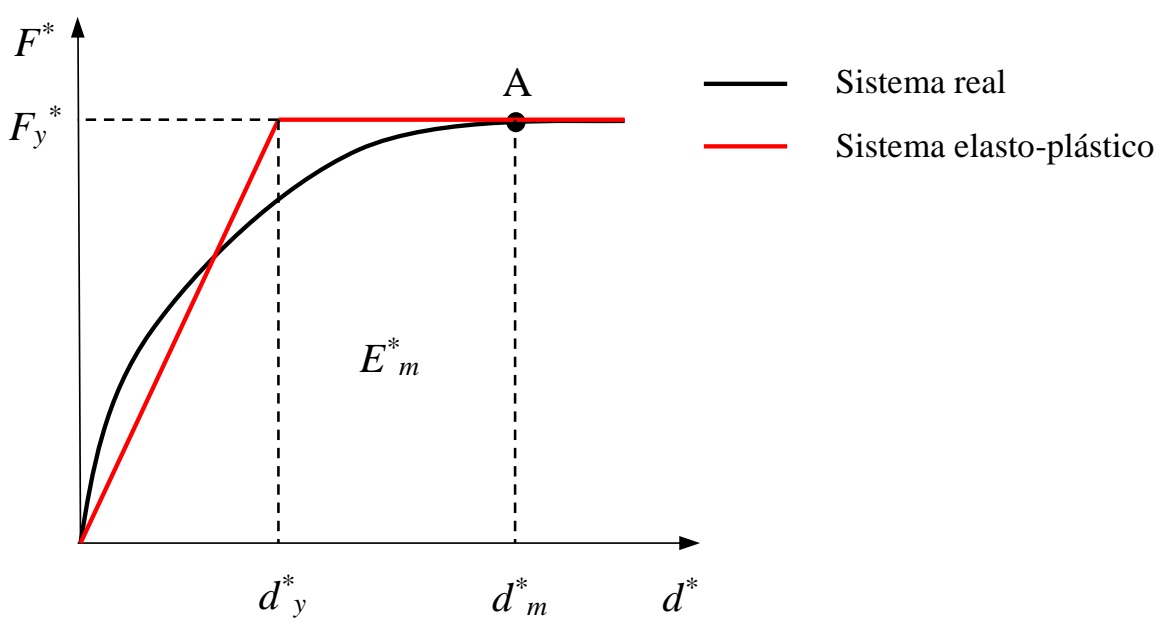

Figura 6.1 Curva de capacidad real y elasto-plástica para el sistema equivalente de un grado de libertad. 
El esfuerzo cortante $F_{y}{ }^{*}$ correspondiente al límite elástico es el actuante en la base cuando se origina el mecanismo plástico, representado por el punto A de la Figura 6.1. La rigidez inicial del sistema elasto-plástico se determina de modo que las áreas bajo las curvas de capacidad del sistema real y elasto-plástico sean iguales. El desplazamiento $d^{*}{ }_{y}$ correspondiente al límite elástico del sistema elasto-plástico viene dado por

$$
d_{y}^{*}=2\left(d_{m}^{*}-\frac{E_{m}^{*}}{F_{y}^{*}}\right)
$$

donde $E^{*}{ }_{m}$ y $d^{*}{ }_{m}$ representan, respectivamente, la energía de deformación y el desplazamiento del sistema real de un grado de libertad al generarse el mecanismo plástico. Para obtener el desplazamiento objetivo $d_{t}{ }^{*}$ del sistema equivalente de un grado de libertad con comportamiento elasto-plástico resulta necesario determinar previamente el período $T^{*}$ y el desplazamiento objetivo $d^{*}$ et de un sistema con comportamiento elástico ilimitado. Estos conceptos vienen dados por

$$
\begin{aligned}
T^{*} & =2 \pi \sqrt{\frac{m^{*} d_{y}^{*}}{F_{y}^{*}}} \\
d_{e t}^{*} & =S_{e}\left(T^{*}\right)\left[\frac{T^{*}}{2 \pi}\right]^{2}
\end{aligned}
$$

siendo $S_{e}\left(T^{*}\right)$ la ordenada del espectro elástico de respuesta horizontal correspondiente al período $T^{*}$.

La determinación del desplazamiento objetivo $d_{t}{ }^{*}$ depende del valor del período $T^{*}$. Así, pueden presentarse los siguientes casos:

a) $T^{*}<T_{C}$ (rango de período corto)

- Si $F_{y}{ }^{*} / m^{*} \geq S_{e}\left(T^{*}\right)$, la respuesta del sistema equivalente de un grado de libertad es elástica, por lo que

$$
d_{t}^{*}=d_{e t}^{*}
$$

- Si $F_{y}{ }^{*} / m^{*}<S_{e}\left(T^{*}\right)$, la respuesta es no lineal, deduciéndose que

$$
d_{t}^{*}=\frac{d_{e t}^{*}}{q_{u}}\left(1+\left(q_{u}-1\right) \frac{T_{C}}{T^{*}}\right) \geq d_{e t}^{*}
$$

siendo $q_{u}$ la relación entre las aceleraciones experimentadas por los sistemas con comportamiento elástico ilimitado $S_{e}\left(T^{*}\right)$ y con resistencia limitada $F_{y}{ }^{*} / m^{*}$

$$
q_{u}=\frac{S_{e}\left(T^{*}\right) m^{*}}{F_{y}^{*}}
$$

b) $T^{*} \geq T_{C}$ (rango de períodos medios y largos) 


$$
d_{t}^{*}=d_{e t}^{*}
$$

Finalmente, el desplazamiento objetivo $d_{t}$ de la estructura real viene dado por

$$
d_{t}=\Gamma d_{t}^{*}
$$

La Figura 6.2 muestra una representación gráfica de los conceptos descritos, donde los espectros de respuesta se muestran en formato aceleración-desplazamiento. El periodo $T^{*}$ se representa mediante una línea radial discontinua que parte del origen de coordenadas.

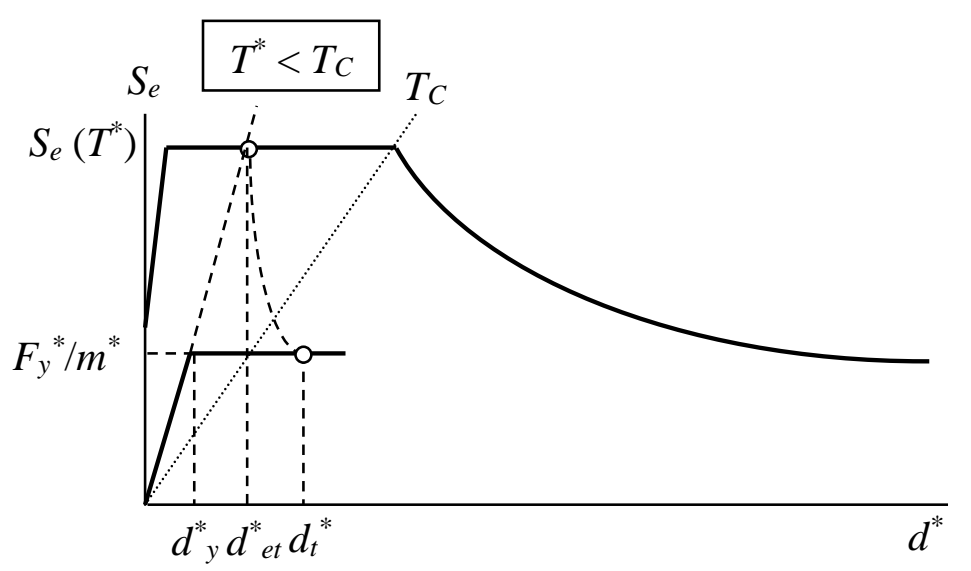

(a)

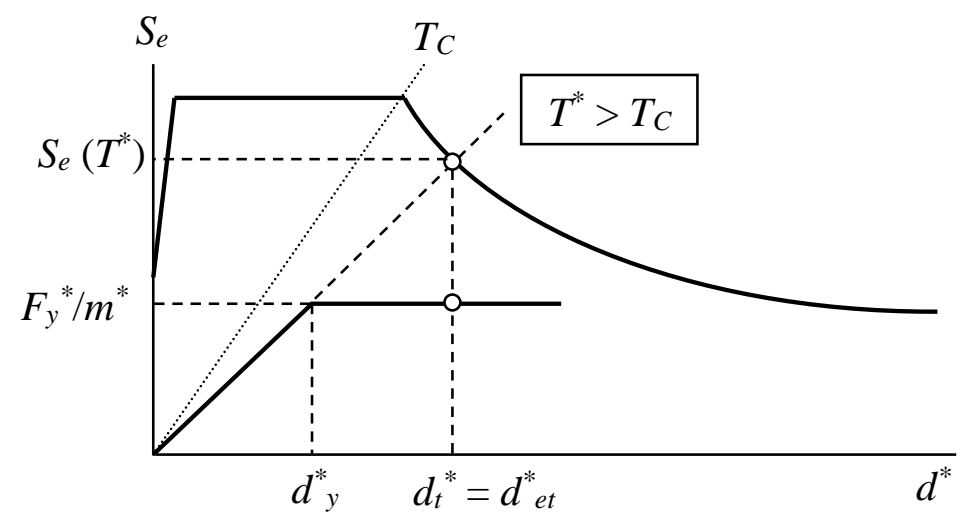

(b)

Figura 6.2 Determinación del desplazamiento objetivo para el sistema equivalente de un grado de libertad. (a) Rango de periodo corto. (b) Rango de periodos medio y largo.

El espectro de respuesta elástica horizontal $S_{e}(T)$ propuesto por el EC-8 viene dado por las ecuaciones (6.20) a (6.23). Su representación gráfica se muestra en la Figura 6.3.

$$
S_{e}(T)=a_{g} S\left[1+\frac{T}{T_{B}}(2,5 \eta-1)\right] \text { si } 0 \leq T \leq T_{B}
$$




$$
\begin{gathered}
S_{e}(T)=2,5 a_{g} S \eta \quad \text { si } T_{B} \leq T \leq T_{C} \\
S_{e}(T)=2,5 a_{g} S \eta \frac{T_{C}}{T} \quad \text { si } T_{C} \leq T \leq T_{D} \\
S_{e}(T)=2,5 a_{g} S \eta \frac{T_{C} T_{D}}{T^{2}} \quad \text { si } T_{D} \leq T \leq 4 \mathrm{~s}
\end{gathered}
$$

donde

$S \quad$ coeficiente de suelo,

$T$ periodo de vibración de un sistema lineal con un grado de libertad,

$T_{B} \quad$ límite inferior del periodo para el tramo de aceleración espectral constante,

$T_{C}$ límite superior del periodo para el tramo de aceleración espectral constante,

$T_{D} \quad$ valor que define el comienzo del tramo de respuesta de desplazamiento constante del espectro.

$a_{g} \quad$ valor de cálculo de la aceleración del suelo en un terreno tipo A, y

$\eta \quad$ coeficiente de corrección del amortiguamiento, con valor $\eta=1$ para un amortiguamiento viscoso del $5 \%$ del amortiguamiento crítico.

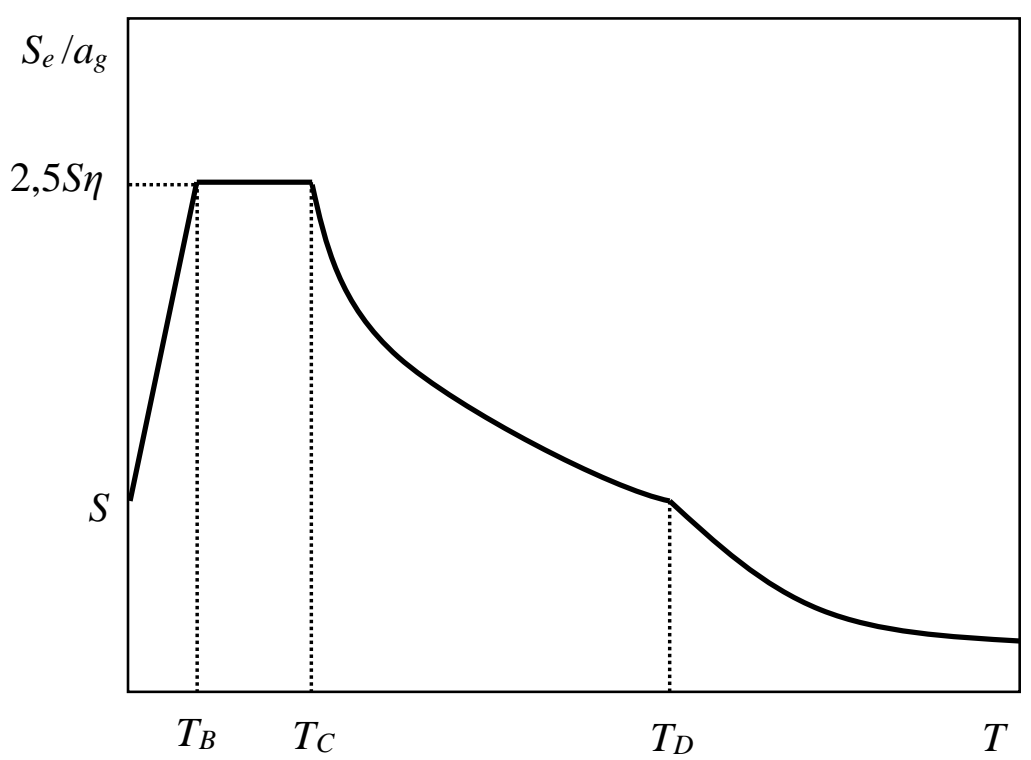

Figura 6.3 Representación gráfica del espectro de respuesta elástica horizontal propuesto por el EC-8.

Los valores de los parámetros $S, T_{B}, T_{C}$ y $T_{D}$ dependen del tipo de espectro y de terreno considerados. El EC-8 contempla dos tipos de espectros, el tipo 1 y el tipo 2, y los siguientes tipos de terreno:

a) terreno A: roca u otra formación geológica similar, incluyendo como máximo cinco metros de material más débil en la superficie, 
b) terreno B: depósitos de arena muy densa, grava o arcilla muy rígida, de al menos algunas decenas de metros de espesor, caracterizados por un aumento gradual de las propiedades mecánicas con la profundidad,

c) terreno $\mathrm{C}$ : depósitos profundos de arena densa o de densidad de media a densa, grava o arcilla dura con espesor de algunas decenas a muchos centenares de metros,

d) terreno D: depósitos de suelos sueltos a medios no cohesionados, con o sin algunas capas blandas cohesivas, o principalmente suelos cohesivos de rigidez débil a firme, y

e) terreno E: perfil de suelo constituido por una capa aluvial con valores de velocidad media de la onda de corte $v_{s}$ correspondientes a terrenos tipo C o D y espesor variable entre 5 y 20 metros, situado sobre un material más rígido con $v_{s}>800 \mathrm{~m} / \mathrm{s}$.

Las Tablas 6.1 y 6.2 muestran respectivamente los valores de los parámetros $S$, $T_{B}, T_{C}$ y $T_{D}$ para los espectros tipo 1 y 2 en función del tipo de terreno. Dichos espectros se muestran gráficamente en la Figura 6.4.

Tabla 6.1 Valores de parámetros para el espectro tipo 1.

\begin{tabular}{ccccc}
\hline Tipo de terreno & $S$ & $T_{B}(\mathrm{~s})$ & $T_{C}(\mathrm{~s})$ & $T_{D}(\mathrm{~s})$ \\
\hline $\mathrm{A}$ & 1,00 & 0,15 & 0,4 & 2,0 \\
$\mathrm{~B}$ & 1,20 & 0,15 & 0,5 & 2,0 \\
$\mathrm{C}$ & 1,15 & 0,20 & 0,6 & 2,0 \\
$\mathrm{D}$ & 1,35 & 0,20 & 0,8 & 2,0 \\
$\mathrm{E}$ & 1,40 & 0,15 & 0,5 & 2,0 \\
\hline
\end{tabular}

Tabla 6.2 Valores de parámetros para el espectro tipo 2.

\begin{tabular}{ccccc}
\hline Tipo de terreno & $S$ & $T_{B}(\mathrm{~s})$ & $T_{C}(\mathrm{~s})$ & $T_{D}(\mathrm{~s})$ \\
\hline $\mathrm{A}$ & 1,00 & 0,05 & 0,25 & 1,2 \\
$\mathrm{~B}$ & 1,35 & 0,05 & 0,25 & 1,2 \\
$\mathrm{C}$ & 1,50 & 0,10 & 0,25 & 1,2 \\
$\mathrm{D}$ & 1,80 & 0,10 & 0,30 & 1,2 \\
$\mathrm{E}$ & 1,60 & 0,05 & 0,25 & 1,2 \\
\hline
\end{tabular}

\subsubsection{Análisis pushover de estructuras de hormigón armado mediante ETABS $®$}

Tras exponer las particularidades del análisis pushover en los apartados 6.2.1 a 6.2.3, se aborda una serie de análisis pushover convencional de estructuras de hormigón armado, empleando los distintos tipos de rótulas plásticas especificadas en el apartado 6.1. El objetivo principal de este planteamiento es evaluar la influencia de dichas rótulas en los 
resultados obtenidos, comparando los siguientes aspectos correspondientes a las estructuras analizadas:

a) curva de capacidad, y

b) valores de esfuerzo cortante sísmico en la base $F_{b}$, desplazamiento del nudo de control $\delta$, aceleración del sistema equivalente de un grado de libertad $S_{a} / g$ y distribución de las rótulas plásticas en los puntos de plastificación global y de colapso de la estructura.

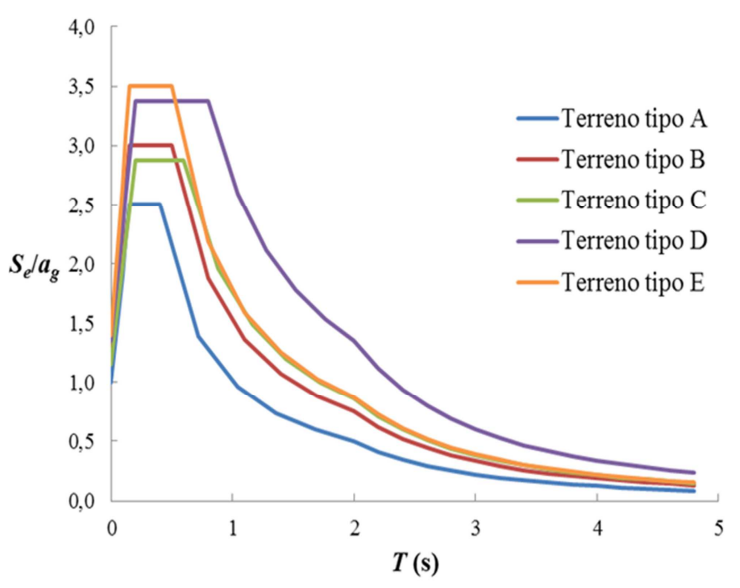

(a)

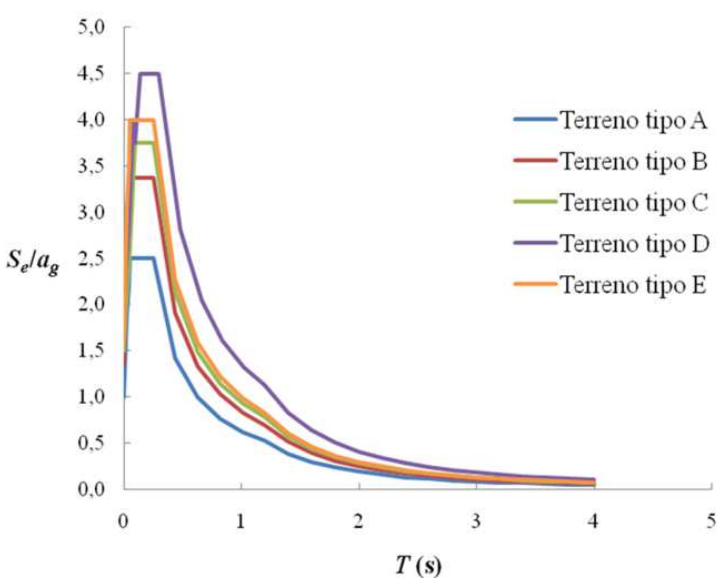

(b)

Figura 6.4 Espectro de respuesta elástica horizontal propuesto por el EC-8 en función del tipo de terreno. (a) Espectro tipo 1. (b) Espectro tipo 2.

Otro aspecto que resulta de interés es la evaluación de las diferencias obtenidas al aplicar los distintos tipos de rótulas plásticas en función del tipo de espectro y de terreno utilizados en el análisis. Concretamente, en este estudio se han considerado los espectros tipo 1 y 2 y los tipos de terreno A, C y D propuestos por el EC-8.

Las diferencias obtenidas al emplear las distintas rótulas plásticas dependen del valor de cálculo de la aceleración del suelo $a_{g}$ introducido en el espectro de respuesta. Para considerar este aspecto se han identificado los mínimos valores de $a_{g}$ que inducen diferencias al aplicar las diferentes rótulas, evaluándose adicionalmente las diferencias obtenidas al realizar análisis específicos para ciertos valores máximos de $a_{g}$, los cuales dependen del tipo de espectro empleado, considerándose

a) $a_{g}=1,0 g$ cuando se emplea el espectro tipo $1, \mathrm{y}$

b) $a_{g}=0,5 g$ al emplear el espectro tipo 2 .

La realización de los análisis pushover mediante el software ETABS® comprende ciertas particularidades que es necesario comentar. Aunque este software no ofrece la posibilidad de implementar subrutinas para realizar análisis específicos, permite la consideración de ciertos aspectos que repercuten significativamente en cuestiones como la precisión de los resultados obtenidos o el proceso de convergencia hacia la solución. Concretamente, los aspectos específicos de ETABS® que se describen para realizar los análisis pushover son 
a) propiedades de los materiales considerados,

b) características de las rótulas plásticas que se generan durante el análisis, y

c) parámetros del análisis no lineal.

\subsubsection{Propiedades de los materiales}

En los análisis realizados se ha considerado un hormigón no confinado con resistencia a compresión simple $f_{c}=25 \mathrm{MPa}$ y un acero con límite elástico $f_{y}=500 \mathrm{MPa}$. El modelo constitutivo considerado para el hormigón es el de Mander, Priestley y Park (1988), habiéndose empleado el modelo de endurecimiento por deformación para el acero de las armaduras.

La Tablas 6.3 y 6.4 muestran, respectivamente, las propiedades consideradas para el hormigón y el acero de las armaduras, siendo

$f_{u}$ tensión de rotura a tracción del acero de las armaduras,

$\gamma_{c}{ }^{\prime} \quad$ peso específico del hormigón,

$\gamma_{s} \quad$ peso específico del acero de las armaduras,

$\mathcal{E}_{c 0}$ deformación de rotura a compresión simple del hormigón,

$\varepsilon_{s u} \quad$ deformación de rotura a tracción del acero de las armaduras, $\mathrm{y}$

$v$ ' coeficiente de Poisson del hormigón

Tabla 6.3 Propiedades del hormigón.

\begin{tabular}{cccccc}
\hline $\begin{array}{c}\gamma_{c}{ }^{\prime} \\
\left(\mathrm{kN} / \mathrm{m}^{3}\right)\end{array}$ & $\begin{array}{c}E_{c} \\
(\mathrm{MPa})\end{array}$ & $v^{\prime}$ & $\begin{array}{c}f_{c} \\
(\mathrm{MPa})\end{array}$ & $\varepsilon_{c 0}$ & $\begin{array}{c}\text { Pendiente } \\
\text { residual }\end{array}$ \\
\hline 25 & 27300 & 0,2 & 25 & 0,002 & $-0,1 E_{c}$ \\
\hline
\end{tabular}

Tabla 6.4 Propiedades del acero de las armaduras.

\begin{tabular}{cccccc}
\hline $\begin{array}{c}\gamma_{s} \\
\left(\mathrm{kN} / \mathrm{m}^{3}\right)\end{array}$ & $\begin{array}{c}E_{s} \\
(\mathrm{MPa})\end{array}$ & $\begin{array}{c}f_{y} \\
(\mathrm{MPa})\end{array}$ & $\begin{array}{c}f_{u} \\
(\mathrm{MPa})\end{array}$ & $\varepsilon_{y}$ & $\varepsilon_{s u}$ \\
\hline 78 & 200000 & 500 & 575 & 0,0025 & 0,075 \\
\hline
\end{tabular}

Las relaciones constitutivas tensión-deformación del hormigón y del acero de las armaduras se muestran gráficamente en las Figuras 6.5 y 6.6. Resulta necesario destacar que la elección de los diagramas constitutivos repercute en el comportamiento de las zonas de la estructura que permanecen en estado elástico durante el análisis. El comportamiento de las zonas plastificadas está gobernado por las rótulas plásticas, cuyas propiedades se definen mediante las expresiones de comportamiento seccional citadas en el apartado 6.1, sin considerar la relación constitutiva de los materiales.

\subsubsection{Definición de rótulas plásticas}

Un aspecto relevante a la hora de realizar un análisis pushover es la elección del tipo de rótula plástica a emplear. En este sentido, ETABS® permite considerar 
a) rótulas plásticas tipo lumped de longitud nula, $\mathrm{y}$

b) rótulas plásticas tipo fiber hinge de longitud finita.

En las rótulas plásticas de tipo fiber hinge, la sección transversal del elemento se divide en una serie de fibras. Dependiendo de su posición en la sección, dicha fibras modelizan el comportamiento constitutivo del acero de las barras de armadura longitudinal, del hormigón del núcleo confinado o del hormigón del recubrimiento. La relación fuerza-deformación de este tipo de rótulas se obtiene mediante la integración del comportamiento constitutivo uniaxial de los materiales que constituyen las diferentes fibras. Además de modelizar el comportamiento de las diferentes partes de la sección, este tipo de rótulas permite considerar la distribución de la plasticidad a lo largo del elemento al imponerse en ellas una cierta longitud.

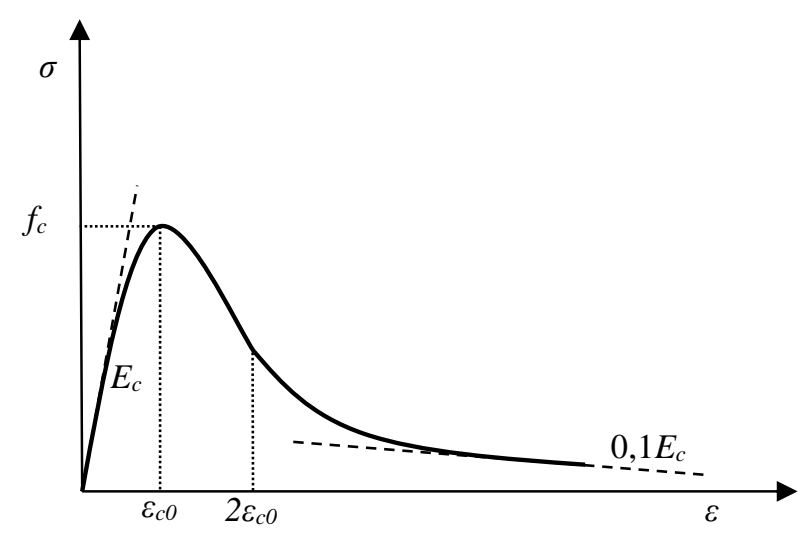

Figura 6.5 Relación constitutiva tensión-deformación del hormigón a compresión.

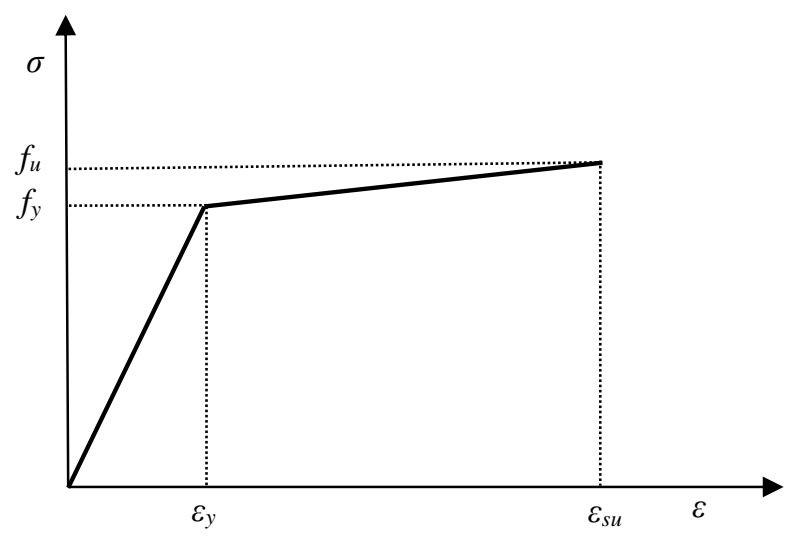

Figura 6.6 Relación constitutiva tensión-deformación del acero de las armaduras a tracción.

Para las rótulas tipo lumped se considera que la plastificación se concentra en secciones cuya localización se establece previamente a la realización del análisis pushover. La relación fuerza-deformación de este tipo de rótulas no se obtiene mediante un análisis de la sección, pudiendo ser definida manualmente por el usuario. Concretamente, ETABS $®$ considera como relación fuerza-deformación para estas rótulas la relación momento flector-giro de la sección del elemento estructural donde se generan. 
De acuerdo con Yön y Calayir (2013), el tipo de rótula plástica considerada en el análisis pushover influye en aspectos como los desplazamientos entre plantas y la rigidez inicial de la curva de capacidad obtenida. Con objeto de emplear las diferentes expresiones de comportamiento seccional propuestas en el apartado 6.1 para obtener las relaciones momento-giro, se han considerado rótulas tipo lumped en los análisis pushover realizados. La Figura 6.7 muestra las posiciones de las posibles rótulas plásticas generadas durante el análisis. Las rótulas se sitúan en los extremos de las vigas y los pilares, en posiciones que ocupan el 5 y el $95 \%$ de las longitudes de los elementos.

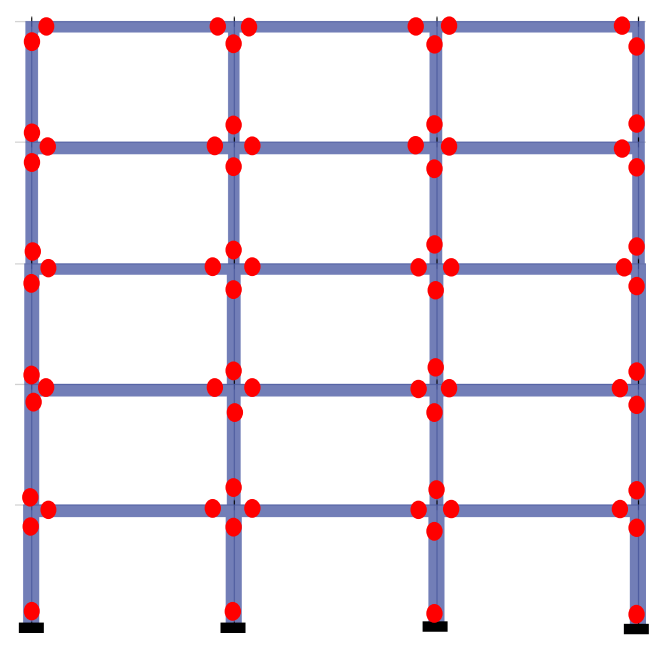

Figura 6.7 Definición de las posiciones de las rótulas plásticas.

La FEMA (2000) propone la relación momento-giro mostrada en la Figura 6.8 para caracterizar las rótulas plásticas de hormigón armado. La relación momento-giro de las rótulas plásticas que ETABS $®$ considera por defecto, aunque se basa en la definida en la FEMA (2000), modeliza el tramo $A B$ previo a la plastificación de la sección y el $C D$ posterior al estado último mediante líneas verticales, tal y como muestra la Figura 6.9 .

Los valores de los parámetros $a, b$ y $c$, mostrados en las Tablas 6.5 y 6.6, dependen de si la rótula plástica se genera en una viga o en un pilar, siendo:

$\mathrm{C}$ sección tipo conforming,

NC sección tipo nonconforming,

$V \quad$ esfuerzo cortante de diseño, y

$\rho_{b a l}$ cuantía de armadura de tracción para que se produzca simultáneamente la plastificación de la armadura de tracción y la máxima deformación de compresión admisible para el hormigón. 


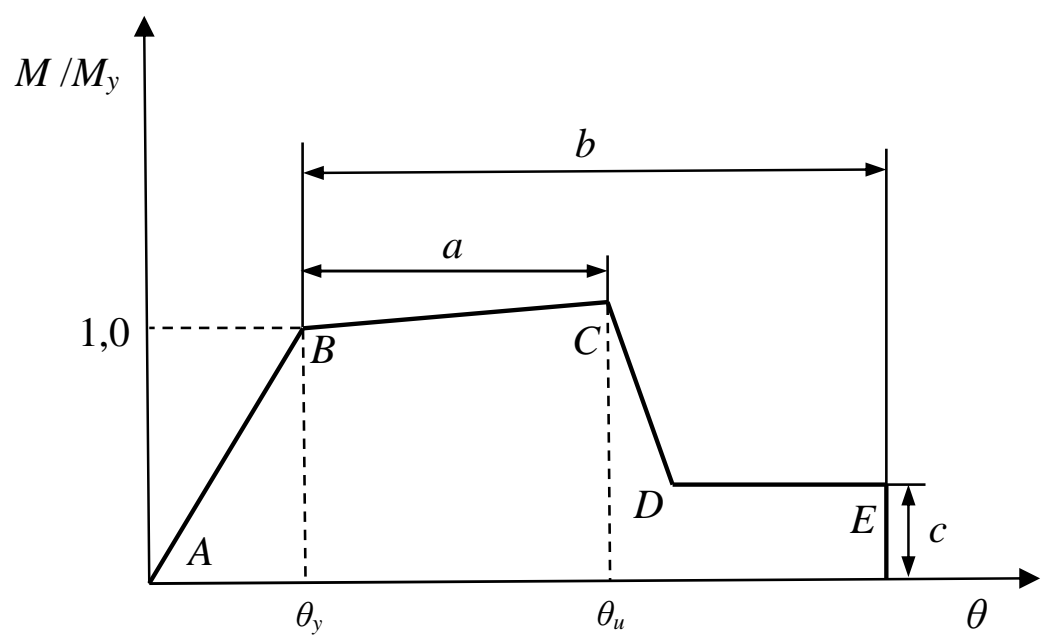

Figura 6.8 Relación momento-giro para rótulas plásticas de hormigón armado según la FEMA (2000).

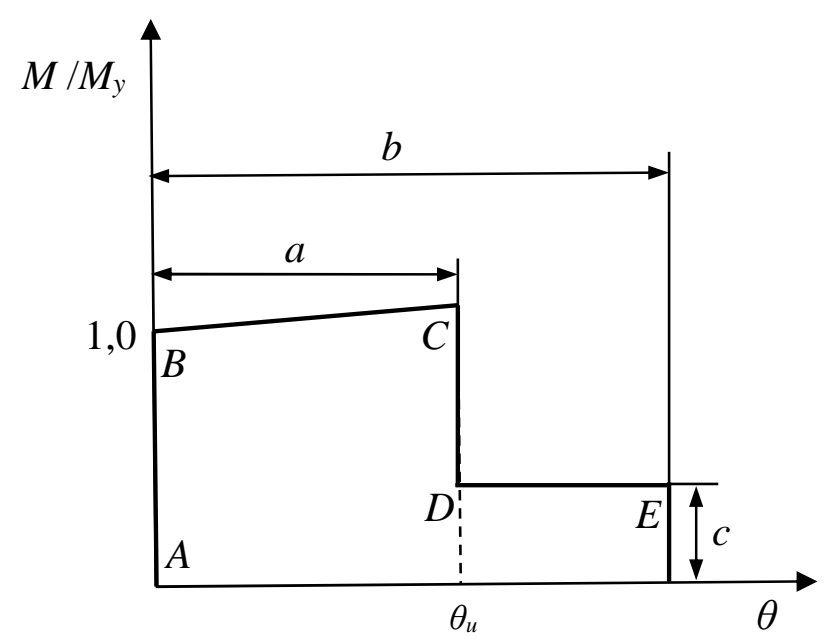

Figura 6.9 Relación momento-giro adoptada por ETABS® para las rótulas plásticas tipo lumped.

Tabla 6.5 Parámetros de las rótulas plásticas para vigas según la FEMA (2000).

\begin{tabular}{cccccc}
\hline$\frac{\rho-\rho^{\prime}}{\rho_{b a l}}$ & Armadura transversal & $\frac{V}{c}$ & & & \\
\cline { 3 - 6 } & & $b d \sqrt{f_{c}}$ & & $b$ & $c$ \\
$\leq 0,0$ & $\mathrm{C}$ & $\leq 3$ & 0,025 & 0,050 & 0,200 \\
$\leq 0,0$ & $\mathrm{C}$ & $\geq 6$ & 0,020 & 0,040 & 0,200 \\
$\geq 0,5$ & $\mathrm{C}$ & $\leq 3$ & 0,020 & 0,030 & 0,200 \\
$\geq 0,5$ & $\mathrm{C}$ & $\geq 6$ & 0,015 & 0,020 & 0,200 \\
$\leq 0,0$ & $\mathrm{NC}$ & $\leq 3$ & 0,020 & 0,030 & 0,200 \\
$\leq 0,0$ & $\mathrm{NC}$ & $\geq 6$ & 0,010 & 0,015 & 0,200 \\
$\geq 0,5$ & $\mathrm{NC}$ & $\leq 3$ & 0,010 & 0,015 & 0,200 \\
$\geq 0,5$ & $\mathrm{NC}$ & $\geq 6$ & 0,005 & 0,010 & 0,200 \\
\hline
\end{tabular}

Al asignar las posiciones de las rótulas plásticas, ETABS® define de forma automática las relaciones momento-giro de dichas rótulas en función de los parámetros 
expuestos en las Tablas 6.5 y 6.6. Si las características de la sección donde se introduce la rótula plástica no se corresponden con ninguno de los casos expuestos en dichas tablas, ETABS ${ }^{\circledR}$ realiza una interpolación lineal para obtener los valores de los parámetros $a, b$ y $c$.

Tabla 6.6 Parámetros de las rótulas plásticas para pilares según la FEMA (2000).

\begin{tabular}{cccccc}
\hline \multirow{2}{*}{$\frac{N}{A f_{c}}$} & Armadura transversal & $\frac{V}{3 \sqrt{f_{c}}}$ & $a$ & $b$ & $c$ \\
\hline$\leq 0,1$ & & $\leq 3$ & 0,020 & 0,030 & 0,200 \\
$\leq 0,1$ & $\mathrm{C}$ & $\geq 6$ & 0,016 & 0,024 & 0,200 \\
$\geq 0,4$ & $\mathrm{C}$ & $\leq 3$ & 0,015 & 0,025 & 0,200 \\
$\geq 0,4$ & $\mathrm{C}$ & $\geq 6$ & 0,012 & 0,020 & 0,200 \\
$\leq 0,1$ & $\mathrm{C}$ & $\leq 3$ & 0,006 & 0,015 & 0,200 \\
$\leq 0,1$ & $\mathrm{NC}$ & $\geq 6$ & 0,005 & 0,012 & 0,200 \\
$\geq 0,4$ & $\mathrm{NC}$ & $\leq 3$ & 0,003 & 0,010 & 0,200 \\
$\geq 0,4$ & $\mathrm{NC}$ & $\geq 6$ & 0,002 & 0,008 & 0,200 \\
\hline
\end{tabular}

Las secciones tipo conforming presentan un espaciamiento de la armadura transversal $s_{h} \leq d / 3$, siendo de tipo nonconforming en caso contrario. Se ha considerado el esfuerzo cortante de diseño $V$ y el esfuerzo axil $N$ correspondientes al caso de carga previo al comienzo del análisis pushover, esto es, al actuar la combinación sísmica de cargas gravitatorias dada por la ecuación (6.5).

Un aspecto relevante a la hora de analizar los resultados obtenidos es la consideración de los distintos niveles de comportamiento o performance level de la estructura. ETABS $®$ contempla tres posibles niveles de comportamiento, definidos en la FEMA (2000):

a) Immediate occupancy IO: la acción sísmica induce en la estructura daños muy limitados. Los sistemas primarios de resistencia frente a cargas verticales y horizontales mantienen sus condiciones de resistencia y rigidez previas a la ocurrencia del sismo. El daño estructural supone un riesgo muy bajo para las personas. Resulta apropiado realizar reparaciones de carácter menor en la estructura, no siendo necesario que las mismas tengan lugar previamente a la reocupación del edificio.

b) Life safety LS: la estructura, aunque sufre daños significativos, presenta un cierto margen hasta el colapso parcial o total, pudiendo resultar inviable su reparación por motivos económicos. A pesar de que el daño experimentado no supone un riesgo de colapso inminente, resulta recomendable realizar reparaciones estructurales o apuntalar la estructura previamente a su reutilización. 
c) Collapse prevention CP: corresponde al estado en el que la estructura está a punto de sufrir el colapso parcial o total. El sistema resistente frente a cargas laterales experimenta una importante degradación en su rigidez y resistencia, produciéndose grandes deformaciones laterales permanentes. Sin embargo, los elementos principales del sistema resistente frente a cargas gravitatorias mantienen sus prestaciones. La reparación de la estructura puede no resultar técnicamente viable, no siendo seguro su reocupación por el riesgo de colapso.

Con objeto de considerar la ductilidad de las secciones de los elementos, en este estudio se emplean rótulas plásticas de control por deformación. En este tipo de rótulas, ETABS $®$ considera los tres niveles de comportamiento anteriormente descritos en función del giro experimentado por la sección, atribuyendo un porcentaje del giro último $\theta_{u}$ a cada nivel de comportamiento. Los porcentajes correspondientes a cada nivel de comportamiento son los mostrados en la Tabla 6.7.

Tabla 6.7 Porcentajes del giro último $\theta_{u}$ atribuidos en ETABS® a los diferentes niveles de comportamiento.

\begin{tabular}{cccc}
\hline & IO & LS & CP \\
\hline Vigas & 40 & 80 & 100 \\
Pilares & 25 & 75 & 100 \\
\hline
\end{tabular}

Las Tablas 6.5 y 6.6 ofrecen valores de parámetros correspondientes a edificios construidos en Estados Unidos, por lo que, de acuerdo con Inel y Ozmen (2006), la aplicación de las rótulas plásticas de ETABS ${ }^{\circledR}$ a estructuras diseñadas según los códigos de otros países puede no reflejar convenientemente el comportamiento no lineal de la estructura. Por tanto, en este estudio se plantean rótulas plásticas alternativas, modelizadas mediante las expresiones de comportamiento seccional propuestas por Biskinis y Fardis (2010a, 2010b) y las ecuaciones ajustadas a la selección de ensayos del capítulo 4. Para definir las características de dichas rótulas, resulta necesario determinar la resistencia y capacidad de deformación de las secciones de los elementos estructurales mediante los valores del momento de plastificación $M_{y} \mathrm{y}$ del giro último $\theta_{u}$. Puesto que no se dispone de expresiones calibradas con ensayos experimentales para determinar el momento último $M_{u}$ de la sección, se ha considerado una relación momento-giro como la mostrada en la Figura 6.10, utilizada en otros estudios (California Department of Transportation, 2013).

\subsubsection{Parámetros del análisis no lineal}

Los análisis pushover realizados comprenden ciertas particularidades de carácter numérico. ETABS $®$ permite definir el valor de una serie de parámetros para controlar los siguientes aspectos:

a) obtención de la solución del problema, 
b) precisión de los resultados obtenidos, $\mathrm{y}$

c) velocidad de convergencia hacia la solución.

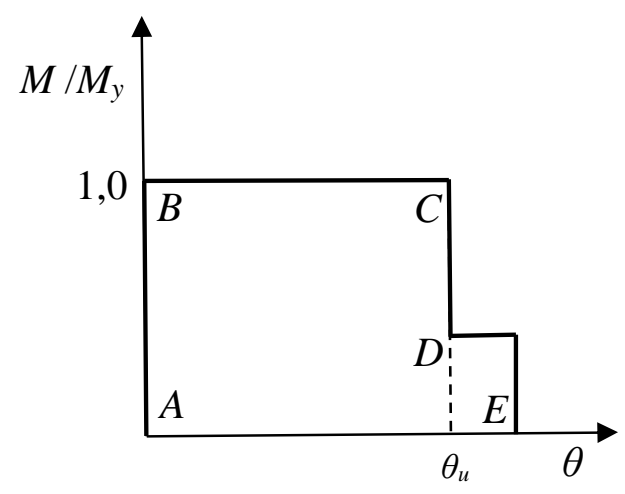

Figura 6.10 Relación momento-giro elasto-plástica para las rótulas plásticas definidas con las expresiones de comportamiento seccional.

Los valores de los diferentes parámetros se han adoptado tras la realización de diversas pruebas. Dos parámetros de especial importancia son el número máximo de pasos del análisis y el número máximo de pasos nulos. El número máximo de pasos establece la cantidad de estados analizados, siendo un factor determinante en la duración del análisis. Por otro lado, ETABS® considera que se ha producido un paso nulo cuando se genera o se descarga una rótula plástica. Resulta necesario escoger un número adecuado de pasos nulos, pues un valor excesivo puede dar lugar a problemas de convergencia al elevar la sensibilidad del análisis y un valor insuficiente puede detener el proceso de búsqueda de la solución. En los análisis realizados se ha considerado un número máximo de pasos de 5000 y un número máximo de pasos nulos de 3000 .

Para alcanzar el equilibrio en cada paso del análisis, resulta necesario realizar una serie de iteraciones, adoptando dos posibles estrategias: rigidez constante o rigidez tangente, la cual se considera mediante el método de Newton-Raphson. ETABS ${ }^{\circ}$ emplea primeramente el método de rigidez constante, aplicando posteriormente el método de Newton-Raphson si no existe convergencia. Resulta necesario definir el número máximo de iteraciones realizadas para cada método, habiéndose considerado 10 iteraciones con rigidez constante y 40 iteraciones tipo Newton-Raphson.

El aspecto más influyente en la convergencia hacia la solución del análisis lo constituye la tolerancia de convergencia, definida como la diferencia entre los esfuerzos generados en la estructura y las fuerzas exteriores aplicadas, existiendo convergencia cuando el valor de dicha diferencia resulta inferior a un cierto porcentaje de las fuerzas exteriores aplicadas. Valores elevados de tolerancia pueden conducir a resultados poco precisos, mientras que una tolerancia excesivamente restrictiva impide la convergencia. 
En general, se ha considerado el valor propuesto por ETABS $®$ de 0,0001, incrementándose este valor hasta 0,01 para aquellos casos que lo requieran.

Otro aspecto de especial relevancia es el método de descarga de las rótulas plásticas. Cuando una rótula plástica se descarga al alcanzar el punto $\mathrm{C}$ en la relación momento-giro de la Figura 6.10, resulta necesario redistribuir los esfuerzos al resto de la estructura. De entre los métodos disponibles, se ha escogido el método de reinicio usando la rigidez secante, según el cual, tras producirse la descarga de una rótula, se descargan todas las rótulas de la estructura, volviendo posteriormente a su estado original según sus propiedades de rigidez secante.

\subsection{EJEMPLOS DE APLICACIÓN}

\subsubsection{Descripción de las estructuras analizadas}

Los análisis pushover se aplican a cuatro estructuras de hormigón armado en las que se han considerado diferentes longitudes de vano y número de plantas con objeto de determinar si la geometría y la altura de la estructura influyen en los resultados obtenidos en los análisis. Las estructuras analizadas se dividen en dos parejas:

a) una pareja de pórticos planos de cinco y ocho plantas con geometría regular, y

b) dos estructuras espaciales de cinco y ocho plantas con longitudes de vano variables.

Con el fin de poder modelizar las rótulas plásticas empleando las expresiones de comportamiento seccional calibradas con la selección de ensayos del capítulo 4, las estructuras analizadas están compuestas por vigas y pilares de sección rectangular.

Los alzados de los pórticos regulares de cinco y ocho plantas se muestran en la Figura 6.11. Estos pórticos presentan tres vanos de cinco metros de longitud y una altura de planta de tres metros. Las secciones transversales de las vigas y los pilares se muestran en las Figuras 6.12 y 6.13. En ambos pórticos se han considerado vigas planas. Domínguez, López-Almansa y Benavent-Climent (2014) ponen de manifiesto la problemática de este tipo de vigas desde el punto de vista sismorresistente, especificando sus inconvenientes cuando el análisis se realiza en el sentido longitudinal y transversal. Según estos autores, las vigas planas presentan los siguientes inconvenientes en su comportamiento longitudinal:

a) los edificios presentan una reducida resistencia y rigidez lateral, siendo insuficiente la ductilidad de las vigas debido a la elevada cuantía de armadura,

b) pueden generarse excesivas compresiones en las bielas del interior de los nudos por su elevada inclinación, y

c) la transmisión de momentos flectores entre las vigas y los pilares presenta dificultades, ya que parte de la armadura longitudinal de las vigas discurre fuera del ancho de los pilares, produciéndose mecanismos frágiles de torsión. 


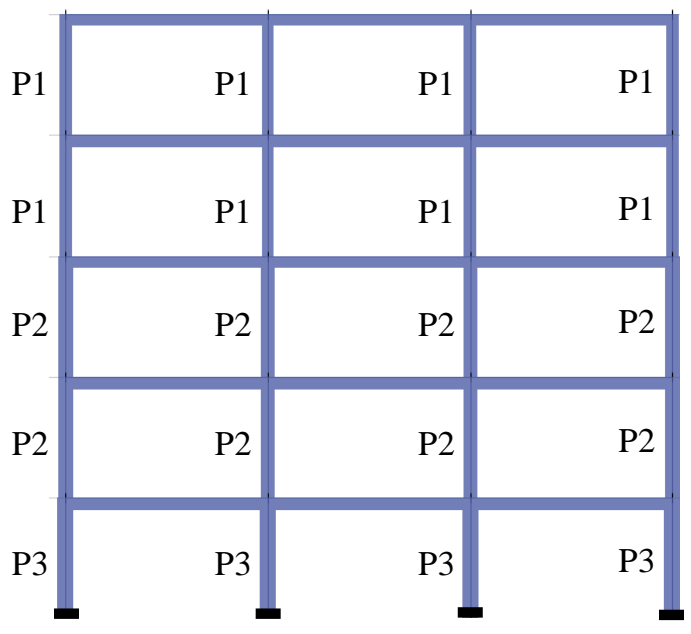

(a)

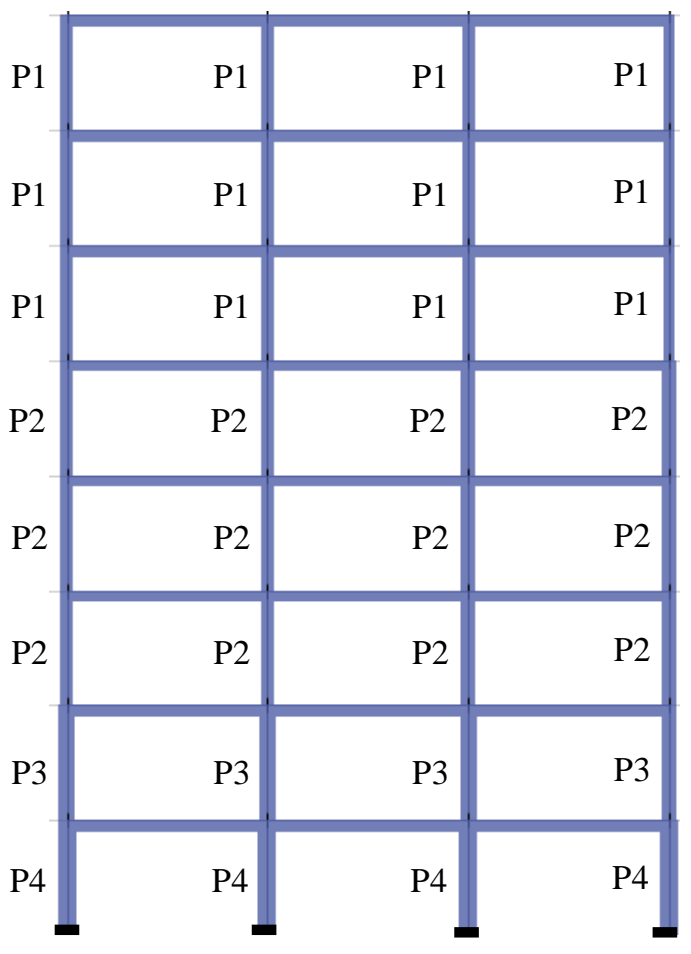

(b)

Figura 6.11 Alzados de los pórticos regulares. (a) Pórtico de cinco plantas. (b) Pórtico de ocho plantas.

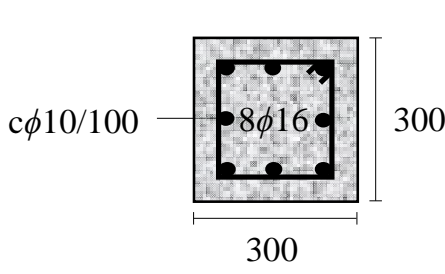

P1

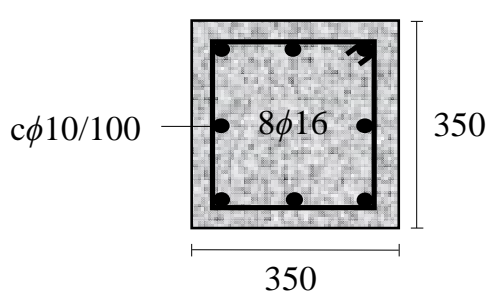

$\mathrm{P} 2$

(a)

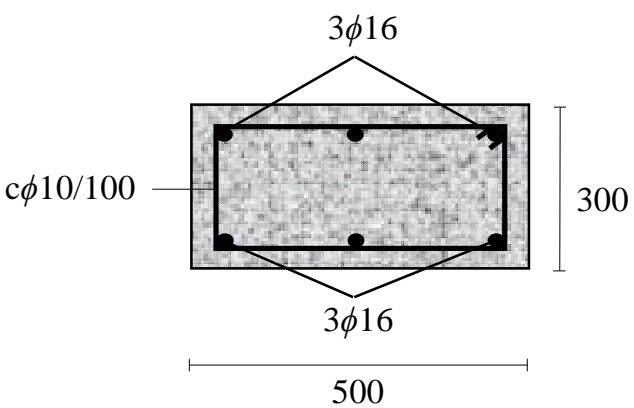

(b)

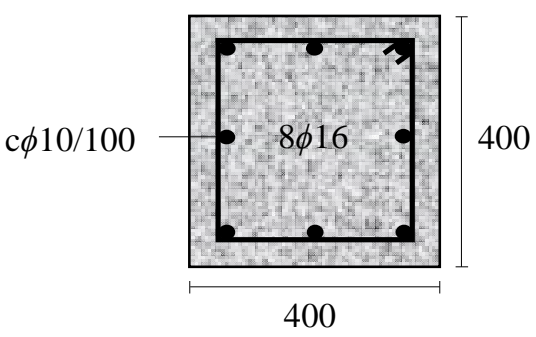

P3 
El comportamiento de las vigas planas en la dirección transversal resulta aún más problemático, puesto que los pórticos son menos resistentes y pueden aparecer problemas de anclaje de las armaduras inferiores de las vigas a los pilares si se produce inversión de momentos. A pesar de estos inconvenientes, se ha considerado en los análisis este tipo de vigas puesto que constituyen una solución empleada con frecuencia en España y en otros países de su entorno al permitir forjados unidireccionales de canto constante, aspecto que presenta ciertas ventajas como el uso de encofrados lisos y la facilidad en el paso de las instalaciones. Los pilares presentan una sección cuadrada y una configuración doblemente simétrica de armadura longitudinal.

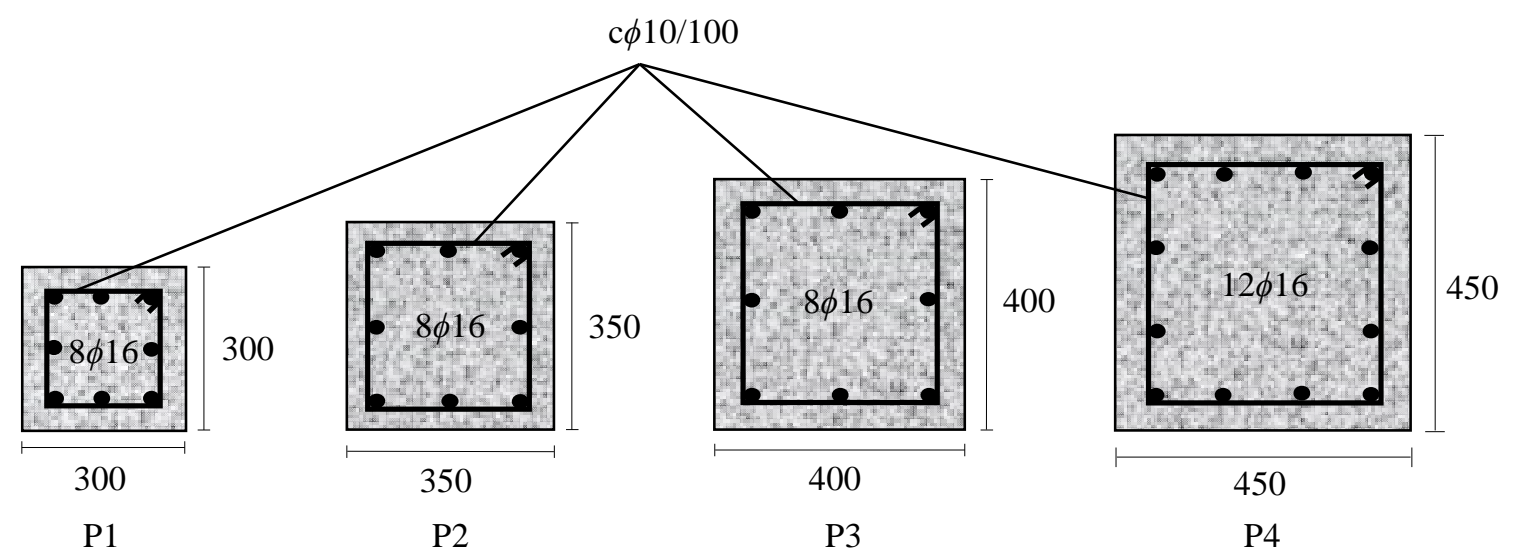

(a)

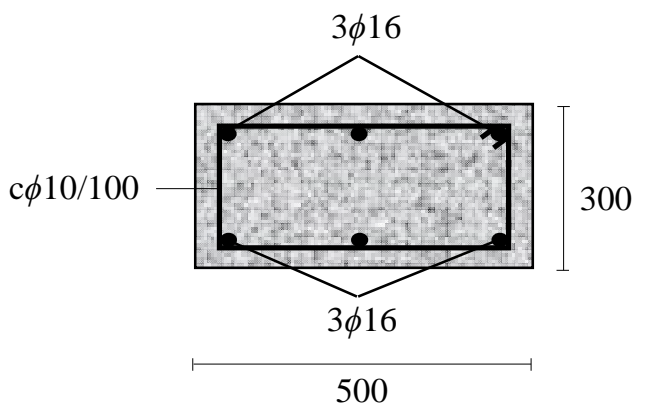

(b)

Figura 6.13 Secciones transversales de los elementos del pórtico regular de ocho plantas.

(a) Pilares. (b) Vigas.

El segundo grupo de estructuras analizadas se muestra gráficamente en la Figura 6.14. Consta de dos estructuras espaciales, cada una compuesta por dos sistemas de pórticos de vigas y pilares dispuestos según dos direcciones perpendiculares. La distribución de vigas y pilares y las dimensiones en planta son idénticas en ambas estructuras, siendo la altura el único aspecto que las diferencia. Los forjados son unidireccionales, estando las viguetas dispuestas según la dirección del eje X. Los pórticos no presentan una geometría regular, siendo variable el valor de las luces entre vanos. Para realizar el análisis pushover de estructuras espaciales, el EC-8 establece la 
posibilidad de emplear dos modelos planos según las dos direcciones horizontales principales si la estructura cumple las condiciones de regularidad en planta indicadas en su apartado 4.2.3.2. Al cumplir las estructuras espaciales de la Figura 6.14 dichas condiciones, y puesto que las viguetas del forjado unidireccional transmiten las cargas únicamente a los pórticos dispuestos según la dirección transversal, se ha analizado para cada estructura un pórtico plano en la dirección Y.

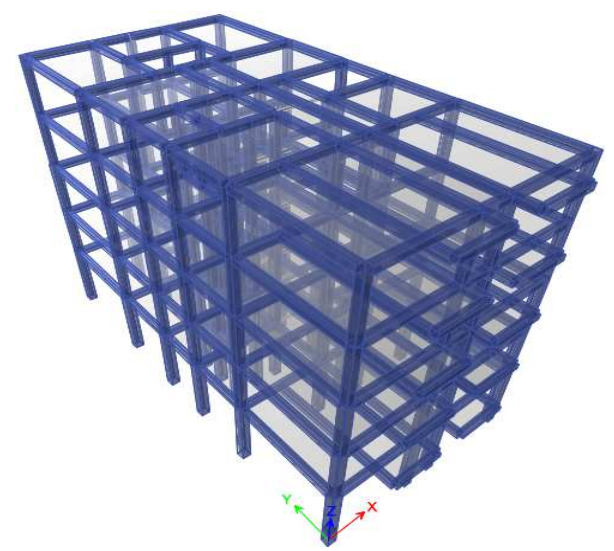

(a)

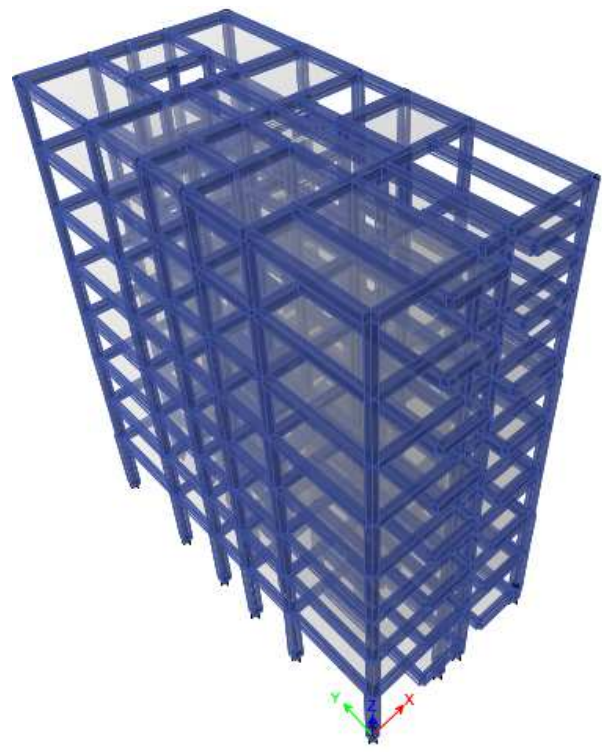

(b)

Figura 6.14 Estructuras espaciales. (a) Cinco plantas. (b) Ocho plantas.

La Figura 6.15 muestra la planta de las estructuras espaciales y la alineación del pórtico A-A' considerado para realizar el análisis pushover. Los alzados de los pórticos analizados se presentan en la Figura 6.16, en los que se ha situado un apoyo en el extremo derecho de las vigas que coarta el movimiento vertical para modelizar el apoyo con las vigas del pórtico B-B'. La altura de las plantas es de cuatro metros para la primera planta y de tres metros para el resto. Las secciones transversales de las vigas y pilares considerados se muestran en las Figuras 6.17 y 6.18. A diferencia de las vigas planas consideradas para los pórticos con geometría regular, en los pórticos espaciales se han introducido vigas con un canto superior a su ancho, las cuales resultan más adecuadas para edificios emplazados en zonas sísmicas. La consideración de ambas tipologías tiene por objeto evaluar las posibles diferencias en su comportamiento. 


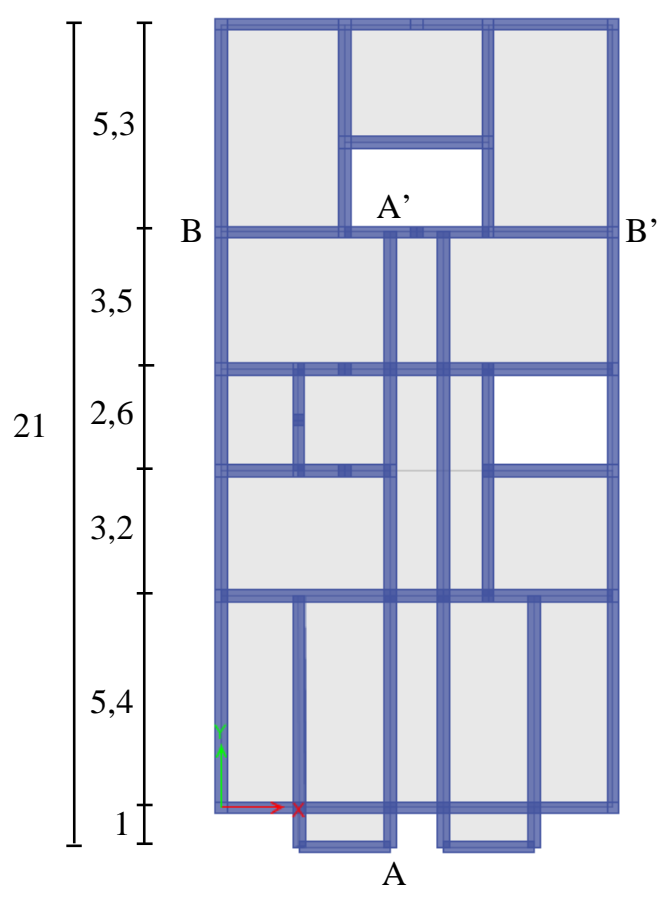

Figura 6.15 Dimensiones en metros de las plantas de las estructuras espaciales y alineación del pórtico A-A' considerado para el análisis pushover.

En lo que respecta a las acciones consideradas en los análisis, resulta necesario destacar que se han considerado cargas gravitatorias de igual valor para las cuatro estructuras analizadas. Se ha supuesto un forjado unidireccional con viguetas de $25 \mathrm{~cm}$ de canto y $5 \mathrm{~cm}$ de capa de compresión y un intereje de $70 \mathrm{~cm}$, siendo el peso propio de este sistema de $4 \mathrm{kN} / \mathrm{m}^{2}$. Respecto al valor de la carga muerta debida al solado, aunque el EC-2 establece valores orientativos acerca de los pesos específicos de los diferentes materiales, no propone valores de peso propio para diferentes configuraciones de suelo, habiéndose adoptado las prescripciones establecidas en la Tabla C.5 del Anexo C del Documento Básico SE-AE (Código Técnico de la Edificación, 2009). Se ha considerado un pavimento cerámico sobre plastón con un espesor total inferior a $8 \mathrm{~cm}$, el cual supone una carga de $1 \mathrm{kN} / \mathrm{m}^{2}$. Para las sobrecargas de uso se ha considerado que las estructuras son de tipo residencial, esto es, categoría A según el EC-1 (European Committee for Standardization, 2002), adoptándose una sobrecarga de uso de $2 \mathrm{kN} / \mathrm{m}^{2}$. La tabiquería se ha considerado introduciendo una sobrecarga de $1 \mathrm{kN} / \mathrm{m}^{2}$. Se ha supuesto que la cubierta de las estructuras analizadas es de tipo I o transitable según el EC-2, aplicándose en la misma la sobrecarga de uso supuesta para el resto de plantas, es decir, $2 \mathrm{kN} / \mathrm{m}^{2}$. 


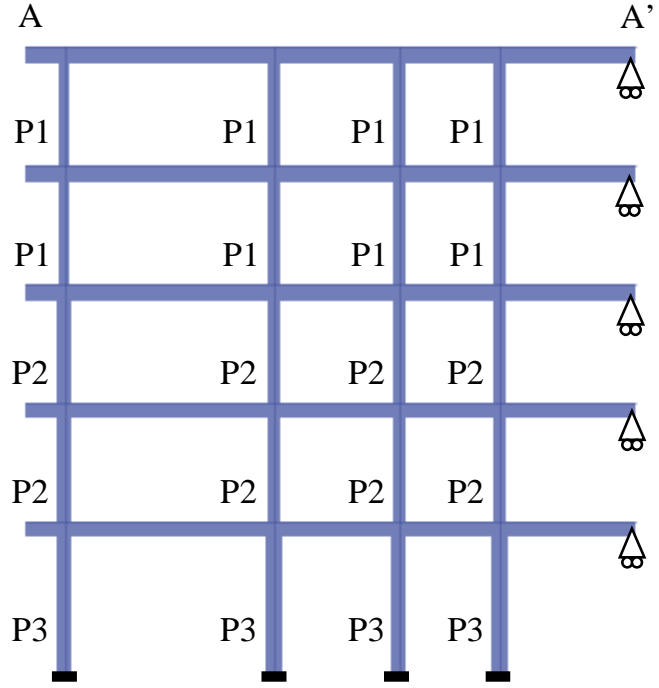

(a)

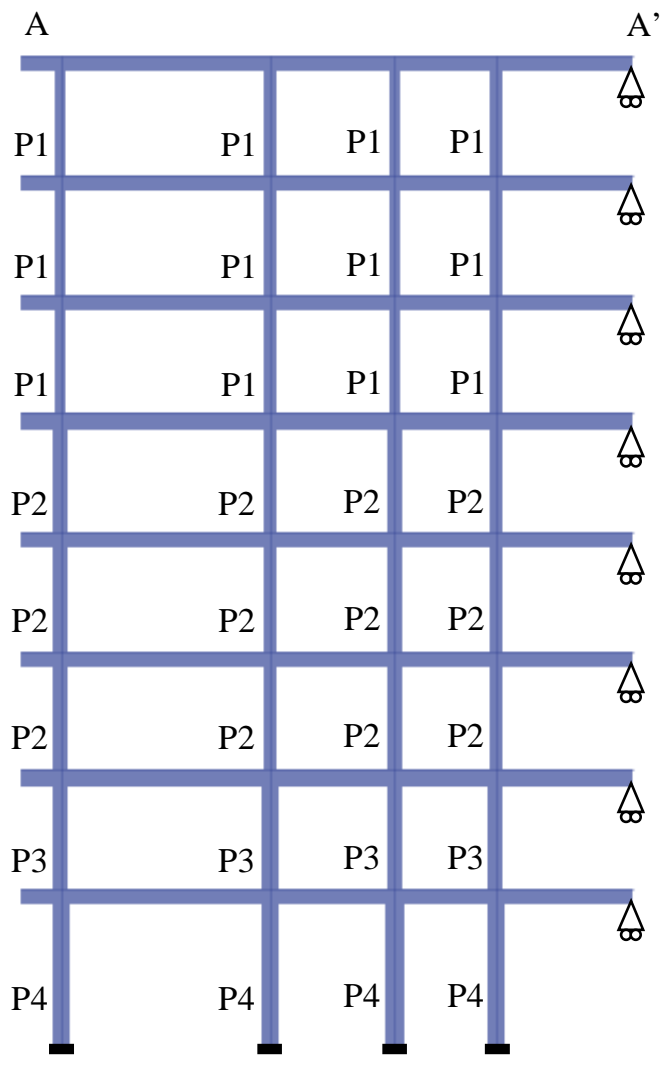

(b)

Figura 6.16 Alzado de los pórticos espaciales. (a) Estructura de cinco plantas. (b) Estructura de ocho plantas.

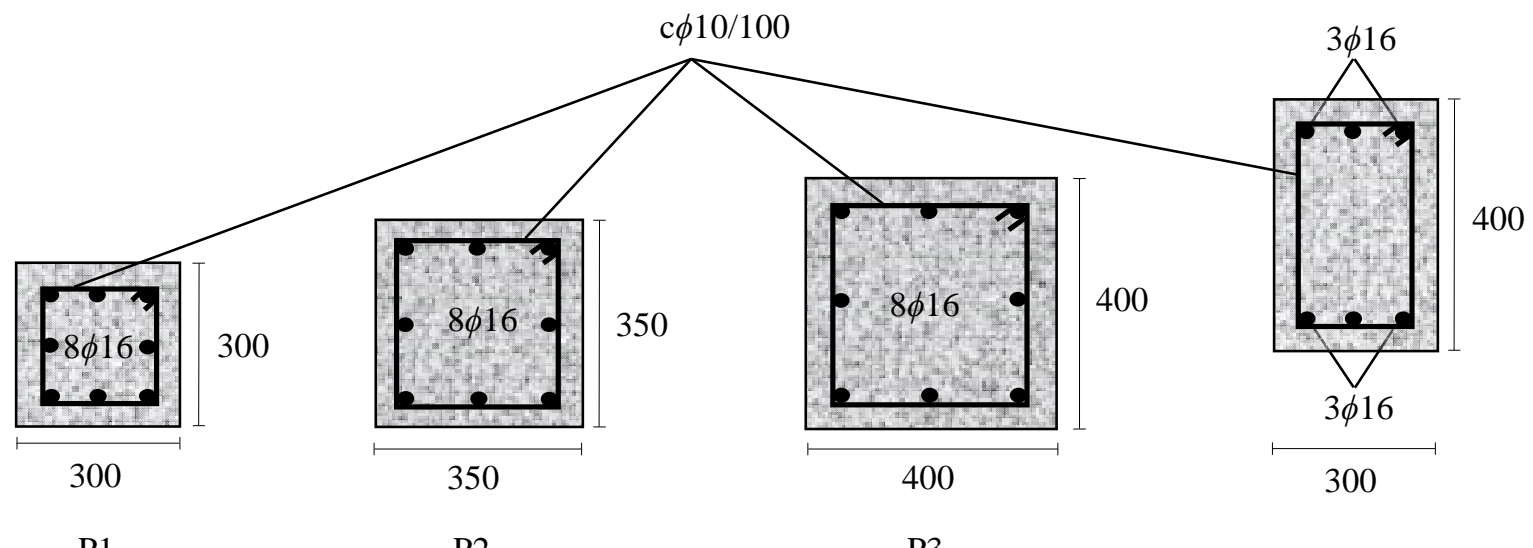

(a)

(b)

Figura 6.17 Secciones transversales de los elementos del pórtico espacial de cinco plantas.

(a) Pilares. (b) Vigas.

Resulta necesario destacar que, tal y como propone el EC-8, el efecto de la fisuración en la rigidez de los elementos previa a la plastificación de la sección se ha considerado imponiendo en las secciones unos valores de rigidez a flexión y esfuerzo cortante del $50 \%$ de los valores correspondientes a la sección sin fisurar. 


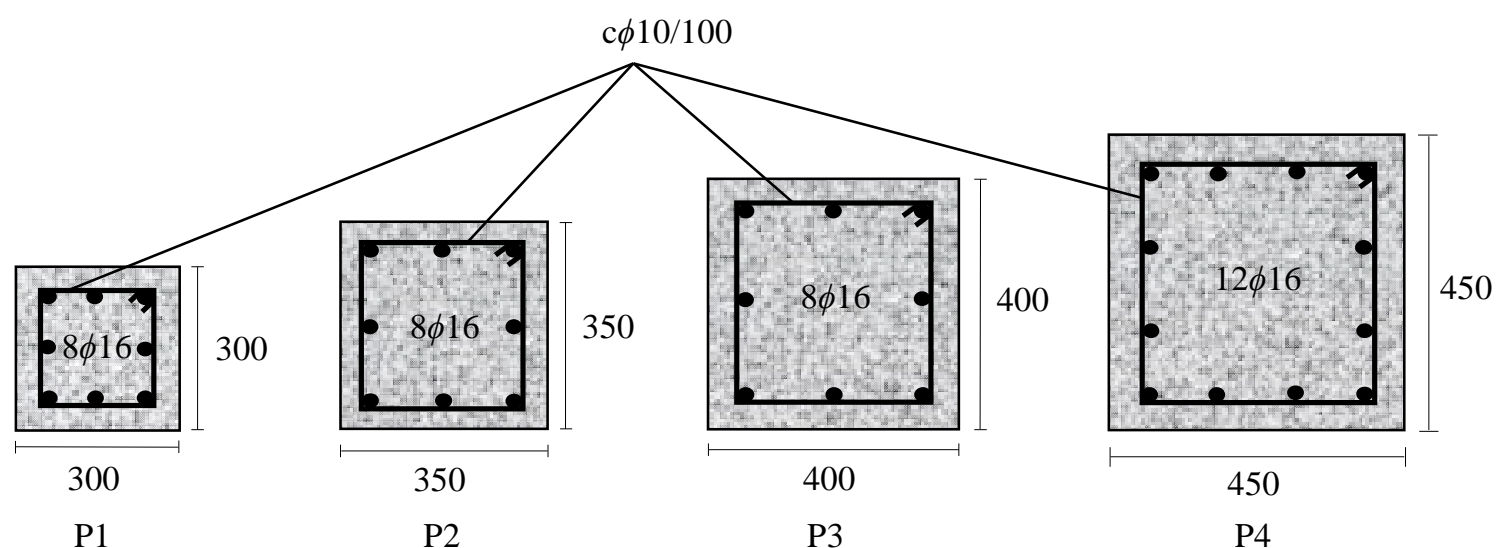

(a)

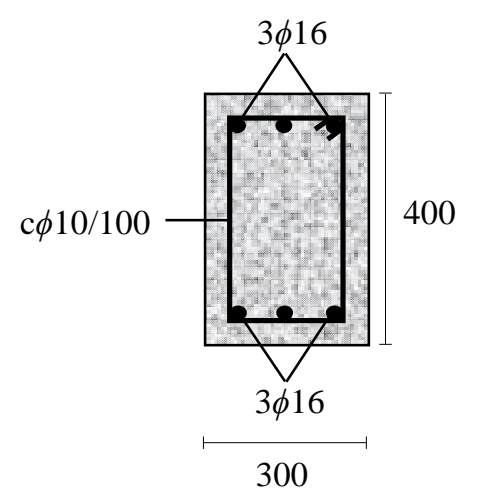

(b)

Figura 6.18 Secciones transversales de los elementos del pórtico espacial de ocho plantas.

(a) Pilares. (b) Vigas.

La Tabla 6.8 muestra los valores de ancho tributario asignados a los diferentes pórticos analizados y las cargas por metro lineal actuantes en cada planta. Las propiedades de los pórticos relativas a las diferentes distribuciones de carga lateral empleadas en los análisis se muestran en las Tablas 6.9 a 6.12. Se han considerado distribuciones de tipo uniforme y modal. La distribución uniforme se obtiene aplicando la ecuación (6.1). Puesto que los pórticos analizados cumplen las condiciones de la ecuación (6.2) y los criterios de regularidad en alzado del apartado 4.2.3.3 del EC-8, es posible aplicar una distribución de fuerzas contemplada en el método de análisis de la fuerza lateral, siendo la distribución modal escogida la que considera la deformada del modo fundamental de vibración, dada por la ecuación (6.3). El análisis pushover se realiza por control de desplazamiento, incrementándose el desplazamiento del nudo de control de la estructura en cada paso del análisis y adaptando los valores de las fuerzas laterales para conseguir dicho desplazamiento. Resulta conveniente destacar que el período fundamental $T_{1}$ de los pórticos analizados se ha obtenido considerando exclusivamente las masas asociadas a las vigas y a los pilares que los constituyen. 
Tabla 6.8 Ancho tributario y cargas por metro lineal para los pórticos analizados.

\begin{tabular}{cccccc}
\hline Estructura & $\begin{array}{c}\text { Ancho } \\
\text { tributario } \\
(\mathrm{m})\end{array}$ & $\begin{array}{c}\text { Peso propio forjado } \\
\text { unidireccional } \\
(\mathrm{kN} / \mathrm{m})\end{array}$ & $\begin{array}{c}\text { Carga muerta } \\
\text { de solado } \\
(\mathrm{kN} / \mathrm{m})\end{array}$ & $\begin{array}{c}\text { Sobrecarga de } \\
\text { uso }(\mathrm{kN} / \mathrm{m})\end{array}$ & $\begin{array}{c}\text { Sobrecarga de } \\
\text { tabiquería } \\
(\mathrm{kN} / \mathrm{m})\end{array}$ \\
\hline $\begin{array}{l}\text { Pórtico } \\
\text { regular }\end{array}$ & 3,0 & 12,0 & 3,0 & 6,0 & 3,0 \\
$\begin{array}{l}\text { Pórtico } \\
\text { espacial }\end{array}$ & 3,2 & 12,8 & 3,2 & 6,4 & 3,2 \\
\hline
\end{tabular}

Tabla 6.9 Características del pórtico regular de cinco plantas para las distribuciones de carga lateral uniforme y modal.

\begin{tabular}{cccccc}
\hline \multirow{2}{*}{$\mathrm{N}^{\text {o }}$ planta } & $\begin{array}{c}m_{i} \\
\text { (ton) }\end{array}$ & $\frac{m_{i}}{\sum m_{i}}$ & $\begin{array}{c}T_{1} \\
(\mathrm{~s})\end{array}$ & $\begin{array}{c}s_{i} \\
(\mathrm{~mm})\end{array}$ & $\frac{s_{i} m_{i}}{\sum s_{i} m_{i}}$ \\
\hline 5 & 34,17 & 0,198 & & 0,200 & 0,273 \\
4 & 33,49 & 0,194 & & 0,200 & 0,268 \\
3 & 34,48 & 0,200 & 0,59 & 0,200 & 0,276 \\
2 & 34,48 & 0,200 & & 0,100 & 0,138 \\
1 & 35,63 & 0,207 & & 0,032 & 0,046 \\
\hline
\end{tabular}

Tabla 6.10 Características del pórtico regular de ocho plantas para las distribuciones de carga lateral uniforme y modal.

\begin{tabular}{|c|c|c|c|c|c|}
\hline $\mathrm{N}^{\mathrm{o}}$ planta & $\begin{array}{c}m_{i} \\
\text { (ton) }\end{array}$ & $\frac{m_{i}}{\sum m_{i}}$ & $\begin{array}{l}T_{l} \\
(\mathrm{~s})\end{array}$ & $\begin{array}{c}s_{i} \\
(\mathrm{~mm})\end{array}$ & $\frac{s_{i} m_{i}}{\sum s_{i} m_{i}}$ \\
\hline 8 & 34,17 & 0,123 & \multirow{8}{*}{0,96} & 0,200 & 0,210 \\
\hline 7 & 33,49 & 0,121 & & 0,200 & 0,206 \\
\hline 6 & 33,49 & 0,121 & & 0,200 & 0,206 \\
\hline 5 & 34,48 & 0,124 & & 0,100 & 0,106 \\
\hline 4 & 34,48 & 0,124 & & 0,100 & 0,106 \\
\hline 3 & 34,48 & 0,124 & & 0,100 & 0,106 \\
\hline 2 & 35,63 & 0,129 & & 0,041 & 0,045 \\
\hline 1 & 36,93 & 0,133 & & 0,014 & 0,015 \\
\hline
\end{tabular}

\subsubsection{Relaciones momento-giro y curvas de plastificación}

La caracterización de las rótulas plásticas mediante las expresiones de comportamiento seccional se realiza modificando las relaciones momento-giro y las curvas de plastificación respecto a lo que ETABS® considera por defecto. 
Tabla 6.11 Características del pórtico espacial de cinco plantas para las distribuciones de carga lateral uniforme y modal.

\begin{tabular}{cccccc}
\hline No planta & $\begin{array}{c}m_{i} \\
\text { (ton) }\end{array}$ & $\begin{array}{c}m_{i} \\
\sum m_{i}\end{array}$ & $\begin{array}{c}T_{l} \\
(\mathrm{~s})\end{array}$ & $\begin{array}{c}s_{i} \\
(\mathrm{~mm})\end{array}$ & $\frac{s_{i} m_{i}}{\sum s_{i} m_{i}}$ \\
\hline 5 & 33,67 & 0,197 & & 0,200 & 0,248 \\
4 & 32,90 & 0,192 & & 0,200 & 0,242 \\
3 & 33,89 & 0,198 & 0,52 & 0,200 & 0,249 \\
2 & 33,89 & 0,198 & & 0,100 & 0,125 \\
1 & 36,68 & 0,214 & & 0,100 & 0,135 \\
\hline
\end{tabular}

Tabla 6.12 Características del pórtico espacial de ocho plantas para las distribuciones de carga lateral uniforme y modal.

\begin{tabular}{cccccc}
\hline $\mathrm{N}^{\mathrm{o}}$ planta & $\begin{array}{c}m_{i} \\
\text { (ton) }\end{array}$ & $\begin{array}{c}m_{i} \\
\sum m_{i}\end{array}$ & $\begin{array}{c}T_{l} \\
(\mathrm{~s})\end{array}$ & $\begin{array}{c}s_{i} \\
(\mathrm{~mm})\end{array}$ & $\frac{s_{i} m_{i}}{\sum s_{i} m_{i}}$ \\
\hline 8 & 33,67 & 0,123 & & 0,200 & 0,195 \\
7 & 32,90 & 0,119 & & 0,200 & 0,190 \\
6 & 32,90 & 0,119 & & 0,200 & 0,190 \\
5 & 33,89 & 0,123 & & 0,100 & 0,098 \\
4 & 33,89 & 0,123 & 0,79 & 0,100 & 0,098 \\
3 & 33,89 & 0,123 & & 0,100 & 0,098 \\
2 & 35,04 & 0,128 & & 0,100 & 0,101 \\
1 & 38,41 & 0,139 & & 0,027 & 0,030 \\
\hline
\end{tabular}

Las Figuras 6.19 y 6.20 muestran las relaciones momento flector-giro de las rótulas plásticas para las vigas y los pilares, obtenidas según diferentes métodos. Para poder comparar las relaciones momento-giro obtenidas mediante los distintos métodos, se ha considerado como valor del momento flector posterior al estado último, representado por el punto D de la Figura 6.10, el propuesto por ETABS®, esto es, el 20 $\%$ del valor máximo. De las Figuras 6.19 y 6.20 se deduce que, para las vigas, los valores del momento de plastificación $M_{y}$ obtenidos al aplicar la expresión de Biskinis y Fardis (2010a) resultan similares a los supuestos por ETABS ${ }^{\circ}$, obteniéndose los máximos valores de $M_{y}$, tanto en las vigas como en los pilares, al emplear las expresiones calibradas con la selección de ensayos. Analizando la capacidad de deformación de las rótulas plásticas, se deduce que, a diferencia de las propuestas por ETABS $®$, las rótulas obtenidas para las expresiones de Biskinis y Fardis (2010a, 2010b) y las expresiones calibradas con la selección de ensayos carecen de capacidad de deformación posterior al estado último, proporcionando las expresiones de Biskinis y Fardis (2010a, 2010b) un valor superior del giro último $\theta_{u}$ de la sección en todos los casos. 


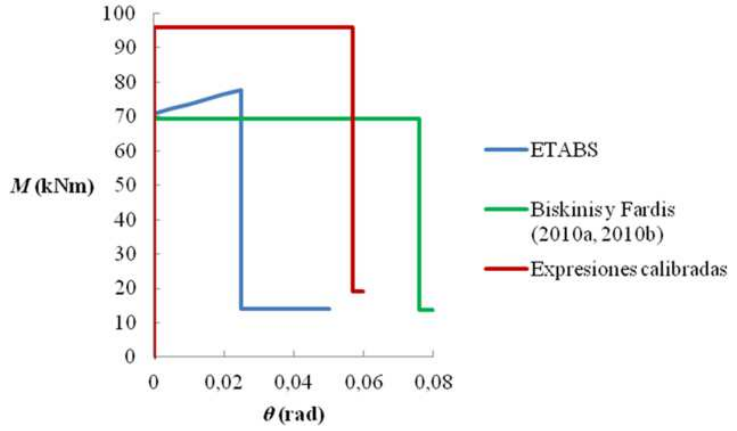

(a)

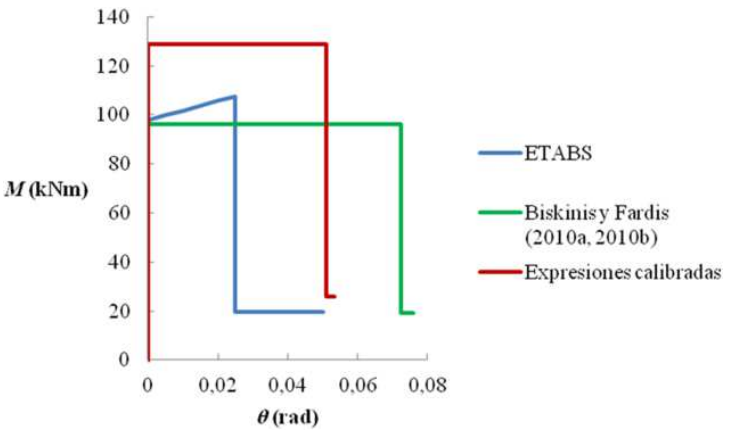

(b)

Figura 6.19 Relaciones momento-giro para las rótulas plásticas generadas en las vigas. (a) Viga $500 \times 300 \mathrm{~mm}$. (b) Viga 300×400 mm.

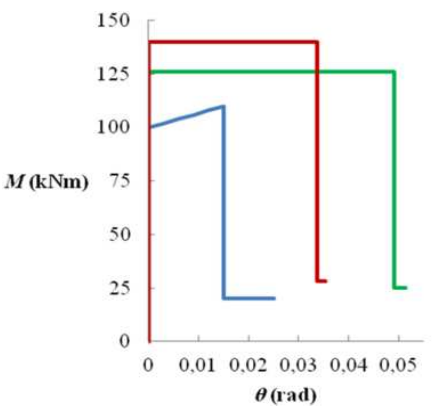

(a)

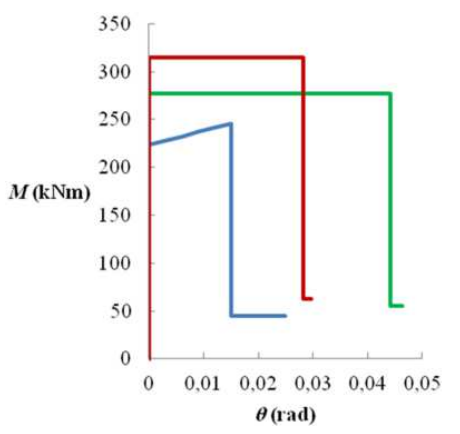

(c)
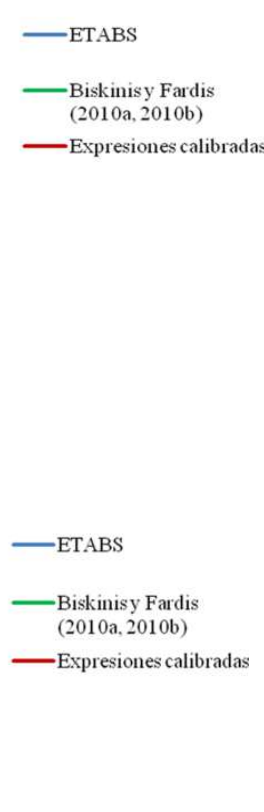

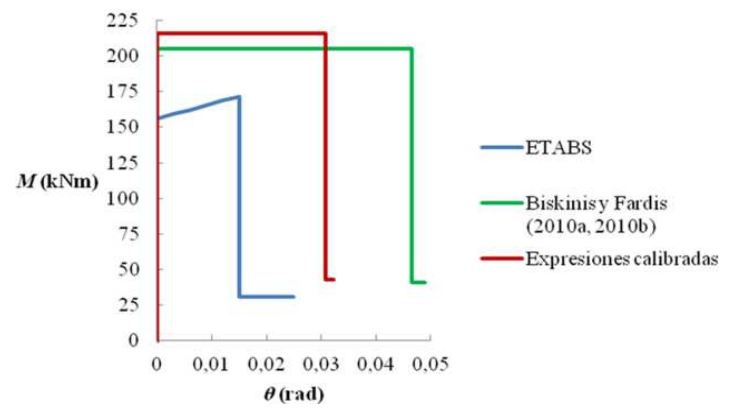

(b)

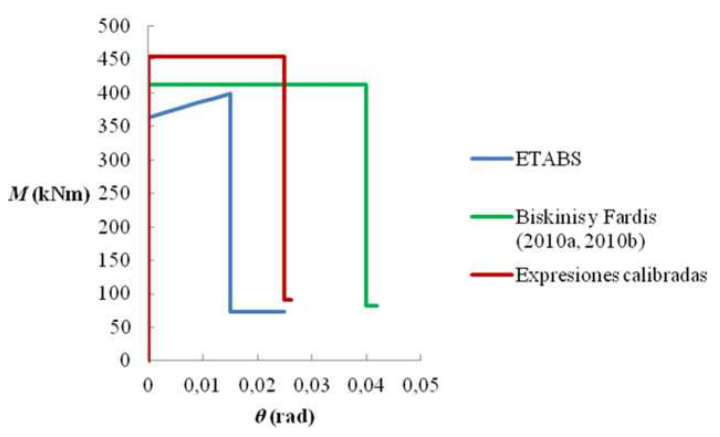

(d)

Figura 6.20 Relaciones momento-giro para las rótulas plásticas generadas en los pilares. (a) Pilar P1. (b) Pilar P2. (c) Pilar P3. (d) Pilar P4.

Puesto que los esfuerzos axiles varían en los pilares durante el análisis, resulta necesario definir curvas de plastificación que modelicen la interacción esfuerzo axilmomento flector de plastificación para caracterizar las rótulas plásticas generadas en estos elementos. A diferencia de otros estudios que suponen constantes los esfuerzos axiles en los pilares (Inel y Ozmen, 2006), se han definido curvas de plastificación empleando las expresiones de comportamiento seccional consideradas en este estudio. La Figura 6.21 muestra las curvas de plastificación obtenidas según los diferentes métodos, donde los esfuerzos axiles presentan un signo positivo para compresión y 
negativo en caso contrario. Resulta necesario destacar que las curvas obtenidas con las expresiones de comportamiento seccional presentan en general valores de momento de plastificación $M_{y}$ superiores a los ofrecidos por ETABS®. Si bien los valores máximos de $M_{y}$ se obtienen para las expresiones calibradas con la selección de ensayos, las expresiones de Biskinis y Fardis (2010a) proporcionan valores de $M_{y}$ superiores para esfuerzos axiles elevados.

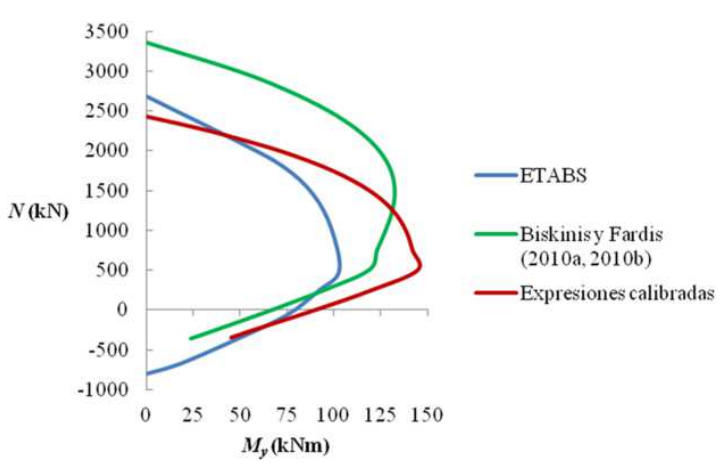

(a)

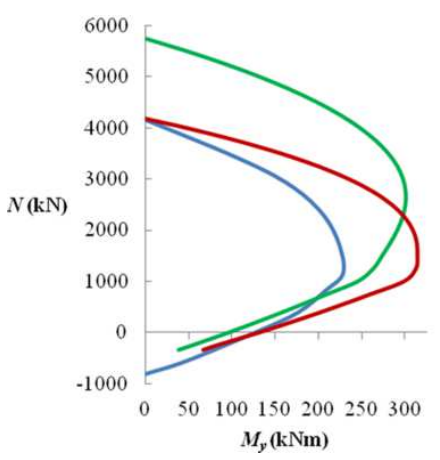

(c)

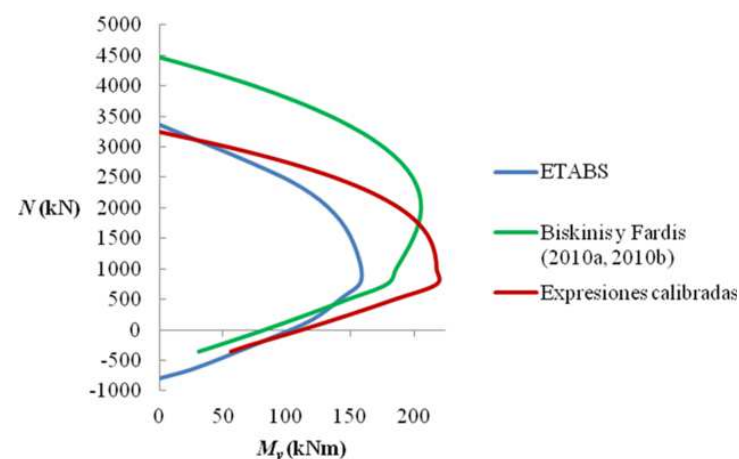

(b)

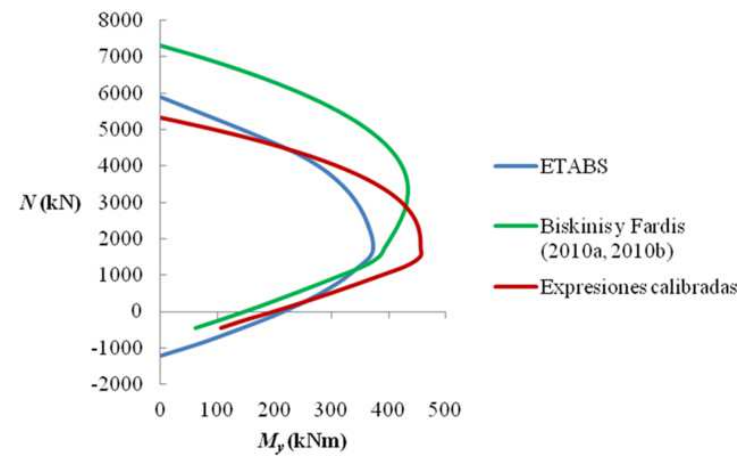

(d)

Figura 6.21 Curvas de plastificación para los pilares de los pórticos. (a) Pilar P1. (b) Pilar P2. (c) Pilar P3. (d) Pilar P4.

\subsubsection{Curvas de capacidad}

Las Figuras 6.22 a 6.25 muestran las curvas de capacidad obtenidas tras realizar los análisis pushover. Para cada estructura analizada se muestran las curvas correspondientes a los casos de carga lateral uniforme y modal. En todos los casos analizados se deduce que las rótulas plásticas de ETABS ${ }^{\circledR}$ y las modelizadas con las expresiones de Biskinis y Fardis (2010a, 2010b) proporcionan valores similares del esfuerzo cortante en la base $F_{b}$ para desplazamientos del nudo de control $\delta$ de hasta 250 mm. Los valores máximos de $F_{b}$ se consiguen al analizar los pórticos espaciales considerando el caso de carga lateral uniforme. Se observa además que, al aumentar $\delta$, se producen disminuciones más acentuadas en los valores de $F_{b}$ para los pórticos espaciales. 
Resulta necesario destacar la relación existente entre $F_{b}$ y el momento de plastificación $M_{y}$ de las rótulas plásticas, aumentando el valor de $F_{b}$ con los valores de $M_{y}$. Este aspecto justifica que los máximos valores de $F_{b}$ se alcancen al considerar las rótulas plásticas modelizadas mediante las expresiones calibradas con la selección de ensayos, pues, de acuerdo con las Figuras 6.19 a 6.21, éstas presentan los mayores valores de $M_{y}$. Otro aspecto relevante lo constituye el hecho de que las curvas de capacidad obtenidas al aplicar las expresiones calibradas con la selección de ensayos y las ecuaciones de Biskinis y Fardis (2010a, 2010b) se cruzan para un cierto valor de $\delta$, ya que, de acuerdo con la Figura 6.21, dichas expresiones pueden adoptar el mismo valor de $M_{y}$ y, en consecuencia, de $F_{b}$.

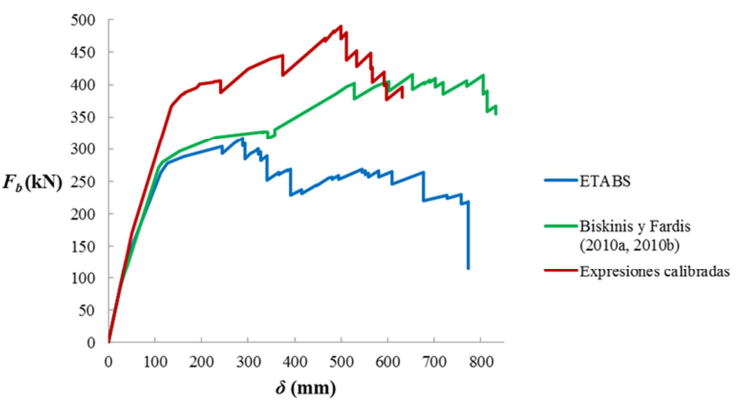

(a)

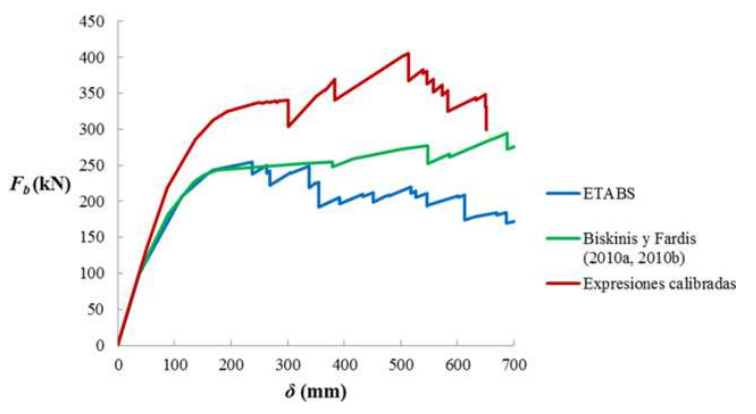

(b)

Figura 6.22 Curvas de capacidad para el pórtico regular de cinco plantas. (a) Carga lateral uniforme. (b) Carga lateral modal.

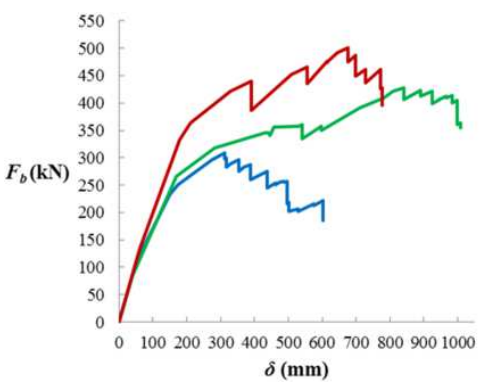

(a)
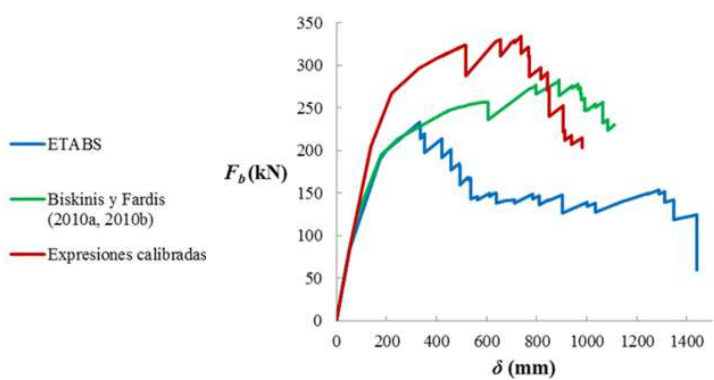

(b)

Figura 6.23 Curvas de capacidad para el pórtico regular de ocho plantas. (a) Carga lateral uniforme. (b) Carga lateral modal.

\subsubsection{Resultados en el punto de plastificación global de la estructura}

Inel y Ozmen (2006) definen la plastificación global de la estructura como el punto de la curva de capacidad en el que la estructura deja de comportarse de forma elástica y lineal. Las Tablas 6.13 a 6.16 muestran los valores obtenidos para el desplazamiento del nudo de control $\delta$, el esfuerzo cortante en la base $F_{b}$ y la aceleración $S_{a} / g$ del sistema equivalente de un grado de libertad en el punto de plastificación global de las estructuras analizadas. 


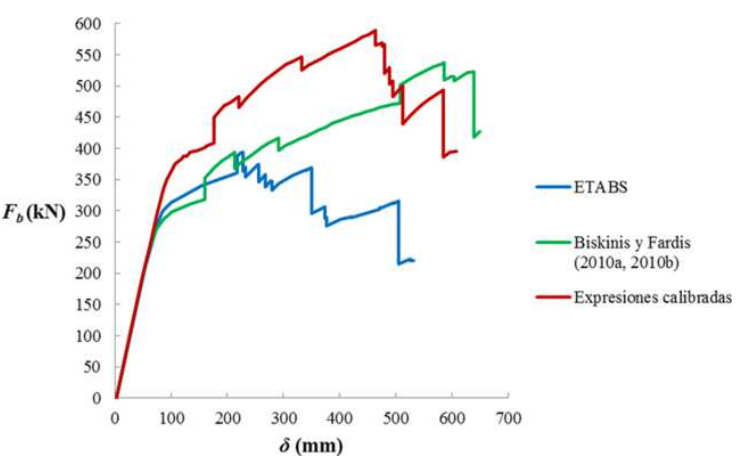

(a)

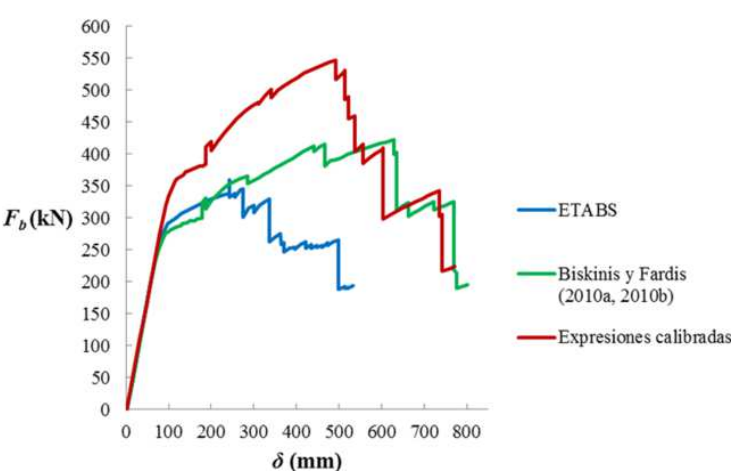

(b)

Figura 6.24 Curvas de capacidad para el pórtico espacial de cinco plantas. (a) Carga lateral uniforme. (b) Carga lateral modal.

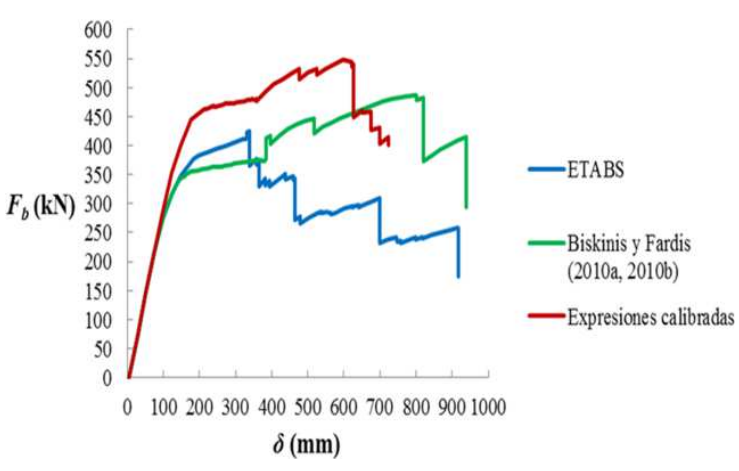

(a)

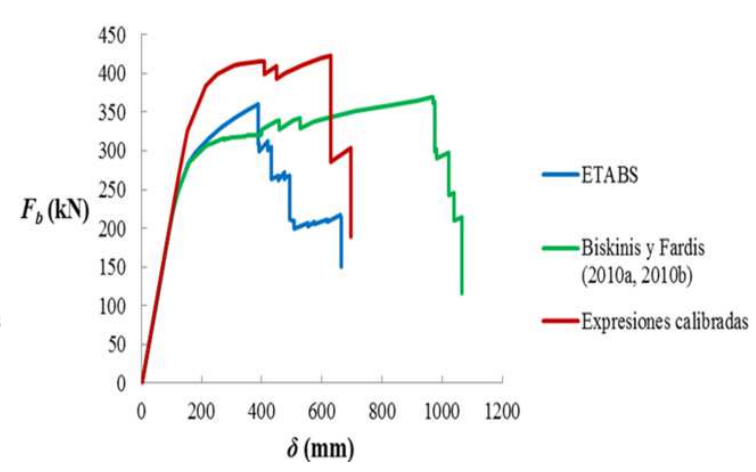

(b)

Figura 6.25 Curvas de capacidad para el pórtico espacial de ocho plantas. (a) Carga lateral uniforme. (b) Carga lateral modal.

Para obtener el valor de $S_{a} / g$, resulta necesario transformar la curva de capacidad $F_{b}-\delta$ en el espectro de capacidad en formato aceleración-desplazamiento $\left(S_{a}-S_{d}\right.$ ') para el sistema equivalente de un grado de libertad. A cada punto $F_{b}-\delta$ de la curva de capacidad le corresponde un punto $S_{a}-S_{d}$ ' del espectro de capacidad, aplicándose las ecuaciones (6.24) y (6.25), propuestas en la ATC-40 (1996):

$$
\begin{gathered}
S_{a}=\frac{F_{b}}{W \alpha_{1}} \\
S_{d}{ }^{\prime}=\frac{\delta}{P F_{1} \phi_{1}}
\end{gathered}
$$

siendo

$P F_{1}$ factor de participación modal correspondiente al modo fundamental,

$W$ peso total de la estructura, considerando las masas obtenidas al aplicar la ecuación (6.5),

$\alpha_{1} \quad$ coeficiente de masa modal correspondiente al modo fundamental, y

$\phi_{1}$ desplazamiento normalizado del nudo de control para el modo fundamental. 
Los resultados mostrados en las Tablas 6.13 a 6.16 corresponden a las distribuciones de carga lateral uniforme y modal y a los diferentes métodos de modelización de las rótulas plásticas. En general se observa que el caso de carga lateral modal obtiene valores de desplazamiento $\delta$ superiores a los ofrecidos al aplicar la carga uniforme, resultando inferiores los valores de $F_{b}$.

Tabla 6.13 Resultados en el punto de plastificación global para el pórtico regular de cinco plantas.

\begin{tabular}{cccccccc}
\hline & \multicolumn{3}{c}{ Cargas uniformes } & \multicolumn{3}{c}{ Cargas modales } \\
Tipo de rótula plástica & $\delta$ & $F_{b}$ & $S_{a} / g$ & $\delta$ & $F_{b}$ & \\
& $(\mathrm{~mm})$ & $(\mathrm{kN})$ & & $(\mathrm{mm})$ & $(\mathrm{kN})$ & $S_{a} / g$ \\
\hline ETABS® & 128 & 278 & 0,197 & 169 & 243 & 0,189 \\
Biskinis y Fardis (2010a, 2010b) & 116 & 279 & 0,204 & 176 & 243 & 0,186 \\
Expresiones calibradas & 138 & 370 & 0,282 & 194 & 324 & 0,250 \\
\hline
\end{tabular}

Tabla 6.14 Resultados en el punto de plastificación global para el pórtico regular de ocho plantas.

\begin{tabular}{cccccccc}
\hline & \multicolumn{3}{c}{ Cargas uniformes } & \multicolumn{3}{c}{ Cargas modales } \\
Tipo de rótula plástica & $\delta$ & $F_{b}$ & & $\delta$ & $F_{b}$ & $S_{a} / g$ \\
& $(\mathrm{~mm})$ & $(\mathrm{kN})$ & $S_{a} / g$ & $(\mathrm{~mm})$ & $(\mathrm{kN})$ & \\
\hline ETABS $®$ & 175 & 251 & 0,109 & 175 & 189 & 0,093 \\
Biskinis y Fardis (2010a, 2010b) & 169 & 267 & 0,118 & 179 & 195 & 0,108 \\
Expresiones calibradas & 210 & 364 & 0,172 & 220 & 267 & 0,127 \\
\hline
\end{tabular}

Las rótulas plásticas modelizadas con las expresiones ajustadas a la selección de ensayos proporcionan los mayores valores de $\delta, F_{b}$ y $S_{a} / g$ para todos los casos analizados, debido a que dichas expresiones ofrecen los mayores valores de $M_{y}$. Al analizar las diferencias en $\delta$ respecto a lo obtenido con otros métodos, las expresiones calibradas proporcionan mayores diferencias al aumentar la altura de la estructura analizada, resultando superiores para el patrón de cargas laterales modales.

Tabla 6.15 Resultados en el punto de plastificación global para el pórtico espacial de cinco plantas.

\begin{tabular}{cccccccc}
\hline & \multicolumn{3}{c}{ Cargas uniformes } & \multicolumn{3}{c}{ Cargas modales } \\
Tipo de rótula plástica & $\delta$ & $F_{b}$ & $S_{a} / g$ & $\delta$ & $F_{b}$ & $S_{a} / g$ \\
& $(\mathrm{~mm})$ & $(\mathrm{kN})$ & & $(\mathrm{mm})$ & $(\mathrm{kN})$ & \\
\hline ETABS® & 99 & 312 & 0,204 & 97 & 290 & 0,202 \\
Biskinis y Fardis (2010a, 2010b) & 101 & 299 & 0,192 & 93 & 276 & 0,189 \\
Expresiones calibradas & 107 & 375 & 0,241 & 118 & 361 & 0,246 \\
\hline
\end{tabular}


Tabla 6.16 Resultados en el punto de plastificación global para el pórtico espacial de ocho plantas.

\begin{tabular}{cccccccc}
\hline & \multicolumn{3}{c}{ Cargas uniformes } & \multicolumn{3}{c}{ Cargas modales } \\
Tipo de rótula plástica & $\delta$ & $F_{b}$ & & & $\delta$ & $F_{b}$ & $S_{a} / g$ \\
& $(\mathrm{~mm})$ & $(\mathrm{kN})$ & $S_{a} / g$ & $(\mathrm{~mm})$ & $(\mathrm{kN})$ & \\
\hline ETABS® & 171 & 368 & 0,074 & 157 & 284 & 0,079 \\
Biskinis y Fardis (2010a, 2010b) & 159 & 348 & 0,071 & 164 & 288 & 0,081 \\
Expresiones calibradas & 177 & 444 & 0,103 & 213 & 384 & 0,101 \\
\hline
\end{tabular}

Las diferencias en $\delta$ oscilan entre el 3 y el $35 \%$ para el pórtico espacial de ocho plantas analizado con cargas laterales uniformes y modales, respectivamente. Ambas diferencias se obtienen respecto a las rótulas plásticas de ETABS®. Las diferencias en $F_{b}$ respecto a otros métodos resultan similares para ambos patrones de carga, aumentando al incrementarse la altura de la estructura. Dichas diferencias están comprendidas entre el $20 \%$ respecto al pórtico espacial de cinco plantas y el $45 \%$ respecto al pórtico regular de ocho plantas, estando ambas estructuras analizadas con las rótulas plásticas de ETABS® y sujetas a cargas laterales uniformes. En lo que respecta a $S_{a} / g$, el patrón de cargas laterales uniformes induce mayores diferencias, incrementándose las mismas con la altura de la estructura. Las diferencias en este parámetro están comprendidas entre el $17 \%$ respecto al pórtico regular de ocho plantas con cargas laterales modales y rótulas modelizadas con las expresiones de Biskinis y Fardis $(2010 \mathrm{a}, 2010 \mathrm{~b})$ y el 57 \% respecto al mismo pórtico con cargas laterales uniformes y rótulas de ETABS®.

Autores como Inel y Ozmen (2006) asignan a los niveles de comportamiento IO, LS y CP el 10, 60 y $90 \%$ del giro último $\theta_{u}$ de la sección del elemento donde se genera la rótula plástica. Con objeto de poder comparar los resultados ofrecidos por los diferentes métodos, en este estudio se han adoptado los porcentajes considerados por ETABS $®$, expuestos en la Tabla 6.7, para las rótulas plásticas modelizadas mediante las expresiones calibradas con la selección de ensayos y las expresiones de Biskinis y Fardis (2010a, 2010b). Los colores asociados a los diferentes niveles de comportamiento y a los puntos B, C, D y E mostrados en la curva de respuesta de la Figura 6.10 se muestran en la Figura 6.26.

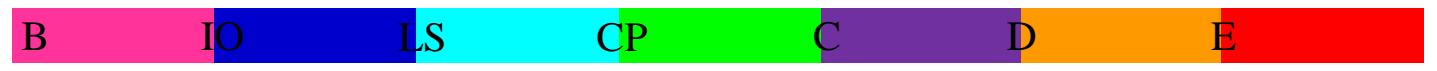

Figura 6.26 Colores asociados a los niveles de comportamiento IO, LS y CP y a los puntos B,

C, D y E de la relación momento-giro de las rótulas plásticas.

Las Figuras 6.27 a 6.34 muestran la distribución de rótulas plásticas generadas en las estructuras para el punto de plastificación global. Para todos los casos analizados las expresiones calibradas con la selección de ensayos proporcionan una distribución de 
rótulas plásticas menos desfavorable que las obtenidas empleando otros métodos, obteniéndose un menor número de rótulas y unos niveles de comportamiento más alejados del colapso, debido a los mayores valores del momento de plastificación $M_{y}$ obtenidos con dichas expresiones.

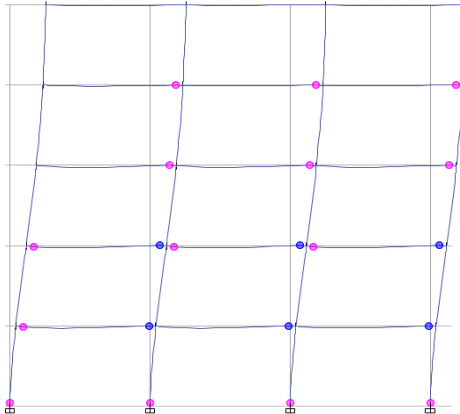

(a)

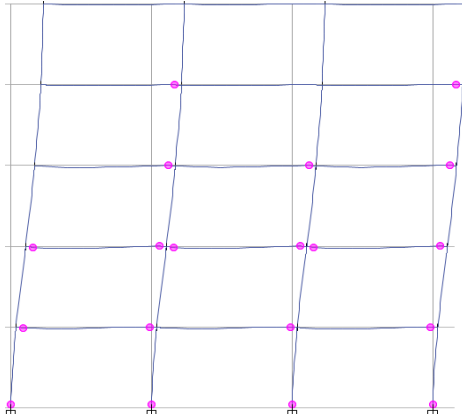

(b)

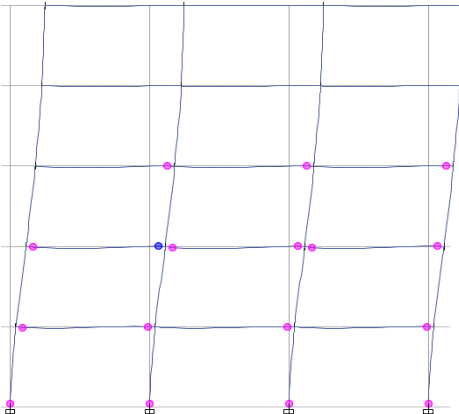

(c)

Figura 6.27 Distribución de rótulas plásticas para el pórtico regular de cinco plantas con cargas laterales uniformes en el punto de plastificación global. (a) ETABS®. (b) Biskinis y Fardis (2010a, 2010b). (c) Expresiones calibradas.

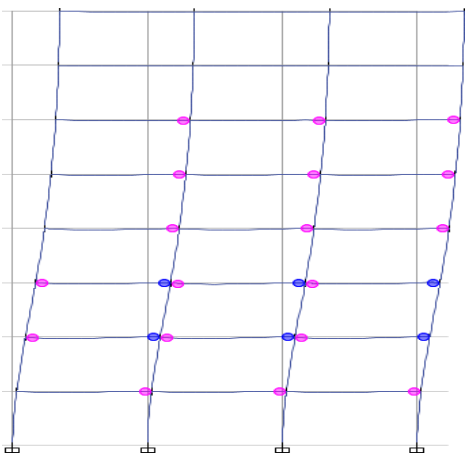

(a)

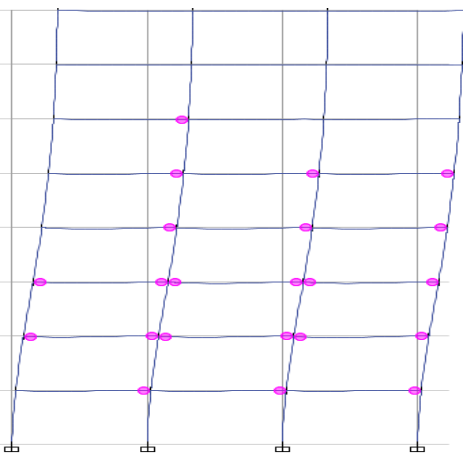

(b)

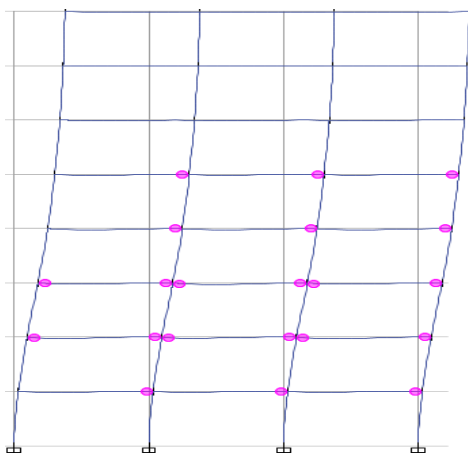

(c)

Figura 6.28 Distribución de rótulas plásticas para el pórtico regular de ocho plantas con cargas laterales uniformes en el punto de plastificación global. (a) ETABS®. (b) Biskinis y Fardis (2010a, 2010b). (c) Expresiones calibradas.

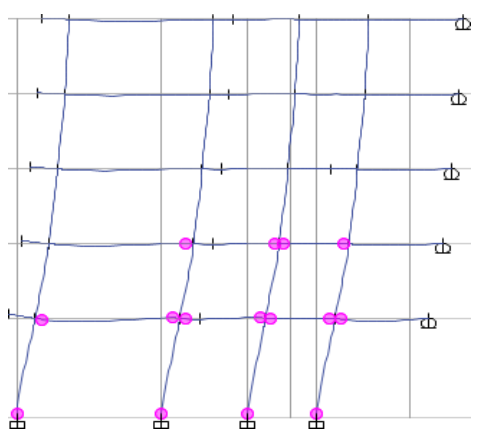

(a)

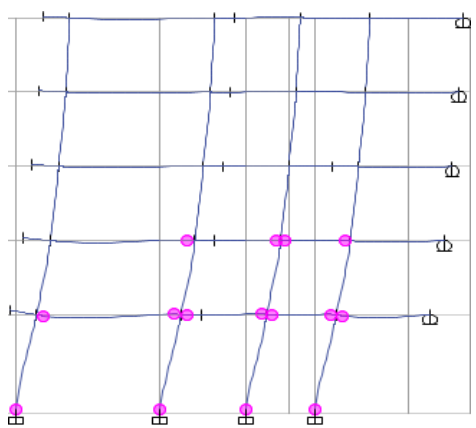

(b)

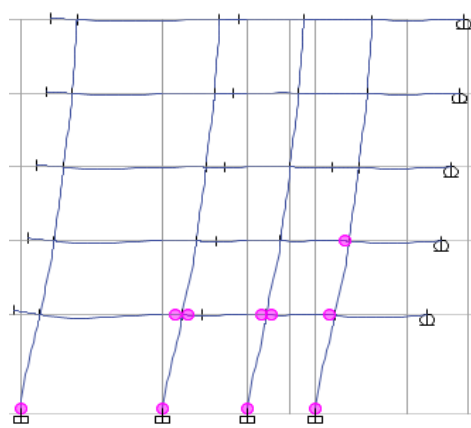

(c)

Figura 6.29 Distribución de rótulas plásticas para el pórtico espacial de cinco plantas con cargas laterales uniformes en el punto de plastificación global. (a) ETABS®. (b) Biskinis y Fardis (2010a, 2010b). (c) Expresiones calibradas. 


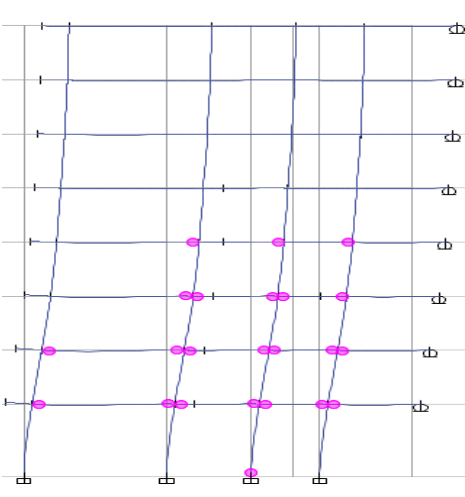

(a)

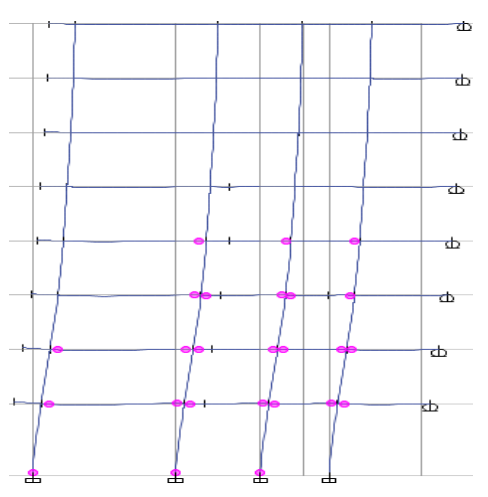

(b)

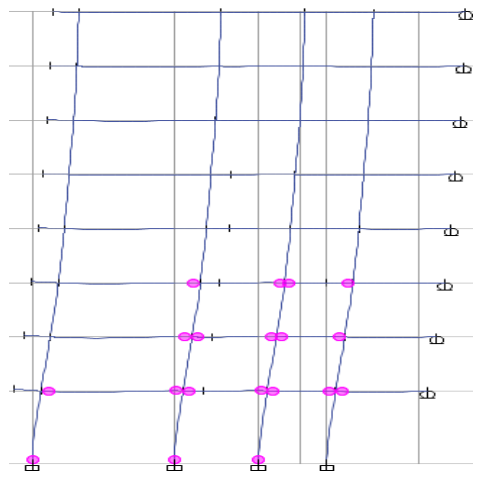

(c)

Figura 6.30 Distribución de rótulas plásticas para el pórtico espacial de ocho plantas con cargas laterales uniformes en el punto de plastificación global. (a) ETABS®. (b) Biskinis y Fardis (2010a, 2010b). (c) Expresiones calibradas.

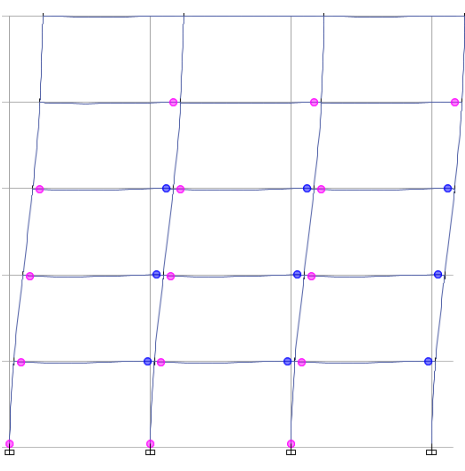

(a)

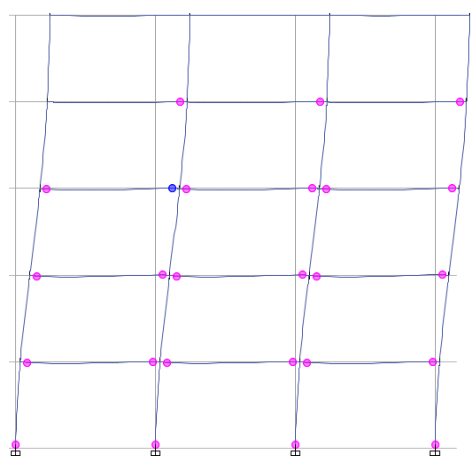

(b)

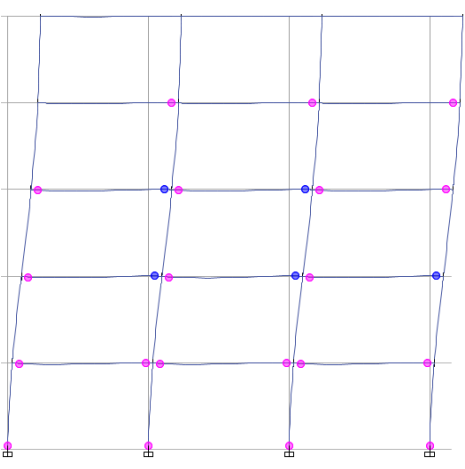

(c)

Figura 6.31 Distribución de rótulas plásticas para el pórtico regular de cinco plantas con cargas laterales modales en el punto de plastificación global. (a) ETABS®. (b) Biskinis y Fardis (2010a, 2010b). (c) Expresiones calibradas.

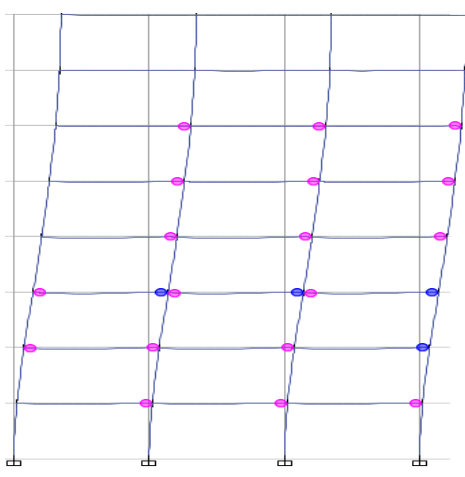

(a)

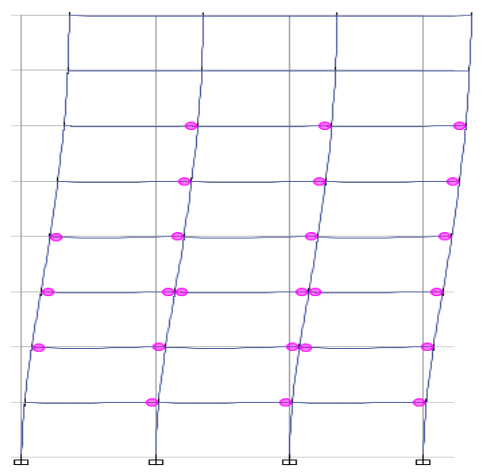

(b)

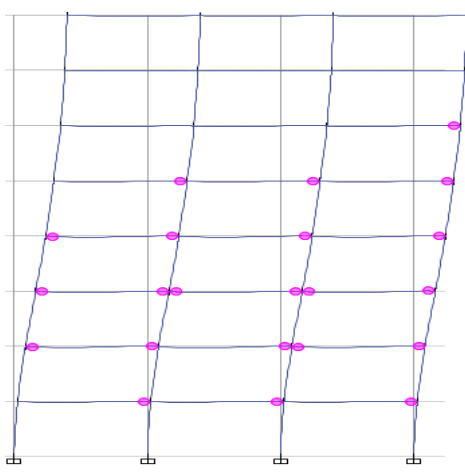

(c)

Figura 6.32 Distribución de rótulas plásticas para el pórtico regular de ocho plantas con cargas laterales modales en el punto de plastificación global. (a) ETABS@. (b) Biskinis y Fardis (2010a, 2010b). (c) Expresiones calibradas. 


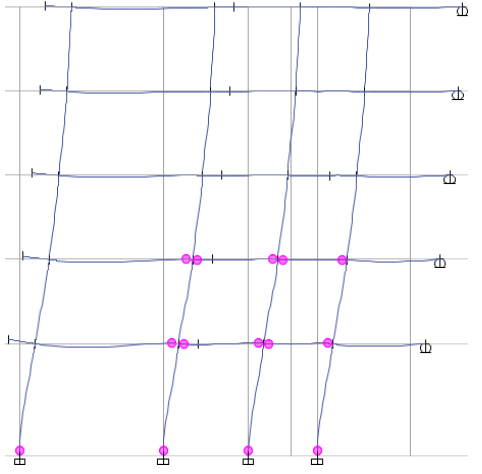

(a)

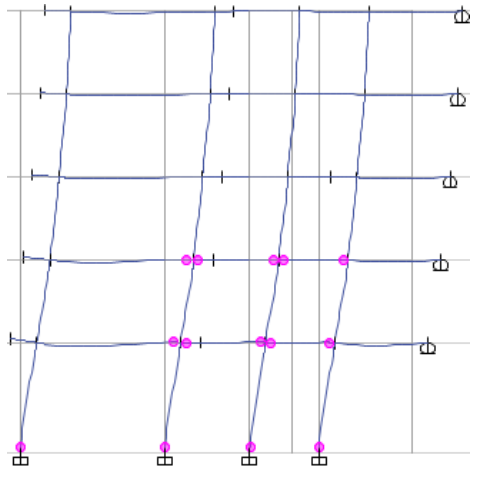

(b)

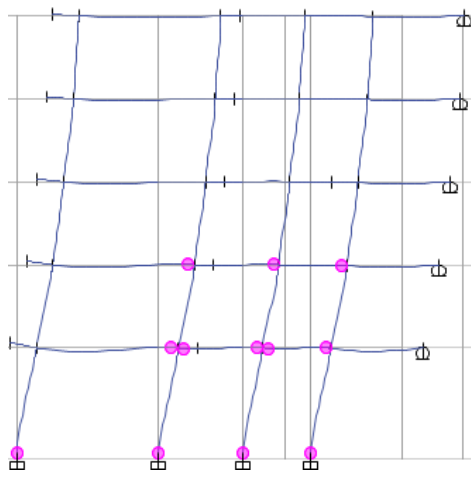

(c)

Figura 6.33 Distribución de rótulas plásticas para el pórtico espacial de cinco plantas con cargas laterales modales en el punto de plastificación global. (a) ETABS®. (b) Biskinis y Fardis (2010a, 2010b). (c) Expresiones calibradas.

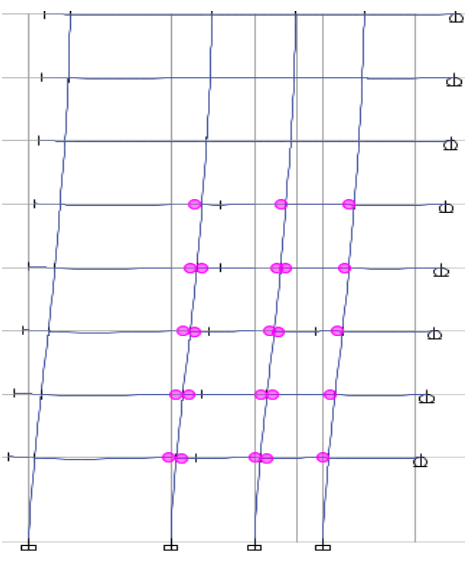

(a)

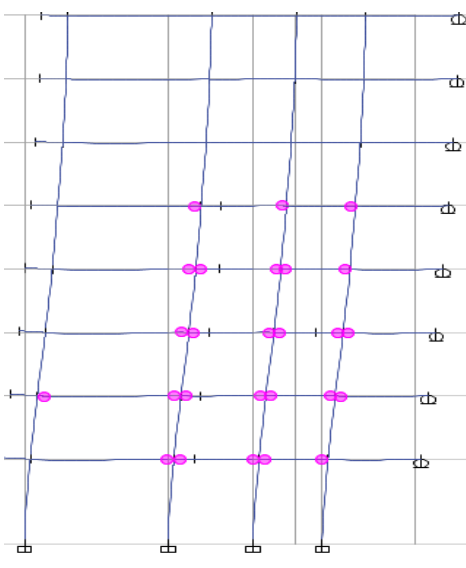

(b)

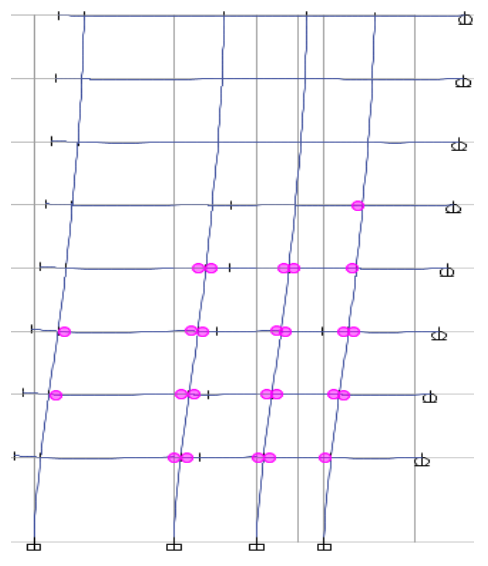

(c)

Figura 6.34 Distribución de rótulas plásticas para el pórtico espacial de ocho plantas con cargas laterales modales en el punto de plastificación global. (a) ETABS®. (b) Biskinis y Fardis (2010a, 2010b). (c) Expresiones calibradas.

\subsubsection{Resultados en el punto de colapso de la estructura}

Resulta de interés la obtención de ciertos parámetros en el punto de colapso de la estructura. El concepto de colapso resulta subjetivo, siendo en ocasiones un aspecto a considerar en función del nivel de seguridad que se quiera adoptar para la estructura. Así, autores como Inel y Ozmen (2006) suponen el colapso de la estructura al producirse en la curva de capacidad una disminución del $20 \%$ en $F_{b}$. Otros, como Panagiotakos y Fardis (2001), consideran que se produce la rotura de la sección transversal de un elemento al registrarse una reducción del $15 \%$ en la carga lateral aplicada en el ensayo. En este estudio se ha considerado que se produce el colapso de las estructuras al tener lugar el primer descenso mayor o igual del $15 \%$ en $F_{b}$. Las Tablas 6.17 a 6.20 muestran los valores obtenidos para el desplazamiento del nudo de control $\delta$, el esfuerzo cortante en la base $F_{b}$ y el valor de la aceleración $S_{a} / g$ 
correspondiente a un sistema equivalente de un grado de libertad en el punto de colapso de las estructuras analizadas, aplicando los dos patrones de carga lateral y los distintos métodos considerados para modelizar las rótulas plásticas. Se ha incluido además el \% de disminución de $F_{b}$ en la curva de capacidad para el punto de colapso. Los valores de $F_{b}$ y $S_{a} / g$ obtenidos resultan superiores para el caso de carga lateral uniforme. Si se analiza la influencia de la altura de la estructura, la mayor flexibilidad de las estructuras de ocho plantas propicia generalmente la obtención de valores superiores de $\delta$ e inferiores para $F_{b}$ y $S_{a} / g$.

Tabla 6.17 Resultados en el punto de colapso para el pórtico regular de cinco plantas.

\begin{tabular}{|c|c|c|c|c|c|c|c|c|}
\hline \multirow[b]{2}{*}{ Tipo de rótula plástica } & \multicolumn{4}{|c|}{ Cargas uniformes } & \multicolumn{4}{|c|}{ Cargas modales } \\
\hline & $\begin{array}{c}\delta \\
(\mathrm{mm})\end{array}$ & $\begin{array}{c}F_{b} \\
(\mathrm{kN})\end{array}$ & $S_{a} / g$ & $\begin{array}{c}\text { Disminución } \\
\text { de } F_{b} \\
(\%)\end{array}$ & $\begin{array}{c}\delta \\
(\mathrm{mm})\end{array}$ & $\begin{array}{c}F_{b} \\
(\mathrm{kN})\end{array}$ & $S_{a} / g$ & $\begin{array}{c}\text { Disminución } \\
\text { de } F_{b} \\
(\%)\end{array}$ \\
\hline ETABS $®$ & 391 & 269 & 0,217 & 15 & 354 & 226 & 0,153 & 15 \\
\hline $\begin{array}{c}\text { Biskinis y Fardis } \\
(2010 a, 2010 b)\end{array}$ & 805 & 414 & 0,308 & 15 & 547 & 277 & 0,212 & 10 \\
\hline Expresiones calibradas & 565 & 449 & 0,345 & 16 & 545 & 380 & 0,299 & 15 \\
\hline
\end{tabular}

Las expresiones de Biskinis y Fardis (2010a, 2010b) obtienen los mayores valores de $\delta$, con diferencias que pueden oscilar entre el $0,37 \%$ respecto a las expresiones calibradas con la selección de ensayos para el pórtico regular de cinco plantas con carga lateral modal y el $151 \%$ respecto a ETABS $®$ para el pórtico espacial de ocho plantas con carga lateral modal. Estas expresiones presentan además los menores porcentajes de disminución de $F_{b}$ para el punto de colapso, habiéndose considerado como excepción valores inferiores al $15 \%$.

Tabla 6.18 Resultados en el punto de colapso para el pórtico regular de ocho plantas.

\begin{tabular}{|c|c|c|c|c|c|c|c|c|}
\hline \multirow[b]{2}{*}{ Tipo de rótula plástica } & \multicolumn{4}{|c|}{ Cargas uniformes } & \multicolumn{4}{|c|}{ Cargas modales } \\
\hline & $\begin{array}{c}\delta \\
(\mathrm{mm})\end{array}$ & $\begin{array}{c}F_{b} \\
(\mathrm{kN})\end{array}$ & $S_{a} / g$ & $\begin{array}{c}\text { Disminución } \\
\text { de } F_{b} \\
(\%) \\
\end{array}$ & $\begin{array}{c}\delta \\
(\mathrm{mm})\end{array}$ & $\begin{array}{c}F_{b} \\
(\mathrm{kN})\end{array}$ & $S_{a} / g$ & $\begin{array}{c}\text { Disminución } \\
\text { de } F_{b} \\
(\%)\end{array}$ \\
\hline ETABS® & 496 & 258 & 0,125 & 22 & 537 & 167 & 0,074 & 14 \\
\hline $\begin{array}{c}\text { Biskinis y Fardis } \\
(2010 a, 2010 b)\end{array}$ & 998 & 405 & 0,216 & 13 & 967 & 278 & 0,144 & 11 \\
\hline Expresiones calibradas & 773 & 462 & 0,242 & 15 & 843 & 291 & 0,138 & 15 \\
\hline
\end{tabular}


Tabla 6.19 Resultados en el punto de colapso para el pórtico espacial de cinco plantas.

\begin{tabular}{|c|c|c|c|c|c|c|c|c|}
\hline \multirow[b]{2}{*}{ Tipo de rótula plástica } & \multicolumn{4}{|c|}{ Cargas uniformes } & \multicolumn{4}{|c|}{ Cargas modales } \\
\hline & $\begin{array}{c}\delta \\
(\mathrm{mm})\end{array}$ & $\begin{array}{c}F_{b} \\
(\mathrm{kN})\end{array}$ & $S_{a} / g$ & $\begin{array}{c}\text { Disminución } \\
\text { de } F_{b} \\
(\%)\end{array}$ & $\begin{array}{c}\delta \\
(\mathrm{mm})\end{array}$ & $\begin{array}{c}F_{b} \\
(\mathrm{kN})\end{array}$ & $S_{a} / g$ & $\begin{array}{c}\text { Disminución } \\
\text { de } F_{b} \\
(\%)\end{array}$ \\
\hline ETABS $®$ & 350 & 369 & 0,247 & 20 & 275 & 345 & 0,230 & 13 \\
\hline $\begin{array}{l}\text { Biskinis y Fardis } \\
(2010 a, 2010 b)\end{array}$ & 640 & 523 & 0,369 & 23 & 630 & 422 & 0,310 & 25 \\
\hline Expresiones calibradas & 480 & 567 & 0,404 & 15 & 515 & 531 & 0,370 & 24 \\
\hline
\end{tabular}

Tabla 6.20 Resultados en el punto de colapso para el pórtico espacial de ocho plantas.

\begin{tabular}{|c|c|c|c|c|c|c|c|c|}
\hline \multirow[b]{2}{*}{ Tipo de rótula plástica } & \multicolumn{4}{|c|}{ Cargas uniformes } & \multicolumn{4}{|c|}{ Cargas modales } \\
\hline & $\begin{array}{c}\delta \\
(\mathrm{mm})\end{array}$ & $\begin{array}{c}F_{b} \\
(\mathrm{kN})\end{array}$ & $S_{a} / g$ & $\begin{array}{c}\text { Disminución } \\
\qquad \begin{array}{c}\text { de } F_{b} \\
(\%)\end{array}\end{array}$ & $\begin{array}{c}\delta \\
(\mathrm{mm})\end{array}$ & $\begin{array}{c}F_{b} \\
(\mathrm{kN})\end{array}$ & $S_{a} / g$ & $\begin{array}{c}\text { Disminución } \\
\qquad \begin{array}{c}\text { de } F_{b} \\
(\%)\end{array}\end{array}$ \\
\hline ETABS $®$ & 463 & 344 & 0,085 & 20 & 388 & 361 & 0,098 & 20 \\
\hline $\begin{array}{l}\text { Biskinis y Fardis } \\
(2010 a, 2010 b)\end{array}$ & 802 & 478 & 0,128 & 22 & 976 & 363 & 0,101 & 20 \\
\hline Expresiones calibradas & 628 & 540 & 0,141 & 17 & 633 & 423 & 0,116 & 32 \\
\hline
\end{tabular}

Las expresiones calibradas con la selección de ensayos proporcionan los mayores valores de $F_{b}$, estando las diferencias comprendidas entre el $5 \%$ respecto a las expresiones de Biskinis y Fardis (2010a, 2010b) empleadas en el pórtico regular de ocho plantas con carga lateral modal y el $79 \%$ para el mismo pórtico analizado con las expresiones de ETABS $®$ y carga lateral uniforme. En cuanto a los valores de $S_{a} / g$, las expresiones calibradas con la selección de ensayos proporcionan valores superiores a los ofrecidos por otros métodos, obteniéndose una diferencia máxima del $95 \%$ respecto a lo obtenido aplicando las expresiones de ETABS® para el pórtico regular de cinco plantas sometido a la distribución de cargas laterales de tipo modal.

La distribución de rótulas plásticas en el punto de colapso para las diferentes estructuras analizadas se muestra en las Figuras 6.35 a 6.42. Al igual que sucede para el punto de plastificación global, las expresiones calibradas con la selección de ensayos proporcionan una distribución de rótulas menos desfavorable que las obtenidas con otras expresiones. Otro aspecto a destacar es que las rótulas plásticas observadas en el punto de colapso aparecen principalmente en las vigas, lo cual resulta indicativo de un adecuado diseño desde el punto de vista sísmico. 


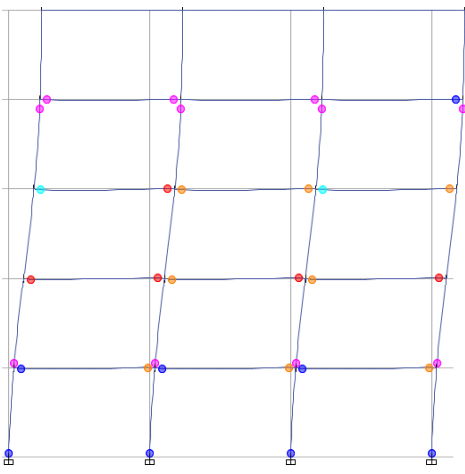

(a)

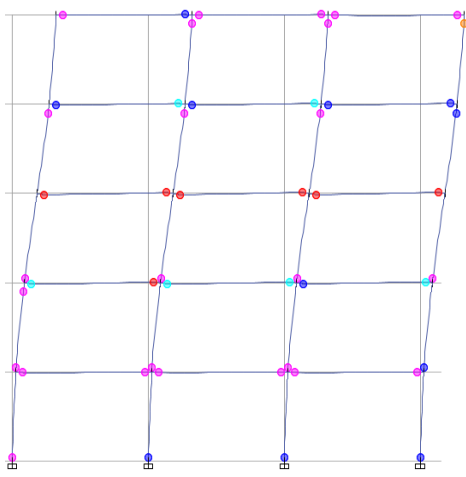

(b)

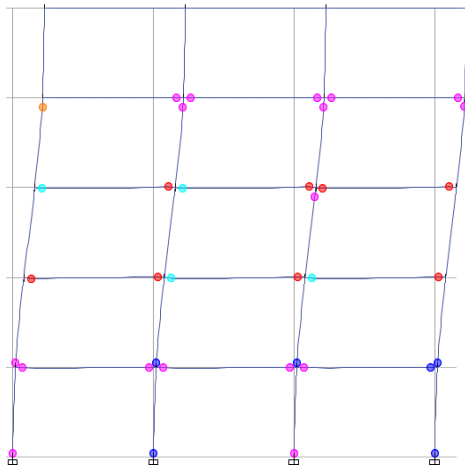

(c)

Figura 6.35 Distribución de rótulas plásticas para el pórtico regular de cinco plantas con cargas laterales uniformes en el punto de colapso. (a) ETABS®. (b) Biskinis y Fardis (2010a, 2010b). (c) Expresiones calibradas.

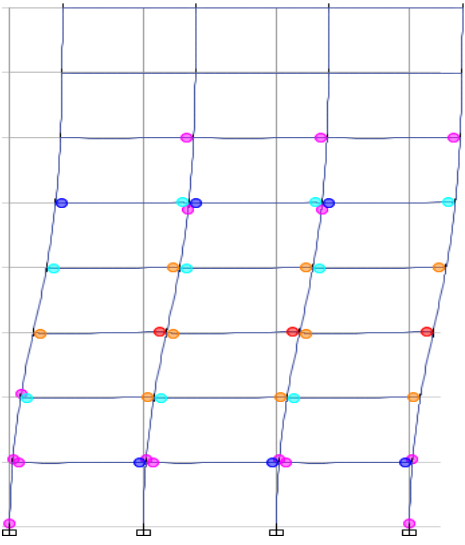

(a)

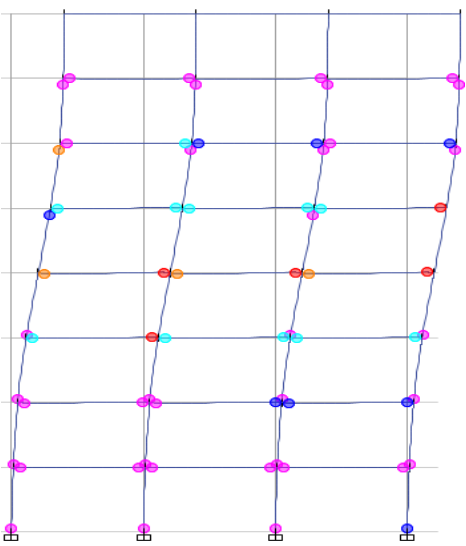

(b)

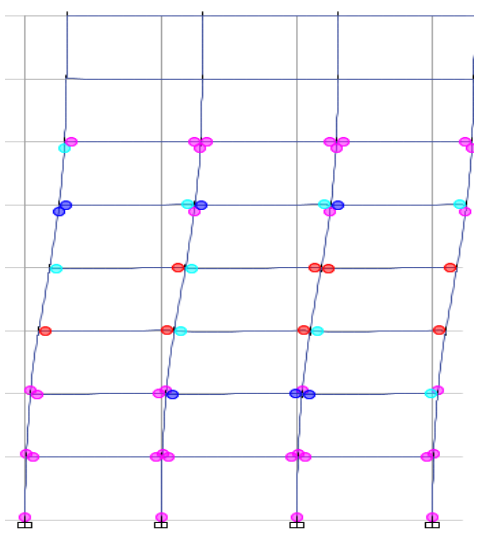

(c)

Figura 6.36 Distribución de rótulas plásticas para el pórtico regular de ocho plantas con cargas laterales uniformes en el punto de colapso. (a) ETABS®. (b) Biskinis y Fardis (2010a, 2010b).

(c) Expresiones calibradas.

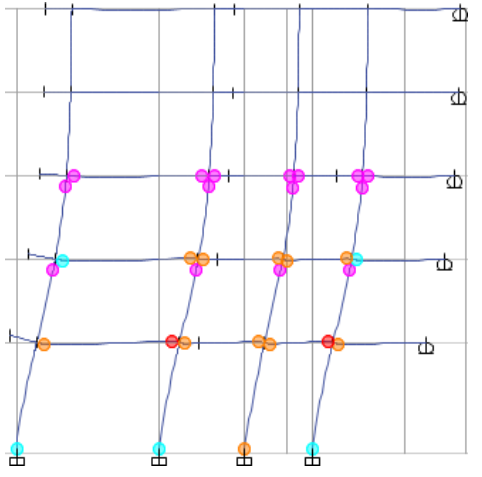

(a)

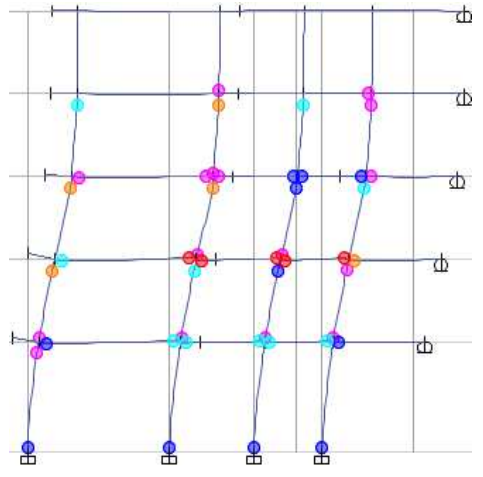

(b)

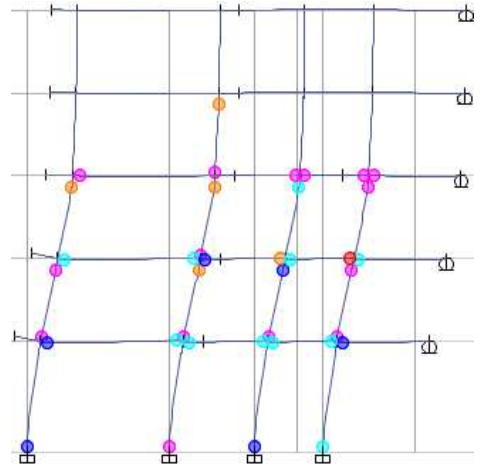

(c)

Figura 6.37 Distribución de rótulas plásticas para el pórtico espacial de cinco plantas con cargas laterales uniformes en el punto de colapso. (a) ETABS®. (b) Biskinis y Fardis (2010a, 2010b). (c) Expresiones calibradas. 


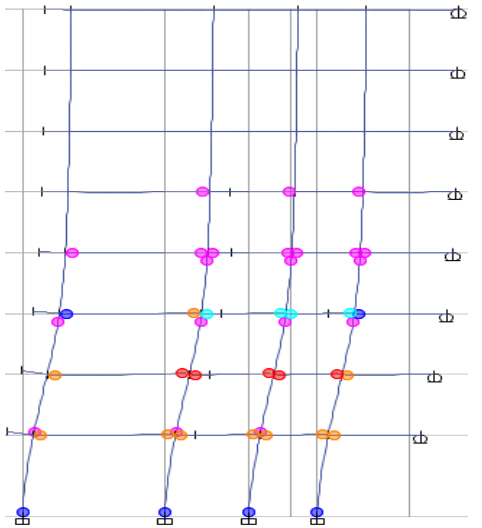

(a)

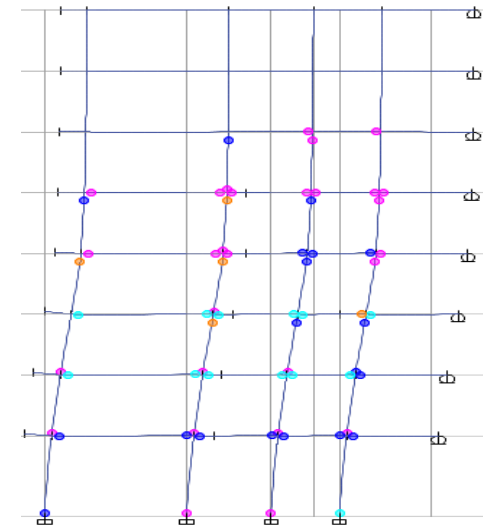

(b)

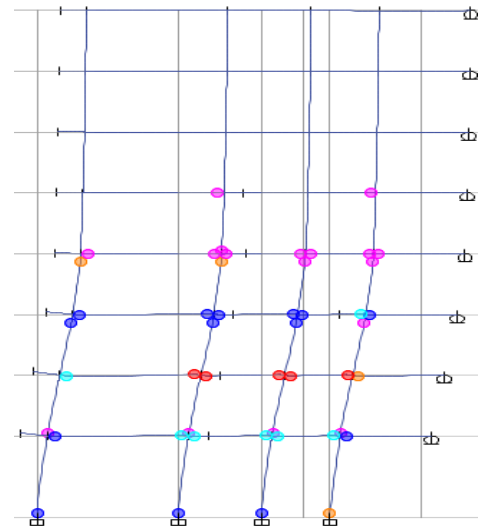

(c)

Figura 6.38 Distribución de rótulas plásticas para el pórtico espacial de ocho plantas con cargas laterales uniformes en el punto de colapso. (a) ETABS®. (b) Biskinis y Fardis (2010a, 2010b).

(c) Expresiones calibradas.

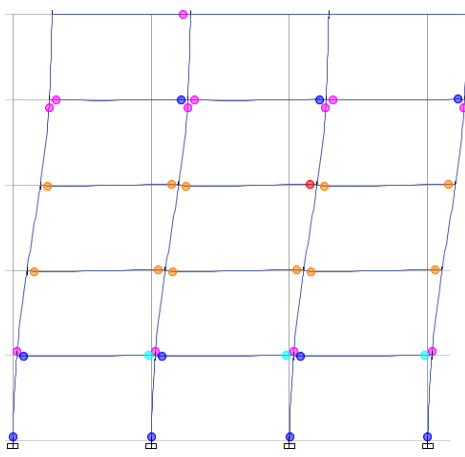

(a)

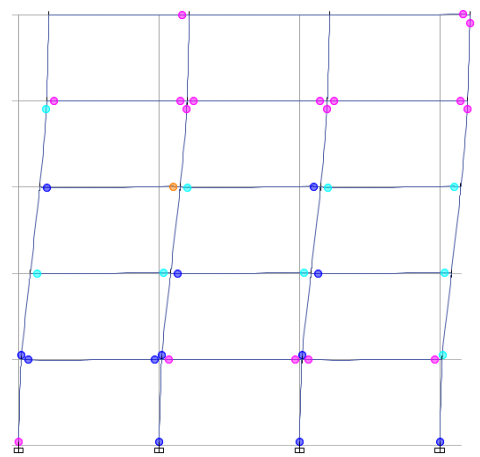

(b)

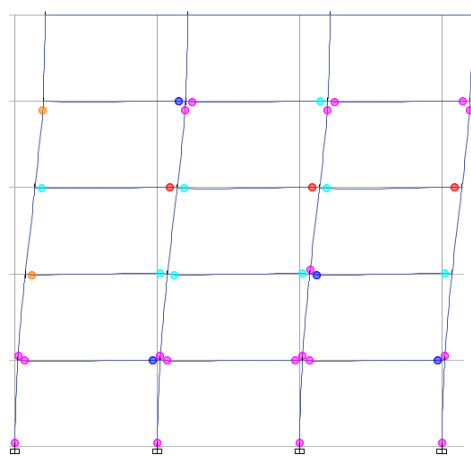

(c)

Figura 6.39 Distribución de rótulas plásticas para el pórtico regular de cinco plantas con cargas laterales modales en el punto de colapso. (a) ETABS®. (b) Biskinis y Fardis (2010a, 2010b).

(c) Expresiones calibradas.

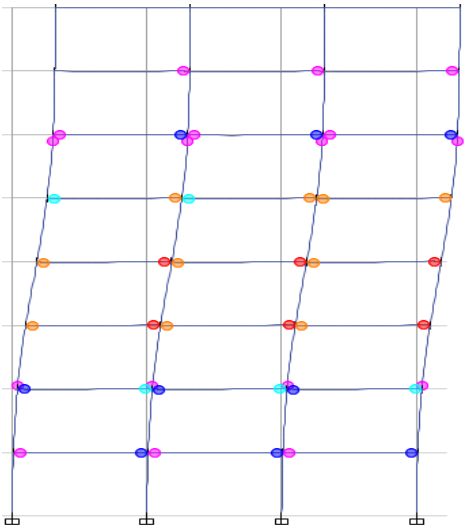

(a)

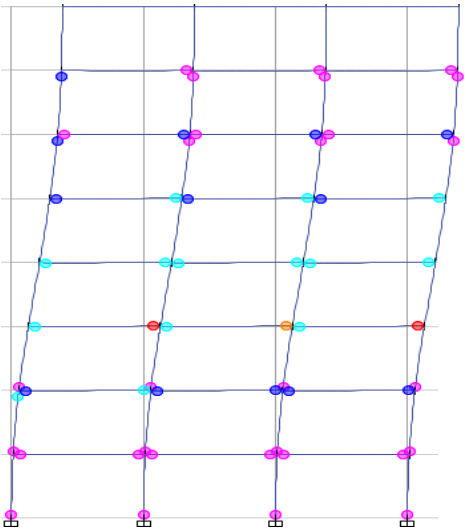

(b)

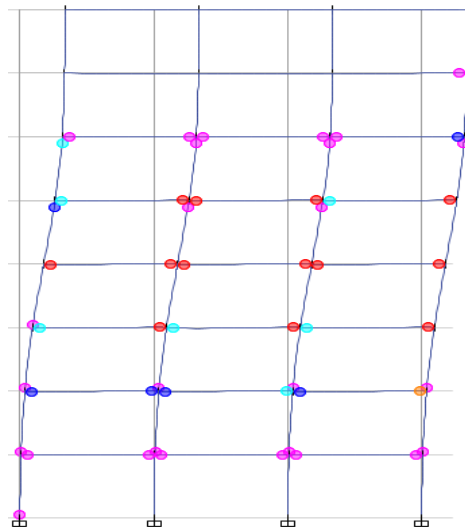

(c)

Figura 6.40 Distribución de rótulas plásticas para el pórtico regular de ocho plantas con cargas laterales modales en el punto de colapso. (a) ETABS®. (b) Biskinis y Fardis (2010a, 2010b).

(c) Expresiones calibradas. 


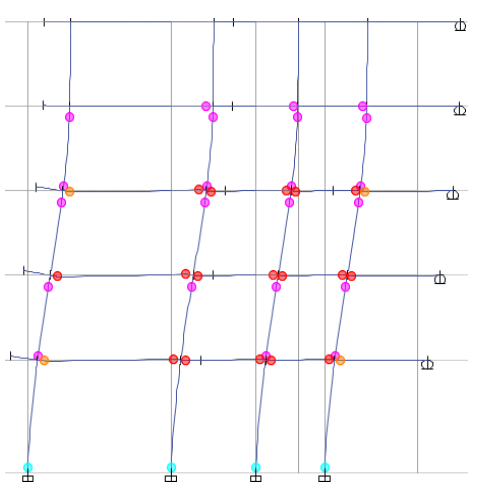

(a)

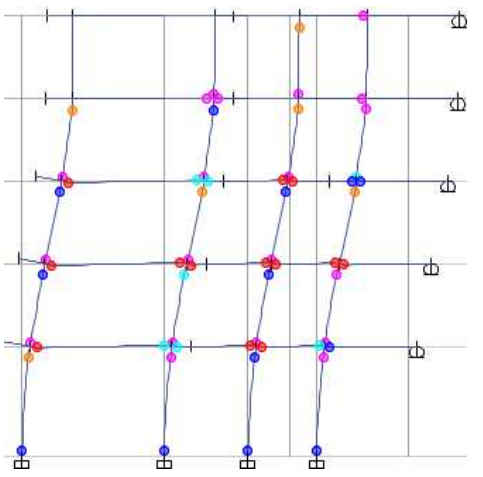

(b)

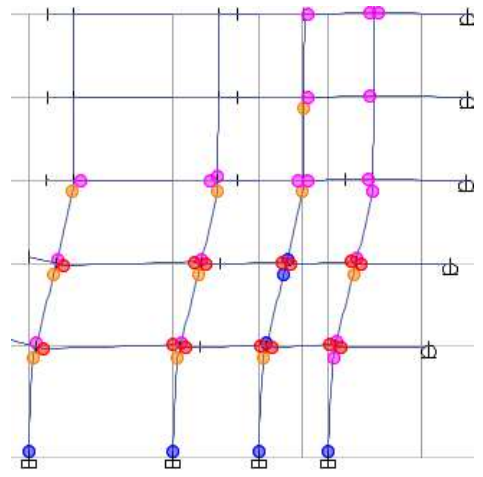

(c)

Figura 6.41 Distribución de rótulas plásticas para el pórtico espacial de cinco plantas con cargas laterales modales en el punto de colapso. (a) ETABS®. (b) Biskinis y Fardis (2010a, 2010b). (c) Expresiones calibradas.

\subsubsection{Influencia del tipo de espectro, tipo de terreno y valor de $a_{g}$}

En este apartado se ha realizado una serie de análisis pushover de las estructuras descritas en el apartado 6.3.1, empleando los diferentes métodos considerados para modelizar las rótulas plásticas y evaluando la influencia de los siguientes aspectos en los resultados obtenidos:

a) tipo de espectro de respuesta elástica horizontal empleado,

b) tipo de terreno considerado, y

c) valor de cálculo de la aceleración del suelo $a_{g}$.

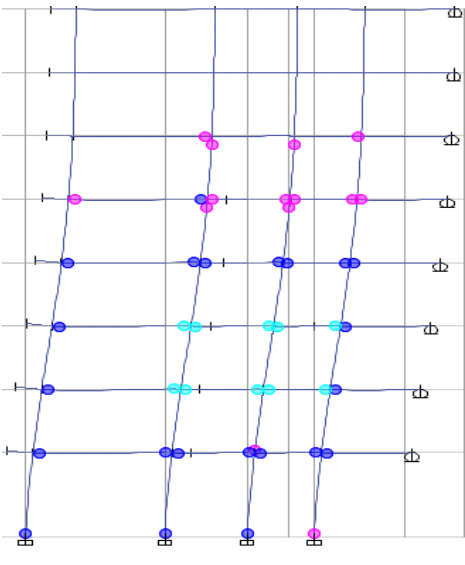

(a)

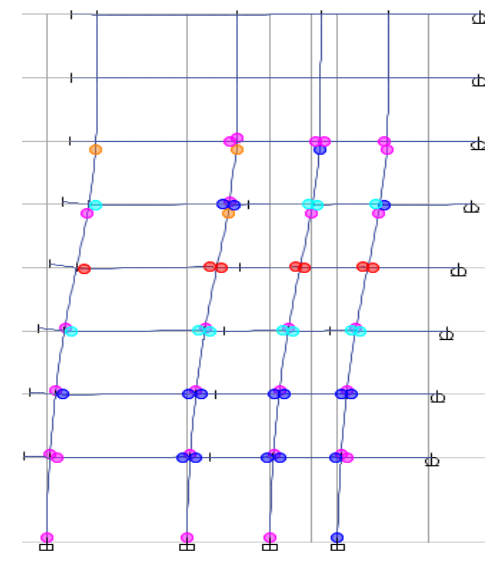

(b)

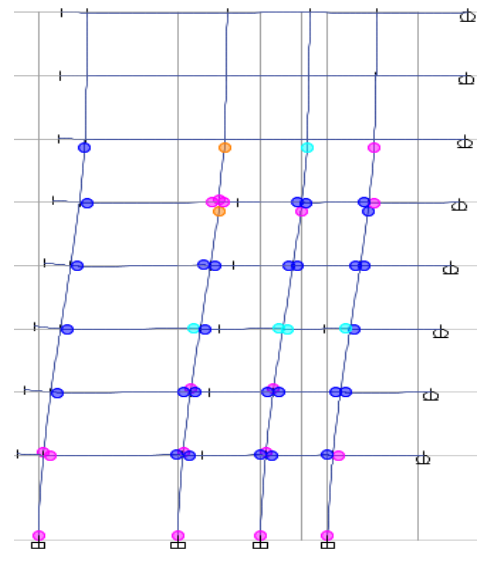

(c)

Figura 6.42 Distribución de rótulas plásticas para el pórtico espacial de ocho plantas con cargas laterales modales en el punto de colapso. (a) ETABS®. (b) Biskinis y Fardis (2010a, 2010b).

(c) Expresiones calibradas.

Se han considerado los espectros tipo 1 y 2 definidos por el EC-8. Con objeto de estudiar el mayor rango posible de tipos de terreno incluidos en el EC-8, se han considerado los terrenos de mejor y peor calidad, esto es, el terreno tipo A y D, 
respectivamente, considerándose adicionalmente el tipo $\mathrm{C}$ por presentar unas características intermedias. Los valores de $a_{g}$ considerados dependen del tipo de espectro empleado, habiéndose realizado los análisis para valores superiores a los máximos registrados hasta la fecha. El EC-8 propone la aplicación del espectro tipo 2 para zonas donde puedan generarse terremotos con ondas superficiales de magnitud $M_{s}$ $\leq 5,5$, empleándose el espectro tipo 1 en caso contrario. De acuerdo con GasparEscribano, Benito y García-Mayordomo (2008), el espectro que debe emplearse para el sureste español es el tipo 2, por lo que para este espectro se ha considerado $a_{g}=0,367 g$ como máximo valor registrado, correspondiente a la aceleración máxima del terremoto de Lorca de Mayo de 2011. Para el espectro tipo 1, aplicable a países con una alta actividad sísmica como Grecia e Italia, se ha considerado como máxima aceleración registrada $a_{g}=0,77 g$, correspondiente al terremoto de Cephalonia de 2014 (ITSAK, 2014). Los valores de $a_{g}$ finalmente considerados son $a_{g}=0,5 g$ cuando se utiliza el espectro tipo 2 y $a_{g}=1,0 g$ al emplear el espectro tipo 1 . Adicionalmente, para cada uno de los casos analizados se ha obtenido el valor de $a_{g}$ que impone un desplazamiento objetivo $d_{t}$ a partir del cual se producen diferencias en los resultados obtenidos al aplicar los diferentes métodos de caracterización de las rótulas plásticas.

Para cada uno de los casos analizados se han obtenido los siguientes aspectos:

a) desplazamientos laterales de las plantas de la estructura,

b) desplomes o drifts entre plantas, y

c) distribución de rótulas plásticas.

De acuerdo con Searer y Freeman (2004), los desplazamientos laterales de las plantas de la estructura y los drifts entre plantas pueden inducir efectos adversos en los elementos que constituyen la estructura, pudiendo afectar también a las estructuras adyacentes si no se limitan sus valores. En este sentido, el EC-8 establece la necesidad de satisfacer el requisito de limitación de daño para una acción sísmica con probabilidad de ocurrencia superior a la correspondiente al requisito de no colapso de la estructura, sin producirse daños ni limitaciones de uso con costes desproporcionados. A la acción sísmica considerada para cumplir el requisito de limitación de daño le corresponde una probabilidad de ser superada $P_{D L R}=10 \%$ en diez años y un período de retorno $T_{D L R}=$ 95 años. El valor de cálculo de la aceleración del suelo para la acción sísmica correspondiente a la comprobación del requisito de limitación de daño es $a_{g}=\gamma_{\mathrm{I}} a_{g R}$, siendo $\gamma_{I}$ el coeficiente de importancia de la estructura y $a_{g R}$ la aceleración máxima de referencia del suelo en un terreno tipo A, establecida en los mapas de sismicidad. Se ha considerado que las estructuras analizadas en esta tesis corresponden a edificios corrientes, a los que el EC-8 atribuye la clase de importancia II y un coeficiente de importancia $\gamma_{\mathrm{I}}=1$.

El EC-8 considera que se satisface el requisito de limitación de daño si, para la acción sísmica correspondiente, los drifts entre plantas cumplen la restricción impuesta 
mediante la ecuación (6.26), aplicable a edificios que carecen de elementos no estructurales

$$
d_{r} \leq \frac{0,010 h^{\prime}}{v}
$$

siendo

$d_{r} \quad$ desplome o drift entre plantas, obtenido como la diferencia entre los desplazamientos laterales superior e inferior de la planta,

$h^{\prime} \quad$ altura de la planta, y

$v \quad$ coeficiente de reducción que considera el período de retorno de la acción sísmica correspondiente al requisito de limitación de daño, con valor $v=0,5$ para edificios con clase de importancia II.

En lo relativo a las estructuras analizadas, la ecuación (6.26) proporciona un valor máximo admisible de drift $d_{r}=60 \mathrm{~mm}$ para plantas de tres metros de altura, resultando $d_{r}=80 \mathrm{~mm}$ si la altura es de cuatro metros.

Para abordar los análisis pushover se ha considerado únicamente la distribución modal de cargas laterales, la cual resulta más adecuada para modelizar el comportamiento del sistema equivalente de un grado de libertad considerado en el método N2.

\subsubsection{Pórtico regular de cinco plantas}

Las Tablas 6.21 y 6.22 muestran respectivamente los valores del desplazamiento objetivo $d_{t}$ y los valores de $a_{g}$ a partir de los cuales existen diferencias en los resultados obtenidos al aplicar los diferentes tipos de rótulas plásticas al pórtico regular de cinco plantas. Según la Tabla 6.21, los valores de $d_{t}$ coinciden para los diferentes tipos de rótulas plásticas si se considera el espectro tipo 2 y el mismo tipo de terreno. La consideración del espectro tipo 1 ofrece valores diferentes según las rótulas empleadas, obteniéndose valores inferiores para las rótulas modelizadas con las expresiones ajustadas a la selección de ensayos. Los valores mínimos de $a_{g}$ que inducen diferencias al aplicar los distintos tipos de rótulas plásticas resultan inferiores cuanto más exigente es la acción sísmica. Este aspecto puede observarse en la Tabla 6.22, donde los menores valores corresponden al espectro tipo 1 y el tipo de terreno D.

$\mathrm{Al}$ analizar los desplazamientos laterales de las diferentes plantas de la estructura, en la Figura 6.43 se observa que los resultados obtenidos para los diferentes tipos de rótulas plásticas resultan similares si se emplea el espectro tipo 2. La Figura 6.44 muestra que, al considerar el espectro tipo 1, los desplazamientos obtenidos con las expresiones de Biskinis y Fardis (2010a, 2010b) y con las expresiones calibradas con la selección de ensayos resultan más desfavorables que los correspondientes a las rótulas plásticas de ETABS®. 
Tabla 6.21 Valores de desplazamiento objetivo $d_{t}$ para el pórtico regular de cinco plantas.

\begin{tabular}{cccccccc}
\hline & \multicolumn{3}{c}{ Espectro tipo 1 } & \multicolumn{3}{c}{ Espectro tipo 2 } \\
Tipo de rótula plástica & \multicolumn{2}{c}{ Tipo de terreno } & \multicolumn{3}{c}{ Tipo de terreno } \\
& A & C & D & A & C & D \\
\hline ETABS® & 434 & 748 & 1171 & 104 & 156 & 225 \\
Biskinis y Fardis (2010a, 2010b ) & 428 & 739 & 1156 & 104 & 156 & 225 \\
Expresiones calibradas & 411 & 710 & 1111 & 104 & 156 & 225 \\
\hline
\end{tabular}

Tabla 6.22 Valores mínimos de aceleración $a_{g}$ para los que existen diferencias entre los diferentes tipos de rótulas plásticas.

\begin{tabular}{ccccccc}
\hline \multicolumn{3}{c}{ Espectro tipo 1 } & \multicolumn{3}{c}{ Espectro tipo 2 } \\
Tipo de terreno & \multicolumn{3}{c}{ Tipo de terreno } \\
A & C & D & A & C & D \\
\hline 0,10 & 0,06 & 0,04 & 0,20 & 0,13 & 0,09 \\
\hline
\end{tabular}

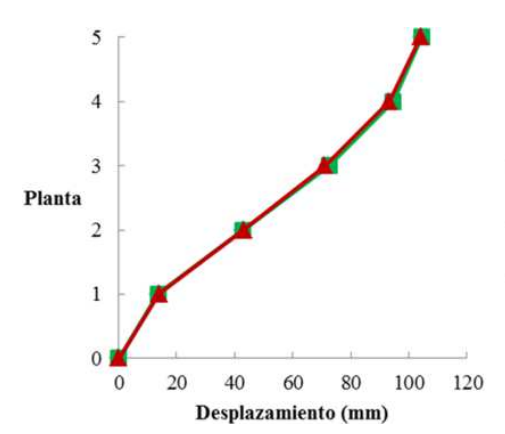

(a)

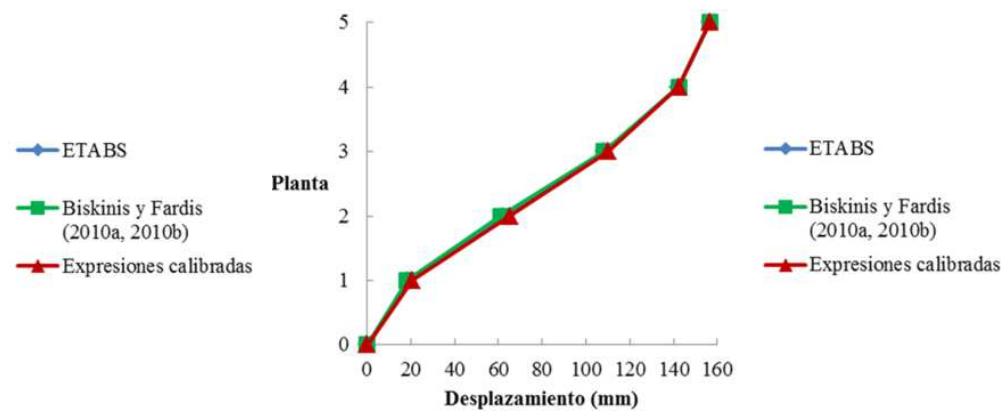

(b)

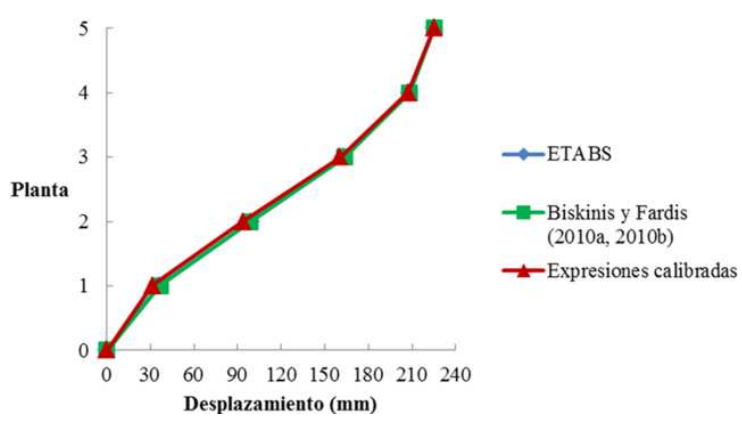

(c)

Figura 6.43 Desplazamientos laterales del pórtico regular de cinco plantas para el espectro tipo 2 y $a_{g}=0,5 g$. (a) Terreno tipo A. (b) Terreno tipo C. (c) Terreno tipo D. 


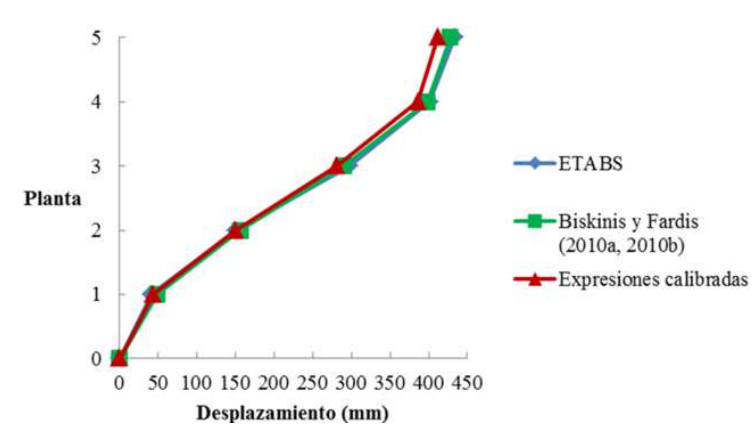

(a)

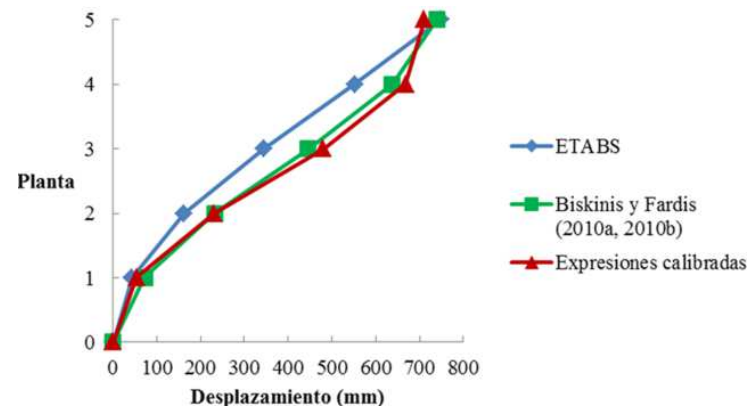

(b)

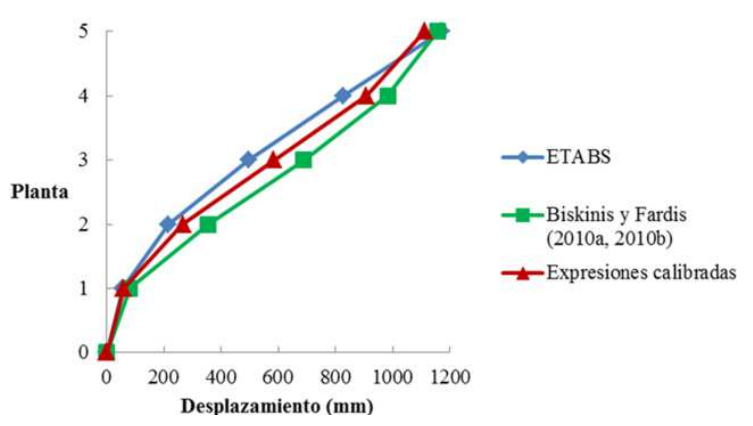

(c)

Figura 6.44 Desplazamientos laterales del pórtico regular de cinco plantas para el espectro tipo 1 y $a_{g}=1,0 g$. (a) Terreno tipo A. (b) Terreno tipo C. (c) Terreno tipo D.

En cuanto a los drifts, mostrados en las Figuras 6.45 y 6.46 , los tres tipos de rótulas plásticas ofrecen valores similares para el espectro tipo 2. Para el espectro tipo 1, las expresiones calibradas obtienen en general valores superiores a los obtenidos con las rótulas plásticas de $\mathrm{ETABS} \circledast$ para las plantas primera a tercera, sucediendo lo contrario para las dos últimas. Las limitaciones impuestas por el EC-8 en los valores del drift se cumplen únicamente para los terrenos tipo A y $\mathrm{C}$ y al emplear el espectro tipo 2.

La distribución de rótulas plásticas en el pórtico regular de cinco plantas para el desplazamiento objetivo $d_{t}$ se muestra en las Figuras 6.47 a 6.52. Se observa un patrón similar de formación de rótulas plásticas para los distintos métodos de modelización de rótulas, generándose dichas rótulas primeramente en las vigas de las plantas inferiores y posteriormente en las vigas de las plantas superiores y en los pilares a medida que la acción sísmica considerada es más desfavorable. Las estructuras con rótulas modelizadas mediante las expresiones calibradas con la selección de ensayos presentan un menor número de rótulas que las modelizadas con los valores por defecto de ETABS $®$, aspecto que puede observarse al no aparecer rótulas plásticas calibradas con la selección de ensayos en las plantas cuarta y quinta de las estructuras mostradas en las Figuras 6.47 y 6.48 y en la quinta planta de las expuestas en las Figuras 6.50 y 6.51. En todos los casos las estructuras que consideran rótulas plásticas modelizadas con las expresiones calibradas presentan unos niveles de comportamiento menos desfavorables. Sin embargo, en las Figuras 6.49 y 6.51 se observa que dichas expresiones propician en 
algunos casos la aparición de rótulas plásticas en los pilares de las plantas intermedias, aspecto que resulta indicativo de una rotura de tipo frágil.

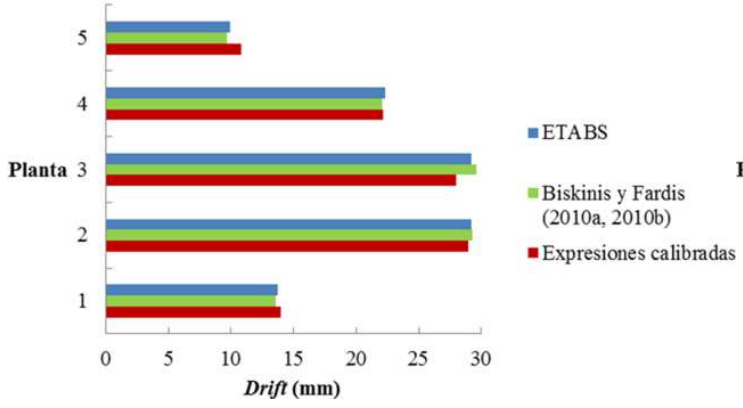

(a)

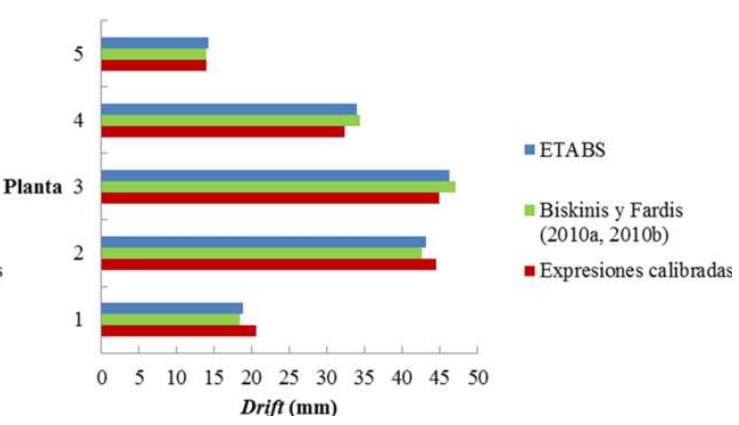

(b)

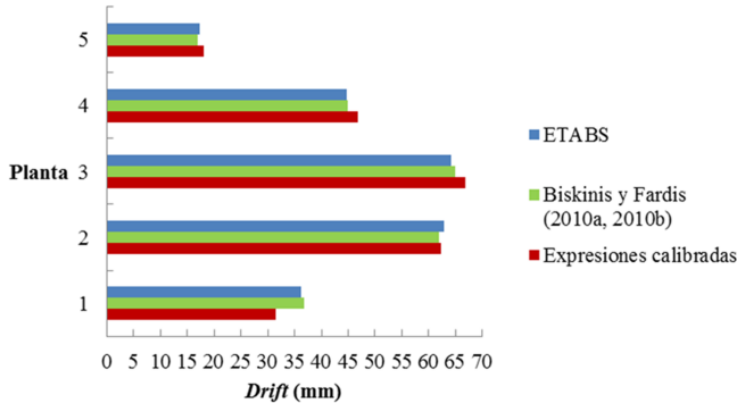

(c)

Figura 6.45 Drifts para el pórtico regular de cinco plantas considerando el espectro tipo 2 y $a_{g}=$ 0,5g. (a) Terreno tipo A. (b) Terreno tipo C. (c) Terreno tipo D.

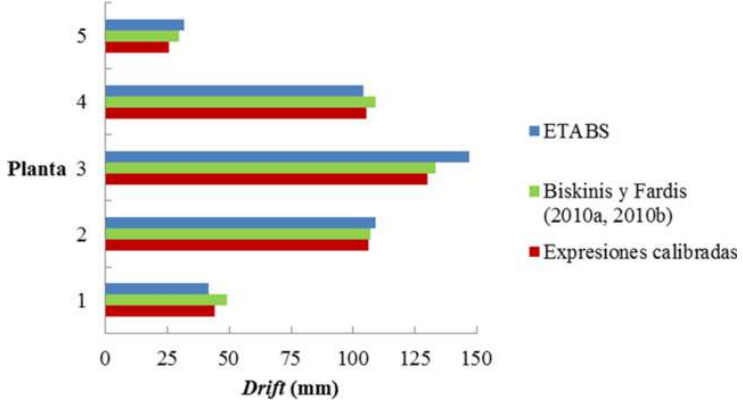

(a)

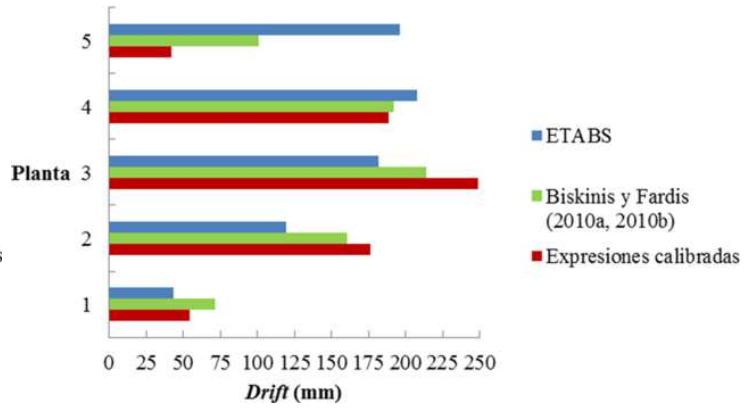

(b)

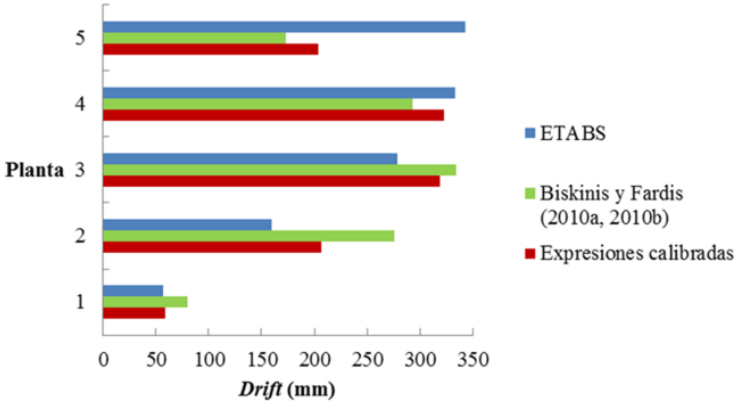

(c)

Figura 6.46 Drifts para el pórtico regular de cinco plantas considerando el espectro tipo 1 y $a_{g}=$ 1,0g. (a) Terreno tipo A. (b) Terreno tipo C. (c) Terreno tipo D. 


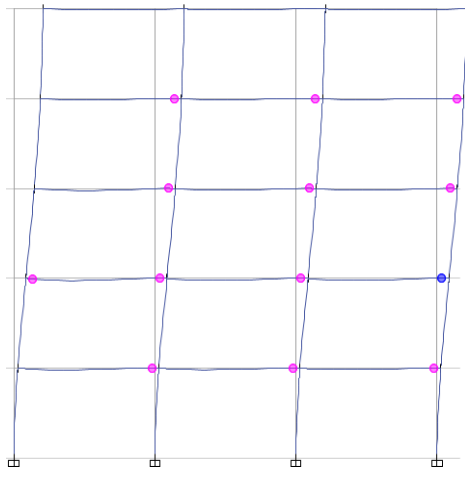

(a)

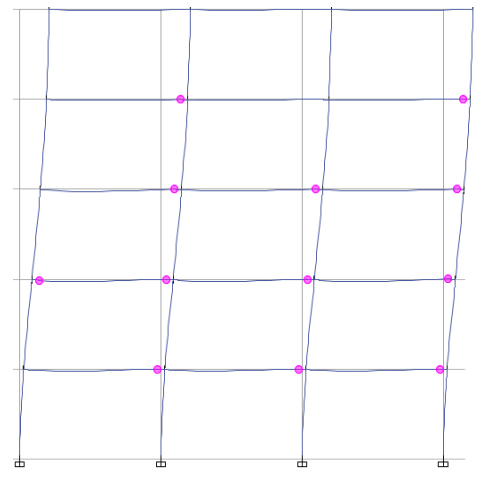

(b)

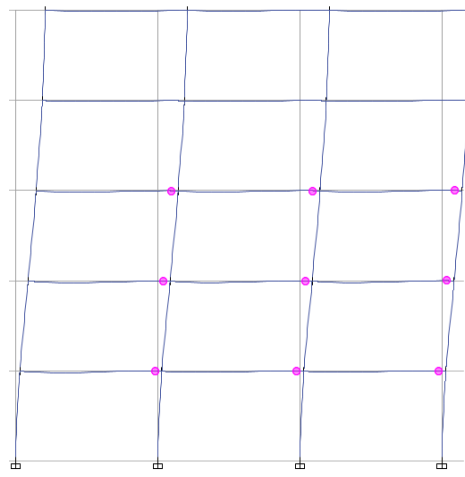

(c)

Figura 6.47 Distribución de rótulas plásticas para el pórtico regular de cinco plantas considerando el espectro tipo 2, $a_{g}=0,5 g$ y terreno tipo A. (a) ETABS®. (b) Biskinis y Fardis (2010a, 2010b). (c) Expresiones calibradas.

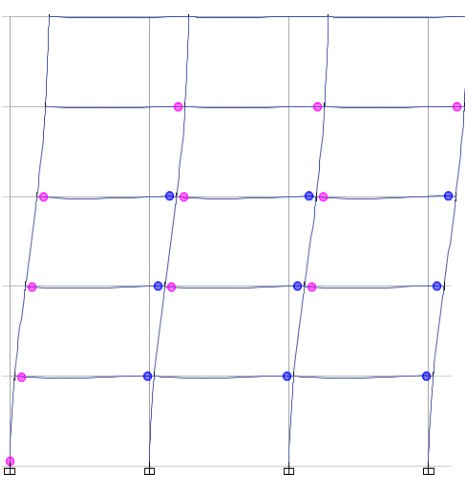

(a)

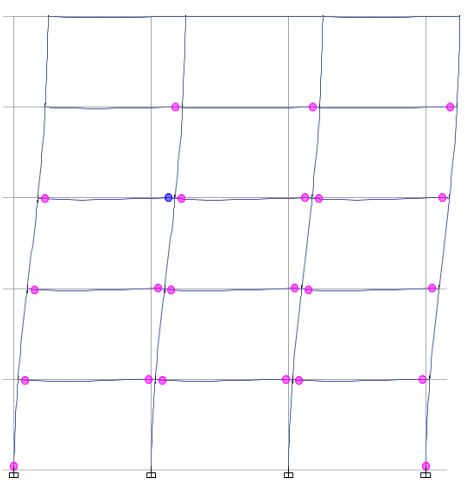

(b)

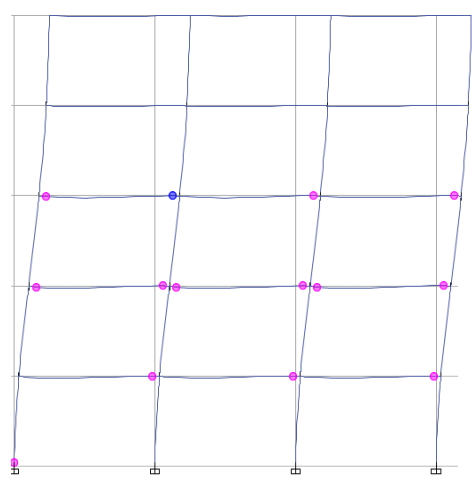

(c)

Figura 6.48 Distribución de rótulas plásticas para el pórtico regular de cinco plantas considerando el espectro tipo 2, $a_{g}=0,5 g$ y terreno tipo C. (a) ETABS®. (b) Biskinis y Fardis (2010a, 2010b). (c) Expresiones calibradas.

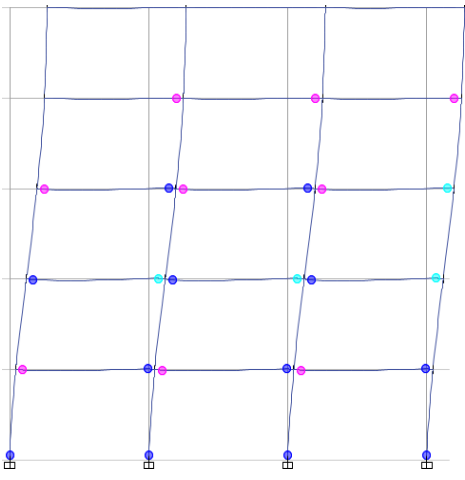

(a)

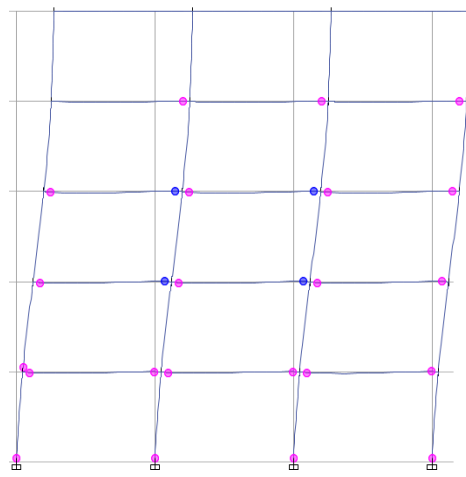

(b)

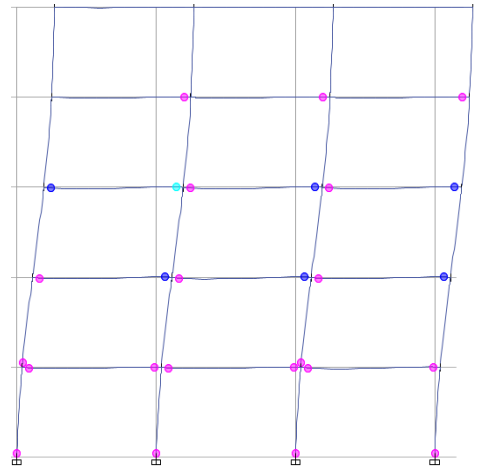

(c)

Figura 6.49 Distribución de rótulas plásticas para el pórtico regular de cinco plantas considerando el espectro tipo 2, $a_{g}=0,5 g$ y terreno tipo D. (a) ETABS®. (b) Biskinis y Fardis (2010a, 2010b). (c) Expresiones calibradas. 


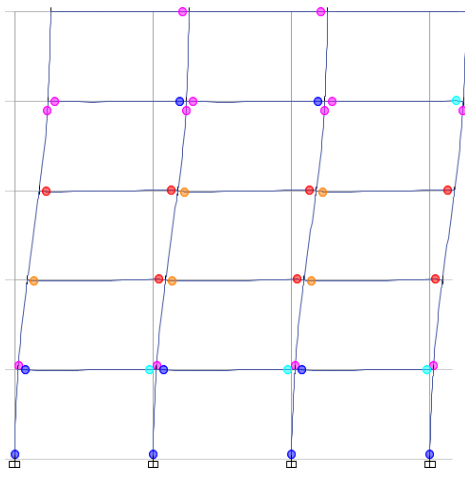

(a)

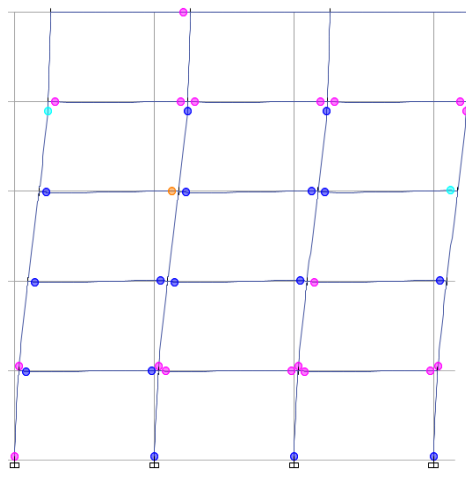

(b)

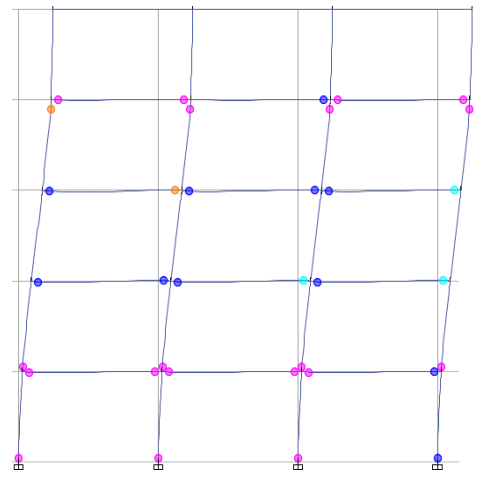

(c)

Figura 6.50 Distribución de rótulas plásticas para el pórtico regular de cinco plantas considerando el espectro tipo 1, $a_{g}=1,0 g$ y terreno tipo A. (a) ETABS®. (b) Biskinis y Fardis (2010a, 2010b). (c) Expresiones calibradas.

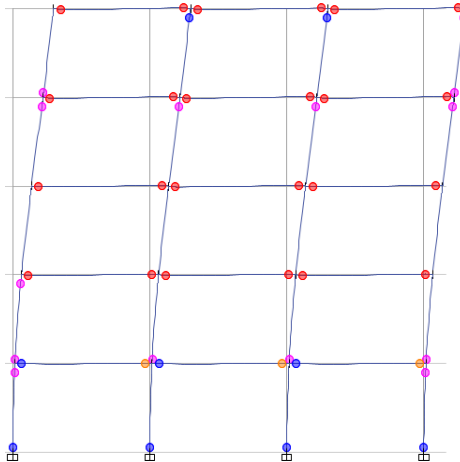

(a)

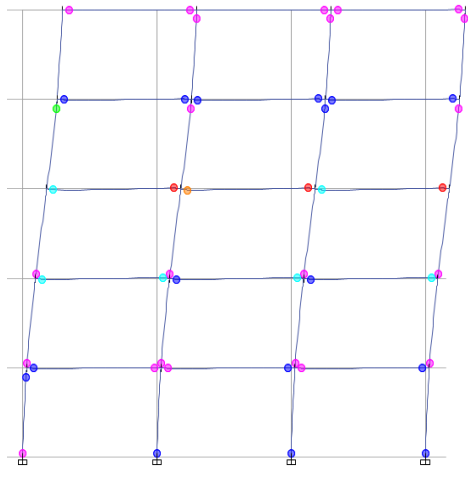

(b)

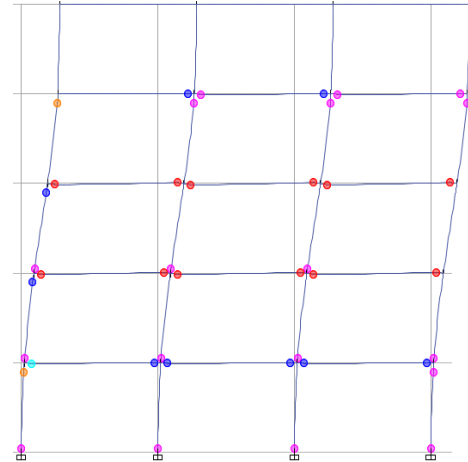

(c)

Figura 6.51 Distribución de rótulas plásticas para el pórtico regular de cinco plantas considerando el espectro tipo $1, a_{g}=1,0 g$ y terreno tipo C. (a) ETABS ${ }^{\circledR}$. (b) Biskinis y Fardis (2010a, 2010b). (c) Expresiones calibradas.

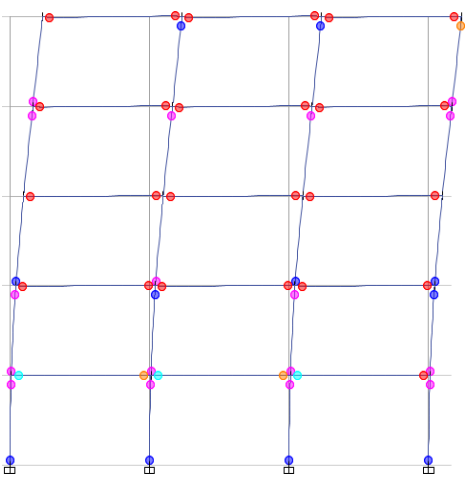

(a)

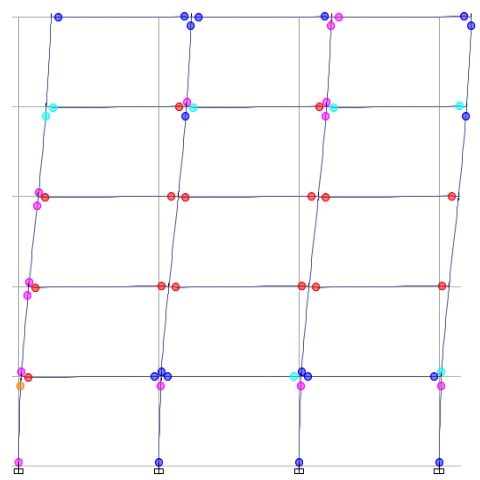

(b)

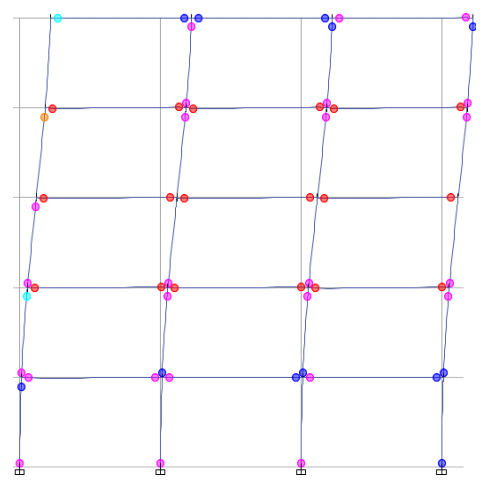

(c)

Figura 6.52 Distribución de rótulas plásticas para el pórtico regular de cinco plantas considerando el espectro tipo 1, $a_{g}=1,0 g$ y terreno tipo D. (a) ETABS $®$. (b) Biskinis y Fardis (2010a, 2010b). (c) Expresiones calibradas. 


\subsubsection{Pórtico regular de ocho plantas}

Los valores de desplazamiento objetivo $d_{t}$ y mínima aceleración que induce diferencias entre los diferentes métodos de modelización de rótulas plásticas para el pórtico regular de ocho plantas se muestran respectivamente en las Tablas 6.23 y 6.24. Para este pórtico los valores obtenidos de $d_{t}$ coinciden al considerar los distintos tipos de rótulas plásticas para un mismo tipo de terreno. Los valores de las Tablas 6.23 y 6.24 resultan superiores a los obtenidos para el pórtico regular de cinco plantas debido a la mayor flexibilidad del pórtico regular de ocho plantas.

Los desplazamientos laterales del pórtico regular de ocho plantas se muestran en las Figuras 6.53 y 6.54. Si bien para el espectro tipo 2 no se observan diferencias al aplicar los distintos tipos de rótulas plásticas, la Figura 6.54 muestra que las rótulas modelizadas con la selección de ensayos obtienen desplazamientos laterales superiores a los obtenidos con otros métodos si se considera el espectro tipo 1.

Tabla 6.23 Valores de desplazamiento objetivo $d_{t}$ para el pórtico regular de ocho plantas.

\begin{tabular}{cccccccc}
\hline & \multicolumn{3}{c}{ Espectro tipo 1 } & \multicolumn{3}{c}{ Espectro tipo 2 } \\
Tipo de rótula plástica & \multicolumn{2}{c}{ Tipo de terreno } & \multicolumn{3}{c}{ Tipo de terreno } \\
& A & C & D & A & C & D \\
\hline ETABS® & 628 & 1084 & 1696 & 118 & 177 & 254 \\
Biskinis y Fardis (2010a, 2010b ) & 628 & 1084 & 1696 & 118 & 177 & 254 \\
Expresiones calibradas & 628 & 1084 & 1696 & 118 & 177 & 254 \\
\hline
\end{tabular}

Tabla 6.24 Valores mínimos de aceleración $a_{g}$ para los que existen diferencias entre los diferentes tipos de rótulas plásticas.

\begin{tabular}{ccccccc}
\hline \multicolumn{3}{c}{ Espectro tipo 1 } & \multicolumn{3}{c}{ Espectro tipo 2 } \\
\multicolumn{2}{c}{ Tipo de terreno } & \multicolumn{3}{c}{ Tipo de terreno } \\
A & C & D & A & C & D \\
\hline 0,12 & 0,07 & 0,05 & 0,30 & 0,20 & 0,14 \\
\hline
\end{tabular}

La Figura 6.55 muestra que los drifts obtenidos al aplicar los distintos tipos de rótulas plásticas resultan similares si se considera el espectro tipo 2. De acuerdo con la Figura 6.56, para el espectro tipo 1 las mayores diferencias se producen en las plantas tercera a sexta para los terrenos tipo A y $\mathrm{C}$ y en las plantas séptima y octava para el terreno tipo D. Los drifts obtenidos para el pórtico regular de cinco plantas resultan superiores a los del pórtico regular de ocho plantas si se considera el espectro tipo 2, sucediendo lo contrario al emplear el espectro tipo 1. Las limitaciones impuestas por el EC-8 en el valor admisible del drift se cumplen para todos los tipos de terreno si se emplea el espectro tipo 2. 


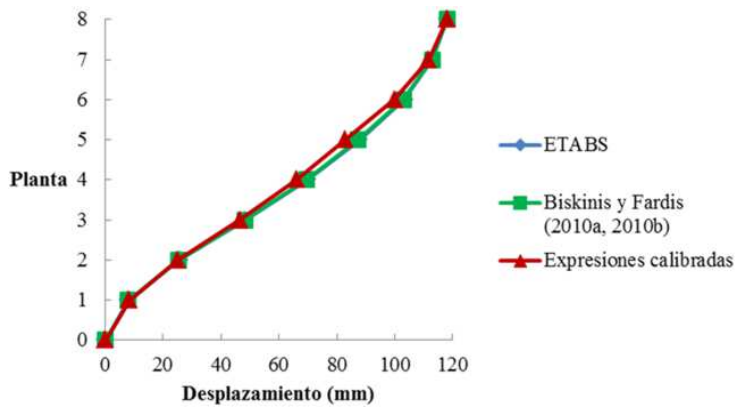

(a)

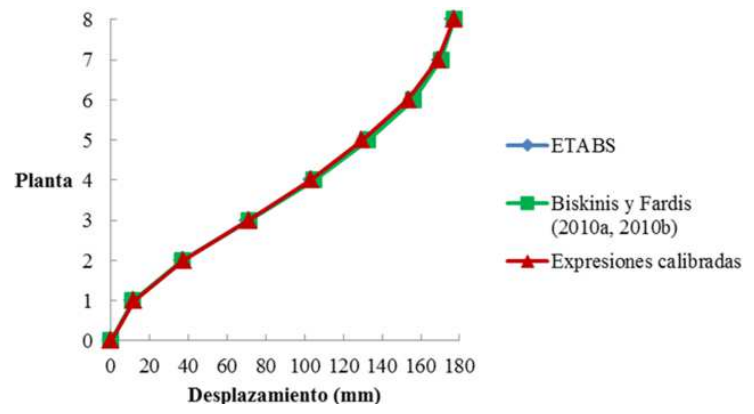

(b)

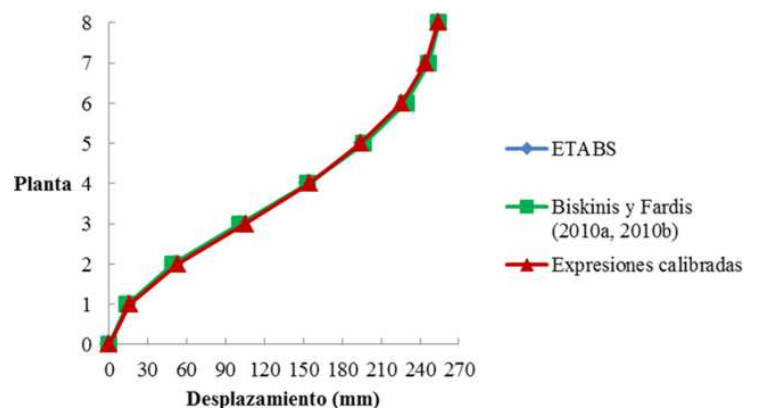

(c)

Figura 6.53 Desplazamientos laterales del pórtico regular de ocho plantas para el espectro tipo 2 y $a_{g}=0,5 g$. (a) Terreno tipo A. (b) Terreno tipo C. (c) Terreno tipo D.

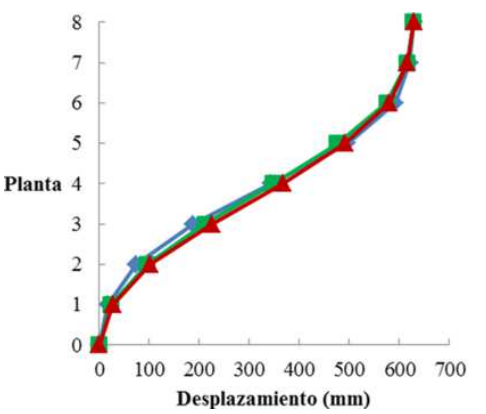

(a)
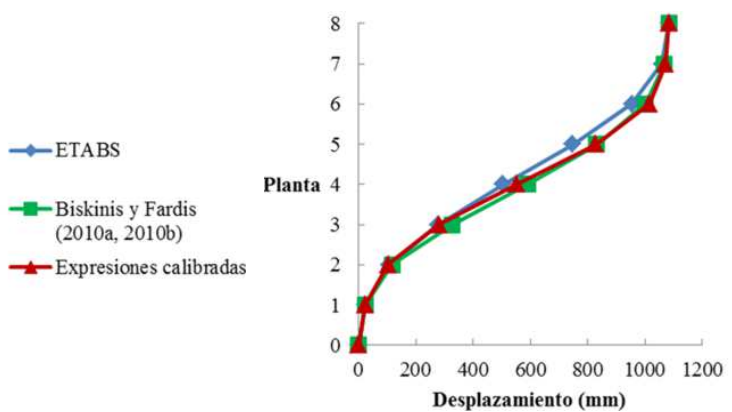

(b)

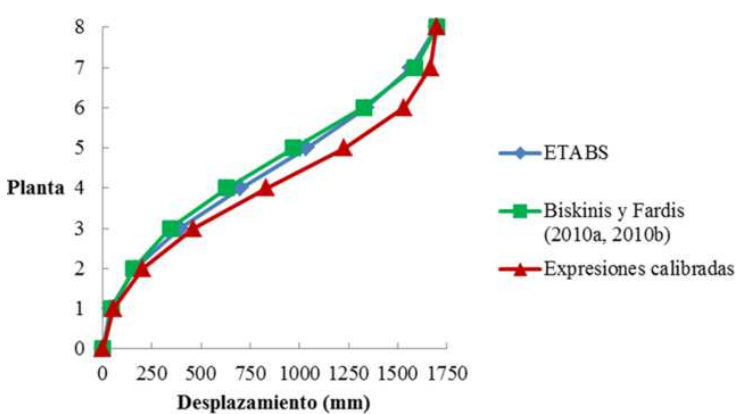

(c)

Figura 6.54 Desplazamientos laterales del pórtico regular de ocho plantas para el espectro tipo 1 y $a_{g}=1,0 g$. (a) Terreno tipo A. (b) Terreno tipo C. (c) Terreno tipo D. 


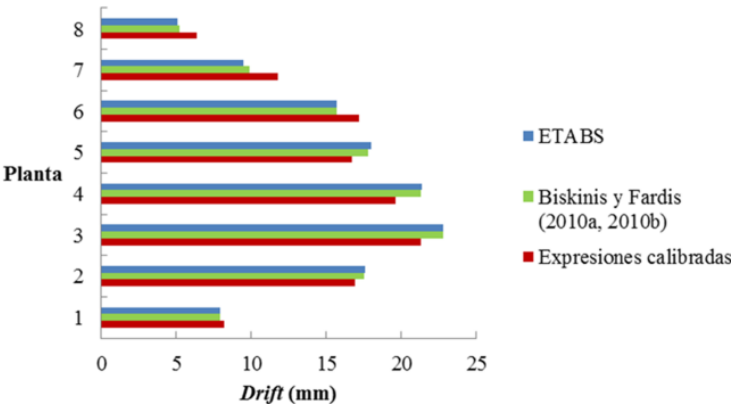

(a)

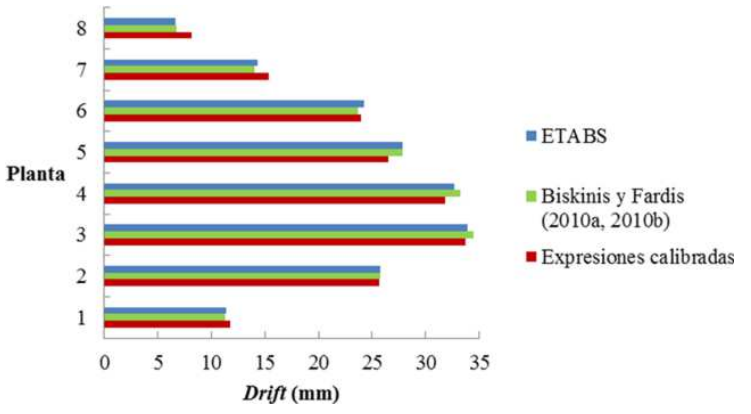

(b)

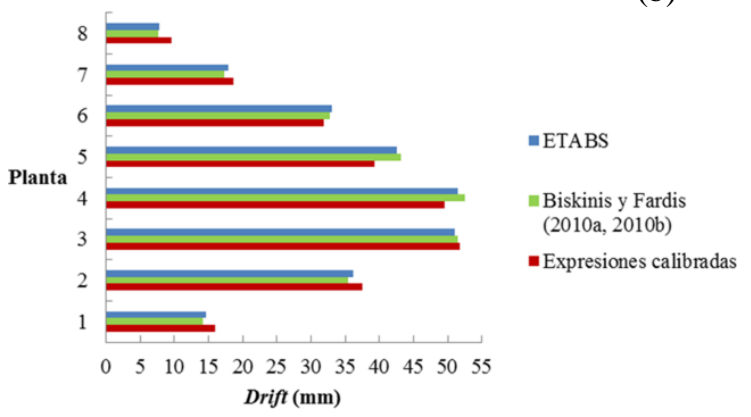

(c)

Figura 6.55 Drifts para el pórtico regular de ocho plantas considerando el espectro tipo 2 y $a_{g}=$ 0,5g. (a) Terreno tipo A. (b) Terreno tipo C. (c) Terreno tipo D.

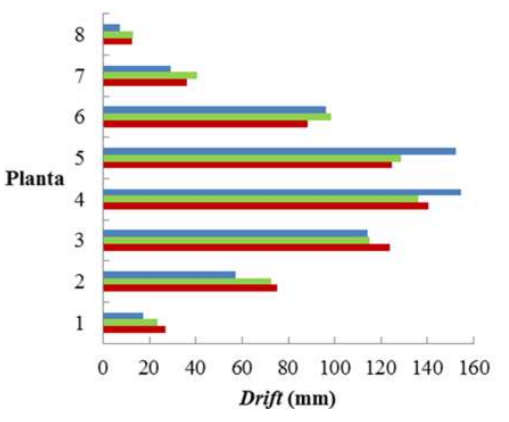

(a)

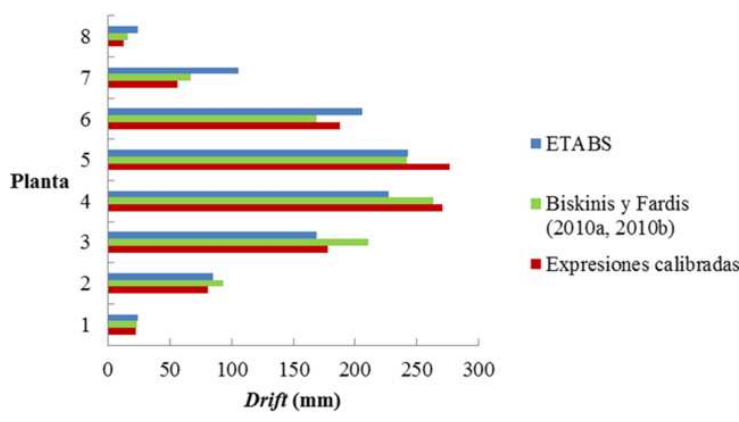

(b)

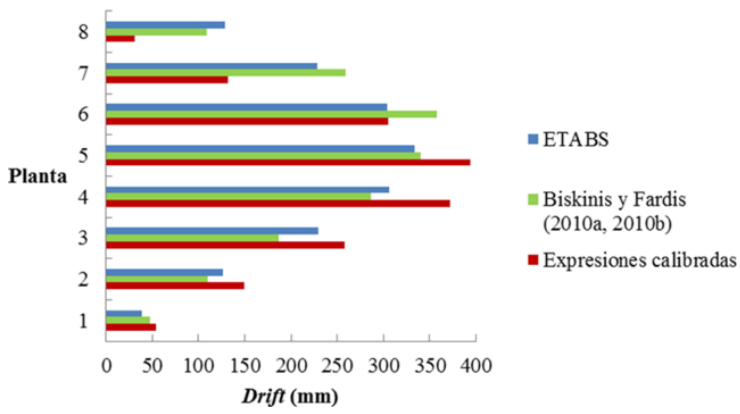

(c)

Figura 6.56 Drifts para el pórtico regular de ocho plantas considerando el espectro tipo 1 y $a_{g}=$ 1,0g. (a) Terreno tipo A. (b) Terreno tipo C. (c) Terreno tipo D. 
Las Figuras 6.57 a 6.62 muestran las distribuciones de rótulas plásticas para el pórtico regular de ocho plantas. Al igual que sucede para el pórtico regular de cinco plantas, las estructuras con rótulas caracterizadas con las expresiones ajustadas a la selección de ensayos presentan un menor número de rótulas plásticas y unos niveles de comportamiento menos exigentes. Resulta necesario destacar que, de acuerdo con las Figuras 6.60 y 6.61, las expresiones de Biskinis y Fardis (2010a, 2010b) presentan una situación más desfavorable respecto a los otros tipos de rótulas al considerar el espectro tipo 1, ya que suponen la aparición de rótulas plásticas en las secciones de empotramiento de los pilares.

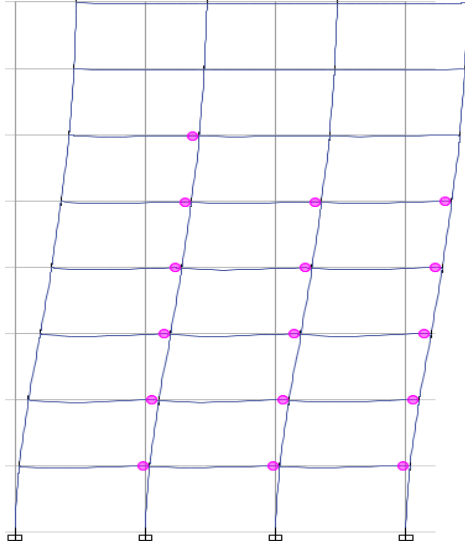

(a)

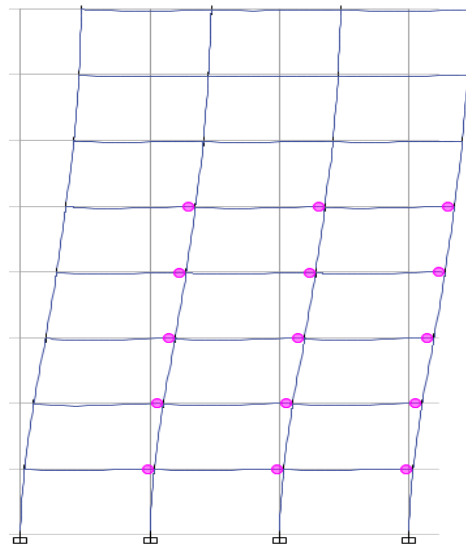

(b)

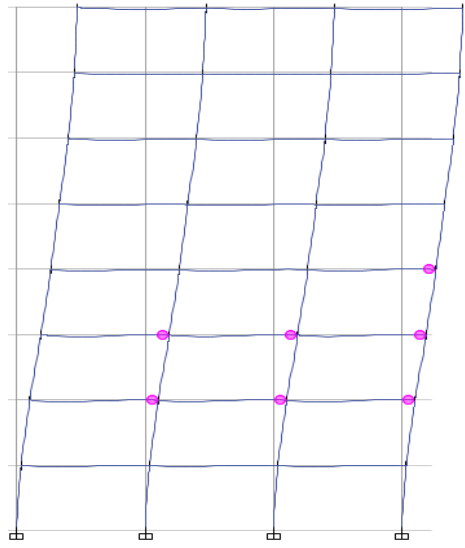

(c)

Figura 6.57 Distribución de rótulas plásticas para el pórtico regular de ocho plantas considerando el espectro tipo 2, $a_{g}=0,5 g$ y terreno tipo A. (a) ETABS®. (b) Biskinis y Fardis (2010a, 2010b). (c) Expresiones calibradas.

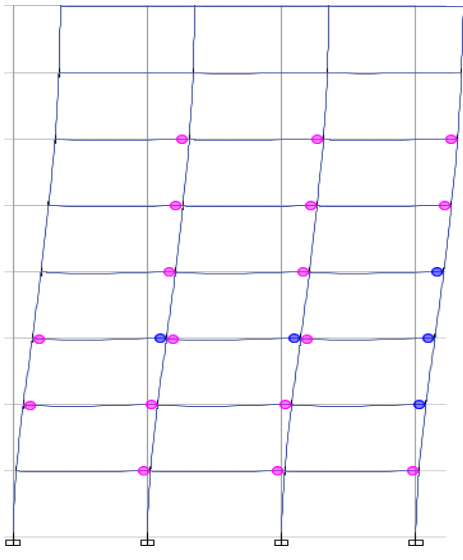

(a)

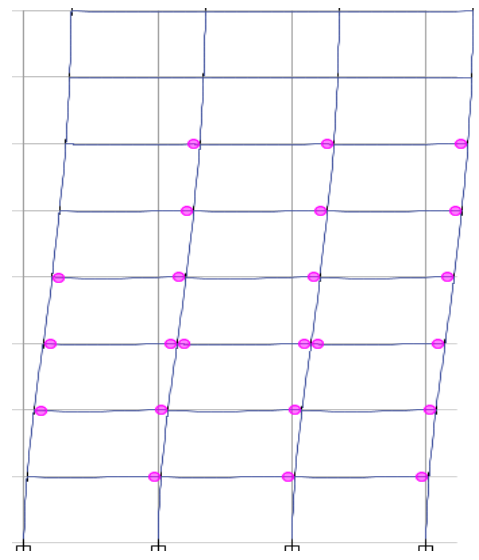

(b)

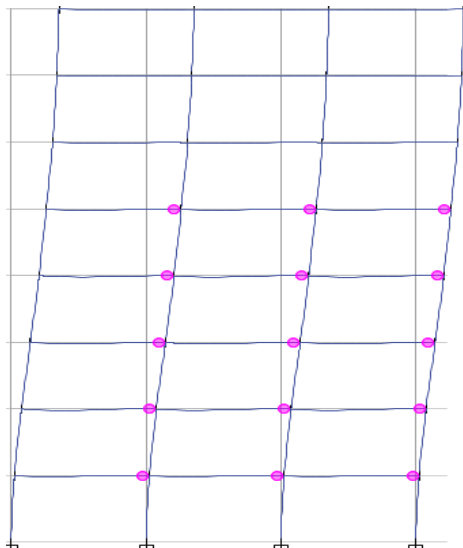

(c)

Figura 6.58 Distribución de rótulas plásticas para el pórtico regular de ocho plantas considerando el espectro tipo 2, $a_{g}=0,5 g$ y terreno tipo C. (a) ETABS ${ }^{\circledR}$. (b) Biskinis y Fardis (2010a, 2010b). (c) Expresiones calibradas. 


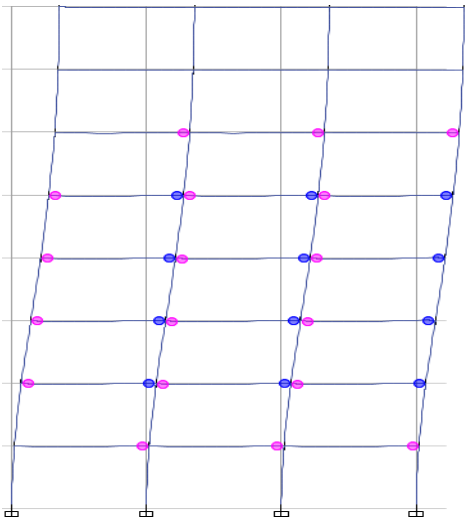

(a)

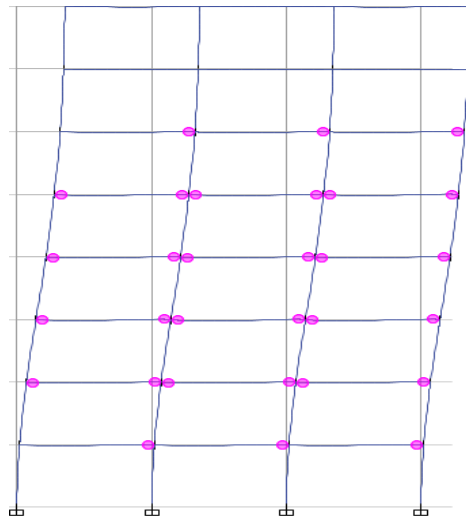

(b)

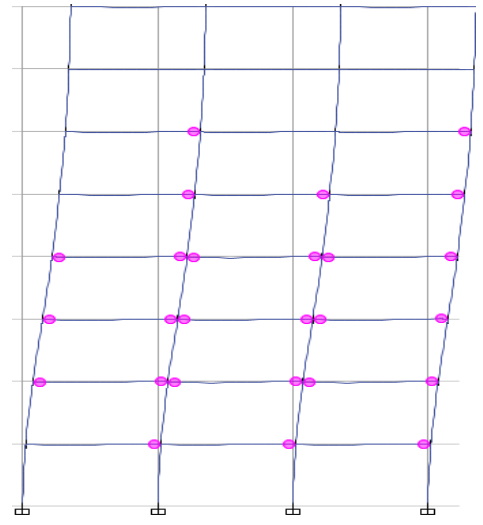

(c)

Figura 6.59 Distribución de rótulas plásticas para el pórtico regular de ocho plantas considerando el espectro tipo 2, $a_{g}=0,5 g$ y terreno tipo D. (a) ETABS®. (b) Biskinis y Fardis (2010a, 2010b). (c) Expresiones calibradas.

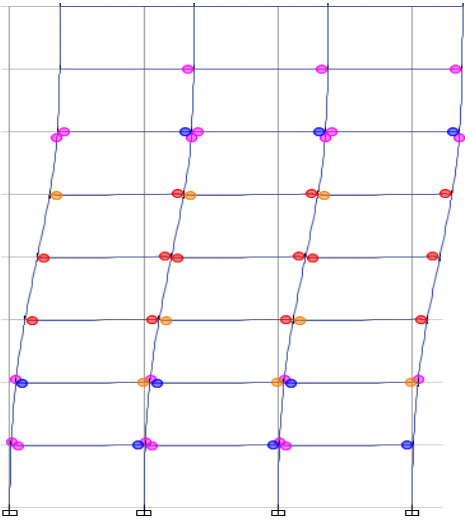

(a)

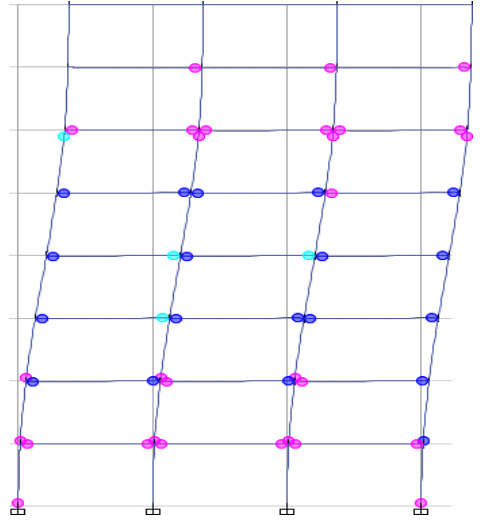

(b)

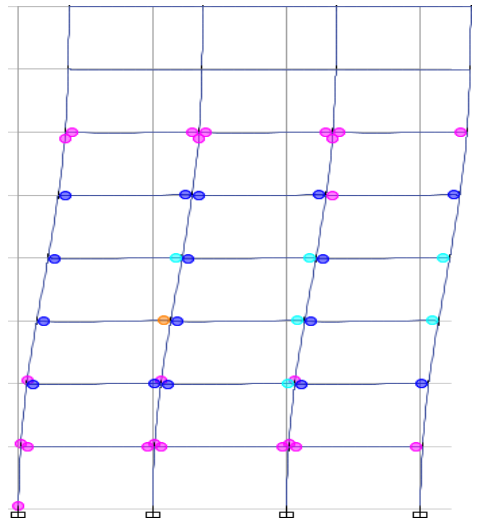

(c)

Figura 6.60 Distribución de rótulas plásticas para el pórtico regular de ocho plantas considerando el espectro tipo 1, $a_{g}=1,0 g$ y terreno tipo A. (a) ETABS®. (b) Biskinis y Fardis (2010a, 2010b). (c) Expresiones calibradas.

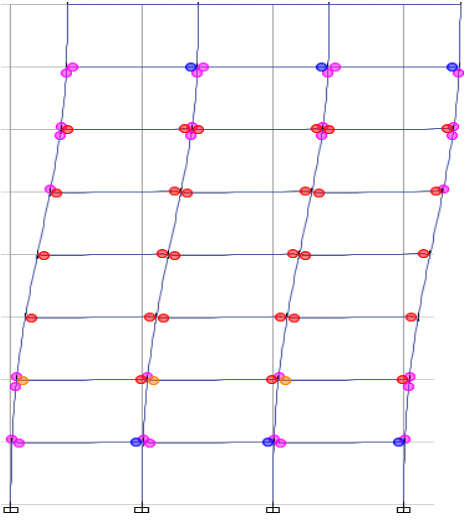

(a)

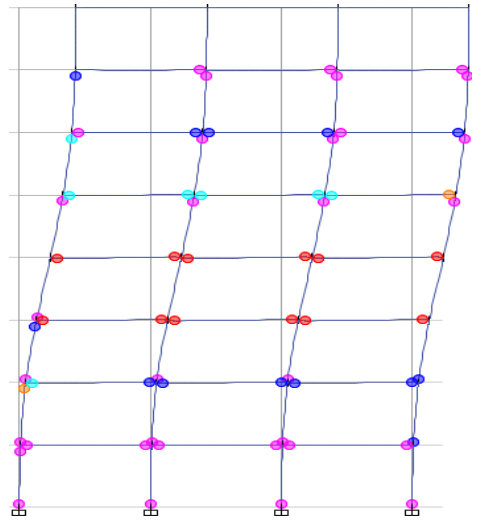

(b)

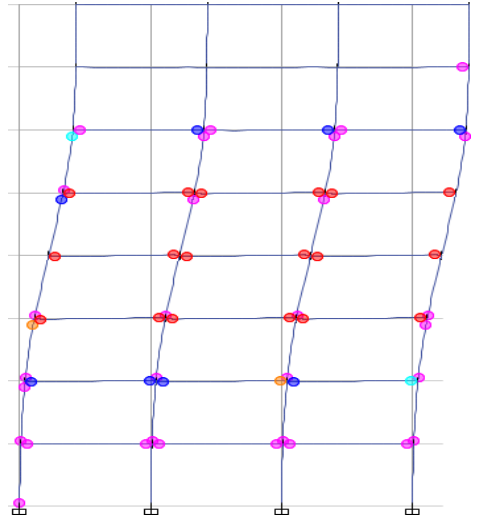

(c)

Figura 6.61 Distribución de rótulas plásticas para el pórtico regular de ocho plantas considerando el espectro tipo $1, a_{g}=1,0 \mathrm{~g}$ y terreno tipo C. (a) ETABS ${ }^{\circledR}$. (b) Biskinis y Fardis (2010a, 2010b). (c) Expresiones calibradas. 


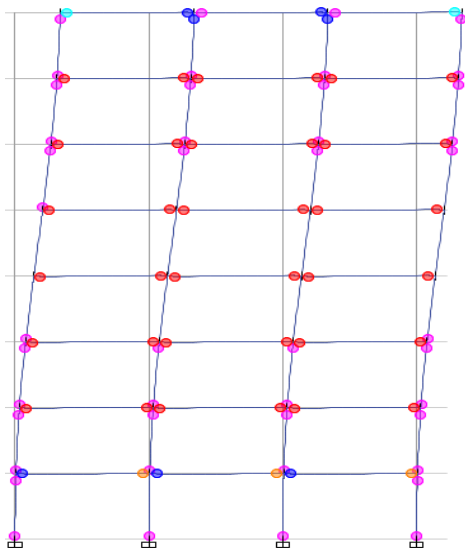

(a)

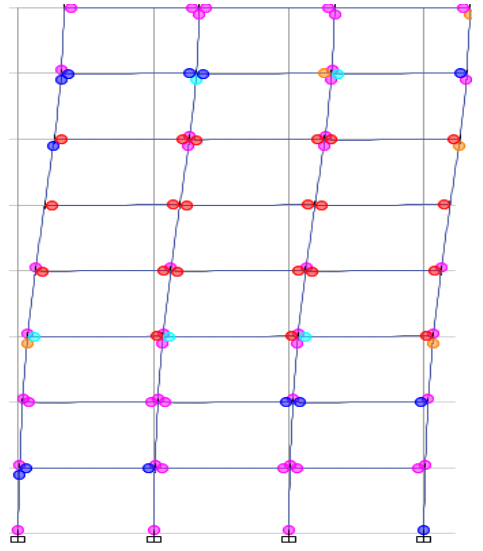

(b)

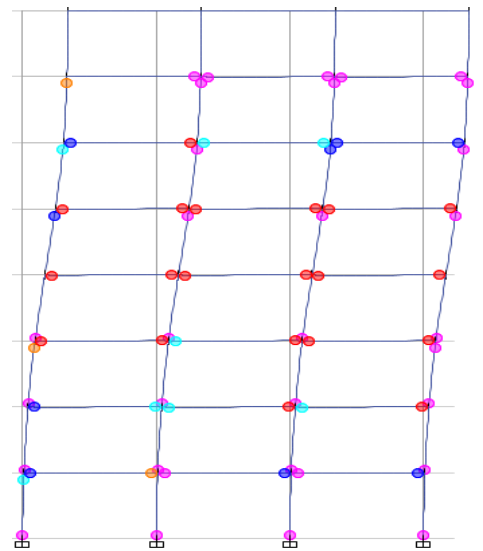

(c)

Figura 6.62 Distribución de rótulas plásticas para el pórtico regular de ocho plantas considerando el espectro tipo 1, $a_{g}=1,0 g$ y terreno tipo D. (a) ETABS®. (b) Biskinis y Fardis (2010a, 2010b). (c) Expresiones calibradas.

\subsubsection{Pórtico espacial de cinco plantas}

Las Tablas 6.25 y 6.26 muestran los valores de desplazamiento objetivo $d_{t}$ y los valores mínimos de $a_{g}$ para los cuales comienzan a apreciarse diferencias en los resultados obtenidos para el pórtico espacial de cinco plantas al aplicar los distintos tipos de rótulas plásticas. Para el espectro tipo 1, las expresiones calibradas con la selección de ensayos obtienen el menor valor de desplazamiento objetivo si se considera el mismo tipo de terreno, obteniéndose los mismos valores si se emplean los distintos tipos de rótulas al considerar el espectro tipo 2. Los valores mínimos de $a_{g}$ resultan superiores a los obtenidos para el pórtico regular de cinco plantas.

Tabla 6.25 Valores de desplazamiento objetivo $d_{t}$ para el pórtico espacial de cinco plantas.

\begin{tabular}{cccccccc}
\hline & \multicolumn{3}{c}{ Espectro tipo 1 } & \multicolumn{3}{c}{ Espectro tipo 2 } \\
Tipo de rótula plástica & \multicolumn{2}{c}{ Tipo de terreno } & \multicolumn{3}{c}{ Tipo de terreno } \\
& A & C & D & A & C & D \\
\hline ETABS $®$ & 397 & 685 & 1072 & 107 & 161 & 231 \\
Biskinis y Fardis (2010a, 2010b $)$ & 388 & 670 & 1048 & 107 & 161 & 231 \\
Expresiones calibradas & 371 & 640 & 1002 & 107 & 161 & 231 \\
\hline
\end{tabular}

Tabla 6.26 Valores mínimos de aceleración $a_{g}$ para los que existen diferencias entre los diferentes tipos de rótulas plásticas.

\begin{tabular}{cccccc}
\hline \multicolumn{3}{c}{ Espectro tipo 1 } & \multicolumn{3}{c}{ Espectro tipo 2 } \\
\multicolumn{2}{r}{ Tipo de terreno } & \multicolumn{3}{c}{ Tipo de terreno } \\
A & C & D & A & C & D \\
\hline 0,16 & 0,10 & 0,06 & 0,28 & 0,19 & 0,13 \\
\hline
\end{tabular}


La Figura 6.63 ofrece los desplazamientos laterales obtenidos para el espectro tipo 2. Si bien no se producen diferencias significativas entre los diferentes tipos de rótulas plásticas, las expresiones calibradas con la selección de ensayos obtienen los menores valores de desplazamiento. La Figura 6.64 muestra que, si se considera el espectro tipo $1 \mathrm{y}$ los terrenos tipo $\mathrm{C}$ y $\mathrm{D}$, las expresiones calibradas ofrecen desplazamientos superiores en las plantas primera a tercera, sucediendo lo contrario en las dos últimas plantas.

La Figura 6.65 muestra los drifts obtenidos considerando las distintas rótulas plásticas y el espectro tipo 2, observándose que sólo se producen diferencias significativas para el terreno tipo D. Los drifts disminuyen al aumentar el número de planta de la estructura. En los resultados correspondientes al espectro tipo 1, mostrados en la Figura 6.66, se observa que las expresiones calibradas obtienen los mayores valores para las plantas segunda y tercera, sucediendo lo contrario para las dos últimas plantas.

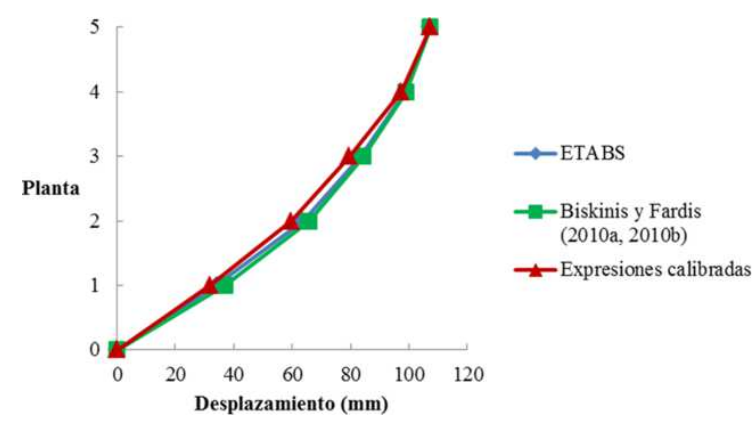

(a)

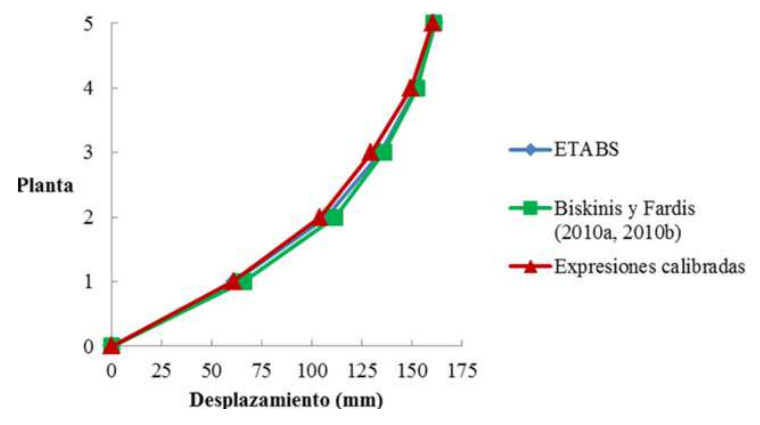

(b)

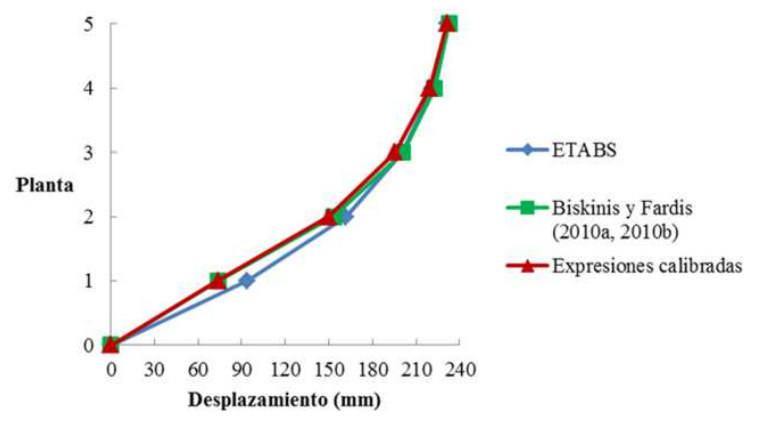

(c)

Figura 6.63 Desplazamientos laterales del pórtico espacial de cinco plantas para el espectro tipo 2 y $a_{g}=0,5 g$. (a) Terreno tipo A. (b) Terreno tipo C. (c) Terreno tipo D.

Las limitaciones impuestas por el EC-8 en los valores del drift se cumplen para el espectro tipo 2 y los terrenos tipo A y C. Para el terreno tipo D, las rótulas plásticas de ETABS ${ }^{\circledR}$ son las únicas que no cumplen las limitaciones. El espectro tipo 1 proporciona valores de drift superiores a los máximos admisibles para todos los casos analizados. 


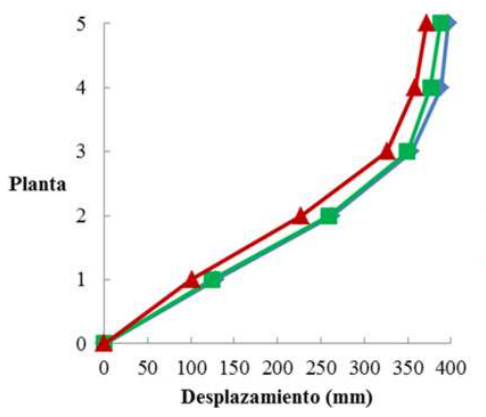

(a)

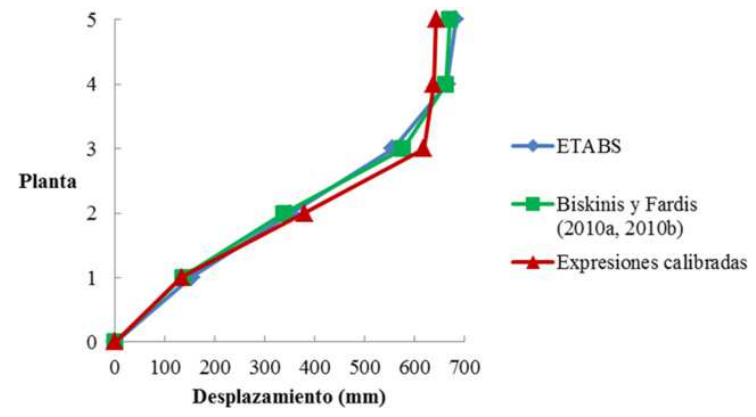

(b)

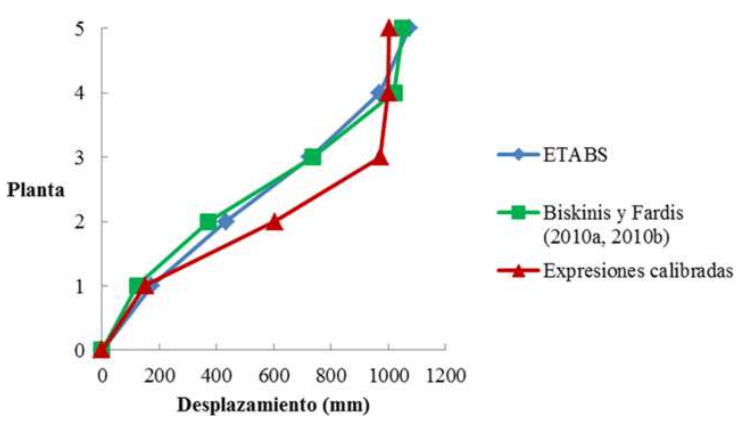

(c)

Figura 6.64 Desplazamientos laterales del pórtico espacial de cinco plantas para el espectro tipo 1 y $a_{g}=1,0 g$. (a) Terreno tipo A. (b) Terreno tipo C. (c) Terreno tipo D.

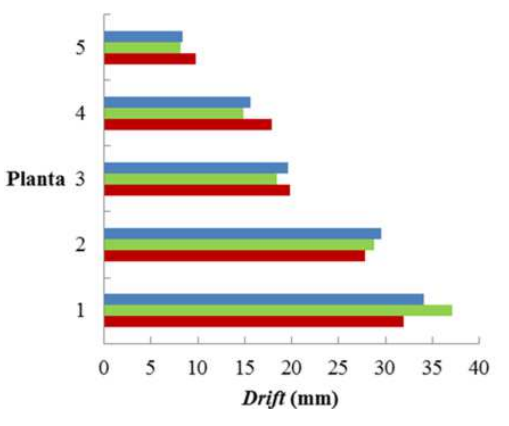

(a)

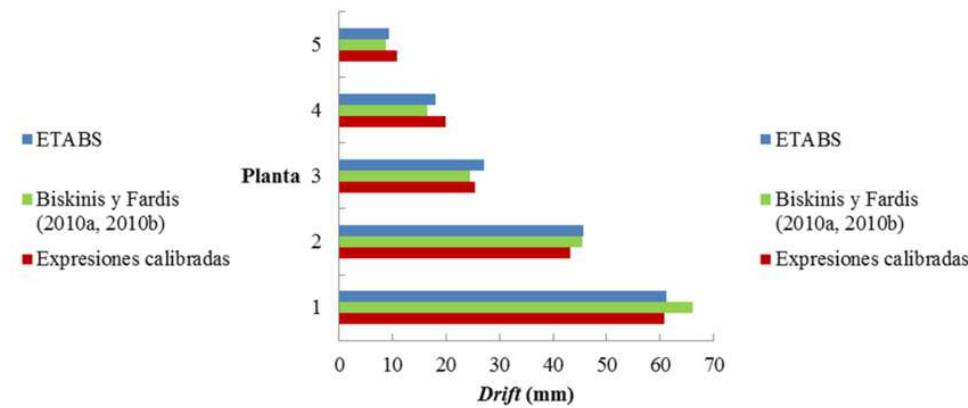

(b)

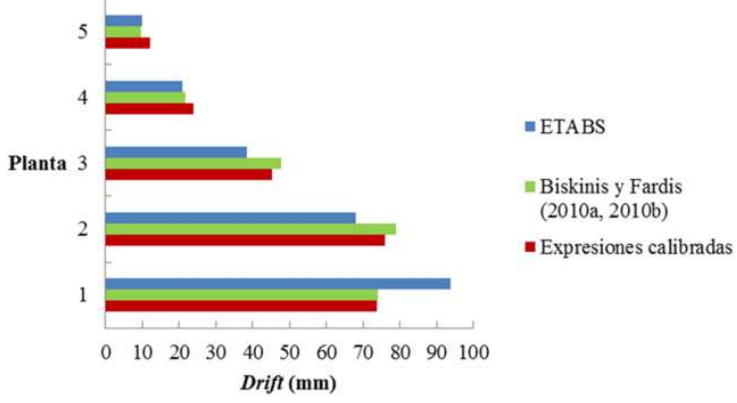

(c)

Figura 6.65 Drifts para el pórtico espacial de cinco plantas considerando el espectro tipo 2 y $a_{g}$ $=0,5 g$. (a) Terreno tipo A. (b) Terreno tipo C. (c) Terreno tipo D. 


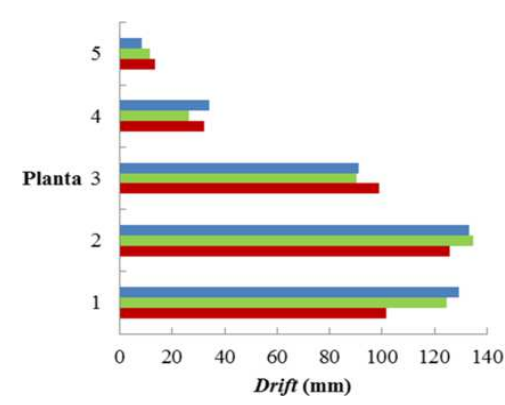

(a)

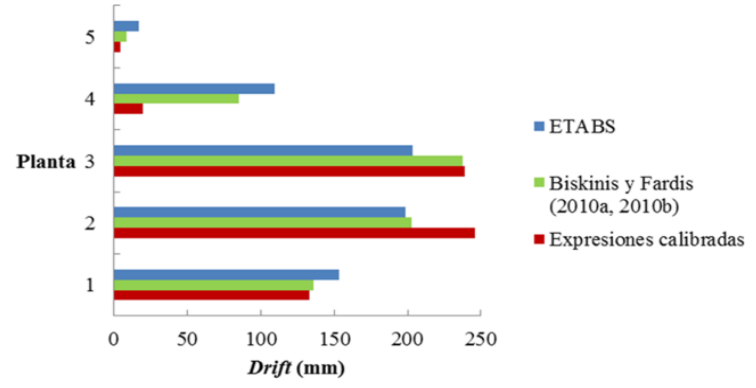

(b)

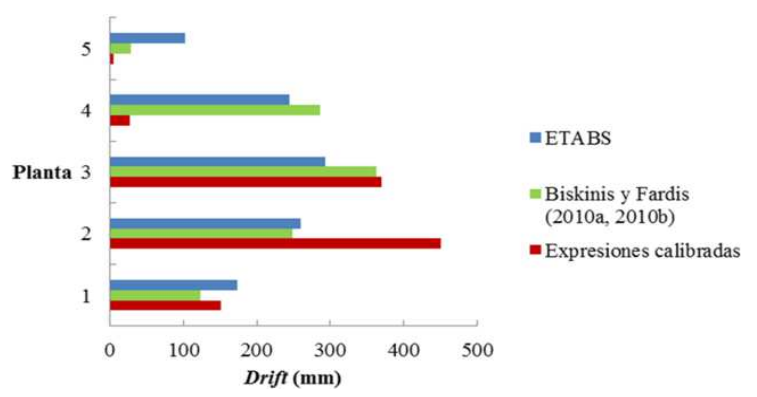

(c)

Figura 6.66 Drifts para el pórtico espacial de cinco plantas considerando el espectro tipo 1 y $a_{g}$ = 1,0g. (a) Terreno tipo A. (b) Terreno tipo C. (c) Terreno tipo D.

Las distribuciones de rótulas plásticas de las Figuras 6.67 a 6.72 muestran las mismas características que las obtenidas para los pórticos regulares de cinco y ocho plantas, esto es, las expresiones calibradas con la selección de ensayos propician la aparición de un menor número de rótulas, las cuales presentan niveles de comportamiento menos exigentes. Sin embargo, dichas expresiones obtienen rótulas en ciertos pilares con niveles de comportamiento más desfavorables que los obtenidos con otros tipos de rótulas, tal y como se observa en las plantas intermedias de las estructuras mostradas en las Figuras 6.70 a 6.72.

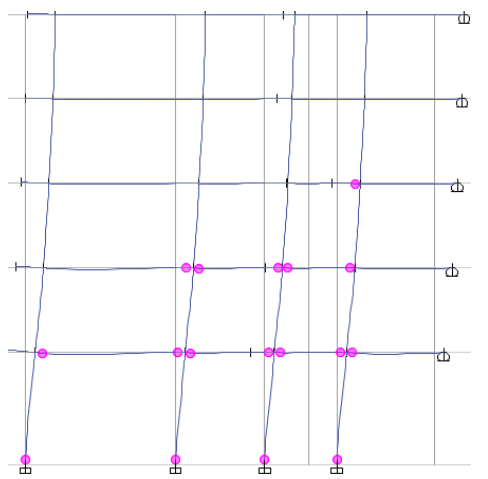

(a)

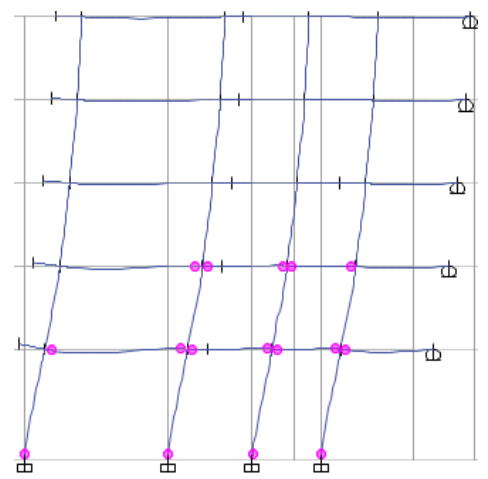

(b)

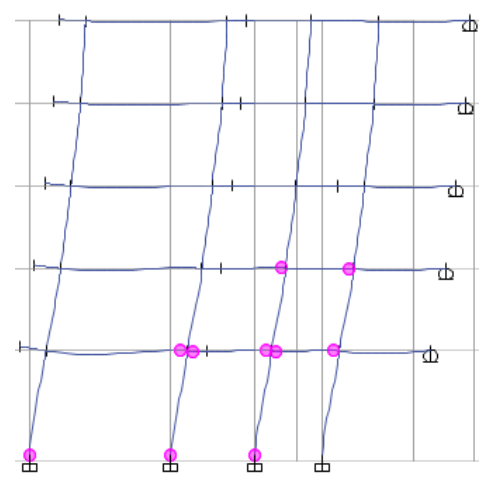

(c)

Figura 6.67 Distribución de rótulas plásticas para el pórtico espacial de cinco plantas considerando el espectro tipo 2, $a_{g}=0,5 g$ y terreno tipo A. (a) ETABS $®$. (b) Biskinis y Fardis (2010a, 2010b). (c) Expresiones calibradas. 


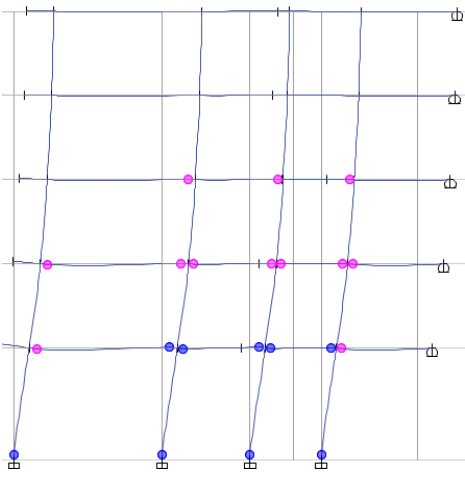

(a)

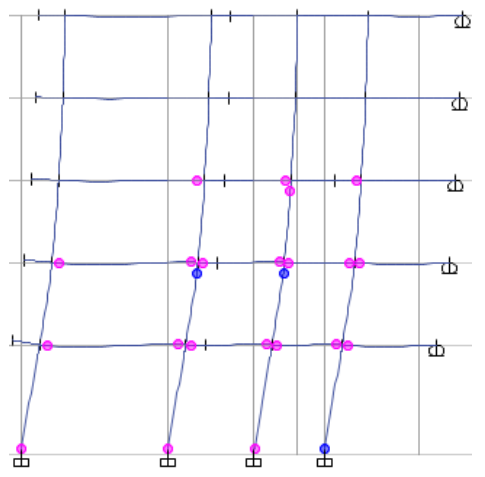

(b)

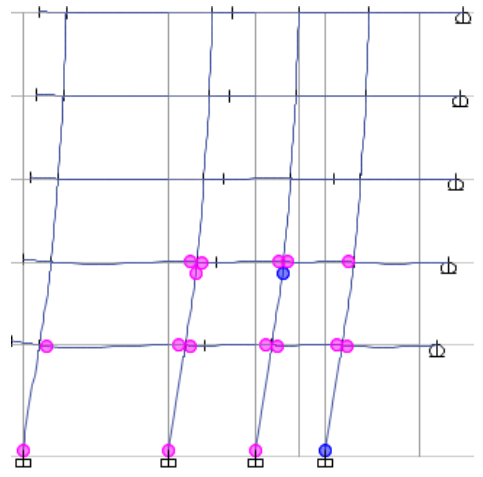

(c)

Figura 6.68 Distribución de rótulas plásticas para el pórtico espacial de cinco plantas considerando el espectro tipo 2, $a_{g}=0,5 g$ y terreno tipo C. (a) ETABS $®$. (b) Biskinis y Fardis (2010a, 2010b). (c) Expresiones calibradas.

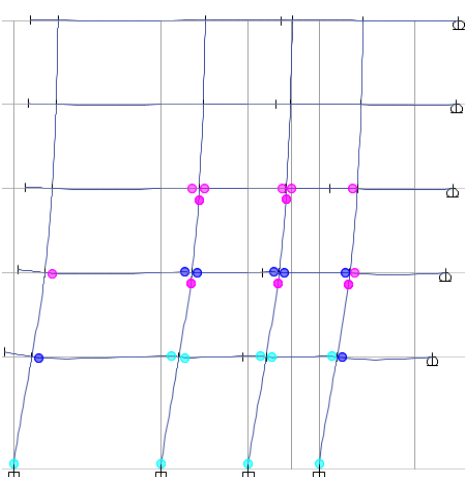

(a)

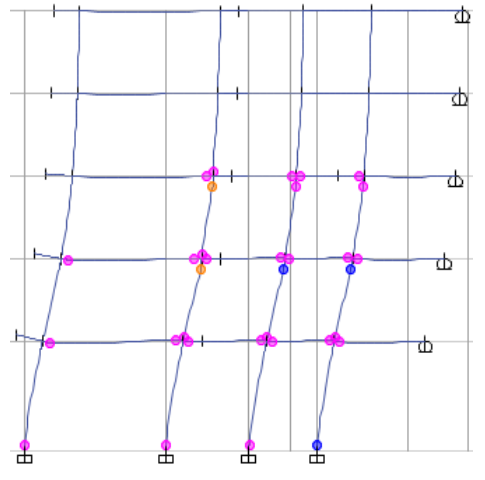

(b)

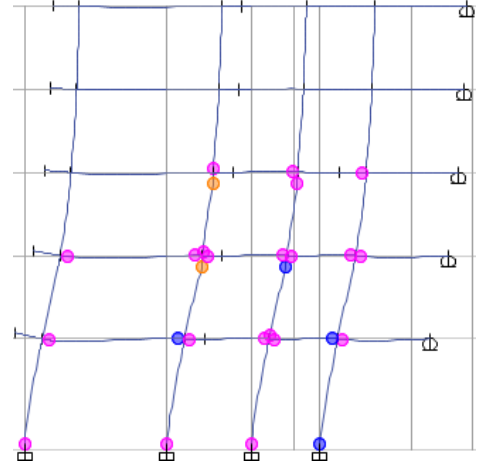

(c)

Figura 6.69 Distribución de rótulas plásticas para el pórtico espacial de cinco plantas considerando el espectro tipo 2, $a_{g}=0,5 g$ y terreno tipo D. (a) ETABS®. (b) Biskinis y Fardis (2010a, 2010b). (c) Expresiones calibradas.

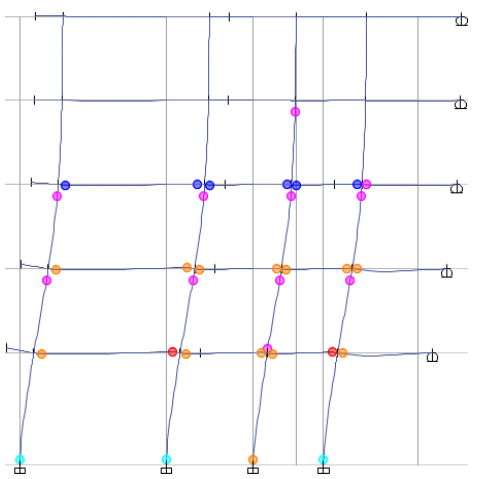

(a)

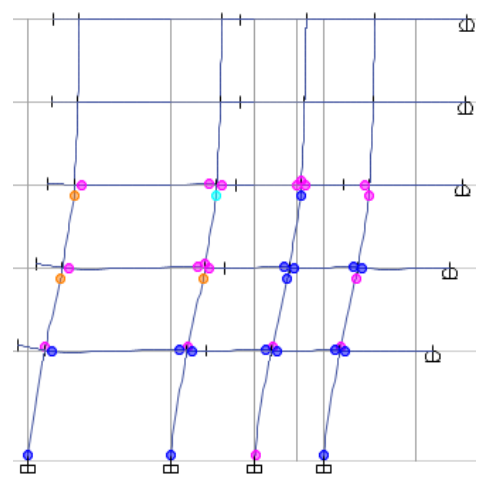

(b)

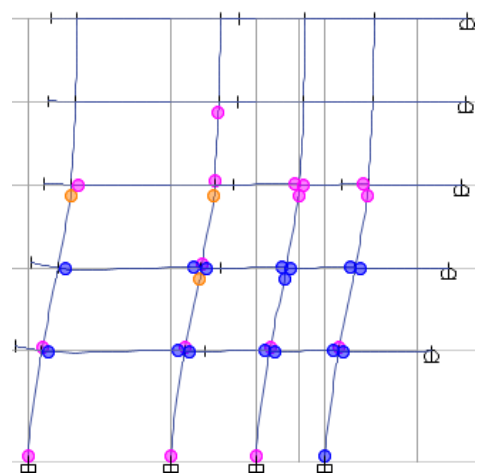

(c)

Figura 6.70 Distribución de rótulas plásticas para el pórtico espacial de cinco plantas considerando el espectro tipo 1, $a_{g}=1,0 g$ y terreno tipo A. (a) ETABS®. (b) Biskinis y Fardis (2010a, 2010b). (c) Expresiones calibradas. 


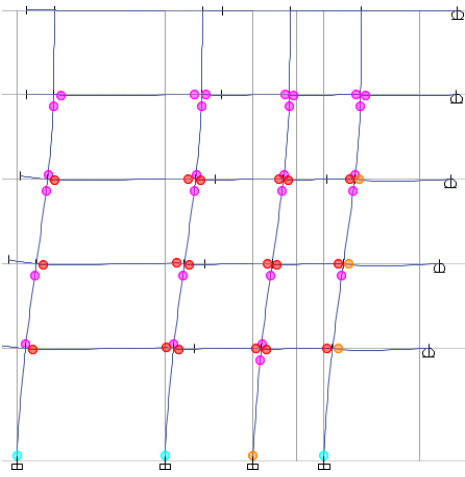

(a)

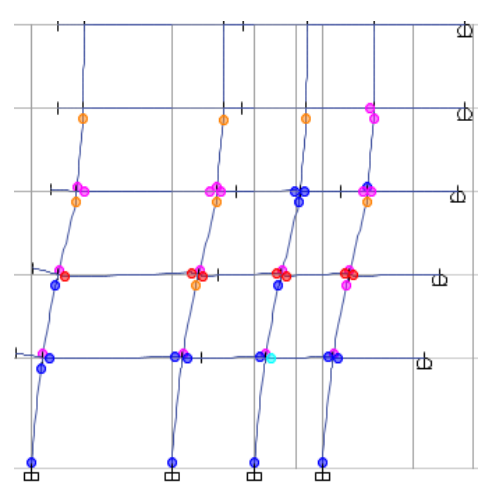

(b)

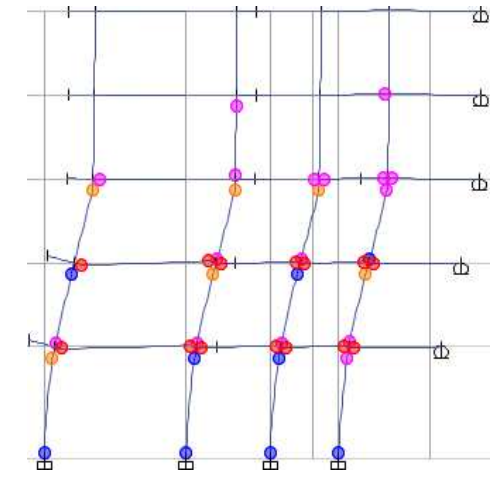

(c)

Figura 6.71 Distribución de rótulas plásticas para el pórtico espacial de cinco plantas considerando el espectro tipo 1, $a_{g}=1,0 g$ y terreno tipo C. (a) ETABS®. (b) Biskinis y Fardis (2010a, 2010b). (c) Expresiones calibradas.

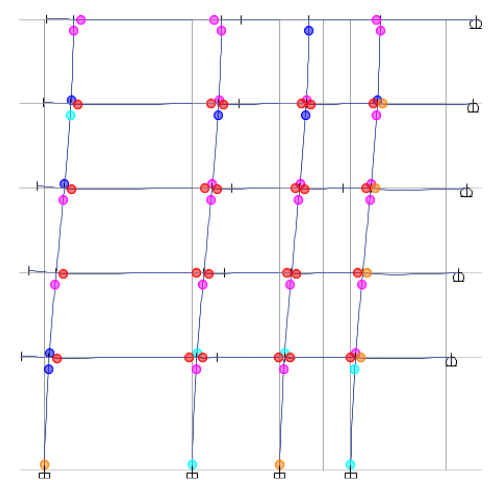

(a)

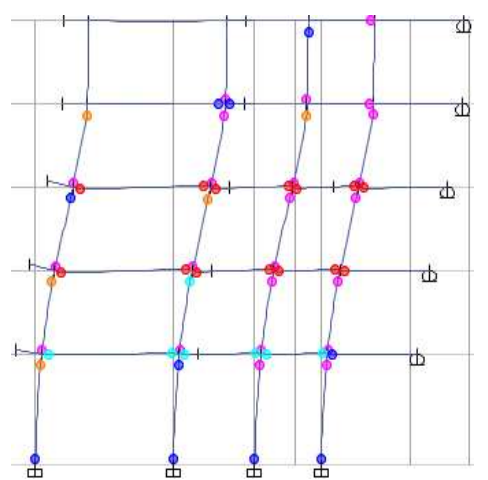

(b)

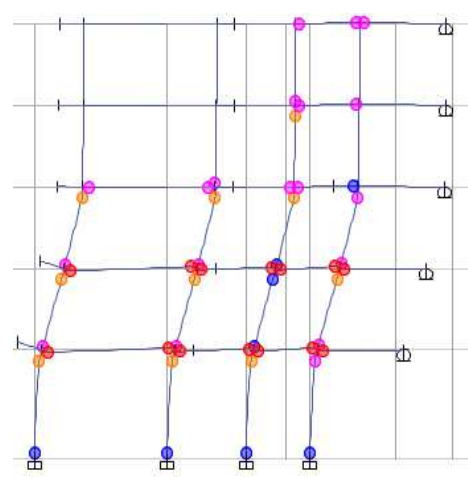

(c)

Figura 6.72 Distribución de rótulas plásticas para el pórtico espacial de cinco plantas considerando el espectro tipo 1, $a_{g}=1,0 g$ y terreno tipo D. (a) ETABS®. (b) Biskinis y Fardis (2010a, 2010b). (c) Expresiones calibradas.

\subsubsection{Pórtico espacial de ocho plantas}

Las Tablas 6.27 y 6.28 muestran los valores de desplazamiento objetivo $d_{t}$ y los valores mínimos de $a_{g}$ para los cuales comienzan a apreciarse diferencias en los resultados obtenidos al aplicar los distintos tipos de rótulas plásticas, correspondientes al pórtico espacial de ocho plantas. Al igual que sucede para los pórticos anteriores, las rótulas modelizadas mediante las expresiones calibradas con la selección de ensayos obtienen valores inferiores de desplazamiento objetivo respecto a otros tipos de rótulas si se emplea el espectro tipo 1. La mayor altura y flexibilidad del pórtico espacial de ocho plantas hace que se obtengan valores mínimos de $a_{\mathrm{g}}$ superiores a los obtenidos para el pórtico espacial de cinco plantas. 
Tabla 6.27 Valores de desplazamiento objetivo $d_{t}$ para el pórtico espacial de ocho plantas.

\begin{tabular}{cccccccc}
\hline & \multicolumn{3}{c}{ Espectro tipo 1 } & \multicolumn{3}{c}{ Espectro tipo 2 } \\
Tipo de rótula plástica & \multicolumn{2}{c}{ Tipo de terreno } & \multicolumn{3}{c}{ Tipo de terreno } \\
& A & C & D & A & C & D \\
\hline ETABS® & 619 & 1068 & 1672 & 120 & 180 & 259 \\
Biskinis y Fardis (2010a, 2010b ) & 613 & 1058 & 1656 & 120 & 180 & 259 \\
Expresiones calibradas & 610 & 1052 & 1646 & 120 & 180 & 259 \\
\hline
\end{tabular}

Tabla 6.28 Valores mínimos de aceleración $a_{g}$ para los que existen diferencias entre los diferentes tipos de rótulas plásticas.

\begin{tabular}{ccccccc}
\hline \multicolumn{3}{c}{ Espectro tipo 1 } & \multicolumn{3}{c}{ Espectro tipo 2 } \\
\multicolumn{2}{c}{ Tipo de terreno } & \multicolumn{3}{c}{ Tipo de terreno } \\
A & C & D & A & C & D \\
\hline 0,18 & 0,11 & 0,06 & 0,41 & 0,30 & 0,21 \\
\hline
\end{tabular}

Las Figuras 6.73 y 6.74 muestran los desplazamientos laterales para los distintos casos analizados. Para el espectro tipo 2 no se aprecian diferencias significativas al aplicar los distintos tipos de rótulas plásticas. Si se considera el espectro tipo 1, las expresiones calibradas con la selección de ensayos obtienen desplazamientos inferiores para las plantas primera a cuarta e iguales o superiores para el resto de plantas.

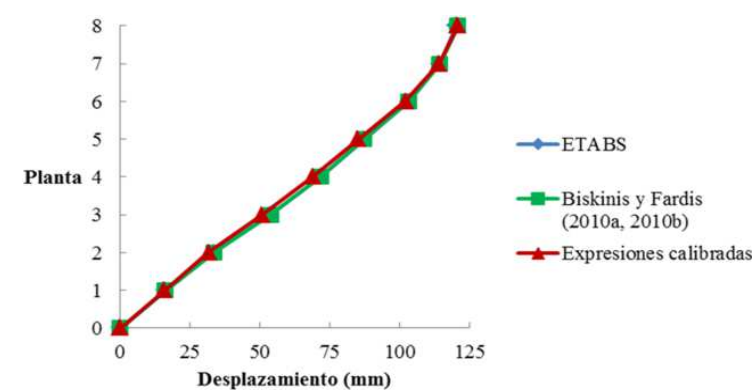

(a)

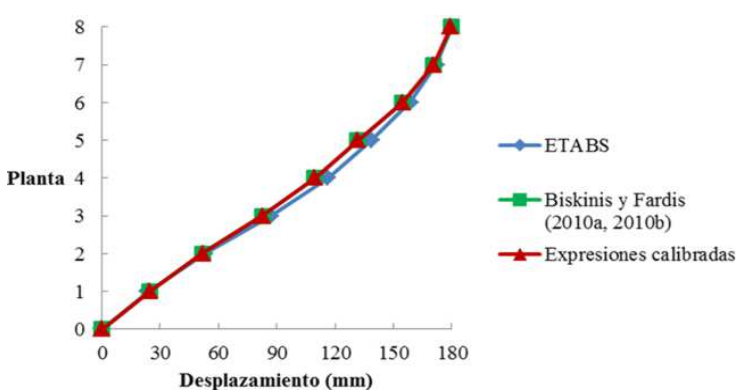

(b)

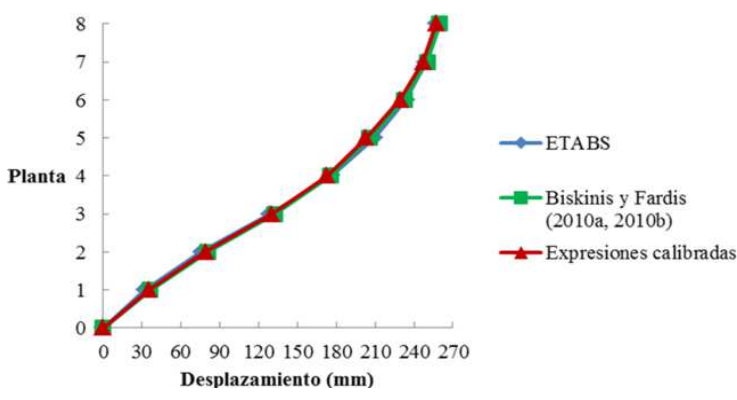

(c)

Figura 6.73 Desplazamientos laterales del pórtico espacial de ocho plantas para el espectro tipo 2 y $a_{g}=0,5 g$. (a) Terreno tipo A. (b) Terreno tipo C. (c) Terreno tipo D. 


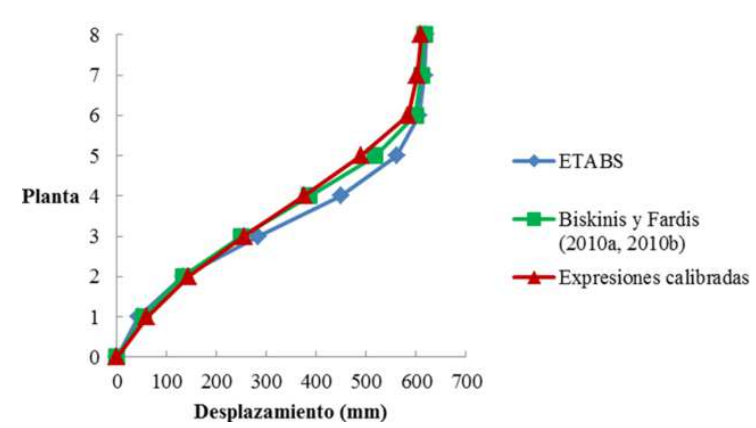

(a)

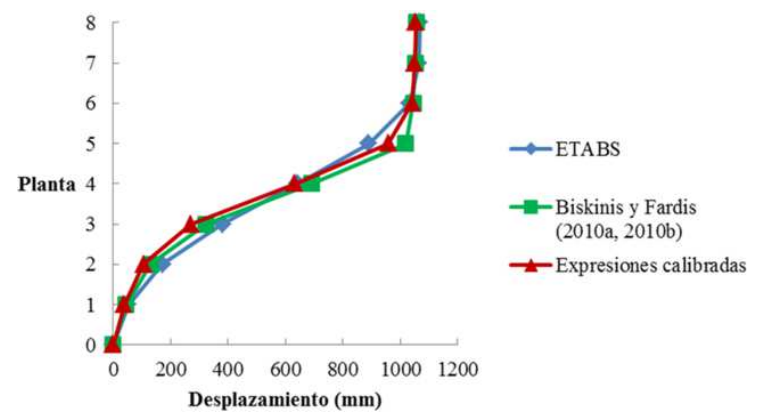

(b)

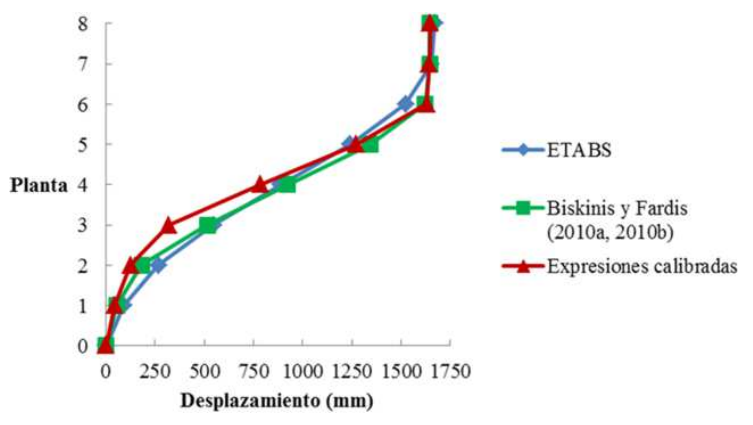

(c)

Figura 6.74 Desplazamientos laterales del pórtico espacial de ocho plantas para el espectro tipo 1 y $a_{g}=1,0 g$. (a) Terreno tipo A. (b) Terreno tipo C. (c) Terreno tipo D.

Los drifts obtenidos para el pórtico espacial de ocho plantas se muestran en las Figuras 6.75 y 6.76. Para el espectro tipo 2 no se aprecian diferencias significativas al aplicar los distintos tipos de rótulas plásticas. Las expresiones calibradas con la selección de ensayos obtienen valores superiores a los obtenidos con las rótulas de ETABS $®$ en las plantas cuarta y quinta para el espectro tipo 1 y los terrenos tipo C y D. Los valores de drift obtenidos satisfacen únicamente las restricciones impuestas por el EC-8 para el espectro tipo 2.

Las Figuras 6.77 a 6.82 muestran las distribuciones de rótulas plásticas para los diferentes casos analizados. De nuevo, las rótulas plásticas modelizadas con las expresiones ajustadas a la selección de ensayos obtienen una situación menos desfavorable que lo ofrecido por otros tipos de rótulas. Destaca el caso en el que se considera el terreno tipo A y el espectro tipo 2, mostrado en la Figura 6.77, en el que no se generan rótulas plásticas al emplear las expresiones calibradas con la selección de ensayos. 


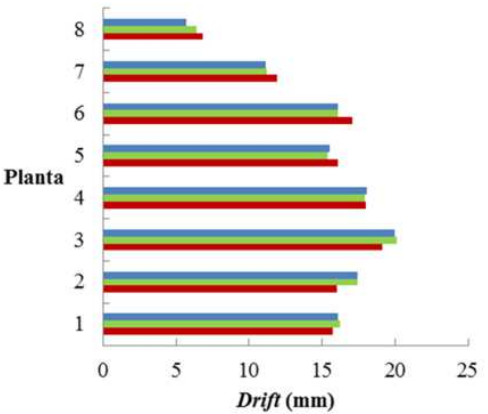

(a)

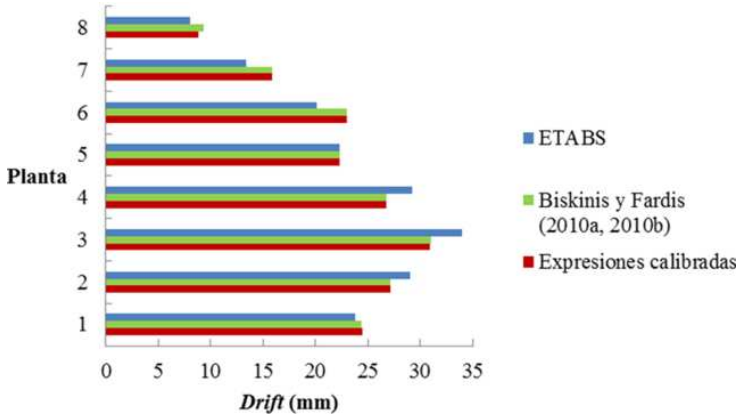

(b)

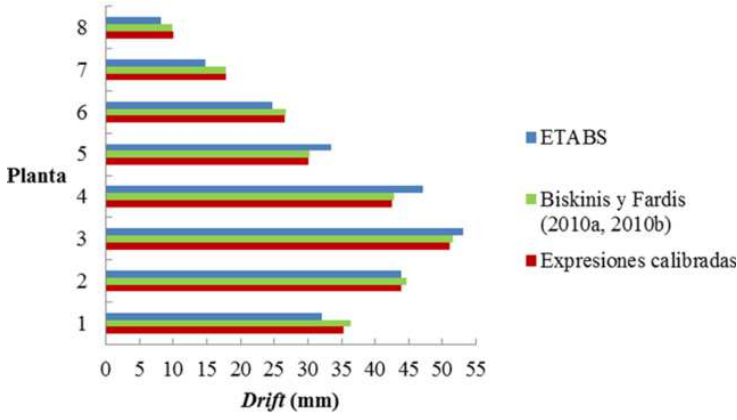

(c)

Figura 6.75 Drifts para el pórtico espacial de ocho plantas con espectro tipo 2 y $a_{g}=0,5 g$.

(a) Terreno tipo A. (b) Terreno tipo C. (c) Terreno tipo D.

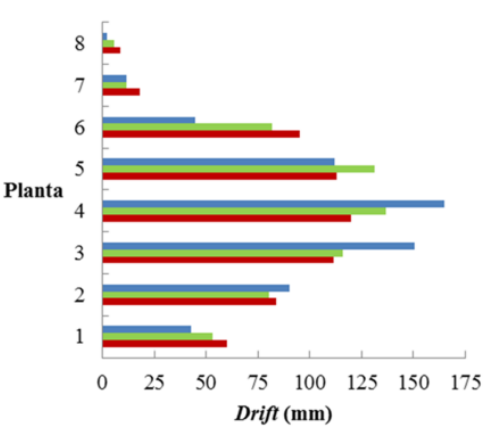

(a)

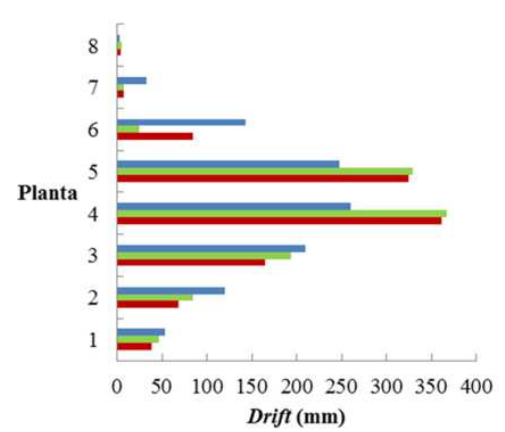

(b)

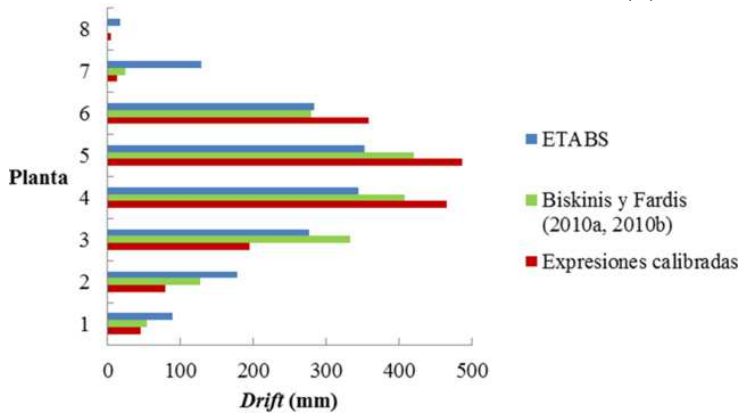

(c)

Figura 6.76 Drifts para el pórtico espacial de ocho plantas con espectro tipo 1 y $a_{g}=1,0 g$.

(a) Terreno tipo A. (b) Terreno tipo C. (c) Terreno tipo D. 


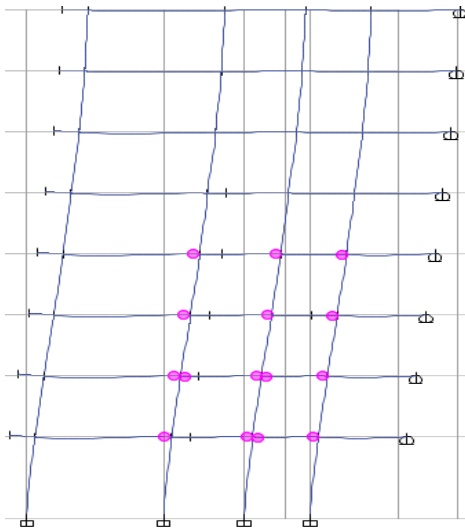

(a)

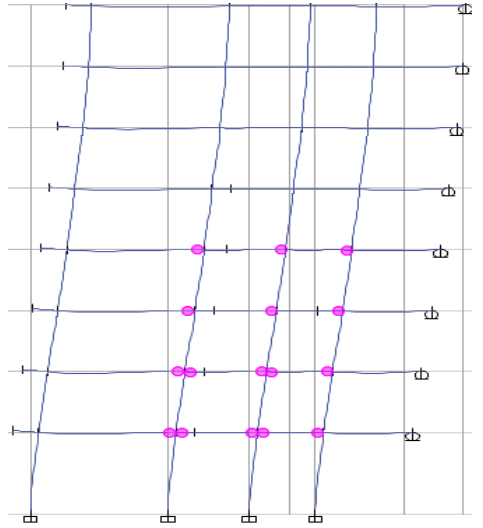

(b)

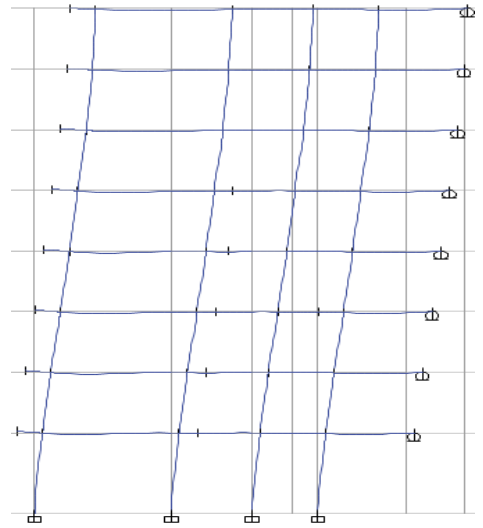

(c)

Figura 6.77 Distribución de rótulas plásticas para el pórtico espacial de ocho plantas considerando el espectro tipo 2, $a_{g}=0,5 g$ y terreno tipo A. (a) ETABS®. (b) Biskinis y Fardis (2010a, 2010b). (c) Expresiones calibradas.

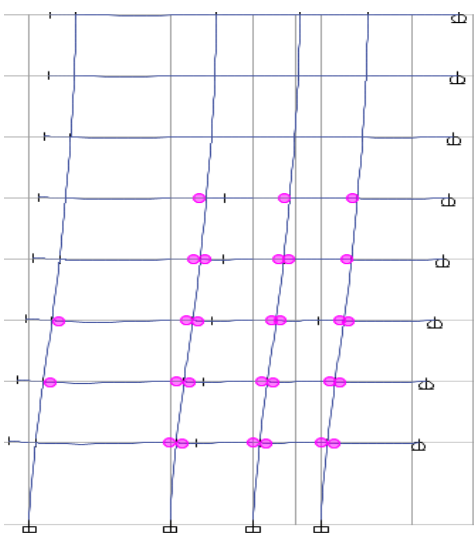

(a)

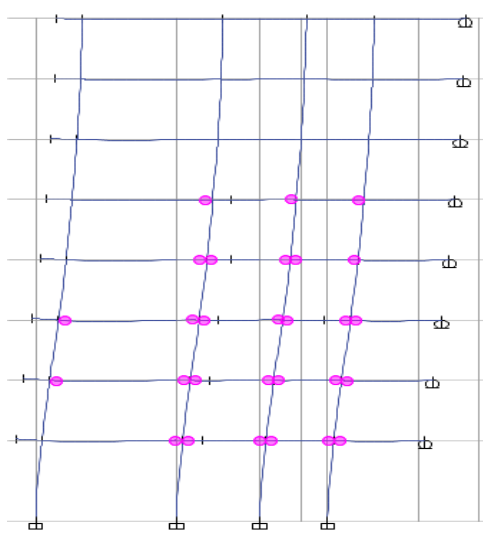

(b)

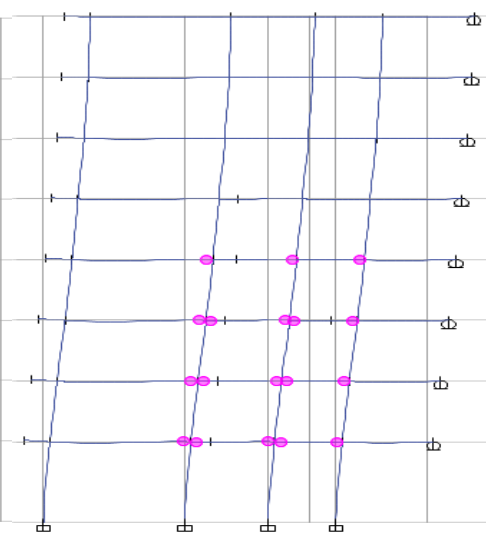

(c)

Figura 6.78 Distribución de rótulas plásticas para el pórtico espacial de ocho plantas considerando el espectro tipo $2, a_{g}=0,5 g$ y terreno tipo C. (a) ETABS $®$. (b) Biskinis y Fardis (2010a, 2010b). (c) Expresiones calibradas.

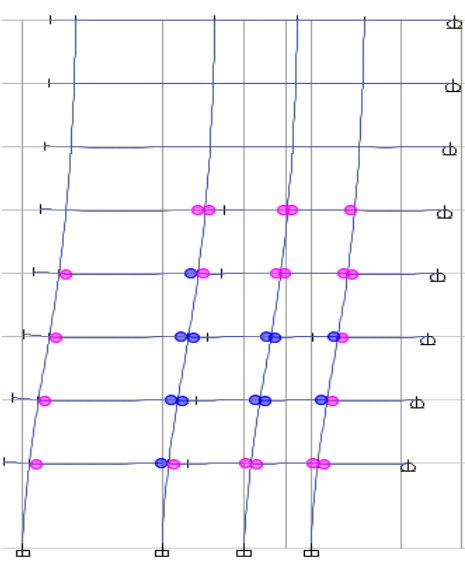

(a)

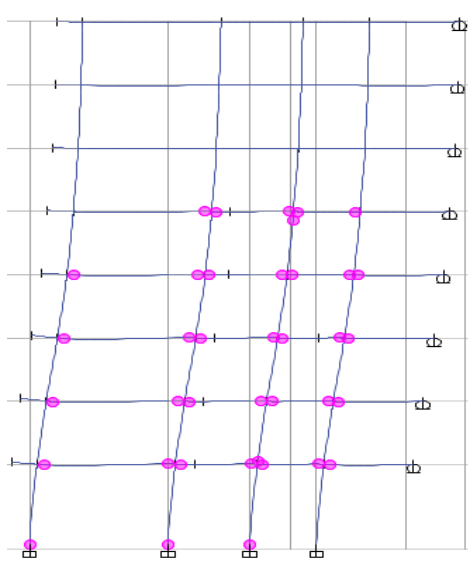

(b)

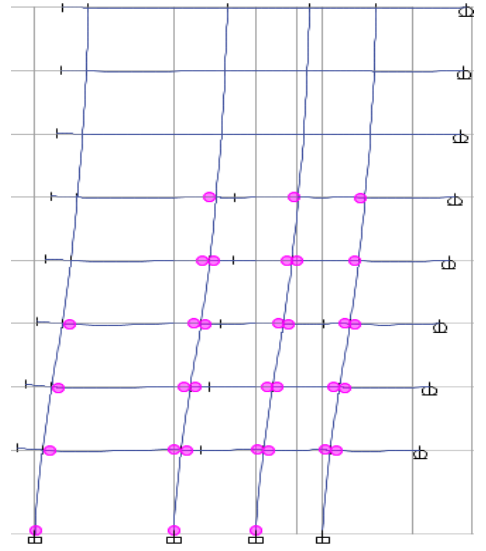

(c)

Figura 6.79 Distribución de rótulas plásticas para el pórtico espacial de ocho plantas considerando el espectro tipo 2, $a_{g}=0,5 g$ y terreno tipo D. (a) ETABS®. (b) Biskinis y Fardis (2010a, 2010b). (c) Expresiones calibradas. 


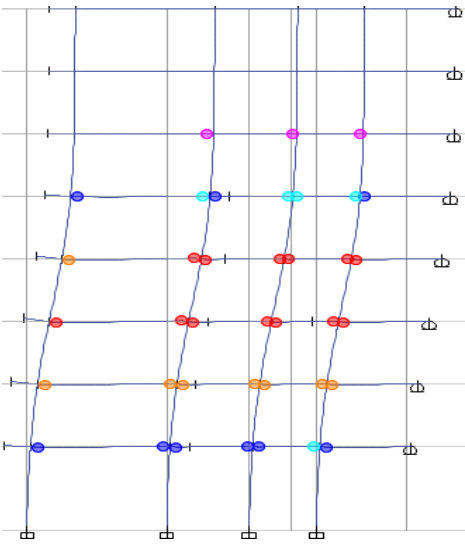

(a)

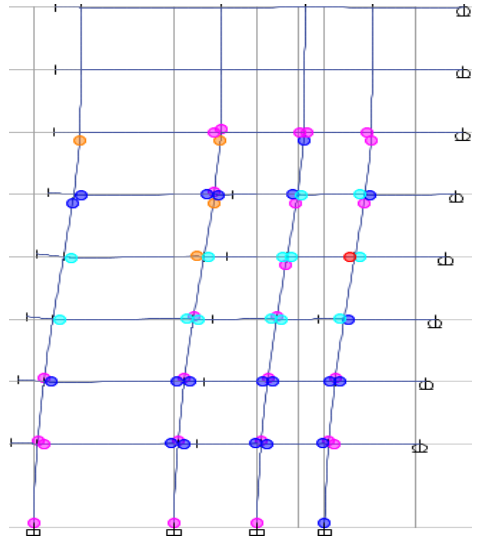

(b)

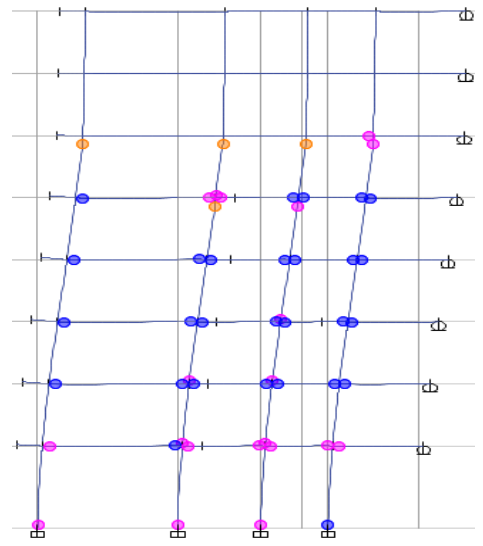

(c)

Figura 6.80 Distribución de rótulas plásticas para el pórtico espacial de ocho plantas considerando el espectro tipo 1, $a_{g}=1,0 g$ y terreno tipo A. (a) ETABS®. (b) Biskinis y Fardis (2010a, 2010b). (c) Expresiones calibradas.

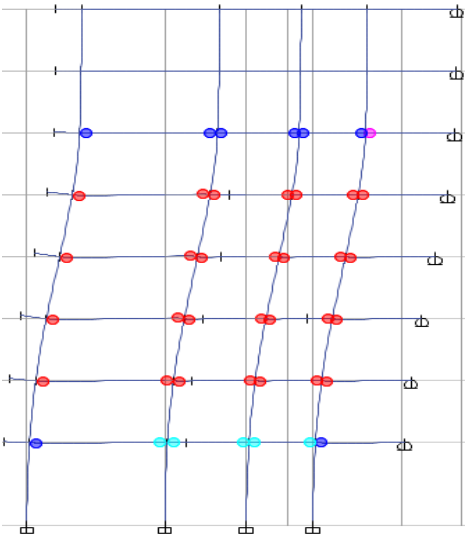

(a)

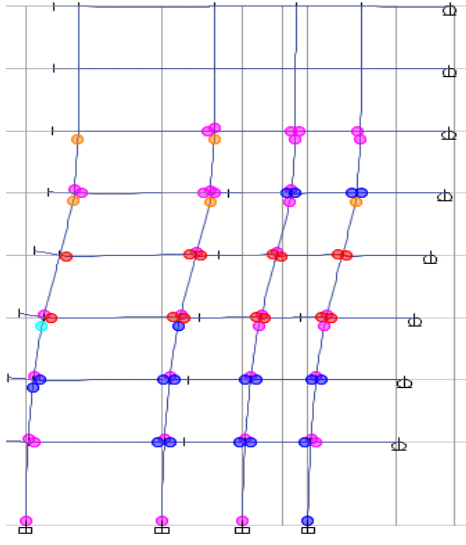

(b)

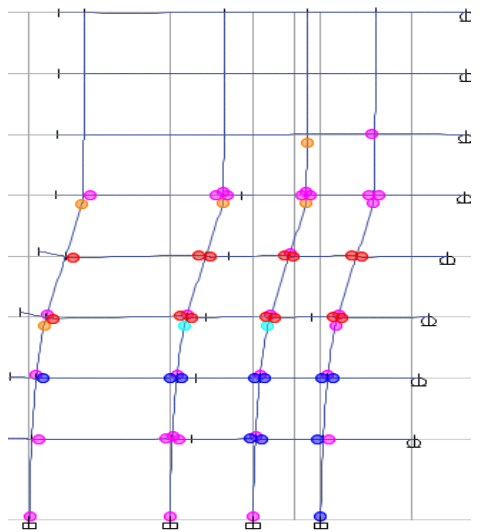

(c)

Figura 6.81 Distribución de rótulas plásticas para el pórtico espacial de ocho plantas considerando el espectro tipo 1, $a_{g}=1,0 g$ y terreno tipo C. (a) ETABS®. (b) Biskinis y Fardis (2010a, 2010b). (c) Expresiones calibradas.

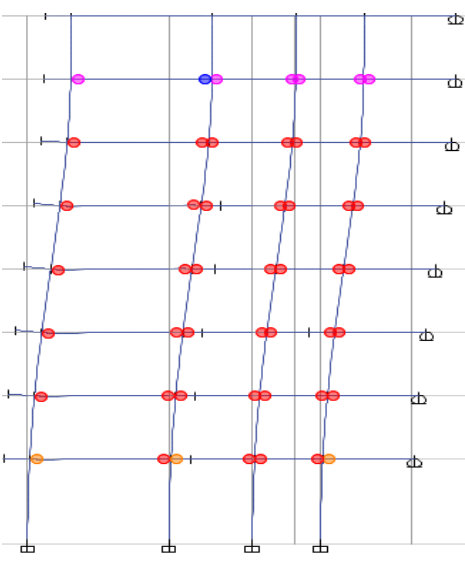

(a)

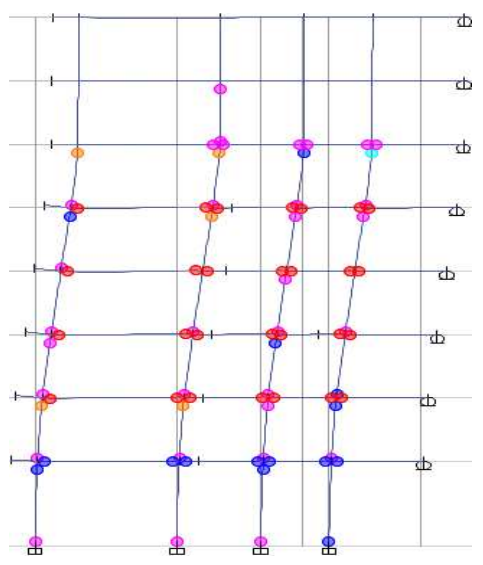

(b)

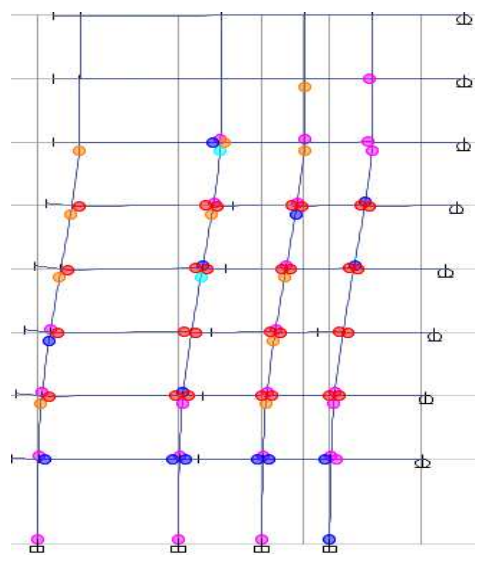

(c)

Figura 6.82 Distribución de rótulas plásticas para el pórtico espacial de ocho plantas considerando el espectro tipo 1, $a_{g}=1,0 g$ y terreno tipo D. (a) ETABS®. (b) Biskinis y Fardis (2010a, 2010b). (c) Expresiones calibradas. 


\subsubsection{Resumen de resultados}

Analizando las relaciones momento-giro de las rótulas plásticas caracterizadas mediante los diferentes métodos, se observa que las expresiones calibradas con la selección de ensayos proporcionan un momento de plastificación $M_{y}$ superior al obtenido con los otros métodos para todas las secciones de los elementos de los pórticos analizados. Para las vigas, la expresión de Biskinis y Fardis (2010a) proporciona valores de $M_{y}$ similares a los que ofrece ETABS® por defecto. En cuanto a la capacidad de deformación de las rótulas plásticas, las expresiones calibradas ofrecen un valor de giro último $\theta_{u}$ intermedio a los valores ofrecidos por la expresión de Biskinis y Fardis (2010b) y ETABS $®$. Además, las expresiones calibradas y las de Biskinis y Fardis (2010a, 2010b) ofrecen unas curvas de plastificación con valores de $M_{y}$ superiores a los que ETABS $®$ proporciona por defecto, correspondiendo los valores máximos a las curvas de plastificación obtenidas con las expresiones calibradas.

En cuanto a las curvas de capacidad obtenidas, las expresiones calibradas con la selección de ensayos proporcionan los valores máximos de esfuerzo cortante en la base $F_{b}$, debido a que dichas expresiones ofrecen los mayores valores del momento de plastificación $M_{y}$ de la sección. Además, las curvas de capacidad obtenidas con las rótulas plásticas modelizadas mediante las expresiones de Biskinis y Fardis (2010a, 2010b) y las expresiones calibradas con la selección de ensayos se cruzan para un cierto valor del desplazamiento $\delta$ del nudo de control de la estructura, aspecto que se justifica por la intersección entre las curvas de plastificación obtenidas mediante ambas expresiones para un cierto valor de esfuerzo axil $N$.

Respecto al punto de plastificación global de la estructura, se observa que, en general, el caso de carga lateral modal obtiene valores de desplazamiento $\delta$ del nudo de control superiores a los proporcionados al aplicar la carga uniforme, resultando inferiores los valores de $F_{b}$. Las rótulas plásticas modelizadas con las expresiones ajustadas a la selección de ensayos proporcionan los mayores valores de $\delta, F_{b}$ y $S_{a} / g$ para todos los casos analizados, debido a que dichas expresiones ofrecen los mayores valores de $M_{y}$. Las diferencias oscilan entre el 3 y el $35 \%$ para el desplazamiento $\delta$ del nudo de control y entre el 20 y el $45 \%$ para los valores del esfuerzo cortante en la base $F_{b}$, obteniéndose dichas diferencias respecto a lo ofrecido por las rótulas plásticas de ETABS®. Las difererencias en los valores de la aceleración del sistema equivalente de un grado de libertad $S_{a} / g$ presentan un valor máximo del $57 \%$, obtenido respecto a las rótulas plásticas de $\mathrm{ETABS}{ }^{\circledR}$. Resulta necesario destacar que las diferencias en $\delta, F_{b} \mathrm{y}$ $S_{a} / g$ aumentan con la altura de la estructura.

En el punto de colapso de la estructura, la mayor flexibilidad de las estructuras al aumentar su altura proporciona valores superiores de $\delta$ e inferiores para $F_{b}$ y $S_{a} / g$. Las rótulas plásticas modelizadas con las expresiones de Biskinis y Fardis (2010a, 2010b) proporcionan los mayores valores de $\delta$ para todos los casos analizados, ya que dichas 
expresiones ofrecen los máximos valores de $\theta_{u}$. Se obtiene una diferencia máxima del $151 \%$ respecto a lo ofrecido por ETABS $®$. Las expresiones calibradas con la selección de ensayos proporcionan los máximos valores de $F_{b}$ y $S_{a} / g$. Las diferencias en $F_{b}$ están comprendidas entre el $5 \%$ respecto a las rótulas caracterizadas con las expresiones de Biskinis y Fardis (2010a, 2010b) y el 79 \% respecto a lo proporcionado por ETABS®. La máxima diferencia obtenida en $S_{a} / g$ es del $95 \%$ respecto a lo ofrecido por ETABS®. Resulta conveniente destacar que, para el punto de plastificación global y de colapso de la estructura, los mayores valores de $M_{y}$ obtenidos con las expresiones calibradas con la selección de ensayos justifican que las distribuciones de rótulas plásticas obtenidas con las expresiones calibradas resulten menos desfavorables que las proporcionadas por los otros métodos.

Al estudiar la influencia del tipo de espectro, el tipo de terreno y el valor de $a_{g}$, en general se observa que los valores de $d_{t}$ obtenidos coinciden al emplear los distintos tipos de rótulas plásticas si se utiliza el espectro tipo 2 . Si se considera el espectro tipo 1 , los valores de $d_{t}$ resultan inferiores para las expresiones calibradas con la selección de ensayos. Otro aspecto a destacar es que los mínimos valores de $a_{g}$ que inducen diferencias entre los diferentes tipos de rótulas plásticas resultan inferiores cuanto más exigente resulta la acción sísmica considerada.

En lo que respecta a los desplazamientos laterales de las plantas, para los pórticos regulares no se aprecian diferencias significativas al aplicar los diferentes tipos de rótulas plásticas si se considera el espectro tipo 2, obteniéndose valores superiores para las expresiones calibradas con la selección de ensayos y las expresiones de Biskinis y Fardis (2010a, 2010b) respecto a lo ofrecido por ETABS® si se emplea el espectro tipo 1. Para los pórticos espaciales, se aprecian diferencias entre los distintos tipos de rótulas aplicando ambos tipos de espectros, obteniéndose diferencias más significativas para el espectro tipo 1 .

Para los drifts, las diferencias más significativas se aprecian para el espectro tipo 1. En general, las limitaciones impuestas por el EC-8 únicamente se satisfacen para los terrenos A y $\mathrm{C}$ al considerar el espectro tipo 2. En cuanto a la plastificación de las estructuras analizadas, las rótulas plásticas caracterizadas con las expresiones calibradas con la selección de ensayos proporcionan unos niveles de comportamiento menos desfavorables que los obtenidos aplicando otros tipos de rótulas. Sin embargo, para ciertos casos, dichas expresiones obtienen rótulas plásticas en pilares intermedios con niveles de comportamiento más desfavorables que los obtenidos con otros tipos de rótulas, lo cual puede resultar indicativo de un tipo de rotura frágil. Conforme la acción sísmica es más exigente, se observa una secuencia de plastificación similar para todos los tipos de rótulas plásticas, apareciendo las primeras rótulas en las vigas de las plantas inferiores y generándose posteriormente en las vigas de las plantas superiores y en los pilares, lo cual resulta indicativo de un adecuado diseño sísmico. 
La mayor capacidad resistente y de deformación de la sección ofrecida por las expresiones ajustadas a la selección de ensayos respecto a lo modelizado en ETABS® justifica la obtención de valores superiores en $F_{b}$ y $\delta$, aspecto que implica una mayor resistencia y capacidad de deformación a nivel estructural. Sin embargo, los valores superiores obtenidos para $S_{a} / g$ implican la introducción de esfuerzos superiores en la estructura.

Estas circunstancias pueden hacer plantearse al ingeniero estructural el método de modelización de rótulas plásticas a introducir en un código comercial de cálculo, como ETABS ${ }^{\circledR}$ en este caso, para la realización de análisis pushover, pudiendo comprobarse si lo que el código considera por defecto conduce a resultados excesivamente conservadores. Dicha comprobación resulta tanto más interesante cuanto mayor es la altura de la estructura y la exigencia de la acción sísmica, aspectos que incrementan las diferencias obtenidas entre los distintos métodos de modelización de rótulas.

Por otro lado, el hecho de que la selección de ensayos se haya realizado imponiendo las prescripciones sísmico-constructivas de los principales códigos nacionales e internacionales hace que las rótulas modelizadas con las expresiones ajustadas a dicha selección tengan un mayor campo de aplicación que las rótulas que un código comercial de cálculo ofrezca por defecto, las cuales pueden estar caracterizadas mediante valores correspondientes a elementos de hormigón armado empleados en un país determinado o grupo de países que emplean una normativa concreta. 



\section{Capítulo 7}

\section{Conclusiones}

\subsection{INTRODUCCIÓN}

Este capítulo tiene por objeto resumir el trabajo de investigación realizado en la tesis, exponer las conclusiones principales y plantear futuras líneas de investigación derivadas del trabajo realizado.

\subsection{TRABAJOS REALIZADOS}

En la elaboración de esta tesis se han realizado los siguientes trabajos:

- Revisión bibliográfica de artículos de revistas, libros y congresos relacionados con la modelización numérica de estructuras de hormigón armado sometidas a acciones sísmicas. Adicionalmente, se han recopilado los trabajos más relevantes relativos al empleo de técnicas de optimización para la calibración de modelos numéricos.

- Descripción de la base de datos empleada como base de ensayos inicial, detallando aspectos como los elementos que la componen, las condiciones de los ensayos y los parámetros considerados en cada ensayo.

- Justificación de la normativa empleada y de los parámetros considerados, con sus rangos correspondientes, para la confección de una selección de ensayos procedentes de la base de ensayos inicial.

- Descripción de las expresiones propuestas por diversos autores para determinar conceptos relativos al estado de plastificación y último de secciones de hormigón armado, como el momento de plastificación $M_{y}$, el giro de plastificación $\theta_{y}$ y el giro último $\theta_{u}$.

- Realización de un estudio de sensibilidad de los diferentes parámetros que intervienen en las expresiones, con objeto de establecer los términos en los que se introducen coeficientes de ajuste $C_{i}$ para calibrar las expresiones con la base de ensayos inicial y con la selección de ensayos. 
- Propuesta de parámetros estadísticos para realizar un estudio comparativo entre los resultados obtenidos al aplicar las expresiones de Panagiotakos y Fardis (2001), las expresiones del EC-8, las expresiones de Biskinis y Fardis (2010a, 2010b) y las expresiones calibradas en esta tesis.

- Empleo de estrategias de optimización para el ajuste y calibración de las diferentes expresiones con las bases de datos utilizadas: algoritmos genéticos para el ajuste con la selección de ensayos y el algoritmo Firefly para la calibración con la base de ensayos inicial. Este algoritmo constituye una técnica novedosa y de uso creciente en la actualidad debido a las ventajas que ofrece frente a otros algoritmos de optimización.

- Evaluación de la influencia de diversas configuraciones de armadura longitudinal y distintos niveles de esfuerzo axil según se empleen las expresiones calibradas con la selección de ensayos, las expresiones de Biskinis y Fardis (2010a, 2010b) y las de un método clásico.

- Análisis estático no lineal (pushover) de cuatro pórticos de hormigón armado, empleando el método N2 propuesto por el EC-8 e implementando tres tipos de rótulas plásticas: (i) las modelizadas mediante las expresiones ajustadas a la selección de ensayos, (ii) las caracterizadas con las expresiones de Biskinis y Fardis (2010a, 2010b), y (iii) las consideradas por defecto en ETABS®, programa empleado para realizar los análisis.

- Modelización de las rótulas plásticas mediante curvas de respuesta momento-giro de tipo elastoplástico, definiendo, además, curvas de plastificación para las rótulas generadas en los pilares, con objeto de considerar la influencia del esfuerzo axil en el valor obtenido para el momento de plastificación de la sección.

- Consideración, en los análisis pushover, de distribuciones de carga lateral uniforme y modal, obteniéndose en ambos casos la curva de capacidad de la estructura. Adicionalmente, se ha obtenido el esfuerzo cortante en la base $F_{b}$, el desplazamiento del nudo de control $\delta$ y la aceleración $S_{a} / g$ del sistema equivalente de un grado de libertad en los puntos de plastificación global y de colapso de la estructura.

- Análisis de la influencia del tipo de terreno y del espectro propuestos por el EC-8 en los resultados obtenidos según las distintas rótulas plásticas implementadas. Para cada tipo de espectro se ha considerado un valor de la aceleración del suelo algo superior a los máximos históricos registrados, y se ha identificado cuál es el valor mínimo de la aceleración del suelo que provoca diferencias al aplicar los distintos tipos de rótulas plásticas.

- Obtención, para cada análisis, de los desplazamientos laterales de las plantas, los desplazamientos relativos o drifts entre plantas, la distribución de rótulas plásticas y los niveles de comportamiento que indican el estado límite alcanzado en la estructura. 


\subsection{CONCLUSIONES}

Del trabajo desarrollado en esta tesis se pueden extraer las siguientes conclusiones:

- La aplicación de técnicas de optimización para calibrar modelos de comportamiento con bases de datos de ensayos experimentales es una práctica numérica escasamente utilizada y restringida prácticamente a la última década. Menor aún es el empleo de algoritmos metaheurísticos en la calibración de modelos numéricos de hormigón armado con ensayos, siendo prácticamente inexistente para uno de los algoritmos empleados en esta tesis: el algoritmo de la luciérnaga o firefly algorithm (FA).

- Se ha constatado la elevada eficiencia del algoritmo FA debido a la subdivisión automática del espacio de búsqueda de la solución en subgrupos, y a la posibilidad de controlar la aleatoriedad del proceso de búsqueda de la solución a medida que se van produciendo las iteraciones. Además, con el algoritmo FA se obtienen mejores resultados con menor tiempo computacional respecto al resto de algoritmos metaheurísticos.

- Las expresiones que se han calibrado con las bases de ensayos para la obtención del momento de plastificación $M_{y}$, el giro de plastificación $\theta_{y}$ y el giro último $\theta_{u}$ de la sección, mejoran los parámetros estadísticos que cuantifican la dispersión y el grado de relación lineal entre los valores obtenidos $x_{\text {pred }} \mathrm{y}$ los valores experimentales $x_{\text {exp }}$ respecto a los ofrecidos por las expresiones propuestas por diversos autores y por el EC-8, lo que implica una mayor precisión en los resultados obtenidos.

- Las mejoras obtenidas resultan más significativas cuando el ajuste de las expresiones se realiza al seleccionar sólo los ensayos que cumplen con los requisitos sísmico-constructivos de la normativa. Destaca una disminución de hasta el $16 \%$ en el coeficiente de variación $C V$ y de hasta el $36 \%$ en el valor absoluto de la suma de las diferencias $S_{d}$, incrementándose el coeficiente de correlación lineal $r$ hasta un $62 \%$.

- La calibración de las expresiones con la selección de ensayos permite reproducir, de un modo muy ajustado, el comportamiento real de elementos de hormigón armado tipo viga y pilar, por lo que resulta adecuada, y muy aconsejable, la aplicación de estas expresiones al análisis y diseño de edificios convencionales de hormigón armado situados en zonas sísmicas.

- Las expresiones calibradas con la selección de ensayos proporcionan una resistencia y una capacidad de deformación del elemento superiores a las ofrecidas por otros métodos, como las expresiones de Biskinis y Fardis (2010a, 2010b) y los métodos clásicos, obteniendo valores de $M_{y}$ y $\theta_{y}$ que 
pueden resultar hasta un $40 \%$ superiores, lo que muestra el carácter conservador existente en estos últimos métodos.

- Las expresiones de comportamiento seccional que determinan momentos y giros en los estados de plastificación y último, tradicionalmente se han utilizado en la adecuación y evaluación sísmica de edificios; no obstante, si estas expresiones se calibran con ensayos experimentales, su implementación resulta de utilidad en el análisis sísmico debido a su eficiencia computacional y a la precisión que ofrecen sus resultados.

- En las curvas de respuesta de las rótulas plásticas generadas en las vigas de los pórticos analizados, la expresión calibrada con la selección de ensayos proporciona valores de $M_{y}$ superiores a los obtenidos al realizar la subdivisión de la sección en fibras. Para las vigas y los pilares, la capacidad de deformación última obtenida con la expresión ajustada a la selección de ensayos adopta un valor intermedio respecto a la obtenida por la expresión de Biskinis y Fardis (2010b) y por el procedimiento implementado en el código de análisis ETABS® (FEMA, 2000). Las curvas de plastificación obtenidas para los pilares con la expresión ajustada a la selección de ensayos denotan una capacidad de plastificación superior a la ofrecida por otros métodos.

- La mayor resistencia proporcionada por las expresiones calibradas con la selección de ensayos conlleva la obtención de curvas de capacidad con valores superiores del esfuerzo cortante en la base $F_{b}$. Este aspecto también se manifiesta en el punto de plastificación global de la estructura, al obtenerse valores superiores del propio cortante $F_{b}$, del desplazamiento del nudo de control de la estructura $\delta$ y de la aceleración del sistema equivalente de un grado de libertad $S_{a} / g$.

- En el punto de colapso de la estructura, si bien los valores de $F_{b}$ y $S_{a} / g$ resultan superiores al emplear las expresiones calibradas con la selección de ensayos, el valor de $\delta$ obtenido indica que las rótulas plásticas modelizadas con dichas expresiones proporcionan una capacidad de deformación última de la estructura intermedia a las obtenidas con las rótulas plásticas definidas en la FEMA (2000) y con las modelizadas mediante las expresiones de Biskinis y Fardis (2010a, 2010b).

- Las diferencias obtenidas al aplicar los distintos tipos de rótulas plásticas en los desplazamientos laterales de las plantas y en los drifts o desplazamientos entre plantas, resultan más significativas si se considera el espectro tipo 1. Las limitaciones impuestas por el EC-8 en los valores de los drifts sólo se satisfacen si se considera el espectro tipo 2 y los terrenos de mejor calidad, es decir, los tipos A y C. El tipo de distribución de carga lateral empleada en los análisis pushover no presenta una influencia significativa en los resultados obtenidos. 
- Los niveles de comportamiento obtenidos con las rótulas plásticas modelizadas mediante las expresiones calibradas propuestas resultan menos desfavorables que los ofrecidos por otros tipos de rótulas. Sin embargo, la consideración de las rótulas modelizadas con dichas expresiones propicia, en algunos casos, la aparición de niveles de comportamiento más desfavorables en ciertos pilares de las plantas intermedias de la estructura, lo cual puede resultar indicativo de un tipo de rotura frágil.

- Los valores superiores de $F_{b}$ y $\delta$ obtenidos al aplicar las rótulas plásticas caracterizadas mediante las expresiones calibradas propuestas respecto a lo proporcionado por la FEMA (2000) implican una mayor resistencia y capacidad de deformación de la estructura; sin embargo, los valores superiores obtenidos para $S_{a} / g$ suponen la introducción de mayores esfuerzos en la misma. Por tanto, las expresiones ajustadas a la selección de ensayos suponen una herramienta para que el ingeniero estructural pueda comprobar si las rótulas plásticas consideradas por defecto en los códigos comerciales de cálculo conducen a resultados excesivamente conservadores. Dicha comprobación resulta más conveniente al aumentar la altura de la estructura y la exigencia de la acción sísmica considerada en el análisis, aspectos que incrementan las diferencias obtenidas entre los distintos métodos.

- Las rótulas plásticas modelizadas mediante las expresiones calibradas propuestas aumentan el campo de aplicación respecto a las disponibles por defecto en los códigos comerciales de cálculo, las cuales suelen estar caracterizadas para una determinada norma de hormigón estructural.

\subsection{TRABAJOS FUTUROS}

Las líneas de investigación que se proponen como continuación al trabajo desarrollado en esta tesis son las siguientes:

- Ampliar la selección de ensayos mediante ensayos adicionales de elementos que cumplan las prescripciones sísmico-constructivas impuestas por las principales normas y códigos de diseño, en los que se midan los valores de la curvatura de plastificación $\phi_{y}$, con objeto de poder realizar la calibración de este concepto con una muestra de ensayos representativa desde el punto de vista estadístico.

- Emplear estrategias de calibración inversa para realizar la identificación de parámetros en las expresiones iniciales de Biskinis y Fardis (2010a, 2010b).

- Desarrollar un diagrama de histéresis, calibrado con ensayos, que presente como curva de respuesta la relación momento-giro obtenida con las expresiones de comportamiento seccional calibradas propuestas. 
- Realizar el análisis seccional de un número representativo de secciones de hormigón armado que presenten diferentes geometrías, armados, resistencias de materiales y esfuerzos, aplicando tanto las expresiones calibradas propuestas como otros métodos existentes en la literatura, efectuando un análisis estadístico y de sensibilidad de las diferencias obtenidas.

- Investigar la respuesta estructural en relación a la curva de capacidad en los análisis pushover, con objeto de identificar los puntos de plastificación global y de colapso en la estructura. 


\section{Referencias bibliográficas}

ACI Committee 318. (2008). Building code requirements for structural concrete (ACI 318-08) and commentary (318R-08). Farmington Hills, USA: American Concrete Institute.

Adriano de Araújo, F. \& Proença, S.P.B. (2008). Application of a lumped dissipation model to reinforced concrete structures with the consideration of residual strains and cycles of hysteresis. Journal of Mechanics of Materials and Structures, 3(5), 1011-1031.

Agrawal, V., Chandwani, V. \& Nagar, R. (2014). Swarm intelligence assisted optimization in structural engineering: a review. INROADS-An International Journal of Jaipur National University, 3(1), 173-180.

Aktan, E., Pecknold, D.A. \& Sozen, M.A. (1974). R/C column earthquake response in two dimensions. Journal of the Structural Division, 100(10), 1999-2015.

Alath, S. \& Kunnath, S.K. (1995). Modeling inelastic shear deformations in RC beamcolumn joints. In: Sture, S. (ed.), Proceedings of the $10^{\text {th }}$ Conference on Engineering Mechanics. (pp. 822-825). New York, USA: American Society of Civil Engineers.

Altoontash, A. (2004). Simulation and damage models for performance assessment of reinforced concrete beam-column joints. [PhD Thesis]. Department of Civil and Environmental Engineering, Stanford University, USA.

Alva, G.M.S. \& Homce, A.L. (2010). Application of lumped dissipation model in nonlinear analysis of reinforced concrete structures. Engineering Structures, 32(4), 974-981. 
Amirineni, K.C. (2009). Fracture properties of fiber reinforced concrete. [Master of Science]. Graduate School College of Engineering, Pennsylvania State University, USA.

Amorim, D.L.N.F., Proença, S.P.B. \& Flórez-López, J. (2013). A model of fracture in reinforced concrete arches based on lumped damage mechanics. International Journal of Solids and Structures, 50(24), 4070-4079.

Amorim, D.L.N.F., Proença, S.P.B. \& Flórez-López, J. (2014). Simplified modeling of cracking and collapse process on frames and arches of reinforced concrete. Ibracon Structures and Materials Journal, 7(1), 100-137.

Anderson, J.C. \& Townsend, W.H. (1977). Models for RC frames with degrading stiffness. Journal of the Structural Division, 103(12), 2361-2376.

Antoniou, S. \& Pinho, R. (2004a). Advantages and limitations of adaptive and nonadaptive force-based pushover procedures. Journal of Earthquake Engineering, $8(4), 497-522$.

Antoniou, S. \& Pinho, R. (2004b). Development and verification of a displacementbased adaptive pushover procedure. Journal of Earthquake Engineering, 8(5), 643661.

Aoyama, H. \& Sugano, T. (1968). A generalized inelastic analysis of reinforced concrete structures based on the tests of members. Recent researches of structural mechanics-contributions in honor of the 60-th birthday of prof. Y. Tsuboi (pp. 1530). Tokyo, Japan: Uno Shoten.

Applied Technology Council. (1996). Seismic evaluation and retrofit of concrete buildings. ATC-40. Redwood City, USA: Applied Technology Council.

ASTM. (2008). ASTM A615/A615M-13. Standard specification for deformed and plain carbon-steel bars for concrete reinforcement. West Conshohocken, USA: American Society of Testing and Material.

Aydinoglu, M.N. (2003). An incremental response spectrum analysis procedure based on inelastic spectral displacements for multi-mode seismic performance evaluation. Bulletin of Earthquake Engineering, 1, 3-36.

Aydogdu, I. \& Saka, M.P. (2012). Ant colony optimization of irregular steel frames including elemental warping effect. Advances in Engineering Software, 44, pp. 150169.

Baber, T. \& Noori, M. (1985). Random vibration of degrading pinching systems. Journal of Engineering Mechanics, 111(8), 1010-1026. 
Baber, T. \& Wen, Y.K. (1981). Random vibrations of hysteretic degrading systems. Journal of the Engineering Mechanics Division, 107(6), 1069-1087.

Badoni, D. \& Makris, N. (1996). Nonlinear response of single piles under lateral inertial and seismic loads. Soil Dynamics and Earthquake Engineering, 15(1), 29-43.

Bae, S. (2005). Seismic performance of full-scale reinforced concrete columns. [PhD Thesis]. Faculty of the Graduate School, University of Texas at Austin, USA.

Baker, A.L.L. \& Amarakone, A.M.N. (1964). Inelastic hyperstatic frames analysis. Proceedings of the International Symposium on the Flexural Mechanics of Reinforced Concrete, ASCE-ACI. (pp. 85-142).

Barrera, A.C. (2012). Estudio experimental y numérico de la capacidad de deformación de soportes esbeltos de hormigón armado. [Tesis Doctoral]. Departamento de Ingeniería de la Construcción y Proyectos de Ingeniería Civil, Universitat Politècnica de València, España.

Benavent-Climent, A., Escolano-Margarit, D. \& Morillas, L. (2014). Shake-table tests of a reinforced concrete frame designed following modern codes: seismic performance and damage evaluation. Earthquake Engineering \& Structural Dynamics, 43(6), 791-810.

Bertero, V.V., Anderson, J.C., Krawinkler, H. \& Miranda, E. (1991). Design guidelines for ductility and drift limits: review of state-of-the-practice and state-of-the-art in ductility and drift-based earthquake-resistant design of buildings. [Report EERC 1991/15]. Earthquake Engineering Research Center, University of California, Berkeley, USA.

Biddah, A. \& Ghobarah, A. (1999). Modelling of shear deformation and bond slip in reinforced concrete joints. Structural Engineering and Mechanics, 7(4), 413-432.

Bigaj, A.J. \& Walraven, J.C. (1993). Size effect on the rotation capacity of plastic hinges in reinforced concrete beams. fib Bulletin 218 (pp. 7-24). Lausanne, Switzerland: Fedération Internationale du Béton.

Biskinis, D. \& Fardis, M.N. (2010a). Deformations at flexural yielding of members with continuous or lap-spliced bars. Structural Concrete, 11(3), 127-138.

Biskinis, D. y Fardis, M.N. (2010b). Flexure-controlled ultimate deformations of members with continuous or lap-spliced bars. Structural Concrete, 11(2), 93-108.

Botez, M.D., Bredean, L.A. \& Ioani, A.M. (2014). Plastic hinge vs. distributed plasticity in the progressive collapse analysis. Acta Technica Napocensis: Civil Engineering \& Architecture, 57(1), 24-36. 
Bouc, R. (1971). Modèle mathémathique d'hystérésis: application aux systèmes à un degré de liberté. Acustica, 24, 16-25.

Bracci, J.M., Kunnath, S.K. \& Reinhorn, A.M. (1997). Seismic performance and retrofit evaluation of reinforced concrete structures. Journal of Structural Engineering, 123(1), 3-10.

Bretschneider, N., Villmann, B. \& Slowik, V. (2007). Inverse analyses of bending tests for determining the properties of strain hardening cement-based materials. In: Carpinteri, A., Gambarova, P.G., Ferro, G. \& Plizzari, G. (eds.), Proceedings of the $6^{\text {th }}$ international Conference on Fracture Mechanics of Concrete and Concrete Structures. (pp. 1419-1427). London, UK: Taylor \& Francis.

California Department of Transportation (Caltrans). (2013). Seismic design criteria. Version 1.7. Sacramento, USA: California Department of Transportation. Retrieved March 13, 2014, from http://www.dot.ca.gov/hq/esc/earthquake_engineering/sdc/documents/SeismicDesign-Criteria-(SDC-1.7-Full-Version,-OEE-Release).pdf

Caner, F.C. \& Bazant, Z.P. (2000). Microplane model M4 for concrete. II: algorithm and calibration. Journal of Engineering Mechanics, 126(9), 954-961.

Carbas, S. \& Saka, M.P. (2012). Optimum topology design of various geometrically nonlinear latticed domes using improved harmony search method. Structural and Multidisciplinary Optimization, 45, 337-350.

Cardinetti, F. (2011). Fiber beam-column models with flexure-shear interaction for nonlinear analysis of reinforced concrete structures. [PhD Thesis]. Università di Bologna, Italia.

Ceroni, F., Pecce, M.R., Di Sarno, L. \& Manfredi, G. (2005). Evaluation of inelastic deformation capacity of RC columns. In: Chiorino, M.A. \& Nanni, A. (eds.), Proceedings of the Symposium at the 2005 ACI Spring Convention. (pp. 177-188). New York, USA: American Concrete Institute.

Chatterjee, A. \& Mahanti, G.K. (2012). Design of a fully digital controlled reconfigurable switched beam conconcentric ring array antenna using firefly and particle swarm optimization algorithm. Progress in Electromagnetics Research, 36, 113-131.

Chipperfield, A.J., Fleming, P.J. \& Fonseca, C.M. (1994). Genetic algorithm tools for control systems engineering. In: Parmee, I.C. (ed.), Proceedings of $1^{\text {st }}$ International Conference on Adaptive Computing in Engineering Design and Control. (pp. 128133). Plymouth, UK. 
Chopra, A.K. \& Goel, R.K. (2002). A modal pushover analysis procedure for estimating seismic demands for buildings. Earthquake Engineering \& Structural Dynamics, 31(3), 561-582.

Chopra, A.K. \& Goel, R.K. (2001). A modal pushover analysis procedure to estimate seismic demands for buildings: theory and preliminary evaluation. [PEER Report 2001/03]. Pacific Earthquake Engineering Research Center, College of Engineering, University of California, Berkeley, USA.

Chung, W., Lam, E., Wu, B. \& Wong, Y. (2007). Hysteresis behavior of rectangular reinforced concrete columns with non-seismic detailing. In: Lyons, R. (ed.), Proceedings of the 2007 Structures Congress: New Horizons and Better Practices. (pp. 1-10). Reston, USA: American Society of Civil Engineers.

Cipollina, A. (1992). Análisis de elementos estructurales de concreto armado mediante la mecánica de la degradación. [Tesis de Magister Scientiae en Ingeniería Estructural]. Facultad de Ingeniería, Universidad de Los Andes, Mérida, Venezuela.

Cipollina, A., Febres-Cedillo, R. \& Flórez-López, J. (1992). A model of plasticity coupled to damage for RC frames. In: Bernal, A. (ed.), Proceedings of the $10^{\text {th }}$ World Conference on Earthquake Engineering. (pp. 4239-4244). Rotterdam, Netherlands: Balkema.

Cipollina, A. \& Flórez López, J. (1995). Modelos simplificados de daño en pórticos de concreto armado. Revista Internacional de Métodos Numéricos para Cálculo y Diseño en Ingeniería, 11(1), 3-22.

Cipollina, A., López-Inojosa, A. \& Flórez-López, J. (1995). A simplified damage mechanics approach to nonlinear analysis of frames. Computers \& Structures, 54(6), 1113-1126.

Clough, R.W. (1966). Effect of stiffness degradation on earthquake ductility requirements. [Report 6614]. Department of Civil Engineering, University of California, Berkeley, USA.

Clough, R.W., Benuska, K.L. \& Wilson, E.L. (1965). Inelastic earthquake response of tall buildings. In: Van Roekel, J.H. (ed.), Proceedings of the Third World Conference on Earthquake Engineering. (pp. 68-89). Wellington, New Zealand: New Zealand National Committee on Earthquake Engineering.

Código Técnico de la Edificación. (2009). Documento básico SE-AE: seguridad estructural. Acciones en la edificación. Madrid, España: Ministerio de Fomento.

Comisión Permanente del Hormigón. (2008). Instrucción de hormigón estructural: EHE-08. Madrid, España: Ministerio de Fomento. 
Comisión Permanente de Normas Sismorresistentes. (2002). Norma de construcción sismorresistente: NCSE-02. Parte general y edificación. Madrid, España: Ministerio de Fomento.

Corley, W.G. (1966). Rotational capacity of reinforced concrete beams. Journal of the Structural Division, 92(5), 121-146.

Corres, H., Martínez, J.L., Pérez, A. y López, J.C. (2014). Prontuario Informático del Hormigón Estructural 3.1.7. [Software]. IECA. (www.ieca.es)

Cosenza, E., Greco, C. \& Manfredi, G. (1992). Evaluation of plastic rotations in $R / C$ beams: comparison between theoretical and experimental results. [Report presented at CEB Task Group 2.2. "Ductility Requirements" Meeting]. Pavia, Italy.

Cosenza, E., Greco, C. \& Manfredi, G. (1991). Theoretical evaluation of inelastic rotations and displacements in the reinforced concrete monodimensional elements. Atti della Accademia Nazionale dei Lincei. Classe di Scienze Fisiche, Matematiche e Naturali. Rendiconti Lincei. Matematica e Applicazioni, 2(3), 249-258.

Cosenza, E., Manfredi, G., Verderame, G.M., Ricci, P., De Carlo, G. \& Masi, A. (2009). Capacity models of RC members with emphasis on sub-standard columns wih plain bars. In: Consenza, E. (ed.), Eurocode 8 perspectives from the Italian standpoint workshop. (pp. 129-144). Napoli, Italia: Doppiavoce.

Darvall, P. \& Mendis, P. (1985). Elastic-plastic softening analysis of plane frames. Journal of Structural Engineering, 111(4), 871-888.

Deierlein, G.G., Reinhorn, A.M. \& Willford, M.R. (2010). Nonlinear structural analysis for seismic design. A guide for practicing engineers. Washington D.C., USA: U.S. Department of Commerce.

De Oliveira e Sousa, J. \& Gettu, R. (2006). Determining the tensile stress-crack opening curve of concrete by inverse analysis. Journal of Engineering Mechanics, 132(2), 141-148.

Díaz, A. (1996). Optimización heurística y redes neuronales. Madrid, España: Paraninfo.

Dogn, E. \& Saka, M.P. (2012). Optimum design of unbraced steel frames to LRFDAISC using particle swarm optimization. Advances in Engineering Software, 46, 27-34.

Domínguez, D., López-Almansa, F. y Benavent-Climent, A. (2014). Comportamiento, para el terremoto de Lorca de 11-05-2011, de edificios de vigas planas proyectados sin tener en cuenta la acción sísmica. Informes de la Construcción, 66(533), 1-9. 
Erol, O.K. \& Eksin, I. (2006). A new optimization method: big bang-big crunch. Advances in Engineering Software, 37(2), 106-111.

ETABS. (2013). [Software]. Extended Three Dimensional Analysis of Building Systems. Berkeley, USA: Computers \& Structures, Inc.

European Committee for Standardization. (2002). EN 1991-1-1, Eurocode 1: actions on structures. Part 1-1: general actions-densities, self-weight, imposed loads for buildings. Brussels, Belgium: European Committee for Standardization.

European Committee for Standardization. (2004a). EN 1992-1-1, Eurocode 2: design of concrete structures. Part 1-1: general rules and rules for buildings. Brussels, Belgium: European Committee for Standardization.

European Committee for Standardization. (2005). EN 1998-3, Eurocode 8: design of structures for earthquake resistance. Part 3: assessment and retrofitting of buildings. Brussels, Belgium: European Committee for Standardization.

European Committee for Standardization. (2004b). EN 1998-1, Eurocode 8: design of structures for earthquake resistance. Part 1: general rules, seismic actions and rules for buildings. Brussels, Belgium: European Committee for Standardization.

Fajfar, P. (2000a). A nonlinear analysis method for performance-based seismic design. Earthquake Spectra, 16(3), 573-592.

Fajfar, P. (2000b). A practical nonlinear method for seismic performance evaluation. In: M. Elgaaly (ed.), Proceedings of Structures Congress 2000: Advanced Technology in Structural Engineering. (pp. 1-8). Reston, USA: American Society of Civil Engineers.

Fajfar, P. \& Fischinger, M. (1988). N2 - A method for non-linear seismic analysis of regular buildings. Proceedings of the $9^{\text {th }}$ World Conference on Earthquake Engineering. (pp. 111-116).

Fajfar, P. \& Fischinger, M. (1987). Non-linear seismic analysis of RC buildings: implications of a case study. European Earthquake Engineering, 1(1), 31-43.

Fajfar, P. \& Gaspersic, P. (1996). The N2 method for the seismic damage analysis of RC buildings. Earthquake Engineering \& Structural Dynamics, 25(1), 31-46.

Faleiro, J., Barbat, A. \& Oller, S. (2005). Plastic damage model for nonlinear reinforced concrete frames analysis. In: Owen, D.R.J., Oñate, E. \& Suárez, B. (eds.),

Proceedings of the VIII International Conference on Computational Plasticity, COMPLAS VIII. (pp. 906-909). Barcelona, Spain: International Center for Numerical Methods in Engineering. 
Faleiro, J., Oller, S. \& Barbat, A.H. (2008). Plastic-damage seismic model for reinforced concrete frames. Computers \& Structures, 86, 581-597.

Fardis, M.N. \& Biskinis, D.E. (2003). Deformation capacity of RC members, as controlled by flexure or shear. In: Kabeyasawa, T. y Shiohara, H. (eds.), Proceedings of International Symposium on Performance-Based Engineering for Earthquake Resistant Structures, a Volume Honoring Prof. Shunsuke Otani. (pp. 511-530).

Fardis, M.N. \& Panagiotakos, T.B. (1996). Hysteretic damping of reinforced concrete elements. In: Sociedad Mexicana de Ingeniería (ed.), $11^{\text {th }}$ World Conference on Earthquake Engineering. Paper 464.

Farhat, F., Nakamura, S. \& Takahashi, K. (2009). Application of genetic algorithm to optimization of buckling restrained braces for seismic upgrading of existing structures. Computers \& Structures, 87, 110-119.

Favvata, M.J., Izzuddin, B.A. \& Karayannis, C.G. (2008). Modelling exterior beamcolumn joints for seismic analysis of RC frame structures. Earthquake Engineering \& Structural Dynamics, 37(13), 1527-1548.

FEMA. (1997). NEHRP guidelines for the seismic rehabilitation of buildings (FEMA Publication 273). Washington D.C., USA: Federal Emergency Management Agency.

FEMA. (2000). Prestandard and commentary for the seismic rehabilitation of buildings (FEMA Publication 356). Washington D.C., USA: Federal Emergency Management Agency.

Feng, Y., Kowalsky, M.J. \& Nau, J.M. (2014). Fiber-based modeling of circular reinforced concrete bridge columns. Journal of Earthquake Engineering, 18(5), 714-734.

FIB. (2003a). Displacement-based seismic design of reinforced concrete buildings. fib bulletin 25. Lausanne, Switzerland: Fedération Internationale du Béton.

FIB. (2012a). Model Code 2010. Final Draft. Volume 2. fib bulletin 66. Lausanne, Switzerland: Fedération Internationale du Béton.

FIB. (2012b). Probabilistic performance-based seismic design. fib bulletin 68. Lausanne, Switzerland: Fedération Internationale du Béton.

FIB. (2003b). Seismic assessment and retrofit of reinforced concrete buildings. fib bulletin 24. Lausanne, Switzerland: Fedération Internationale du Béton. 
Filippou, F.C. \& Issa, A. (1988). Nonlinear analysis of reinforced concrete frames under cyclic load reversals. [Report UCB/EERC 88-12]. Earthquake Engineering Research Center, University of California, Berkeley, USA.

Filippou, F.C., Popov, E.P. \& Bertero, V.V. (1983). Effects of bond deterioration on hysteretic behaviour of reinforced concrete joints. [Report UCB/EERC-83/19]. Earthquake Engineering Research Center, University of California, Berkeley, USA.

Flórez-López, J. (1993a). Calcul simplifié de portiques endommageables. Revue Européenne des Éléments, 2(1), 47-74.

Flórez-López, J. (1993b). Modelos de daño concentrado para la simulación numérica del colapso de pórticos planos. Revista Internacional de Métodos Numéricos para Cálculo y Diseño en Ingeniería, 9(2), 123-139.

Flórez-López, J. (1995). Simplified model of unilateral damage for RC frames. Journal of Structural Engineering, 121(12), 1765-1772.

Flórez-López, J. (1996). Un modelo del comportamiento histerético de elementos de concreto armado basado en la teoría del daño concentrado. Revista Internacional de Métodos Numéricos para Cálculo y Diseño en Ingeniería, 12(4), 411-426.

Freeman, S.A., Nicoletti, J.P \& Tyrell, J.V. (1975). Evaluations of existing buildings for seismic risk: a case study of Puget Sound Naval Shipyard, Bremerton, Washington. Proceedings of U.S. National Conference on Earthquake Engineering. (pp. 113122). Ann Arbor, USA: Earthquake Engineering Research Institute.

Galal, K.E. \& Ghobarah, A. (2003). Flexural and shear hysteretic behaviour of reinforced concrete columns with variable axial load. Engineering Structures, 25(11), 1353-1367.

Gandomi, A.H., Yang, X.S. \& Alavi, A.H. (2011). Mixed variable structural optimization using Firefly Algorithm. Computers \& Structures, 89(23-24), 23252336.

García Reyes, L.E. (1998). Dinámica estructural aplicada al diseño sísmico. Bogotá, Colombia: Universidad de Los Andes.

Gaspar-Escribano, J., Benito, B. \& García-Mayordomo, J. (2008). Hazard-consistent response spectra in the Region of Murcia (Southeast Spain): comparison to earthquake-resistant provisions. Bulletin of Earthquake Engineering, 6(2), 179-196.

Geem, Z.W., Kim, J.H. \& Loganathan, G.V. (2001). A new heuristic optimization agorithm: harmony search. Simulation, 76(2), 60-68. 
Gharaibeh, N., Freeman, T., Saliminejad, S., Wimsatt, A., Chang-Albitres, C., Nazarian, S., Abdallah, I., Weissmann, J., Weissmann, A.J., Papagiannakis, A.T. \& Gurganus, C. (2012). Evaluation and development of pavement scores, performance models and needs estimates for the TXDOT pavement management information systemFinal Report. Texas, USA: Texas A\&M Transportation Institute.

Gholizadeh, S., Sheidaii, M.R. \& Farajzadeh, S. (2012). Seismic design of double layer grids by neural networks. International Journal of Optimization in Civil Engineering, 2, 29-45.

Giberson, M.F. (1967). The response of nonlinear multi-story structures subjected to earthquake excitation. [PhD Thesis]. California Institute of Technology at Pasadena, California, USA.

Giberson, M.F. (1969). Two nonlinear beams with definitions of ductility. Journal of the Structural Division, 95(2), 137-157.

Goldberg, D.E. (1989). Genetic algorithms in search, optimization and machine learning. Boston, USA: Addison-Wesley Longman.

Gomes, H.M. (2011). Truss optimization with dynamic constraints using a particle swarm algorithm. Expert Systems with Applications, 38, 957-968.

Goodman, L.E. \& Klumpp, J.H. (1955). Analysis of slip damping with reference to turbine-blade vibration. Journal of Applied Mechanics, 23(3), 421-429.

Grassl, P. \& Jirasek, M. (2006). Damage-plastic model for concrete failure. International Journal of Solids and Structures, 43(22-23), 7166-7196.

Grassl, P., Xenos, D., Nyström, U., Rempling, R. \& Gylltoft, K. (2013). CDPM2: A damage-plasticity approach to modelling the failure of concrete. International Journal of Solids and Structures, 50(24), 3805-3816.

Griffith, A.A. (1921). The phenomena of rupture and flow in solids. Philosophical Transactions of the Royal Society of London. Series A, Containing Papers of a Mathematical or Physical Character, 221, 163-198.

Guedes, J., Pegon, P. \& Pinto, A.V. (1994). A fiber-Timoshenko beam element in CASTEM 2000. [Special Publication I.94.31]. Joint Research Center in Ispra, Italy.

Gupta, B. \& Kunnath, S.K. (2000). Adaptive spectra-based pushover procedure for seismic evaluation of structures. Earthquake Spectra, 16(2), 367-392.

Haskett, M., Oehlers, D.J., Ali, M.S.M. \& Wu, C. (2009). Rigid body moment-rotation mechanism for reinforced concrete beam hinges. Engineering Structures, 31(5), 1032-1041. 
Heo, Y. \& Kunnath, S.K. (2013). Damage-based seismic performance evaluation of reinforced concrete frames. International Journal of Concrete Structures and Materials, 7(3), 175-182.

Holland, J.H. (1975). Adaptation in natural and artificial systems: an introductory analysis with applications to biology, control and artificial intelligence. Michigan, USA: University of Michigan Press.

Hopper, M.W. (2009). Analytical models for the nonlinear seismic response of reinforced concrete frames. [Master of Science]. College of Engineering, Pennsylvania State University, USA.

Hughes, T.J. (1987). The finite element method. Linear static and dynamic finite element analysis. New Jersey, USA: Prentice-Hall, Englewood Cliffs.

Hwang, S.J. \& Lee, H.J. (1999). Analytical model for predicting shear strengths of exterior reinforced concrete beam-column joints for seismic resistance. ACI Structural Journal, 96(5), 846-858.

Ibarra, L.F., Medina, R.A. \& Krawinkler, H. (2005). Hysteretic models that incorporate strength and stiffness deterioration. Earthquake Engineering \& Structural Dynamics, 34(12), 1489-1511.

Ikhouane, F., Mañosa, V. \& Rodellar, J. (2007). Dynamic properties of the hysteretic Bouc-Wen model. Systems \& Control Letters, 56(3), 197-205.

Imbeault, F.A. \& Nielsen, N.N. (1973). Effect of degrading stiffness on the response of multistory frames subjected to earthquakes. In: Da Schio, L. (ed.), Proceedings of the Fifth World Conference on Earthquake Engineering. (pp. 1756-1765). Rome, Italy: EDIGRAF.

Inel, M. \& Ozmen, H.B. (2006). Effects of plastic hinge properties in nonlinear analysis of reinforced concrete buildings. Engineering Structures, 28(11), 1494-1502.

Institute of Engineering Seismoloy \& Earthquake Engineering (ITSAK). (2014). Cephalonia earthquake: effects on soil and built environment in combination with the January 26, 2014 (M6.1) event. Thessaloniki, Greece: Ministry of Infrastructures Transportation and Networks.

Ismail, M., Ikhouane, F. \& Rodellar, J. (2009). The hysteresis Bouc-Wen model, a survey. Archives of Computational Methods in Engineering, 16(2), 161-188.

Isobe, D. \& Tsuda, M. (2003). Seismic collapse analysis of reinforced concrete framed structures using the finite element method. Earthquake Engineering \& Structural Dynamics, 32(13), 2027-2046. 
Ivorra, S., Pallarés, F.J. \& Adam, J.M. (2009). Experimental and numerical results from the seismic study of a masonry bell tower. Advances in Structural Engineering, 12(2), 287-293.

Jan, T.S., Liu, M.W. \& Kao, Y.C. (2004). An upper-bound pushover analysis procedure for estimating seismic demands of high-rise buildings. Engineering Structures, 26(1), 117-128.

Jorquera, J.J. \& Manterola, J. (2012). An iterative form-finding method for antifunicular shapes in spatial arch bridges. Computers \& Structures, 108-109, 42-60.

Kaba, S. \& Mahin, S.A. (1984). Refined modeling of reinforced concrete columns of seismic analysis. [Report EERS 84-03]. Earthquake Engineering Research Center, University of California, Berkeley, USA.

Kachanov, L.M. (1958). On creep rupture time. Izvestia Akademii Nauk SSSR, 8, 26-31.

Kaewkulchai, G. \& Williamson, E.B. (2004). Beam element formulation and solution procedure for dynamic progressive collapse analysis. Computers \& Structures, 82(7-8), 639-651.

Kaveh, A. \& Ahangaran, M. (2012). Discrete cost optimization of composite floor system using social harmony search model. Applied Soft Computing, 12, 372-381.

Kaveh, A. \& Talatahari, S. (2010a). An improved ant colony optimization for the design of planar steel frames. Engineering Structures, 32, 864-873.

Kaveh, A. \& Talatahari, S. (2010b). A novel heuristic optimization method: charged system search. Acta Mechanica, 213(3-4), 267-289.

Keshavarzian, M. \& Schnobrich, W.C (1984). Computed nonlinear seismic response of $R / C$ wall-frame structures. [Structural Research Series 515]. Department of Civil Engineering, University of Illinois at Urbana-Champaign, USA.

Kilar, V. \& Fajfar, P. (1997). Simple push-over analysis of asymmetric buildings. Earthquake Engineering \& Structural Dynamics, 26(2), 233-249.

Kim, S.P. \& Kurama, Y.C. (2008). An alternative pushover analysis procedure to estimate seismic displacement demands. Engineering Structures, 30(12), 37933807.

Kirtrick, S., Gelatt, C.D. \& Vecchi, M.P. (1983). Optimization by simulated annealing. Science, 220(4598), 671-680.

Krawinkler, H. (1995). New trends in seismic design methodology. In: Duma, G. (ed.), Proceedings of the $10^{\text {th }}$ European Conference on Earthquake Engineering. (pp. 821-830). Rotterdam, Netherlands: Balkema. 
Krawinkler, H. (1996). Pushover analysis: why, how, when and when not to use it. Proceedings of the $65^{\text {th }}$ Annual Convention: 1996 SEAOC Convention. Sacramento, USA: Structural Engineers Asociation of California.

Krawinkler, H. \& Seneviratna, G.D.P.K. (1998). Pros and cons of a pushover analysis of seismic performance evaluation. Engineering Structures, 20(4-6), 452-464.

Kucerová, A. (2007). Identification of nonlinear mechanical model parameters based on softcomputing methods. [PhD Thesis]. Laboratoire de M'ecanique et Technologie, Ecole Normale Supérieure de Cachan, France.

Kucerová, A. \& Leps, M. (2014). Soft computing-based calibration of microplane M4 model parameters: methodology and validation. Advances in Engineering Software, $72,226-235$.

Kucerová, A. \& Mares, T. (2010). Self-adaptive artificial neural network in numerical models calibration. In: Diamantaras, K., Duch, W. \& Iliadis, L.S. (eds.), $20^{\text {th }}$ International Conference on Artificial neural networks-ICANN 2010. (pp. 347351). Berlin, Germany: Springer-Verlag.

Kwak, H.G. \& Kim, S.P. (2010). Simplified monotonic moment-curvature relation considering fixed-end rotation and axial force effect. Engineering Structures, 32(1), 69-79.

Kyakula, M. \& Wilkinson, S. (2004). An improved spread plasticity model for inelastic analysis of R/C frames subjected to seismic loading. In: 13WCEE Secretariat (ed.), Proceedings of $13^{\text {th }}$ World Conference on Earthquake Engineering. Paper 933.

La Borderie, C. (1991). Phénomènes unilatéraux dans un matériau endommageable: modélisation et application à l'analyse de structures en béton. [PhD Thesis]. Université de Paris, France.

Lai, S., Will, G. \& Otani, S. (1984). Model for inelastic biaxial bending of concrete members. Journal of Structural Engineering, 110(11), 2563-2584.

Lam, S.S.E., Wu, B., Wong, Y.L., Wang, Z.Y., Liu, Z.Q. \& Li, C.S. (2003). Drift capacity of rectangular reinforced concrete columns with low lateral confinement and high-axial load. Journal of Structural Engineering, 129(6), 733-742.

Langer, P. (1987). Verdrehfahigkeit plastizierter tragwerksbereiche im stahlbetonbau. $[\mathrm{PhD}$ Thesis]. Insititut für Werkstoffe im Bauwesen, Universität Stuttgart, Deutschland. (En alemán).

Lawson, R.S., Vance, V.L. \& Krawinkler, H. (1994). Nonlinear static push-over analysis-why, when and how?. Proceedings of the $5^{\text {th }}$ US National Conference on Earthquake Engineering. (pp. 283-292). Oakland, USA: Earthquake Engineering Research Institute. 
Légeron, F., Paultre, P. \& Mazars, J. (2005). Damage mechanics modeling of nonlinear seismic behaviour of concrete structures. Journal of Structural Engineering, 131(6), 946-955.

Lehký, D., Kersner, Z. \& Novák, D. (2012). Determination of statistical material parameters of concrete using fracture test and inverse analysis based on FraMePID3PB tool. In: Vorechovský, M., Sadílek, V., Seitl, S., Veselý, V., Muhanna, R.L. \& Mullen, R.L. (eds.), Proceedings of the $5^{\text {th }}$ International Conference on Reliable Engineering Computing (REC 2012). (pp. 261-270). Brno, Czech Republic: LITERA.

Lehman, D.E. (1998). Performance-based seismic design of well-confined concrete columns. [PhD Thesis]. Department of Civil and Environmental Engineering, University of California, Berkeley, USA.

Lehman, D.E. \& Moehle, J.P. (2000). Seismic performance of well-confined concrete bridge columns. [PEER Report 1998/01]. Pacific Earthquake Engineering Research Center, University of California, Berkeley, USA.

Li, L. (1995). Rotationsfähigkeit von plastischen gelenken im stahl und spannbetonbau. [PhD Thesis]. Insititut für Werkstoffe im Bauwesen, Universität Stuttgart, Deutschland. (En alemán).

Li, L.J., Huang, Z.B. \& Liu, F. (2009). A heuristic particle swarm optimization method for truss structures with discrete variables. Computers \& Structures, 87, 435-443.

Li, S., Liu, Y., He, X. \& Zhou, Y. (2003). Parameter identification procedures for the concrete dam based on ant colony optimization. In: Yuan, Y.S., Shah, S.P. \& Lü, H.L. (eds.), Proceedings of the International Conference on Advances in Concrete and Structures (pp. 1428-1433). Bagneux, France: RILEM Publications.

Li, Z. \& Hatzigeorgiou, G.D. (2012). Seismic damage analysis of RC structures using fiber beam-column elements. Soil Dynamics and Earthquake Engineering, 32(1), 103-110.

Lin, P., Liu, X., Chen, H.X. \& Kim, J. (2014). Ant colony optimization analysis on overall stability of high arch dam basis of field monitoring. The Scientific World Journal, 2014, 1-14.

López-Almansa, F., Alfarah, B. \& Oller, S. (2014). Numerical simulation of RC frame testing with damaged plasticity model. Comparison with simplified models. Proceedings of the $2^{\text {nd }}$ European Conference on Earthquake Engineering and Seismology. (pp. 1-12). 
López-Inojosa, A. (1995). Grandes desplazamientos y deformaciones en modelos simplificados de daño. [Tesis de Magister Scientiae en Ingeniería Estructural]. Facultad de Ingeniería, Universidad de Los Andes, Mérida, Venezuela.

López-López, A.T. (2012). Estudio comparativo de modelos de comportamiento histerético de rótulas plásticas de hormigón armado para su implementación en modelos estructurales para el cálculo sísmico. [Trabajo de investigación CST/MIH-0X]. Departamento de Ingeniería de la Construcción y Proyectos de Ingeniería Civil, Universitat Politècnica de València, España.

López, M.A. (2004). A review of existing pushover methods for 2-D reinforced concrete buildings. [PhD Thesis]. Istituto Universitario di Studi Superiori, Università degli Studi di Pavia, Italia.

Lowes, L. \& Altoontash, A. (2003). Modelling reinforced-concrete beam-column joints subjected to cyclic loading. Journal of Structural Engineering, 129(12), 1686-1697.

Luh, G.C. \& Lin, C.Y. (2009). Structural topology optimization using ant colony optimization algorithm. Applied Soft Computing, 9, 1343-1353.

Mahin, S.A. \& Bertero, V.V. (1975). An evaluation of some methods for predicting seismic behavior of reinforced concrete buildings. [Report EERC 75-5]. Earthquake Engineering Research Center, University of California, Berkeley, USA.

Mander, J.B., Priestley, M.J.N. \& Park, R. (1984). Seismic design of bridge piers. [Research Report 84-2]. Department of Civil Engineering, University of Canterbury, New Zealand.

Mander, J., Priestley, M. \& Park, R. (1988). Theoretical stress-strain model for confined concrete. Journal of Structural Engineering, 114(8), 1804-1826.

Manfredi, G. (1995). Some remarks on the size effect on the rotation capacity. [Report presented at CEB Task Group 2.2. "Ductility Requirements" meeting]. Delft, Netherlands.

Marante, M.E. \& Flórez-López, J. (2002). Model of damage for RC elements subjected to biaxial bending. Engineering Structures, 24(9), 1141-1152.

Marante, M.E. \& Flórez-López, J. (2003). Three-dimensional analysis of reinforced concrete frames based on lumped damage mechanics. International Journal of Solids and Structures, 40(19), 5109-5123.

Marín, J. (1988). Computing double integrals with moments of area. Boletín Técnico IMME, 76, 144-155. 
Marquardt, D.W. (1963). An algorithm for least-squares estimation of nonlinear parameters. Journal of the Society for Industrial and Applied Mathematics, 11(2), 431-441.

Martinelli, L. (1998). Modellazione di pile di ponte in C.A. a travata soggetti ad eccitazione sismica. [PhD Thesis]. Dipartimento di Ingegneria Strutturale, Politecnico di Milano, Italia.

Martínez, F.J., González-Vidosa, F. \& Hospitaler, A. (2011). A parametric study of piers for motorway bridge viaducts. Revista Internacional de Métodos Numéricos para Cálculo y Diseño en Ingeniería, 27(3), 236-260.

Maruyama, M. \& Jirsa, J.O. (1979). Shear behavior of reinforced concrete members under bidirectional reversed lateral loading. [CESRL Report 78-1]. Structures Research Laboratory, University of Texas at Austin, USA.

MATLAB. (2013). [Software]. MATrix LABoratory. Version R2013b. Natick, USA: MathWorks.

Mattock, A.H. (1967). Discussion of "Rotational capacity of reinforced concrete beams" by W.G. Corley. Journal of the Structural Division, 93(2), 519-522.

Mattock, A.H. (1965). Rotational capacity of hinging regions in reinforced concrete beams. Proceedings of the International Symposium on the Flexural Mechanics of Reinforced Concrete. (pp. 143-181). New York, USA: American Society of Civil Engineers.

Mazars, J. (1986). A description of micro- and macroscale damage of concrete structures. Engineering Fracture Mechanics, 25(5-6), 729-737.

Mazars, J. (1984). Application de la mécanique de l'endommagement au comportement non linéaire et à la rupture du béton de structure. [PhD Thesis]. Laboratoire de Mecanique et Technologie, Université Pierre et Marie Curie, Paris, France.

Mazars, J., Kotronis, P., Ragueneau, F. \& Casaux, G. (2006). Using multifiber beams to account for shear and torsion: applications to concrete structural elements. Computer Methods in Applied Mechanics and Engineering, 195(52), 7264-7281.

Mazza, F. \& Mazza, M. (2010). Nonlinear analysis of spatial framed structures by a lumped plasticity model based on the Haar-Kàrmàn principle. Computational Mechanics, 45(6), 647-664.

Mazza, F. \& Mazza, M. (2012). Nonlinear modeling and analysis of R.C. framed buildings located in a near-fault area. The Open Construction \& Building Technology Journal, 6, 346-354. 
Mazza, F. \& Mazza, M. (2011). Nonlinear modeling and analysis of R.C. spatial frames to study the effects of the vertical component of near-fault ground motions. In: Papadrakakis, M., Fragiadakis, M. \& Plevris, V. (eds.), Proceedings of the Third ECCOMAS Thematic Conference on Computational Methods in Structural Dynamics and Earthquake Engineering. (pp. 314-327). Athens, Greece: National Technical University of Athens.

Men, J., Lu, X.Z., Song, E.X. \& Chen, Z.Y. (2006). Application of multi-layer model in shell wall computation. International Journal of Protective Structures, 28(3), 9-13.

Mestrovic, D., Cizmar, D. \& Pende, M. (2008). Non linear analysis of structures according to new european design code. Proceedings of the $14^{\text {th }}$ World Conference on Earthquake Engineering. St. Louis, USA: Mira Digital Publishing.

Moghadam, A.S. \& Tso, W.K. (1996). Damage assessment of eccentric multistory buildings using 3D pushover analysis. In: Sociedad Mexicana de Ingeniería (ed.), Proceedings of the 11 $11^{\text {th }}$ World Conference on Earthquake Engineering. Paper 997. Oxford, UK: Pergamon.

Moghadam, A.S. \& Tso, W.K. (2000). Pushover analysis for asymmetric and set-back multi-story buildings. Proceedings of the $12^{\text {th }}$ World Conference on Earthquake Engineering. (pp. 1093-2001). Upper Hutt, New Zealand: New Zealand Society for Earthquake Engineering.

Moghadam, A.S. \& Tso, W.K. (1998). Pushover analysis for asymmetrical multi-story buildings. Proceedings of the $6^{\text {th }}$ U.S. National Conference on Earthquake Engineering. Oakland, USA: Earthquake Engineering Research Institute.

Mosavi, A.A., Sedarat, H., O’Connor, S.M., Emami-Naeini, A. \& Lynch, J. (2014). Calibrating a high-fidelity finite element model of a highway bridge using a multivariable sensitivity-based optimisation approach. Structure and Infraestructure Engineering: Maintenance, Management, Life-Cycle Design and Performance, 10(5), 627-642.

Mühlenbein, H., Schomisch, M. \& Born, J. (1991). The parallel genetic algorithm as function optimizer. Parallel computing, 17(6-7), 619-632.

Muttoni, A., Schwartz, J. \& Thürlimann, B. (1989). Bemessen und konstruieren von stahlbetontragwerken mit spannungsfeldern. [AK Reinforced Concrete Lecture]. Abteilung für Bauingenieurwesen, Institut für Baustatik und Konstruktion, ETH Zürich, Schweiz. (En alemán).

Neuenhofer, A. \& Filippou, F.C. (1997). Evaluation of nonlinear frame finite-element models. Journal of Structural Engineering, 123(7), 958-966. 
Nimtawat, A. \& Nanakorn, P. (2009). Automated layout design of beam-slab floors using a genetic algorithm. Computers \& Structures, 87, 1308-1330.

Nogueiro, P., Bento, R. \& da Silva, L.S. (2006). Evaluation of the ductility demand in partial strength steel structures in seismic areas using non-linear static analyses. Proceedings of the $11^{\text {th }}$ International Conference on Metal Structures. London, UK: Taylor \& Francis.

Novelli, V.I. (2008). The unloading stiffness of reinforced concrete members. [Master Degree in Earthquake Engineering \& Engineering Seismology]. Istituto Universitario di Studi Superiori di Pavia, Università degli Studi di Pavia, Italia.

Oesterle, R.G., Aristizabal-Ochoa, J.D., Fiorato, A.E., Russell, H.G. \& Corley, W.G. (1979). Earthquake resistant structural walls: tests of isolated walls. Phase II. Skokie, USA: Portland Cement Association.

Oftadeh, R., Mahjoob, M.J. \& Shariatpanahi, M. (2010). A novel meta-heuristic optimization algorithm inspired by group hunting of animals: hunting search. Computers \& Mathematics with Applications, 60(7), 2087-2098.

Oldfield, M., Ouyang, H. \& Mottershead, J.E. (2005) Simplified models of bolted joints under harmonic loading. Computers \& Structures, 84(1-2), 25-33.

Orakcal, K. \& Wallace, J.W. (2004). Modeling of slender reinforced concrete walls. In: Canadian Association for Earthquake Engineering (ed.), Proceedings of the $13^{\text {th }}$ World Conference on Earthquake Engineering. Paper 555. Vancouver, Canada: 13 WCEE Secretariat Publisher.

Osman, I.H. \& Kelly, J.P. (1996). Meta-Heuristics: theory and applications. Boston, USA: Kluwer Academic.

Østergaard, L. (2003). Early-age fracture mechanics and cracking of concrete. [PhD Thesis]. Department of Civil Engineering, Technical University of Denmark, Lyngby, Denmark.

Otani, S. (1974). Inelastic analysis of R/C frame structures. Journal of the Structural Division, 100(7), 1433-1449.

Otani, S. (1975). SAKE: a computer program for inelastic response of $R / C$ frames to earthquakes. [Structural Research Series 413]. College of Engineering, University of Illinois at Urbana-Champaign, USA.

Otani, S., Nagai, S. \& Aoyama, H. (1996). Load-deformation relationship of highstrength reinforced concrete beams. In: Wight, J.K. \& Kreger, M.E. (eds.), Proceedings of Mete A. Sozen Symposium: a tribute from his students. (pp. 35-52). Michigan, USA: American Concrete Institute. 
Ou, Y., Song, J., Wang, P., Adidharma, L., Chang, K. \& Lee, G. (2014). Ground motion duration effects on hysteretic behavior of reinforced concrete bridge columns. Journal of Structural Engineering, 140(3).

Ozcebe, G. \& Saatcioglu, M. (1989). Hysteretic shear model for reinforced concrete members. Journal of Structural Engineering, 115(1), 132-148.

Panagiotakos, T.B. \& Fardis, M.N. (2001). Deformations of reinforced concrete members at yielding and ultimate. ACI Structural Journal, 98(2), 135-148.

Park, R. \& Paulay, T. (1974). Reinforced concrete structures. London, UK: John Wiley \& Sons.

Park, Y. \& Ang, A. (1985). Mechanistic seismic damage model for reinforced concrete. Journal of Structural Engineering, 111(4), 722-739.

Paulay, T. \& Priestley, M.J.N. (1992). Seismic design of reinforced concrete and masonry buildings. New York, UK: John Wiley \& Sons.

Pecknold, D.A. (1974). Inelastic structural response to 2D ground motion. Journal of the Engineering Mechanics Division, 100(5), 949-963.

Peng, Q. \& Liu, W. (2013). Inverse analysis of related parameters in calculation of concrete drying shrinkage based on ANSYS design optimization. Journal of Materials in Civil Engineering, 25(6), 683-692.

Perdomo, M.E., Picón, R., Marante, M.E., Hild, F., Roux, S. \& Flórez-López, J. (2013). Experimental analysis and mathematical modelling of fracture in $\mathrm{RC}$ elements with any aspect ratio. Engineering Structures, 46, 407-416.

Petrangeli, M., Pinto, P. \& Ciampi, V. (1999). Fiber element for cyclic bending and shear of R/C structures. I: theory. Journal of Engineering Mechanics, 125(9), 9941001 .

Petschke, T., Corres, H., Ezeberry, J.I., Pérez, A. \& Recupero, A. (2013). Expanding the classic moment-curvature relation by a new perspective onto its axial strain. Computers and Concrete, 11(6), 515-529.

Pommerening, D. (1996). Ein beitrag zum nichtlinearen materialverhalten und zur schnittgrößenermittlung im massivbau. [PhD Thesis]. Technische Hochschule Darmstadt, Deutschland. (En alemán).

Poursha, M., Khoshnoudian, F. \& Moghadam, A.S. (2009). A consecutive modal pushover procedure for estimating the seismic demands of tall buildings. Engineering Structures, 31(2), 591-599.

Powell, G.H. (1972). Notes on inelastic dynamic analysis of tall buildings. Berkeley, USA: University of California. 
Powell, G.H. \& Chen, P. (1986). 3D beam-column element with generalized plastic hinges. Journal of Engineering Mechanics, 112(7), 627-641.

Prager, W. \& Hodge, P.G. (1951). Theory of perfectly plastic solids. New York, USA: John Wiley \& Sons.

Priestley, M.J.N. (1998). Displacement-based approaches to rational limit states design of new structures. In: Bisch, P., Labbé, P. \& Pecker, A. (eds.), Proceedings of the $11^{\text {th }}$ European Conference on Earthquake Engineering. (pp. 317-335). Rotterdam, Netherlands: Balkema.

Priestley, M.J.N. \& Park, R. (1987). Strength and ductility of concrete bridge columns under seismic loading. ACI Structural Journal, 84(1), 61-76.

Priestley, M.J.N., Seible, F. \& Calvi, G.M. (1996). Seismic design and retrofit of bridges. London, UK: John Wiley \& Sons.

Ramberg, W. \& Osgood, W.R. (1943). Description of stress-strain curves by three parameters. [Technical Note 902]. Washington D.C., USA: National Advisory Committee for Aeronautics.

Remino, M. (2004). Shear modelling of reinforced concrete structures. [PhD Thesis]. Dipartimento di Ingegneria Civile, Università degli studi di Brescia, Italia.

Rodrigues, H., Romao, X., Andrade-Campos, A., Varum, H., Arêde, A., \& Costa, A.G. (2012). Simplified hysteretic model for the representation of the biaxial bending response of RC columns. Engineering Structures, 44, 146-158.

Roelfstra, P.E. \& Wittmann, F.H. (1986). Numerical method to link strain-softening with failure of concrete. In: Wittmann, F.H. (ed.), Fracture toughness and fracture energy of concrete. (pp. 163-175). Amsterdam, Netherlands: Elsevier Science Publishers.

Roh, H., Reinhorn, A.M. \& Lee, J.S. (2012). Power spread plasticity model for inelastic analysis of reinforced concrete structures. Engineering Structures, 39, 48-161.

Rose, B.W. (2001). A constitutive model for the analysis of reinforced concrete beamcolumns subjected to lateral loads. [PhD Thesis]. Civil, Environmental and Architectural Engineering Department, University of Colorado, Boulder, USA.

Saatcioglu, M., Alsiwat, J. \& Ozcebe, G. (1992). Hysteretic behavior of anchorage slip in R/C members. Journal of Structural Engineering, 118(9), 2439-2458.

Saatcioglu, M. \& Ozcebe, G. (1988). Response of reinforced concrete columns to simulated seismic loading. ACI Structural Journal, 86(1), 3-12. 
Saatcioglu, M., Ozcebe, G. \& Lee, B.C.K. (1988). Tests of reinforced concrete columns under uniaxial and biaxial load reversals. [Technical Report]. Department of Civil Engineering, University of Toronto, Canada.

Saiidi, M. (1982). Hysteresis models for reinforced concrete. Journal of the Structural Division, 108(5), 1077-1087.

Saiidi, M. \& Sozen, M.A. (1979). Simple and complex models for nonlinear seismic response of reinforced concrete structures. [Structural Research Series SRS-465]. College of Engineering, University of Illinois at Urbana-Champaign, USA.

Saiidi, M. \& Sozen, M.A. (1981). Simple nonlinear seismic analysis of R/C structures. Journal of the Structural Division, 107(5), 937-953.

Salajegheh, E. \& Gholizadeh, S. (2012). Structural seismic optimization using metaheuristics and neural networks: a review. Computational Technology Reviews, 5, 109-137.

Saka, M.P. (2007). Optimum topological design of geometrically nonlinear single layer latticed domes using coupled genetic algorithm. Computers \& Structures, 85, 1635 1646.

Sasaki, K.K., Freeman, S.A. \& Paret, T.F. (1998). Multi-mode pushover procedure (MMP) - a method to identify the effects of higher modes in a pushover analysis. Proceedings of the $6^{\text {th }}$ US National Conference on Earthquake Engineering. (pp. 112). Oakland, USA: Earthquake Engineering Research Institute.

Sawyer, H.A. (1964). Design of concrete frames for two failure states. Proceedings of the International Symposium on the Flexural Mechanics of Reinforced Concrete, ASCE-ACI. (pp. 405-431).

Schlaich, J. \& Schäfer, K. (1984). Konstruieren im stahlbetonbau. Berlin, Deutschland: Verlag Wilhelm Ernst und Sohn K.G.

Scott, M.H. \& Fenves, G.L. (2006). Plastic hinge integration methods for force-based beam-column elements. Journal of Structural Engineering, 132(2), 244-252.

Searer, G.R. \& Freeman, S.A. (2004). Design drift requirements for long-period structures. In: Canadian Association for Earthquake Engineering (ed.), Proceedings of the $13^{\text {th }}$ World Conference on Earthquake Engineering. Paper 3292. Vancouver, Canada: 13 WCEE Secretariat Publisher.

Sengupta, P. \& Li, B. (2011). Hysteresis behavior of reinforced concrete non-ductile beam-column joints. In: Ma, Q. \& Deam, B. (eds.), Proceedings of the $9^{\text {th }}$ Pacific Conference on Earthquake Engineering Building and Earthquake-Resilient Society. Paper 109. Auckland, New Zealand: PCEE. 
Sengupta, P. \& Li, B. (2013). Modified Bouc-Wen model for hysteresis behaviour of RC beam-column joints with limited transverse reinforcement. Engineering Structures, 46, 392-406.

Setzler, E.J. (2005). Modeling the behavior of lightly reinforced concrete columns subjected to lateral loads. [M.S. Thesis]. The Ohio State University, Columbus, USA.

Sezen, H. \& Chowdhury, T. (2009). Hysteretic model for reinforced concrete columns including the effect of shear and axial load failure. Journal of Structural Engineering, 135(2), 139-146.

Sezen, H. \& Moehle, J.P. (2003). Bond-slip behavior of reinforced concrete members. In: Fedération Internationale du Béton (ed.), Proceedings of the Fib Symposium on Concrete Structures in Seismic Regions. (pp. 1-10). Athens, Greece: Technical Chamber of Greece.

Shakir, A. \& Rogowsky, D.M. (2000). Evaluation of ductility and allowable moment redistribution in reinforced concrete structures. Canadian Journal of Civil Engineering, 27(6), 1286-1299.

Shin, M. \& LaFave, J.M. (2004). Testing and modelling for cyclic joint shear deformations in RC beam-column connections. In: Canadian Association for Earthquake Engineering (ed.), Proceedings of the $13^{\text {th }}$ World Conference on Earthquake Engineering. Paper 0301. Vancouver, Canada: 13 WCEE Secretariat Publisher.

Sigrist, V. (1995). Zum verformungsvermögen von stahlbetonträgern. [PhD Thesis]. Institut für Baustatik und Konstruktion, Eidgenössische Technische Hochschule Zürich, Schweiz. (En alemán).

Soleimani, D. (1979). Reinforced concrete ductile frames under earthquake loadings with stiffness degradation. [PhD Thesis]. Civil Engineering Department, University of California, Berkeley, USA.

Soleimani, D., Popov, E.P. \& Bertero, V.V. (1979). Nonlinear beam model for R/C frame analysis. In: Technical Council on Computing and Information Technology (ed.), Proceedings of the $7^{\text {th }}$ ASCE Conference on Electronic Computation. New York, USA: American Society of Civil Engineers.

Spacone, E., Ciampi, V. \& Filippou, F.C. (1996). Mixed formulation of nonlinear beam finite element. Computers \& Structures, 58(1), 71-83.

Spacone, E., Filippou, F.C. \& Taucer, F.F (1996). A fiber beam-column model for nonlinear analysis of RC frames. Part I. Formulation. Earthquake Engineering \& Structural Dynamics, 25(7), 711-725. 
Starkweather, T., Whitley, D. \& Mathias, K. (1990). Optimization using distributed genetic algorithms. In: Schwefel, H.P. \& Männer, R. (eds.), Proceedings of Parallel Problem Solving from Nature. (pp. 176-185). Berlin, Germany: Springer-Verlag.

Statgraphics. (2013). [Software]. Version 16.2. Warrenton, USA: Statpoint Technologies, Inc.

Stavroulakis, G.E., Bolzon, G., Waszczyszyn, Z. \& Ziemianski, L. (2003). Inverse analysis. Comprehensive structural integrity (pp. 685-718). Oxford, UK: ELSEVIER Pergamon.

Taghavipour, S., Majid, T.A. \& Liang, L.T. (2013). Effect of different lateral load distribution on pushover analysis. Australian Journal of Basic and Applied Sciences, 7(4), 133-142.

Takayanagi, T., Derecho, A.T. \& Corley, W.G. (1979). Analysis of inelastic shear deformation effects in R/C structural wall systems. Proceedings of the Nonlinear Design of Concrete Structures, CSCE-ASCE-ACI-CEB International Symposium. (pp. 545-579). Ontario, Canada: University of Waterloo Press.

Takayanagi, T. \& Schnobrich, W.C. (1976). Computed behavior of reinforced concrete coupled shear walls. [Civil Engineering Studies SRS-434]. College of Engineering, University of Illinois at Urbana-Champaign, USA.

Takayanagi, T. \& Schnobrich, W.C. (1979). Non-linear analysis of coupled wall systems. Earthquake Engineering \& Structural Dynamics, 7(1), 1-22.

Takeda, T., Sozen, M.A. \& Nielsen, N.N. (1970). Reinforced concrete response to simulated earthquakes. Journal of the Structural Division, 96(12), 2557-2573.

Takizawa, H. \& Aoyama, H. (1976). Biaxial effects in modelling earthquake response of R/C structures. Earthquake Engineering \& Structural Dynamics, 4(6), 523-552.

Tanabashi, R. (1956). Studies on the non-linear vibrations of structures subjected to destructive earthquakes. In: Clough, R.W. (ed.), Proceedings of the World Conference on Earthquake Engineering.

Thomson, E., Bendito, A. \& Flórez-López, J. (1998). Simplified model of low cycle fatigue for RC frames. Journal of Structural Engineering, 124(9), 1082-1085.

Thomson, E. \& Flórez-López, J. (2004). A simplified damage model for shear dominated reinforced concrete walls under lateral forces. In: Canadian Association for Earthquake Engineering (ed.), Proceedings of the $13^{\text {th }}$ World Conference on Earthquake Engineering. Paper 844. Vancouver, Canada: 13 WCEE Secretariat Publisher. 
Thomson, E.D., Perdomo, M.E., Picón, R., Marante, M.E. \& Flórez-López, J. (2009). Simplified model for damage in squat RC shear walls. Engineering Structures, 31(10), 2215-2223.

Tian, M. \& Zhou, J. (2004). Inversing mechanical parameters of concrete gravity dams using ant colony optimization. In: Dorigo, M., Birattari, M., Blum, C., Gambardella, L.M., Mondada, F. \& Stützle, T. (eds.), Proceedings of the $4^{\text {th }}$ International Workshop, ANTS 2004. (pp. 358-365). Berlin, Germany: SpringerVerlag.

Tomás, A. \& Martí, P. (2010). Shape and size optimisation of concrete shells. Engineering Structures, 32(6), 1650-1658.

Tomás, A., Parra, C.J. \& Huesca, J.A. (2015). Edificaciones con estructura de hormigón. In: Frías, A. \& Guzmán, J.A. (eds.), Evaluación rápida de daños en emergencias. Protocolos de activación y actuación del grupo de evaluación de daños. (pp. 110-168). Murcia: Dirección General de Seguridad Ciudadana y Emergencias, Consejería de Presidencia y Empleo.

Topcu, I.B. \& Saridemir, M. (2008). Prediction of rubberized concrete properties using artificial neural network and fuzzy logic. Construction and Building Materials, 22, 532-540.

Torrenti, J.M., Pijaudier-Cabot, G. \& Reynouard, J.M. (2010). Mechanical behavior of concrete. London, UK: ISTE-John Wiley \& Sons.

Van Dorsselaer, N., Lapoujade, V., Nahas, G., Ciree, B., Tarallo, F. \& Rambach, J.M. (2013). LS-OPT parameters identification on concrete sample tests for an impact simulation on concrete slab. FEA Information Engineering Journal, 2(12), 4-11.

Vecchio, F.J. (2000). Disturbed stress field model for reinforced concrete: formulation. Journal of Structural Engineering, 126(9), 1070-1077.

Vecchio, F.J. \& Collins, M.P. (1988). Predicting the response of reinforced concrete beams subjected to shear using modified compression field theory. ACI Structural Journal, 85(27), 258-268.

Veletsos, A.S., Newmark, N.M. \& Chelapati, C.V. (1965). Deformation spectra for elastic and elastoplastic systems subjected to ground shock and earthquake motions. In: Van Roekel, J.H. (ed.), Proceedings of the Third World Congress on Earthquake Engineering. (pp. 663-682). Auckland, New Zealand: New Zealand National Committee on Earthquake Engineering.

Verderame, G.M., Ricci, P., Manfredi, G. \& Cosenza, E. (2010). Ultimate chord rotation of $\mathrm{RC}$ columns with smooth bars: some considerations about EC8 prescriptions. Bulletin of Earthquake Engineering, 8(6), 1351-1373. 
Villmann, B., Slowik, V., Wang, P. \& Wittmann, F.H. (2013). Infinitesimal shrinkage as determined by inverse analysis based on drying and shrinkage tests. In: FranzJosef, U., Hamlin, J.M. \& Pellenq, R.J.M. (eds.), Proceedings of the $9^{\text {th }}$ International Conference on Creep, Shrinkage and Durability Mechanics: a Tribute to Zdenek P. Bazant. (pp. 158-165). Reston, USA: American Society of Civil Engineers.

Villmann, B., Villmann, T. \& Slowik, V. (2004). Determination of softening curves by backward analyses of experiments and optimization using evolutionay algorithms. In: Li, V. (ed.), Proceedings of the $5^{\text {th }}$ International Conference on Fracture Mechanics of Concrete and Concrete Structures. (pp. 439-445). Vail, USA: FRAMCOS.

Voyiadjis, G.Z., Taqieddin, Z.N. \& Kattan, P.I. (2008). Anisotropic damage-plasticity model for concrete. International Journal of Plasticity, 24(10), 1946-1965.

Wang, M.L. \& Shah, S.P. (2006). Reinforced concrete hysteresis model based on the damage concept. Earthquake Engineering \& Structural Dynamics, 15(8), 993-1003.

Wen, Y.K. (1976). Method for random vibration of hysteretic systems. Journal of the Engineering Mechanics Division, 102(2), 249-263.

Xu, S.Y. \& Zhang, J. (2011). Hysteretic shear-flexure interaction model of reinforced concrete columns for seismic response assesment of bridges. Earthquake Engineering \& Structural Dynamics, 40(3), 315-337.

Yang, X.S. (2010). A new metaheuristic bat-inspired algorithm. Nature inspired cooperative strategies for optimization (NISCO 2010) (pp. 65-74). Berlin, Germany: Springer-Verlag.

Yang, X.S. (2009). Firefly algorithms for multimodal optimization. In: Watanabe, O. y Zeugmann, T. (eds.), Stochastic algorithms: foundations and applications. (pp. 169-178). Berlin, Germany: Springer-Verlag.

Yang, X.S. \& Deb, S. (2009). Cuckoo search via Lévy flights. In: Ajith, A., Carvalho, A., Herrera, F. \& Vijayalakshmi, P. (eds.), Proceedings of the World Congress on Nature \& Biologically Inspired Computing (NaBIC 2009). (pp. 210-214). New York, USA: Institute of Electrical and Electronics Engineers Publications.

Yang, X.S. \& He, X. (2013). Firefly algorithm: recent advances and applications. International Journal of Swarm Intelligence, 1(1), 36-50.

Yön, B. \& Calayir, Y. (2013). Pushover analysis of a reinforced concrete building according to various hinge models. Proceedings of the $2^{\text {nd }}$ International Balkans Conference on Challenges of Civil Engineering, BCCCE. 
Youssef, M. \& Ghobarah, A. (2001). Modelling of RC beam-column joints and structural walls. Journal of Earthquake Engineering, 5(1), 93-111.

Zahn, F.A. (1985). Design of reinforced concrete bridge columns for strength and ductility. [PhD Thesis]. Department of Civil Engineering, University of Canterbury, New Zealand.

Zeris, C. \& Mahin, S. (1988). Analysis of reinforced concrete beam-columns under uniaxial excitation. Journal of Structural Engineering, 114(4), 804-820.

Zeris, C. \& Mahin, S. (1991). Behavior of reinforced concrete structures subjected to biaxial excitation. Journal of Structural Engineering, 117(9), 2657-2673.

Zhang, J., Xu, S.Y. \& Tang, Y. (2011). Inelastic displacement demand of bridge columns considering shear-flexure interaction. Earthquake Engineering \& Structural Dynamics, 40(7), 731-748.

Zhou, Y., Ye, M., Yang, Z. \& Ou, L. (2011). Hysteresis analysis of RC columns based on fiber cross-section model. Advanced Materials Research, 255, 3770-3774.

Zienkiewicz, O.C. \& Taylor, R.L. (2000). The finite element method. Volume 1: the basis. Oxford, UK: Butterworth-Heinemann. 


\section{Publicaciones del doctorando relacionadas con esta tesis}

López, A.T., Tomás, A. \& Sánchez-Olivares, G. Section behaviour based on tests complying with seismic construction requirements. Computers and Concrete; artículo en revisión, remitido en octubre de 2013.

López, A.T., Tomás, A. \& Sánchez-Olivares, G. (2014). Formulation for the adjustment of RC-section behaviour to experimental tests. In: Bicanic, N., Mang, H., Meschke, G. \& de Borst, R. (eds.), Proceedings of EUROC 2014: Computational Modelling of Concrete Structures. (pp. 947-956). St. Anton am Arlberg, Austria. London, UK: Taylor \& Francis.

López, A.T., Tomás, A. y Sánchez-Olivares, G. (2014). Modelos de comportamiento último y de plastificación de secciones de hormigón ajustados con ensayos cumpliendo requerimientos constructivos y sísmicos. En: Asociación Científica del Hormigón Estructural (ed.), Actas del VI Congreso Internacional de Estructuras ACHE. Madrid, España: ACHE.

López-López, A.T., Tomás, A. y Sánchez-Olivares, G. (2014). Estimación del comportamiento de secciones de hormigón armado mediante calibración con ensayos experimentales. Dyna, 90(2), 210-218. 
López-López, A.T. \& Tomás, A. (2015). Nonlinear seismic analysis of concrete buildings considering different models of plastic hinges. Proceedings of the $3 \mathrm{rd}$ International Conference on Mechanical Models in Structural Engineering. Comunicación aceptada. Sevilla, España.

López, A.T., Tomás, A. \& Sánchez-Olivares, G. Behaviour of reinforced concrete sections based on tests complying with seismic construction requirements. Engineering Structures; artículo en revisión, remitido en abril de 2015.

López, A.T. \& Tomás, A. Influence of adjusted models of plastic hinges in nonlinear behaviour of reinforced concrete buildings. Computers and Structures; artículo en revisión, remitido en mayo de 2015. 